Every day, scientists and engineers at Argonne National Laboratory conduct transformational research that has lasting impacts on society. They do this by leveraging the laboratory's broad capabilitiesin materials discovery, chemistry, physics and biology, engineering of advanced energy systems, computation and analysisand world-renowned scientific user facilities. 


\section{DIRECTOR'S MESSAGE}

Laboratory Directed Research and Development (LDRD) at Department of Energy (DOE) laboratories creates new program opportunities for the Department and enhances the labs' ability to support the Department's multiple mission areas. This report on fiscal year 2017 LDRD program activities at Argonne National Laboratory demonstrates the value and productivity of LDRD and is presented for Department review. LDRD-supported research projects represent Argonne's commitment to delivering high impact science and building foundations for technologies that will shape our nation's future.

Reports on projects aligned to each of DOE's critical mission areas are included in this compilation. In all cases, LDRD has been central to Argonne establishing research efforts that mature as part of anticipated growth areas for the laboratory and DOE. Most of Argonne's major program initiatives, as well as many of our user facilities, began as innovative concepts granted LDRD funding. They have grown into critical resources for delivering world-class research in the basic energy sciences and beyond.

As Argonne looks to the future, we use our LDRD program to align our research investments to our strategic initiatives. Our LDRD commitments also are designed to attract the best minds from across the world to join our science and engineering teams. As you will see in this year's report, Argonne LDRD-funded research includes advanced computing, novel materials synthesis, and detectors and imaging research spanning from the single atom to the universe. Just as LDRD has supported past exploratory research leading to the Advanced Photon Source, Argonne Leadership Computing Facility and Joint Center for Energy Storage Research, we are confident that in these pages are the beginnings of our lab's next major scientific facilities and programs.

I attest that in managing the LDRD program, Argonne has adhered without exception to the requirements of DOE Order $413.2 \mathrm{C}$ and associated guidelines. Our program management operates with a strategy of continuous quality improvement. While meeting all reporting requirements throughout FY 2017, the LDRD Program Office implemented process enhancements that keep LDRD closely aligned to our strategic initiatives and better serve all stakeholders.

I am pleased to deliver this report on our LDRD FY 2017 activities, as it demonstrates how Argonne's exemplary research, operations, and people support our strategic initiatives and, in turn, the strategic initiatives of DOE.

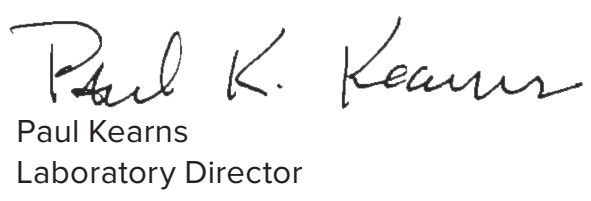




\section{LABORATORY OVERVIEW}

Argonne National Laboratory, as a U.S. Department of Energy (DOE) national laboratory, is dedicated to science that changes the world. Building on our proud history of discovery and innovation, we seek to be known for our ideas and for safely delivering lasting impact on society through our exemplary research and operations.

Argonne accelerates innovation in science and technology for its primary sponsor, the DOE Office of Science (SC), as well as for DOE's Office of Energy Efficiency and Renewable Energy (EERE), DOE's National Nuclear Security Administration (NNSA), and a number of other federal agencies.
As a national laboratory, Argonne concentrates on scientific and technological challenges that can be addressed only through sustained, interdisciplinary focus at a national scale. Argonne's five major initiatives, as described in its laboratory plan, are Hard X-Ray Sciences, Advanced Computing, Materials and Chemistry, Manufacturing Science and Engineering, and Universe as Our Lab. 


\section{TABLE OF CONTENTS}

PROGRAM OVERVIEW V v

LDRD MANAGEMENT PROCESS vi vi vis

FISCAL YEAR 2017 LDRD PROGRAM COMPONENTS vii

RESEARCH REPORTS BY COMPONENT

LDRD Innovate 1

LDRD Swift 30

LDRD Named Fellows

LDRD Prime

Applied Energy and Sustainable Transportation 75

Biological and Environmental Science Capability Development 86

Hard X-ray Sciences $\quad 94$

Materials and Molecules to Manufacturing 105

$\begin{array}{ll}\text { National and Global Security } & 118\end{array}$

Next Generation Computing 124

$\begin{array}{ll}\text { Universe as a Lab (ULab) } & 140\end{array}$

$\begin{array}{ll}\text { Other Novel R\&D } & 144\end{array}$

$\begin{array}{ll}\text { PROGRAM ASSESSMENT } & 159\end{array}$

$\begin{array}{ll}\text { Performance Metrics } & 160\end{array}$

$\begin{array}{ll}\text { LDRD Project Characteristics } & 162\end{array}$

$\begin{array}{ll}\text { APPENDIX } & 163\end{array}$

Intellectual Property Events Occuring in FY 2017

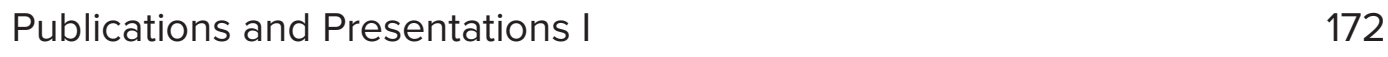

Publications and Presentations II 222

$\begin{array}{ll}\text { Author Index } & 238\end{array}$ 
PROGRAM OVERVIEW 


\section{PROGRAM OVERVIEW}

Argonne's Laboratory Directed Research and Development (LDRD) program encourages the development of novel technical concepts, enhances the Laboratory's research and development (R\&D) capabilities, and enables pursuit of strategic laboratory goals.

Argonne's LDRD projects are proposal based and peer reviewed, supporting ideas that require advanced exploration so they can be sufficiently developed to pursue support through normal programmatic channels. Among the aims of the projects supported by the LDRD program are the establishment of engineering proofs of principle, assessment of design feasibility for prospective facilities, development of instrumentation or computational methods or systems, and discoveries in fundamental science and exploratory development.

The projects supported by the LDRD program are distributed across the major mission areas at Argonne, as indicated in the Laboratory's LDRD Plan for fiscal year (FY) 2017. All LDRD projects have demonstrable ties to one or more of the science, energy, environment, and national security missions of the U.S. Department of Energy (DOE) and its National Nuclear Security Administration (NNSA), and many are also relevant to the missions of other federal agencies that sponsor work at Argonne. A natural consequence of the more "applied" types of projects is their concurrent relevance to industry.
In addition to their relevance to strategic and programmatic activities, LDRD projects readily align with science and technology areas where Argonne's technical staff are trained and excel in supporting existing programs. The reports in this document illustrate the expertise that is often drawn from multiple technical areas to pursue the goals of each project. This multidisciplinary approach is one of Argonne's hallmarks, and reflects the synergistic manner in which both LDRD and programmatic work are pursued.

The FY 2017 DOE approved funding cap for LDRD was $\$ 36.0$ million, or about 4.8\% of Argonne's estimated FY 2017 operating plus capital budgets. Actual expenditure amounted to $\$ 33.0$ million, which includes an overhead levy of approximately $30 \%$.

Individual brief project reports for FY 2017 are the focus of this document. 


\section{LDRD MANAGEMENT PROCESS}

Argonne's R\&D activities are organized under four Associate Laboratory Directors (ALDs), who oversee the research conducted in some 16 programmatic divisions. Responsibility for management and oversight of LDRD during FY 2017 resides with the Deputy Laboratory Director for Science and the Director for Strategy, Performance and Risk (SPR). This senior management team makes final recommendations to the Laboratory Director concerning the LDRD program investment and management.

As described below, Argonne's LDRD program has consisted of the following components: LDRD Innovate, LDRD Named Fellows, LDRD Swift, and LDRD Prime. All LDRD projects under all components align with DOE mission areas and core competencies of the Laboratory. The LDRD Prime and Named Fellows projects are also explicitly aligned with initiatives within Argonne's Strategic Plan, whereas LDRD Innovate and Swift projects may pursue more wide-ranging exploratory research. Every year, the Laboratory Director determines the appropriate balance of funding among the various components. In addition, a Director-Designated Exceptional Opportunities fund (a portion of the total allowed expenditure held in reserve to handle midyear opportunities, strategic hires for LDRD projects, and LDRD collaborative arrangements) may support projects within any of the LDRD components. All final decisions concerning Argonne's LDRD program reside with the Laboratory Director. Certain responsibilities regarding funding, oversight, proposal evaluation, and project direction are delegated.

The LDRD Program Office reviews all proposals to ensure their adherence to DOE Order 413.2C and associated guidelines, as well as to Laboratory administrative procedures.

The LDRD program is funded lab wide through the Laboratory's indirect budget, which derives from a levy against all program operating and equipment budgets. Although two or three year durations are proposed for many projects, funding levels and project selection are determined annually and based on technical progress and the Laboratory's strategic goals and resources.
LDRD funds may be used in collaborative arrangements with third parties to enhance the application or scope of the research, with attendant beneficial interactions. To effect these arrangements, the Laboratory has in place DOE approved procedures and instruments, such as Cooperative Research and Development Agreements (CRADAs), subcontracts, and site access Guest Agreements. These instruments and procedures enable the application of LDRD funds while ensuring that appropriate terms and conditions are applied thereto, including those related to intellectual property protection.

Line managers in Argonne's programmatic divisions are responsible for monitoring the progress and performance of LDRD research during execution. LDRD Prime Focus Area contacts, line managers, and subject matter experts confirm that midyear progress reviews have occurred and indicate whether any remedial action or advice is needed. The responsibility for the actual conduct of all LDRD projects, including associated environmental safety and health requirements, resides with the ALDs and their line managers.

During FY 2017, all program management requirements were satisfied. Reports required by DOE, including the Annual Report for FY 2016, the LDRD Program Plan for FY 2018, and project data uploaded to the DOE CFO (Office of the Chief Financial Officer) database, were submitted completely and in a timely fashion.

Concurrence request forms (also referred to as "data sheets" in relevant guidance documents) were submitted electronically for each FY 2017 LDRD project to DOE's Argonne Site Office (ASO) to obtain the DOE Site Manager's concurrence before work on the project proceeded. 


\section{FISCAL YEAR 2017 LDRD PROGRAM COMPONENTS}

\section{LDRD INNOVATE}

The LDRD Innovate component invests in a full spectrum of investigator initiated proposals across the Laboratory in DOE mission related science and engineering areas. This component provides an avenue for R\&D staff to propose highly innovative projects in research areas outside the purview of the Prime Focus Areas. Proposals are subjected to peer review by the LDRD Innovate Advisory Board to assess their scientific and technical quality. The board is composed of scientists and engineers who have substantial expertise in their technical fields, are familiar with the peer review process, and represent a broad cross section of the Laboratory.

The Advisory Board conducts a two-stage review process for all LDRD Innovate project proposals. An initial peer review of one-page pre-proposals results in a smaller number of proposals that advance for further review. The second stage consists of a more extensive peer review conducted by the Advisory Board. The Advisory Board also solicits proposals for continuation of a project into a second or third year. Proposals for continuation are assessed according to progress made against previously proposed milestones. A list of proposals recommended for funding are then given to the Laboratory Director for selection and approval.

The LDRD Innovate review process for new proposal submissions is supported by the Laboratory's research community, which serves as a source of volunteer subject matter experts to help the Advisory Board review proposals. Brief critiques of proposals are sent to the proposing investigators based on the review feedback.

\section{LDRD SWIFT}

The LDRD Swift component provides an avenue for R\&D staff to conduct short-term research with a targeted funding opportunity in mind, as well as a means for researchers to explore ideas before developing a full proposal. Projects funded through this component area have a maximum one-year duration. As with the LDRD Innovate component, the LDRD Swift component invests in a full spectrum of proposals across all mission-related science and engineering areas.

Proposals are evaluated based on the targeted funding opportunity, the potential return on investment, and the urgency of the funding request. The preliminary review is conducted by the LDRD Program Office. Those proposals meeting these minimum criteria are then subjected to peer review to assess their scientific and technical merit. A list of proposals is then provided to the Laboratory Director for consideration for funding.

\section{LDRD NAMED FELLOWS}

The LDRD Named Fellows component aims to support the scientific or engineering research of exceptional early career scientists and engineers. Working with an Argonne sponsor (a senior member of the research staff), LDRD Named Fellows carry out work that is either at the forefront of new research areas or is synergistic with current research efforts.

There is a Named Fellows Review Committee in place to assess the scientific and technical merit of each proposal. The Committee is composed of one member from each technical division. The outcome of this review process is a list of finalists provided to the Laboratory Director for final approval.

\section{LDRD PRIME}

The largest component of Argonne's program is LDRD Prime, which emphasizes R\&D explicitly aligned with the Laboratory's major initiatives as defined by senior management (the Laboratory Director, Deputy Laboratory for Science, and ALDs) in support of Argonne's strategic plan. Strategic goals are reevaluated periodically and revised as necessary.

The choice of Focus Areas under the LDRD Prime component for FY 2017 reflects the major initiatives; the state of development of relevant technical fields; the potential value of advancing those fields to DOE/ NNSA and the nation; and the compatibility of the fields with existing facilities, capabilities, and staff expertise at Argonne.

For FY 2017, the individual ALD organizations issued a preliminary call to Laboratory staff for LDRD Prime concept papers in the individual Focus Areas.

Researchers seeking funding under this component of the program were encouraged to limit the duration of their proposed projects to 24 months, although exceptions could be made for a third year of funding if necessary.

Informed by reviews of the concept papers, a subsequent invitation for full proposal submission produced a welldefined portfolio of strategic research proposals. Each 
LDRD Prime proposal undergoes an extensive review process by committees impaneled for that purpose. Prioritized results are then assembled by the LDRD Program Office and provided to the Laboratory Director who makes final funding decisions. Some projects topically associated with an LDRD Prime Focus Area, as well as other innovative projects, were reviewed and selected by the Laboratory Director outside the normal annual LDRD call for proposals and review cycle.

The selected FY 2017 Focus Areas capture the Laboratory's capabilities and fundamental knowledge in areas of strategic importance and address the future mission needs of the Laboratory and DOE/NNSA. Our work in high-performance computing, X-ray science, and R\&D on detectors and sensors exemplifies crosscutting capabilities that contribute to a broad spectrum of Focus Areas. In addition, integration of basic and applied approaches is often an important feature of the LDRD projects selected for support under this program component.

These Focus Areas are described below.

\section{APPLIED ENERGY AND SUSTAINABLE TRANSPORTATION}

Key topics include sustainable transportation, the water-energy nexus, advanced manufacturing, and renewable energy.

\section{BIOLOGICAL AND ENVIRONMENTAL SCIENCE CAPABILITY DEVELOPMENT}

Key topics include accelerating protein function determination, next-generation climate research facility, and systems science, specifically the application of systems science to urban science and laying the foundation for a next-generation ecological experiment for urban climate.

\section{HARD X-RAY SCIENCES}

Key topics include research and development in support of the Advanced Photon Source (APS) upgrade project, science enablers, and future hard X-ray sources.

\section{MATERIALS AND MOLECULES TO MANUFACTURING}

Key areas include materials and molecules design and discovery (M2D2), biologically based materials, computational chemistry and materials (CCM), the integrated imaging initiative $\left(\mathrm{I}^{3}\right)$, and manufacturing.

\section{NATIONAL AND GLOBAL SECURITY}

Key topics include the science of secure and resilient infrastructure and infrastructure modeling and simulation.

\section{NEXT GENERATION COMPUTING}

Key topics include (1) creating the capability to integrate advanced computing with the APS to create the world's first light source that is tightly coupled to a world-class supercomputing system; (2) continuing the push for new computational science and engineering applications that will transform scientific research; (3) developing new computational approaches to problem solving that integrate deep learning, scalable data analytics or machine learning into scientific workflows; and (4) advancing new computational models and architectures that may become more important in the post-Moore's Law era such as approximate computing, neuromorphic computing, and quantum computing.

\section{NUCLEAR ENERGY SCIENCE AND TECHNOLOGY}

Key topics include increased understanding of nuclear energy phenomena and behaviors, development of advanced technologies and concepts, and nuclear energy system modeling and analysis.

\section{UNIVERSE AS A LAB (ULAB)}

Key topics include cosmic microwave background (CMB); and cosmology/particle theory, data analysis, and computation. 


\section{RESEARCH REPORTS BY COMPONENT}

LDRD Innovate

2015-078-R2 Josephson Plasma Wave-Based Ultra- High-Frequency Electronics ............................................... 2

2015-091-R2 Next-Generation Natural Gas Adsorbent through Rational Design and Modeling .............................3

2015-096-R2 Understanding Atomic-Scale Uranium Interactions under Severe Accident Conditions ..................4

2015-121-R2 Development of Advanced $\mathrm{VO}_{2}$ Nano-Composite Thermochromic Materials for High-

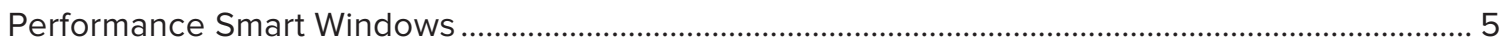

2016-001-R1 Structure and Dynamics of Chiral Molecules and Radicals .............................................................. 6

2016-010-R1 A Theory of Out-of-Equilibrium Phase Transitions ................................................................................

2016-020-R1 Nano-Mechanical Delivery of Biomolecules into Live Bacterial Cells ............................................... 8

2016-023-R1 Real-Time Monitoring of Material Structure Evolution in Additive Manufacturing Processes ......... 9

2016-027-R1 A Novel Gas-Filled Microchannel Plate (GF-MCP) X-ray Polarimetry Imager ....................................10

2016-048-R1 A Missing Protein in the Bacterial Methylmercury Pathway ................................................................11

2016-054-R1 Perovskite Halide-Based Intermediate-Band Solar Cells..................................................................12

2016-063-R1 Efficient Droplet-Based Environmental Mechanical Energy Harvesting through Reverse Electrowetting ........................................................................................................ 13

2016-069-R1 GO-IN-EM - Genetic algorithm Optimization of INterface structure from Electron Microscopy ....14

2016-082-R1 Top-Down Fabrication of Large Area Monolayers of 2D Materials ......................................................15

2016-092-R1 Spin-Vortex-Based Non-Volatile Superconducting Memory................................................................16

2016-094-R1 Ordered Core-Shell Nanostructure for Transverse Thermoelectric Applications .............................17

2016-098-R1 Images from Inner Space: Exposing Quantum Mechanics within Nucleons and Nuclei ..................18

2017-002-NO Developing Superconducting $\mathrm{MgB}_{2}$ Films on Copper Radio-Frequency

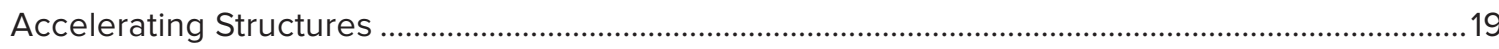

2017-004-NO The Missing Link in XTIP: Synergy of Experiments and Theory for Argonne's

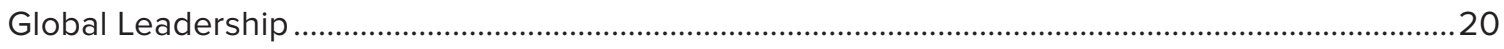

2017-007-NO A Novel Method of Longitudinal Bunch Shaping by Double-Emittance Exchange .........................21

2017-012-NO Integrating High-Throughput Computation and Wet-Chemistry Synthesis for

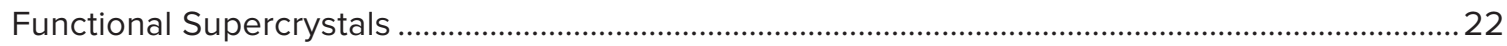

2017-013-NO Atomic Layer Deposition of Silicon Carbide for Nuclear Applications ................................................23

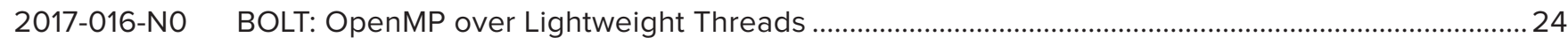

2017-017-NO A Continuously Refinable Mesh, Limited-Area Atmospheric Model .................................................25

2017-019-NO Bonding Dissimilar Materials Using Nanoparticles/Nanofilm as Eutectic Compounds ..................26

2017-022-NO Engineered Interfaces for Gallium Oxide Power Semiconductor Devices......................................... 27 
2017-023-NO

2017-026-NO

LDRD Swift

2016-240-R1

2017-098-NO

2017-099-NO

2017-100-NO

2017-101-NO

2017-102-NO

2017-103-NO

2017-107-NO

2017-108-NO

2017-109-NO

2017-110-NO

2017-113-NO

2017-116-NO

2017-118-NO

2017-131-NO

2017-137-NO

2017-141-NO

2017-152-NO

2017-154-NO

2017-159-NO

2017-160-NO

2017-161-NO

2017-162-NO

2017-163-NO

2017-164-NO

2017-165-NO
New Techniques to Manipulate Rare Isotopes Using Adaptive Optics 28

Realizing a Gate-Tunable Kinetic Inductance for a Transmon Qubit Using $\mathrm{SrTiO}_{3}$. 29

Recovery of Critical Materials from Post-Consumer Electronics 31

X-ray Investigation of the Potential of Pressure-Assisted Atomization Technology

for Medical Inhaler Sprays

Toward the Reduction of U.S. Petroleum Consumption by Fueling Light-Duty Engines

with Propane .33

High-Entropy Alloys for Advanced Nuclear Reactors .34

Enhancing Computational Tools for Polynomial Optimization Problems Relevant to Networked Systems. .35

Multiscale Modeling to Understand Ion-Diffusion-Induced Degradation in Photovoltaics. 36

Application of Combined Rheology and X-ray Scattering to the Paint Industry for Optimizing the Formulation Process 37

Building a Battery Recycling Model within GREET and BatPaC .38

Validating Replicability of Waggle Urban Deployments .39

Foxhunt Proof-of-Concept Demonstration 40

Argonne Extended Range Friction Tribometer..... 41

Ultra-High Efficiency Fuel Cell-Heat Engine Hybrids 42

Stretchable Conductive Ink for Energy Harvesting Device .43

Improvement of in situ Nuclear Fuel Characterization Capability at APS .44

Argonne's UAS Capability: Application Framework Development and Benchmarking .45

Accurate, Real-Time Categorization of Unmanned Aircraft Systems (UASs)

in an Urban Environment through Application of Deep Learning Strategies Leveraging Distributed Computing Technology. 46

Applying Community Network Analysis to Generalize Microbial Assembly Rules 47 Immersive 3D Visualization of Military Complex Systems 48

Tribology of Graphene at Elevated Temperatures. 49

Production of Cellulose Nanocrystals from Miscanthus x Giganteus .50

Neuromorphic Architectures for Dynamic and Reactive Learning Based on Neuromodulation....51 A Mechanically Based Antenna for RF-Incompatible Environments .52

Novel Gallium Oxide Devices for Microwave and RF Technology .53

Biofilms and Human-Made Surfaces: How Microorganisms Attach to and Influence the World around Us. .54

Simulating the Dynamics of Gene Drive Propagation through Populations... .55

Global Survey of CRISPR-CAS Systems in Archaeal and Bacterial Species. 56 


\section{LDRD Prime - Named Fellows}

2016-180-R1 Substitutional Growth of Lead Halide Perovskites for Solar Cells ..................................................58

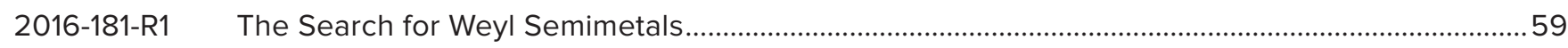

2016-183-R1 Understanding and Controlling Charge, Spin, Pseudospins, and Lattice Degrees

of Freedom in Layered Transition Metal Dichalcogenides .................................................................60

2016-184-R1 Investigation of Solid-Liquid Interfaces in Energy Materials: Interfacing Multi-Scale

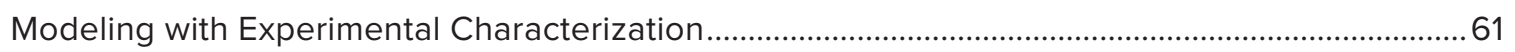

2016-185-R1 Charge Transport in Nanostructured Materials from ab initio Simulations .....................................62

2016-186-R1 Ultrafast Spectroscopy of Nanometer-Scale Heterojunctions Fabricated by Self-Assembly ........ 63

2016-187-R1 Coherent X-ray Investigations of Defect Dynamics in Next-Generation

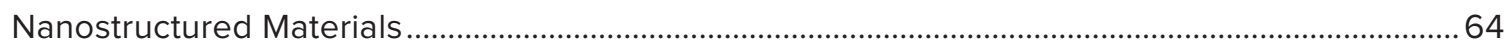

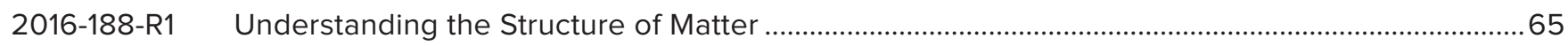

2017-029-NO Novel Devices and Systems for Neuromorphic Computing .............................................................66

2017-031-NO Ecological Organic Photovoltaics Using Water-Borne Semiconductor Nanoparticles.....................67

2017-093-NO Beehive: A Dynamic Execution Environment for Performance, Power, and Resilience

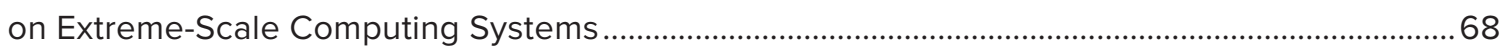

2017-094-NO Microstructural Simulations of Stable, Conjugated Polymer Glasses................................................69

2017-095-NO Combining Electrochemistry and Ultrafast Spectroscopies: Real-Time Characterization of Multi-Electron/Proton Intermediates in Hydrogen- and Oxygen-Evolving Catalysts .................. 70

2017-096-NO On the Colloidal Suspension of Lithium Clusters in Molten Lithium Chloride.................................... 71

2017-097-NO A Universal Data Analytics Platform for Science .............................................................................. 72

2017-157-NO Transport and Photophysical Processes in Hybrid Perovskites for Energy Applications ................ 73

2017-158-NO Real-Time Control of Urban Drainage Systems................................................................................ 74

\section{LDRD Applied Energy and Sustainable Transportation}

2015-151-R2 Chemical Vapor Processing for Additive Manufacturing .................................................................... 76

2015-157-R2 Sustainable Transportation: Novel Bio-Derived Fuel Additives for Improved Vehicle Efficiency ..77

2015-159-R2 Large-Scale Modeling and Simulation for an Adaptive and Resilient Power Grid............................. 78

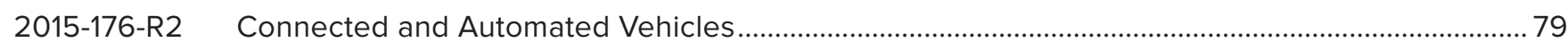

2016-126-R1 Advanced Control Algorithms for Improving Energy Consumption of Connected

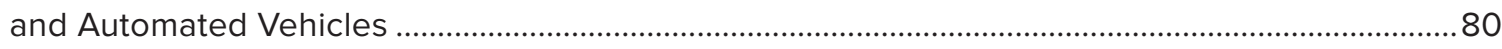

2016-152-R1 Integrated Water-Energy Systems Assessment Framework (IWESAF) for Water-Energy

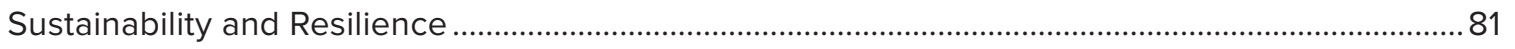

2016-175-R1 VERIFI 2.0: Next-Generation Engine/Fuel Simulation Codes ............................................................82

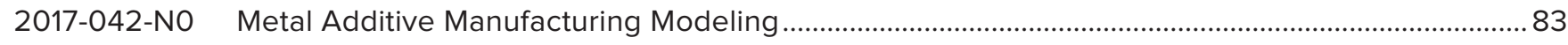

2017-055-NO Improving Cost and Energy Efficiency of Nontraditional Water Desalination through Innovative Material and Process Integration ........................................................................... 84

2017-084-NO Advancing Additive Manufacturing of Metal Alloys: From Fundamental Principles to Durable Components .......................................................................................................................... 85 


\begin{tabular}{|c|c|}
\hline $15-171-R 2$ & 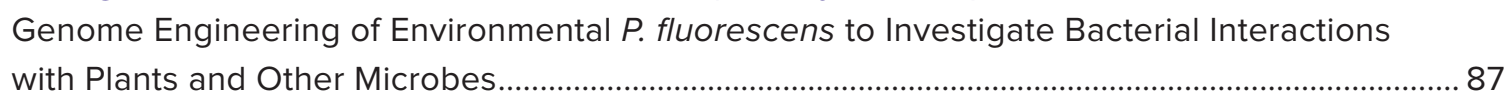 \\
\hline 15-179-R2 & 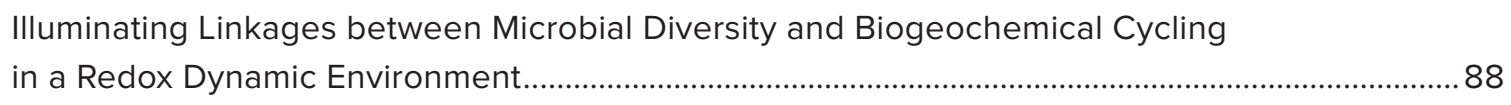 \\
\hline $15-180-R 2$ & $\begin{array}{l}\text { Functional Analysis of Proteins from a Key Signaling Network Involved } \\
\text { in Plant-Growth-Promoting Bacteria .................................................................... }\end{array}$ \\
\hline $16-139-R 1$ & Models to Observations, a Digital Atmospheric Library (MODAL)............ \\
\hline $16-165-R 1$ & Establishing a Proof-of-Concept for Protein Function Discovery Initiative ............ \\
\hline 2017-051-NO & Integrating Atmospheric, Ecological, and Biogeochemical Monitoring in Wetlands ........................92 \\
\hline 017-087-NO & $\begin{array}{l}\text { Linking Climate to Water: Implementing a 4-km Regional Climate Model } \\
\text { with Hydrologic Model Coupling (WRF-Hydro) Using Argonne's HPC Resources...... }\end{array}$ \\
\hline \multicolumn{2}{|c|}{ LDRD Prime - Hard X-ray Sciences } \\
\hline 2015-147-R2 & $\begin{array}{l}\text { Development of a Compact 352-MHz/12-kW CW Solid State RF Power Amplifier } \\
\text { System for Accelerators }\end{array}$ \\
\hline 2015-150-R2 & $\begin{array}{l}\text { Unraveling Mesoscale Spatial-Temporal Correlations in Materials Using Coherent } \\
\text { X-ray Probes }\end{array}$ \\
\hline 2015-153-R2 & The Velociprobe: Ultra-High-Resolution Ptychographic Hard X-ray Nanoprobe............ \\
\hline 2015- & 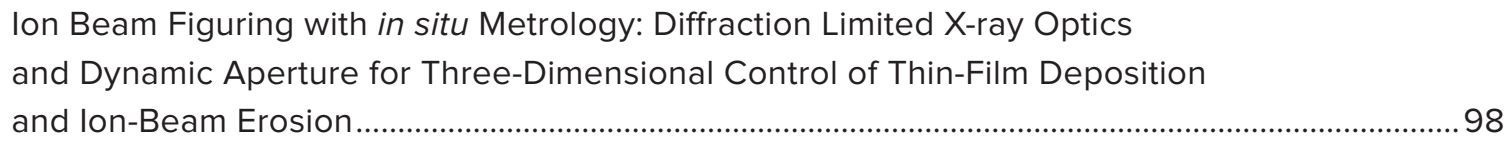 \\
\hline 2016-150-R1 & A Conveyer Belt of Nanoliter to Picoliter Droplets for Hard X-ray Pump-Probe Experiments .......99 \\
\hline 2017-028-NO & nducting Undulator. \\
\hline 2017-073-NO & 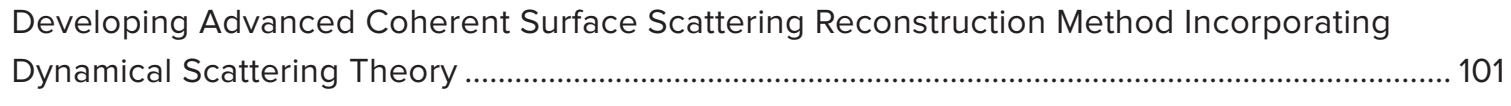 \\
\hline 2017-080-NO & 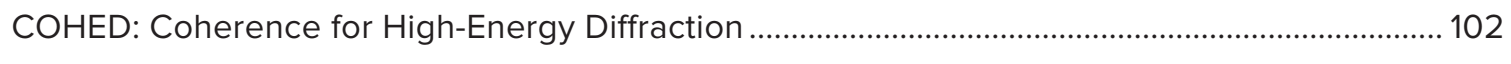 \\
\hline 2017-091-NO & celerator for a High-Repetition Rate Free-Elec \\
\hline 2017-153-NO & Soft Matter Visualization and Characterization by Electron Optical Beam Lines ..............................104 \\
\hline \multicolumn{2}{|c|}{ LDRD Prime - Materials and Molecules to Manufacturing } \\
\hline 2014-191-R3 & Defect-Localized Spins in Semiconductors for Quantum Optc \\
\hline 2014-192-R3 & Computational Spectroscopy of Heterogeneous Interfaces...... \\
\hline 2016-131-R1 & 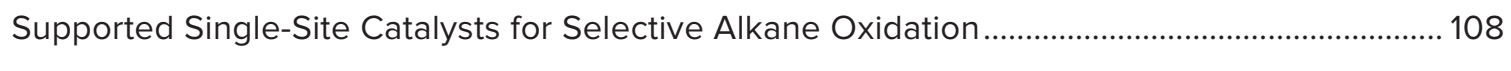 \\
\hline 2016- & naging Emission and Thermal Absorption (META). \\
\hline 2017-032-NO & Catalysts Modeled after Nature's Enzymes \\
\hline 2017-033-NO & s for the Energy-Water Nexus.... \\
\hline & tures with Ultra-S \\
\hline
\end{tabular}




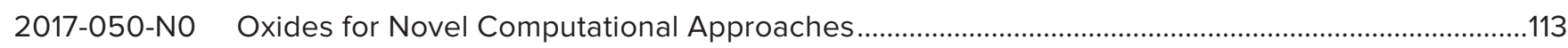

2017-061-NO Toward an Artificial Neuron - Non-Covalent Synaptic Assemblies ....................................................114

2017-082-NO Developing Hierarchical Multi-Functional Hybrid Polymer-Protein Structures

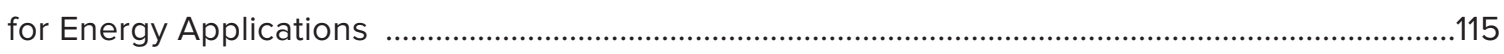

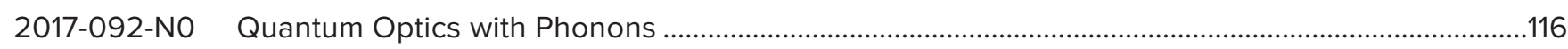

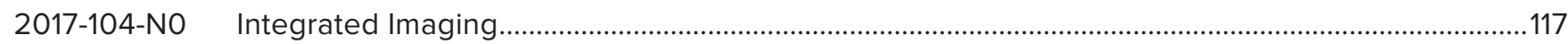

LDRD Prime - National and Global Security

2016-123-R1 New ${ }^{6}$ Li-Rich Semiconductors for Neutron Detection........................................................................119

2016-136-R1 A Novel Interferometric Terahertz Phase Imager (THz-PI) for National Security Applications .... 120

2016-173-R1 Fabrication and Testing of a Borosilicate Microchannel Plate Thermal Neutron Detector with Optimized Geometry ...............................................................................................................121

2017-076-NO Miniaturized High-Efficiency RF Energy Harvesting ........................................................................ 122

2017-088-NO Understanding Resilient Infrastructure Dependencies and Interdependencies through Advanced Optimization and Simulation ..............................................................................123

LDRD Prime - Next Generation Computing

2016-135-R1 Event-Based Monte Carlo Transport Methods for Next-Generation Node Architectures ............ 125

2016-148-R1 Re-form: Leveraging FPGA Reconfigurability and Floating-Point Capabilities for Next-Generation Computing Systems....................................................................................... 126

2016-157-R1 Data-Driven Multiscale Coupled Urban Systems Modeling ….........................................................127

2017-034-NO The Perfect Thermodynamics of Imperfect Materials ..........................................................................128

2017-049-NO SLIK-D: Scalable Machine Learning Infrastructure for Knowledge Discovery ................................ 129

2017-057-NO An Exascale Application for Simulating Urban Boundary Layers......................................................130

2017-063-NO Enabling Multidimensional X-ray Nano-Tomography .......................................................................131

2017-066-NO End-to-End Genome Annotation and Phenotype Prediction with Deep Learning …......................132

LDRD Prime - Nuclear Energy Science and Technology

2016-140-R1 Development of Analysis Methods for Non-Destructive Evaluation of Concrete Degradation in Light Water Reactors...................................................................................................134

2016-158-R1 The Production of Medical Isotopes Using the Argonne Electron LINAC ........................................135

2016-159-R1 Additive Manufacturing for Nuclear Energy Applications................................................................136

2017-030-NO Conceptual Design of a Flexible Spectrum Test Reactor (FSTR)....................................................137

2017-040-NO Development of Molten Salt Reactor Analysis Computation Tools

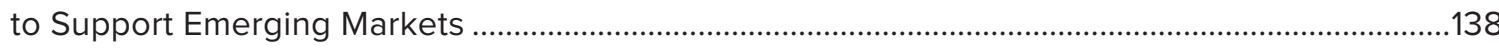

2017-041-NO Technological Improvement to Increase Scalability of Ex-Vessel Melt Coolability and Concrete Interaction Experiments 
LDRD Prime - Universe as a Lab (ULab)

2017-058-NO A Strategic Scientific Program to Establish Argonne Leadership in the Development of the Future Electron-Ion Collider. 141

2017-105-NO Superconducting Detectors for Future CMB Experiments …........................................................142

2017-106-NO Exploring the Universe: Large-Scale Structure to the First Stars ...................................................143

Other Novel R\&D

2015-168-R2 The Computational Design of New Functional Materials from Complex Transition Metal Oxides .145

2015-169-R2 Agent-Based Behavioral Modeling of Ebola Spread in Chicago and Other Large

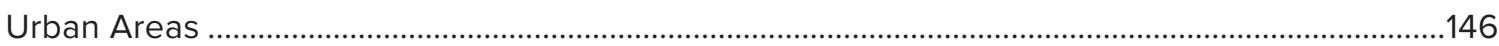

2015-170-R2 Biomimetic Approaches for Water-Smart Landscapes …....................................................................147

2015-173-R2 Isotope Geochemistry via Sn Isotope Fractionation Using Inelastic X-ray Scattering of Synchrotron Radiation .148

2015-174-R2 Conversion of $C_{2}$ and $C_{3}$ Paraffins into Liquid-Phase Products ....................................................149

2015-178-R2 Towards Ionotronics: First-Principles Strategies for Coupling Electronic and Ionic Properties in Complex Oxides 150

2015-181-R2 Fine-Resolution Reconstruction of Large Volumes of a Brain............................................................151

2016-120-R1 New Thin Film Oxide, Chalcogenide and Oxy-Chalcogenide Materials Discovery ....................... 152

2016-179-R1 Development of a Pre-Conceptual Design of a New Tracking System for CLAS12 Detector.......153

2016-182-R1 Core-Shell Nanowire Magnetic/Ferroelectric Multiferroic Heterostructure

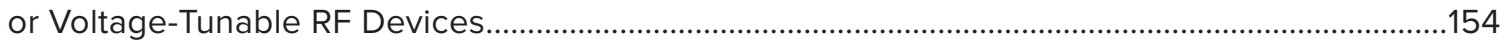

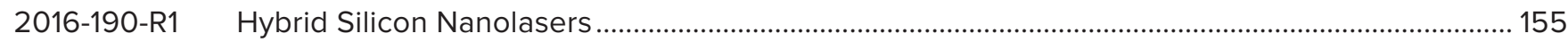

2016-191-R1 Exploring Next-Generation Coherent X-ray Science ...................................................................... 156

2017-155-NO Coherent X-Ray Studies of Phase Transitions in the Complex Oxides ............................................157

2017-156-NO Scalable Data Movement for Data-Centric Supercomputing …........................................................ 158 


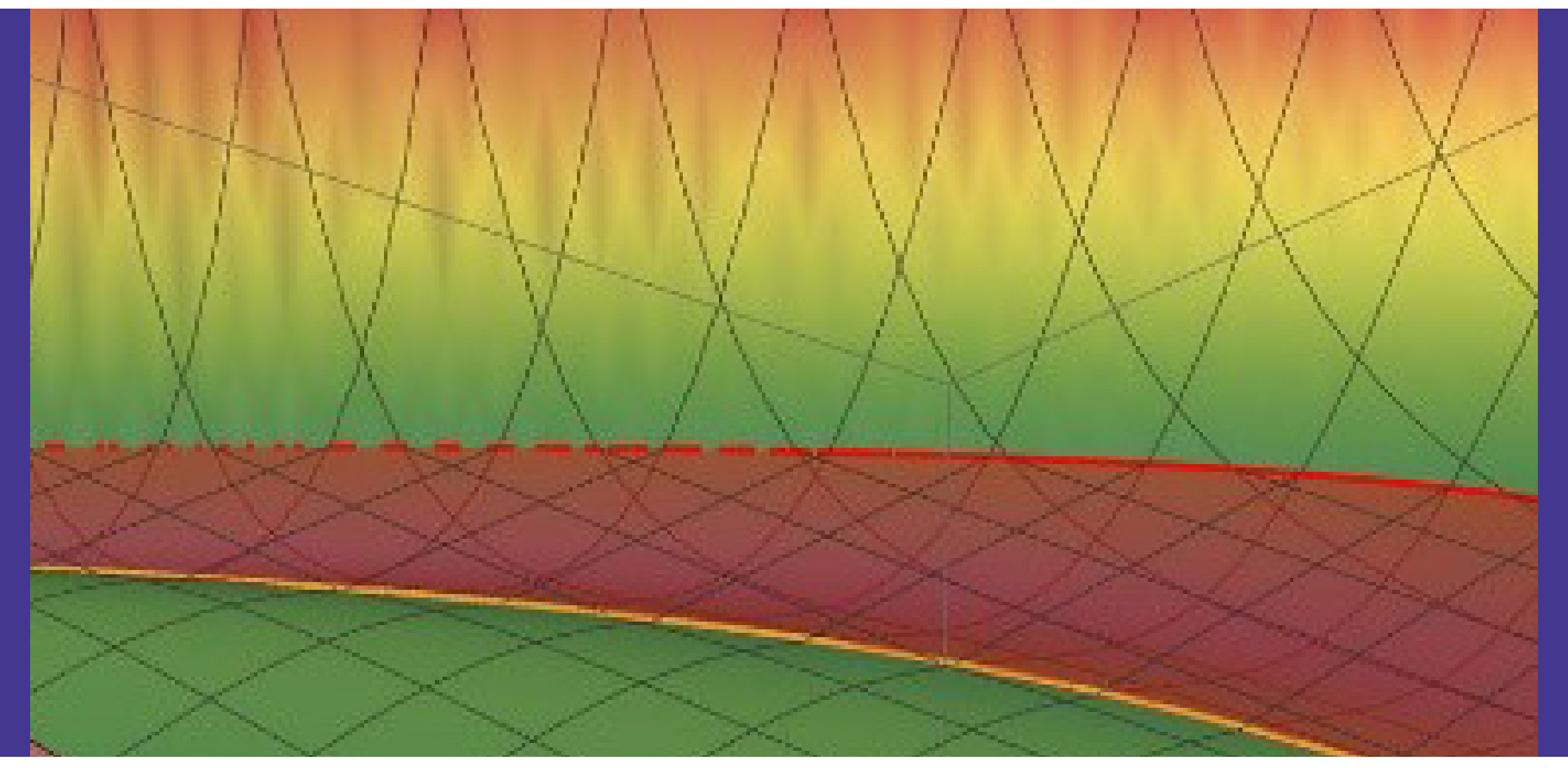

\section{LDRD INNOVATE}


Josephson Plasma Wave-Based UltraHigh-Frequency Electronics

\author{
2015-078-R2 \\ Alexei Koshelev and Vitalii Vlasko-Vlasov
}

\section{PROJECT DESCRIPTION}

Operation at frequencies of $100 \mathrm{GHz}$ and above is beyond the reach of conventional electronics. However, this frequency range is the next frontier for high-bandwidth communications and high-speed signal processing. Certain devices constructed from high-temperature superconductors promise to be naturally suited for operation at these frequencies.

Extremely anisotropic high-temperature superconductors, such as $\mathrm{Bi}_{2} \mathrm{Sr}_{2} \mathrm{CaCu}_{2} \mathrm{O}_{8}(\mathrm{Bi}-2212)$, contain $\mathrm{CuO}_{2}$ planes alternating with insulating barrier layers. These naturally formed Josephson junctions can be used for DC-to-AC conversion at terahertz frequencies, allowing them to act as very compact sources and detectors of electromagnetic radiation in the "terahertz gap" range spanning approximately 0.3 to $1.3 \mathrm{THz}$. These periodic structures are also predicted to act as nonlinear waveguides, allowing the propagation of so-called Josephson plasma waves (JPWs) with switchable transmission characteristics at frequencies of $100 \mathrm{GHz}$ and above. These various properties can thus be exploited for novel optoelectronic integrated circuits that incorporate components such as interferometers, ridge waveguides, and mixers for which the unique properties of layered superconductors can be fully exploited. Such systems would be of particular value for ultra-high-bandwidth telecommunications. In the course of this project, we propose to demonstrate proof-of-concept for such devices, by testing the mixing and controllability of JPWs in the $\mathrm{Bi}_{2} \mathrm{Sr}_{2} \mathrm{CaCu}_{2} \mathrm{O}_{8}$ devices that we will construct. We also plan to test a new technique for growing high-quality thin films of $\mathrm{Bi}_{2} \mathrm{Sr}_{2} \mathrm{CaCu}_{2} \mathrm{O}_{8}$, with a view to enabling wafer-scale fabrication of JPW devices.

\section{MISSION RELEVANCE}

This project is relevant to DOE's missions in national security, environmental quality, and science.

The rapidly emerging field of $\mathrm{THz}$ science and technology holds immense promise for diverse areas, including high-speed electronics, high-bandwidth communications, environmental monitoring, security, manufacturing, and pharmaceuticals. Directly related to DOE's missions in basic science are the development of new materials platforms for high-speed electronics and the energy-efficient generation of electromagnetic nonlinearites.

\section{RESULTS AND ACCOMPLISHMENTS}

In FY 2017, we further focused on growth of thick Bi-2212 films by liquid-phase epitaxy (LPE), optimization of growth conditions to achieve high-quality films, and their characterization. We have successfully grown high-quality thick films by LPE at a growth temperature of $\sim 870^{\circ} \mathrm{C}$ on $\mathrm{MgO}$ substrates. X-ray diffraction (XRD) and energy-dispersive $\mathrm{X}$-ray spectroscopy (EDS) analyses confirm single-phase structure and high c-axis alignment of the films.

Figure 1a shows the largest single-crystalline grain on a 1- $\mu$ m-thick Bi-2212 film. To study the c-axis transport properties of the films, we have fabricated a chip on an annealed film, including three intrinsic Josephson junction (IJJ) stacks with sizes of $20 \times 20 \mu \mathrm{m}^{2}$ and $30 \times 90 \mu \mathrm{m}^{2}$ in a four-terminal configuration (see Figure $1 \mathrm{~b}$ ). The temperature dependence of resistance for one of the small mesas shown in Figure 1c demonstrates a sharp superconducting transition around $85 \mathrm{~K}$. The current-voltage characteristic of the small mesa at $15 \mathrm{~K}$ in Figure 1d shows the hysteretic multi-branch structure including all resistive switching characteristics of each IJJ. The critical current density $\left(J_{c}\right)$ in the IJJs stack is approximately $600 \mathrm{~A} / \mathrm{cm}^{2}$. The values of $T_{c}$ and $J_{c}$ are close to the values of slightly underdoped $\mathrm{Bi}-2212$ single crystals. These encouraging results indicate that thick films grown by LPE are potential candidates for manufacturing integrated Josephson devices on a single chip.
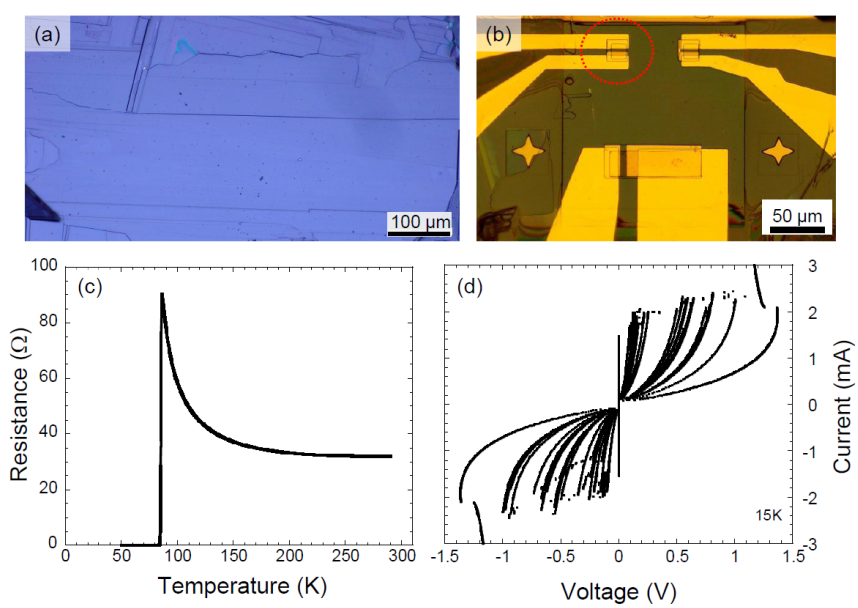

Figure 1. (a) Polarized light image of an as-grown Bi-2212 film on MgO substrate. (b) An optical picture of a manufactured chip on the film including three mesas with 4-point sample geometry (sizes: $20 \times 20 \mu \mathrm{m}^{2}, 30 \times 90 \mu \mathrm{m}^{2}$ ). (c-d) The c-axis R-T and I-V characterizations of a small mesa (left) on the chip.

\section{PROPOSED FUTURE WORK}

This is the last year of the project. We plan to submit a proposal to the Air Force Office of Scientific Research (AFOSR) based on this work. 


\section{Next-Generation Natural Gas Adsorbent through Rational Design and Modeling}

\author{
2015-091-R2 \\ Di-Jia Liu and Rajesh K. Ahluwalia
}

\section{PROJECT DESCRIPTION}

This project has focused on developing a new synthetic method and fundamental understanding that could lead to the next generation of three-dimensional (3D) porous polymeric network (PPN) materials for gas adsorption, separation, and other energy-environmental applications. A PPN is an all-organic framework formed by crosslinking a molecular "strut" with a stereo-contorted molecular node. The scaffolding through covalent bonds between these building units creates unique properties. PPNs have (1) high surface areas and porosity when properly prepared, potentially significantly higher than those of traditional zeolites; (2) light weight from hydrocarbon-only frameworks; and (3) high tolerance of heat and contaminants due to the covalent bonds.

In this project, we prepared PPNs with high specific surface area and controlled microporosity. Molecular struts with different chelating functional groups were incorporated into PPNs with the desired surface properties. We used advanced characterization techniques to study how a gas adsorbent such as methane $\left(\mathrm{CH}_{4}\right)$ interacts with the PPN, and also the optimal structural/physical properties for maximum usable storage capacity. Project outcomes will be crucial to realizing the full potential of PPN-based gas adsorbents, catalysts, and photocatalysts. The new PPNs will also have broad potential uses in gas separation, catalysis, and other energy-efficiency-related applications.

\section{MISSION RELEVANCE}

This project is relevant to DOE's mission in energy and environment. It focuses on developing a new class of porous polymers for natural gas (NG) storage applications. The project is highly relevant to DOE's mission in reducing dependence on foreign oil and securing a national energy supply. In addition to gas storage, the new PPNs derived from this project could be applied in gas separation, catalysis, and other applications related to energy conversion and environmental protection.

\section{RESULTS AND ACCOMPLISHMENTS}

During FY 2016, we designed more than 30 PPNs

using different monomers and crosslinking

chemistries. We also explored application of metallated PPNs as new precursors for preparing precious-metal-free electrocatalysts.
In FY 2017, we accomplished several major tasks in synthesis and explored a variety of PPNs for NG storage and other applications. One example is the design and synthesis of the first PPN with a sulfur-containing contorted core as the linker. We successfully prepared the sulfur-substituted contorted core, spirodithiophene, as the monomer, as shown by the reaction scheme in Figure 1. This monomer was subsequently used to form a PPN with high aromatic sulfur content through a crosslinking reaction. The PPN has high application potential in methane storage, as well as in heavy element removal in water purification. We also prepared several metallated PPNs based on the concept that doped metal in PPNs could enhance methane adsorption through a charge-induced dipole interaction. For example, we designed a cobalt (Co)-platinum (Pt) bimetallic polyporphyrin (PPN). It was prepared by end-to-end crosslinking of Co-ligated and Pt-ligated porphyrin monomers at a 1:1 ratio through a directionally controlled coupling reaction. Our preliminary laboratory study showed that the PPN has promising efficiency in light harvesting and electron transfer during photocatalytic carbon dioxide $\left(\mathrm{CO}_{2}\right)$ conversion.
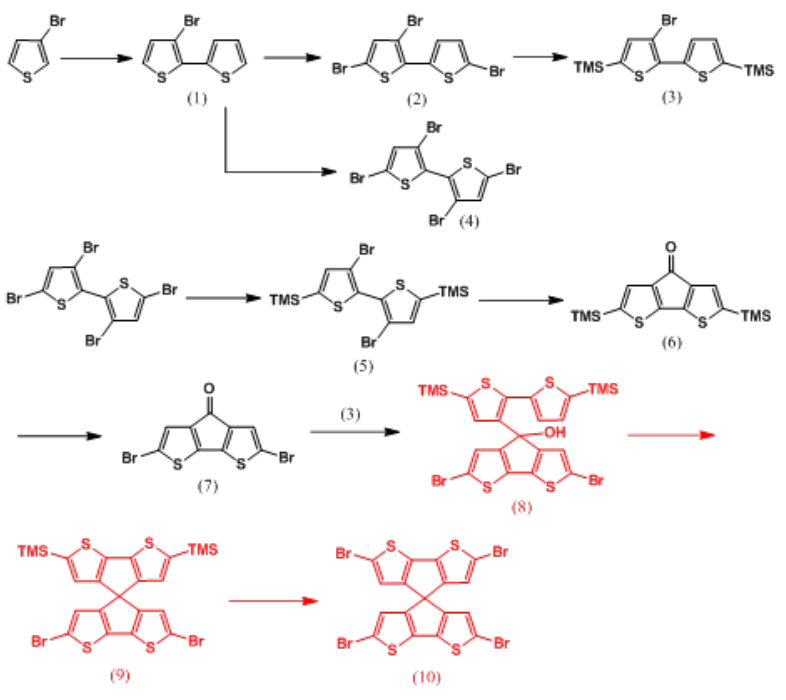

Figure 1. Synthetic scheme of preparing a representative spirodithiophene monomer for a new sulfur-containing PPN.

\section{PROPOSED FUTURE WORK}

This is the final year of this LDRD project. We will, however, continue to develop the technology by seeking support from DOE and the private sector by leveraging the results obtained through this study. 


\section{Understanding Atomic-Scale Uranium Interactions under Severe Accident Conditions}

\section{5-096-R2}

Chris J. Benmore and Mark A. Williamson

\section{PROJECT DESCRIPTION}

A key safety concern in nuclear reactors is the melting and subsequent leakage of radioactive uranium dioxide $\left(\mathrm{UO}_{2}\right)$ as it reacts with its zirconium $(\mathrm{Zr})$ cladding to form a substance known as corium. The atomic structure of the liquid state is the key to understanding the properties of molten nuclear fuel, yet until this work, there were no published structural data on liquid $\cup_{2}$. Part of the reason for the lack of data is the very high melting temperature of $\mathrm{UO}_{2}$ : 3140 kelvin $(\mathrm{K})$. The main objective of the research is to develop a fundamental understanding of the physics of molten nuclear systems, as well as the high-temperature crystalline phases, by using high-energy $X$-rays at Argonne's Advanced Photon Source and ultra-high temperature melting techniques. To solve the problem of accessing samples at high temperatures, we used an aerodynamic levitator system with laser heating to attain temperatures above $3300 \mathrm{~K}$ in a radiologically controlled, hermetically sealed chamber (Figure 1). Thus, with no solid contact surface, the chemical purity of the very hot sample could be maintained.

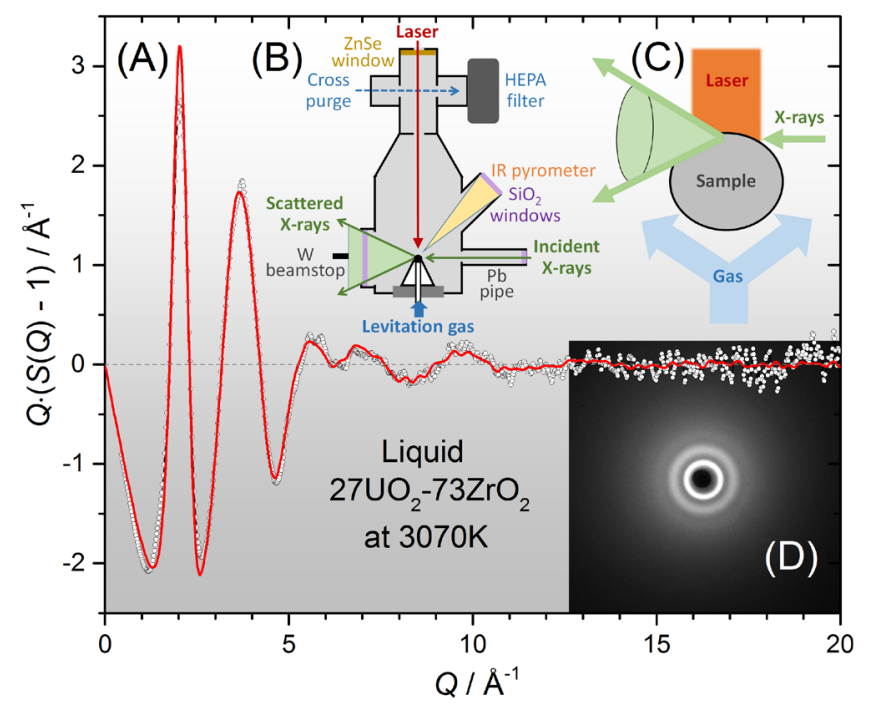

Figure 1. Comparison of measured (circles) and MD modeled (red curves) $X$-ray diffraction data: (A) X-ray interference functions, (B) schematic of levitator within sealed chamber, (C) X-rays impinging on the top few hundred microns of levitated sample, (D) closeup of two-dimensional X-ray data.

\section{MISSION RELEVANCE}

This project supports DOE missions in nuclear energy, where DOE's Office of Nuclear Energy is actively pursuing the development of accident-tolerant fuels. Performing hazard and accident analyses of nuclear fuels is paramount in establishing nuclear safety requirements. The primary motivation for this study was the exclusive use of $\mathrm{UO}_{2}$ in nuclear power reactors operating today, given that even mixed-oxide fuel is typically $90 \mathrm{wt} \% \cup_{2}$. Insight into the behavior of liquid $\mathrm{UO}_{2}$ and its interaction with $\mathrm{ZrO}_{2}$ at high temperatures will provide guidance in selecting an optimum fuel composition, additives to stabilize the fuel, and cladding material. Because computer modeling of materials is an important component of the fuels development program, experimentally derived interatomic potentials are essential for model development. Similarly, reactor safety codes under development by the U.S. Nuclear Regulatory Commission will benefit from this project's experimental structural data.

\section{RESULTS AND ACCOMPLISHMENTS}

High-energy X-ray diffraction from levitated droplets of molten corium have been used to validate classical molecular dynamics models of high-temperature liquids. Atomistic models based upon existing $\mathrm{UO}_{2}$ interatomic potentials have been refined against our measured $\mathrm{X}$-ray structure factors. Investigations into hypo-stoichiometric $\mathrm{UO}_{2-x}$ in reducing gas atmospheres are found to be consistent with a partial change in the uranium oxidation state to $U^{3+}$. Properties including thermal expansion, self-diffusion coefficients, and melt viscosities show very little dependence on the $\mathrm{U} / \mathrm{Zr}$ ratio in the $\mathrm{UO}_{2}-\mathrm{ZrO}_{2}$ system and confirm the extremely fluid and highly penetrating nature of these liquids. Corium liquids are therefore capable of traveling large distances within a reactor vessel during meltdown, independent of the amount of cladding incorporated into the molten fuel. A mismatch between the metal-oxygen coordination numbers of molten $\mathrm{UO}_{2}$ and $\mathrm{ZrO}_{2}$ results in their variation in composition. This behavior is explained on the basis of the stabilization of the various 3- and 4-fold oxygen sites present in the melts. We also observed temperature-dependent coordination numbers, which we suggest are a primary mechanism for liquid thermal expansion.

\section{PROPOSED FUTURE WORK}

Our experimental verification of interatomic potentials for molten corium suggests their suitability for larger-scale simulations, including melt-solid interface interaction, incorporation and behavior of fission products, and use in finite element codes as part of multiscale reactor models. 


\section{Development of Advanced $\mathrm{VO}_{2}$ Nano-Composite Thermochromic Materials for High-Performance Smart Windows}

\author{
2015-121-R2 \\ Jie Li, Leah Guzowski, Ralph Muehleisen, and Xiaojie Yan
}

\section{PROJECT DESCRIPTION}

Buildings consume more than $40 \%$ of the world's energy, primarily for heating, cooling, and lighting. Vast amounts of energy are consumed to cool spaces heated by the sun via windows. Nano-vanadium dioxide $\left(\mathrm{VO}_{2}\right)$ is a promising material for making windows "smart" and energy efficient: its unique thermochromic function selectively blocks infrared solar heat while allowing visible light to pass through.

This project synthesized thermochromic $\mathrm{VO}_{2}$ (M-phase) micro-nano-particles for smart-window film applications. By developing a novel flow-reaction system, we have been able to manufacture the nanoparticles continuously. After nano-coating with silicon dioxide $\left(\mathrm{SiO}_{2}\right)$ for enhanced durability and reduced light scattering, the nanoparticles can be incorporated into a dielectric host to make a novel window film. The particle size, shape, and phase can be controlled by tuning chemical kinetics and transport. Our continuous-flow synthesis technology not only enables the mass production of the particulate materials, but effectively intensifies the reaction, reducing the synthesis time from days to minutes.

\section{MISSION RELEVANCE}

This project supports DOE missions of improving national energy security and protecting the environment. It aligns well with the DOE Secretary's emphasis on energy efficiency and the DOE/Energy Efficiency and Renewable Energy (EERE) Building Technology Office's focus. Our advanced green processing technology also supports the DOE/EERE Advanced Manufacturing Office's mission in additive manufacturing, in which nano-powders are typically used as raw materials. The potential beneficiaries of this technology include building owners, window film manufacturers, automobile makers, sensor and detector producers, and the aerospace industry, as well as the DOD and defense industry where the $\mathrm{VO}_{2}$ material can be used to protect equipment from high-power lasers.

\section{PROPOSED FUTURE WORK}

We have discussed commercializing this technology with several potential industry partners

\section{RESULTS AND ACCOMPLISHMENTS}

In FY 2015 and FY 2016, we conducted nano- $\mathrm{VO}_{2}$ syntheses using a commercial microwave and a micro-flow reactor and concluded that those reactors are not suited to mass synthesis of nano-VO 2 . In FY 2017, we developed a novel, continuous-flow, hot-waterfluidized, suspension reaction system. By tuning reaction temperature, precursor concentration, and residence time, pure $\mathrm{M}$-phase $\mathrm{VO}_{2}$ nano- and micro-particles were successfully fabricated for the first time in a continuous-flow system. The liquid suspension containing $\mathrm{VO}_{2}(\mathrm{M})$ particles flowed from this system continuously, yet with a significantly reduced residence time of less than 10 min-much shorter than that obtained using a traditional autoclave reactor, in which the residence time was measured in days.

In addition, three methods were tested to enable transient mixing between reactant and heating water, which is required for an ultrafast reaction: (1) fluidized glass beads, (2) dynamic mixers, and (3) pulsed flow. With the first method, the materials synthesized tended to become attached to the glass beads. The cross impinging mixer showed better control of morphologies and size distribution of the resulting particles (Figure 1) than a center-T mixer, while maintaining the same low metal-insulator transition (MIT) conversion temperatures of $55^{\circ} \mathrm{C}$ (heating). Pulsed flow led to enhanced mixing in which pulsing amplitude and frequencies play essential roles, affecting particle phase and morphologies. At a frequency of $4 \mathrm{~Hz}, \mathrm{VO}_{2}$ particles with a spherical shape and a uniform 200-nm diameter were achieved.

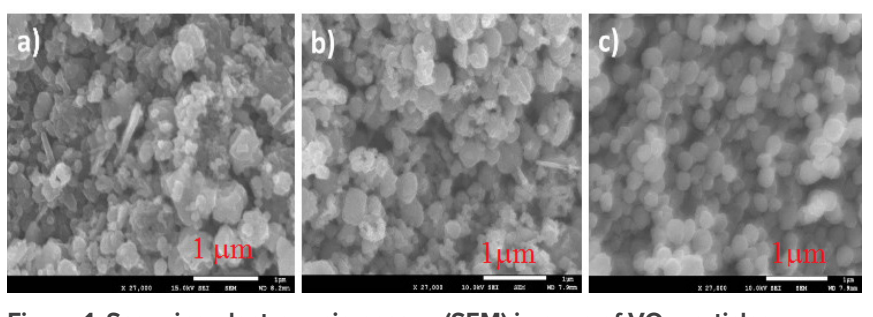

Figure 1. Scanning electron microscopy (SEM) images of $\mathrm{VO}_{2}$ particles synthesized using hot water fluidized suspension reactor with (a) a traditional T mixer, (b) an impinging mixer, and (c) an impinging mixer and pulse flow.

Finally, tungsten (W) was successfully doped into the $\mathrm{VO}_{2}$ particles by using our novel flow synthesis system to bring down the MIT to a comfortable room temperature. Differential scanning calorimetry analysis demonstrated that the Tc was reduced to $32^{\circ} \mathrm{C}$ (heating) and $22^{\circ} \mathrm{C}$ (cooling). The lower Tc is necessary to make the micro-nano-particles useful for customer applications. 
Structure and Dynamics of Chiral Molecules and Radicals

\author{
2016-001-R1 \\ Stephen T. Pratt and Ananya Sen
}

\section{PROJECT DESCRIPTION}

The deoxyribonucleic acid (DNA) nucleosides and many of the amino acids that form proteins are chiral molecules with non-superimposable mirror images, or "handedness." In general, the chemical and physical properties of the two forms, or enantiomers, are the same, although they interact differently with polarized light. In this project, we are using photoelectron imaging techniques, tunable ultraviolet light, and photoelectron angular distributions to study the stability and selective detection of chiral molecules, as well as of chiral radicals. This project has already led to an improved understanding of optically induced circular dichroism, and is providing a foundation for the development of new tools to monitor and characterize chiral molecules and radicals.

\section{MISSION RELEVANCE}

This project supports the DOE mission in science by providing the foundation for new detection methods for chiral chemicals, which are ubiquitous in our natural world. Because the methods can be used to study time-dependent processes, the work helps open up the study of dynamical processes in chiral systems, as well as how chiral systems can evolve into non-chiral racemic mixtures. The methods should be equally applicable to stable molecules and reactive species like free radicals, which play an important role in many biological, chemical, and environmental systems. Finally, the techniques developed in this project have analogues in which the laboratory laser systems are replaced with synchrotron or free-electron laser sources, and thus could find significant utility at DOE light sources.

\section{RESULTS AND ACCOMPLISHMENTS}

In FY 2017, we focused on the study of circular dichroism effects induced by laser excitation with circularly polarized light. This allowed us to understand how the techniques we used to probe samples could influence our results. In particular, excitation with linearly or circularly polarized light can induce an alignment or orientation in the sample that can result in circular dichroism. It will be important to distinguish this effect from effects produced by the inherent chirality of the sample. This distinction will be particularly important in our planned studies of chiral photofragments (in particular, molecular radicals) and excited states of chiral molecules produced by laser excitation in the coming year. We collaborated on this work with Professor Katharine Reid of the University of Nottingham, UK. We recently completed this study of optically induced circular dichroism and published results in the Journal of Chemical Physics. We are now confident in our ability to distinguish between circular dichroism resulting from the excitation process and circular dichroism resulting from inherent chirality.

\section{PROPOSED FUTURE WORK}

In the final year of this project, we will focus on two topics. First, we will record photoelectron circular dichroism data for the two enantiomers of propylene oxide and compare these data to existing circular dichroism data recorded using synchrotron radiation. Next, we will record photoelectron circular dichroism data for enantiomers of 1-bromo-2-methylbutane, a molecule which has not been characterized previously. This molecule is an ideal candidate for studies of chiral radicals because the primary photodissociation process is expected to yield the chiral 2-methylbut-1-yl radical and a bromine atom, which should be easy to study with our imaging spectrometer. This photodissociation process will be interesting because both the photofragment angular distributions and the photoelectron angular distributions of the radical should display circular dichroism. Our goal is to complete the studies on 1-bromo-2-methylbutane and its radical fragment by the end of FY 2018.

If our approach for characterizing chiral molecules and radicals proves successful, we will submit a proposal to the National Institutes of Health for longer-term support to develop the methods and to generalize them to address various chiral pharmaceuticals. The National Oceanic and Atmospheric Administration or the U.S. Environmental Protection Agency may provide funding for studies of the reactions of chiral radicals in the atmosphere. The work on excited-state chirality may also lead to funding relevant to stereospecific synthesis. 


\section{A Theory of Out-of-Equilibrium Phase Transitions}

\author{
2016-010-R1 \\ Valerii Vinokour and Alexey Galda
}

\section{PROJECT DESCRIPTION}

This project aims to develop a new research direction that embraces important recent developments in physics of the non-equilibrium state and leads us to an innovative transformational science describing energy and spin transmission. The basis of this science is the theory of strongly non-equilibrium macroscopic quantum systems. The theory will provide a quantitative description of far-from-equilibrium quantum phenomena and enable discovery of new effects applicable to new energy technologies.

We develop a rigorous formalism using a non-Hermitian Hamiltonian approach, which we successfully apply to a wide range of non-equilibrium quantum systems. We achieve our objective by introducing a complex "Hamiltonian"- the mathematical non-Hermitian operator corresponding to the total energy of the system. The dissipation and non-equilibrium forces in the system are then incorporated in its imaginary part. This approach provides a method to compensate for the losses in real experimental systems.

We focus on non-Hermitian Hamiltonians endowed with parity-time (PT) symmetry, i.e., invariant under simultaneous parity transformation, $\chi \rightarrow-\chi$, and time reversal, $t \rightarrow-t, i \rightarrow-i$. Such systems experience a field-driven non-equilibrium phase transition associated with the spontaneous breaking of PT symmetry, which leads to a plethora of topologically nontrivial dynamic and thermodynamic phase transitions. The loss of the PT symmetry marks a transition from the steady state to nonstationary dynamics and manifests as a bifurcation in the spectrum of the non-Hermitian Hamiltonian describing the system. The corresponding phase transitions are topologically protected with respect to small perturbations, preserving the symmetry of the Hamiltonian. This is the essence of our breakthrough in the quantitative description of out-of-equilibrium phase transitions.

In the framework of our project, we apply this technique to the dynamic (field-driven) Mott transition and to the dissipative spin-transfer torque-driven dynamics of classical and quantum low-dimensional spin systems.

\section{MISSION RELEVANCE}

This project is relevant to DOE's basic science mission and advances Argonne's mission of exercising control over far-from-equilibrium processes.

\section{RESULTS AND ACCOMPLISHMENTS}

We studied the universality and critical behavior of the dynamical Mott transition in a system with long-range interactions. We established equivalence between dynamic and thermodynamic properties of non-equilibrium phase transitions in classical spin systems and found that the fundamental Lee-Yang approach, which describes thermodynamic phase transitions in spin chains via the distribution of zeros of the partition function, can be generalized on the out-of-equilibrium physics. See Figure 1.

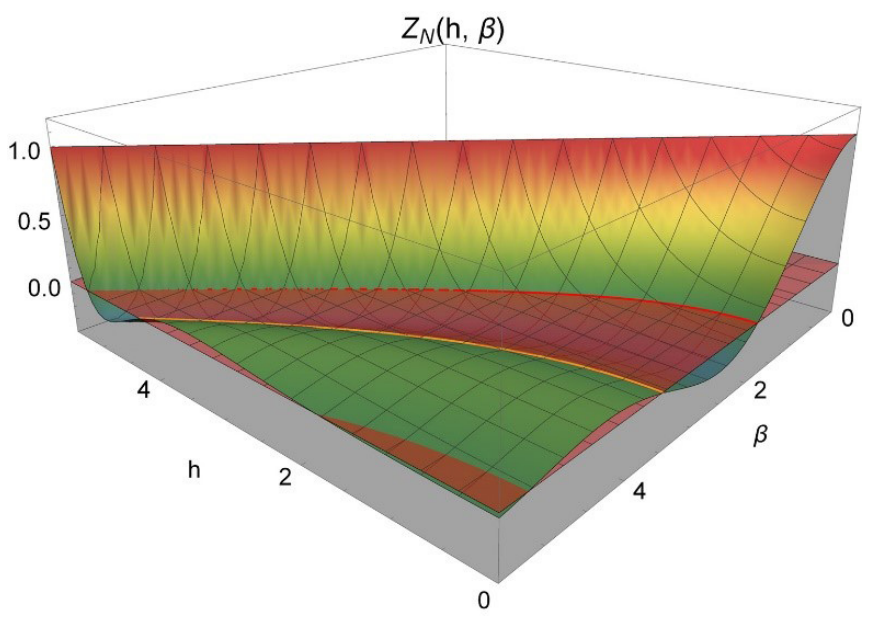

Figure 1. Partition function of a $P T$-symmetric Heisenberg spin model showing Lee-Yang zeros in the parametric region of broken $P T$ symmetry $(h<\beta)$, establishing connection of dynamic and thermodynamic phase transitions in classical spin chains.

\section{PROPOSED FUTURE WORK}

In FY 2018, we plan to develop chaos theory of non-equilibrium dynamic systems described by non-Hermitian Hamiltonians and study the connection between singularities of PT-symmetric Hamiltonians, PT symmetry breaking, and topologically non-trivial phases in one- and two-dimensional strongly correlated systems. 


\section{Nano-Mechanical Delivery of Biomolecules into Live Bacterial Cells}

\author{
2016-020-R1 \\ Philip Laible and Andrey Sokolov
}

\section{PROJECT DESCRIPTION}

Bacteria play different roles in the environment, interacting beneficially or detrimentally (e.g., disease-causing) with hosts. Our understanding of positive or negative interactions is limited by the fact that over half of the sequenced genes have unknown functions. Linking genes to specific microbial functions generally requires the ability to inactivate target genes in an organism and observe the consequences. However, techniques to deliver biomolecules into bacterial cells for such characterization efforts are very limited. They are mainly available for only a few dozen bacterial species. Here, we are developing novel, potentially universal methods for delivery of biomolecules into cells that are based on nano-mechanical disruption of the bacterial cell wall. Such methods have the potential to harness the diverse, mostly untapped biosynthetic capabilities of environmental microbes for biomanufacturing.

\section{MISSION RELEVANCE}

This project addresses problems that are at the core of the DOE Office of Biological and Environmental Research Genomic Science program. These problems include understanding the basis of bacterial communities and ecosystems at a molecular level and discovering protein functions at large scale. The proposed method fits current objectives of both the Bioenergy Technologies Office in the Office of Energy Efficiency and Renewable Energy and biotechnology companies, as it opens new avenues for biomanufacturing and industrial biotechnology.

\section{RESULTS AND ACCOMPLISHMENTS}

We have mastered and scaled the fabrication processes of nanotextured surfaces such that we can obtain various topographies in a controlled manner. We are employing synthetic analogues of dragonfly wings with surfaces characterized by nanopillars that exhibit antimicrobial properties. We reproducibly tuned the shapes and densities of the nanopillars to elicit differences in their bactericidal properties. We have investigated the interaction of these materials with a range of genera using cell viability tests (Figure 1), along with confocal and scanning electron microscopies (Figure 2). All materials containing sharp nanopillars of lengths above $3 \mu \mathrm{m}$ showed generic bactericidal properties-killing rapidly with exceptional efficiency. In contrast, the shorter $(\leq 2 \mu \mathrm{m})$ and blunter nanopillars killed less efficiently with variable potency among the bacterial types studied, thus affording selectivity in killing. The mechanism of action of the two types of nanopillars was found to be different. Our control over silicon fabrication allows for modulation of nanopillar interaction and biomolecule delivery, thus opening new avenues of research for materials use-from applications envisioned for medicine to those involving selective modulation of microbial colonization. We also chemically modified the nanotextured surfaces after synthesis to facilitate binding of biomolecules in the first step of delivery into living bacterial cells.

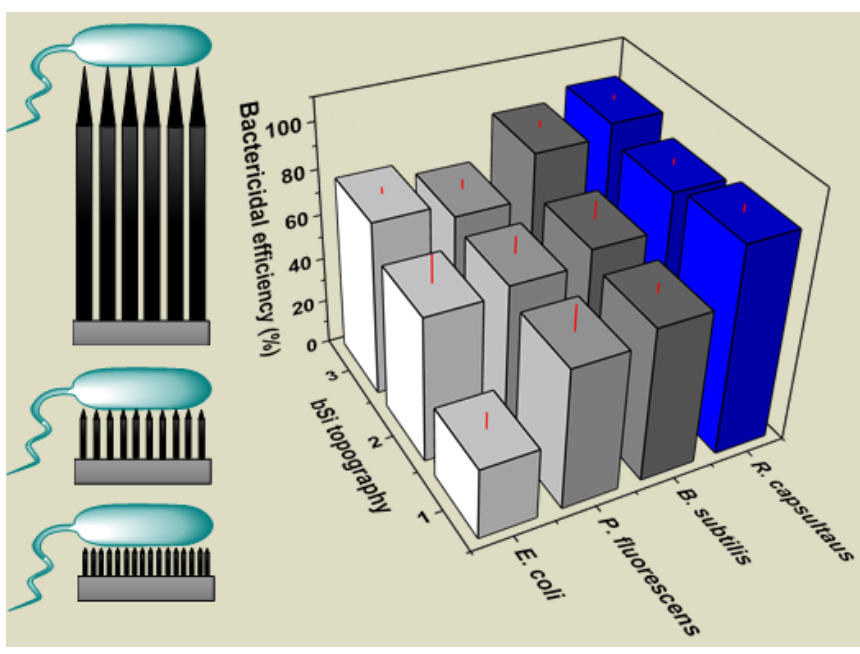

Figure 1. Bactericidal efficiencies (\%) of various nanotextured surfaces (1-3) towards four bacteria species showing differences in material performance.

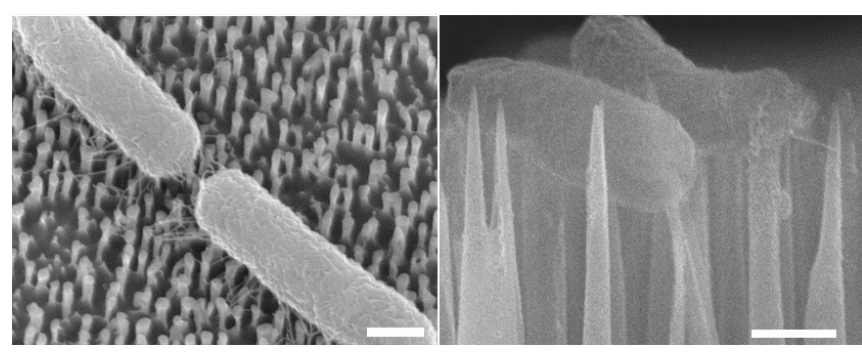

Figure 2. Scanning electron microscopy images of $E$. coli cells interacting on blunt (left) and sharp (right) nanopillars of black silico

\section{PROPOSED FUTURE WORK}

We will continue our discovery of material attributes and process conditions for delivery of biocompatible molecules into a range of bacterial cell types. The short-term focus will be proof-of-principle demonstration of release of immobilized molecules, followed by miniaturization of the methodology through development of a microfluidics-based device. 


\section{Real-Time Monitoring of Material Structure Evolution in Additive Manufacturing Processes}

\section{6-023-R1}

Tao Sun, Kamel Fezzaa, Haidan Wen, and Cang Zhao

\section{PROJECT DESCRIPTION}

Additive manufacturing ( $\mathrm{AM}$, also known as 3D printing) of metallic materials has experienced tremendous growth over the past decade. Laser powder bed fusion (LPBF) has become the most extensively used metal AM technique because this method can make geometrically complex parts with high dimensional precision. Although the LPBF process is conceptually simple, it involves many highly dynamic physical phenomena because of its extremely high heating and cooling rates. Often, the complex laser-metal interaction results in a product with a rough surface, significant porosity and residual stress, and/or unfavorable phase and grain structures. These microstructural defects can largely reduce the strength and corrosion resistance-as well as degrade the fatigue performance-of additively manufactured parts.

To understand the mechanisms responsible for the formation of these structural defects, it is imperative to develop and apply in situ characterization techniques to probe the dynamic microstructural evolution during the LPBF process. However, developing these techniques has been extremely challenging. The most advanced characterization method reported so far, which uses a high-speed visible-light camera, can monitor only what happens above the surface of the powder bed. To address this issue, we are utilizing the intense high-energy X-rays and state-of-the-art high-speed instruments at the Advanced Photon Source (APS) to study the LPBF process. The high penetration power of hard X-rays allows us to image through millimeter-sized metals and watch the microstructure evolution and defect formation inside the samples with unprecedented spatial and temporal resolutions. See Figure 1.

\section{MISSION RELEVANCE}

The experiment platform at the APS developed in this project enables transformative studies on laser AM processes. For the first time, dynamic microstructure parameters of the sample in a LPBF process can be measured experimentally. By further enhancing the beamline capabilities and expanding the user community of DOE's synchrotron facilities, this research aligns well with the core mission of DOE's Office of Science-Basic
Energy Sciences. Development of material- and energy-efficient manufacturing processes for building high-quality products is the major goal of DOE's Office of Energy Efficiency and Renewable Energy-Advanced Manufacturing. In addition, AM has been identified as a critical area for further R\&D by DOE's Office of Nuclear Energy and National Nuclear Security Administration, as well as the Department of Defense, National Institute of Standards and Technology, and National Aeronautics and Space Administration, because of its relevance to nuclear energy, national security, measurement science, and space exploration. In industry, many aerospace, automobile, defense, and medical companies are investing in AM technologies.

\section{RESULTS AND ACCOMPLISHMENTS}

In the second year of this project, we (1) integrated a laser scanner into our sample chamber; (2) conducted high-speed X-ray imaging and diffraction experiments, and in particular, demonstrated the feasibility of imaging the laser AM process with a rate of 10 million frames per second; (3) developed computer codes for extracting quantitative structural information from X-ray data on melt pool dynamics, depression zone fluctuation, powder ejection velocity, solidification rate, and phase transformation rate; and (4) achieved direct observation of the formation of various defects, including powder-induced porosity, trapped-gas porosity, keyhole porosity, and hot cracks.
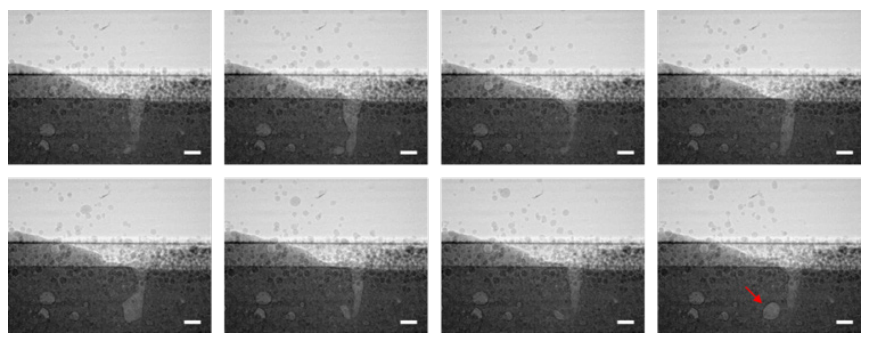

Figure 1. Dynamic X-ray images showing the formation of a keyhole pore (indicated by the red arrow in the last frame) in the LPBF processing of Ti-6Al-4V. The frame rate of this data series is $45.3 \mathrm{kHz}$, and the exposure time for each image is $100 \mathrm{ps}$. All scale bars are $100 \mu \mathrm{m}$.

\section{PROPOSED FUTURE WORK}

In the third year of this project, we plan to further improve the instrument and data analysis approach to gain better stability and efficiency. Specifically, we will (1) design and build a new sample chamber with integrated thermal and chemical sensors; (2) apply dictionary-learning algorithms to aid feature identification and segmentation of X-ray images and diffraction patterns; and (3) apply deep-learning algorithms to correlate the measurable physical phenomena in LPBF and establish a rigorous predictive framework. 


\section{A Novel Gas-Filled Microchannel Plate (GF-MCP) $\mathrm{X}$-ray Polarimetry Imager}

\section{6-027-R1}

Shaolin “Allen” Liao, Marcellinus Demarteau, Daniel Haskel, and Anil Mane

\section{PROJECT DESCRIPTION}

This project is to develop a novel gas-filled microchannel plate (GF-MCP) X-ray polarimeter for X-ray polarization imaging. The project integrates $R \& D$ expertise from four Argonne divisions: Nuclear Engineering, High Energy Physics, Energy Systems, and X-ray Science. The working principle of the GF-MCP X-ray polarimeter is to use the device to detect electron trajectories (from hundreds of microns to a few millimeters long) initiated by a polarized X-ray photon along its polarization direction within a neon/ argon gas medium. A gas medium is ideal for such an application because of its long X-ray photoelectron track and many tuning mechanisms, like gas species and gas pressure. The GF-MCP X-ray polarimeter aims to detect the projection of such electron trajectories onto the MCP readout/imaging plane. We investigated two imaging methods: (1) optics imaging based on photon emission due to the electron gas ionization process, and (2) direct electron trajectory stripline readout. The GF-MCP X-ray polarimeter has a potential detection efficiency $>90 \%$. The GF-MCP can be modified for detection and tracking of other types of particles, such as fast neutrons for national security applications.

\section{MISSION RELEVANCE}

This project relates closely to DOE's mission in basic science, which includes polarized X-ray and astrophysics R\&D, and has national security applications within the National Nuclear Security Administration.

\section{RESULTS AND ACCOMPLISHMENTS} In FY 2017 we:

$\square$ Designed and fabricated a chamber-based GF-MCP $X$-ray polarimeter prototype. Figure 1 shows the prototype laboratory assembly, consisting of an electric drift plate, the MCP, spacers, and their supporting structure.

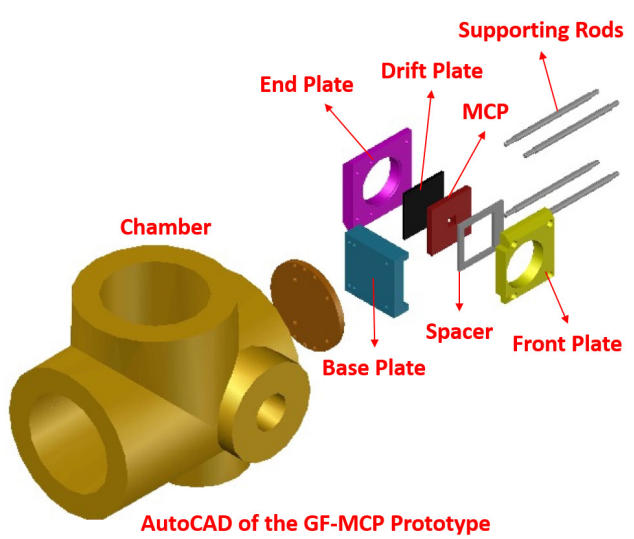

a)

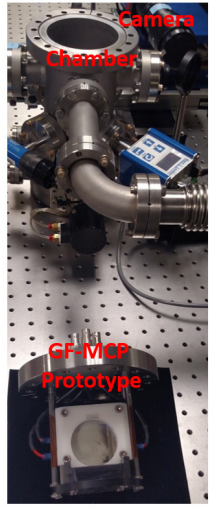

b)
Figure 1. The GF-MCP X-ray polarimeter: (a) AutoCAD drawing of the prototype and gas chamber; (b) photograph of laboratory prototype.

$\square$ Integrated the prototype with the gas chamber, optics instrument, and electronic circuit.

$\square$ Performed laboratory characterization of the GF-MCP gas discharge process.

$\square$ Our synchrotron X-ray test of the GF-MCP X-ray polarimeter prototype at the Advanced Photon Source (APS) detected a promising electric signal (Figure 2): we can clearly see that the detected current is linearly proportional to the effective drift voltage.

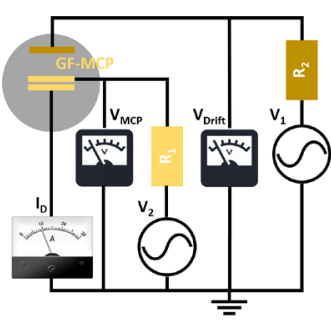

a)

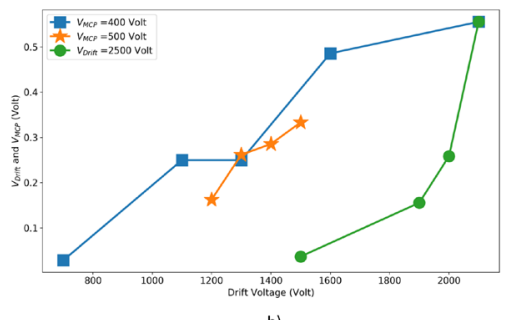

b)
Figure 2. APS experiment on the GF-MCP X-ray polarimeter prototype: (a) schematic of electronic circuit connection; (b) chart of current, $I_{D}$, detected by the GF-MCP X-ray polarimeter for different drift voltages, $V_{\text {Drift, }}$ with $V_{\mathrm{MCP}}=400 \mathrm{~V}$ and $V_{\mathrm{MCP}}=500 \mathrm{~V}$; and for different effective drift voltages, $V_{\text {eff }}=V_{\text {Drift }}-V_{\text {MCP }}$ when $V_{\text {Drift }}=\mathbf{2 5 0 0 ~ V}$.

\section{PROPOSED FUTURE WORK}

Based on experimental results with the GF-MCP $X$-ray polarimeter prototype, our future R\&D will focus on (1) laboratory tests of various MCP prototypes;

(2) fabrication and testing of a high-resolution (50-micrometer pixel size) electric stripline readout imaging system; (3) synchrotron X-ray testing of the optimized prototype at the APS; and (4) publication of peer-reviewed journal/conference papers and lectures, as well as submission of patent applications as appropriate. 


\section{A Missing Protein in the Bacterial Methylmercury Pathway}

\author{
2016-048-R1 \\ P. Raj Pokkuluri, Marianne Schiffer, and Rosemarie Wilton
}

\section{PROJECT DESCRIPTION}

The formation of methylmercury, an extremely toxic compound, is a biologically mediated process carried out by some bacterial species. The two proteins known to be responsible for this transformation reside in the interior of the bacterial cells, in the cytoplasm. The mystery that remains is how mercury from outside the cell is acquired by these bacteria and transported into the cytoplasm. We have targeted our research to fill this information gap. Relying on the previous observations that mercury uptake requires energy and is inhibited by the presence of some bivalent metal ions, such as $\mathrm{Zn}^{2+}$ and $\mathrm{Cd}^{2+}$, we hypothesized that periplasmic solute-binding proteins (SBPs) that are known to transport $\mathrm{Zn}^{2+}$ may be responsible for mercury transport into these bacteria. To establish this requirement for SBPs, we studied $\mathrm{Zn}^{2+}$-binding SBPs from two mercury-methylating bacteria.

\section{MISSION RELEVANCE}

Understanding the transformations and cycling of mercury is of interest to the DOE Subsurface Biogeochemical Research program of the Office of Biological and Environmental Research, as well as the National Institutes of Health and the U.S. Environmental Protection Agency. Methylmercury, a potent neurotoxin, poses a serious risk to human health and the ecosystem. This project contributes to fundamental science in the bacterial transformation pathways of mercury to methylmercury.

\section{RESULTS AND ACCOMPLISHMENTS}

In FY 2016, using recombinant DNA methods, we prepared the $\mathrm{Zn}^{2+}$-binding SBP proteins (ZnuA) from two mercury-methylating organisms, G. sulfurreducens (ZnuA_Gs) and D. desulfuricans (ZnuA_Dd). The G. sulfurreducens protein was produced in two different forms, one full-length (ZnuA_Gs) and the other with an $\mathrm{N}$-terminal truncations (ZnuAtr_Gs). The following paragraphs summarize our FY 2017 work.

Inductively coupled plasma mass spectrometry analysis: The SBP proteins under study, ZnuA_Gs and ZnuA_Dd, were expected to bind $\mathrm{Zn}^{2+}$ and/or $\mathrm{Mn}^{2+}$. E. coli has a native ZnuA protein (homologous to our target SBPs), which has two $\mathrm{Zn}^{2+}$ binding sites. Therefore, it is important to determine whether our target SBP proteins prepared by recombinant methods, using $E$. coli as the expression host, contain any metal ions bound to the protein as isolated. Analysis of the two SBPs by Argonne's Analytical Chemistry Laboratory did not reveal the presence of stoichiometric amounts of transition metal ions ( $\mathrm{V}, \mathrm{Cr}, \mathrm{Mn}$, $\mathrm{Fe}, \mathrm{Co}, \mathrm{Ni}, \mathrm{Cu}, \mathrm{Zn}$, and $\mathrm{Cd}$ ).

Crystallization: Crystallization trials with all three proteins in the presence of zinc acetate were unsuccessful.

Metal binding by differential scanning fluorometry: Differential scanning fluorometry (DSF) is a rapid ligand screening method that requires only small amounts of purified protein. The technique is based on the binding of fluorescent dye molecules to proteins upon thermal denaturation. Bound ligands tend to stabilize proteins in their folded forms, and therefore in the presence of an authentic ligand, the midpoint of a protein's thermal denaturation profile $\left(T_{m}\right)$ shifts to higher temperatures. We conducted DSF studies with the SBP proteins ZnuA_Gs and ZnuA_Dd with various metal ions. We observed positive shifts in $T_{m}$ values of the SBP proteins in the presence of $\mathrm{Zn}^{2+}, \mathrm{Hg}^{2+}$, and $\mathrm{Co}^{2+}$, indicating protein interaction with the three metal ions. To corroborate results of the rapid DSF screens, we undertook similar thermal stability studies with and without metal ions using the technique of circular dichroism (CD) spectroscopy.

Metal binding with CD spectroscopy: The unfolding of the protein's secondary structure was monitored by the $\alpha$-helix band at $222 \mathrm{~nm}$ as a function of temperature. A significant thermal stabilization of the protein (signified by a positive shift in $T_{m}$ ) in the presence of metal ions is highly indicative of a specific interaction of the protein with the metal ions. The $C D$ study demonstrated a moderate increase in stability for ZnuA_Gs with $\mathrm{Hg}^{2+}\left(\Delta \mathrm{T}_{\mathrm{m}}=2.5^{\circ} \mathrm{C}\right)$, but a significant increase in stability with $\mathrm{Zn}^{2+}\left(\Delta \mathrm{T}_{\mathrm{m}}=6.5^{\circ} \mathrm{C}\right)$, which is expected because the biological function of ZnuA_Gs is likely to be high-affinity $\mathrm{Zn}^{2+}$ transport into the cell. A similar study with ZnuA_Dd did not show any shift in $T_{m}$ in the presence of the metal ions $\mathrm{Zn}^{2+}$ and $\mathrm{Hg}^{2+}$.

In conclusion, the SBP ZnuA from G. sulfurreducens could potentially interact with mercury and may be involved in its uptake.

\section{PROPOSED FUTURE WORK}

This project has ended in FY 2017. Gene deletion studies are needed to confirm the role of $\mathrm{ZnuA}$ in $\mathrm{Hg}^{2+}$ uptake by G. sulfurreducens. 


\section{Perovskite Halide-Based Intermediate-Band Solar Cells}

\author{
2016-054-R1 \\ Alex Martinson and Maria K. Chan
}

\section{PROJECT DESCRIPTION}

Solar cells whose absorbers are intermediate-band (IB) media are designed to retain the high output voltages of large-bandgap semiconductors while harvesting significantly more of the solar spectrum. By using the intermediate band as a stepping stone, electron-hole pairs may be generated from photons with sub-bandgap energy to pump electrons directly from the valence band to the conduction band. However, development of an IB absorber layer with outstanding photophysical properties and novel substitutional control will likely be required to achieve the excited-state lifetimes necessary for successful charge extraction. Therefore, we are computationally screening, synthesizing, and characterizing the optoelectronic properties of a new class of bulk IB materials based on perovskite halides. By utilizing the rapidly expanding base of perovskite halide materials known to exhibit exceptional optoelectronic properties and the predictive power of $a b$ initio density functional theory, we are developing an IB absorber with nearly ideal band gap and IB energy level spacing. The objective is to probe the extent to which doped or mixed hybrid perovskites satisfy the demands of an IB solar absorber.

\section{MISSION RELEVANCE}

This project is relevant to the mission of DOE's Solar Energy Technologies Office. From a practical perspective, if a perovskite halide IB material can be fabricated into an efficient photovoltaic (PV) device, there is an opportunity to continue the remarkable rise of PV efficiency beyond the $30 \%$ ceiling. Higher PV efficiencies would have a compounding effect owing to reductions in the overall cost (\$/watt) of PV devices, including land use, hardware, installation, and maintenance. Indeed, even small gains in efficiency, combined with the outstanding scalability, low cost, and high efficiency (22\%) of existing hybrid perovskite PV devices, would achieve the economics needed to drive widespread displacement of the current energy infrastructure by solar technology.

\section{RESULTS AND ACCOMPLISHMENTS}

Ultra-fast transient absorption (TA) and time-correlated single photon counting (TCSPC) of luminescence were applied to probe the carrier dynamics of the most promising absorber layer, Co-substituted methylammonium lead bromide $\left(\mathrm{MAPbBr}_{3}\right)$. The time-resolved dynamics of absorption, free carrier generation, and relaxation are of paramount importance to IB PV device operation. Time-resolved photoluminescence of films revealed little to no loss of excited state lifetimes upon Co substitution in $\mathrm{MAPb}_{1-x} \mathrm{Co}_{x} \mathrm{Br}_{3}$. For IB PVs, it is important to retain the long photo-excited-state lifetimes inherent in perovskite films; therefore, this result demonstrates promise for creating high-quality IB materials from the Co-substituted films. However, TA studies revealed that excitation of the new low-energy absorption feature (potentially to or from an intermediate band) does not produce excitation features characteristic of the parent material, but instead two-photon effects, Figure 1. Therefore, we infer an internal transition from the Co-induced density of states which did not act as stepping stone to excitation of the conduction/valence bands, but instead motivates the more direct approach discussed below.

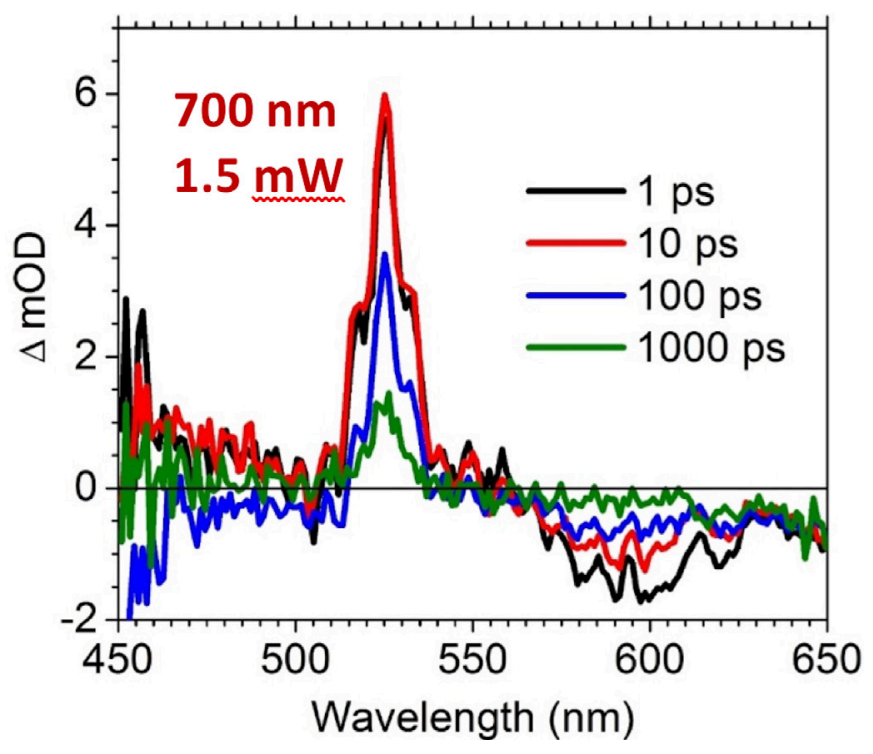

Figure 1. Pump power dependence of the parent band edge absorbance (unit of optical density) upon pumping the Co-induced absorption feature.

\section{PROPOSED FUTURE WORK}

The next generation of perovskite-based IB absorbers will utilize well-defined domains (ligated 2D slabs or quantum dots) with a relatively small bandgap within a sea of larger-bandgap bulk perovskite. These structures are analogous to the most successful IB PV to dateInAs quantum dots in a sea of GaAs. Achieving solvent co-solubility while maintaining bi-phase stability will require new ligand chemistry as well as new deposition approaches. In FY 2018, both TA and TCSPC of luminescence will be used to probe the carrier dynamics of the most promising composites. 
Efficient Droplet-Based Environmental Mechanical Energy Harvesting through Reverse Electrowetting

\section{6-063-R1}

Alexey Snezhko and Gasper Kokot

\section{PROJECT DESCRIPTION}

Microfluidic droplets exploiting the reverse electrowetting process promise a high areal energy harvesting efficiency. The method is based on generating electric energy by changing the overlap between the droplet and the electrode interface. The change in overlap is caused by a mechanical actuation coming from a vibration, shearing, or change in pressure.

The goal of the project is to develop and optimize a microfluidic-based environmental mechanical-energy harvester with higher efficiency compared to currently available mechanical actuation mechanisms. To maximize the efficiency of the portable droplet-based energy harvesters, optimal surface-to-droplet volume ratio for the electrodes, electrode patterning schemes, and energy-harvesting-layer stacking are being determined. Typical sizes of the droplets are being scaled down to the microscale. These advances will constitute an important step towards commercially viable droplet-based energy harvesting.

\section{MISSION RELEVANCE}

One of the primary missions of DOE is the development of novel renewable energy sources. Energy harvesting from mechanical movements opens up the possibility of extracting energy from vibrating car engines, buildings, bridges, or land next to a highway. Electricity gained in this way from dispersed local sources would lower the burden on the U.S. electric grid. On a smaller scale, the otherwise wasted mechanical energy from a walking person can also be harvested. Mechanical-energy harvesting can potentially provide a valuable portable energy source "on the go" for multiple mobile devices and applications, such as cell phones, laptops, and pacemakers. Current realizations of vibrational-energy harvesting either suffer from low efficiency or are unsuitable for large-scale production. There is a pressing need for innovations that would combine high-efficiency energy harvesting with the possibility of widespread implementation.

\section{RESULTS AND ACCOMPLISHMENTS}

We performed droplet generation tests by a T-junction micro-droplet generation method (apparatus shown in Figure 1) and concluded that the droplet size and spacing control provided by that method become challenging for narrow channels (below $50 \mu \mathrm{m}$ ). To provide better droplet generation control at the microscale, we redesigned the microfluidic channels towards a more complicated flow-focusing micro-droplet generation method. To stabilize the flow, we incorporated hydrodynamic damping channels for each inlet. We also performed tests with a non-toxic ionic liquid (a room-temperature liquid salt, 1-ethyl-3-methylimidayolium ethyl sulfate) as a conductive liquid to replace the liquid metal Galinstan, which is easily oxidized. Using silicon oil as a carrier solution, the method achieved stable droplet generation with channel widths down to $30 \mu \mathrm{m}$. The project is now at the stage of energy-generation testing and optimization.

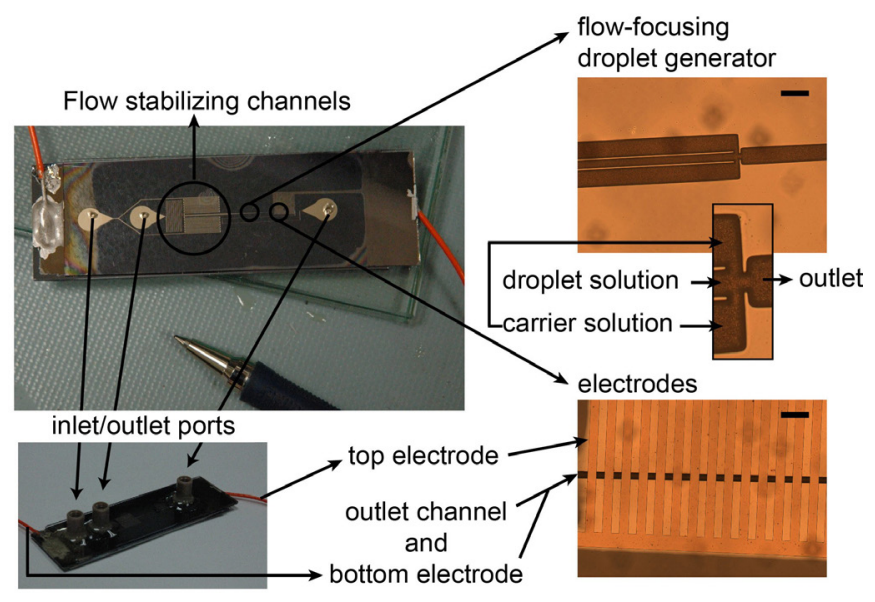

Figure 1. Microdroplet-based energy-harvesting device.

\section{PROPOSED FUTURE WORK}

In FY 2018 we will continue:

$\square$ Optimization of the channel and carrier solutions to minimize contact angle pinning and charge trapping in the channel with droplets.

$\square$ Channel design optimization for different mechanical-energy harvesting scenarios (vibration and bending).

$\square$ Development of a prototype high-volumetric (stack) energy harvester. 


\section{GO-IN-EM - Genetic algorithm Optimization of INterface structure from Electron Microscopy}

\section{6-069-R1}

Maria K. Chan, Fatih Sen, and Jianguo Wen

\section{PROJECT DESCRIPTION}

Solid-solid interfaces are important for materials properties in a wide variety of systems, yet the characterization of these interfaces via both experiments and computational modeling is challenging. In particular, the interfacial region is poorly imaged in electron microscopy, even if resolution is atomic away from the interface. In this project, we use a combination of atomistic modeling and multi-objective genetic algorithms, in conjunction with transmission electron microscopy (TEM) or scanning TEM (STEM), and computer-vision-based image matching algorithms, to solve this problem and produce three-dimensional (3D) atomistic structures of complex solid-solid interfaces.

\section{MISSION RELEVANCE}

Enhancing materials understanding, design, and development is central to DOE's energy security and scientific discovery missions. As use-inspired science, this work falls under the purview of DOE Basic Energy Sciences. This work improves materials characterization, including in situ and operando studies, by automating the construction of 3D atomistic models from two-dimensional (2D) electron microscopy. Such models will allow for rapid understanding of solid-solid interfaces and prediction of properties from the atomistic structure. The central theme of achieving rapid materials understanding and design is also a key focus of the Materials Genome and National Strategic Computing Initiatives.

\section{RESULTS AND ACCOMPLISHMENTS}

The key accomplishments in various components of the project include the following: our team (1) improved resolution in experimental TEM images using phase-contrast imaging; (2) performed parallel high-throughput computation of simulated TEM/STEM images from atomistic models, including a dataset covering all structure prototypes in the inorganic crystal structure database; (3) developed signature-based image matching algorithms, borrowing ideas from computer vision algorithms, which allowed us to quantitatively rank the similarity between simulated and experimental images; (4) developed and implemented the Python-porting of the "Interface Genie" code, which allows the building of initial grain boundary/ interface atomistic models having the correct boundary conditions away from the interface; and (5) developed a Python-based, modular multi-objective genetic algorithm and basin-hopping code that allows the iterative determination of atomistic structure from a combination of energies (from first principles or empirical potential calculations) and matching of the simulated image to a TEM/STEM image. The image matching, TEM/STEM simulation, and optimization codes have been used in concert to solve for the 3D structure of a CdTe grain boundary (Figure 1). With a straightforward addition to the modular code developed in this project, the combined energy-experiment optimization approach has also been applied to other experimental characterization techniques, most notably, X-ray pair distribution functions.

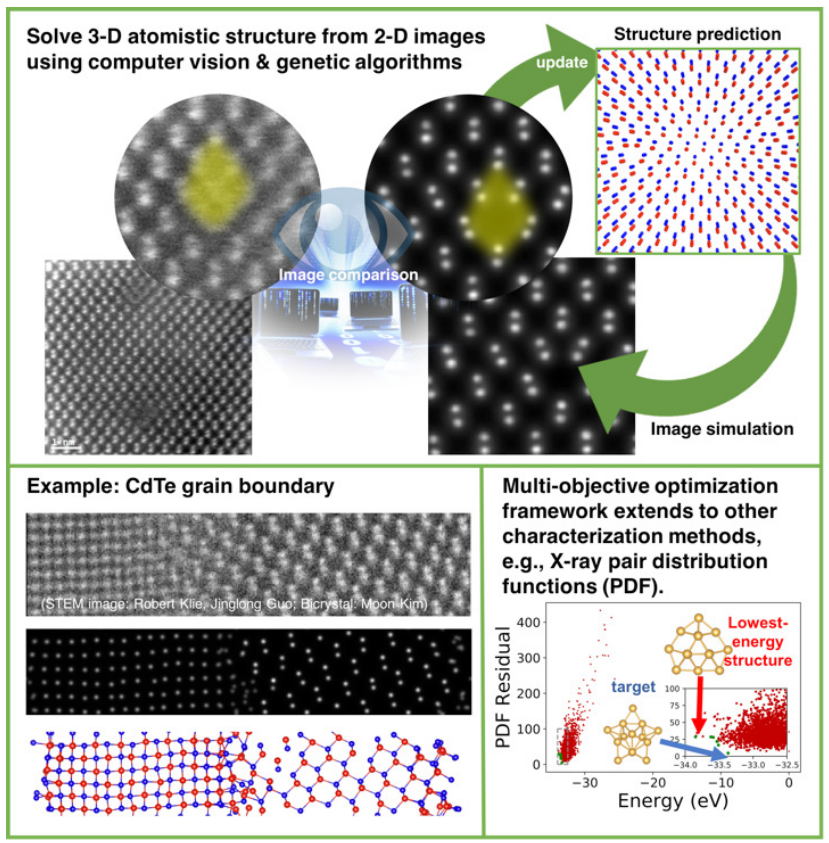

Figure 1. (Top) Approach of the GO-IN-EM project, which seeks to incorporate computer vision algorithms, atomistic simulations, and iterative optimization to produce 3D atomistic structures from 2D electron microscopy images of interfaces. (Bottom, left) An example is CdTe grain boundaries (image credit: Robert Klie, Jinglong Guo (UIC)). (Bottom, right) The approach extends to other materials characterization methods, including X-ray pair distribution functions.

\section{PROPOSED FUTURE WORK}

In FY 2018, we will further test the image similarity comparison code and complete the integration of Interface Genie with image comparison and multi-objective optimization codes. To treat a larger variety of interfaces, we will incorporate grand-canonical sampling into the codes, allowing variations in the composition at the interfaces. Testing of the codes will be carried out for oxide interfaces and embedded metallic nanoparticles. 


\section{Top-Down Fabrication of Large Area Monolayers of 2D Materials}

\author{
2016-082-R1 \\ Subramanian Sankaranarayanan, Mrinal Bera, \\ and Mathew Cherukara
}

\section{PROJECT DESCRIPTION}

The search for novel two-dimensional (2D) materials beyond graphene has attracted considerable attention because of their exotic physical properties, like the room-temperature quantum Hall effect, charge density waves, high-temperature superconductivity, superlubricity, and high carrier mobility, when the thickness is reduced to a monolayer or a few layers. Although these unique properties have led to prototype devices and applications, the eventual transition into commercial technologies requires large-area, scalable, and controllable growth of monolayers or a few layers. Fundamental insights into the growth and synthesis of 2D materials are needed to enable scalable and rapid formation of monolayer and single-crystal 2D materials. The state of the art relies heavily on chemical vapor deposition (CVD), which has recently been optimized to grow large-area 2D materials. The success of CVD is, however, dependent on the availability of precursors and optimized conditions like temperature, pressure, and air flow. As such, the CVD process is limited in scope. We are developing a new top-down electrochemical exfoliation method that would allow for synthesis of large-area monolayers of a broad class of 2D materials.

\section{MISSION RELEVANCE}

The proposed research is relevant to the low-power electronics area, which is a strategic energy-related mission of DOE. Success could open the doors for the industrial implementation of 2D materials that could find application in large screen displays for televisions and smartphones, tunable optical detectors, mechanical resonators, and even gas sensors. In the short term, the method developed in this project could be used in the design and fabrication of innovative nanoscale devices. In addition, the project could create opportunities for research into recently observed proximity effects for all 2D materials, which would be relevant to DOE basic science. The research may also be of interest to the National Science Foundation (NSF) program on Designing Materials to Revolutionize and Engineer our Future (DMREF) and the Semiconductor Research Corp. (SRC) program on Nanoelectronics COmputing REsearch (NCORE). In addition, it will likely be an important component of upcoming proposals for Energy Frontier Research Centers.

\section{RESULTS AND ACCOMPLISHMENTS}

During FY 2017, we have made remarkable progress (both experimental and theoretical) towards developing a very simple, cheap, fast, and scalable process for preparing large-area single-crystal monolayers of 2D materials, specifically dichalcogenides, based on electrochemical exfoliation. Simultaneously, using reactive atomistic simulations, we explored the growth of large-area 2D materials via both the CVD and electrochemical exfoliation methods. We have also performed detailed $X$-ray characterization of the 2D samples and continuum simulations to understand the effects of the synthesis route and underlying substrate on the structure (lattice strain, corrugations, etc.) and thereby the properties/ performance of devices utilizing these 2D materials. See Figure 1. Overall, our experimental and theoretical findings are laying the foundation for the first rational route to preparing practical quantities of monolayer, processable single-crystal and large-area 2D materials, facilitating the fabrication of 2D-based devices, and further catalyzing the use of layered 2D materials in batteries, supercapacitors, photovoltaics, catalysis, and environmental remediation.

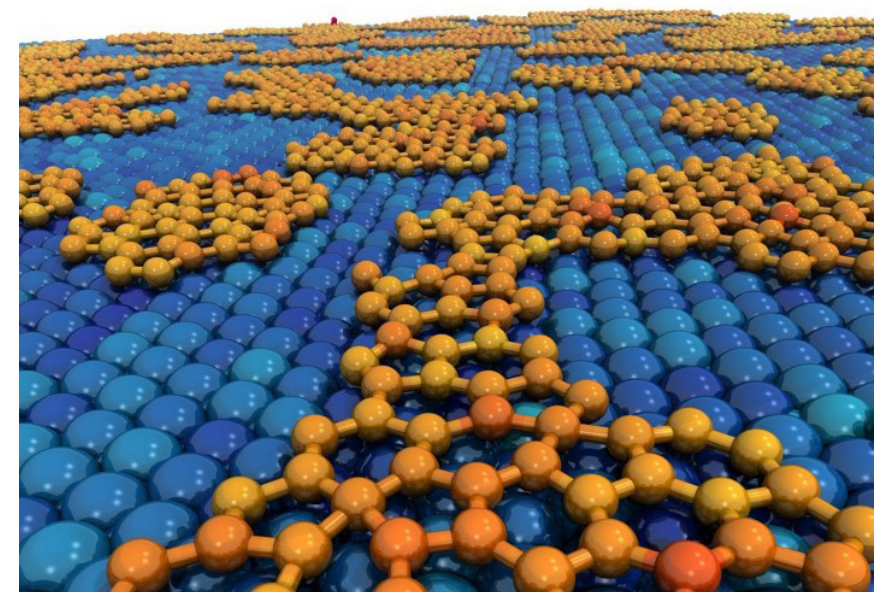

Figure 1. Large-scale molecular dynamics simulations performed for growth of silicene on an iridium substrate.

\section{PROPOSED FUTURE WORK}

This project ended in FY 2017. An important ongoing and future direction for this research will be to develop a machine-learning-based computational workflow tool for training force-fields (FFs) for 2D materials of interest. We anticipate that this will be one of the first large-scale computational 2D materials discovery tools that will combine first-principles physics with the strengths of state-of-the-art machine learning. 


\section{Spin-Vortex-Based Non-Volatile Superconducting Memory}

\author{
2016-092-R1 \\ Valentine Novosad and Sergi Lendinez
}

\section{PROJECT DESCRIPTION}

In recent years we have witnessed an explosion of high performance computing accompanied by the highest growth rate of energy consumption. The centralization of the computing in data centers and use of novel computational paradigms has opened up an opportunity to improve the energy efficiency. One particularly promising technology is superconducting computing, which offers the prospect of moving information without loss over zero-resistance channels. Development of novel cryogenic data storage elements could be an important step forward opening up new and exciting possibilities in this field.

In this project we are developing a hybrid memory architecture that marries the best features of classic magnetic memory with high-fidelity superconducting electronics. The concept has significant advantages over existing technologies. First, since this scheme relies on resonant spin excitations, it requires much lower power to alter the state of a memory element than the state-of-the-art spin-torque-based systems. Second, the dynamic switching is in the range of microwave frequencies, so pulses with these frequencies are readily available in the logic boards. Third, a readout scheme for a multiplexed superconducting quantum interference device (SQUID) makes the integration density scalable with the Q-factor of the cells and the reduced nanomagnet size (increased resonant frequency of vortex core oscillation). Finally, the memory is non-volatile up to the Curie temperature of the magnetic structure, i.e., above room temperature.

\section{MISSION RELEVANCE}

The scope of this proposal is well aligned with the mission of the DOE Office of Science to deliver scientific discoveries that transform our understanding of nature and advance the energy, economic, and national security of the United States. This proposal is also pertinent to the materials science and engineering programs of the DOE Office of Basic Energy Sciences, which encompass development of materials with novel structures, functions, and properties. Someday, superconducting computers could solve problems impossible for today's and even tomorrow's most powerful supercomputers.
The ability to design, develop, test, and implement novel superconducting memory architectures will be a major step forward, opening new and exciting possibilities for future R\&D on superconducting computing and pushing forward world-class science.

\section{RESULTS AND ACCOMPLISHMENTS}

Thus, the focus is on exploring the dynamic response and manipulation of spin vortices at low temperatures.

We performed extensive micromagnetic modeling and experimental work at room temperature. We established that core ordering could be achieved even when the excitation frequency is off resonance, provided the excitation amplitude is increased accordingly. This important finding confirms that some (inevitable) dot-to-dot variations in realistic memory arrays will not prevent us from selective "writing" or "reading" of the magnetic state via resonant excitation. We also found that the vortex core eigenfrequency will be influenced by the magnetic field screening effect from the superconducting strip line. In FY 2017 we explored how to control the resonance frequencies by, for example, altering the dot topography (e.g., without changing the overall geometry of the element). By using pre-patterned substrates, we obtained a strong geometric confinement effect, which was confirmed experimentally and numerically. This result is important, as it allows us to significantly expand the dynamic range of future memory cells.

Furthermore, several kinds of magnetic materials, including thin films of nanocrystalline Fe-Ni-Mo, amorphous Fe-Co-B, and single-crystal Fe-Co-Mn-Si Heusler alloy, were prepared. We developed an insert-type setup for low-temperature measurements of the ferromagnetic resonance. We designed and purchased custom SQUIDs and electronics for readout on a He-3 cryostat, and fabricated superconducting nanoloops on top of magnetic dots to detect the magnetic flux. Finally, we developed all the steps necessary to build our own Josephson junctions.

\section{PROPOSED FUTURE WORK}

In FY 2018 we plan to characterize the pickup nanoloops' sensitivity using the SQUID readout electronics at low temperatures. We will also start the fabrication of the array of elements and the readout scheme. Ultimately, we aim to optimize the operation of the readout scheme with new superconducting materials to achieve low electrical losses and better stability. We will verify the capability to select one element of the array by the combined application of two microwave pulses. Finally, we will combine the nanoloop readout with the chain of elements and verify the operation of the entire memory array. 
Ordered Core-Shell Nanostructure for Transverse Thermoelectric Applications

\author{
2016-094-R1 \\ Kaizhong Gao, Dileep Singh, Ana M. Aragon, \\ and Bahadir Kucukgok
}

\section{PROJECT DESCRIPTION}

For conventional thermoelectric (TE) materials, where heat flow and electric current flow are in the same direction, there is always a trade-off between a material's thermal conductivity (which needs to be low) and electrical conductivity (which needs to be high). The transverse thermoelectric effect (TTE) has attracted attention because of its unique mechanism, in which the electrical and thermal flows are perpendicular to each other. This project is exploring the design and materials for TTE devices. One can apply a thermal gradient and get a perpendicular electric current. In theory, this approach may enable more efficient applications for converting heat into electrical energy, if appropriate materials can be developed and the design can be optimized.

\section{MISSION RELEVANCE}

This project is directly aligned with DOE missions in energy and the environment, given that the technology will utilize wasted energy to generate new power and will have a beneficial environmental impact.

The most direct application of TTE devices is to build a thermoelectric generator (TEG). U.S. government funding agencies interested in self-powered sensorsan important application of TEGs-include the Defense Advanced Research Projects Agency, National Science Foundation, Defense Threat Reduction Agency, the DOE Office of Energy Efficiency and Renewable Energy, Advanced Research Projects Agency-Energy (autonomous vehicles program), and others. Many firms are also interested in this technology, including the automotive manufacturers (Ford, Tesla, etc.) that are working on autonomous vehicles. Moreover, conformal TEGs can provide near-limitless opportunities for energy generation in many engineering applications. More than $60 \%$ of the energy used in work and life activities is wasted as heat, yet technologies for thermal energy harvesting and recycling are grossly under-developed. The ubiquitous nature of waste heat offers limitless opportunities to design TEG devices for energy recovery at every level of human activity. One of the most outstanding opportunities is to design conformal TEGs for harvesting waste heat from vehicles, appliances, and consumer electronics, with conversion into electricity for self-powered devices.

\section{RESULTS AND ACCOMPLISHMENTS}

For this project, FeCo nanofibers with diameters of 25-30 nm were prepared using electrospinning and a two-step post-spinning heat treatment. The nanofibers were coated with $\mathrm{ZnO}$ by atomic layer deposition (ALD) to build a core-shell structure. The nanofibers were then aligned to create an ordered core-shell structure for TE measurements. Transmission electron microscopy (TEM) was performed on the core-shell nanofibers to evaluate the quality of the ALD film. The nanofibers form a thin-film layer (Figure 1). A test setup has been prepared for measurement of TE properties such as the Seebeck coefficient, electrical conductivity, and temperature-dependent carrier concentration.

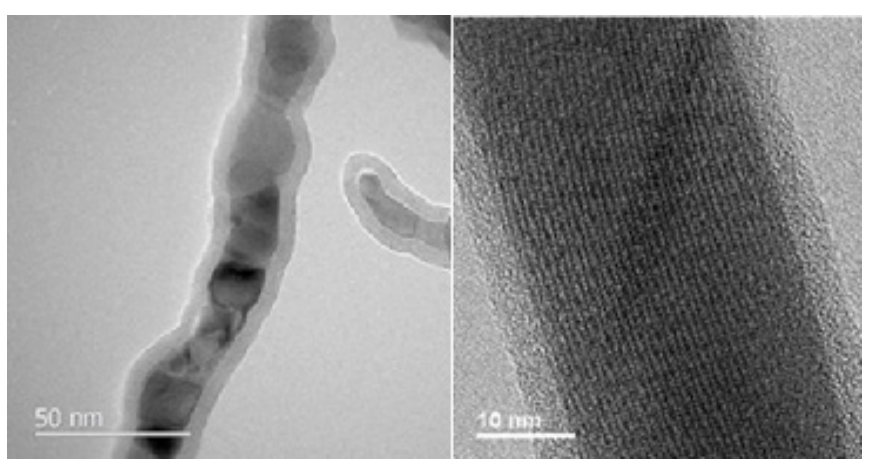

Figure 1. TEM images of FeCo nanofibers with a 7-nm ALD ZnO coating.

\section{PROPOSED FUTURE WORK}

Future work includes continuing to develop nanofibers with the above-described core-shell structure and to utilize those fibers to measure TE and electric properties, then looking into TEG design. The team, working in collaboration with Purdue University (Prof. Lu), will compare the initial results to conventional longitudinal TE properties, such as the Seebeck coefficient and thermal and electric conductivities. This approach will then be used to further explore potential applications of TTE devices. 


\section{Images from Inner Space: Exposing Quantum Mechanics within Nucleons and Nuclei}

\author{
2016-098-R1 \\ lan Cloët and John Arrington
}

\section{PROJECT DESCRIPTION}

Almost all the Universe's visible matter is constituted from protons and neutrons; but even after a century of striking progress in subatomic physics, an intimate understanding of these principal building blocks is still lacking. The challenge resides in quantum chromodynamics (QCD), the strong-interaction piece of the Standard Model of particle physics. A comprehensive approach to describing the internal structure of protons, neutrons, and other hadrons has recently emerged. This framework encodes our knowledge of hadrons and even nuclei in the Wigner distributions of the fundamental constituents (the quarks and gluons), which is a quantum mechanical concept analogous to the classical notion of a phase space distribution. From the Wigner distributions, a natural interpretation of measured observables is provided via construction of quantities known as generalized parton distributions (GPDs) and transverse momentum-dependent parton distributions (TMDs): GPDs are the key to a spatial tomography of hadrons, and TMDs allow for their momentum tomography. A new generation of experiments (at, for example, Jefferson Lab and CERN) will provide the empirical information necessary to develop a phenomenology of Wigner distributions. However, this work will only provide detailed information about the Standard Model's strong-interaction sector if these distributions are also calculated in a framework with a well-defined connection to QCD. The goal of this project is to fulfill this goal by directly calculating the tomography of the quarks and gluons inside hadrons and nuclei.

\section{MISSION RELEVANCE}

This project is relevant to the mission of the DOE Office of Nuclear Physics to discover, explore, and understand all forms of nuclear matter. To further this mission, Jefferson Lab underwent a $\$ 370$ million upgrade that doubled the energy of its electron beam from 6 to $12 \mathrm{GeV}$. Over $50 \%$ of allocated running time at the upgraded Jefferson Lab is dedicated to exposing the quark and gluon tomography of nucleons and nuclei. However, access to this tomography is not direct, and to develop these 3D images will require significant theoretical guidance, such as that undertaken as part of this project.

\section{RESULTS AND ACCOMPLISHMENTS}

Sub-atomic particles have an intrinsic quantum mechanical property called "spin," which can have integer or half-integer values, depending on the type of particle. The lightest bound state in QCD, the pion, has spin zero and, therefore, cannot be polarized, whereas protons and neutrons have spin $1 / 2$ and therefore have two polarization states, $\lambda= \pm 1 / 2$. The only (stable) strong-interaction targets with spin greater than $1 / 2$ are atomic nuclei, the deuteron being a very important example. To a good approximation, the deuteron is a bound state of one proton and one neutron and has spin equal to one. This means it has three polarization states: vector $(\lambda= \pm 1)$ and tensor $(\lambda=0)$ polarization. There are many facets of QCD that only appear in particles that have tensor polarization, e.g., new structure functions in deep inelastic scattering and three new leading-twist TMDs and GPDs.

For the first time, we derived the TMD structure of a spin-one target. This enabled the unambiguous identification of all 9 T-even spin-one TMDs. Using the Dyson-Schwinger-equations (DSEs), we then calculated each of these nine TMDs for the simplest spin-one target, namely, the $\rho$-meson. Figure 1 illustrates our result for a spin-one TMD associated with its tensor polarization. The unique structure, with a single maximum and two minima, is a consequence of non-zero orbital angular momentum components in the hadron wave function. Understanding orbital angular momentum is one of the most important goals at Jefferson Lab and the proposed Electron Ion Collider (EIC), and these results demonstrate that having access to targets with tensor polarization may be crucial to the realization of these goals.

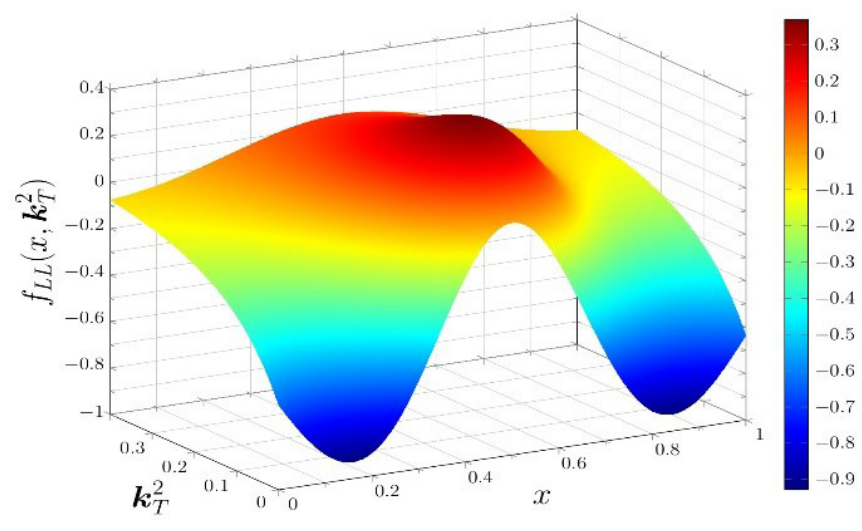

Figure 1. Illustration of a TMD associated with the tensor polarization of a spin-one target.

\section{PROPOSED FUTURE WORK}

With this detailed tomography of the deuteron, the focus now is to further extend the tomographic studies to other nuclei. These investigations will address questions that are at the very heart of QCD, for example, how do the strongest known forces in nature arrange partons within nucleons and nuclei? 


\section{Developing Superconducting $\mathrm{MgB}_{2}$ Films on Copper Radio- Frequency Accelerating Structures}

\section{7-002-NO}

Alireza Nassiri, David Mandia, and Angel Yangus-Gil

\section{PROJECT DESCRIPTION}

Magnesium diboride $\left(\mathrm{MgB}_{2}\right)$ is a promising

"intermediate-T $T_{c}$ " binary intermetallic superconducting material that could provide low surface resistivity at higher temperatures than niobium (Nb). The two-gap nature of $\mathrm{MgB}_{2}$ is reported by several studies. Experimental evidence indicates that a higher energy gap (6-7 meV) is dominant in the superconductivity of $\mathrm{MgB}_{2}$ and has a stronger coupling to phonons; lower energy gaps (1-4 meV) are less important to superconductivity and exhibit much weaker coupling. Using an energy gap of about $6 \mathrm{meV}$ (critical temperature of about $39.4 \mathrm{~K}$ ), a radio-frequency (RF) structure (cavity) coated in $\mathrm{MgB}_{2}$ film would have the same surface resistivity at about $8 \mathrm{~K}$ that a niobium cavity would have at $2 \mathrm{~K}$. The main objective of this research is to develop and demonstrate deposition of $\mathrm{MgB}_{2}$ thin films on bare copper or on niobium RF cavities employing plasma-enhanced atomic layer deposition (PE-ALD). We also aim to continue using hybrid-physical chemical vapor deposition (HPCVD) with improved coating processes for better surface quality and lower RF surface resistivity.

\section{MISSION RELEVANCE}

The project is relevant to the DOE mission in energy. If $\mathrm{MgB}_{2}$ films can be developed to achieve their full promise, significant cost savings would be possible in cryogenic cooling systems because it would be possible to cool with low-temperature helium gas instead of liquid helium. A typical cryogenic plant for $2-K$ cavity operation requires a complex refrigeration plant, vacuum pumping system, gas compressors, and gas storage tanks. A gaseous helium plant is far less complex and considerably less expensive. In addition, operating costs with gaseous helium cooling would be significantly lower than with liquid helium. Cavities fabricated out of copper with an $\mathrm{MgB}_{2}$ surface instead of $\mathrm{Nb}$ should be far less expensive than pure $\mathrm{Nb}$ cavities. For smaller installations, cryocoolers could eliminate the need to install a complete cryogenic plant. Our goal at the end of this project is to test, characterize, and report on the superconducting RF properties of $\mathrm{MgB}_{2}$ coated cavities.

\section{RESULTS AND ACCOMPLISHMENTS}

We successfully demonstrated that a uniform $\mathrm{MgB}_{2}$ coating with good superconducting properties can be achieved on the inner walls of commercially available copper tubes utilizing HPCVD. The copper tubes were not oxygen-free, so the outgassing of oxygen may have had detrimental effects on the film properties shown here (see Figures 1 and 2). This outcome can be avoided by using oxygen-free copper in future studies.

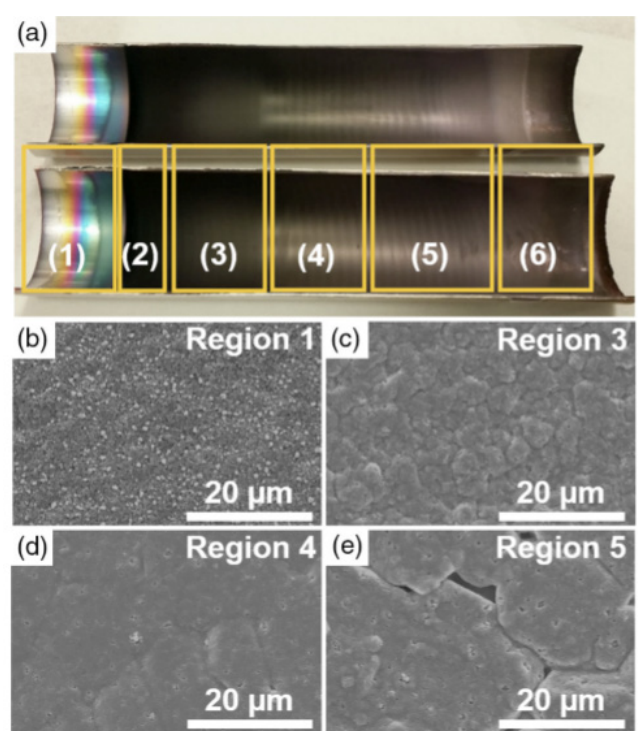

Figure 1. (a) Photo of $\sim 850-\mathrm{nm}$-thick, $\mathrm{MgB}_{2}$-coated $\mathrm{Cu}$ tube halves. (b)-(e) SEM images of $\mathrm{MgB}_{2}$ on $\mathrm{Cu}$ samples cut out from regions 2, 3, 4, and 5 , respectively.

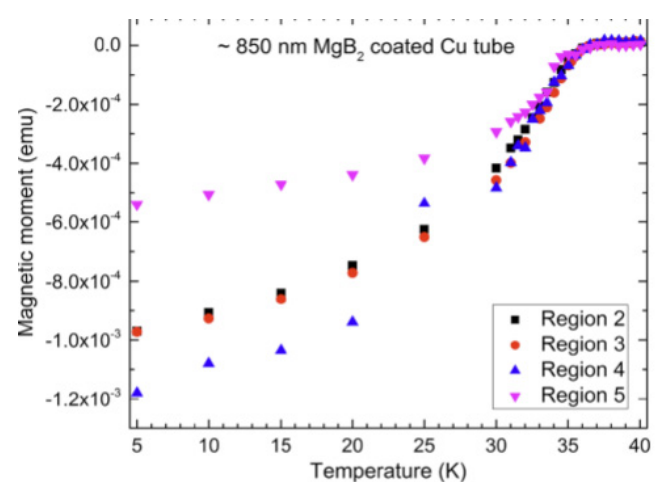

Figure 2. Magnetic moment vs. temperature curves of samples from regions 2, 3, 4, and 5 of a $\sim 850-n m$-thick, $\mathrm{MgB}_{2}$-coated Cu tube.

\section{PROPOSED FUTURE WORK}

We plan to demonstrate $\mathrm{MgB}_{2}$ coatings on 2-inch-diameter copper samples utilizing both thermaland PE-ALD. We will measure these samples to determine whether their surface properties are suited for superconducting RF structures. We will then apply the ALD technique to coat the interior of a $3-\mathrm{GHz}$ copper resonant structure suitable for a superconducting RF accelerator. 


\section{The Missing Link in XTIP: Synergy of Experiments and Theory for Argonne's Global Leadership}

\author{
2017-004-NO \\ Volker Rose, Larry A. Curtiss, Saw Wai Hla, Anh Ngo, \\ and Nozomi Shirato
}

\section{PROJECT DESCRIPTION}

We are exploring experimental and theoretical methods to develop new capabilities for the future XTIP beamline at the Advanced Photon Source. In particular, we are focusing on local three-dimensional (3D) molecular imaging assisted by X-ray standing waves (XSWs), which cannot be achieved by any other technique available today.

\section{MISSION RELEVANCE}

This project is relevant to DOE's basic science mission. Despite the great experimental accomplishments of Argonne's Synchrotron X-ray Scanning Tunneling Microscopy (SX-STM) program, the lack of comprehensive theoretical exploration seriously jeopardized the progress of unique SX-STM research. This project aims to overcome this limitation by germinating a strong synergy between experimental and theoretical SX-STM research, and to enable intimate collaboration between experimental and theoretical groups in three different Argonne divisions.

\section{RESULTS AND ACCOMPLISHMENTS}

We developed theoretical concepts that further our understanding of the fundamental mechanisms in SX-STM, particularly in combination with XSWs. Our novel code utilizes dynamical X-ray diffraction theory, together with density functional theory (DFT), to enable accurate atomic simulation models. We applied the code to study the herringbone reconstruction of $\mathrm{Au}(111)$, as well as Co nanoclusters on Au(111). In addition, we used multilayers and sub-monolayers of iron (Fe) and cobalt (Co) on gold (Au) single crystals to study the localization of SX-STM data. For example, Figure 1 shows the results of XSW fingerprinting a Co multilayer film on Au(111). It provides the first theoretical description of experimental XSW observations in SX-STM. Such measurements have not been reported in the literature so far. First, we carried out $a b$ initio spin-polarized DFT calculations to determine the theoretical structure of Co layers physisorbed on the $\mathrm{Au}(111)$ surface. The calculations predict an average Co-Au distance at the interface of $0.27 \mathrm{~nm}$. The Co layers adopt a hexagonal close-packed structure. A ferromagnetic phase then emerges, driven by the lattice mismatch that enhances the magnetic moment of the Co atoms. In order to entangle the SX-STM signal of Co and the $\mathrm{Au}(111)$ substrate, our code simulates the normalized photoelectron yield for the atomic-scale emission. This treatment makes the identification of $\mathrm{Co}$ and Au peaks possible in the experimental data. Furthermore, the adsorption geometry can be derived from this analysis. In addition to the high spatial resolution perpendicular to the sample surface (also achieved in conventional XSW studies), SX-STM provides ultra-high spatial resolution in the $x$ - and $y$-dimensions.

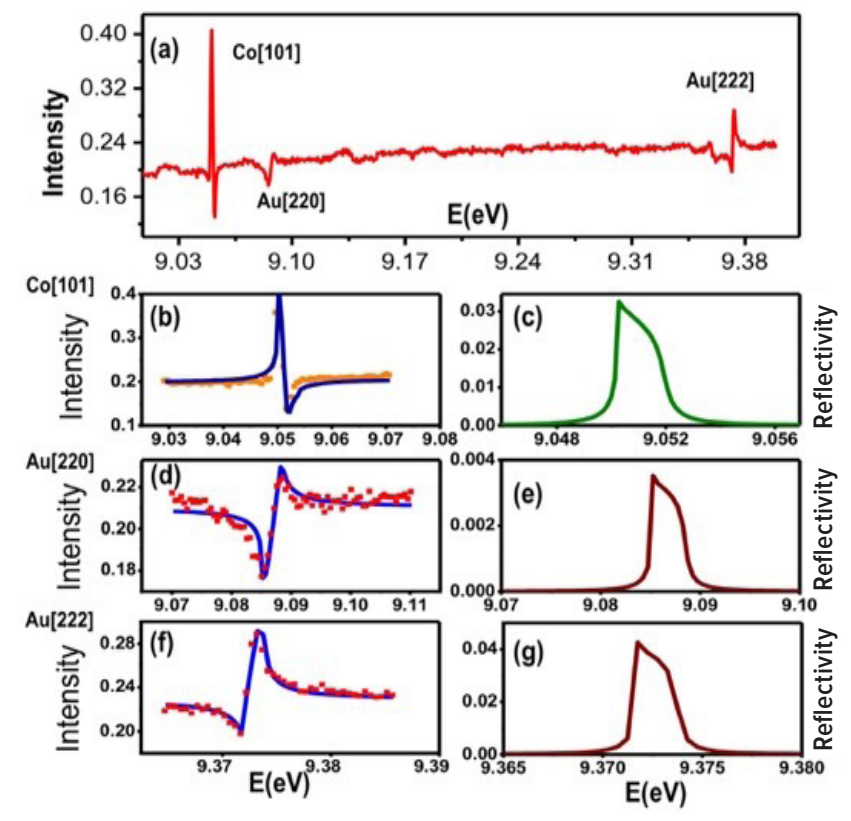

Figure 1. (a) Measurement (red line) of XSW peaks as a function of incident photon energy of a Co multilayer film on Au(111). (b) Co intensity peak, and (d, f) Au intensity peaks. The blue curves show the theoretical calculations using our novel code. The red squares represent experimental data obtained with our SX-STM system at the Advanced Photon Source. (c, e, g) Corresponding theoretical reflectivity calculations.

\section{PROPOSED FUTURE WORK}

Experimental and theoretical work will extend to the study of molecules. We already demonstrated the power of our theoretical framework utilizing Co nanoclusters that served as a model system for smaller molecules. Now, we will grow TBrPP-Co molecules on $\mathrm{Au}, \mathrm{Cu}$, and Ag substrates. The goal is to pinpoint individual Co atoms inside of single molecules, one molecule at a time, and investigate the quantum properties up close. After establishing the sample preparation for the molecular system under ultra-high vacuum conditions, we will improve the fabrication of XSW generators that consist of multilayer films. Single crystals and multilayer films will allow us to extend our experiments to a wide range of $X$-ray photon energies from a few hundred electron volts to several kilo-electron volts. 


\section{A Novel Method of Longitudinal Bunch Shaping by Double-Emittance Exchange}

\author{
2017-007-NO \\ John Power and Yine Sun
}

\section{PROJECT DESCRIPTION}

We are exploring a method to suppress the coherent synchrotron radiation (CSR) effect in an emittance exchange (EEX) beamline. CSR is known to be the main limiting factor of precision longitudinal bunch shaping (LBS), which is an enabling method for advanced accelerator physics. In order to study this method we are installing a double-EEX beamline, composed of two EEX beamlines, equipped with FODO (Focus-DriftDefocus-Drift) beam optics in the middle.

\section{MISSION RELEVANCE}

A double-EEX beamline enables precision LBS and is a gateway technology for a host of accelerator applications relevant to the DOE's science mission, including high-energy physics, basic energy science, and the National Nuclear Security Administration. Applications relevant to OHEP mission to develop a future linear collider include highly efficient dielectric wakefield acceleration and plasma wakefield acceleration. Applications relevant to OBES mission to develop future radiation source for coherent $X$-rays include high-power terahertz radiation; efficient bunch compressors and stretchers; microbunch trains for harmonic generation; frequency upshifting and downshifting of radiation; and others.

\section{RESULTS AND ACCOMPLISHMENTS}

As the first year of this project was the construction phase, our accomplishments are primarily hardware oriented. (1) We finalized the designs of variable-gap vacuum chambers and associated diagnostics needed to study CSR suppression. This required a trade-off study between CSR suppression vs. beam transmission. The results of this study are shown in Figure 1. (2) We designed a high-precision emittance diagnostic system based on emittance slits to allow us to study the CSR suppression. We also completed the final design of the double-EEX beamline (Figure 2). We are currently fabricating all of this equipment and expect to install the beamline in FY 2018.

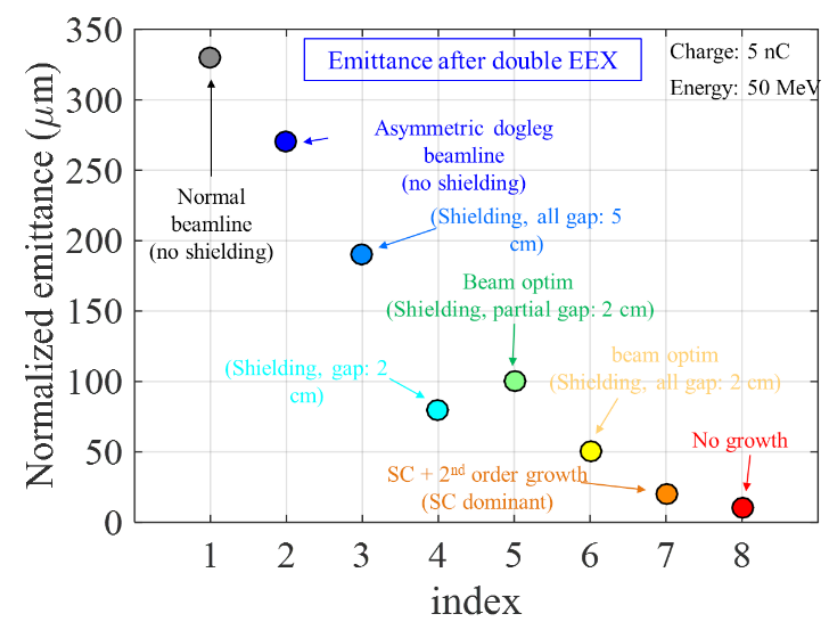

Figure 1. Study to reduce emittance growth by CSR suppression.

\section{PROPOSED FUTURE WORK}

The second year of the project will be the experimental phase. Our next steps are to (1) install the double-EEX beamline in the Argonne Wakefield Accelerator (AWA) bunker; (2) commission the beamline; and (3) begin the CSR study. CSR suppression has previously been studied in chicanes; however, our project will be the first to study CSR suppression in a phase space rotation regime.

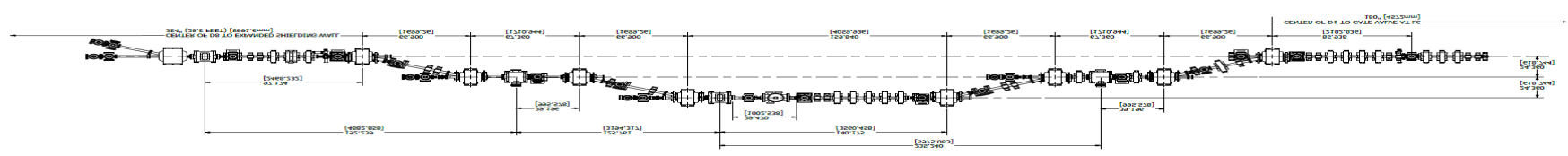

Figure 2. AWA double EEX layout. 


\section{Integrating High-Throughput Computation and Wet-Chemistry Synthesis for Functional Supercrystals}

\author{
2017-012-NO
}

Badri Narayanan, Wei Jiang, Xiao-Min Lin, Yuzi Liu, Subramanian Sankaranarayanan, and Elena Shevchenko

\section{PROJECT DESCRIPTION}

The aim of this work is to design and synthesize periodic arrays of nanoparticle (NP) building blocks (supercrystals, or SCs) with enhanced functionality for a wide variety of applications in water filtration, energy, electronics, and other devices. To achieve this, we integrate computational materials design via classical models, machine learning, wet-chemistry synthesis, and state-of-the-art X-ray and electron characterization. Our overarching goals are to (1) gain a fundamental understanding of the various physical processes controlling the self-assembly of NPs, and the morphology and symmetry of the formed SCs; (2) identify NP/ligand combinations, and the experimental conditions best suited to obtaining a specific structural organization in the final SC; and (3) understand the role of ligand dynamics in governing the thermo-mechanical response of SCs. This effort will provide design principles to engineer novel ordered NP architectures with prescribed mechanical, structural, thermal, and chemical properties.

\section{MISSION RELEVANCE}

This project is relevant to DOE's basic science mission in materials discovery design and synthesis, and in controlling matter and energy at the nano- and molecular scales. The project offers a unique opportunity to advance the field of nanoscale meta-materials synthesis, and to enhance soft-materials research to solve energy challenges.

\section{RESULTS AND ACCOMPLISHMENTS}

During FY 2017, we gained significant molecular insights into thermal and mechanical properties of gold (Au)-based NP superlattices, in both two and three dimensions. We synthesized free-standing membranes (monolayers) of colloidal Au NPs (<10-nm thick) with remarkable stiffness. We found that these membranes remain strong and resilient up to unprecedented high temperatures. Our molecular dynamics simulations revealed that this improved resilience is due to temperature-induced changes in ligand arrangement around NPs (Figure 1). In the as-synthesized membranes (at $7^{\circ} \mathrm{C}$ ), the ligands are asymmetrically distributed at the air-water interface. Upon heating the membrane to $\sim 87^{\circ} \mathrm{C}$, the low ligand coverage and high mobility cause the ligands to distribute evenly. This uniform ligand distribution persists upon cooling and subsequent heat-cool cycles; thereby, making the membrane resilient to temperature changes. Large-scale coarse-grained molecular dynamics simulations also elucidated the effect of ligand coverage and dynamics on the response of three-dimensional Au SCs to compression-release cycles. At high ligand coverage, the SC exhibit no hysteresis in structure (i.e., lattice constants) during compress-release cycles due to reversible re-organization of ligands (i.e., compaction and restoration). On the other hand, low coverage results in permanent re-organization of ligands into localized patches, which causes significant structural hysteresis.

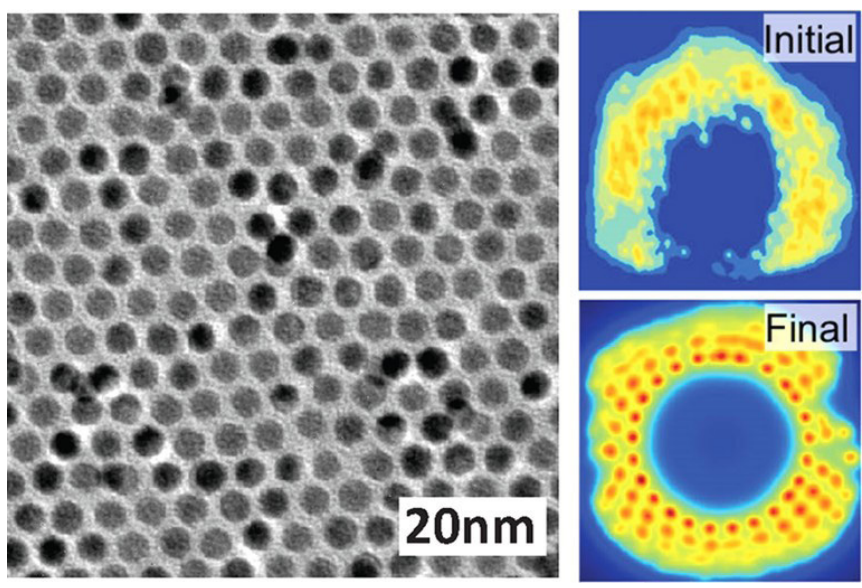

Figure 1. Left: Transmission electron microscopy image of the synthesized freestanding NP monolayer. Right: Distribution of ligands around a typical NP before (initial) and after (final) the heat-cool cycle.

\section{PROPOSED FUTURE WORK}

Our work illustrated the key role played by ligand coverage and ligand dynamics on the structure of SCs in different environmental conditions (e.g., temperature, applied pressure). This creates the opportunity to explore new SC symmetries that can be produced by tuning the self-assembly conditions. Our next steps include: (1) synthesizing SCs with different symmetries and pressure response and characterizing using small-angle X-ray spectroscopy; (2) developing accurate coarse-grained models for various ligand systems;

(3) building automated workflows to interface our machine learning predictive framework with popular molecular dynamics packages LAMMPS and NAMD, which are commonly employed for soft matter systems; and (4) leveraging machine learning-based sampling, and atomistically informed accurate coarse-grained descriptions to identify the various Au NP supercrystal symmetries that form at different NP sizes and ligand coverages. 


\section{Atomic Layer Deposition of Silicon Carbide for Nuclear Applications}

\section{7-013-NO}

Zhi-Gang Mei, Sumit Bhattacharya, Aaron Oaks, Abdellatif M. Yacout, and Angel Yanguas-Gil

\section{PROJECT DESCRIPTION}

This project focuses on theoretical design and computational screening of precursors for atomic layer deposition (ALD) of a silicon carbide (SiC) coating for accident-tolerant nuclear fuel claddings. Nuclear claddings protect the fuel from the surrounding environment and are critical to the severe accident tolerance level of a reactor. An SiC coating by ALD has great potential for survival in an environment created by severe accidents. However, there is no known method for performing ALD of $\mathrm{SiC}$ at low temperatures. Our goal is to develop a computational approach to identify novel chemical precursors for achieving ALD of SiC, optimize deposition conditions, and validate proposed precursors and reaction routines using designed experiments. The developed computational approach can be applied to identify novel precursors for other technically important coatings, as well.

\section{MISSION RELEVANCE}

This project supports DOE's missions in energy and national security. The development of accident-tolerant nuclear fuel claddings using ALD of SiC coating addresses-at a fundamental level-challenges in nuclear energy that are directly related to the current grand challenge of realizing sustainable energy. This project explores the feasibility of ALD of $\mathrm{SiC}$, a technologically important material with various applications. Achieving the successful ALD of $\mathrm{SiC}$ at low temperatures could significantly increase the number of materials that could be deposited using this method.

\section{RESULTS AND ACCOMPLISHMENTS}

The major goal of this project is to discover promising chemical precursors for ALD of SiC using high-throughput computational screening. The following is a summary of the major accomplishments in FY 2017. First, we constructed a large chemical reaction database of silicon- and carbon-containing molecules with chemical properties calculated using density function theory (DFT). From the high-throughput calculations of the reaction energetics, we identified promising silicon and carbon precursors. We then performed detailed DFT calculations to investigate the ALD reaction mechanism and surface reactivity of these precursors. Calculations show that some of the down-selected precursors can form stable $\mathrm{Si}-\mathrm{C}$ bonds during the ALD reactions. With the chemical reaction database, we applied machine learning to develop a chemical reaction prediction model using a novel fingerprint for molecules. The developed model can be used to screen the chemical databases, such as the PubChem database, to explore a wider chemical space for more promising precursors. Second, we developed a multiscale model to simulate the infiltration of tubular arrays coated with highly porous materials. Through multiscale simulations, we explored the scale up of the infiltration of tube claddings using both self-limited (ALD) and non-self-limited processes (chemical vapor deposition). Figure 1 shows that the large surface area of the porous cladding tube greatly enhances the effective surface reactivity though effective reaction probability. Our goal is to predict the scalability of the coating process beyond our existing experimental capabilities, providing metrics such as throughput and precursor utilization as a function of the fundamental parameters of growth chemistry. Third, we designed and constructed a brand-new ALD setup to validate the down-selected precursors from high-throughput computations.

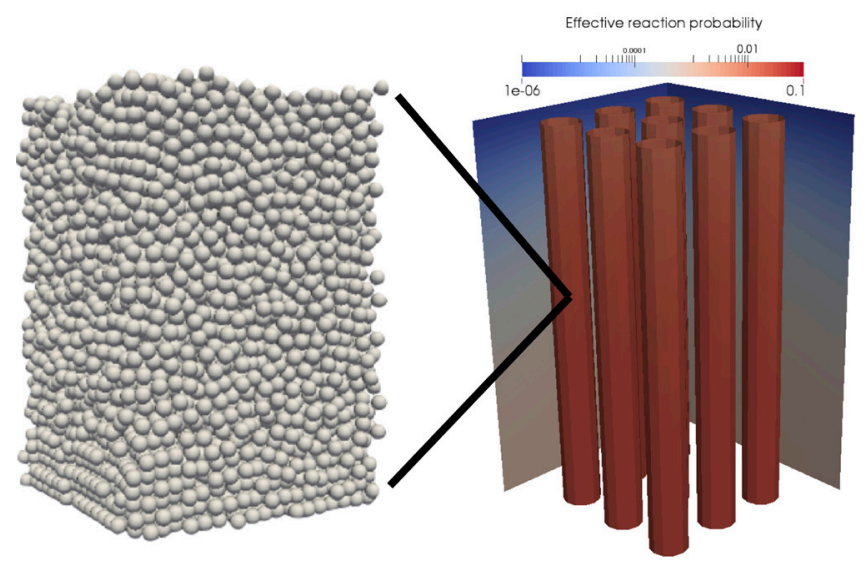

Figure 1. Through multiscale simulations by integrating transport at the nano- and reactor scales, we explored the scale-up of the infiltration of tube claddings using both self-limited and non-self-limited processes.

\section{PROPOSED FUTURE WORK}

In FY 2018, we will continue to develop chemical reaction prediction models and apply machine learning to screen the available chemical database for the most promising precursors. We will also develop kinetic Monte Carlo (kMC) models for ALD reactions using the energetics and kinetics from DFT calculations, and we will develop reactor- and feature-scale simulations of the ALD process by interfacing with the developed kMC models. Finally, we will design experiments to verify the proposed deposition conditions and ALD reaction routes. 


\section{BOLT: OpenMP over Lightweight Threads}

\author{
2017-016-NO \\ Pavan Balaji and Abdelhalim Amer
}

\section{PROJECT DESCRIPTION}

OpenMP (Open Multi-Processing) is the most widely used programming system on shared-memory multicore and many-core computers, including DOE supercomputers. Going forward, applications will need to express fine-grained levels of parallelism in order to harness the increasingly massive hardware concurrency and to interoperate well with MPI (Message Passing Interface), the predominant internode programming model on these systems. Existing OpenMP implementations, such as those provided by the Intel ${ }^{\oplus}$ commercial compilers or the open-source GNU and LLVM ones, however, do a poor job of handing fine-grained parallelism and interoperation with other programming systems, such as MPI. This inefficiency is the product of relying on heavyweight operating system (OS)-level threads (such as Pthreads) underneath, and thus incurs expensive thread management costs. We proposed BOLT, an OpenMP runtime that overcomes these issues by leveraging an extremely lightweight threading system. Under BOLT, traditional coarse-grained parallel applications perform well, and fine-grained parallel applications perform significantly better than when running under other OpenMP runtimes, including commercial ones.

\section{MISSION RELEVANCE}

Several DOE, U.S. Department of Defense (DOD), and National Science Foundation (NSF) applications rely on OpenMP directly or indirectly (through external dependencies, such as linear algebra libraries) to harness the power of parallel computing systems and reduce the time to solution of critical problems. Thus, an efficient OpenMP implementation is extremely important for the success of several government missions. In particular, the vast majority of applications from the DOE Exascale Computing Project depend on OpenMP for shared-memory parallel execution and on MPI for internode communication. Thus, BOLT can help improve the efficiency of a large number of applications across many government agencies.

\section{RESULTS AND ACCOMPLISHMENTS}

We designed and implemented a BOLT prototype and performed initial correctness and performance testing. BOLT shows promising preliminary results in terms of robustness and performance. Specifically, BOLT significantly outperforms the competitor Pthread-based Intel and GNU OpenMP runtimes. For instance, our evaluation of a popular and fast multiple-method implementation, which generates nested parallelism by offloading linear algebra operations to Intel MKL (Math Kernel Library), performs roughly three times better when using BOLT instead of the original Intel OpenMP runtime (Figure 1). From a robustness perspective, BOLT can be considered production quality: it successfully passes most of the OpenUH test suite; real-world applications such as the Accelerated Climate Modeling for Energy (ACME) application have reported using BOLT successfully; and BOLT has been deployed on large-scale production systems such as Theta at Argonne National Laboratory.

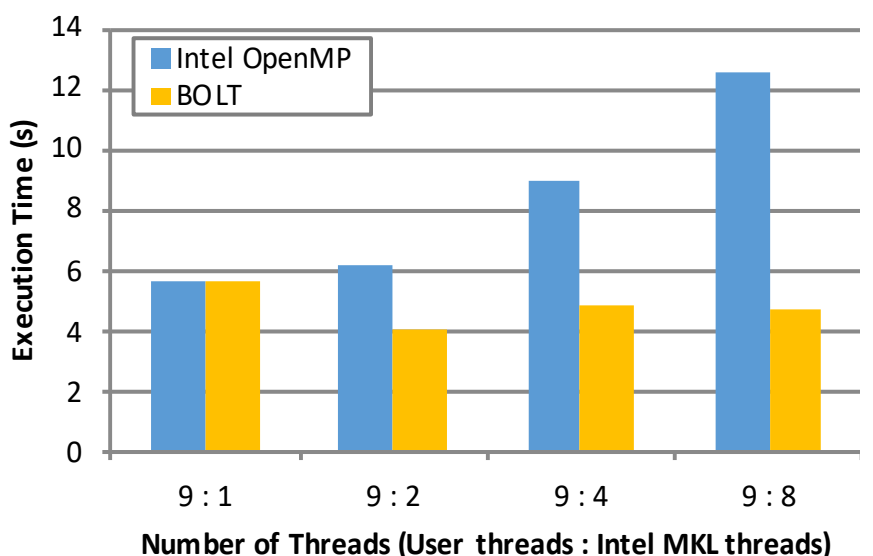

Figure 1. Performance comparison of BOLT against the Intel OpenMP runtime on a 36-core Intel Xeon machine with a real-world nested parallelism case. The application being used is an implementation of the fast multipole method that offloads linear algebra routines to Intel MKL, which also uses OpenMP internally.

The BOLT compiler and runtime source codes are available online. The results of our investigations were reported in a paper published in the prestigious journal IEEE Transactions on Parallel and Distributed Systems. Furthermore, several technical and keynote talks about BOLT have been given in various venues during the past fiscal year.

\section{PROPOSED FUTURE WORK}

We plan to enhance BOLT over the next fiscal year with advanced performance optimizations at the compiler and runtime levels. We are conducting more rigorous evaluations of BOLT with benchmarks and applications, and we will work with end users to obtain performance feedback. We also plan to improve the interoperability layer between BOLT and MPI and potentially propose extensions to the OpenMP standard to alleviate fundamental issues that implementations cannot overcome. 


\section{A Continuously Refinable Mesh, Limited-Area Atmospheric Model}

\author{
2017-017-NO \\ Jiali Wang, Emil Constantinescu, Iulian Grindeanu, \\ Robert Jacob, Veerabhadra Kotamarthi, and Vijay Mahadevan
}

\section{PROJECT DESCRIPTION}

This project is developing a refinable mesh that extends from the mesoscale to the microscale over a limited area. It will provide a test bed for understanding the climate and earth system and the relationship between climate change and human life, as well as ecosystems. This effort will also significantly reduce required computing resources and allow researchers to focus on small areas at a very high spatial resolution while providing details of the regional scale flow.

Prediction of severe weather, development of wind and solar energy sources, and assessments of infrastructure vulnerability all require numerically stable and computationally efficient models of the earth system, from mesoscale (kilometers) to microscale (meters). Current earth system models that attempt to capture mesoscale to microscale interactions primarily use a nesting approach, in which one or more higher-resolution domain(s) are placed inside a coarser resolution domain. For example, achieving a refinement of a factor of 10 (from $1 \mathrm{~km}$ to $100 \mathrm{~m}$ ) will require approximately three levels of nesting. Each nest introduces a new numerical boundary and loss of information across this boundary.

We propose to develop a code base that bridges the scales from mesoscale to microscale using a continuously refined mesh that can allow for local refinement from kilometers and scales with well-established physics parameterization to meters, where the flow is completely resolved without the need for nesting. The National Center for Atmospheric Research and the DOE are developing models that have such capabilities (e.g., Model Prediction Across Scales (MPAS) and the Energy Exascale Earth System Model [E3SM]). However, these are global-scale models. Running global models at very high resolution is computationally expensive. This project focuses only on the area of interest and allows continuous refinement from kilometers to meters using much less costly computational resources. The decrease in computational burden may support improvements such as longer simulation run times and the addition of members within ensembles.

\section{MISSION RELEVANCE}

The DOE research community can apply this modeling system to address DOE missions, such as energy security and environmental quality. It will provide knowledge related to environmental issues to inform decision makers for climate risk management.

There is an urgent need to develop cross-scale capabilities for simulating atmospheric phenomena, and in particular the impacts of climate change on human life and ecosystems.

\section{RESULTS AND ACCOMPLISHMENTS}

We adapted an existing mesh refinement algorithm to create grid refinements for the MPAS-Atmosphere over the continental United States (CONUS) (Figure 1). We worked with E3SM, focusing on variable-mesh methodologies to assist E3SM in reducing model bias over the refined area by smoothing the boundary conditions of the refined mesh. We set up and built three E3SM cases to investigate the value added by high-spatial resolution and data assimilation over the refined region. We found that, although the refined mesh does not affect global circulation, it does improve model performance over the refined area, especially for orographic forced fields such as precipitation and clouds.

This project considers the CONUS only for mesh refinement, but the approach developed can be applied flexibly to any other regions in which E3SM is interested.

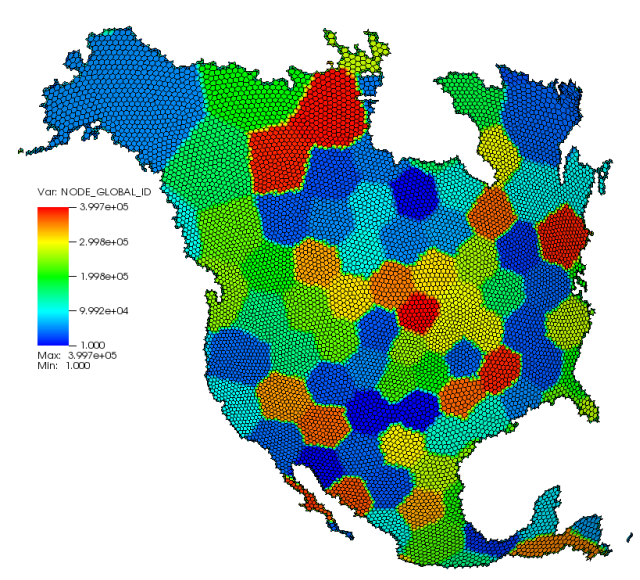

Figure 1. A regional refined mesh generated using a parallel meshing algorithm over land that we developed based on an existing approach used by MPAS-Ocean.

\section{PROPOSED FUTURE WORK}

We will implement our generated mesh into an existing solver and develop a boundary description package for limited-area MPAS. We will explore smoothing techniques by introducing observations to the boundaries of the refined domain for E3SM. 


\section{Bonding Dissimilar Materials Using Nanoparticles/Nanofilm as Eutectic Compounds}

\author{
2017-019-NO \\ Kamlesh Suthar, Lahsen Assoufid, H. Christopher Fry, \\ and XianRong Huang
}

\section{PROJECT DESCRIPTION}

We are working to develop a virtually stress-free bonding technique, using low-melting-temperature nanoparticles and/or nanofilms of reactive metals as a source of heat and intermetallic compound for bonding purposes. The proposed bonding methods can reduce or eliminate residual stresses in next-generation X-ray optics, accelerator components, and power-electronics packaging applications, enabling them to withstand higher operating temperatures. The proposed techniques will utilize two distinctive characteristics of nanomaterials: (1) their tendency to melt at much lower temperatures than corresponding bulk materials and (2) their aggressive reactivity that can generate the localized high temperatures required for bonding. Nanomaterials' lower melting and bonding temperatures can reduce or eliminate residual stresses generated in the bulk material while bonding two materials with different thermal expansion coefficients. The nanoscale materials will impregnate within the material and the new bond will assume properties of the bulk material, including its higher melting temperature. The high reactivity of nanomaterials can create high temperatures via reactions between incompatible mixtures of nanomaterials. Because the reaction is quick and local, it can reduce the amount of heat released into the bulk material. This significant reduction in heat intake reduces the degree of recrystallization, strain, and stress effects that occur during bonding. Bonds can be formed via coalescing of eutectic compounds of the nanoparticles or nanofilms and the bulk constituent materials.

For the $\mathrm{X}$-ray optics to efficiently diffract $\mathrm{X}$-rays and preserve the incident $X$-ray beam wave-front, it is critical that crystal lattice distortions be minimized. Efficient cooling can reduce distortions in the X-ray optics. The proposed techniques can produce bonding in less than a second, so the bonding can be achieved without heating the entire $X$-ray optics assembly. Our research could result in a dramatic improvement of optics behavior via efficient thermal management.

\section{MISSION RELEVANCE}

The proposed technique supports the DOE science mission and would have wide-ranging applications at
DOE laboratories like Argonne, as well as in industry. Components fabricated using this technique could have a significantly larger safety margin, allowing for future increases in operating parameters. There is a growing demand for efficient and economical packaging of power electronics, and high-temperature, high-power, wide-bandgap semiconductor devices based on SiC (silicon carbide) and GaN (gallium nitride) that could create further interest in the kind of bonding techniques that we will develop. Reported breakthroughs in very-low-temperature brazing or localized high-temperature bonding using nanoparticles or reactive nanomaterials can address both environmental concerns and cost reduction.

\section{RESULTS AND ACCOMPLISHMENTS}

There are two major achievements in the last year:

(1) bonding of Si crystal with copper plates, and

(2) production of curved mirror surfaces (we have filed a patent based on this success). Figure 1 shows a successful bonding of a Si crystal with two copper plates.

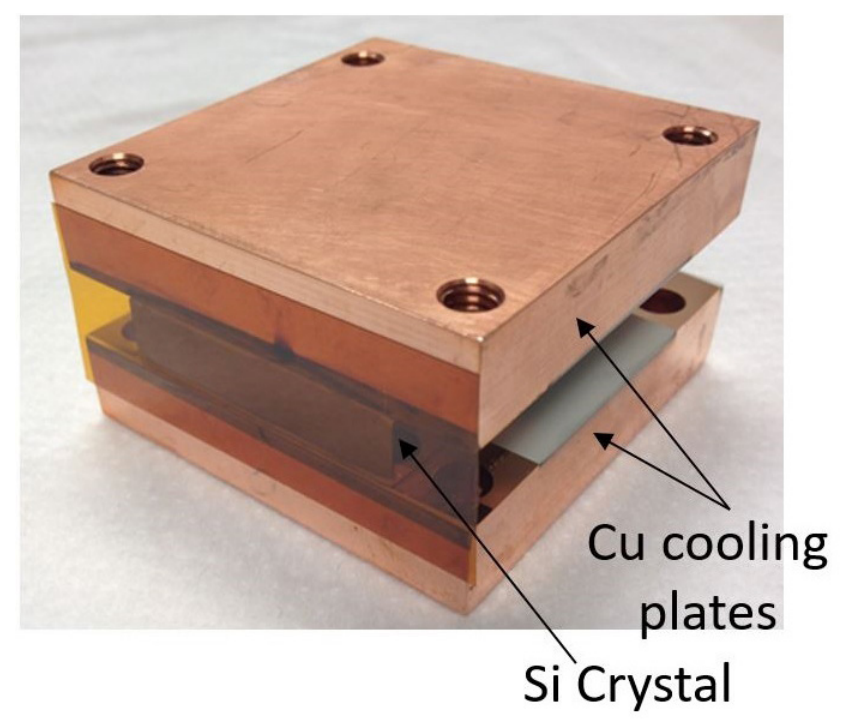

Figure 1. Examples of bonded optics using nanobonding technique.

\section{PROPOSED FUTURE WORK}

In FY 2018, we will investigate the shapes and forms of nanomaterials that can be applied to joining components of any shape and to any combination of materials. This will allow us to bond materials at the room temperature and onsite without elaborative setup such as used for the brazing/welding purposes. We continue working toward producing energetic nanomaterials that can act as a heat source for the bonding - allowing it to spread over complex shaped joint. The team is also working on the utilization of porous nanomaterials at the interface that can enhance bonding via facilitating the melt materials to flow in porous structure throughout the bonding interface. 


\section{Engineered Interfaces for Gallium Oxide Power Semiconductor Devices}

\author{
2017-022-NO \\ Angel Yanguas-Gil
}

\section{PROJECT DESCRIPTION}

Gallium oxide $\left(\mathrm{Ga}_{2} \mathrm{O}_{3}\right)$ has the potential to replace silicon carbide ( $\mathrm{SiC}$ ) and gallium nitride ( $\mathrm{GaN})$ as the material of choice for advanced power semiconductor devices. $\mathrm{Ga}_{2} \mathrm{O}_{3}$ is a wide-bandgap semiconductor material whose crystal growth process allows for a much lower density of defects than those achievable in $\mathrm{SiC}$ and $\mathrm{GaN}$. The ability to form high quality, stable interfaces is one of the key requirements for power devices. Because development of $\mathrm{Ga}_{2} \mathrm{O}_{3}$-based devices is still in an early stage, the nature of these interfaces with either oxides or metal contacts and the ways of achieving stable interfaces at high operating temperatures are not well understood. The aim of this research is to develop a rational approach to the design of high-quality interfaces. Through the combination of in situ and electrical characterization of test structures, we will probe the properties and the stability of interfaces that are critical for power devices based on $\mathrm{Ga}_{2} \mathrm{O}_{3}$.

\section{MISSION RELEVANCE}

This project is relevant to DOE's energy and national security missions. Power devices are at the core of every single technology involving the manipulation of electricity, from energy generation to its final point of use. Wide-bandgap semiconductors will enable higher energy efficiencies, as well as circuits and architectures that are smaller and able to operate in a wider range of conditions that are inaccessible to devices based on silicon.

In addition to DOE's interest in this type of material, the U.S. Department of Defense (DoD) has been invested in the development of $\mathrm{Ga}_{2} \mathrm{O}_{3}$ devices, with the U.S. Air Force taking a leadership role in $\mathrm{Ga}_{2} \mathrm{O}_{3}$ device research in the United States.

\section{RESULTS AND ACCOMPLISHMENTS}

In FY 2017 we carried out electronic, chemical, and microstructural characterization of $\mathrm{Ga}_{2} \mathrm{O}_{3}$ interfaces with various dielectrics, including aluminum oxide $\left(\mathrm{Al}_{2} \mathrm{O}_{3}\right.$, hafnium oxide $\left(\mathrm{HfO}_{2}\right)$, and zirconium oxide $\left(\mathrm{ZrO}_{2}\right)$ and their laminates. We determined the band alignment between $\mathrm{Ga}_{2} \mathrm{O}_{3}$ and the dielectrics, and we carried out ex situ studies using X-ray absorption spectroscopy at the APS to probe the microstructure and thermal stability of the amorphous dielectrics. These studies allowed us to understand the impact that the choice of chemical precursors used for the synthesis of the dielectrics have on the passivation of interfacial defects that are present at the $\mathrm{Ga}_{2} \mathrm{O}_{3}$ surface.

The band alignment results are consistent with the formation of a Type II interface between $\mathrm{HfO}_{2}$ and $\mathrm{Ga}_{2} \mathrm{O}_{3}$, with a conduction band offset of $1.3 \mathrm{eV}$ (Figure 1). From a microstructural point of view, $\mathrm{Ga}_{2} \mathrm{O}_{3}$ templates the crystallinity of $\mathrm{HfO}_{2}$, resulting on polycrystalline films at experimental conditions where the films are typically amorphous when grown on other semiconductors such as $\mathrm{Ga}_{2} \mathrm{O}_{3}$ and $\mathrm{Si}$. This can have a negative impact on the electronic properties of the interface as grain boundaries enhance leakage currents in the type of heterostructures that are relevant for advanced semiconductor devices.

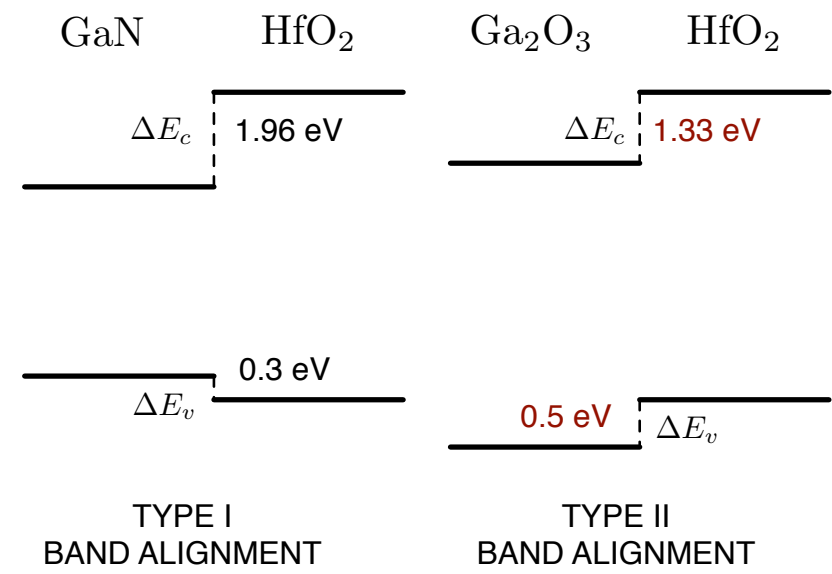

Figure 1. The band alignment of ALD hafnium oxide with gallium oxide as determined by photoelectron spectroscopy, showing a Type II band alignment. A comparison with gallium nitride is also shown.

Through photoluminescence characterization we evaluated the impact of surface roughness in the non-radiative recombination of charge carriers near the surface of the semiconductor.

\section{PROPOSED FUTURE WORK}

In FY 2018, we aim to rationalize the interface design of $\mathrm{Ga}_{2} \mathrm{O}_{3}$ and identify mechanisms for interfacial degradation at high temperatures that could illuminate the potential of $\mathrm{Ga}_{2} \mathrm{O}_{3}$ for high-temperature power electronics. In situ characterization of the early stages of growth of various gate dielectrics on single-crystal $\mathrm{Ga}_{2} \mathrm{O}_{3}$ will be measured at the APS to understand how interfaces are formed. To better understand interfacial stability we will perform electrical characterization of metal-dielectric-semiconductor test structures. Finally, we will characterize the role of plasma etching-induced faceting at the surface on the electronic properties of the gallium oxide-dielectric interface. 


\section{New Techniques to Manipulate Rare Isotopes Using Adaptive Optics}

\section{7-023-NO}

Michael Bishof, Matthew Dietrich, and Peter Mueller

\section{PROJECT DESCRIPTION}

This project aims to leverage the novel capabilities of adaptive optics to address the unique challenges facing experiments that manipulate laser-cooled, rare, radioactive isotopes. Atom scarcity and radioactive decay require new manipulation techniques that minimize preparation time and atom loss. The adaptive optics technique has the potential to greatly expand the capabilities of optical dipole traps (ODTs), a tool commonly used to confine and transport various species of atoms, molecules, and mesoscopic objects.

The ODT forms at the focus of an intense laser beam, where atoms are attracted to the intensity maximum. Long-range ( 1-m) ODT transport currently requires physical translation of a focusing optic along an ultra-low vibration translation stage. In addition to being slow, this technique introduces vibrations that cause atom heating and loss.

The goal of this project is to develop techniques based on adaptive optics that achieve a reliable, near-unity transfer efficiency from initial atom collection to the final measurement region and dramatically reduce the amount of time required to prepare atoms for precision measurements.

\section{MISSION RELEVANCE}

This project is relevant to DOE's mission in science. The Argonne Medium Energy Physics Group is a world-leading group that specializes in precision measurement of rare isotopes. The investigators are uniquely suited to achieve the goals of this project due to their extensive experience with cold atom trapping techniques and, in particular, their expertise in long-range ODT transportation of cold atoms.

In the short term, the success of this project will immediately enhance the scientific impact of the ongoing ${ }^{225}$ Ra electric dipole moment (EDM) experiment. The techniques we develop also could be used in many other current investigations using laser-trapped neutral atoms, especially those that use rare or short-lived isotopes such as ${ }^{6} \mathrm{He},{ }^{38 \mathrm{~m}} \mathrm{~K}$, and various isotopes of francium. Over the long term, the techniques we develop will enable future investigations that were previously impossible due to short isotope lifetimes or low production cross-sections. This project will positively impact research possibilities for isotopes that can be produced at future facilities such as the Facility for Rare Isotope Beams. By leveraging techniques developed in this research, Argonne scientists will expand the frontiers of nuclear physics and precision measurement science.

\section{RESULTS AND ACCOMPLISHMENTS}

During the first fiscal year of this two-year project, we designed and constructed an optical system to achieve long-range cold atom transport and trap size manipulation using two variable focal length (VFL) lenses. We also designed and constructed a mechanical support for the optical system based on active vibration isolation legs to reduce vibrations on the optical elements and thereby reduce atom loss due to vibration-induced heating.

Using the optical system, we calibrated the adaptive lenses to compensate for temperature-dependent effects in the lenses. Based on this initial calibration and stock control electronics, we are able to achieve meter-scale trap translation using arbitrary translation functions.

\section{PROPOSED FUTURE WORK}

In the second year of the project, we will complete our construction of new lens-control electronics that improve trap position resolution, which is necessary to avoid atom heating during transport. We will build new control software for the control electronics that allows simultaneous control of trap position and trap size by manipulating two VFL lenses simultaneously. We will also test our system at the high laser power $(50 \mathrm{~W})$ that is necessary for trapping ${ }^{226} \mathrm{Ra}$ atoms using 1,550-nm light.

In the second half of the year, we will install our instrument in the radium EDM apparatus and use the new capabilities of our adaptive trap to optimize atom transport from the trapping chamber to an environmentally shielded science chamber, located approximately $1 \mathrm{~m}$ away. Our goal is to improve atom survival and reduce the time necessary for atom preparation and transportation.

Improvements that we demonstrate using ${ }^{226}$ Ra will have a significant impact on a broad spectrum of cold atom experiments investigating nuclear structure and fundamental symmetries. 


\section{Realizing a Gate-Tunable Kinetic Inductance for a Transmon Qubit Using $\mathrm{SrTiO}_{3}$}

\section{7-026-NO}

Anand Bhattacharya, Jidong Jiang, and Alex Martinson

\section{PROJECT DESCRIPTION}

This project seeks to create a novel device-a gatetunable inductance based on a low-carrier-density superconductor, $\mathrm{SrTiO}_{3}$. The basis for a quantum computer is a qubit, or quantum bit that can take a value of 0 or 1 or an arbitrary quantum superposition of these states. One of the leading contenders for implementation of qubits involves superconducting Josephson junctions. The technology currently used for creating these Josephson junctions was developed more than 30 years ago. With modern-day materials and nanofabrication capabilities, it may be possible to implement new Josephson device concepts that would significantly improve the performance and scalability of a quantum computer based on Josephson junctions.

Electrostatic gating forms the basis for conventional transistors, in which a gate electric field is used to tune the carrier density of a semiconductor to vastly change its capacity to carry electric currents. If we translate this concept to a superconducting channel, we can tune its ability to carry a dissipationless super-current, and thus also its "kinetic inductance." This tuning would form a critical circuit element in a particular realization of a quantum computer involving a superconducting "transmon." Electrostatic gating enables local tuning of superconducting properties instead of the more global tuning that is typically accomplished using magnetic fields in superconducting qubits. While unconventional approaches such as ionic liquid gating have been used to gate superconducting materials, in our project we seek to accomplish this using a conventional dielectric. This approach will enable fast switching and facile scalability. Realizing such a gated device involves development of a novel material-a very-low-carrier-density superconductor -and fabrication of an appropriately gated structure that would enable transmon operation.

\section{MISSION RELEVANCE}

This project is relevant to DOE's mission in basic science. Quantum computing has been identified as an area where new developments could revolutionize information technology and computation, areas of vital strategic importance as we enter the "beyond Moore" post-silicon era. Our research will impact one of the priority areas in fundamental research: new materials related to quantum computing.

\section{RESULTS AND ACCOMPLISHMENTS}

We have been able to prepare samples that are superconducting at very low carrier (electron) densities, $n<1 \times 10^{18} / \mathrm{cm}^{3}$, using very carefully reduced single crystals of $\mathrm{SrTiO}_{3}$ where $\mathrm{O}$ vacancies lead to doping with electrons. In fact, our samples display superconductivity at carrier densities lower than any reported in the literature (see Figure 1), with very high mobilities. Achieving this low density is a key step for creating gated devices, because electrostatic gating is only effective at these ultralow densities. We have also developed all of the process steps including depositing the gate dielectric and making good electrical contacts to the very low-doped material, which are essential to developing a gated device. In 2017, bulk superconducting samples were extensively characterized both at Argonne and the National High Magnetic Field Laboratory (MagLab) at high magnetic fields and low temperatures, allowing us to form a comprehensive picture of the electronic properties of $\mathrm{SrTiO}_{3}$.

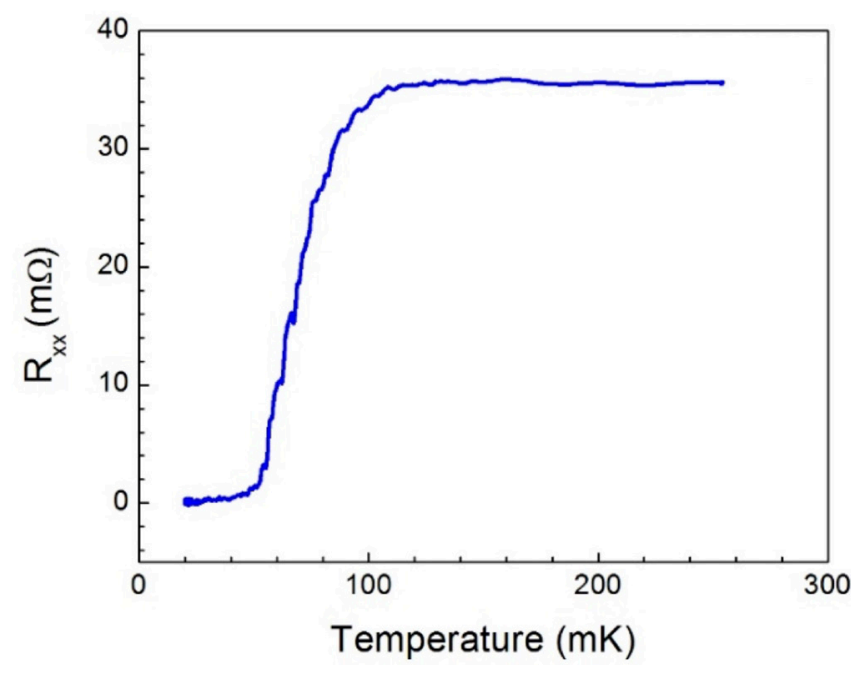

Figure 1. Superconducting transition in low carrier density $\mathrm{SrTiO}_{3}$, $n=6.6 \times 10^{17} / \mathrm{cm}^{3}$.

We have fabricated our first set of field-effect devices, and these will be tested in FY 2018 at MagLab and Argonne.

\section{PROPOSED FUTURE WORK}

In FY 2018, we will create and characterize gate-tunable devices to establish proof of concept for our idea. Going forward, we seek to explore other low-carrier-density superconductors beyond $\mathrm{SrTiO}_{3}$, including superconducting semimetals. 


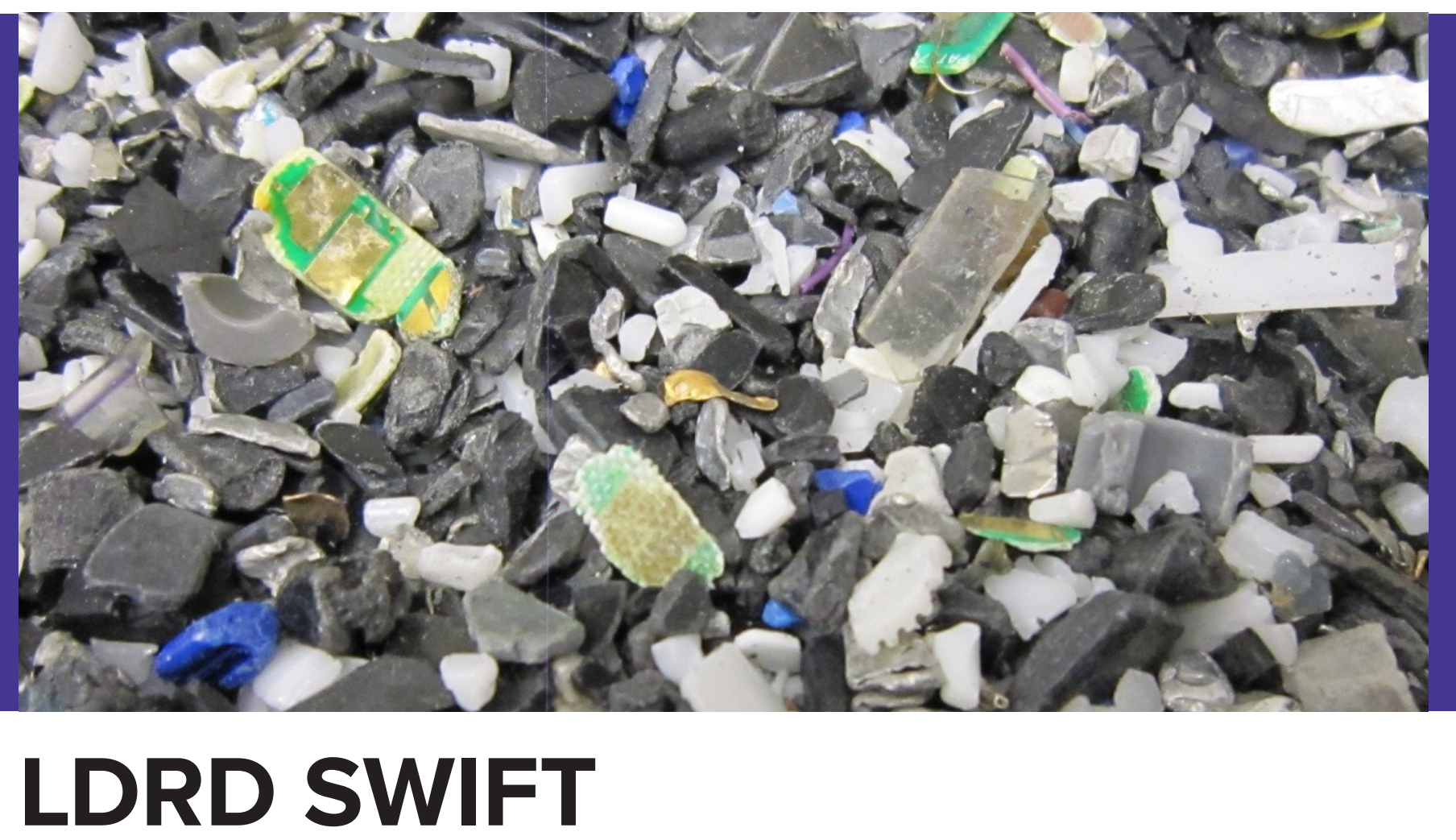




\section{Recovery of Critical Materials from Post- Consumer Electronics}

\author{
2016-240-R1 \\ John Hryn and Jeffrey Spangenberger
}

\section{PROJECT DESCRIPTION}

The objective of this project was to adapt technology developed at Argonne for the separation and recovery of polymers from mixed automotive plastics to the separation and recovery of polymers and other materials from obsolete electronics. Process operating conditions were developed to demonstrate proof-of-concept for recovering valuable and critical materials from obsolete electronics (Figure 1). The key technical barriers to the development of this process technology are the yield and selectivity of the process for specific polymers relative to the market requirements for product purity and consistency. The research conducted in this project directly addressed these barriers. We characterized the materials, developed a process model and design configuration, and developed and verified process operating conditions.

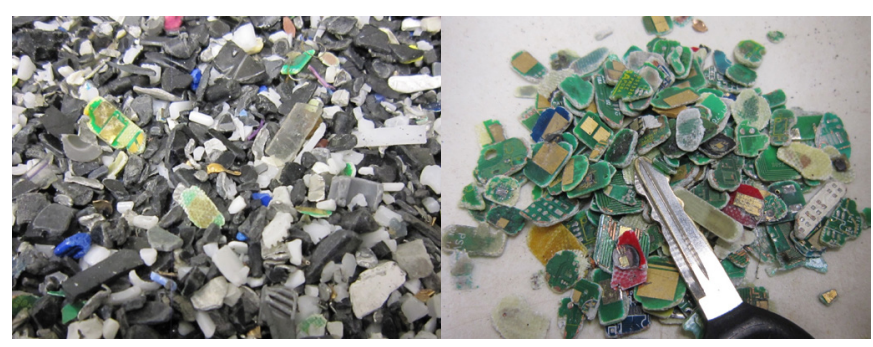

Figure 1. Critical materials concentrated in printed-circuit-board scrap recovered from a mixed polymer waste stream (car key shown for size reference).

The basic process configuration for separation and recovery of materials from obsolete consumer electronics consists of two primary systems. The first is mechanical separation: conventional magnetic separation and size-reduction technology to produce a metal-free polymer concentrate from bulk electronics scrap (e-scrap). This step is followed by polymer separation: a series of modular wet separation tanks (designed and patented by Argonne) in which the polymer concentrate is sequentially separated into constituent polymers using flotation/elutriation techniques (patented by Argonne) to selectively recover the polymers at high yield and at the purities necessary for their re-use in value-added market applications.

Most of the metals in the obsolete electronics are recovered in the mechanical separation system (we also point out that, owing to their intense magnetic properties, rare-earth magnets that are used in electronics equipment can be isolated from the metals fraction for separate recycling, if sufficient volumes can be recovered). Any residual metals (primarily nonferrous) in the polymer concentrate are recovered in one of the downstream separation tanks. The process is effective for selectively recovering polymers of similar densities through surface modification of the specific polymers by $\mathrm{pH}$ and surface tension adjustment, combined with the appropriate elutriation velocity of the solution. The elutriation process is controlled by solution flow into a process tank in which the plastics are isolated in a column through which the solution flows. Solution flow is controlled by recirculating solution pump rates. Plastics that sink to the bottom of the tank are conveyed out with a screw conveyor and then discharged or conveyed to the next separation stage. Plastics that rise in the elutriation column are carried with the solution to a conventional spin-dryer that discharges the plastics and recovers the solution to be pumped back into the separation tank. Process solutions are continuously recycled in the process.

\section{MISSION RELEVANCE}

The project addresses DOE missions in energy and the environment. The mining and processing of energy-intensive materials consume more than 8 quads annually; up to $50 \%$ of these materials (equivalent to 4 quads) is estimated to be disposed of in landfills within one year of production. Internal DOE analysis shows that by increasing the recycling rate of key material classes by $30 \%$ and improving the energy efficiency of secondary feedstock processing by $30 \%$, energy savings of up to 1.6 quads can be achieved.

\section{RESULTS AND ACCOMPLISHMENTS}

We evaluated the recovery rate of various materials in electronic scrap using our versatile material recovery pilot plant. The feed material processed for this project consisted of electronics of all types, including printers, copiers, electric scooters, digital keyboards, and others. In FY 2016, the work demonstrated the recovery of ferrous and non-ferrous metals and a polymer concentrate equating to nearly $30 \%$ of the feed material. In FY 2017, the polymer concentrate was processed further to recover various classes of plastics. Circuit board material, also targeted as a potential concentrate, was generated with a content of about $25 \%$ by weight.

\section{PROPOSED FUTURE WORK}

Two proposals were prepared for follow-on funding, with decisions expected in FY 2018. 


\section{X-ray Investigation of the Potential of Pressure-Assisted Atomization Technology for Medical Inhaler Sprays}

\author{
2017-098-NO \\ Christopher Powell and Daniel Duke
}

\section{PROJECT DESCRIPTION}

The main reason for the inefficiency of medical inhaler devices is that the fundamental physics of the sprays are poorly understood. This stasis is mainly because the region inside and near the nozzle, which constitutes the initial conditions of spray formation, is a very challenging measurement environment (Figure 1). Laser diagnostics have difficulty penetrating this region because of the high number density of the droplets (multiple scattering). The small droplet sizes (hundreds of nanometers to several microns) are below the diffraction limit of conventional Rayleigh/Mie scattering and Schlieren imaging systems.

The objectives of this project were to develop and demonstrate a series of X-ray measurements of droplet size and flow morphology in pressurized medical sprays, and to use these measurements to test the efficacy of pressure-assisted atomization techniques to improve the performance of existing spray devices. In addition, the goal of the project has been to demonstrate a capacity to perform novel X-ray-based measurements of medical inhaler devices that would be sufficient to attract industrial and academic funding interest.

\section{MISSION RELEVANCE}

The project is relevant to DOE's mission in basic science. Aerosol and spray delivery are some of the safest and most cost-effective means of drug delivery available today. However, medical aerosols and sprays (e.g., asthma inhalers) are severely limited by poor control of the final particle or droplet size. In the case of inhaled aerosols, only $10 \%$ to $40 \%$ of the drug actually reaches the lung tissues. Over-size particles impact the throat, and under-size particles are exhaled. Similar aerodynamic problems occur in topical and nasal sprays. Not only are the sprays inefficient, but their applicability is constrained to a limited number of drugs because of poor dose control.

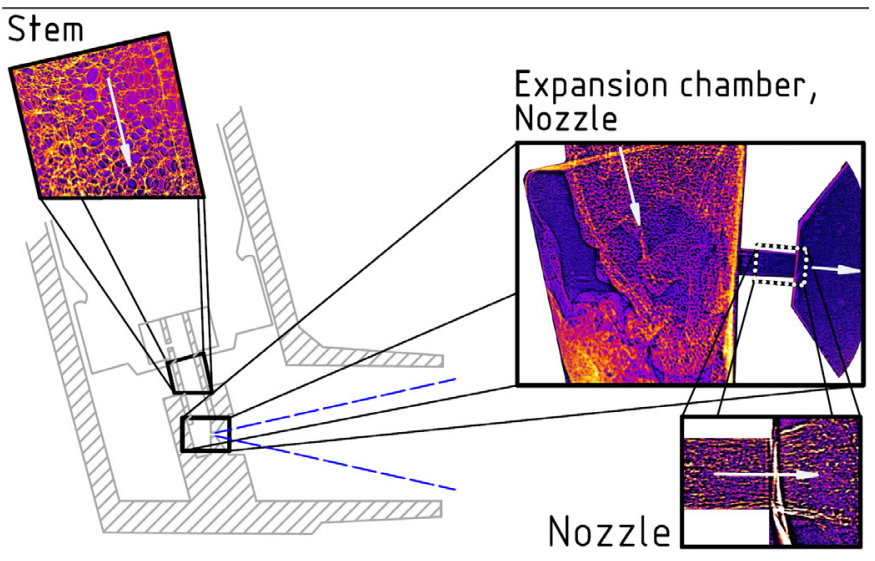

Figure 1. False-color $X$-ray phase contrast images reveal the complexity of the internal fluid flow in the stem, expansion chamber, and nozzle region of an $85 \%$ HFA $134 a, 15 \%$ ethanol pMDI spray. Changes in color are indicative of complex multiphase flow features.

\section{RESULTS AND ACCOMPLISHMENTS}

X-ray experiments at the Advanced Photon Source (APS) were completed in late 2016 and early 2017, including phase-contrast imaging, time-resolved $\mathrm{X}$-ray radiography and ultra-small-angle X-ray scattering. The measurements showed that the internal flows of pressurized metered dose inhalers (pMDIs) contain large volumes of vapor that originate upstream of the valve stem and dominate the volume in the expansion chamber. Propellant-only flows are characterized by a continuous vapor core that extends from the valve stem into the sump, and large droplets that are not in the respirable range are produced by liquid discharged from the nozzle when the base of the sump fills with liquid. The inclusion of ethanol substantially alters the internal flow structure by preventing bubble coalescence, additionally stabilizing the spray. Quantification of the spray density showed that at all times in the injection, the nozzle exit area is predominantly occupied by vapor, with peak liquid volume fractions around $10 \%$. Combining radiography measurements with a multiphase flow model can predict the time-variant mass flow rate and provide experimental validation of thermodynamic models used for pMDI modeling. This research highlights the importance of the internal flow structure on spray formation from suspension and solution pMDIs.

\section{PROPOSED FUTURE WORK}

This project was competed in FY 2017. 


\section{Toward the Reduction of U.S. Petroleum Consumption by Fueling Light-Duty Engines with Propane}

\author{
2017-099-NO
}

Riccardo Scarcelli

\section{PROJECT DESCRIPTION}

This project aims at improving Argonne's experimental and numerical capabilities regarding the use of propane as fuel for ground transportation. Most of the budget allocated for this project was used to purchase a flexible and mobile fuel supply system dedicated to liquid propane/liquefied petroleum gas (LPG) operation and to integrate the new fuel system into our test cell, where a single-cylinder research engine, representative of light-duty transportation engines, is being operated with conventional (gasoline) and alternative (natural gas) fuels. The goal of the modeling task of this project is to expand the computational fluid dynamics (CFD) toolkit used at Argonne for engine simulations (CONVERGE) to include liquid propane in the list of available fuels for parcel simulations and to initiate calculations of direct injection (DI) of propane.

\section{MISSION RELEVANCE}

This project is relevant to DOE's energy mission. By completing the tasks described above, the proper numerical and experimental tools will be built, and preliminary results will be generated that place Argonne in a strategic position to quickly respond to the demand of advanced fuel engine concepts involving propane, which will help support DOE's mission of reducing petroleum consumption.

\section{RESULTS AND ACCOMPLISHMENTS}

A mobile fuel cart dedicated to propane DI use was commissioned to Re-Sol and delivered during FY 2017. The cart was connected to the engine, fuel tank, and ventilation pipes. The cart (Figure 1, left) is intrinsically safe as far as the locations of electrical connectors and the fuel area are concerned. The main issue identified (i.e., fuel venting at the end of daily operation and during emergencies) was solved by connecting the fuel cart to a pipe that forces engine exhaust gases outside of the building through a spark-proof fan. The worst-case scenario fuel volume to be vented and the dilution tolerance in the exhaust pipe were calculated to validate the assumption of safe operation.

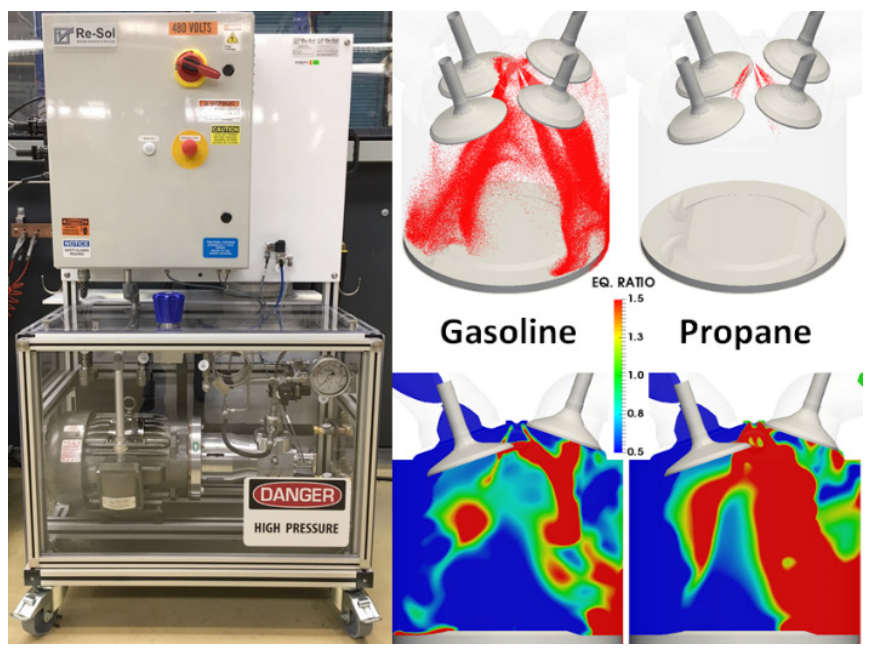

Figure 1. Propane DI cart (left) and preliminary CFD calculations of propane $\mathrm{DI}$ (right) showing different mixture formation processes with respect to gasoline.

Numerical simulations using the CONVERGE software were expanded to account for liquid propane as a fuel and were initiated to provide insight into the effects of the fuel on mixture formation. Propane liquid data were included in the latest version of CONVERGE (2.4), which allowed simulating the DI of propane and comparing the results to the gasoline DI baseline. Figure 1 (right) shows that evaporation is much more pronounced for the propane case as compared to gasoline, which can be observed by looking at the liquid spray visualized in the top CFD image. This has an impact on mixture formation, as shown in the bottom part of the CFD image.

The Lagrangian calculations carried out for this project do not properly account for flash boiling. Indeed, it can be shown by theoretical calculations that for the operating condition simulated here (2,000 RPM (rev/min) and 6 bar indicated mean effective pressure, which corresponds to typical part-load engine operation), gasoline will likely not flash boil, whereas propane will.

\section{PROPOSED FUTURE WORK}

Future work will consist of:

1. Running the single-cylinder engine with propane DI and experimentally evaluating mixture formation strategies.

2. Conducting fundamental CFD studies and developing Lagrangian flash-boiling models for propane DI.

The mobile fuel cart will be utilized at the Advanced Photon Source to conduct fundamental X-ray investigations of fuel injection and mixture formation, with a specific focus on the occurrence of flash boiling. This approach will help deliver a proper dataset for flash-boiling model development. 


\section{High-Entropy Alloys for Advanced Nuclear Reactors}

\author{
2017-100-NO
}

Wei-Ying Chen, Yiren Chen, and Krishnamurti Natesan

\section{PROJECT DESCRIPTION}

Recently, high-entropy alloys (HEAs) have attracted wide research interest in the nuclear materials community because of the potential radiation and corrosion resistance resulting from their unique characteristics of high entropy, sluggish diffusion, and distorted lattice. The goal of this project is to validate this hypothesis and to evaluate the potential of HEAs for use in advanced sodium fast nuclear reactors by comparing them to 316 stainless steels (the structural materials used in current sodium-cooled reactors).

CoCrFeMnNi and $\mathrm{Al}_{0.3} \mathrm{CoCrFeNi}$, the two most-studied HEAs, and $316 \mathrm{H}$ (a high-carbon version of 316 stainless steel) were selected for this project. In situ ion irradiation and high-energy electron irradiation were performed in the Intermediate-Voltage Electron Microscopy facility at Argonne National Laboratory and High-Voltage Electron Microscopy facility in Hokkaido University in Japan, respectively, to investigate the irradiation process in HEAs. Irradiation parameters (e.g., irradiation temperature) were chosen to simulate the conditions in light water reactors and sodium-cooled fast reactors. The irradiation-induced microstructural modification was examined with electron microscopy to reveal the difference between HEAs and $316 \mathrm{H}$ stainless steels.

The elemental segregation at irradiation-induced defect clusters in HEAs was studied with atom probe tomography at Oak Ridge National Laboratory's Center for Nanophase Materials Sciences. With the atomic resolution of this technique, the distribution of each constituent element around the nanosized irradiation-induced defect clusters was revealed.

HEA samples were exposed to high-temperature liquid sodium for various periods of time to study their sodium compatibility. The weight and thickness losses were measured to investigate the effect of liquid sodium on the corrosion rate of the materials. The results were compared with results on $316 \mathrm{H}$ from DOE's Advanced Reactor Concepts Program.

\section{MISSION RELEVANCE}

This project is highly relevant to DOE's mission in nuclear energy. Recently, HEAs have been recognized by DOE's Office of Advanced Reactor Technologies as a new candidate structural material for use in advanced molten salt reactors because of their high-temperature strength and corrosion resistance. The results of this work provide information on the radiation performance and dissolution-controlled corrosion of HEAs at a temperature close to the design temperature of advanced molten salt reactors (i.e., $700^{\circ} \mathrm{C}$ ).

\section{RESULTS AND ACCOMPLISHMENTS}

For the irradiation performance studies, we performed in situ ion and high-energy electron irradiation experiments. With ion irradiation, the irradiation-induced damage at light water reactor temperature, $300^{\circ} \mathrm{C}$, in HEAs and $316 \mathrm{H}$ was comparable in terms of the size and density of irradiation-induced dislocation loops. In contrast, as shown in Figure 1, at higher irradiation temperatures, $500^{\circ} \mathrm{C}$ and $600^{\circ} \mathrm{C}$, the size of irradiation-induced dislocation loops was evidently different between HEAs and $316 \mathrm{H}$. With high-energy electron irradiation, the presence of carbon impurity in $316 \mathrm{H}$ had an effect on the shape of dislocation loops, while no such effects were observed in HEAs. An elemental segregation study using atom probe tomography was performed on CoCrFeMnNi irradiated at $300^{\circ} \mathrm{C}$ and $550^{\circ} \mathrm{C}$ to $1 \mathrm{dpa}$. The results indicated no observable segregation at dislocation loops at $300^{\circ} \mathrm{C}$. In contrast, significant segregation was observed at the dislocation loops at $550^{\circ} \mathrm{C}$.
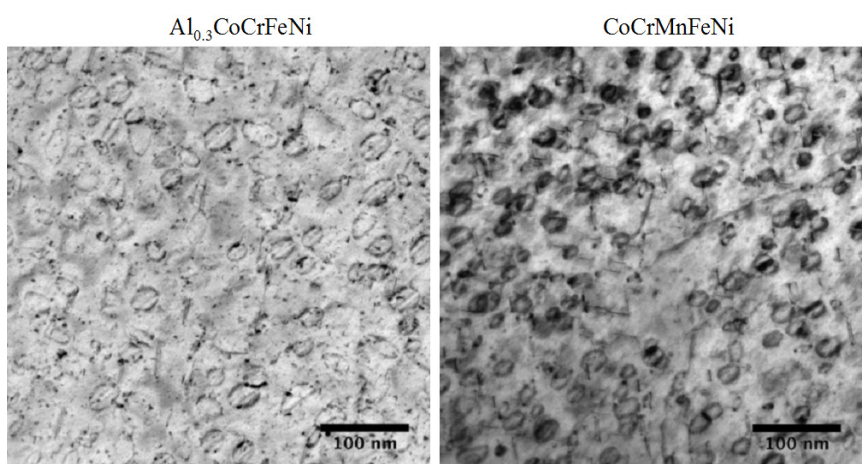

Figure 1. Microstructures after $1 \mathrm{MeV} \mathrm{Kr}$ ion irradiation to $1 \mathrm{dpa}$ at $500^{\circ} \mathrm{C}$.

Sodium compatibility was investigated by immersing $\mathrm{CoCrFeMnNi}$ and $\mathrm{Al}_{0.3} \mathrm{CoCrFeNi}$ in liquid sodium at $650^{\circ} \mathrm{C}$ for 1,000 hours. The results showed that there was no change in the weight or thickness of HEAs after this exposure. This result is comparable to that for $316 \mathrm{H}$.

\section{PROPOSED FUTURE WORK}

This project ended in FY 2017. The future focus of research on HEAs in nuclear applications should be oriented to applications in molten salt nuclear reactors with attention to the HEAs' compatibility with molten salt, high-temperature strength, and irradiation resistance at $700^{\circ} \mathrm{C}$ and higher. 
Enhancing Computational Tools for Polynomial Optimization Problems Relevant to Networked Systems

\section{7-101-NO}

\section{Daniel K. Molzahn}

\section{PROJECT DESCRIPTION}

A variety of physical systems are modeled using polynomial equations defined over a network (e.g., electric power systems, natural gas networks, transportation systems). Accordingly, efficient operation of these systems requires the solution of polynomial optimization problems, which can be very challenging (non-convex and NP-hard in general).

Recently proposed convex relaxation techniques hold great promise for solving polynomial optimization problems. In particular, so-called "moment/sum-ofsquares" hierarchies of convex relaxations provide a rigorous approach for solving polynomial optimization problems. While applying these hierarchies directly is computationally intractable for large-scale systems, the principal investigator's previous research efforts have developed methods for exploiting problem sparsity and selectively applying computationally difficult constraints to particularly challenging regions of the problem (see Figure 1).

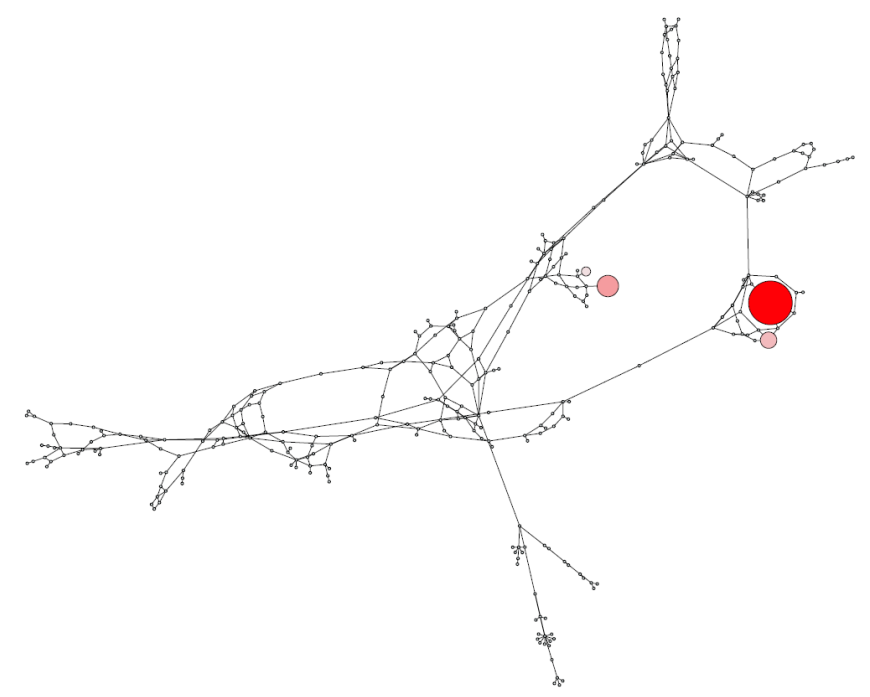

Figure 1. Selective application of computationally intensive constraints (red dots) in a polynomial optimization problem for an electric power system.

While these methods have been applied solely to optimization problems relevant to electric power systems, they have the potential to be much more broadly applicable. The key challenge in extension to a broader class of problems is the lack of a flexible computational tool that implements these methods. This project developed such a tool, thus laying the groundwork for applying moment/sum-of-squares hierarchies both to speed development of further applications in electric power systems and to optimize other large-scale networked systems.

\section{MISSION RELEVANCE}

By improving the optimization of networked systems, this project is relevant to DOE's energy mission, particularly infrastructure modernization with respect to electricity, natural gas, and water systems. This work is closely aligned with projects for control of electric transmission and distribution systems as part of the Grid Modernization Laboratory Consortium (GMLC).

\section{RESULTS AND ACCOMPLISHMENTS}

The computational tools developed in this project enable the application of moment/sum-of-squares relaxation hierarchies to polynomial optimization problems relevant to a variety of networked systems. Several key accomplishments include:

$\square$ Creating an input/output format for polynomial optimization problems that enables fast computational performance while remaining general and understandable in order to speed future developments.

$\square$ Bypassing the need for a modeling language by directly interfacing with the solver in order to improve computational speed. By precisely controlling the problem formulation, this accomplishment provides the added benefit of significantly improved numerical accuracy as compared to previous implementations.

$\square$ Extending recently developed techniques ("clique merging") for speeding computations to general polynomial optimization problems over networks.

This project also facilitated several international collaborations. The computational tools were developed with Dr. Cedric Josz from the University of Toulouse. Results were presented at a January 2018 workshop titled "Power Systems: Semi-Algebraic Techniques for Optimal Power Flow and Stability Assessment" hosted in Paris by RTE, the French electric transmission system operator.

\section{FUTURE WORK}

This project was completed in FY 2017. The computational tools developed in this project will be used to support several ongoing projects, including the GMLC Control Theory project 1.4 .10 for optimally controlling electric distribution systems. 


\section{Multiscale Modeling to Understand Ion-Diffusion- Induced Degradation in Photovoltaics}

\author{
2017-102-NO
}

Maria K. Chan

\section{PROJECT DESCRIPTION}

Despite increasing photovoltaic (PV) efficiency, because of the balance-of-system costs, panels must have a long service life to be cost competitive with fossil fuels. Dominant PV technologies utilizing silicon (Si) and cadmium telluride (CdTe) suffer from long-term performance degradation due to impurity migration. This project focuses on the density functional theory (DFT) modeling of the detrimental effects of impurities on PV performance. We use DFT calculations to systematically understand degradation and devise mitigation strategies for improving PV durability.

\section{MISSION RELEVANCE}

The project is relevant to DOE missions in clean energy, science and innovation. Degradation of PV modules is currently the biggest limitation for solar energy deployment, owing to recent progress in other metrics. The understanding and mitigation of failure-inducing processes will therefore benefit the DOE mission of renewable energy generation, which in the long term has the advantages of limiting carbon emission and climate change, fostering energy independence, and creating jobs. At a more immediate time scale, the ability to translate first-principles modeling of defects into device-level performance benefits Argonne's materials modeling community, and will strengthen its ability to carry out predictive modeling that is integrated with materials characterization.

\section{RESULTS AND ACCOMPLISHMENTS}

Our first focus is on sodium (Na) impurities in Si PVs, which are considered to play an important role in potential-induced degradation (PID), a significant cause of solar cell degradation and failure. Shorting that occurs as a result of $\mathrm{Na}$ accumulation at extended defects has been suggested as a culprit in PID. Because it is not clear how extended defects are decorated by $\mathrm{Na}$ impurities, we used first-principles DFT calculations to investigate the thermodynamics and kinetics of $\mathrm{Na}$ in the most common grain boundaries within Si, namely, extended defects such as intrinsic stacking faults (SFs) and $\Sigma 3$ (111) grain boundaries (GBs) (Figure 1, top). We found that $\mathrm{Na}$ impurities segregate from the bulk into these GBs, and that the energy barrier that must be overcome for $\mathrm{Na}$ to escape from the GBs is substantial and similar to the sum of the barrier energy in bulk Si and the segregation energy to the SF. Surprisingly, we found that the migration barrier for $\mathrm{Na}$ diffusion within the GBs is even higher than the energy barrier for escaping them. The results suggest that these extended defects likely accumulate $\mathrm{Na}$ as the impurities segregate to them through the bulk, but do not act as a network for Na diffusion. This means that it is the bulk density of these extended defects, rather than their network structure, that is important for determining $P V$ degradation. We also investigated the effects of water impurities on hybrid perovskite PVs. We found that the intercalation of water into methylammonium lead iodide is thermodynamically favorable under standard conditions and causes an increase in the band gap, the results of which are consistent with observations in low-pressure experiments.

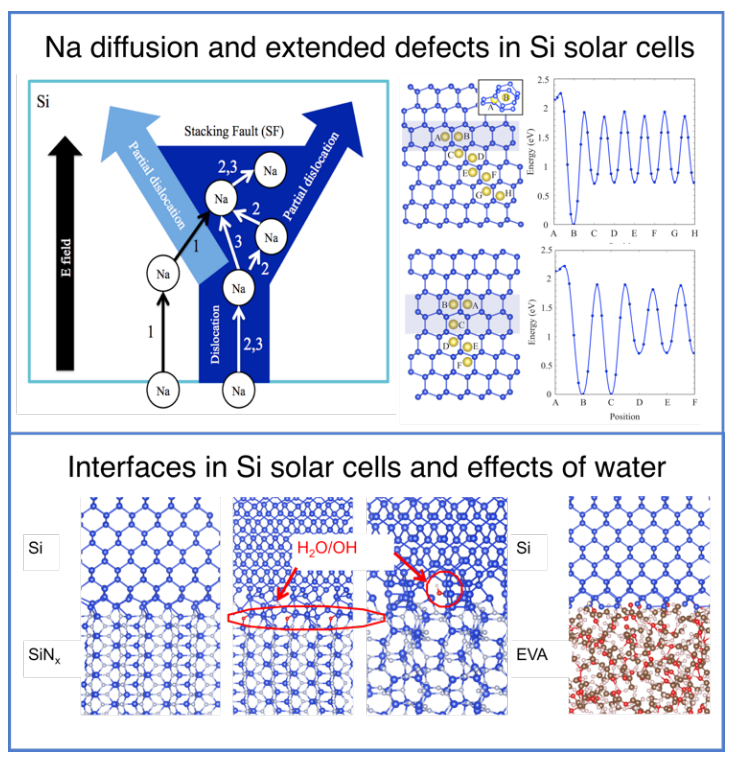

Figure 1. (Top) Different mechanisms of Na impurity diffusion in Si extended defects; diffusion barriers at $\Sigma 3$ twins and intrinsic stacking faults. (Bottom) Atomistic models for different interfaces in Si solar cells, including Si nitrides and ethylene-vinyl acetate (EVA) encapsulants.

\section{PROPOSED FUTURE WORK}

Under a SunShot (Office of Energy Efficiency and Renewable Energy)-funded follow-on project, we will investigate the effects of water impurities at interfaces on the degradation of Si PVs (Figure 1, bottom). The segregation, diffusion, and decomposition of water impurities at various interfaces will be investigated with DFT and molecular dynamics and compared with experimental measurements. 


\section{Application of Combined Rheology and X-ray Scattering to the Paint Industry for Optimizing the Formulation Process}

\author{
2017-103-NO
}

\author{
Jonghun Lee, Xiao-Min Lin, Suresh Narayanan, \\ and Alec Sandy
}

\section{PROJECT DESCRIPTION}

This project aims at applying microstructural information on paints measured by X-ray scattering to improve the paint formulation process. The paint and coating industry is a large industrial sector in the United States; domestic production in 2014 was estimated to be $\$ 24$ billion. Despite a seemingly mature market, this industry actively invests in research and development to satisfy changing environmental regulations and to optimize cost and performance. A major challenge in paint formulation is the flocculation or agglomeration of titania particles during the mixing process in the production step, and during the drying process in the application step. The opacity of paints prevents optical measurement from providing information on their microstructure. Instead, we use ultra-small-angle X-ray scattering (USAXS) to study the dispersion stability of titania particles in liquid paint samples and the evolution of microstructures during the drying process.

\section{MISSION RELEVANCE}

This project is directly relevant to the DOE mission in basic science and is also relevant to environmental quality where coating/protection of surfaces is concerned. The concept of correlating the effect of applied shear with the microstructure and dynamics of materials, using the technique of Rheology-SAXS-XPCS, was developed at the Advanced Photon Source (APS) under the auspices of an earlier LDRD project. This project extends this capability to the study of coatings and to paint in particular.

\section{RESULTS AND ACCOMPLISHMENTS}

The evolution of microstructures of paint during the drying process was studied using USAXS at APS Sector 9-ID-C. Titania particles, $300 \mathrm{~nm}$ in size, play a key role in the hiding effect (i.e., making paints white) and are a major paint component. X-ray scattering from titania particles overwhelms the scattering from other components in a paint sample, and thus USAXS reveals their microstructure. We found that the microstructure of the sample changes rapidly in the first 10 min after application. Figure 1(a) shows that over the first $10 \mathrm{~min}$, the peak of the structure factor shifts from 0.014 to $0.017 \mathrm{~nm}^{-1}$. Figure 1(b) shows the change in the average of titania particle-particle distance over $1 \mathrm{~h}$. The change in the thickness of paint films during drying could also be measured using $X$-ray transmission. The thickness of the paint film was found to shrink by $25 \%$ when completely dried, compared to its initial thickness. It also showed that the paint sample dries rapidly over the first $10 \mathrm{~min}$, and that the thickness does not change thereafter; these observations are consistent with the trend observed in Figure 1. We have demonstrated that USAXS can offer a powerful method of investigating the microstructures of paints.

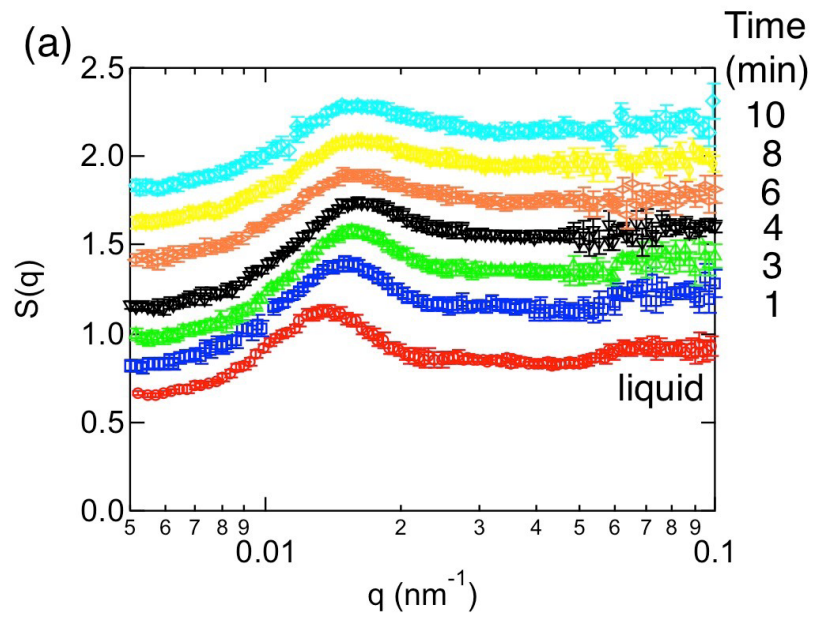

(b)

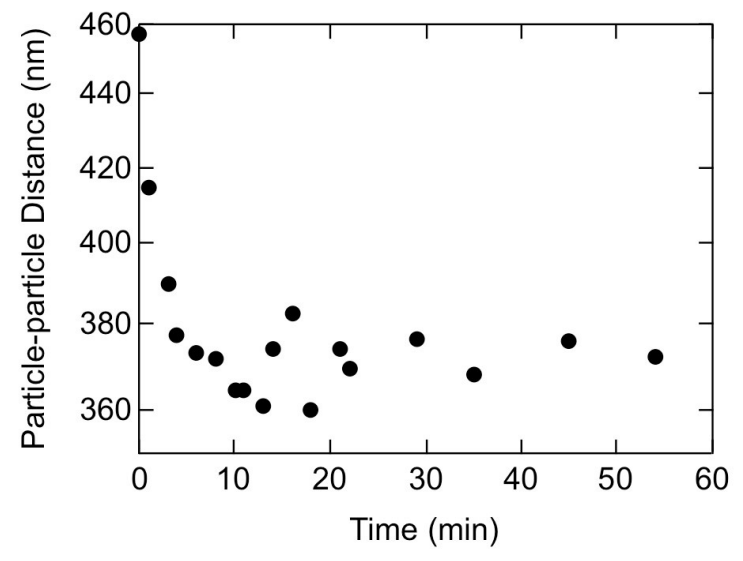

Figure 1. Evolution of microstructure of paint during the drying process. (a) Structure factor (S(q)) of paint; (b) particle-particle distance measured from the peak of the structure factor.

\section{PROPOSED FUTURE WORK}

This project ended in FY 2017. We have exchanged this information with our collaborator at Behr Process Corporation. We also plan to work with Argonne's Technology Commercialization and Partnerships Division to reach out to other paint companies. 


\section{Building a Battery Recycling Model within GREET and BatPaC}

\author{
2017-107-NO \\ Jeffrey Spangenberger and Aude Hubaud
}

\section{PROJECT DESCRIPTION}

Currently, the use of lithium-ion batteries (LIBs) is dominated by consumer electronics (CE) products, and some estimates state a recycling rate of as little as $5 \%$. The use of LIBs in plug-in electric vehicles, however, is quickly increasing and will soon overtake CE market quantities. Vehicle batteries will be recycled at a much higher rate than with CEs due to regulations, and, thus, given that 160,000 plug-in electric vehicles were sold in the United States in 2016 with sales greatly increasing, we are faced with the challenge of managing this waste stream in the near future. Currently, it costs more money to have a plug-in vehicle battery properly treated at the end of the battery's life than can be recovered through revenue from the recycled products.

The goal of this project is to develop a closed-loop battery recycling model to expedite the development of a cost-effective and environmentally friendly end-of-life infrastructure for LIBs. To do this, a spreadsheet-based model, designed to be flexible and extensible, will be constructed in such a way as to break down the individual stages of a battery's life. These stages include manufacture, collection, recycle, and ultimately reuse in new battery manufacture (see Figure 1). Information obtained using Argonne's Battery Performance and Cost (BatPaC) and Greenhouse gases, Regulated Emissions, and Energy use in Transportation (GREET) models has been incorporated into the model.

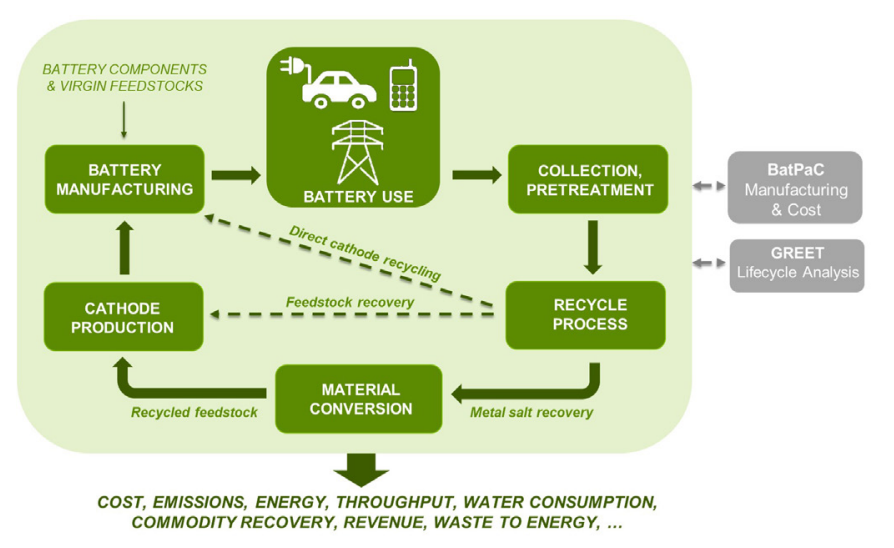

Figure 1. Stages of the closed-loop battery life cycle model and examples of model outputs.

\section{MISSION RELEVANCE}

This project is relevant to DOE's missions in energy and the environment. The use of the successfully completed battery recycling model will allow industry to achieve the goal of reaching the most cost-effective and environmentally friendly route to end-of-life battery management. The successful implementation of an efficient infrastructure within the United States will provide increased security to the country by reducing its reliance on other countries for highly sought-after battery resources.

\section{RESULTS AND ACCOMPLISHMENTS}

This work has concluded with the completion of a baseline closed-loop battery recycling model. Three basic battery recycling routes are included in the model, as well as several different LIB cathode chemistries. The output of the model provides cost, energy, and environmental impacts at the various stages of the life cycle. It also allows for a comparison of these values between batteries made from all virgin materials and those made with any amount of recycled cathode content.

\section{PROPOSED FUTURE WORK}

This project was completed in FY 2017. The baseline model has been used as the starting point for a customized model at the request of a company through a Cooperative Research and Development Agreement (CRADA) project. This project is currently under way. In addition, DOE's Vehicle Technologies Office has started funding further development of this model.

Future work will focus on improving the accuracy of the model's outputs. Higher nickel content cathode materials such as nickel cobalt aluminum and high nickel cobalt manganese oxides will be added to the model's capabilities. We expect to help other stakeholders customize the model for their needs. One example involves adding a proposed recycling process to the model so that it can be evaluated for its cost effectiveness and its impact on the environment. Another example entails the evaluation of a newly proposed battery chemistry to be sure that there are no issues that arise resulting in the prevention of sustainable recycling at end of life. 


\section{Validating Replicability of Waggle Urban Deployments}

\section{7-108-NO}

\section{Charlie Catlett, Pete Beckman, and Rajesh Sankaran}

\section{PROJECT DESCRIPTION}

We will partner with the City of Detroit to install an urban sensing platform that demonstrates the replicability of the Array of Things (AOT) project in Chicago and the scalability of Argonne's underlying Waggle platform to remote installations. Waggle is an open source hardware and software architecture that supports placement of sensors in harsh locations and provides "edge computing" capability, enabling real-time machine learning and other analytics embedded in the field units ("nodes"). Specifically, the goal is to investigate the long-term reliability of the Waggle platform and the optimal placement and density of sensor nodes to measure urban challenges including (1) air quality measurement, (2) traffic and pedestrian flow, and (3) flood detection.

With the early visibility and success of the Array of Things project in Chicago, more than 100 cities, universities, companies, and national laboratories have requested information about pilot projects using the underlying Waggle platform. Based on the unique capabilities of the platform, this widespread interest presents an opportunity for Argonne's Waggle system to become the platform of choice for field measurement in cities and nonurban settings. It is essential, then, that we understand scalability and deployment strategies that will effectively measure critical urban factors such as local air quality and transportation.

\section{MISSION RELEVANCE}

This project is relevant to the DOE missions in areas including environmental sciences (climate, air quality, flooding), transportation (traffic flow and safety), and national security (e.g., machine learning to detect drones with upward-facing camera, detecting gases or other airborne chemicals).

\section{RESULTS AND ACCOMPLISHMENTS}

Working with the City of Detroit's transportation, information technology, and public health commissioners the team developed a deployment plan to place 16 nodes in Southwest Detroit in order to support the evaluation of placement and density strategies for air quality measurement. These nodes will be installed during early
2018, enabling data analysis during the second half of FY 2018.

In this part of the city, the Department of Public Health is conducting an asthma study among youth from low-income areas.

The team formed a partnership with the Cook County Department of Environmental Control in order to place two nodes at a Federal Reference Method (FRM) site in Chicago (See Figure 1). This installation, completed in February 2018, will provide reference data with which to characterize the performance of each air quality sensor.

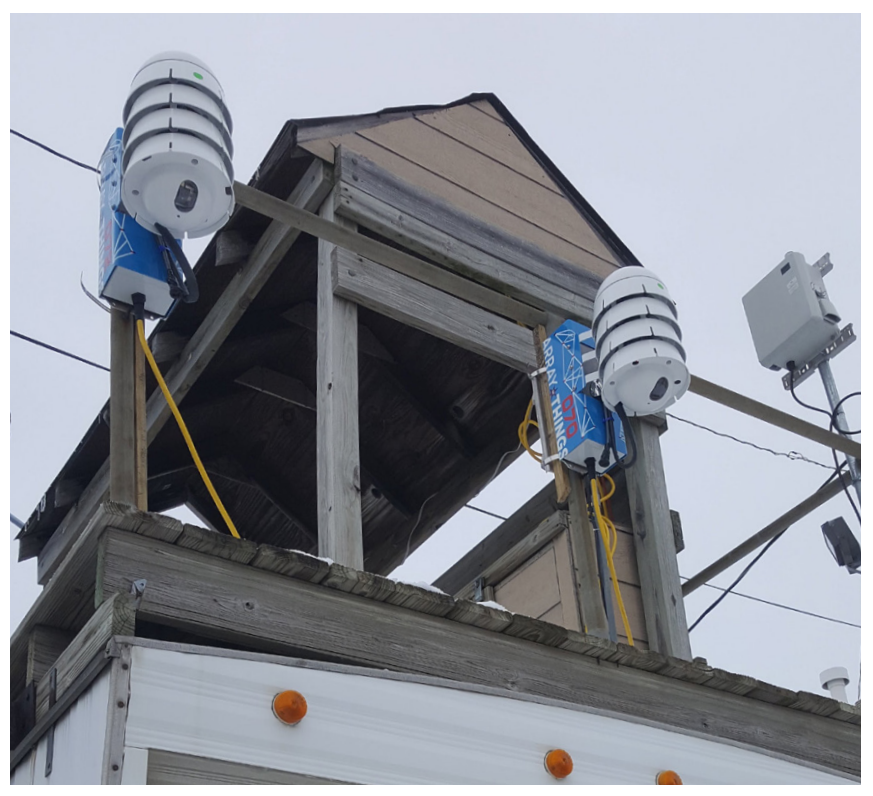

Figure 1: Argonne's Waggle platform hosts sensors and embedded ("edge") computing. Image shows two nodes at a Federal Reference Method site used for air quality sensor calibration.

\section{PROPOSED FUTURE WORK}

In FY 2018, data from the Detroit deployment will examine several sensor placement options to determine optimal strategies for placement of nodes for air quality as well as for capturing traffic flow via a street-facing camera.

Already the early collaborative work planning air quality placement strategies has led the City of Detroit to select the platform for a project funded by the U.S. Department of Transportation. The results from this LDRD project will feed into placement strategies for that project as well as for over a dozen pilot projects in other cities during 2018 alone. The Argonne platform and specifically the AoT packaging is being included in multiple NSF proposals from the University of Chicago, Northwestern, University of Texas, and Northeastern University. 


\section{Foxhunt Proof-of-Concept Demonstration}

\section{7-109-NO}

Pete Heine, Doug Johnson, Ron Orzel, and Scott Parent

\section{PROJECT DESCRIPTION}

Weapon of Mass Destruction (WMD) development programs rely on international procurement of dual-use equipment, materials, and technology. Strategic trade controls regulating exports from the United States are enforced by several federal law enforcement agencies.

Every year these agencies convict or sanction individuals and entities involved in illicitly exporting strategically sensitive goods from the United States. Documents related to these cases represent a rich and largely untapped source of data that could be analyzed to reveal trends in smuggling activities and uncover parties that may still be involved in illegal activities.

Foxhunt will identify actionable information related to the entities involved, modus operandi, commodities sought, methods of transportation, and routes and destinations. Analysts, investigators, and other export control and law enforcement personnel use such information to generate investigative leads, perform better risk assessments, and create more effective targeting profiles.

Publicly available government documents from export enforcement agencies related to closed cases will be obtained, processed, and analyzed. This project supported development of a proof-of-concept demo as a basis for obtaining external project funding.

\section{MISSION RELEVANCE}

The project is relevant to DOE missions in national and global security. Foxhunt will support future counter-proliferation efforts by identifying individuals, organizations, schemes, and modus operandi related to known proliferation activity.

\section{RESULTS AND ACCOMPLISHMENTS}

As a result of project funding, researchers were able to create an illicit procurement ontology and prototype database that demonstrated the potentially valuable information that could be garnered from court documents. This information can be input into the database through a collaboration of subject matter experts (SMEs) with software designed by Argonne engineers that uses natural language processing and machine learning to acquire unstructured text and data.
Based on the proof-of-concept demonstration, the U.S. Department of Defense (DoD), Office of the Secretary (OSD), provided initial funding for a software prototype. The Foxhunt team successfully demonstrated that prototype to OSD in December 2017. The success of that initial effort resulted in a 5-year, up-to- $\$ 5 \mathrm{M}$, umbrella agreement that will fund Argonne SMEs and the further development of the Foxhunt database through calendar year (CY) 2022.

\section{PROPOSED FUTURE WORK}

Foxhunt will be developed as an integral part of DoD's Project Merlin environment, a DoD effort to combine multiple sources of information that can be accessed through one search portal by DoD analysts who are working on counter-proliferation issues.

Initially, Foxhunt is projected to be one of the 13 sources of information available to analysts. A working version of the software prototype is expected to be completed in 2018. By the end of 2018, the database is expected be populated with a majority of documents related to violations of U.S. export controls since 2005. Argonnebased SMEs will also be assisting DoD analysts in their analysis of illicit proliferation networks.

From CY 2019 through CY 2022, Foxhunt will continue to identify and acquire publicly available documents from official U.S. government sources on U.S. export control violations that resulted in criminal prosecutions or administrative penalties. Interest in Foxhunt and Merlin among federal law enforcement agencies will likely lead to efforts to identify and attempt to acquire selected additional investigative case files for Foxhunt. The software tool used by SMEs to process case documents will also support other projects Argonne projects. There also may be interest in developing this software for other mission-critical tasks in the DoD environment.

It is hoped that Foxhunt has a lifespan into the foreseeable future, as it becomes a valuable asset to analysts, researchers, and law enforcement officials. 


\section{Argonne Extended Range Friction Tribometer}

\section{7-110-NO}

Robert Erck, Nicholaos Demas, and George Fenske

\section{PROJECT DESCRIPTION}

It is critical to test engine lubricants at temperatures above $70-100^{\circ} \mathrm{C}$ at high speeds to simulate real engine conditions. High speeds and temperatures are necessary to ensure that the surface chemistries are identical to those experienced in actual use. The Argonne tribology section has a variety of state-of-the-art test rigs to simulate friction conditions, but the rigs are limited to boundary and mixed-lubrication applications, especially applications operating at temperatures greater than $70^{\circ} \mathrm{C}$. The scope of work of this project is to purchase, receive, and install PCS Instruments' model MTM (Mini-Traction Machine) and use the machine to investigate rheological behavior of lubricating oils.

Data generated by the MTM will be directly useful for ongoing projects and for future projects that will use its capability to characterize lubricating oils and additives. The major benefit of this project is the development of the Extended Range Tribometer capability that will enable simulation of the high-speed conditions found in many tribological applications. This capability will permit not only simulation of environments commonly found in many applications, but also to evaluate and optimize technologies developed by Argonne scientists (e.g., diamond-like carbon coatings, other coatings, materials, and additives).

The benefits of greater fuel efficiency include lower operating costs and increased energy security, and the beneficiaries are end users: heavy truck fleets, automotive users, and industrial manufacturers. Future collaborators may be vendors of lubricants who can deliver solutions to original equipment manufacturers.

\section{MISSION RELEVANCE}

This project is relevant to DOE missions in energy and the environment. One of DOE's major goals is to increase the energy efficiency of transportation and industrial systems, and thereby reduce petroleum dependency and greenhouse gases.

\section{RESULTS AND ACCOMPLISHMENTS}

In FY 2017, the system was purchased, received, installed, and accepted. Performance was validated, and the system met performance targets. Figure 1 shows an example of traction test data obtained during installation, to validate the performance of the tribometer. Not shown are test data validating Stribeck curve acquisition.

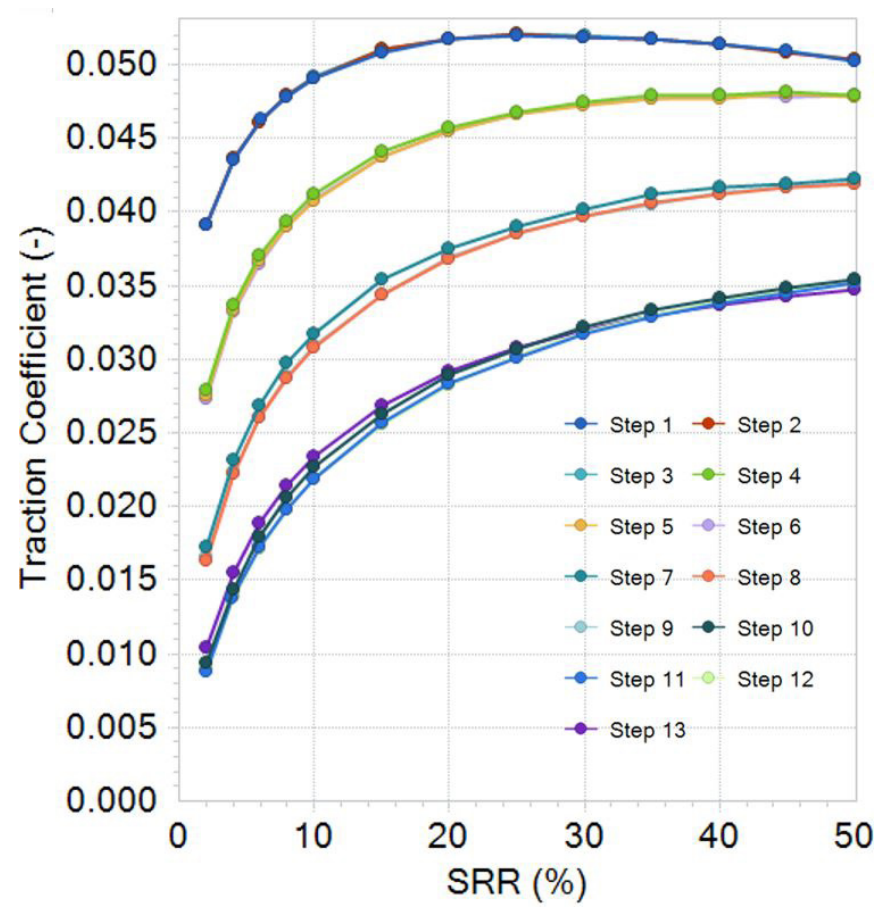

Figure 1. Traction run of MTM from validation tests. (Note: $S R R=$ slide-to-roll ratio $=200\left(u_{1}-u_{2}\right) /\left(u_{1}+u_{2}\right)$ where $u_{1}$ and $u_{2}$ are the speeds of the upper and lower specimens, respectively.

\section{PROPOSED FUTURE WORK}

In FY 2018, work will be conducted to answer outstanding questions on the rheological behavior of seven lubricating oils with different molecular structures: biobased soybean polyvalerate, biobased soybean polypropionate, palm poly-(di-n-bytyl) phosphonate, mineral oil, polyalkylene glycol, polyalphaolefin, and phosphate ester.

The capability developed under this project also resulted in planned strategic partnership projects for lubricant characterization with Croda International and Afton Chemical.

Due to the success of this project, under Round 3 of the Small Business Vouchers (SBV) program, Argonne was selected to partner on an SBV proposal, submitted by Precision Polyolefin LLC, to characterize the tribological performance of the company's proprietary polymer, which has unique attributes that can potentially improve the shear stability and volatility of fuel-efficient lubricants. 


\section{Ultra-High Efficiency Fuel Cell-Heat Engine Hybrids}

\section{7-113-NO}

Sreenath Gupta, Rajesh K. Ahluwalia, and Theodore Krause

\section{PROJECT DESCRIPTION}

An efficient way of using our nation's natural gas deposits is through the widespread deployment of distributed generation (DG). A technology that transcends the shortcomings of prime movers in existing DG technologies is a fuel cell operating in tandem with a heat engine. Through a systems modeling approach, a potential fuel cell-heat engine (FCHE) hybrid scheme will be identified, which makes effective use of the waste heat from the fuel cell and the heat engine. Additional efficiency gains are possible by harnessing energy from the unused fuel (anode off-gas) from the fuel cell.

\section{MISSION RELEVANCE}

This project is relevant to the DOE mission in energy. Overall, the objectives of this effort conform to DOE's goal to reduce U.S. petroleum consumption. Moreover, they are the same as that of the Advanced Research Projects Agency-Energy (ARPA-E) program, which is to develop FCHE hybrids featuring, namely, efficiency $>70 \%$, initial cost $<\$ 1,800 / \mathrm{kW}$, and low water consumption. Key technologies developed in this effort may help improve the performance of stationary as well as on-road medium-duty and heavy-duty natural gas engines. As a result, efforts pursued under this project will be of interest to the DOE-Office of Vehicle Technologies and the Advanced Manufacturing Office.

\section{RESULTS AND ACCOMPLISHMENTS}

A thorough literature search showed that out of all of the variants of fuel cells, solid-oxide fuel cells (SOFCs) offer the most amenable architecture for hybridization with a heat engine. In addition, the team identified a novel technology for a natural gas reformer that could offer pure hydrogen as the fuel for the SOFC.
Subsequently, a few potential SOFC-microturbine (SOFC-MT) and SOFC-reciprocating engine (SOFC-RE) hybrid systems were devised and evaluated by leveraging the existing systems' modeling capabilities at the Nuclear Engineering Division of Argonne. The final optimal SOFC-RE hybrid system consisted of (1) optimal operation of the SOFC using hydrogen fuel, (2) anode gas recirculation, and (3) a turbocharged reciprocating engine capable of utilizing dilute fuel-air mixtures. Computer simulations of this hybrid system show that it achieves the efficiency and cost targets. In response to a funding opportunity announcement by ARPA-E, a concept paper based on these findings was submitted for further evaluation.

In parallel to the above modeling effort, a test bed is being prepared to evaluate the performance of some prototype natural gas reformers/gas separators. By the end of FY 2017, all of the required instrumentation was gathered, and a contract to a potential supplier was issued to supply two prototypes of the reformer/gas separator.

\section{PROPOSED FUTURE WORK}

FY 2018 activities will include:

$\square$ The FCHE-RE system architecture will be further refined for higher efficiency and lower cost;

$\square$ Prototypes of the novel fuel reformer/gas separator will be evaluated for the purity of hydrogen produced and power and heat requirements; and

$\square$ Candidate technologies for AC/DC and DC/AC power electronics will be identified that are cost-effective for integration with the FCHE hybrid. 


\section{Stretchable Conductive Ink for Energy Harvesting Device}

\author{
2017-116-NO
}

Yunsong Xie

\section{PROJECT DESCRIPTION}

Conductors are key components of radiofrequency (RF) electronics that transfer electrical signals and power. Traditional conductors used in RF devices are rigid metallic materials, such as copper $(\mathrm{Cu})$, silver $(\mathrm{Ag})$, and gold $(\mathrm{Au})$. This project is attempting to develop a material that is both highly conductive and stretchable. The method is to fabricate a composite material consisting of an ionic liquid, a block-copolymer, and metal nanoparticles. The nanoparticle-percolated network provides the high conductivity. The ionic liquid produces a conductive matrix that maintains the integrity of the conducting network even under large shape deformation. The block-copolymer serves as the binder to solidify the composite, making the composite material self-standing and easy to handle.

\section{MISSION RELEVANCE}

This project is relevant to DOE's mission in national and global security by enabling ultra-deformable RF devices, as it aims to develop a highly conductive and stretchable iono-elastomer composite material that can be used as the conductor of the RF devices. An ultra-deformable RF device, especially an RF antenna, can be easily transported when inactive and operates with high gain/large directivity during operation. Functional ultra-deformable RF devices increase the survival probability of remotely located units, such as solders, robots, and drones, by enabling them to travel without being slowed down by an extra burden while maintaining successful communication with distant friendly units.

\section{RESULTS AND ACCOMPLISHMENTS}

We have successfully developed the iono-elastomer by mixing the co-polymer Pluronic ${ }^{\circledR}$ F123 and ionic liquid ethylammonium nitrate. Microstructural, electrical, and mechanical properties have been carefully studied in order to achieve a successful conductive ink product. During the study, we found that the microstructure of the iono-elastomer changes drastically during elongation, based on an observed pattern in a small-angle X-ray scattering measurement. This was the first time such an interesting physical property was observed for this material.
The study of the electrical properties of the iono-elastomer revealed that during the elongation test, its resistance increase is slower than the theoretically calculated curve using the traditional material model (see Figure 1). This finding indicates that the conductivity of the iono-elastomer increases as the material is being elongated, which is extremely important for the conductive ink application.

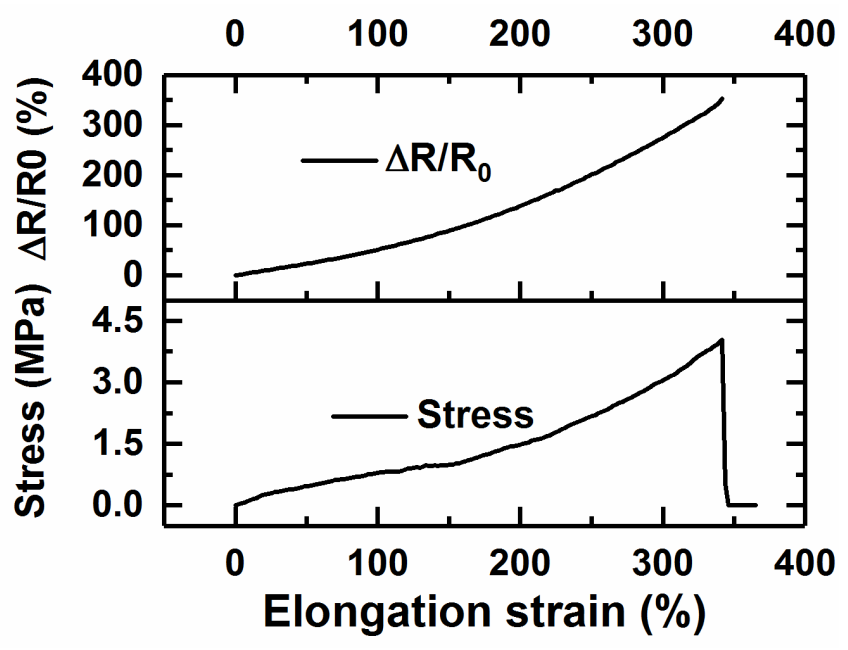

Figure 1. The resistance and stress change of the iono-elastomer when elongated up to break.

\section{PROPOSED FUTURE WORK}

The next step of this project is to integrate metal nanoparticles with the developed iono-elastomer. Thanks to the increase in conductivity upon elongation, we expect that an RF device based on this conductive ink would have minimal performance deterioration when being structurally deformed.

We also found that the resistance of the iono-elastomer changes monotonically as it is being stretched. The resistance change at a small elongation level $(<50 \%)$ is mostly linear with respect to the elongation. Therefore, we are planning to use this property to construct a wearable strain sensor that is able to detect body motion. Such a device has wide potential application in both the medical and robotic communities. Multiple funding agents have released solicitation calls regarding such applications, such as the Defense Advanced Research Projects Agency, the U.S. Air Force, and FlexTech. We are planning to submit proposals for funding to support further research. 


\section{Improvement of in situ Nuclear Fuel Characterization Capability at APS}

\author{
2017-118-NO \\ Yinbin Miao, Kun Mo, and Abdellatif M. Yacout
}

\section{PROJECT DESCRIPTION}

This project aims to utilize the unique material characterization capabilities of the Advanced Photon Source (APS) to investigate microstructure evolution in nuclear fuels in support of the development of the advanced fuel performance code, MARMOT. Its unique high-energy and high-brightness features make the APS powerful in the microstructure characterization of advanced materials. However, radiation hazards of nuclear fuel materials limit the application of the APS in this area. Experimental devices dedicated to nuclear fuel materials must be developed to have strict containment of radioactive material in order to improve the nuclear fuel characterization capability at the APS. In this project, test apparatuses with in situ investigative capabilities for nuclear fuels were designed, built, and tested for the APS to enable future in situ measurements using synchrotron-based X-ray techniques.

\section{MISSION RELEVANCE}

The data produced by the APS investigations using the apparatuses developed in this study are valuable for the development of the MARMOT code, which is a crucial component of the DOE Nuclear Energy Advanced Modeling and Simulation program. MARMOT is an advanced fuel performance code that is based on fundamental microstructural mechanisms in nuclear fuels. Therefore, the improvement of MARMOT relies on microstructural characterization results with well-controlled conditions for verification and validation purposes, especially kinetic results collected from in situ experiments. Thus, the success of this project will definitely advance MARMOT development, helping to enable precise prediction of nuclear fuel performance, and thus advance nuclear power applications.

\section{RESULTS AND ACCOMPLISHMENTS}

Two apparatuses with advanced in situ synchrotron investigative capabilities were designed and built. The apparatuses feature double-containment of nuclear fuel materials to address safety concerns. Each apparatus is also equipped with a high-efficiency particulate air filter and a precise pressure gauge to enable continuous pumping during in situ measurements so that sample oxidation at elevated temperatures can be minimized by maintaining high vacuum. The apparatuses were tested at the APS at up to $900^{\circ} \mathrm{C}$ for 8 hours to demonstrate their capability and reliability during a radiation safety review (see Figure 1). Based on the success of those tests, a systematic experiment plan was made, and APS proposals requesting beam time were composed and submitted.
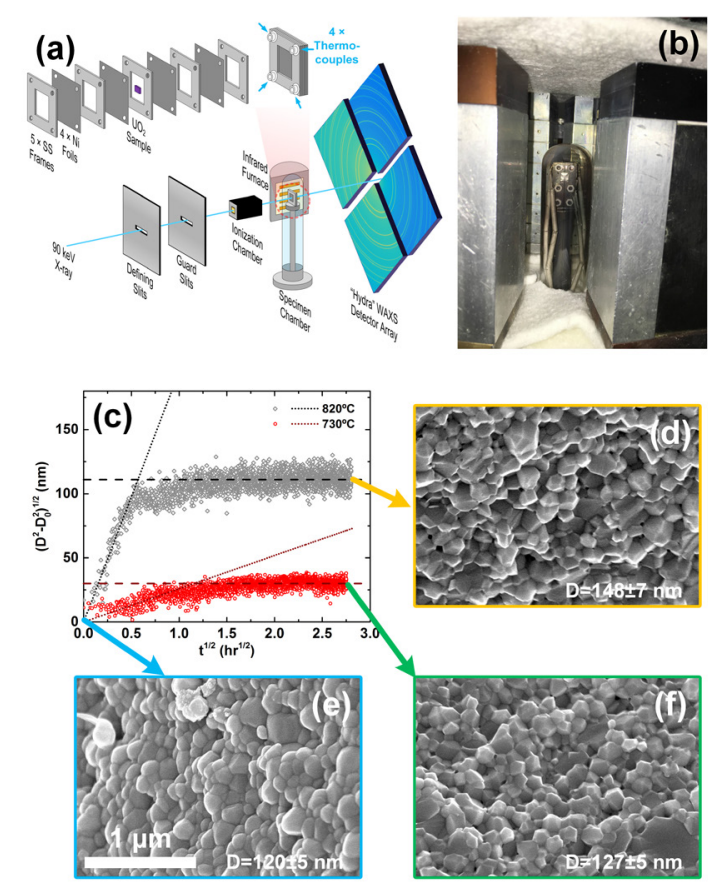

Figure 1. Preliminary data collected using the in situ APS apparatuses: (a) experimental setup for the in situ APS grain-growth measurement; (b) apparatus in furnace; (c) measured grain-growth kinetics; (d) scanning electron microscopy (SEM) image of the sample after 8-hour annealing at $820^{\circ} \mathrm{C}$; (e) SEM image of the sample before annealing; (f) SEM image of the sample after 8-hour annealing at $730^{\circ} \mathrm{C}$.

\section{PROPOSED FUTURE WORK}

The grain growth kinetics of nanocrystalline nuclear fuels at elevated temperatures (up to $900^{\circ} \mathrm{C}$ ) will be measured using the apparatuses developed in this project. A variety of nuclear fuel materials, including conventional fuels $\left(\cup_{2}\right)$ and accident-tolerant fuels $\left(\mathrm{U}_{3} \mathrm{Si}_{2}\right)$, will be tested. The results will be valuable in developing corresponding grain-growth models in MARMOT. 


\section{Argonne's UAS Capability: Application Framework Development and Benchmarking}

\author{
2017-131-NO
}

Yuki Hamada

\section{PROJECT DESCRIPTION}

Although unmanned aerial systems (UASs) have become increasingly popular, the effective use of UASs faces various scientific and technical challenges. Those challenges are often universal across a range of applications including environmental science research and projects. The goal of this study is to develop a framework for using UAS applications in a collection of environmental observations to support environmental decision-making. For this project, a series of UAS-aided and field-data collection experiments was designed that target current and future environmental research needs. Through implementation of these experiments, we will benchmark our capabilities in developing UAS-supported environmental observation designs and in conducting such data collection activities.

\section{MISSION RELEVANCE}

This project is relevant to the DOE mission in science. UAS is recognized as being integral to the future of timely, flexible, and cost-effective collection of detailed environmental data. Such data collection may be associated with, but not limited to, infrastructure mapping and monitoring, natural resource management, and environmental cleanup. UAS-aided environmental observations will likely become a means of supporting both basic and applied science in the near future.

DOE recognizes UASs as a valuable means of environmental observation and sampling for scientific research. Since 2016, DOE's Biological and Environmental Research has been funding the development of UAS-compatible hyperspectral cameras through the Small Business Innovation Research program. The UAS capability could greatly support the monitoring and assessment of bioenergy crop production currently funded by the DOE Offices of Energy Efficiency and Renewable Energy and Bioenergy Technologies. Other DOE national laboratories (e.g., Oak Ridge, Idaho, and Brookhaven) are actively pursuing the development of expertise in UAS applications. Developing Argonne's UAS capability could improve large-scale collaborative work among these national laboratories.
A UAS capability is also relevant to other federal agencies with land management responsibilities, including the U.S. Department of Defense and the Bureau of Land Management.

\section{RESULTS AND ACCOMPLISHMENTS}

In FY 2017, we conducted ten image collection missions that focused on UAS-aided topographic-survey and vegetation-change monitoring applications. The missions aimed to collect aerial images and field observations for the experiments, to improve our effectiveness in mission planning and execution for operational UAS applications. Using the UAS collections, we initiated: (1) an exploration of optimal specifications and application-specific parameter settings for image collection and processing, and (2) the testing of image transformation for extracting target information.

For the topographic survey, we collected stereoscopic images of objects with known dimensions in various sizes to test the accuracy and precision of UAS imaging for estimating the lengths and volumes of the objects using a photogrammetric technique. Data analyses are currently in progress. For vegetation-change analysis, test plots simulating an arid landscape were established, and time series images were collected using a UAS-mounted camera (Figure 1). These images are currently undergoing spectral transformation analyses to test the feasibility of using UAS-aided observations for monitoring vegetation conditions.

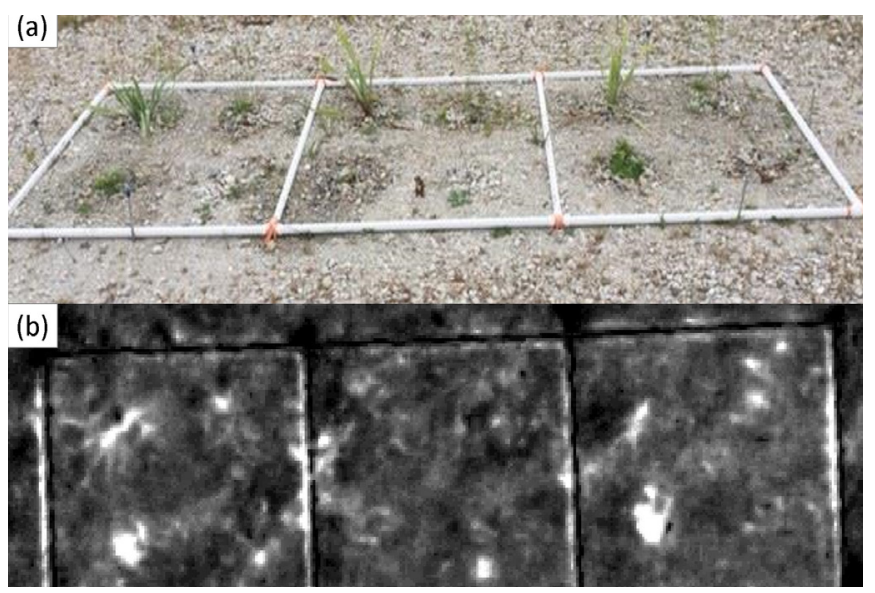

Figure 1. Example of data collected in the project: (a) field picture of vegetation plots, and (b) Image enhanced for green vegetation.

\section{PROPOSED FUTURE WORK}

Using images collected during FY 2017 and applying lessons learned so far, we will complete image analysis and develop the UAS application framework and protocols. We will also develop and test UAS capabilities for other applications. 
Accurate, Real-Time Categorization of Unmanned Aircraft Systems (UASs) in an Urban Environment through Application of Deep Learning Strategies Leveraging Distributed Computing Technology

\author{
2017-137-NO
}

Adam Szymanski

\section{PROJECT DESCRIPTION}

This project will advance development of a distributed image-based (electro-optical) UAS detection system utilizing upward-facing video cameras on large-scale urban sensor networks using advanced machine learning (ML) techniques, such as deep learning (DL). The project has focused on collecting training data and developing advanced $\mathrm{ML}$ algorithms for real-time embedded processing and recognition. The ultimate goal is to advance the system enough to demonstrate its capability at a relevant facility. To accomplish this goal, the major tasks include building data collection devices (distributed sensor nodes), collecting and tagging training data, developing advanced ML algorithms, developing a partnership with a demo facility, building sensor nodes and deploying them to the facility, and testing/ demonstrating the system.

\section{MISSION RELEVANCE}

Several aspects of this program are relevant to DOE missions, including national security, machine learning, and edge computing. Counter-UAS and UAS detection are national security problems of growing concern. Despite a large amount of existing work in this area by the U.S. Department of Homeland Security (DHS) and the U.S. Department of Defense (DoD), there are a number of gaps in existing systems that are still areas of research. This project focuses on urban detection of non-radiofrequency-emitting UASs, while advancing ML techniques. The DL models developed through this program will advance current capabilities for using high-performance computing (HPC) resources to train deep neural networks. By utilizing the Argonne-based program Waggle, this project will provide a concrete example of a distributed advanced edge-computing system.

\section{RESULTS AND ACCOMPLISHMENTS}

To date, this program has accomplished several things. In the area of data collection and tagging, we have purchased and built custom data collection nodes based on the Waggle platform; collected a significant amount of skyward-facing video data representing birds, airplanes, drones (flown by Argonne staff using platforms from the growing Argonne aviary), and other objects; and processed and assembled a tagged data set of 3,000 training images. We have also progressed in developing algorithms and models for detection and classification, including the creation of a modified algorithm to collect video data from nodes; made updates to tracking algorithms based on tests performed on collected data; and begun generating/training new DL classification models with newly collected images.

We have also made significant progress in creating a partnership for program demonstration with the Chicago Bears and Soldier Field management teams to install and demonstrate the program at the stadium and around the Museum Campus. We have had several meetings with teams from Soldier Field, the Chicago Department of Transportation, the Chicago Office of Emergency Management and Communication, and DHS to prepare and discuss the installation. We have started the process of entering into a Cooperative Research and Development Agreement between Argonne and Soldier Field that will allow us to both demonstrate the program and utilize the deployed nodes as a testbed for data collection and continuing algorithm development.

\section{PROPOSED FUTURE WORK}

As mentioned above, we have collected a large amount of training data; however, we will need to continue data collection efforts. This work will include continuing drone flights by Argonne staff both on campus and off, Web scraping of posted videos, and developing a cooperative relationship with other DoD organizations that have expressed interest in sharing data for common goals. We will also need to refine and continue development of a DL classification algorithm utilizing the Mathematics and Computer Science Division's HPC DL pipeline. We will use several DL libraries as well as several network structures and compare model results to create the most effective classifier while balancing performance of the edge-computing hardware. To complete the demonstration, we will also need to purchase and construct fully functional Waggle nodes and deploy them to the Soldier Field/Museum Campus. Once this is accomplished, we will collect data and further refine the algorithms to work in a deployed environment. 


\section{Applying Community Network Analysis to Generalize Microbial Assembly Rules}

\author{
2017-141-NO \\ Pamela Weisenhorn

\section{PROJECT DESCRIPTION}

This project examines whether stressful environmental conditions result in more tightly knit, co-dependent microbial communities when compared to nearby but less stressful environments. Understanding how microbial communities are structured in relation to environmental stress is critical for the eventual prediction and management of these communities in many contexts.

To address this question, comparison of microbial community structure and the nature of interspecific interactions among samples collected along flooding and salt gradients is done (Figure 1). These samples will be provided by collaborators working in these areas across the United States.

This project involves (1) identification and coordination of sample collection with collaborators, (2) deoxyribonucleic acid (DNA) extraction and sequencing, (3) microbial interaction network analysis, and (4) analysis of patterns in community structure along stress gradients, followed by presentation and publication of the results.

\section{MISSION RELEVANCE}

DOE's Genomic Science Program has five key objectives, three of which are touched on by the interaction network-based approach applied in this project, namely: a network-based approach to microbial ecology allows generalization and scaling of biological processes from organisms to complex communities, aids in the development of fundamental understanding and design rules governing microbial communities, and presents the potential to use network parameters to improve modeling capabilities and advance predictive understanding and manipulation of microbiomes. In addition, this approach addresses the DOE Climate and Environmental Sciences Division goal of advancing fundamental understanding of coupled biogeochemical processes in complex environments to enable systems-level environmental prediction, specifically by examining a potential diagnostic signature of microbial community composition across a spatial scale of relevance to Earth system models.
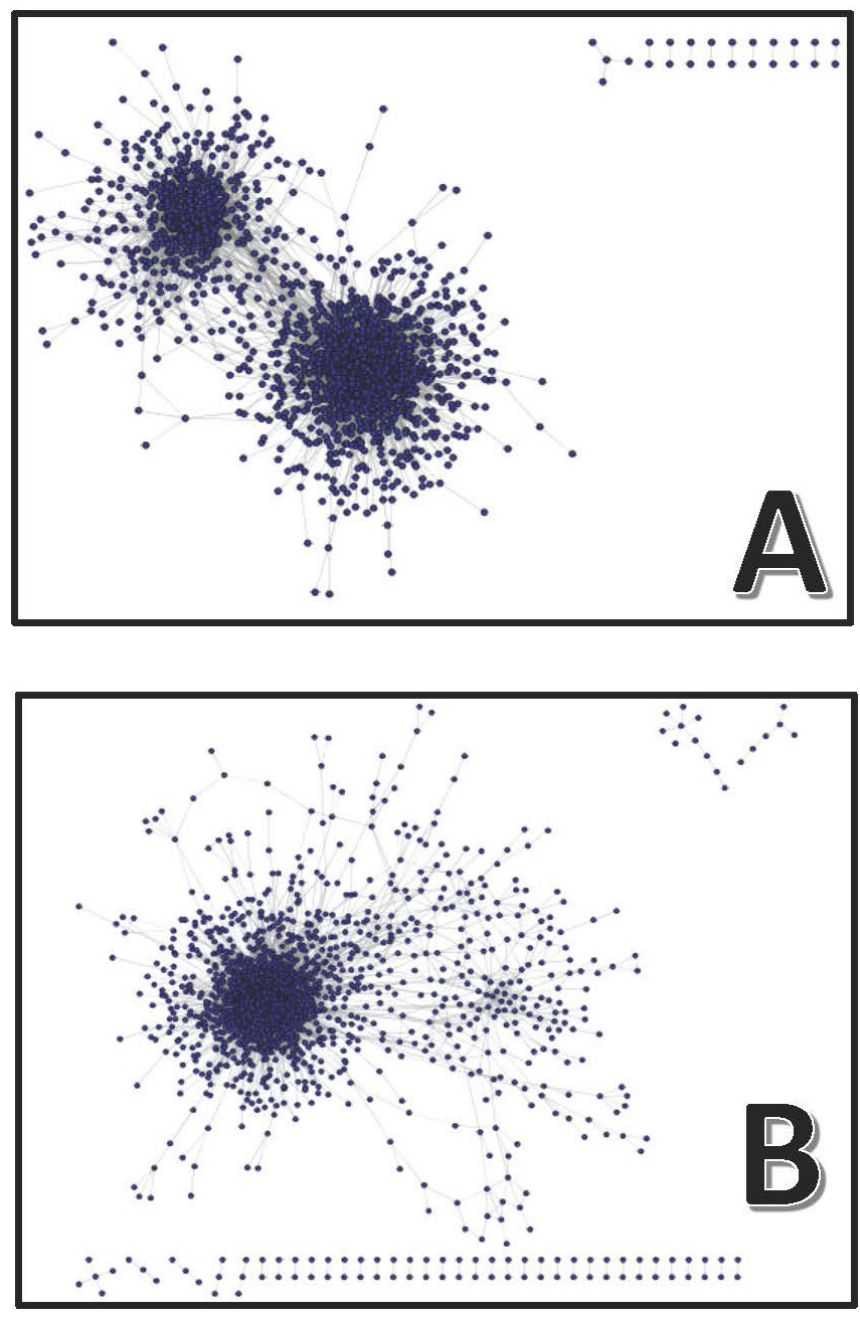

Figure 1. Preliminary data showing shifts in microbial interaction network structure of surface sediments from a freshwater wetland in response to seasonal variation. A) Interaction network in January, 2017 when surface water was frozen and plants were inactive, limiting carbon and oxygen in the sediments. B) Interaction network in March, 2017 at the start of the growing season, supplying both carbon and oxygen to the sediments.

\section{RESULTS AND ACCOMPLISHMENTS}

In the first three months since this project was funded, collaborators' interest has been confirmed and their availability for sampling and collection of associated metadata has been discussed.

\section{PROPOSED FUTURE WORK}

In the next nine months, the sample and metadata collection protocol will be finalized, samples from collaborators will be received, extracting and sequencing DNA will be performed, and microbial interaction network and stress-response pattern analyses will be conducted. This work will then be prepared for presentation and publication in the following year. 


\section{Immersive 3D Visualization of Military Complex Systems}

\author{
2017-152-NO
}

Brian Craig, John Murphy, and Michael Samsa

\section{PROJECT DESCRIPTION}

The goal of this project is to develop a three-dimensional (3D) virtual reality visualization capability for demonstration to the Joint Chiefs of Staff and the United States Central Command (USCENTCOM). The project consists of multiple phases, including the following:

\footnotetext{
$\square$ Perform systemic problem structuring to diagnose and structure the complexity of the problems being addressed. The results are used to inform how the 3D immersive visualization of systems data fits into the overall systemic problem space.

$\square$ Translate unstructured data into the 3D visualization structure to allow for integration into the 3D environment.

$\square$ Develop interactive data exploration and manipulation capabilities.

$\square$ Build a capability to connect visualization to dynamic data generated by simulations.

$\square$ Present a mobile demonstration to Operations Directorate (J3) and USCENTCOM sponsors.
}

\section{MISSION RELEVANCE}

This project is relevant to DOE missions in national security and systems science. Developing this prototype provides a way to demonstrate how the 3D visualization done by Argonne enhances the military's existing efforts in complex adaptive modeling and simulation, structured system mapping, and general complex data analytics. Because these types of complex systems data are becoming more and more important for decision-making in the military (as well as across government and industry), the ability to enhance the understanding and exploration of these data will bring a high-impact capability to this sponsor, in addition to many others. In addition, it is our belief that complex problem structuring for Argonne itself and the visualization of the Laboratory as a complex adaptive system could greatly enhance our ability to develop systemic strategies.

\section{RESULTS AND ACCOMPLISHMENTS}

Under this project, the Argonne team successfully created a prototype version of an advanced 3D visualization environment. The team integrated data from multiple sources, including data on entities and relationships automatically generated by the Air Force Research Laboratory, and hand-coded thematic relationships provided by a contractor performing the data gathering. The team also incorporated outside data from simulations run by the United States Army Training and Doctrine Command (TRADOC). After creation of the $3 \mathrm{D}$ visualization environment, the team successfully demonstrated the capability to numerous potential sponsors, including notable demonstrations to USCENTCOM and the Office of the Under Secretary of Defense for Intelligence.

The team received a Letter of Appreciation from the Joint Chiefs of Staff, commending the effort we expended to develop and deliver the successful demonstration of the $3 \mathrm{D}$ visualization system.

\section{PROPOSED FUTURE WORK}

Argonne is currently in the process of assisting TRADOC with the process of migrating the visualization software onto systems operated by the U.S Department of Defense (DoD). Once the initial software has been approved for use on the DoD systems, we will make an effort to coordinate any potential future funding opportunities from TRADOC/Defense Advanced Research Projects Agency. 


\section{Tribology of Graphene at Elevated Temperatures}

\section{7-154-NO}

\section{Anirudha V. Sumant}

\section{PROJECT DESCRIPTION}

A high-temperature module was acquired for the Center for Nanoscale Materials' (CNM) tribometer and for pin-on-disc tribological studies of stainless steel sliding at elevated temperatures $\left(24^{\circ} \mathrm{C}-300^{\circ} \mathrm{C}\right)$ with and without graphene lubricant. Included in the investigations were molecular dynamics simulation studies to model graphene interactions at elevated temperatures. Together, the experimental and theoretical studies will establish the feasibility of graphene as a lubricant for metal contacts at elevated temperatures in air. These data will greatly help to elucidate the graphene degradation mechanism in air at elevated temperature and will provide new insight into how graphene could be used as a solid lubricant in metal-forming applications, which is of great interest for automotive applications.

\section{MISSION RELEVANCE}

The project is relevant to DOE's Vehicle Technologies Office mission to reduce energy wasted in friction and to help keep the environment clean. The data obtained were very important in forming a collaboration with industry partner Magna International, Inc., which led to receipt of a DOE Technology Commercialization Fund (TCF) grant to carry out further studies for metal-forming applications in the automotive industry.

\section{RESULTS AND ACCOMPLISHMENTS}

The new high-temperature stage was acquired and installed in the multifunctional tribometer at the CNM. Various tribological tests were carried out on stainless steel surfaces with and without graphene lubricant by varying the temperature from room temperature to $300^{\circ} \mathrm{C}$ in air. The wear track was characterized using Raman spectroscopy and confocal microscopy to investigate surface modification during sliding.
Figure 1 shows pin-on-disc tribology test results for a diamond-like-carbon (DLC)-coated stainless steel ball sliding against a stainless steel disc coated with a few layers of graphene. The friction coefficient was found to decrease by more than $20 \%$ with the increase in temperature from room temperature to $300^{\circ} \mathrm{C}$. Molecular dynamics simulations carried out separately agreed with the experimental results. The simulation studies further predicted superlubricity for graphene sliding against graphene in vacuum at elevated temperatures under certain tribological conditions, which is an important finding of these studies.

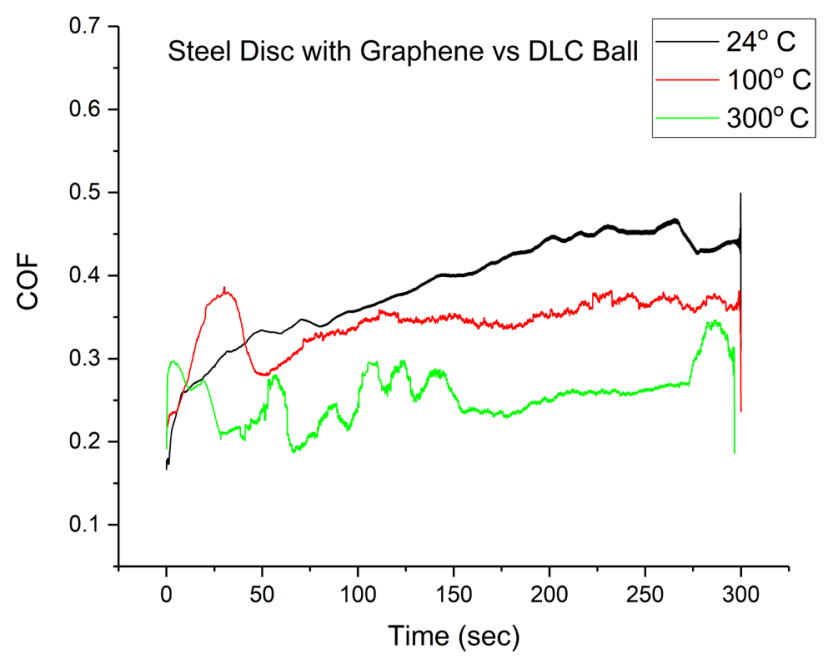

Figure 1. Pin-on-disc tribotesting result demonstrating decreases in friction with increases in temperature (from room temperature to $300^{\circ} \mathrm{C}$ ) when graphene is used as a solid lubricant.

\section{PROPOSED FUTURE WORK}

The new DOE-TCF project funding $(\$ 640,000)$ was awarded to carry out further studies in collaboration with industry partner Magna International, Inc. The future studies will be part of a new Cooperative Research and Development Agreement with Magna International, Inc. 


\section{Production of Cellulose Nanocrystals from Miscanthus x Giganteus}

\author{
2017-159-NO \\ Gregory Krumdick, Erik Dahl, and Krzysztof Pupek
}

\section{PROJECT DESCRIPTION}

Demand for environmentally friendly materials to replace petroleum-based products is on the rise. Cellulose nanocrystals (CNCs) are produced from the fibers of plants and have numerous uses. Currently, the only commercially available CNCs are produced from wood. Producing $\mathrm{CNC}$ from Miscanthus $\times$ Giganteus (MxG), a large grass species, has the potential to be far more efficient while negating concerns about deforestation. MxG can also produce a longer, more valuable crystal.

The purpose of this project is to develop an advanced large-scale process to produce cellulose nanocrystals from MxG. This work was initiated in the University of Chicago's Institute for Molecular Engineering (IME) by Stuart Rowan. This project is a collaboration between IME and Argonne that provides the opportunity to share expertise and strengthen the relationship between both organizations.

The process will be developed by taking the laboratory batch process from the gram to the kilogram scale. To achieve this goal, the following objectives must be met. An industrial process must be developed that produces CNCs that match the properties of the small-scale process. The process should produce crystals that are $5 \mathrm{~nm}$ wide and 200-300 nm long. The overall crystallinity of the product should be greater than $80 \%$. The process must be optimized for efficiency to minimize waste production and energy use.

\section{MISSION RELEVANCE}

This project is relevant to DOE missions in basic science, energy, and the environment. Because of the inherent properties of CNCs, their surface structure is easily modified. The crystals are also very strong and exhibit high thermal stability. This strength and customizability allows CNCs to be used in many applications. CNCs have applications for advanced battery and catalyst material, as well as additives to change the properties of other materials.

Thus, the wide range of uses for CNCs means that the relevance of this project for domestic industry cannot be overstated. Aloterra-a company that produces raw pulp, packaging, and sorbents from its $M \times G$ crop-has expressed strong interest in licensing the intellectual property that results from this project.

\section{RESULTS AND ACCOMPLISHMENTS}

The production of CNCs can be broken down into four steps (see Figure 1). We used a systematic approach to process research and development using the design of experiment methodology, and produced improved procedures for the first three steps. We adapted the new low-waste, scalable procedures to real-world practice. This process meets the kilogram-scale milestone while reducing waste generation by $60 \%$ and yielding $1.3 \mathrm{~kg}$ of $\mathrm{CNC}$ in a single batch.

We discovered that the size distribution of the crystals can be controlled by varying the reaction conditions. This finding raises the possibility of tailoring the crystal size for specific applications.

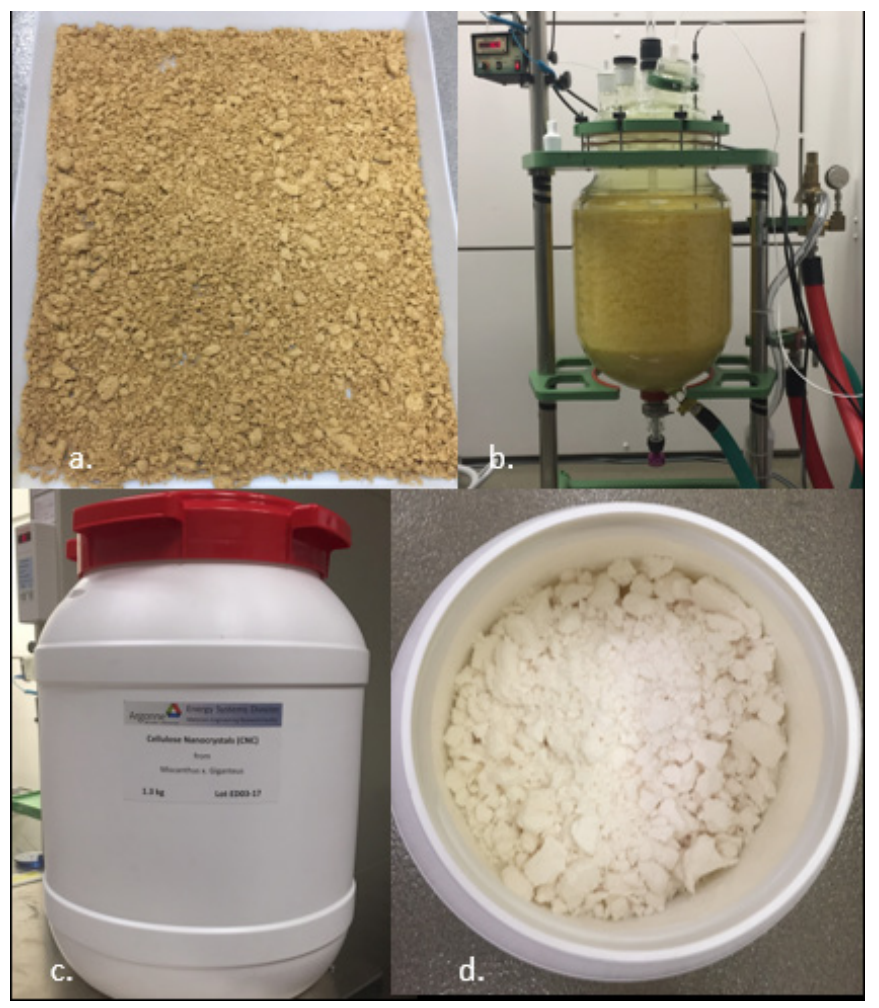

Figure 1. (a) Raw pulp, (b) CNC creation in process in a 50-L reactor, and (c), (d) 1.3-kg batch of CNCs.

\section{PROPOSED FUTURE WORK}

In FY 2018, we will continue work on the fourth step of the process, which involves modifying the surface of the crystals to broaden the possible applications of the CNCs. We will develop viable reaction, separation, and purification processes for kilogram-scale and larger production. 


\section{Neuromorphic Architectures for Dynamic and Reactive Learning Based on Neuromodulation}

\author{
2017-160-NO
}

Angel Yanguas-Gil

\section{PROJECT DESCRIPTION}

This project focuses on developing architectures for neuro-inspired systems that can react to a changing environment.

The key innovative aspect of this project is the exploration of modulatory interactions to control the dynamics of neurons. These types of interactions are crucial for biological systems, but they had not been significantly explored in the open literature.

The proposed work was carried out in two tasks: the identification of suitable design principles for modulatory interactions, and the identification of suitable architectures at the hardware and material levels capable of implementing the desired functionality.

The goal of this project is to develop the design principles for systems capable of reactive learning and to identify the suitable architectures and devices that could be used to implement such systems.

\section{MISSION RELEVANCE}

This project is relevant to DOE missions in basic science. The development of smart systems able to adapt and respond to a changing environment is crucial for areas such as autonomous vehicles and drones, smart sensors, and robotics. Consequently, this is an area of interest for national security, advanced manufacturing, and environmental applications.

Within the U.S. Department of Defense there has been a strong interest in this type of application, from agencies including the Defense Advanced Research Projects Agency (DARPA) and the U.S. Air Force.

More broadly, in the last several years there has been renewed interest in neuromorphic computing. Although this proposal does not focus on high-performance computing applications, there is a strong overlap between the two fields.

\section{RESULTS AND ACCOMPLISHMENTS}

The key accomplishments of this project are as follows:

1. We developed a connection between spiking neural networks (biologically inspired) and a recurrent artificial network (a key component for machine learning) and demonstrated the functional equivalence of the two models (Figure 1). This result allows us to design heterogeneous models incorporating both spiking and non-spiking architectures, and to accelerate the emulation of large spiking systems.

2. We implemented modulatory interactions on an artificial neural network and demonstrated the ability to perform navigational tasks using associative and reinforcement learning through a simple system inspired by the olfactory system of insects.

3. We identified hardware and material architectures that could implement some of the key functionalities demonstrated by simulations.
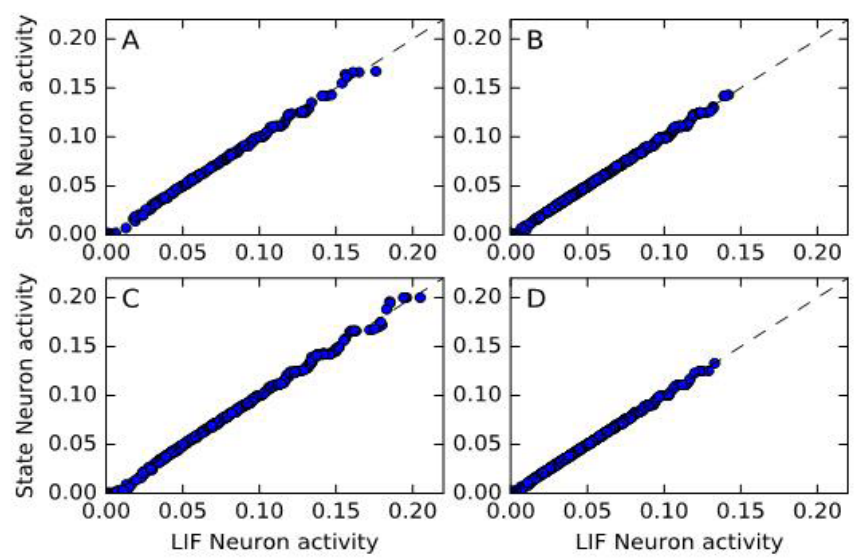

Figure 1. Correlation between neuron activity in a network of leaky integrate-and-fire (LIF) spiking neurons and the activity of their recurrent analogs, showing the functional equivalence between the two models.

\section{PROPOSED FUTURE WORK}

This fiscal year, we prepared a response to DARPA's Lifelong Learning Machines (L2M) program that builds on the concepts developed in this proposal. The team involves researchers from Argonne National Laboratory, Sandia National Laboratory, and the Rochester Institute of Technology, with Argonne acting as lead. Our proposal was selected and is currently in the contract negotiation phase. The proposed budget is approximately $\$ 3.6$ million over 4 years.

We will also seek further funding, either through internal or external channels, aimed at designing and fabricating devices and architectures that implement the design principles developed in this proposal. 


\section{A Mechanically Based Antenna for RF-Incompatible Environments}

\author{
2017-161-NO \\ Kaizhong Gao
}

\section{PROJECT DESCRIPTION}

This project will develop a component technology to enable a high-charge-capacity antenna for radiofrequency $(\mathrm{RF})$ radiation at low frequency or a new form of solid-state supercapacitor for energy storage. For mechanically based antenna, a large charge density antenna is required. We proposed the new design based on magnetic nanofibers and the atomic layer deposition (ALD) process. Our simulation showed that a large surface area-to-volume ratio should be expected, which will potentially enable a large charge density. In applications where nanofibers cannot be utilized, we identified an alternative design with a large dielectric constant interlayer. We also identified an integration path compatible with the semiconductor vacuum process. This path will allow the future integration of energy storage component with the processor and memory, which will enable future miniaturization of Internet of Things (IOT) devices.

\section{MISSION RELEVANCE}

Our project directly aligns with DOE's mission in national security. Progress in this field is also relevant to DOE missions in energy and the environment, because progress here will drastically reduce the energy consumption of electronic devices, which accounts for the majority of energy costs to date. In modern devices, each device spends a significant amount of energy to move the data and then to compute. In addition, lots of energy dissipates on the way from the power source to the memory and computing nodes. For many applications, particularly the IOT, it is desirable to be able to integrate the power supply (batteries/supercapacitors), memory, and processors into the same chip. In addition, modern RF communication at low frequency is of great interest for defense and national security. In recent years, multiple programs focused on developing technologies to enable this approach.

\section{RESULTS AND ACCOMPLISHMENTS}

For this project, we developed an initial design, modeled a solid-state supercapacitor design, and filed a patent application. For the parallel capacitor, the team worked with a partner and built the initial capacitor with high dielectric constant materials, with permeability to be higher than 1000, to enable a higher voltage drop and energy density. We obtained the initial sample and will conduct the initial test in 2018. In addition, our team developed a wafer integration process, which will ultimately allow us to integrate an energy storage component into the chipset.

We prepared iron-cobalt (FeCo) nanofibers with diameters of 25-30 nm using electrospinning and a two-step, post-spinning heat treatment (Figure 1). The nanofibers at this dimension have extremely high surface area to volume ratio. Since they are magnetic fibers, they are automatically attracted to each other, which provides a good contact and can serve as an ideal electrode. We coated the nanofibers with a platinum-hafnium oxide-platinum $\left(\mathrm{Pt} / \mathrm{HfO}_{2} / \mathrm{Pt}\right)$ trilayer using ALD to fulfill their function as capacitor media. For initial validation, the interlayer is chosen to have a high dielectric constant. The figure shows the bright-field transmission electron microscopy (TEM) images of the nanofibers with and without an ALD coating. The energy dispersive X-ray (EDX) spectrum of the coated nanofibers showed $\mathrm{Pt}$ and $\mathrm{Hf}$ signals, indicating that both $\mathrm{Pt}$ and $\mathrm{HfO}_{2}$ were deposited. The future effort will focus on optimizing the growth condition of $\mathrm{HfO}_{2}$ on $\mathrm{Pt}$.
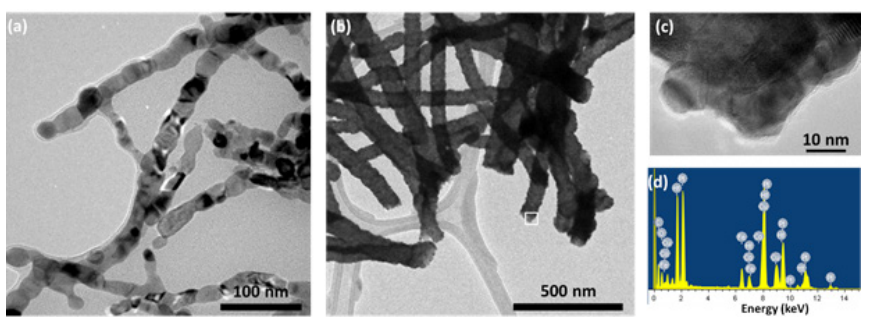

Figure 1. TEM images of the FeCo nanofibers (a) with and (b) without ALD coating. (c) A higher magnification image of the area enclosed by a white box in (b). (d) EDX spectrum of the nanofibers.

\section{PROPOSED FUTURE WORK}

In FY 2018, we will continue to develop a nanofiber-based solid-state supercapacitor. We will develop and build our test capability to characterize different types of capacitors under different conditions, including low temperatures (77K or $4 \mathrm{~K}$ ). We will continue to develop the ALD process and enable high-capacity solid-state supercapacitors and develop processes for wafer integration. 


\section{Novel Gallium Oxide Devices for Microwave and RF Technology}

\author{
2017-162-NO \\ John Hryn and Angel Yanguas-Gil
}

\section{PROJECT DESCRIPTION}

The goal of this project is to develop the capabilities for designing and fabricating a gallium oxide $\left(\mathrm{Ga}_{2} \mathrm{O}_{3}\right)$ device at Argonne National Laboratory. The project consists of the following two tasks: (1) leveraging the capabilities at the Center of Nanoscale Materials (CNM) to develop a workflow for the fabrication of $\mathrm{Ga}_{2} \mathrm{O}_{3}$ devices, combined with (2) performing modeling and simulations focused on developing a better understanding of the trade-offs that $\mathrm{Ga}_{2} \mathrm{O}_{3}$ offers for microwave and radio-frequency (RF) devices.

\section{MISSION RELEVANCE}

This project is relevant to DOE's energy mission. Wide bandgap (WBG) semiconductors have enormous potential for revolutionizing the control and transmission of electrical power. Of these, $\mathrm{Ga}_{2} \mathrm{O}_{3}$ in particular has garnered a lot of interest as a novel substrate for power devices. The superior Baliga's figure of merit, high breakdown field, excellent material properties, and the ability to grow high-quality bulk crystals from a melt make $\mathrm{Ga}_{2} \mathrm{O}_{3}$ a very attractive candidate to compete with or extend the frequency, power range, and reliability of devices based on silicon or gallium nitride ( $\mathrm{GaN}$ ). Therefore, gaining an understanding of the synthesis and processing of WBG materials such as $\mathrm{Ga}_{2} \mathrm{O}_{3}$ will enable higher-performing devices.

\section{RESULTS AND ACCOMPLISHMENTS}

We established a proof-of-concept process for the fabrication of $\mathrm{Ga}_{2} \mathrm{O}_{3}$-based devices, from epitaxial substrates up to the contact, leveraging the tools available at the CNM. The range of device sizes and architectures are compatible with the fabrication and patterning capabilities available at a cleanroom. Our workflow was able to incorporate the technology being developed at Argonne to control the interfacial behavior between the semiconductor material and the gate dielectric, taking advantage of input from our industrial partner to ensure that the final designs were compatible with those currently used in industry. The existing approach focused on the development of horizontal metal-oxide semiconductor field-effect transistors, which only require back-end-of-line processing consistent with existing capabilities. In addition, we were able to leverage existing device simulation tools (Atlas from Silvaco TCAD software), as well as spice-level type of models to understand the limiting factors and potential advantages of gallium oxide with respect to $\mathrm{GaN}$ and gallium arsenide(GaAs).

To further understand the benefits of $\mathrm{Ga}_{2} \mathrm{O}_{3}$ vs $\mathrm{GaN}$, vibrational modes of $\mathrm{Ga}_{2} \mathrm{O}_{3}$ were characterized using Raman spectroscopy to determine the phases, crystal quality, and peak shifts due to strain-stress. Figure 1 shows the three vibrational groups of gallium oxide.

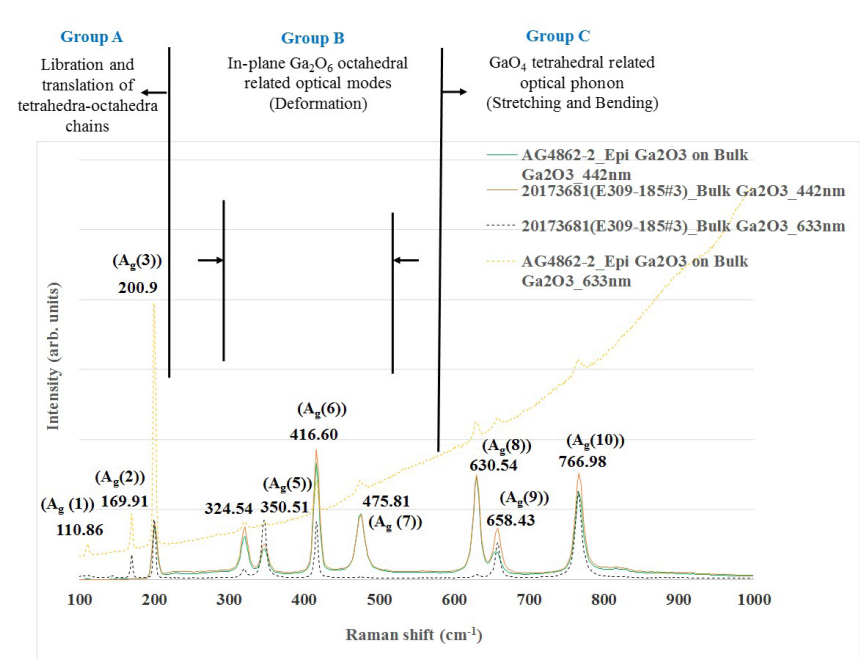

Figure 1. Vibrational groups of gallium oxide.

There are essentially three groups of vibration modes for the optical phonons of $\beta-\mathrm{Ga}_{2} \mathrm{O}_{3} \cdot \beta-\mathrm{Ga}_{2} \mathrm{O}_{3}$ has a monoclinic structure and belongs to the $\mathrm{C} 2 \mathrm{~h}$ space group. The Group $C$ phonon modes and Group A phonon mode $\left[A_{g}(3)\right]$ of $\mathrm{Ga}_{2} \mathrm{O}_{3}$ Epi on bulk $\mathrm{Ga}_{2} \mathrm{O}_{3}$ are slightly red shifted and blue shifted, respectively, compared to bulk $\mathrm{Ga}_{2} \mathrm{O}_{3}$, which indicate a corresponding tensile stress (Group C) and comprehensive stress induced in the interface (Group A).

The data suggest that gallium oxide contains significantly fewer defects than typically found in GaN devices. In summary, this project established a new capability at Argonne in $\mathrm{Ga}_{2} \mathrm{O}_{3}$ device design and fabrication.

\section{PROPOSED FUTURE WORK}

This project was completed in 2017. 
Biofilms and Human-Made Surfaces: How Microorganisms Attach to and Influence the World around Us

\section{7-163-NO}

\section{Dionysios Antonopoulos, Marie-Francoise Gros, and Kenneth Kemner}

\section{PROJECT DESCRIPTION}

Biofilms are a prevalent form of microbial life. A biofilm is a community of surface-associated microbial cells encased in an extracellular polymeric substance matrix, the formation of which is triggered by a variety of environmental chemical and mechanical cues. Biofilms form on a wide range of material surfaces, from natural surfaces to human-made metals, concretes, plastics, or resins. Naturally occurring microbial community assembly typically follows environmental cues; however, chemical and physical properties affect biofilm development and persistence. Because microorganisms naturally occur as communities, there are potentially multiple models for biofilm formation in different settings. Not understood are what environmental component(s) specifically trigger biofilm formation to begin with, what subsequent combination of biotic-abiotic interactions ultimately maintain and keep biofilms in check, and what effect these processes have on the degradation of the surfaces to which they are attached.

This project is focused on developing an experimental platform and workflow to investigate the role of biofilms in material surface degradation. We will create an observational platform and a set of probes to visually track members of a community during this process, which will allow us to model how microbial populations coordinate their activities within a community to alter their local environment. Our initial proof-of-principle will generate high-resolution, real-time images of the spatial distribution of the engineered community as it develops on defined materials with different amounts of surface roughness, as well as high-resolution images of the materials themselves to evaluate a biofilm's impact (e.g., degree of material corrosion).

\section{MISSION RELEVANCE}

This project supports DOE's environmental quality mission. Developing this unique capability will advance several DOE mission interests, including waste storage, environmental contamination (fate and transport), and bioenergy and biofuel feedstock (biofilm formation and interactions with plant roots). This project will also promote development of new capabilities and interactions between disciplines that will lead to new knowledge in the area of environmental microbiology. It should also provide opportunities to work with numerous industries where biocorrosion and biofouling are significant challenges.

\section{RESULTS AND ACCOMPLISHMENTS}

We screened previously enriched communities of low complexity derived from the environment (temperate and tropical forest soils) for their ability to form biofilms in the laboratory. Many of these "minimized communities" form biofilms; however, they do so at different rates based on their composition. Monitoring the development of a colorimetric proxy for biofilm formation over a 72-hour period, we found that the minimized communities sourced from tropical forest soils formed a greater and prolonged amount of biofilm over this period. This may be attributable to the greater abundance of specific keystone bacterial species present in the communities sourced from the tropical forest soils that differ from those sourced from temperate forest soils.

We also used confocal microscopy to evaluate the main parameters (densities, thickness, surface area) of biofilm architecture on abiotic surfaces by tracking bacteria (Pseudomonas species) that have been engineered to emit light (fluorescence). Last, we designed experiments to investigate the colonization of concrete by bacteria and subsequent modifications to the concrete resulting from the surface colonization.

\section{PROPOSED FUTURE WORK}

We plan to test the evaluated minimized communities for their ability to maintain a biofilm in a liquid flow over prolonged periods to enable visualization with microscopy. After introducing engineered bacteria that emit light to our minimized communities we will employ a biofilm-dedicated microfluidic system to observe and characterize the properties of mono- and multi-species biofilms on various manmade surfaces, including concrete. We will then use X-ray and/or electron microscopy measurements to image the concrete surfaces after colonization.

This integrated system will enable the future study of a wide variety of materials and environmental contexts including, but not limited to, environmental contamination (fate and transport), waste storage, infrastructure corrosion (e.g., piping), and polymer degradation (e.g., plastics). 


\section{Simulating the Dynamics of Gene Drive Propagation through Populations}

\author{
2017-164-NO \\ Charles Macal
}

\section{PROJECT DESCRIPTION}

Gene drive is a technique that promotes the inheritance of a particular gene to increase its prevalence in a population. Applications of gene drive include preventing the spread of insects that carry pathogens, such as Aedes mosquitoes that transmit dengue fever, Zika, and chikungunya diseases. Additional applications include controlling invasive species and eliminating pesticide resistance. There are many unknowns concerning the potential impacts of engineered gene drives in the wild. Important scientific questions pertain to whether a modified gene can sustain itself through multiple generations, or whether natural processes will mitigate against the ability of an edited gene to sustain itself. We are developing the capability to assess the population effects of gene editing technology by modeling the dynamics of transmission and inheritance of edited genes through a population. We are designing and developing a computational agent-based model (ABM) to study the propagation of gene drive through a population.

\section{MISSION RELEVANCE}

The project is relevant to the DOE missions in basic science and national security. Few scientific studies have been done, and virtually no analytical tools or models exist, to quantify the effects of gene drive on a population. This project addresses a specific research need identified by DOE.

\section{RESULTS AND ACCOMPLISHMENTS}

We have developed an initial ABM for gene drive propagation through a mosquito population and are applying it to the Chicago region as a test case (Figure 1). We identified the main object classes of the model (mosquitoes by life stage, geospatial regional patches) and the attribute definitions of each class. We conducted an extensive literature review of mosquito lifecycles and gene drive techniques as applied to mosquitoes. We developed population models of mosquito maturation through their lifecycle stages, including egg, larval, pupal and adult stages. We created environment and habitat models to reflect location-specific environmental factors that drive mosquito population dynamics (Figure 2).

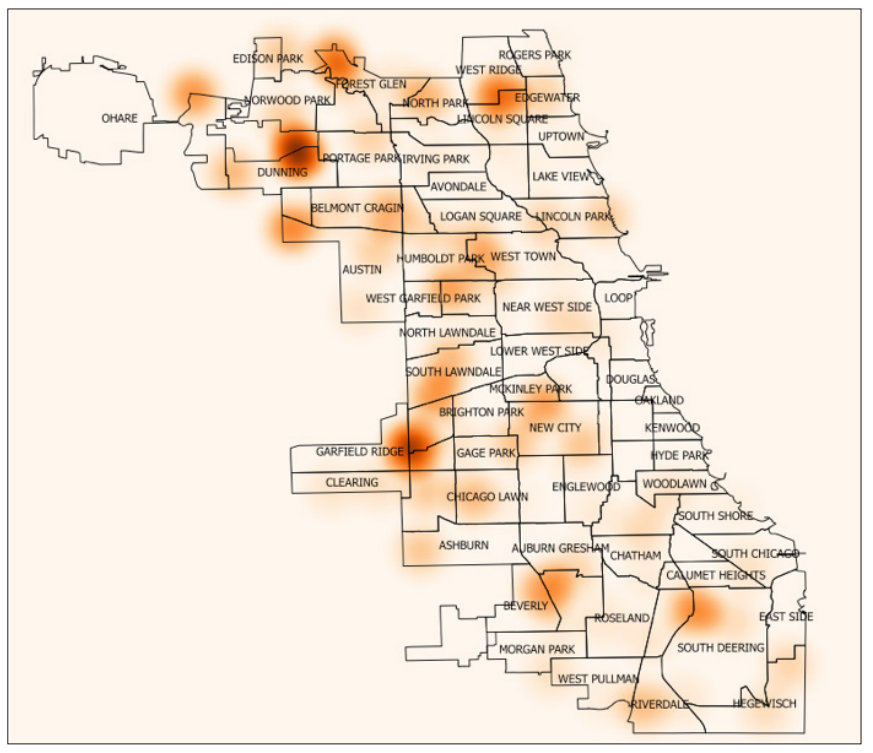

Figure 1. Geospatial Representation of Mosquito Density.

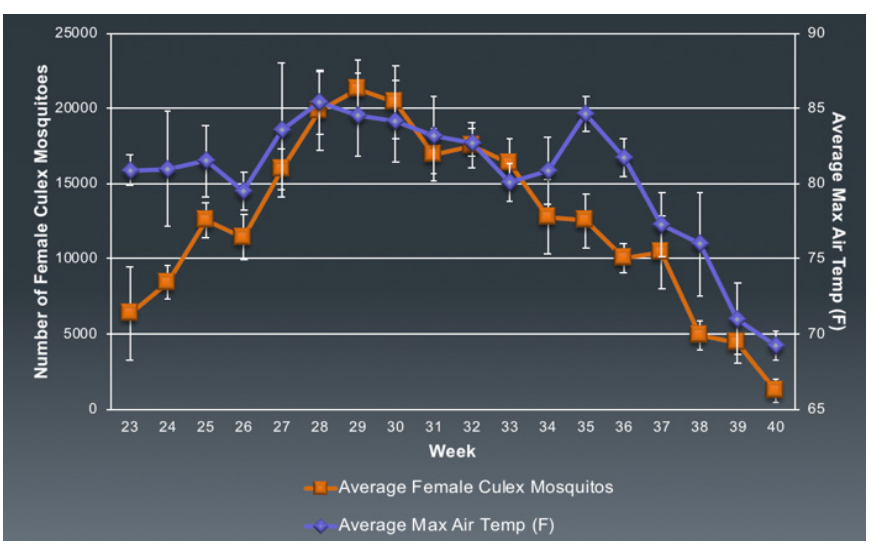

Figure 2. Relationship between Mosquito Abundance and Environmental Factors: Temperature.

\section{PROPOSED FUTURE WORK}

In FY 2018, we will use the capability of ABM to model the individuals in the mosquito population, and their behaviors and interactions. We will model mosquito lifecycles and the genetic processes that operate in propagating change through populations. Each agent's characteristics will include the representation of its genome; a key challenge is to identify and represent the minimal amount of information to represent the genomes of individual mosquitoes and how the genomes change and evolve over time due to crossover (mating) and mutation processes. We will consider transmission interventions including introduced infection with Wolbachia bacteria and gene editing techniques, such as CRISPR-Cas9 (Clustered Regularly Interspaced Short Palindromic Repeats, protein-9 nuclease). 


\section{Global Survey of CRISPR- CAS Systems in Archaeal and Bacterial Species}

\author{
2017-165-NO \\ James Davis and Maulik Shukla
}

\section{PROJECT DESCRIPTION}

Bacteria and archaea have evolved molecular mechanisms that confer immunity to invading genetic elements. One important mechanism for this is CRISPR (Clustered Regularly Interspaced Short Palindromic Repeats)-Cas (CRISPR associated system), which enables the incorporation of short foreign deoxyribonucleic acid (DNA) fragments into the host genome, essentially enabling the host to memorize the genetic composition of viruses that it has encountered and thereby providing immunity.

The goal of this project is to understand the global scope of naturally occurring CRISPR-Cas; provide the necessary background for identifying new, naturally and non-naturally occurring systems; and provide insights into the history of virus infection across the bacterial and archaeal domains. The technical objectives of this work are to (1) study the evolution of CRISPR-Cas in nature, (2) identify signs of CRISPR-Cas engineering, and (3) characterize anti-CRISPR proteins, which are naturally occurring proteins that inhibit CRISPR-Cas.

\section{MISSION RELEVANCE}

This project is relevant to DOE's missions in basic science and national security. Recent advances in understanding the mechanism of action and structural modifications of the CRISPR-Cas system, and specifically the Cas9 protein, are at the basis of significant advances in gene-editing technology. This has also been the cause of great concern in areas of national security, as outlined in a recent President's Council of Advisors on Science and Technology report, and has gained the attention of the DOE's Office of Science and the National Nuclear Security Administration.

\section{RESULTS AND ACCOMPLISHMENTS}

The search for CRISPR-Cas systems separates logically into two operations: the search for CRISPR elements and the search for Cas proteins. Because CRISPR elements must be differentiated from common genomic repeats, we evaluated bespoke algorithms for finding CRISPRs on a set of well-characterized genomes. Overall, the algorithms are similar (Figure 1), but this led to proposed updates in our RAST (Rapid Annotation using Subsystem Technology) CRISPR-finding software. We also searched the literature for characterized Cas proteins to seed searches for homologs in PATRIC (PAThosystem Resource Integration Center), a collection of more than 120,000 genomes. We found 2,035 Cas protein-related families, representing a total collection of 7,725,067 nonredundant, Cas-related proteins.

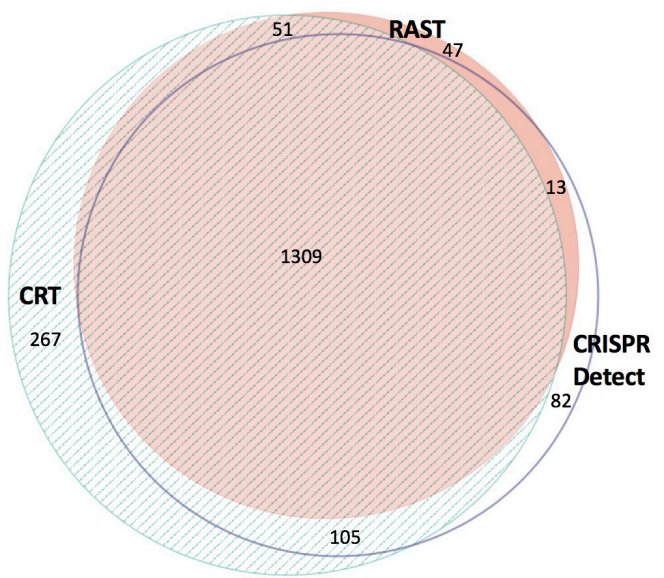

Figure 1. The result of using three popular CRISPR detection tools on a well-curated set of 1,000 diverse bacterial and archaeal genomes. All three tools find 1,309 elements; numbers for other parts of the Venn diagram are shown.

\section{PROPOSED FUTURE WORK}

We plan to update the CRISPR finding tool in RAST. We will use this tool to find CRISPRs in user genomes and to predict all CRISPR regions in PATRIC, making them available to the public. The collection of Cas proteins will be used to improve annotations in RAST. Using the same protocol that we used for identifying Cas proteins, we will identify anti-CRISPR protein families, generate a large collection of their homologs, and use them to improve existing annotations.

We will evaluate methods for identifying CRISPR elements in metagenomic data. We will also develop a protocol for identifying and possibly assembling Cas and anti-CRISPR genes from the reads in the metagenomic data.

We will study the collection of CRISPRs, Cas proteins, and anti-CRISPR proteins to establish a baseline for understanding how to identify evidence of genetic engineering. For example, we will analyze the collection of CRISPR spacers from PATRIC to determine the frequency of naturally occurring homology to eukaryotic or non-phage DNA. These studies will provide the framework for determining whether Cas and anti-CRISPR proteins are being found outside of their natural environmental and evolutionary context, or if human or eukaryotic DNA has been inserted into a CRISPR array. 


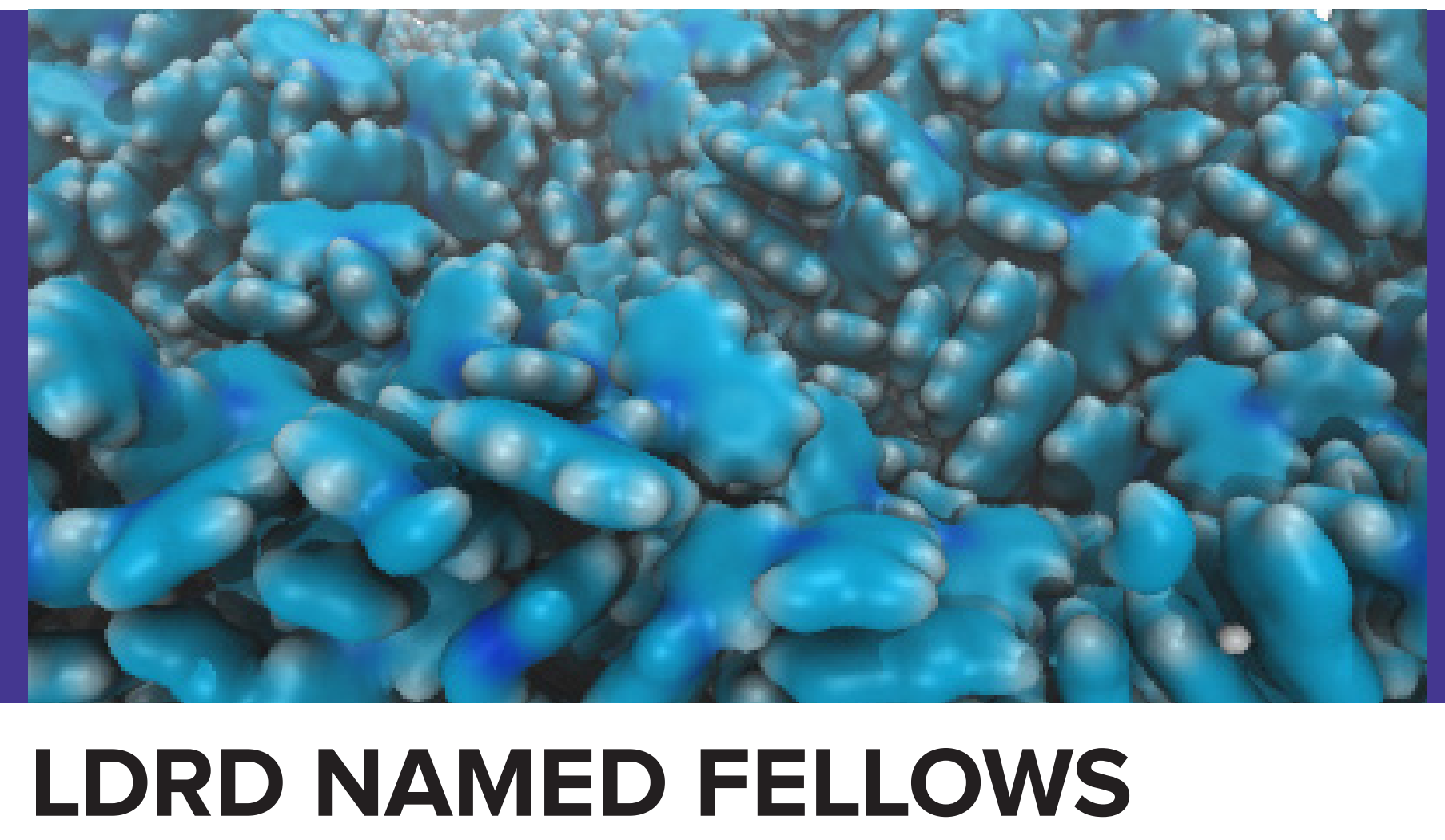




\section{Substitutional Growth of Lead Halide Perovskites for Solar Cells*}

\author{
2016-180-R1 \\ Muge Acik and Saw Wai Hla
}

\section{PROJECT DESCRIPTION}

Typically, the growth of lead halide perovskites to fabricate solar cell devices involves spin coating the precursors onto oxide/polymer films, followed by annealing. However, use of toxic solvents and high temperatures can spoil the film's properties by introducing interfacial imperfections, which hinder device stability and performance. Solution approaches to date have not been able to avoid unmanageable surface reactions, non-stoichiometric dopants, and phase segregation. The growth mechanisms are also ambiguous. This research introduces a "greener" solution-processing (in situ) approach that uses alcohols as polar protic solvents for perovskite growth at low reaction temperatures. The main scope of this work is to address processing issues (i.e., poor stability of perovskites) and to understand new growth mechanisms through a series of experimental and theoretical studies.

\section{MISSION RELEVANCE}

This research is relevant to DOE's energy and environmental missions. The scientific focus on the fundamental understanding of perovskite crystal growth by developing environmentally benign processes targets DOE's Basic Energy Sciences scope. Our experimental findings support innovative efforts to address environmental problems for energy technologies leading to discoveries of eco-friendly processes.

\section{RESULTS AND ACCOMPLISHMENTS}

In FY 2017, our studies focused on the growth of lead halide perovskites on graphene oxide (GO). They revealed chemical interactions between halide precursors of perovskites and GO thin films that reduced GO chemically and introduced structural defects into the perovskite/ GO interfaces, thus determining the perovskite growth yield. We employed a novel, alcohol-based, substitutional growth (SG) method for methylammonium lead iodide $\left(\mathrm{MAPbl}_{3}\right)$ perovskites in polar protic solvents. With this method, the perovskite growth in solution-phase was determined by the type of solvent (protic vs. aprotic) and the polarity effect. The alcohols not only lowered the reaction temperature by catalyzing the growth but also significantly improved the reaction yield. We used multiple analytical techniques to characterize the resulting $\mathrm{MAPbl}_{3}$ perovskites. We found that the $\mathrm{MAPbl}_{3}$ perovskite crystals in alcohol dispersions were in the tetragonal phase oriented as indicated with the (110) peaks shown in Figure 1i, and with a minimum residual inorganic component $\left(<10 \% \mathrm{Pbl}_{2}\right)$ reflecting an efficient perovskite conversion. The grain size of perovskites was associated with the variation in the crystal size reducing from micro- to nano-meter scales as the binding affinity of the alcohol was lowered. The resulting high reflectivity ( 85-90\%) $\mathrm{MAPbl}_{3}$ was metallic-lead-free (Figure 1ii) with a high chemical stability in air for up to 1.5 months, and a high thermal stability up to $\sim 300^{\circ} \mathrm{C}$. Theoretical calculations for $\mathrm{MAPbl}_{3}$ also confirmed the proposed SG mechanism by demonstrating that the reactions between alcohols and $\mathrm{MA}^{+}$cations were highly exothermic and thereby favorable. In contrast to growth in alcohols, the perovskite growth did not occur in non-polar (toluene) or in polar aprotic solvents. In addition to the $\mathrm{MAPbl}_{3}$ growth, the SG technique was employed for the growth of cubic methylammonium lead bromide/chloride ( $\mathrm{MAPbBr}_{3} /$ $\mathrm{MAPbCl}_{3}$ ) perovskites, and their mixed halides. This work has reached a key milestone in the introduction of a novel, environmentally benign approach. We have applied for a patent for the development of lead halide perovskites using the SG method.
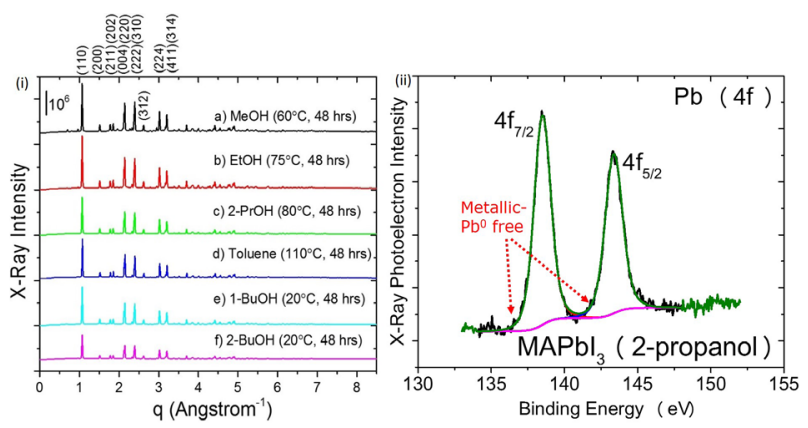

Figure 1. (i) In situ high-energy x-ray, (ii) x-ray photoelectron spectra of MAPbI3.

\section{PROPOSED FUTURE WORK}

In FY 2018, we will explore new, energy-efficient, low-cost, and optimizable solutions. This breakthrough method will open new paths to highly reproducible fabrication processes for large-scale deposition of lead halides, and could eventually bring opportunities for manufacturing flexible devices through low-cost techniques. The Japan Aerospace Exploration Agency will fund a separate project and use SG-driven perovskites for further investigation of solar device designs.

*Formerly "In situ Polarized Spectroscopy of Optically Transparent TrGO-Polymer Solar Cells" 


\section{The Search for Weyl Semimetals}

\author{
2016-181-R1
}

\section{Nirmal Ghimire and John Mitchell}

\section{PROJECT DESCRIPTION}

Graphene is the most celebrated example of a two-dimensional (2D) semimetal, where valence and conduction bands touch at the Fermi energy at what is known as a Dirac point. A close cousin of graphene is the so-called Weyl semimetal. Like graphene, its band structure has a pair of bands crossing at certain points called Weyl nodes at the Fermi energy. Unlike graphene, this system is three dimensional (3D) and is more stable than graphene against small perturbations, making it more attractive as a candidate platform for low-power electronics. The objective of this project is to explore these novel materials and their properties.

After the discovery of the TaAs family of compounds as Weyl semimetals, subsequent investigations showed defining characteristics of these compounds, including extremely large magnetoresistance (XMR), high charge carrier mobility, quantum oscillations, and negative longitudinal magnetoresistance (LMR). We focused our work on addressing the following key issues in Weyl semimetal physics: (1) understanding why properties like XMR and LMR can be found in compounds lacking Weyl nodes, (2) studying the effect of magnetism in such compounds, and (3) discovering and studying new, simpler Weyl semimetals (i.e., Weyl semimetals with fewer Weyl nodes). This study will allow us to understand the unique transport behavior of Weyl semimetals, thus providing essential input to assessing applicability of Weyl materials for future information technology platforms.

\section{MISSION RELEVANCE}

This project lies at the intersection of two recently identified high-priority DOE Basic Energy Sciences research directions-quantum and topological matter and crystal synthesis. Through recent Basic Research Needs workshops, both topics have been emphasized as impacting the DOE science mission of understanding and controlling matter at the atomic level.

\section{RESULTS AND ACCOMPLISHMENTS}

We synthesized single crystals of Yttrium Antimony (YSb) and carried out transport properties measurements together with electronic structure calculations. Through the magnetotransport studies, we found that YSb, like isostructural Lanthanum Antimony (LaSb) and Lanthanum Bismuth (LaBi), has a large unsaturating magnetoresistance; large Hall mobility; quantum oscillation; and a magnetic-field-induced, metal-insulator-like transition followed by the resistivity saturation plateau. We questioned these previously observed behaviors in LaSb to determine whether they are related to surface conduction, possibly resulting from some sort of topological protection on the surface. Our experimental results indicated that the saturation plateau cannot be unambiguously assigned to the surface conduction in YSb.

Both ARPES and magnetotransport measurements explain the XMR behavior in YSb, suggesting that XMR is not necessarily the characteristic feature of Weyl semimetals only, but can also be observed in a trivial semimetal. We have recently synthesized and studied a non-centrosymmetric antiferromagnet $\mathrm{CoNb}_{3} \mathrm{~S}_{6}$ in order to find a system that simultaneously contains Weyl nodes and magnetic ordering. In this compound we have found large anomalous Hall effect where the coefficient of the anomalous Hall resistivity is unusually enhanced as compared to that in simple ferromagnets such as Iron. We attribute the origin of this unusually large anomalous Hall effect, to non-trivial spin texture, topological electronic behavior, or a combination of the two (Figure 1). Band structure calculations indicate the potential role of Weyl or Dirac points, pointing to future ARPES experiments on this compound. (a)

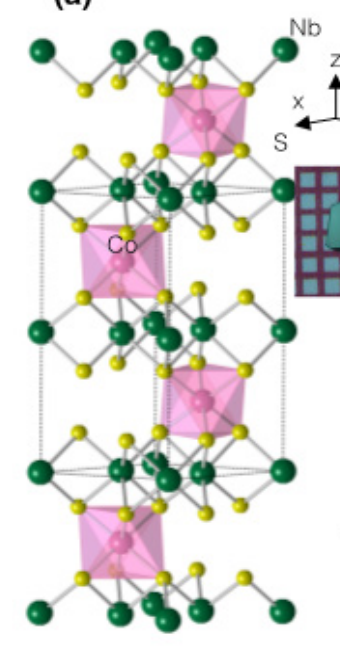

(b)
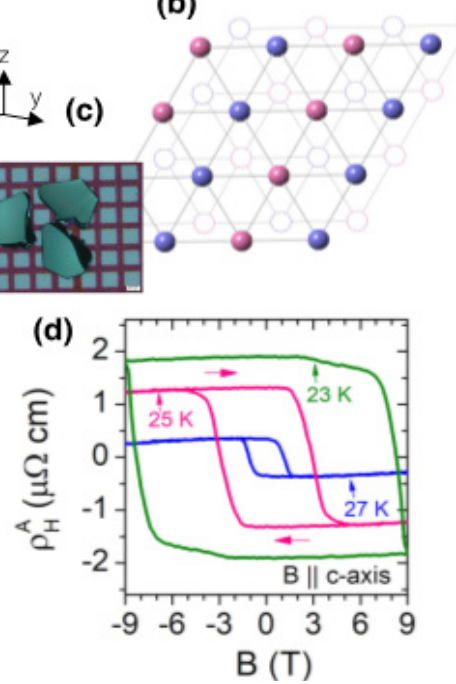

Figure 1. (a) Crystal structure of $\mathrm{CoNb}_{3} \mathrm{~S}_{6}$. (b) Two layers of Co atoms. Solid spheres are the Co atoms on the top layer and the broken circles represent $\mathrm{Co}$ atoms on the bottom layer. $\mathrm{CoNb}_{3} \mathrm{~S}_{6}$ orders antiferromagnetically below $\sim 28 \mathrm{~K}$, so the pink and blue color represent the parallel and antiparallel spins of this ordered magnetic state. (c) Photograph of crystals of $\mathrm{CoNb}_{3} \mathrm{~S}_{6}$ on a 1-mm $\times$ 1-mm grid. (d) Anomalous Hall effect measured in $\mathrm{CoNb}_{3} \mathrm{~S}_{6}$.

PROPOSED FUTURE WORK

This project ended in FY 2017. 
Understanding and Controlling Charge, Spin, Pseudospins, and Lattice Degrees of Freedom in Layered Transition Metal Dichalcogenides

\section{6-183-R1 \\ Qi Zhang and Haidan Wen \\ PROJECT DESCRIPTION}

Recently, two-dimensional (2D) materials systems (e.g., transition metal dichalcogenides or TMDs), have emerged as extraordinary systems because of their coupled quantum degrees of freedom, including spin, valley pseudospin, and layer pseudospins, with electrical and optical accessibility. To advance optoelectronics and new-concept devices for efficient and low-power information processing, it is critical to understand the fundamental dynamics of these electronic degrees of freedom.

To address this challenge, we propose to (1) establish a state-of-the-art optical pump THz spectroscopy system, (2) use this THz system to explore quantum physics in the novel 2D van der Waals materials (including TMDs and 2D magnets) and other novel 2D systems (e.g., 2D Rashba interface in spintronic heterostructures), and (3) unveil the ultrafast lattice dynamics of laser-induced phase transitions in TMDs with recently developed ultrafast hard X-ray nanodiffraction.

\section{MISSION RELEVANCE}

This project is relevant to DOE missions in science. This success of this project will enable versatile pump-probe techniques at $\mathrm{THz}$ frequencies-essential tools particularly important to ultrafast electronic characterization in nanoscale electronic materials. The proposed study of ultrafast dynamics in layered transition metal dichalcogenides will answer key questions about how the structural reconfiguration of 2D materials influences its electronic properties. The knowledge acquired from the proposed work will also strengthen the momentum of understanding exotic magnetic phenomena.

\section{RESULTS AND ACCOMPLISHMENTS}

First, we studied ultrafast lattice dynamics in TMDs by optical-pump X-ray probe spectroscopy at the LCLS. We discovered a surprisingly fast (5-ps) lateral lattice expansion in monolayer $\mathrm{WSe}_{2}$ flakes after intense optical excitation. These unusually fast lattice dynamics may be related to the rippling structure of the $\mathrm{WSe}_{2}$.

Second, we demonstrated that a femtosecond spin current pulse can generate $\mathrm{THz}$ transients at Rashba interfaces between two non-magnetic materials (Ag/Bi). Our results is the first ultrafast demonstration of inverse Edelstein effect.

Third, we also achieved a gate-tunable, ultrafast spin-to-charge current conversion in 2D electron systems at oxide interfaces (i.e., $\mathrm{LaAlO}_{3} / \mathrm{SrTiO}_{3}$ ) (Figure 1), which provide promising platforms for achieving fast, tunable, and efficient spin-charge inter-conversion. Our findings extend ultrafast spin-charge conversion to oxides-based systems, thus providing a crucial link between ultrafast spintronics and correlated phenomena in complex oxides. Our research opens new opportunities to controllable spintronic processes for device application.
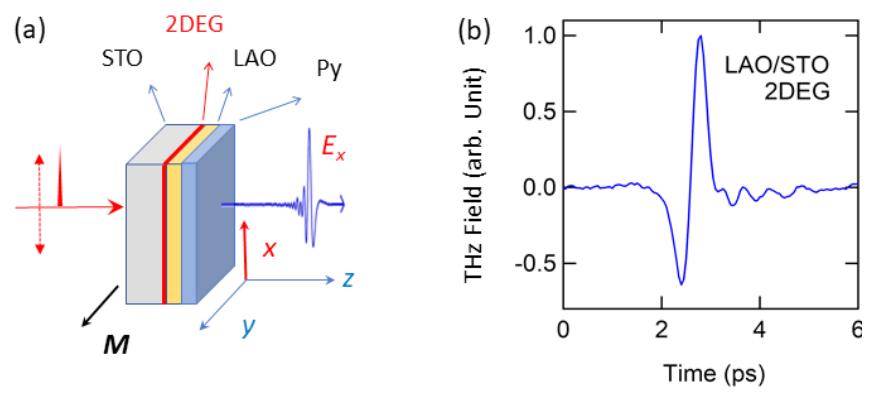

Figure 1. (a) Schematic diagram for $\mathrm{THz}$ generation at an oxide interface. (LAO, STO, Py, and 2DEG stands for $\mathrm{LaAlO}_{3}, \mathrm{SrTiO}_{3}$, permalloy, and 2D electron gas, respectively. $M$ is the magnetization). (b) Typical emitted $\mathrm{THz}$ pulse waveform from the oxide interface.

Last, we have established an optical-pump $\mathrm{THz}$ probe cryogenic spectroscopy system at Argonne's Center for Nanoscale Materials laser lab. This system is a powerful tool for exploring various elementary excitations at meV energy scale, in novel quantum materials.

\section{PROPOSED FUTURE WORK}

We have three areas of focus for FY 2018. First, we will study the low-temperature elementary excitations and their dynamics in van der Waals materials (e.g., WTe ${ }_{2}$ ) and strong correlated materials with the cryogenic $\mathrm{THz}$ spectroscopy system.

Second, we will use intense $\mathrm{THz}$ pulses to trigger ultrafast dynamics or even phase transitions of correlated states in 2D materials.

Third, we will use intense THz pulses to generate sub-ps, pure-spin current burst via inverse spin Hall effect at the 2D Rashba interface. 


\section{Investigation of Solid-Liquid Interfaces in Energy Materials: Interfacing Multi-Scale Modeling with Experimental Characterization}

\author{
2016-184-R1
}

Kendra Letchworth Weaver, Maria K. Chan, and Paul Fenter

\section{PROJECT DESCRIPTION}

Electrochemical energy storage and conversion devices, such as batteries and solar-to-fuel technology, must become more efficient and less costly. However, the complexity of the interface between the charged electrode surface and the fluid electrolyte presents a challenge. Studying these devices under operating conditions requires bridging length-scales from the atomic scale to the macroscopic scale of the devices themselves. The highly scalable yet accurate joint density functional theory (JDFT) approach complements existing molecular dynamics techniques to offer a predictive multiscale description of the solid-liquid interface that can be integrated with experimental measurements. This pioneering work broadens basic understanding of fundamental processes at the electrochemical interface, including ion solvation, catalysis, and electrode stability to dissolution, offering crucial insights into a wide range of energy materials.

\section{MISSION RELEVANCE}

Modeling solid-liquid interfaces is significant in various energy applications, including energy storage, photocatalysis, corrosion, and dissolution-areas of concern to the DOE energy security mission. Predictive modeling, especially from first principles, is a key focus of DOE's Computational Materials Science report. Our work is relevant to both the Basic Energy Sciences and Advanced Scientific Computing Research programs of DOE.

\section{RESULTS AND ACCOMPLISHMENTS}

Batteries: Creating an artificial electrode-electrolyte interface from self-assembled monolayers of phosphonic acids offers a promising avenue to preventing capacity loss in Li-ion batteries owing to metal dissolution from the cathode. We demonstrated that our JDFT-based continuum model for battery electrolytes, in combination with classical molecular dynamics, simultaneously captures both the formation of a stable monolayer on the $\mathrm{LiMn}_{2} \mathrm{O}_{4}$ surface and the interaction of the phosphonate molecules with the electrolyte. Our simulations
(Figure 1) capture experimentally observed trends in solubility and cyclic voltammetry of the coated $\mathrm{LiMn}_{2} \mathrm{O}_{4}$ surface, demonstrating how adjusting the length and functionalization of the phosphonates can maximize Li-ion conductivity but minimize cathode dissolution. This research was part of a collaboration with the Center for Electrochemical Energy Science (CEES).
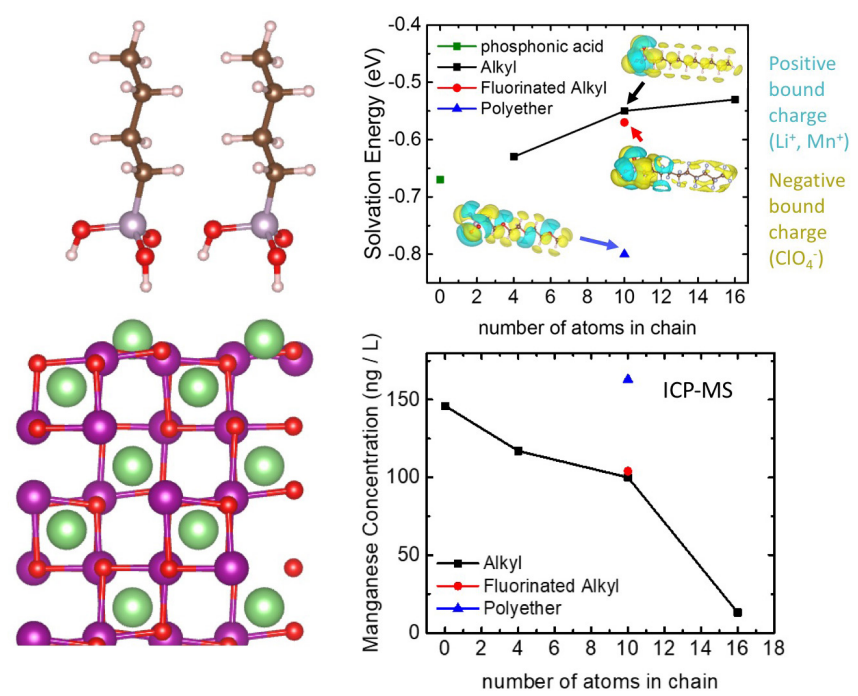

Figure 1. (left) Self-assembled monolayer of phosphonates on $\mathrm{LiMn}_{2} \mathrm{O}_{4}$ battery cathode to prevent Mn dissolution. (right, top) Theoretical solvation energies of different functionalized chains in polyethylene carbonate: $1 \mathrm{M} \mathrm{LiClO}_{4}$ electrolyte, which correlate inversely with (right, bottom) the amount of Mn dissolved from cathode after cycling, demonstrating the importance of wettability in performance of protective coatings.

Catalysis: JDFT studies of hydrogen, chlorine, and hydroxyl diffusion on a platinum surface, with and without the presence of an overlayer of graphene, probed the role of the electrolyte in reaction pathways for hydrogen evolution and oxygen reduction. These studies elucidate the fundamental electrochemistry underlying fuel cell technology.

Combining Theory and Experiment: X-ray reflectivity measurements can predict interfacial electron densities with sub-Angstrom resolution. We directly computed $X$-ray reflectivity signals from JDFT and molecular dynamics simulations of the water $/ \mathrm{Al}_{2} \mathrm{O}_{3}$ (001) interface. Comparing our calculations directly with experimental reflectivity measurements generated insight unavailable from experiment or theory alone regarding chemical composition, thermal motion, and bonding at the interface.

\section{PROPOSED FUTURE WORK}

Batteries with multivalent working electrolytes like magnesium $\left(\mathrm{Mg}^{2+}\right)$ or aluminum $\left(\mathrm{Al}^{3+}\right)$, which carry double or triple the charge of $\mathrm{Li}^{+}$, could theoretically store much more energy. In FY 2018, JDFT calculations of grapheneliquid interfaces will be combined with $\mathrm{X}$-ray reflectivity to predict ion solvation structure near model electrodes. 
Charge Transport in Nanostructured Materials from $a b$ initio Simulations

\author{
2016-185-R1 \\ Marton Andras Voeroes and Larry A. Curtiss
}

\section{PROJECT DESCRIPTION}

One of the major challenges in predicting materials and processes for efficient conversion of solar energy or for energy storage is the optimization of charge transport through heterogeneous interfaces. We proposed to develop a unified platform, based on first principles methods, to investigate electron and hole transport in nanocomposite materials with complex interfaces. We focus on nanostructured systems for solar energy conversion; however, we have developed methods and codes that are general and may be applied to the study of other energy conversion or storage processes.

We plan to devise a unified scheme to investigate charge transport both in the band and hopping regimes, with the intermediate regime treated in an approximate, although non-empirical and hierarchical, manner. In the case of nanoparticle (NP) arrays, we developed ab initio codes to optimize and tune material properties to achieve high carrier mobilities while maintaining the Coulomb interaction-enhanced carrier multiplication (CM) effect.

This study builds on our previous experience in developing methods and codes to compute CM rates from first principles in NPs and in studying NP arrays and embedded NPs. First, we will be working to adapt the Marcus theory to nanocomposites and to generate structural models of simple systems for validation purposes. Band transport will be described using Boltzmann transport theory. An ultimate goal of the project is to connect the hierarchical model of electron transport with our previously developed CM code. Such a platform will be of general applicability to investigating transport at heterogeneous interfaces, including solid/ electrolyte interfaces, and relevant for optimization of batteries.

\section{MISSION RELEVANCE}

Charge transport processes are ubiquitous in materialsrelevant energy conversion and storage. Thus, our goals of understanding and engineering charge transfer and charge transport processes in complex nanostructured materials are relevant to the energy mission of DOE.

\section{RESULTS AND ACCOMPLISHMENTS}

We finished the core implementation of the Marcus theory of hopping transport into the Quantum ESPRESSO code. This implementation is based on constrained density functional theory (CDFT). The first applications of describing charge transport in NP arrays were published in a series of three papers.

In parallel, in collaboration with Gergely Zimanyi and his student, Luman Qu from the University of California Davis, we studied hopping transport in NP arrays using a semi-empirical kinetic Monte Carlo method. We found that recent measurements on enhanced charge transport may be explained by emerging percolation channels in the NP films.

Finally, we studied disproportionation reactions in an exemplary battery cathode material, $\mathrm{LiMn}_{2} \mathrm{O}_{4}$ (LMO). LMO is a promising cathode material for Li-ion batteries. Dissolution of manganese from LMO into the electrolyte, however, limits its cycle life. Although the widely accepted mechanism of dissolution requires charge disproportionation of $\mathrm{Mn}(\mathrm{III})$, the atomistic details of such processes have remained elusive. Using first-principles simulations, we provided insights into the mechanism of disproportionation. See Figure 1.

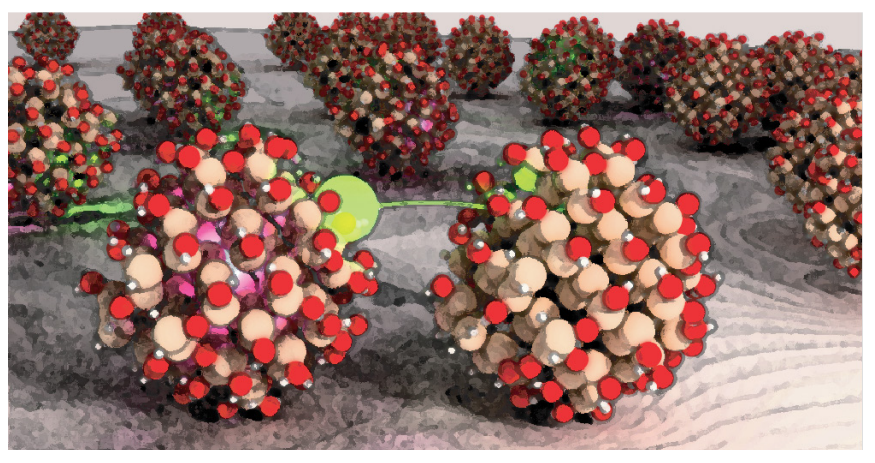

Figure 1. Illustration of charge transfer in a nanoparticle solid.

\section{PROPOSED FUTURE WORK}

In the final year of this project, we are planning to continue working on the CDFT code. In particular, we will implement CDFT gradients, which will allow optimization of the system under constraints. Further, we will implement and test constrained density functional perturbation theory, which may help in finding the optimal strength of the potential more quickly.

As described above, we have studied disproportionation in bulk LMO; however, the general understanding is that disproportionation originates from the surfaces. In collaboration with the Jeffrey P. Greeley group from Purdue University, we will be investigating the relevance of this process by looking at several different LMO surfaces. 


\section{Ultrafast Spectroscopy of Nanometer-Scale Heterojunctions Fabricated by Self-Assembly}

\author{
2016-186-R1 \\ Benjamin T. Diroll and Richard D. Schaller
}

\section{PROJECT DESCRIPTION}

This project concerns using time-resolved spectroscopic techniques to investigate the properties of solids constructed from colloidal nanomaterials. These materials, due to their small size, have large surface-to-volume ratios and therefore allow the formation of solids with large interfacial regions between different materials-i.e., heterojunctions. Furthermore, deposition of these materials into solid thin films can be achieved through many simple solution-processing techniques. Many of these processes in which charge and energy flow from one component of a device to another occur on time-scales of femtoseconds to picoseconds, which are typically experimentally accessible only with optical spectroscopic methods. In this work, semiconductor nanocrystals of many compositions are studied both in isolation and in composites with intimate heterojunctions.

\section{MISSION RELEVANCE}

This project is relevant to DOE missions in energy and basic science. Junctions between two different materials are at the heart of many technologies, and understanding how they affect the transfer of energy and charge at the nanometer-length scale offers a step toward designing higher-performance optoelectronic devices, such as solar cells, LEDs (light-emitting diodes), lasers, and detectors.

\section{RESULTS AND ACCOMPLISHMENTS}

The primary accomplishments of this project are in three areas, summarized below:

(1) Both high- and low-temperature properties of lead trihalide perovskites of cesium and methylammonium were examined to ascertain the fundamental properties of these materials, which hold great promise for solar photovoltaic and solid-state lighting applications. Of particular note, we found that nanometer-sized particles of methylammonium lead iodide have a thermodynamic stability distinct from that of bulk materials and consequently significantly different optoelectronic properties at low temperature.

(2) Building on previous work exploiting optical control of optical absorption features arising from free electrons and holes of heavily doped materials, heavily doped silicon was examined to show related effects. Self-assembled films of silicon particles prepared via plasma deposition were doped with boron impurities that generate a localized surface plasmon resonance. Resonant excitation of the samples at $2500 \mathrm{~nm}$ results in heating and subsequent cooling of the free hole plasma on an ultrafast time-scale of less than 1 picosecond. By tuning the wavelength to $2000 \mathrm{~nm}$, both heating of the hole plasma and carrier generation processes occur, resulting in a time-dependent mid-infrared optical response which is tunable with power and excitation wavelength (Figure 1).

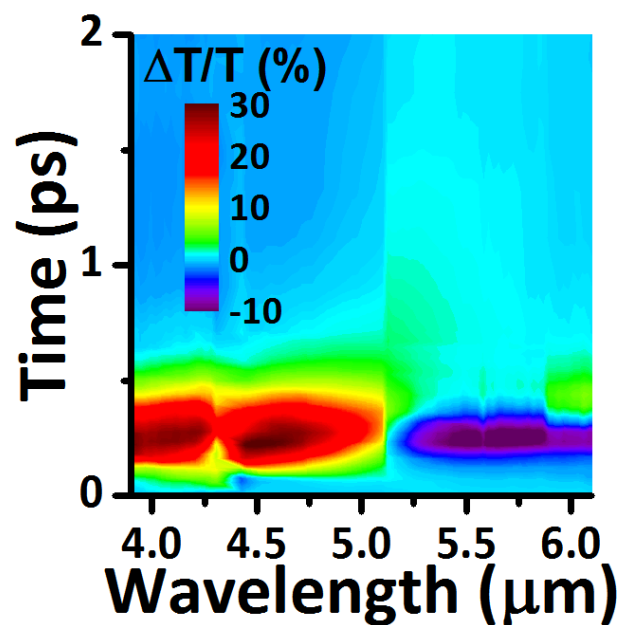

Figure 1. Time versus wavelength plot of the ultrafast response of heavily-doped silicon nanocrystals.

(3) Atomically precise synthesis of heterostructure materials with control of each layer of cations and anions was achieved for a limited number of compositions. Self-assembly of atomically-precise layers of new heterojunction material consisting of cadmium selenide, zinc sulfide, and cadmium sulfide with precise control over layer thickness was controlled using individual self-limiting reactions of cations and anions in separate reaction steps separated by "purge" processes to eliminate excess reagent. Rather than typical epitaxial surfaces, this work used two-dimensional colloidal materials as the atomically-precise substrate material from which more complex heterojunctions were grown. This work was used in particular to construct new light-emitting materials in the blue spectral region, including new lasers, using femtosecond optical pumping of the samples. The samples exhibited fluence-dependent photoluminescence dynamics. Placed within a reflective laser cavity, these materials showed lasing behavior in the blue spectral region.

\section{PROPOSED FUTURE WORK}

In future projects, we will continue our work on atomically precise synthesis, expanding the chemical compatibility of the method to unlock new in device applications. 


\section{Coherent X-ray Investigations of Defect Dynamics in Next-Generation Nanostructured Materials}

\author{
2016-187-R1
}

Andrew Ulvestad and Brian Stephenson

PROJECT DESCRIPTION

Defect engineering, or the rational design and optimization of desired functionalities through deliberate defect manipulation, can be used to design new nanomaterial properties. However, it is limited in scope owing to the inability of current probes to characterize defect dynamics under operando conditions in threedimensional (3D) detail. In Bragg coherent diffractive imaging (BCDI), nanoscale shape and strain are imaged in $3 \mathrm{D}$ detail by using a computer algorithm to invert single particle diffraction patterns obtained with a coherent X-ray beam. BCDI thus avoids the problems of ensemble measurements, which convolve effects due to particle shape and size diversity, and it is capable of 4D imaging of real devices in real time with nanoscale resolution to reveal strain evolution. Two focus areas were investigated-defect dynamics during phase transformations and defect influence on interfacial processes-which demonstrated the power of BCDI to provide the fundamental materials understanding needed to develop next-generation nanostructured materials.

\section{MISSION RELEVANCE}

This project furthers the DOE Basic Energy Sciences (BES) mission to design, discover, and synthesize new materials through atomic-scale control. It addresses the Grand Challenge, "How do we design and perfect atom- and energy-efficient synthesis of revolutionary new forms of matter with tailored properties?" The results contribute to the scientific cases for high-brightness X-ray facilities such as the Advanced Photon Source Upgrade.

\section{RESULTS AND ACCOMPLISHMENTS}

We made significant strides in understanding how defects behave at the nanoscale by studying two systems (Figure 1): defect dynamics in grains in polycrystalline films, which govern properties such as strength and catalytic activity, and the hydriding phase transformation in the palladium $(\mathrm{Pd})$ used in hydrogen storage and purification.

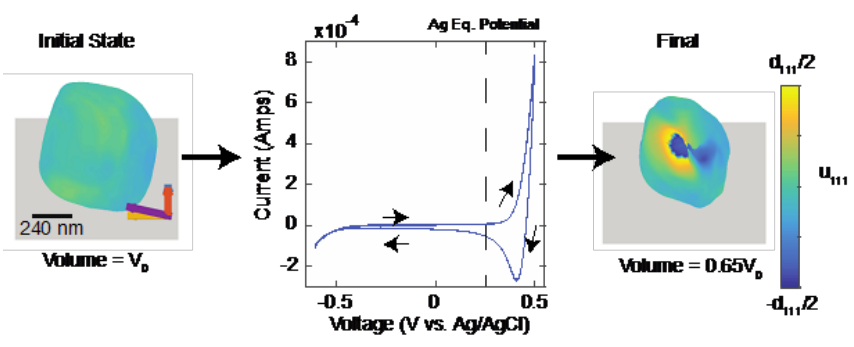

Figure 1. Demonstration of fast dissolution at the dislocation termination points on the particle surface by using in situ Bragg coherent diffractive imaging combined with electrochemistry.

Grains and their defects govern the properties of polycrystalline materials, such as structural materials (high-strength alloys) and catalysts. We demonstrated how coherent $\mathrm{X}$-rays can be used to isolate an individual grain from its neighbors to understand how the defects on that particular grain govern its response to external stimuli. (In this case, temperature is the stimulus that drives grain growth.) We also demonstrated that defects in nanoparticles are much more mobile than originally thought. By utilizing large Pd nanoparticles, we showed how they nucleate defects attributable to strain induced during hydrogen storage. We stopped the storage process midway and allowed the hydrogen to release from the palladium lattice. Surprisingly, the defects that were induced during the storage were actually eliminated during the release! This points to fundamentally interesting structure-function relationships in these particles and potentially to ways to engineer longer-lived hydrogen storage schemes.

\section{PROPOSED FUTURE WORK}

On the basis of this work, we submitted a proposal to DOE BES for the Synchrotron Radiation Studies Field Work Proposal (FWP). The results and future directions of this work will also be incorporated into an early career award proposal to DOE BES. 


\section{Understanding the Structure of Matter}

\author{
2016-188-R1
}

Cédric J. Mezrag and Craig D. Roberts

\section{PROJECT DESCRIPTION}

While the fact that almost all the visible mass of the universe comes from nucleons and nuclei is well established today, the underlying mechanisms generating that mass are still not understood. Today we know that nucleons are composed from particles that are (almost) massless at very high energy, described by a theory called quantum chromodynamics (QCD). A dynamical mechanism is, therefore, at work here to generate both the mass and the structure of the nucleon in terms of its almost massless constituents. The goal of this project is to understand the structure of the nucleon, and more generally of hadrons, to shed light on the above-mentioned mechanisms. Three complementary distributions are therefore considered: distribution amplitudes (DAs), generalized parton distributions (GPDs), and transverse momentum distributions (TMDs). The DAs describe the one-dimensional structure of the nucleon in momentum space, whereas GPDs and TMDs describe its 3D structure. More precisely, GPDs deal with the positions of quarks and gluons inside the nucleon, while TMDs focus on their momenta (see Figure 1). The results will be achieved when (1) a greatly enhanced hadron physics facility at the Thomas Jefferson National Accelerator Facility (Jefferson Lab) is about to begin operations with programs dedicated in large part to the measurement of GPDs and TMDs, and (2) the community is developing the science case for an electron-ion collider, which must be informed by reliable predictions from continuum-QCD.

\section{MISSION RELEVANCE}

This work addresses fundamental science issues at the heart of programs sponsored by DOE's Office of Nuclear Physics. For example, the results are enabling concrete predictions to be made for basic properties of the proton and neutron that will be measured using the upgraded accelerator at Jefferson Lab, and these predictions would have been impossible had the new techniques not been developed.

\section{RESULTS AND ACCOMPLISHMENTS}

The proton and its first radial excitation are baryons, constituted from three valence quarks; and this work delivered the first quantum field theory calculations of their valence-quark distribution amplitudes. The results establish that the proton's amplitude is a broad, concave function; and the details reveal the presence and character of strong correlations within these systems. They will be paradigm changing. An article describing the analysis has been submitted for publication.

We also made a breakthrough in computing the QCD effective coupling within the low-energy area, where perturbative expansion no longer works. We allied non-perturbative continuum and lattice-regularized QCD techniques to extract this coupling, which exhibits an inflexion point and remains finite at low energy, contrary to perturbative computations. Our results are in striking agreement with experimental extractions and will be used in the future for testing fundamental predictions of the Standard Model.

Our third achievement has been made in the field of GPDs. In addition to our model computations of the pion GPDs, we have managed for the first time to build a framework allowing a GPD model to fulfill a priori all of the required theoretical constraints. This work is submitted for publication.

Last but not least, we published work on the structure of virtual composite particles, demonstrating that experiments at Jefferson Laboratory can reliably use a baryon's meson-cloud to provide the targets for forthcoming measurements of pion and kaon electromagnetic form factors.

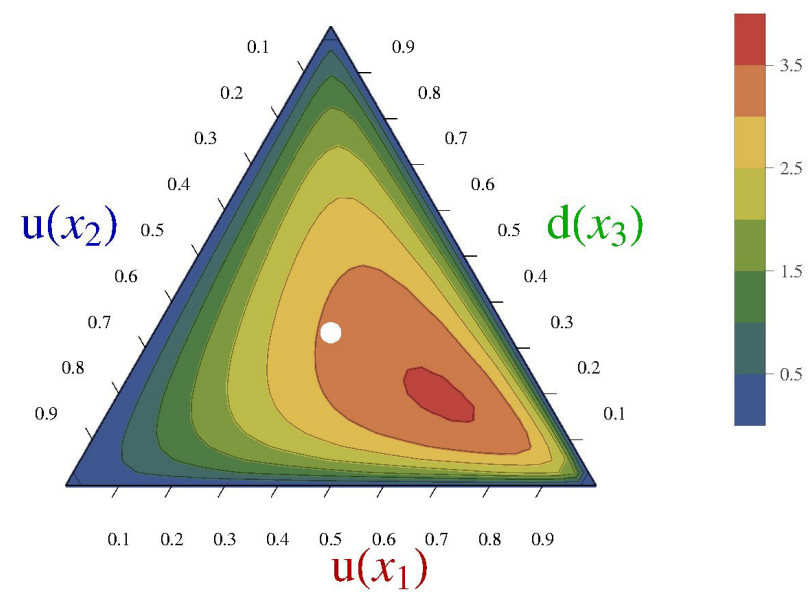

Figure 1. Ternary diagram showing the nucleon DA as a function of the momentum fractions $x 1, \times 2$, and $\times 3$ carried by the $u, u$, and $d$ quarks, respectively. The white dot denotes the center of the triangle, where every quark shares one third of the total nucleon momentum. The momentum is more likely to be carried by quarks 1 and 3 rather than quark 2, as the red area indicates a larger probability amplitude. The fact that the distribution is positive-definitely is consistent with the nucleon being the ground state of baryons.

PROPOSED FUTURE WORK

This project concluded in FY 2017. 
Novel Devices and Systems for Neuromorphic Computing

\author{
2017-029-NO
}

Jianqiang Lin

\section{PROJECT DESCRIPTION}

As conventional von Neumann computers based on digital silicon CMOS technology reach their performance limits, a paradigm shift in computational systems is becoming necessary to sustain the growth of information technology in our modern society. Neuro-inspired computation can be an attractive alternative to conventional computation, because of its massively parallel approach, reconfigurability, and energy efficiency. However, in order to make dramatic performance improvements with neuromorphic systems, novel material systems must be developed. One of the major goals of this project is to develop novel nanostructured materials to implement ultra-low-power artificial neurons for neuromorphic circuits.

\section{MISSION RELEVANCE}

This project is relevant to the DOE mission in basic science. Materials with the properties of the electrically driven insulator-to-metal transition (E-IMT) are promising candidates for neuromorphic circuit components such as integrate-and-fire artificial neurons. However, to date, E-IMT materials are limited in their performance. This work investigates the physics and technology required to improve E-IMT performance and investigate its suitability for constructing artificial neurons and neuromorphic circuits.

\section{RESULTS AND ACCOMPLISHMENTS}

The current on/off ratio, denoted by $\mathrm{M}_{\mathrm{E}}$, and voltage scalability are important metrics to gauge the performance of the nanoscale E-IMT structures fabricated in the Center for Nanoscale Materials. The E-IMT material is $\mathrm{VO}_{2}$ grown on a sapphire substrate. For the first time, we have demonstrated and studied a nanoscale E-IMT structure with a length of $40 \mathrm{~nm}$ and a width of $200 \mathrm{~nm}$ (shown in Figure 1a). The E-IMT in this work demonstrates an on/off current ratio $\left(M_{E}\right)$ above 3,000, which is 10 times better than the existing technology and sets a new record for this class of materials. In addition, the structure shows a record low operating voltage of $0.2 \mathrm{~V}$. Figure $1 \mathrm{~b}$ shows how the performance metrics of our E-IMT structure compare to existing data published in the literature.

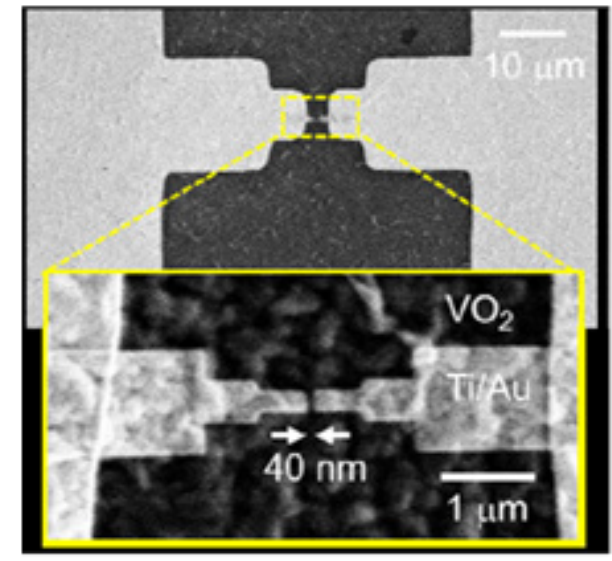

(a)

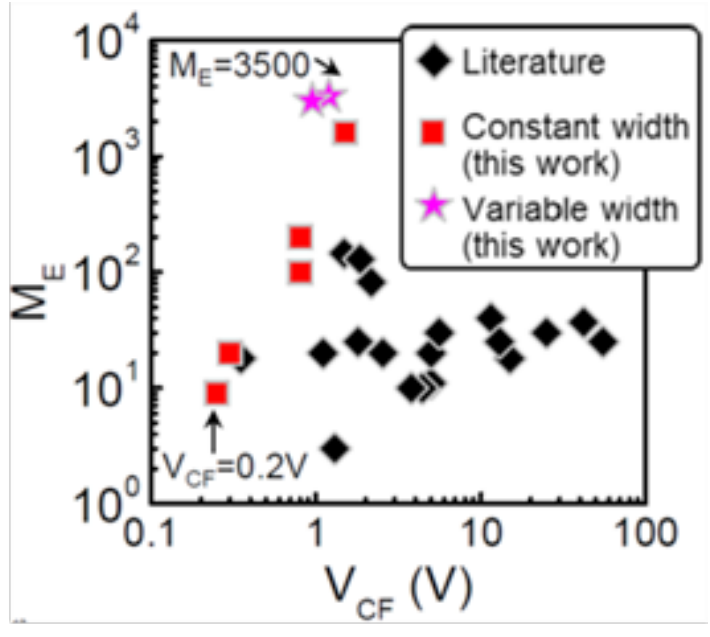

(b)

Figure 1. (a) Scanning electron microscopy (SEM) top view of the E-IMT structure built in this work. Inset: enlarged view at the center of the structure. The length (L) is $40 \mathrm{~nm}$. (b) Benchmarking of the key metrics for the material against data from the literature. This work demonstrates a record low voltage and high $\mathrm{M}_{\mathrm{E}}$ operation.

\section{PROPOSED FUTURE WORK}

In FY 2018, this project will extend our knowledge of performance optimization in E-IMT, including important metrics such as the on/off ratio, operating voltage, and switching speed. We believe that this new nanoscale E-IMT structure is the key for further performance breakthroughs.

Following the performance optimization, this project will aim to develop a physics-based compact model for E-IMT operation, and we will continue exploring new applications enabled by the unique nonlinear properties of E-IMT. To further this goal, we will investigate the low-voltage artificial neuron. 


\section{Ecological Organic Photovoltaics Using Water- Borne Semiconductor Nanoparticles}

\author{
2017-031-NO
}

Yu Jin Kim and Byeongdu Lee

\section{PROJECT DESCRIPTION}

This project focuses on the following: successful synthesis of water-borne nanoparticles (NPs); realization of water-soluble NPs using organic semiconductors via surfactants; optimization of the NPs via smart surfactant engineering; the study of charge carrier properties, particularly charge separation in the NPs; and achieving high-efficiency of organic photovoltaics using the NPs.

\section{MISSION RELEVANCE}

This project is relevant to DOE missions in energy and the environment. In recent years, research in organic photovoltaics (OPVs) has attracted a great deal of attention. However, to date, harmful chlorinated solvents have been the most common choice in high-performance OPVs. Thus, safe and environmentally friendly processed OPVs are needed. Eco-friendly OPVs-particularly water-based OPVs-could revolutionize the global energy picture.

\section{RESULTS AND ACCOMPLISHMENTS}

We identified a new n-type surfactant, PEG-C60, and we investigated its structural formation in a water-based solution. The PEG-C60 surfactant, which had C60 molecules grafted onto polyethylene glycol chains, was studied using a small-angle $X$-ray scattering to reveal its structure. We found that PEG-C60 formed a cluster of aggregated double-layer micelles (Figure 1a).

We synthesized $\mathrm{p}-\mathrm{n}$ heterostructured NPs. Using the poly(3-hexylthiophene) (P3HT) polymer as a p-type compound and the PEG-C60 as an n-type surfactant, we synthesized $p-n$ heterostructured NPs. They showed a P3HT:core-PEG-C60:shell morphology (Figure 1b).

We synthesized the NPs as a function of PEG-C60 or P3HT concentration to investigate their new particle shapes, sizes, and/or conformities. The NPs systematically showed smaller and more circular shapes as PEG-C60 concentration increased. However, when we increased the P3HT concentration, they exhibited larger and more random particle structures. As the structural morphology of the NPs is changed, their charge separation ability also dramatically varied. (a)
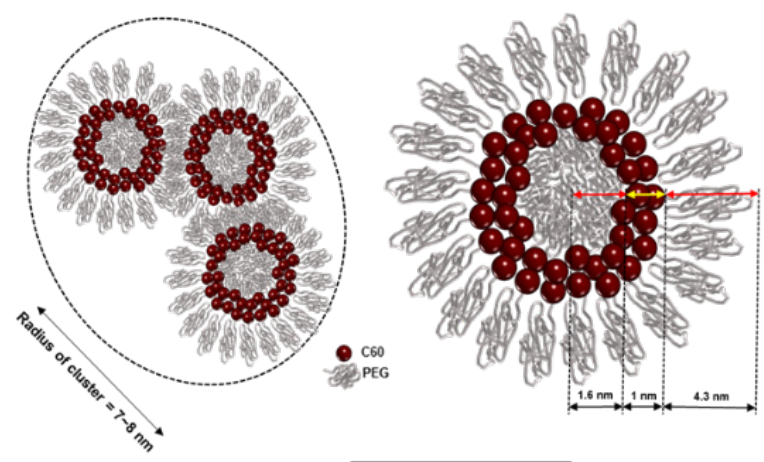

(b)

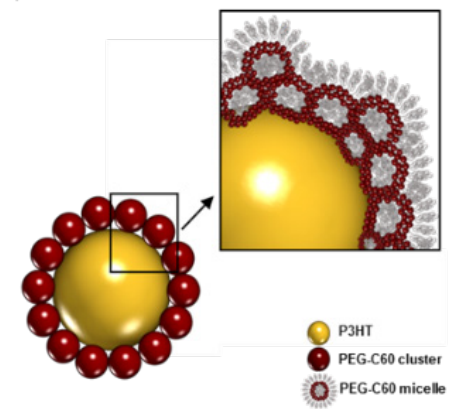

Figure 1. (a) Cluster model of PEG-C60 micelles (left) and detailed PEG-C60 structure (right). (b) Suggested structure of the $\mathrm{p}-\mathrm{n}$ heterostructure NPs.

We synthesized SDS- or C16E10-based P3HT:phenyl-C61-butyric acid methyl ester (PCBM) NPS (SDS and C16E10 are representative surfactants in the water-borne NP field) to compare to our system. They formed $180-\mathrm{nm}$ particles, which is similar to previously reported results. When we compared charge separation effects, however, a higher quenching efficiency appeared in our new heterostructured NPS.

\section{PROPOSED FUTURE WORK}

We will conduct additional research on enhanced charge separation effects in the NPs. The core regions of pure PEG-C60 micelles have the PEG polymer, thereby they have charge-trapping sites because the PEG is an insulating polymer. Therefore, we will attempt to synthesize the PEG-C60 micelles with C60 molecules in the core part. We will then use the micelle to synthesize p-n heterostructured NPs with p-type compounds; these will show boosted charge separation ability compared to the previous heterostructured NPs.

We will fabricate and investigate photovoltaic cells and their performance using the synthesized NPs. We will also investigate structure-property relationships involving the film structures and efficiencies for devices made using the NPs. 
Beehive: A Dynamic Execution Environment for Performance, Power, and Resilience on Extreme-Scale Computing Systems

\section{7-093-NO}

\section{Min Si and Pavan Balaji}

\section{PROJECT DESCRIPTION}

In this project, we will develop a dynamic execution model called "Beehive" that can balance performance, resilience, and power awareness. The central idea of Beehive is based on overdecomposition, where the model is not bound by the available hardware processing units. Beehive will utilize a combination of operating systems (OSs) and runtime techniques to expose such overdecomposition as distinct operating system processes, called Beehive processes. The Beehive dynamic execution environment consists of four primary components: low-level process scheduling, intelligent task scheduling, integration with system fault tolerance software, and power management tools.

\section{MISSION RELEVANCE}

This project is relevant to DOE's mission in basic science. Power and other technological limitations in the next generation of large supercomputers do not bear "free performance." As supercomputing architectures undergo fundamental changes, transformational changes in the programming models are needed. Key challenges will be programmability, performance, and power consumption centered on support of complex, dynamic DOE applications; these challenges will be addressed through this project.

\section{RESULTS AND ACCOMPLISHMENTS}

We carried out two activities during FY 2017 related to process address-space sharing and dynamic adaptation of message passing interface (MPI) asynchronous progress.

Address-space sharing of processes is the core technique to support lightweight process oversubscription and user-level process scheduling in Beehive. We are collaborating with AICS RIKEN (Japan) to exploit this concept. We implemented a portable address-spacesharing model, called Process-in-Process (PiP), and utilized it in several scenarios, including MPI intranode communication, on-node parallel programming as a replacement of threads, and a data-sharing technique used with in situ programming for scientific simulations.

Beehive is designed to overcome the complications associated with dynamic and irregular MPI applications. Casper is a process-based asynchronous progress model for irregular MPI remote memory access (RMA); however, it cannot handle multiphase applications well because of its static message redirection design. To understand what the upper limit of this traditional OS process-based approach is, we studied a dynamic adaptation technique within Casper. The dynamic approach enables asynchronous message redirection only when the cost of user computation becomes dominant, and it disables redirection when the execution moves into a communication-intensive phase. Figure 1 shows the improved performance by using the dynamic approach in the multiphase NWChem quantum chemistry application in a $\operatorname{CCSD}(T)$ computation for the tetracene molecule.

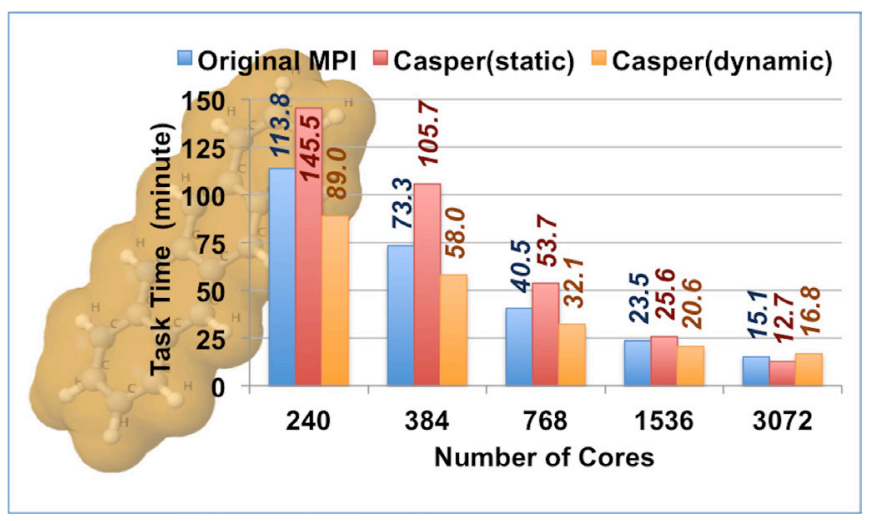

Figure 1. Performance improvement in multiphase NWChem CCSD(T) for tetracene with aug-cc-pVDZ basis set on the National Energy Research Scientific Computing Center Edison supercomputer.

\section{PROPOSED FUTURE WORK}

The project will continue in FY 2018 and FY 2019. Anticipated activities include development of Beehive low-level infrastructure based on the portable process address-space-sharing technique (PiP). It provides the functionality of process oversubscription and user-level scheduling. Optimization of MPI dynamic asynchronous communication on top of Beehive will be conducted, as well as MPI runtime optimization by using the concept of process oversubscription and user-level scheduling. We will perform lightweight checkpointing and dynamic migration within existing fault-resilience software. 


\section{Microstructural Simulations of Stable, Conjugated Polymer Glasses}

\author{
2017-094-NO \\ Nicholas E. Jackson, Juan de Pablo, \\ and Venkatram Vishwanath
}

\section{PROJECT DESCRIPTION}

The rational design of glassy organic semiconducting films requires a multiscale computational approach to elucidate structure-function relationships critical to enabling industrial relevance. The goal of this project is the development of new computational approaches to directly simulate the impact of processing conditions on the optoelectronic properties of organic semiconducting films. Our multiscale approach involves the combination of $a b$ initio electronic structure theory, molecular dynamics and Monte Carlo simulations, and supervised machine learning to expedite the design of these materials' mechanical, thermal, and optoelectronic properties.

\section{MISSION RELEVANCE}

The development of multiscale computational techniques on exascale computing frameworks to understand optoelectronically functional materials is directly related to recent DOE calls for Exascale Computing, Co-Design Centers, Scientific Discovery through Advanced Computing (SciDAC) partnerships, and Energy Frontier Research Centers (EFRCs). This project will also generate considerable interest from the electronics industry.

\section{RESULTS AND ACCOMPLISHMENTS}

In FY 2017, we developed a supervised machine learning technique to map electronic degrees of freedom directly to coarse-grained models, enabling electronic structure calculations on coarse-grained representations at negligible cost.

We successfully performed the first atomistic simulation of a vapor-deposited organic semiconductor and conducted a proof-of-principle study demonstrating that charge transport metrics can be directly influenced via atomistic simulations controlling deposition conditions.

We developed an anisotropic coarse-grained model for organic semiconductors capable of accelerating molecular dynamics simulations by a factor of $\sim 10$ to 20 , while maintaining degrees of freedom required to accurately describe the electronic structure.
We elucidated the mechanism of collapse for polyelectrolytes in the presence of various solvent and salt environments-a result critical for the examination of conjugated polyelectrolyte films.

We derived a coarse-grained model that correctly describes counterion fluctuations to describe polyelectrolyte materials at length scales relevant to the simulation of polyelectrolyte rheology, a critical element of optoelectronic material processing.

Finally, we developed a novel Monte Carlo simulation algorithm using multiple auxiliary potential energy functions capable of accelerating simulations of glassy organic semiconducting materials.

\section{PROPOSED FUTURE WORK}

FY 2018 goals include simulating the vapor deposition of a common organic semiconducting molecule, 4,4'-Bis(N-carbazolyl)-1,1'-biphenyl (mCP), analyzing the impact of tuning vapor deposition parameters on the charge transport performance of the material, and correlating these results with experimental scattering and mobility measurements.

We will extend the multiple auxiliary potential energy functions method to non-Boltzmann distribution functions, thus increasing the utility of this method for the advanced sampling of glassy materials.

We will apply our novel supervised machine learning methodology to map the electronic properties of organic semiconducting films, specifically $\mathrm{mCP}$, to larger-scale, multi-molecule coarse-grained descriptors, and attempt to correlate these descriptors with experimental observables derived from scattering experiments (Figure 1). A new coarse-grained polyelectrolyte model will be applied to a conjugated polyelectrolyte material, and the impact of processing conditions on the resulting optoelectronic functionality will be simulated directly.

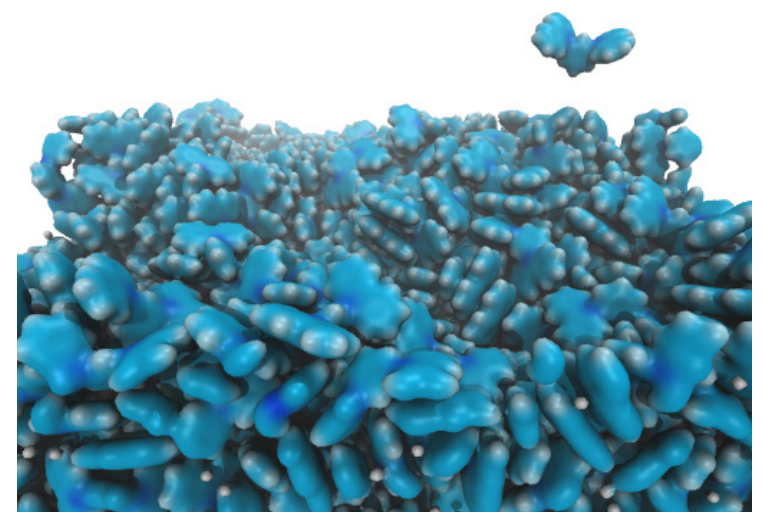

Figure 1. Film of mCP vapor deposited computationally. The molecule at the top of the image indicates a "hot" molecule being deposited onto the existing surface. 


\section{Combining Electrochemistry and Ultrafast Spectroscopies: Real-Time Characterization of Multi-Electron/Proton Intermediates in Hydrogen- and Oxygen-Evolving Catalysts}

\author{
2017-095-NO
}

Ryan G. Hadt

\section{PROJECT DESCRIPTION}

Solar-driven water oxidation represents an attractive means of providing high-energy electrons that can be stored in the form of chemical bonds, that is, solar fuels $\left(\mathrm{H}_{2}\right)$. However, the reactive states of water-oxidation and proton-reduction catalysts remain poorly understood. The goal of this research is to spectroscopically and computationally define reactive states and elusive reaction intermediates that drive solar energy conversion and solar fuel catalysis. To accomplish this objective, we are combining electrochemical methods with advanced spectroscopic techniques to either poise or photo-trigger the formation of reactive intermediates that could not be studied through traditional rapid mixing or freeze-quench methods. By further combining experiment and theory, we aim to define the key geometric and electronic structure contributions to water oxidation, proton reduction, and the formation of $\mathrm{O}-\mathrm{O}$ and $\mathrm{H}-\mathrm{H}$ bonds.

\section{MISSION RELEVANCE}

This project is relevant to DOE's missions in energy, the environment, basic science, and the Solar Energy and Photochemistry and Catalysis programs in the Chemical Sciences, Geosciences, and Biosciences Division of DOE's Office of Basic Energy Sciences (BES). DOE's BES reports on Grand Challenges for Science, and recent basic research needs reports on synthesis, carbon capture, and catalysis feature priority research directed toward the achievement of hierarchical self-assembling architectures and host-guest chemistries for the control of multistep, energy-converting reactions using earth-abundant metals.

\section{RESULTS AND ACCOMPLISHMENTS}

Both homogeneous and heterogeneous transition metal compounds can carry out the water oxidation reaction. However, in all cases, highly oxidizing (e.g., high-valent) states are formed, and the high reactivity of these species results in transient lifetimes. Therefore, one outstanding problem in the field of solar fuels generation is the structural and electronic characterization of high-valent, active sites capable of $\mathrm{O}-\mathrm{O}$ bond formation.

To address this problem, we have recently combined electrochemistry with in situ X-ray spectroscopies to define the electronic structures of active molecular and heterogeneous oxygen-evolving catalysts. Our work has allowed for the capture and characterization of a high-valent $\mathrm{Co}(\mathrm{IV})_{2}$ oxidation state in a $\mathrm{Co}(\mathrm{III})_{2} \mathrm{Co}(\mathrm{IV})_{2}$ transition metal oxide compound, $\left(\mathrm{CO}_{4} \mathrm{O}_{4}\right)$. Importantly, this is the first and only direct electronic analog of the proposed active site in arguably the most highly studied water oxidation catalyst, cobalt phosphate (CoPi). Both in situ X-ray absorption and near-infrared (IR) absorption spectra were coupled to theory for further insights, and we found that the unpaired electrons in the Co $(\mathrm{IV})_{2}$ state were antiferromagnetically coupled. This result was important because it allowed for parallels to be drawn between molecular and heterogeneous catalysis of the oxygen-evolution reaction for the first time. We showed that the antiferromagnetic coupling provides a mechanism to promote a simultaneous transfer of two electrons with opposite spins, which results in a singlet potential energy surface for $\mathrm{O}-\mathrm{O}$ bond formation with a low-energy spin barrier.

In addition, near-IR absorption spectroscopy allowed for the observation and modeling of intervalence charge transfer (IVCT) transitions, which correspond to light-induced electron transfer between the electronically coupled metals in both the $\mathrm{Co}(\mathrm{III})_{3} \mathrm{Co}(\mathrm{IV})$ and $\mathrm{Co}(\mathrm{III})_{2} \mathrm{Co}(\mathrm{IV})_{2}$ states. From the analyses of IVCT bands, we showed that the formation of a Co(IV) ${ }_{2}$ state (and antiferromagnetism) results in the localization of electron holes. This localization can be utilized to drive $\mathrm{O}-\mathrm{O}$ bond formation and oxygen evolution at catalyst surfaces (Figure 1).

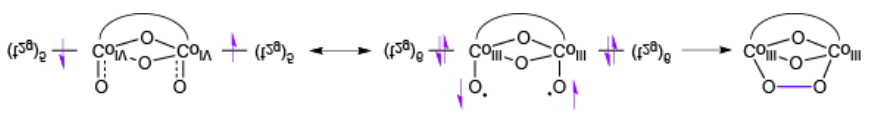

Figure 1. Electronic considerations for the mechanism of $\mathrm{O}-\mathrm{O}$ bond formation by intramolecular coupling of oxyl radicals on the catalyst surface.

\section{PROPOSED FUTURE WORK}

Future work will focus on coupling electrochemistry and high-resolution spectroscopies, such as in situ high-energy resolved, fluorescence-detected X-ray absorption, X-ray emission, and resonant X-ray emission spectroscopies, as well as resonant inelastic X-ray scattering. In addition, we will explore coupling electrochemistry to in situ transient absorption spectroscopy. These studies will allow for the further characterization of highly reactive, transient intermediates that carry out the conversion of solar energy to fuels. 


\section{On the Colloidal Suspension of Lithium Clusters in Molten Lithium Chloride}

\author{
2017-096-NO
}

Augustus Merwin, Chris J. Benmore, and Mark A. Williamson

\section{PROJECT DESCRIPTION}

The generation and presence of elemental lithium in the molten lithium chloride electrolyte used in the electrolytic reduction of oxide nuclear fuels is an impediment to the integration of light water reactor fuels into pyroprocessing fuel cycles. This project is investigating molten solutions of lithium chloride and elemental lithium ( $\mathrm{LiCl}-\mathrm{Li})$ by using laboratory electrochemical techniques and synchrotron-based, hard X-ray analysis to advance pyroprocessing, as well as the basic sciences of lithium chemistry and metal-salt solutions.

\section{MISSION RELEVANCE}

Lithium chemistry, specifically in electrolytes and in molten salts, is of high-level interest to several DOE missions, including energy storage, advanced nuclear energy systems, and basic science. This work relates directly to existing programs in the treatment of nuclear fuels and molten salt batteries. Furthermore, the experimental capabilities developed in these studies are of fundamental importance to future programs in molten salt chemistry as they apply to nuclear technologies.

\section{RESULTS AND ACCOMPLISHMENTS}

To date, this research has yielded practical results on methods of monitoring nuclear fuel cycle unit operations, in addition to fundamental data on the nature of molten metal-salt solutions. The electrochemical behavior of the interface between Li metal and molten $\mathrm{LiCl}$ has been characterized for the first time. This behavior has been utilized to develop an electrochemical method for quantitatively determining the endpoint of the oxide reduction process. This advancement to process monitoring has significant engineering implications and the potential to increase the efficiency of advanced fuel cycles.

An apparatus has been developed to conduct simultaneous synchrotron and electrochemical characterization of molten salts. A plot of the interatomic spacing, or liquid structure, of molten $\mathrm{LiCl}$ recorded using this apparatus is shown in Figure 1. By measuring the changes in melt structure which occur as a result of electrochemical formation of metallic $\mathrm{Li}$ in molten $\mathrm{LiCl}$, this work has recorded preliminary evidence of the existence of delocalized electron states in the resulting metal-salt solution. These electron states are observed by recording slight deviations in the fluid structure shown in Figure 1 as metallic Li is introduced into the melt. Understanding the physical chemistry of this system will help researchers understand the mechanism by which Li metal is dissolved and lost in the pyrochemical treatment of nuclear fuels, and potentially enable an increase in operational efficiencies by suppressing these effects.

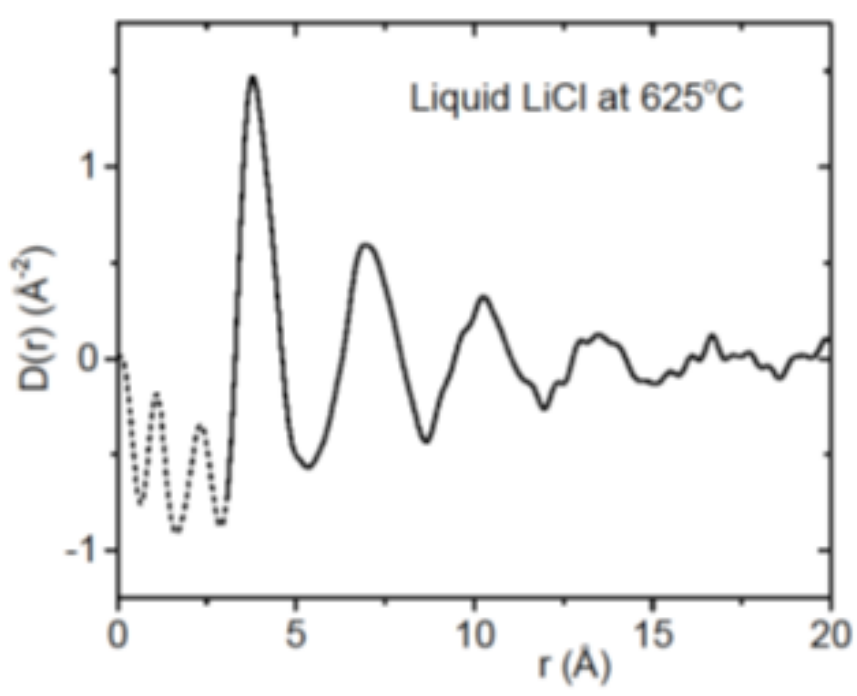

Figure 1. The pair distribution function $D(r)$ of molten $\mathrm{LiCl}$ recorded during electrochemical testing in the melt. This is the first reported combined electrochemical and hard X-ray analysis of a molten salt.

\section{PROPOSED FUTURE WORK}

It remains unknown if the electron solvation observed in molten $\mathrm{LiCl}-\mathrm{Li}$ solutions is a general phenomenon of metal-salt solutions or if it is unique. Future studies will use high-energy $X$-ray diffraction to probe the structure of molten solutions of the chlorides of sodium, cesium, calcium, and magnesium ( $\mathrm{NaCl}-\mathrm{Na}, \mathrm{CsCl}-\mathrm{Cs}, \mathrm{CaCl}_{2}-\mathrm{Ca}$, and $\mathrm{MgCl}_{2}-\mathrm{Mg}$ ). 


\section{A Universal Data Analytics Platform for Science}

\author{
2017-097-NO
}

Ryan Chard and lan Foster

\section{PROJECT DESCRIPTION}

Scientific discovery is increasingly reliant on big data, computation and data science, and large-scale computing infrastructure. Efficiently and reliably managing, curating, synthesizing, and analyzing data are challenges almost every scientific domain faces. This project aims to make scientific data readily available and accessible, regardless of repository location. It will enable analysis of this distributed and diverse data via cutting-edge analytics tools, and it will transparently employ autonomic computing methodologies to optimize the execution of analytics tools on a broad set of large-scale computing infrastructures. To accomplish these goals, we are developing a software-defined cyberinfrastructure (SDCI) solution to enable researchers to define high-level policies and automate much of the scientific data life cycle. We have implemented a preliminary SDCI solution and are now developing technologies to leverage our programmable fabric and facilitate the analysis of arbitrary data across diverse compute infrastructures.

\section{MISSION RELEVANCE}

This project is relevant to DOE's mission in basic science. It has the potential to impact a wide range of Argonne research interests across almost every computational discipline. In particular, our platform will enable users to automate much of the research data life cycle, in turn enforcing best practices (cataloging, sharing, and purging), federating access to distributed data and tools, and increasing the value of Argonne's cyberinfrastructure investments by removing barriers to entry. This platform will democratize access to advanced capabilities, thus enabling a large number of researchers to access and use novel and best practice analytics techniques on leadership-class computing systems.

\section{RESULTS AND ACCOMPLISHMENTS}

Our primary achievement to date is the development and implementation of the Ripple service. Ripple realizes our $\mathrm{SDCl}$ vision by enabling the instrumentation of existing infrastructure to establish an active and responsive system of programmable devices. Users are empowered to define high-level, if-trigger-then-action-style rules to act on data in response to data-driven events (e.g., file creation, data transfer) and to automate data management and analysis tasks. We have developed novel monitoring solutions and enabled the use of SDCl capabilities over leadership-class storage infrastructure. We have also integrated Ripple's execution environment with UChicago's Parsl project to abstract compute environments and enable Ripple's application to multiple high-performance computing resources.

Ripple has been applied to a number of use cases, including the automation of the Kasthuri Group's neuroscience research. To achieve this, Ripple has been deployed at both the Advanced Photon Source (APS) (on beamline 32-ID) and the Argonne Leadership Computing Facility's visualization cluster, Cooley. Data are automatically detected on creation at the APS; transferred to Cooley; and reconstructed, cataloged, shared, and returned to the biologists at the APS. A similar flow has been established to automate reconstructions from Lawrence Berkeley Laboratory's Advanced Light Source using the National Energy Research Scientific Computing Center's computing resources. Overall, Ripple has automated more than 15,000 actions, analyzed terabytes of data, and automated computational processes, thereby simplifying analytics.

\section{PROPOSED FUTURE WORK}

We have numerous work directions to pursue over the next year. Our primary research direction relates to the development of the analytics platform. The analytics platform will leverage the SDCl fabric to automate the end-to-end analysis of data as they are generated (Figure 1). Our platform will expose common analytics models (e.g., MapReduce, HTC) and integrate compute resources. Our key milestones are to (1) develop a policy engine to facilitate workload placement decisions based on data locality and cost, (2) extend Ripple application programming interfaces to accommodate the analytics platform, (3) develop the analytics platform by leveraging Ripple's capabilities, and (4) continue integration with research groups to facilitate use cases and grow adoption.

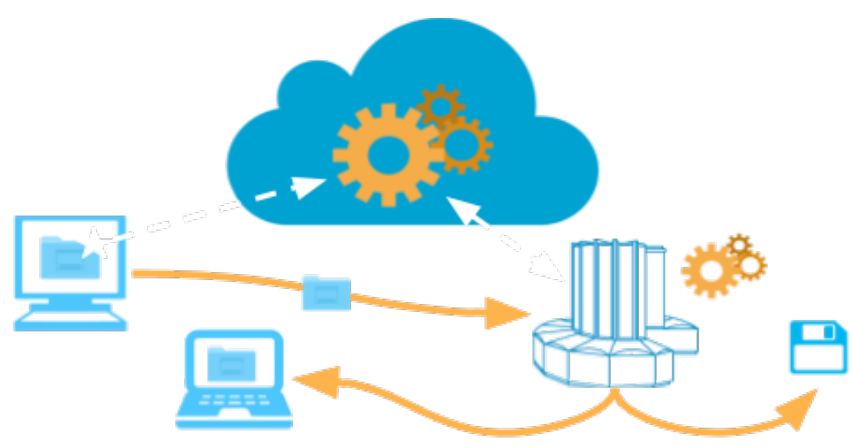

Figure 1. High level view of the proposed analytics platform. 


\section{Transport and Photophysical Processes in Hybrid Perovskites for Energy Applications}

\author{
2017-157-NO
}

Peijun Guo and Richard D. Schaller

\section{PROJECT DESCRIPTION}

Hybrid metal-halide perovskites (HPs) - generally described by the formula $A B X_{3}$, where $A$ is an organic cation (e.g., methylammonium), B is a metal (e.g., lead $[\mathrm{Pb}]$ ), and $\mathrm{X}$ is an anion (e.g., chlorine $[\mathrm{Cl}]$, bromine $[\mathrm{Br}]$, or iodine [I])-are emerging semiconductor materials that hold great promise to deliver cheaper and more efficient photovoltaics. These compounds straddle the divide between organic and inorganic semiconductors, and exhibit excellent optical and electronic properties, especially long carrier lifetimes and low-cost solution processability. Despite efforts centered on improving material processing and device fabrication, it remains unclear how HPs can maintain such exceptional optoelectronic properties even in polycrystalline thin films processed at near room temperature. This project focuses on the fundamental understanding of charge transport and the photophysical properties of HPs. Using advanced spectroscopic techniques, we will investigate charge carrier generation, scattering, and recombination pathways. We will particularly focus on understanding the electronic and vibrational coupling between the inorganic and organic sub-lattices in HPs, and how the dynamic motions of the organic component, which we will vary, can affect the charge transport.

\section{MISSION RELEVANCE}

This research is relevant to DOE missions in energy and the environment. It will provide insights into structure-property relationships in this emerging class of organic-inorganic hybrid materials and yield general principles for designing materials with desired properties for applications ranging from low-cost photovoltaics and solid-state lighting to photocatalysis. Such efforts may provide practical pathways to clean and sustainable energy.

\section{RESULTS AND ACCOMPLISHMENTS}

Using time-resolved visible-pump, infrared-probe spectroscopy, we monitored the timescales of lattice heating in methylammonium lead iodide and revealed the weak phonon-phonon interactions between the inorganic and organic sub-lattices and slow thermal equilibration. Using temperature-dependent transient reflection spectroscopy, we evaluated the velocity of coherent acoustic phonons (which are lattice vibrations) over a wide range of temperatures and uncovered significant structural fluctuation of the perovskites, which can impact the optoelectronic property and thermal conductivity.

We also investigated the variation of acoustic phonon velocities with the number of perovskite layers in two-dimensional hybrid perovskites and studied how the van der Waals bonding impacts the heat transport in this class of materials and beyond.

\section{PROPOSED FUTURE WORK}

In the next phase, we will continue our investigation of two-dimensional hybrid perovskites (2D-HPs) with a focus on several aspects:

1. Studying thermal stability. These materials have enabled efficient and air-stable solar cells as well as light-emitting diodes. As a result, the evaluation of material performance at elevated temperature is important for the deployment of such material in large-scale applications.

2. Elucidating the response of excitons and free carriers and the impact of quantum confinement as a function of temperature.

3. Mapping the static and transient optical properties, especially the refractive indices of these materials. The obtained optical properties are crucial for the design of 2D-HP-based optical devices. 


\section{Real-Time Control of Urban Drainage Systems}

\section{7-158-NO}

\section{Sara P. Rimer and Charles Macal}

\section{PROJECT DESCRIPTION}

This project seeks to advance the methods for real-time monitoring and control of urban drainage systems by developing adaptive hydraulic modeling techniques. The ability to reduce combined-sewer overflow (CSO) events is an issue that challenges more than 800 U.S. municipalities. When the volume of a combined sewer system or wastewater treatment plant is exceeded, untreated wastewater then overflows (a CSO event) into nearby streams, rivers, or other water bodies, which causes localized urban flooding and pollution. The likelihood and impact of CSO events are exacerbated by urbanization, population growth, climate change, aging infrastructure, and system complexity. Thus, there is an urgent need for urban areas to manage CSO events. Traditionally, CSO events have been mitigated via time-intensive and expensive structural interventions such as retention basins or sewer separation. These can reduce CSO events, but are costly, arduous, and provide only a fixed solution to a dynamic problem.

Real-time control (RTC) of urban drainage systems using sensor and actuator networks has been an inexpensive and versatile alternative to traditional CSO intervention (Figure 1). In particular, retrofitting individual stormwater elements for sensing and automated active distributed control has been shown to significantly reduce the volume of discharge during CSO events, with some RTC models demonstrating a reduction upwards of $90 \%$ compared to traditional passive systems. As more stormwater elements become retrofitted for RTC, system-level RTC across complete watersheds is attainable. However, when considering the diverse set of control needs of each of these individual stormwater elements, such system-level RTC becomes a far more complex problem. To address such diverse control needs, this project utilizes agent-based modeling such that each individual stormwater element is treated as an autonomous agent with diverse decision-making capabilities.

This project is carried out in collaboration with the Real-Time Water Systems Laboratory at the University of Michigan.

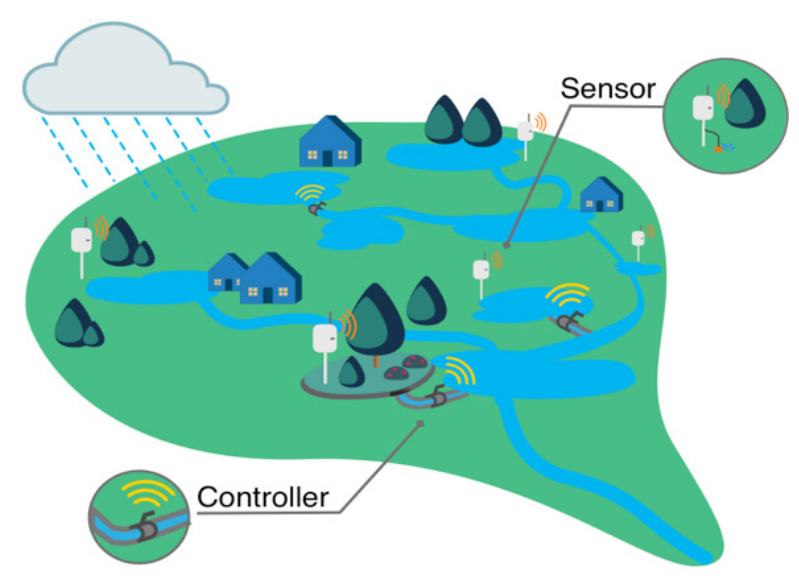

Figure 1. Example of an intelligent watershed with sensor and actuator control. Source: Kerkez et al. (2016).

\section{MISSION RELEVANCE}

This project is relevant to DOE's basic science and national security missions. The development of computational tools for real-time sensing and control of dynamic and complex engineered systems is a fundamental question that is relevant to DOE's mission of advanced scientific computing research, which we are advancing through the scientific understanding of urban stormwater infrastructure by making use of high-performance modeling and simulation. In addition, improving urban drainage systems so they can immediately and adaptively respond to stormwater threats is vital to the resilience and security of our nation's urban infrastructure.

\section{RESULTS AND ACCOMPLISHMENTS}

This project began in the latter part of FY 2017, so the majority of the accomplishments for this year have consisted of initiating and fostering relevant collaborations and community partners who will be a part of the ensuing years. These partners include university collaborators, municipal partners, and professional networks.

\section{PROPOSED FUTURE WORK}

In FY 2018, we will develop an agent-based decentralized control model based on a resilient control systems approach in which agents will represent individual hydraulic elements that make control decisions based on their underlying hydraulic principles and their interaction with each other. In addition, we will collaborate with the City of Chicago's Array of Things project to install hydraulic sensors throughout the city to test potential impacts and installations of urban stormwater RTC mechanisms. 


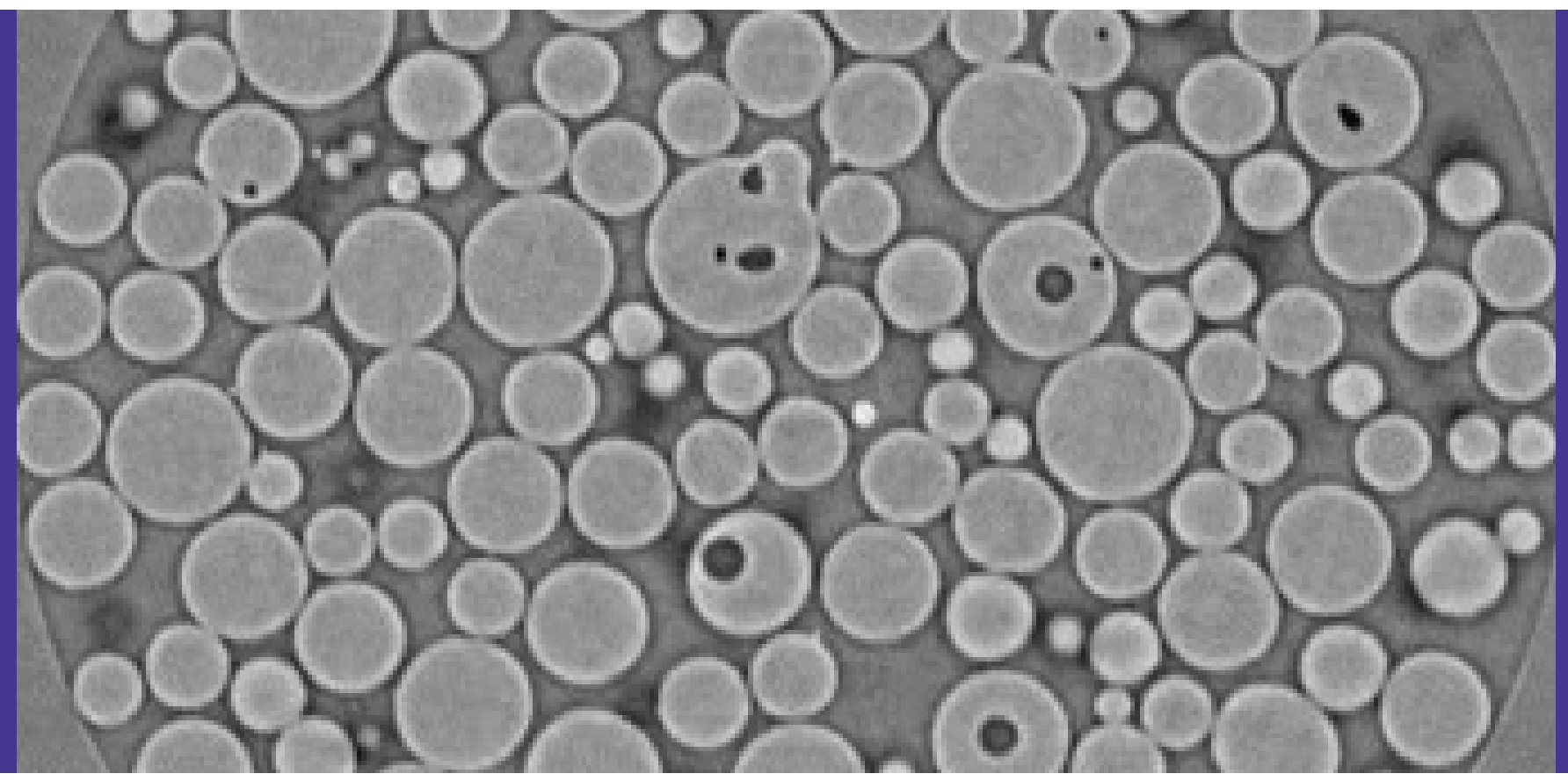

\section{LDRD PRIME -}

APPLIED ENERGY

AND SUSTAINABLE

TRANSPORTATION 


\section{Chemical Vapor Processing for Additive Manufacturing}

\author{
2015-151-R2
}

Jeffrey Elam, Seth Darling, and Randall E. Winans

\section{PROJECT DESCRIPTION}

We are in the midst of what is arguably the most significant transformation in manufacturing since the industrial revolution: the advent and proliferation of additive manufacturing. Additive manufacturing, also called three-dimensional (3D) printing, creates solid, three-dimensional objects from digital models by depositing a series of thin, patterned layers. Since the 1980s, 3D printing has been used primarily for rapid prototyping, but recent advances in printer size, speed, and printable materials are pushing this technology toward manufacturing in a range of industries, including defense, aerospace, automotive, construction, and medical equipment. A variety of 3D printing technologies has emerged, but all have limitations. For instance, the most common 3D printing medium is organic polymers, which are cheap, light, and versatile. However, for applications such as engine parts, electronic components, or medical implants, polymers typically lack the necessary robustness, electrical properties, and chemical compatibility.

To address these problems, we proposed to develop chemical vapor processing for additive manufacturing (CVPAM, Figure 1). In this technique, the desired part is first 3D printed and then post-processed by treating with chemically reactive vapors to either (1) deposit a coating, (2) infuse the bulk of the part to enhance the material properties, or (3) densify the part. This LDRD project aimed to develop CVPAM to the point of attracting industrial partners for eventual commercialization. The first task was to perform a targeted survey of 3D printing media and chemical infiltration methods. Next, we selected the most promising processes for development and refinement and evaluated coating equipment, chemistry, and methodology. Finally, we will pursue commercial applications for CVPAM technology.

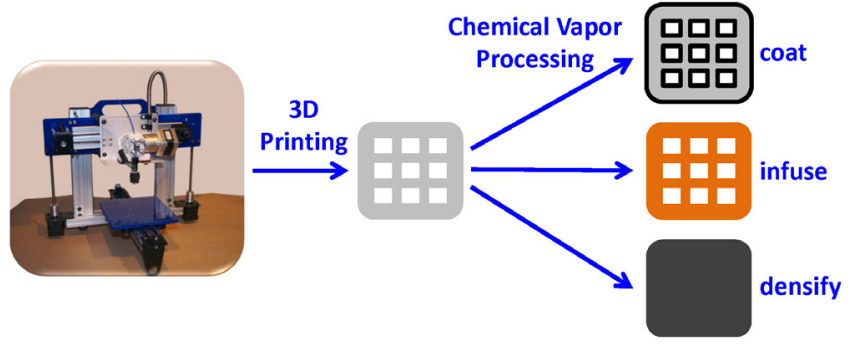

Figure 1. Chemical vapor processing for additive manufacturing (CVPAM): After 3D printing, a part is subjected to chemically reactive vapors to either (1) coat all exposed surfaces (e.g., for biocompatibility), (2) infuse with inorganic material (e.g., for added stiffness or conductivity), or (3) densify (e.g., to fill voids in a sintered metal).

\section{MISSION RELEVANCE}

This project aligns with DOE missions in energy and sustainability. Industrial beneficiaries will include 3D printer suppliers that can incorporate the CVPAM technology into their products, industrial coating equipment manufacturers, and chemical suppliers. Industries such aerospace, automotive, and medical equipment will also benefit through the numerous advantages of additive manufacturing, including shorter lead times, easy customization, and reduced materials usage. Finally, the U.S. economy will benefit from CVPAM because innovations in additive manufacturing will help counterbalance the low labor costs in countries such as China and India.

\section{RESULTS AND ACCOMPLISHMENTS}

In this project, we have engaged local suppliers of 3D printers for production to discuss their needs and the relevance of our technology to their industry. We purchased and installed a development-scale 3D printer and printed a variety of test structures and coated them using atomic layer deposition (ALD). We examined the parts using scanning electron microscopy and four-point probe conductivity measurements, and studied the infusion of polymers by ALD precursors using in situ grazing incidence small angle X-ray scattering (GISAXS). We have examined the mechanical properties of infiltrated 3D printed parts using nanoindentation measurements and explored the suitability of CVPAM to impart corrosion resistance to 3D printed parts for an annular centrifugal contactor. Finally, we have used CVPAM to impart electrical conductivity to prototype 3D printed detectors under development at Argonne.

\section{PROPOSED FUTURE WORK}

This project is completed. 


\section{Sustainable Transportation: Novel Bio-Derived Fuel Additives for Improved Vehicle Efficiency}

\author{
2015-157-R2
}

S. Scott Goldsborough, Stephen Ciatti,
Patricia Ignacio-de Leon, Phillip Laible,
Raghu Sivaramakrishnan, and Robert Tranter

\section{PROJECT DESCRIPTION}

The objective of this project was to discover or develop novel, bio-derived fuel additives to be used at small concentrations (e.g., 1\% by volume [v/v]) to tailor the chemical kinetic pathways that control autoignition of petroleum-based fuels. Such additives could be employed in internal combustion (IC) engines to help meet evolving performance targets and legislated mandates, significantly increase overall vehicle efficiencies, and augment the use of domestically produced biomass feedstocks within the transportation sector. While IC engine-based powertrains may be replaced in the longer term, this work will facilitate near-term gains.

Major tasks included the following: (1) utilizing a heuristics-based approach to identify and screen molecules/classes of molecules capable of boosting octane or improving cetane, and evaluating the autoignition behavior of various additive-fuel blends via rapid compression machine (RCM) tests; (2) characterizing the decomposition kinetics of the targeted molecules using $a b$ initio theory and modeling and investigating additive-fuel interactions using chemical kinetic modeling; (3) biosynthesizing and separating bench-scale levels of select targets; and (4) scaling up sustainable, carbon-neutral biosynthesis efforts and chemical upgrading processes to produce sufficient volumes.

\section{MISSION RELEVANCE}

This work is related to DOE energy and environmental mission areas, as well as to a number of the programs of DOE offices, including the Energy Efficiency and Renewable Energy's (EERE's) Co-Optima initiative, Basic Energy Sciences (BES), and Biological and Environmental Research (BER). This project directly addresses urgent national needs to improve the sustainability of the U.S. transportation system through improved IC engine/ vehicle efficiency and the use of sustainably produced, bio-derived components. Fuel economy gains are projected using bio-derived fuel additives that are compatible with combustion technologies expected to dominate in the near and medium terms.

\section{RESULTS AND ACCOMPLISHMENTS}

The RCM database consists of more than 45 different additive candidates (alcohols, esters, ethers, ketones, furans, aromatics, and others), and was extended in FY 2017 with additional compounds. The functional behavior of one promising candidate, for which intellectual property protection is being sought, was further probed. Screening was first undertaken by blending the components into a full-boiling-range gasoline at $10 \% \mathrm{v} / \mathrm{v}$ and then comparing the results against ethanol, the current octane booster of choice. Data-mining techniques were applied to derive empirical understanding of the relationships between additive molecular structure and perturbative performance. Engine tests were conducted in a gasoline compression ignition engine over a limited operating range where the most promising additive was doped into the fuel.

A chemical kinetic model was developed to understand the combustion behavior of one promising additive, with the model extended to cover interactions with a gasoline-representative surrogate. Comprehensive tests were conducted in the RCM over a wide range of conditions to acquire validation data for the chemical kinetic model. Oxidative behavior of the pure compound was measured, and it was blended at various levels $(0,5$, $10 \%)$ with a single-component surrogate.

To biologically produce fuel additives using negative value material as feedstock, strains of purple, non-sulfur bacteria that can accumulate chemical precursors at up to $75 \%$ of their total cell mass were isolated. Growth rates on renewable waste streams were comparable to growth on purified carbohydrates. High-throughput fluorescent assays were established to non-invasively quantify precursors in live cell cultures. Conditions for the biosynthesis of larger carbon chains and reactive substituents were explored. Additive synthesis routes that proceeded by polymer extraction from the bacteria, acid-catalyzed monomer valorization, and catalytic upgrading were identified.

\section{PROPOSED FUTURE WORK}

The team plans to continue aspects of this work with funding from DOE EERE and BER. 


\section{Large-Scale Modeling and Simulation for an Adaptive and Resilient Power Grid}

\author{
2015-159-R2 \\ Jianhui Wang, Chen Chen, James Kavicky, Sven Leyffer, \\ and Feng Qiu
}

\section{PROJECT DESCRIPTION}

Hurricane Sandy and other recent power outages under extreme weather conditions have revealed that our electric power grid is not sufficiently planned and operated to be resilient to large-scale events. A well-prepared and executed response to such incidents can prevent possible outages, reduce the time to restore power to consumers, and potentially lead to significant economic savings. Maintaining the resilience of the power grid is critical to our nation's energy security and sustainability. This becomes even more critical as climate change alters the frequency and severity of future extreme weather events.

This project aims to develop a tool with models and algorithms to improve power grid resilience, such as quick recovery from outages. Key tasks include optimizing system sectionalization, generator restarting, and load serving after outages; cascading outage simulation and optimal mitigation planning to prevent cascading outages; and system restoration with renewable energy participation.

We have identified primary concerns and scientific challenges in power system restoration with our industrial partners; we also built a set of mathematical models to formulate major decision-making problems in restoration and developed solution algorithms; and we developed a web-based graphical user interface (GUI) to enable operators to experiment with restoration planning under various scenarios. See Figure 1.

\section{MISSION RELEVANCE}

"Resiliency in energy infrastructure" is a key mission for DOE's Office of Electricity and Energy Reliability (OE). Specifically:

[t]he Infrastructure Security and Energy Restoration Division (ISER under OE) leads efforts for securing the U.S. energy infrastructure against all hazards, reducing the impact of disruptive events, and responding to and facilitating recovery from energy disruptions, in collaboration with all levels of industry and State and local governments.
This project addresses the quick recovery capability of the electricity grid by providing a set of tools that helps grid operators better plan and analyze their restoration actions. This project also aligns with the missions of the Federal Emergency Management Agency (FEMA), which is "to build, sustain and improve our capability to prepare for, protect against, respond to, recover from and mitigate all hazards."

\section{RESULTS AND ACCOMPLISHMENTS}

We developed a power system restoration planning tool with a web-based GUI named EGRIP (electric grid resilience improvement program), which includes models and algorithms for a number of decision-making problems in system restoration, such as system sectionalization and generator restarting. A number of algorithms are also in the project results, such as a state-of-the-art algorithm for solving alternating current optimal power flow (ACOPF). The tool helped FEMA perform restoration exercises on industrial-scale data. EGRIP was also demonstrated to the Electric Reliability Council of Texas (ERCOT), which showed interest in working with Argonne to improve EGRIP.

A number of models and algorithms were also developed for other resilience problems, such as distribution system restoration with mobile generators, transmission system restoration with renewable generation, cascading outage simulation software, and an optimization-based simulation tool to mitigate cascading outage risks.

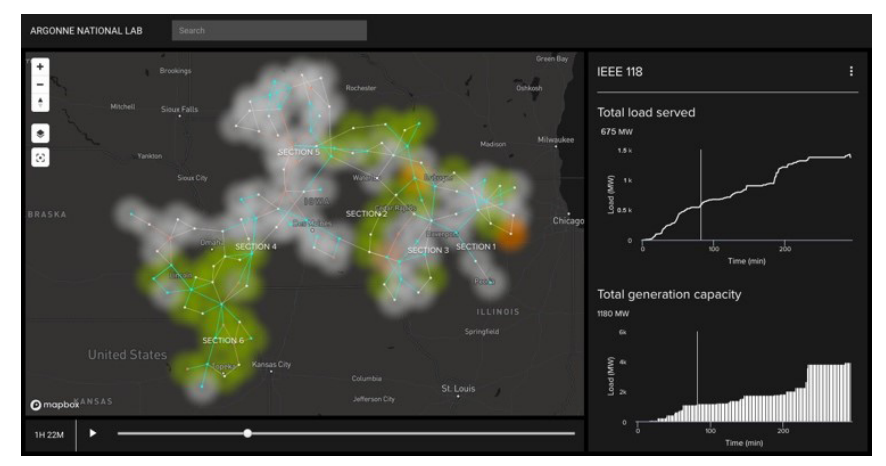

Figure 1. EGRIP's web-based GUI showing the progress of a restoration to operators. The panel on the right shows the time series of total loads picked up and total generation capacity restored. The figure on the geographic map shows a snapshot of the restoration; green areas: electricity service restored; grey areas: service not restored yet; and orange areas: service being restored. A timeline at the bottom can help operators examine any moment of the restoration progress.

\section{PROPOSED FUTURE WORK}

Future work includes enhancing computational performance, integrating cascading simulation into EGRIP, solving restoration with repair crew routing, and developing interface other features. 


\section{Connected and Automated Vehicles}

\section{5-176-R2}

Aymeric Rousseau, Joshua Auld, and Dominik Karbowski

\section{PROJECT DESCRIPTION}

Connected and automated vehicles (CAVs) are bound to transform on-highway transportation in the near future. Vehicles will be able to communicate with each other and with the surrounding infrastructure as some or all of the tasks incumbent upon drivers will gradually shift to automated controllers. Although the R\&D on the underlying technology has been many years in the making, the impact that CAVs may have on energy use-whether it is positive through more efficient driving and reduced congestion or negative through increased travel demand-has been largely overlooked. Advanced high-fidelity simulation tools are needed not only to evaluate impacts before actual implementation, but also to identify system-level control strategies that will improve mobility and reduce energy use. The objective of this project is to develop an integrated model combining both traffic flow models and advanced vehicle models.

\section{MISSION RELEVANCE}

The main benefit of the integrated model we propose would be to quantify the energy impact of Smart Mobility technologies, one of the main focuses of DOE's energy mission. Most of the research in the area of intelligent transportation systems has been targeted at mobility and safety. In another point of view, systems researchers have been focusing on energy efficiency on standardized cycles. However, it has not been possible so far to assess global energy performance in a real-world transportation network scenario. Our focus has been to quantify the energy impact of advanced vehicle technologies.

\section{RESULTS AND ACCOMPLISHMENTS}

In FY 2015, we focused on the development of new capabilities including the following:

$\square$ A new traffic flow model was developed in POLARIS to enable the analysis of platooning's effect on mobility.

$\square$ The development of a new multi-vehicle platform was initiated to allow vehicle-to-vehicle simulations. The first application included the development of a Cooperative Adaptive Cruise Control algorithm.

$\square$ A POLARIS model for the Ann Arbor metropolitan area was developed to leverage existing vehicle datasets from deployments by DOE and the Department of Transportation.
In FY 2016, we developed an improved stochastic simulator for POLARIS-generated trajectories that allows us to bypass the commercial micro-simulator tool. We have developed a process to automatically and efficiently run and analyze hundreds of thousands or millions of runs through high-performance computing. The faster process allows us to handle scenarios that include much larger scales. Multiple improvements were implemented in POLARIS for the router and traffic flow models as well as the overall code.

During FY 2017, we focused on the development of new capabilities, including the following:

$\square$ Modified the POLARIS code to allow for future large-scale simulations (such as model parameter externalization, LINUX compilation for HPC) and future deployment. We

○ externalized parameters;

- established an environment for development and testing of POLARIS on many core processors (e.g., Intel Xeon Phi), which will equip Argonne's next supercomputer, Aurora;

- adapted portions of the POLARIS code to Linux solvers and performed code optimization; and

○ initiated deployment to facilitate POLARIS adoption.

$\square$ Enhanced POLARIS capabilities to expand the list of potential CAVs use cases that researchers can evaluate. We

o developed vehicle assignment models for improved energy use forecasting and

- modeled travel activities and choices to capture how travelers use mobility solutions.

$\square$ Automated the POLARIS $\rightarrow$ Autonomie process and deployed it with AMBER (used POLARIS results to estimate energy).

\section{PROPOSED FUTURE WORK}

This project ended in FY 2017. To build on the successes of this project, we will explore new sources of support to continue the core development of POLARIS and its complete migration to high-performance computing (HPC). 


\section{Advanced Control Algorithms for Improving Energy Consumption of Connected and Automated Vehicles}

\author{
2016-126-R1
}

Jeffrey Larson, Joshua Auld, Mahmoud Javanmardi, Dominik Karbowski, and Todd Munson

\section{PROJECT DESCRIPTION}

Connected and automated vehicles (CAVs) offer opportunities for improved energy efficiency. For example, some CAVs may be able to "platoon," that is, one vehicle may follow another vehicle closely to decrease its aerodynamic drag and reduce its fuel consumption. In this project, we are developing optimization models that route vehicles in a manner that minimizes their collective fuel use. We incorporated these optimization models within POLARIS, Argonne's agent-based modeling framework, in order to understand the effect of platooning vehicles on system-wide traffic flow. A major accomplishment for this year was the development of optimization models that can simulate vehicles traversing road segments at different speeds. This capability more accurately represents the real world while also increasing the opportunities for platooning. Another accomplishment was the introduction of platooning-capable vehicles into POLARIS, which will allow researchers to study the effect of CAVs on traffic within a large metropolitan road network.

\section{MISSION RELEVANCE}

This project contributes to DOE's mission to develop and deploy large-scale, energy-efficient solutions within all of the nation's energy sectors. Individuals and companies use billions of gallons of fuel every year; studying the effects of CAVs will determine whether platooning technologies may result in significant economic and environmental savings. This research is also relevant to transportation network companies (e.g., Uber/Lyft) and freight/logistics companies that wish to incorporate platoon formation/dissolution when routing vehicles.

\section{RESULTS AND ACCOMPLISHMENTS}

We built optimization models from the ground up and extended POLARIS to model the behavior and effects of platooning vehicles. We studied the effects of optimal platooning routing and coordination in the greater-Chicago road network. In 2017, we extended these models to allow vehicles to travel at non-freeflow speeds. The principal finding from our optimization models was that as vehicles increase the percentage of time that they are willing to pause during their commute (thereby allowing for coordinated platoon formation), the amount of fuel saved also increases (see Figure 1). Note that the solution quality after 5 min is nearly equal to the computed lower bound in this large-scale study of the Chicago road network.

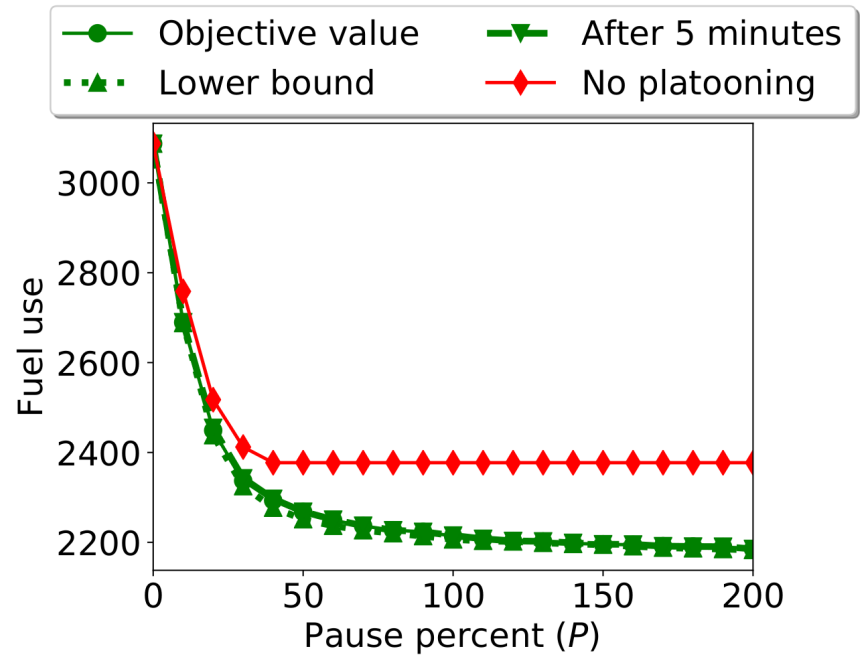

Figure 1. As the willingness-to-wait time for CAVs increases, the collective amount of fuel used decreases. Though the time-to-solution can be large, the solution quality after $\mathbf{5}$ min is near optimal.

\section{PROPOSED FUTURE WORK}

Work in FY 2018 will focus on integrating our optimization models inside the POLARIS framework. Past studies required capturing the state of the road network within POLARIS and saving it to a separate file. We then ran our optimization models offline, and loaded the resulting vehicle routes back into POLARIS. This step was useful when debugging our models and improving POLARIS, but requires a significant amount of data manipulation by hand. The handoff between POLARIS and our optimization models meant that only isolated cases could be studied. To accurately study the system-wide effect of platooning vehicles in real time, POLARIS must repeatedly solve large optimal-routing problems. This ability will be incorporated into POLARIS as a result of this project. We are seeking follow-on funding from the DOE Vehicle Technologies Office to extend our optimization models and support incorporating further optimization methods within POLARIS. 


\section{Integrated Water-Energy Systems Assessment Framework (IWESAF) for Water-Energy Sustainability and Resilience}

\author{
2016-152-R1
}

Eugene Yan, Getnet Betrie, Vladimir Koritarov, Matthew Mahalik, Feng Qiu, Thomas Veselka, May Wu, and Zhi Zhou

\section{PROJECT DESCRIPTION}

Energy-water systems provide critical services to the nation's manufacturing and economy. These systems are highly dynamic and involve various physical processes and human activities across multiple scales. The future growth of energy-water demands, projected increases in climate and hydrologic extremes, rapidly evolving technologies, and changing policy exacerbate energywater system interactions, complexity, and uncertainty. The goal of this project is to develop a regional integrated water-energy systems assessment framework (IWESAF) that provides flexible and extensible energy-water nexus (EWN) assessment tools to evaluate and enhance the sustainability, reliability, and resilience of energywater systems. The main technical objectives include: (1) developing a modeling framework that incorporates existing and new tools that represent energy and end-user sectors, physical and thermal processes with hydrologic cycles, and the management practices and human activities relevant to EWN; (2) creating an IWESAF coupling process that provides temporal and spatial coupling among system components and facilitates simulation of cross-domain feedback; (3) formulating data-modeling processes that characterize intensity, duration, and frequency of climate and hydrologic extremes and optimize power generation operation, power grid functioning, and planning in response to extremes and market values; and (4) validating IWESAF's functionality for various scenarios.

\section{MISSION RELEVANCE}

The IWESAF developed from this project will support DOE's EWN crosscut effort and will make it possible to (1) investigate the complex, dynamic interactions of energy and water systems; (2) improve our understanding of the interactions, which will lead to more resilient, robust, and reliable energy-water systems and support DOE's energy security goals; and (3) provide a basis for identifying regionally appropriate technologies and improving aging energy-water infrastructure.

\section{RESULTS AND ACCOMPLISHMENTS}

In the first year of the project, we built a database for energy and water systems and developed some key system components, including regional hydrologic and riverine thermal regime modeling, a climate and hydrologic extremes generator, an initial module for thermal power plant simulation, and a power grid test system. This year, we have made significant progress in improving or developing the modeling tools for the remaining key system components:

Risk-based reservoir adaptive management model. The model for hydropower plants incorporates a flexible multilayered approach that relies on user inputs and risk preferences and penalizes risky solutions while rewarding those that perform well under a wide range of futures.

Thermal power plant simulator. Our improved process-based model includes a power plant database, heat balance, and cooling system modules to simulate thermodynamic states through the process and to quantify the impacts of water availability and influent water temperature on electricity generation output, water withdrawal/consumption, and effluent water temperature.

Energy-water economic model. The model uses a numerical equilibrium framework to evaluate the economic consequences (both unintended and intended) of water or energy policies and their interactions under extreme events.

Power grid system model. Our grid system model includes thermo-, hydro-, solar, and wind power plants and linkages to other model layers (e.g., water and economic systems) (Figure 1) to facilitate dynamic interactions among water and power plants and to evaluate short-term operation and long-term planning.

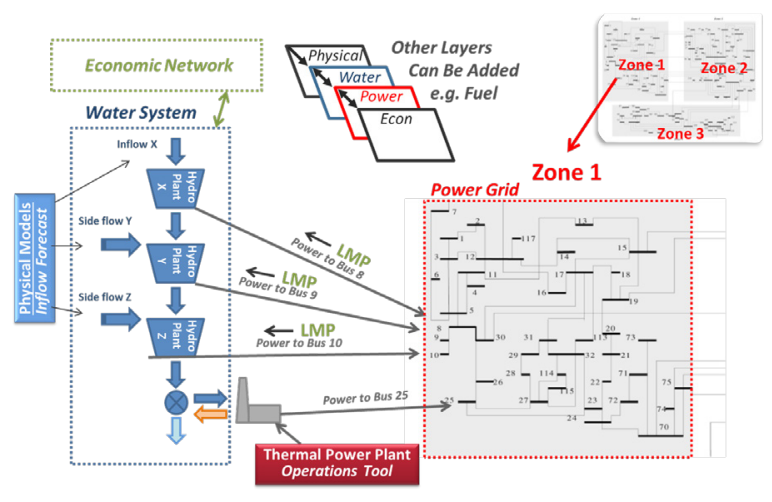

Figure 1. Power grid system integration.

\section{PROPOSED FUTURE WORK}

A case study will be performed to test model integration, identify system responses, and assess resilience and optimization strategies. 


\section{VERIFI 2.0: Next-Generation Engine/Fuel Simulation Codes}

\author{
2016-175-R1
}

Douglas Longman, Ray Bair, Paul Fischer, Stephen Klippenstein, Misun Min, Stephen T. Pratt, Sibendu Som, Ananias Tomboulides, and Al Wagner

\section{PROJECT DESCRIPTION}

VERIFI (Virtual Engine Research Institute and Fuels Initiative) 2.0 undertakes the development of a well-integrated suite of massively parallel simulation codes for internal combustion engines and their fuel systems that (1) provide a substantial improvement in computational combustion and predictive engine simulation, (2) are well tuned for the next decade of computer architectures, and (3) are capable of greatly accelerating the design of future engines and fuel systems. This major advance over the existing VERIFI codes will yield a unique capability for DOE labs and academia to understand the science required to enhance engine efficiency, and for industry to engineer the engine and fuel combustion concepts of the future in a timely fashion.

\section{MISSION RELEVANCE}

This program is relevant to DOE's energy security mission. New DOE programs, such as the Office of Energy Efficiency and Renewable Energy's Co-Optimization of Fuels and Engines (Co-Optima), which began in FY 2016, would be prime beneficiaries of the VERIFI simulation capability. We will actively pursue engagement with DOE programs like the existing Advanced Scientific Computing Research (ASCR) co-design centers. Engine manufacturers will also benefit from these Argonne-developed codes, which will enable predictive engine and combustion simulations with new fuels of interest.

\section{RESULTS AND ACCOMPLISHMENTS}

Work in the first year (FY 2016) focused on both improving computational fluid dynamics (CFD) modeling capabilities as well as new fuel chemistry kinetics modeling capabilities. In FY 2017, the enhancement of Argonne's open source CFD software, NEK5000, to add additional features required for internal combustion engine simulation focused on combusting fuel jet models. However, before modeling combusting fuel jets in natural gas direct injection spark ignition engine applications, we first set up Nek5000, the highly scalable spectral element method (SEM) code, to perform large eddy simulation (LES) of a non-reacting turbulent isothermal jet. A systematic study of the influence of grid size, numerical order of discretization, and inflow perturbation schemes on the LES results was performed. The accuracy and computational expense of Nek5000 were compared with those of a low-order code with a similar number of grid points. It was shown that the Nek5000 SEM can obtain significantly higher accuracy than the finite volume method solution, albeit at 2.5-7.0 times higher computational expense. However, the superior scaling performance of the SEM code was utilized to reduce the turnaround time by using a larger number of computing cores, achieving 81\% parallel efficiency on 32,768 CPU cores of ALCF/Theta (15th order, total $616 \mathrm{M}$ grid points). This approach reduced the overall time-to-solution by a third.

The primary accomplishment relative to the fuel chemistry aspect of this work is the development of the EStokTP software. This software couples commercial electronic structure codes with our own in-house chemical kinetics code. This code greatly facilitates large-scale, high-accuracy simulations of chemical reaction kinetics. Recent effort has focused on preparing the code for public release including writing a detailed manual, among other things. Codes for connecting such simulations to simple molecular-geometry descriptions were also implemented. The ability to use the fundamental aspects of gas phase chemical physics to automatically predict the kinetics of chemical reactions allows accurate predictions of the fuel chemical kinetics occurring in an internal combustion engine. This has not been previously possible since the conditions inside an engine (temperature, pressure, air/ fuel ratio, and presence of combustion byproducts) is continuously changing. This forces the current state of the art to compromise by using specific conditions for the chemical kinetics models used within the engine simulation modeling codes.

\section{PROPOSED FUTURE WORK}

Our effort on this project played an instrumental role in the successful addition of our automated fuel chemistry component to the Exascale Computing Program, "Transforming Combustion Science and Technology with Exascale Simulation." This addition is a new ASCR-funded project at $\$ 800,000$ per year. 


\section{Metal Additive Manufacturing Modeling}

\section{7-042-NO}

\author{
Marius Stan, Shashikant Aithal, Dileep Singh, \\ and Angel Yanguas-Gil
}

\section{PROJECT DESCRIPTION}

The objective of this project is to produce models of metal additive manufacturing (AM) covering both powder and wire feedstocks that run at speeds needed to meet practical industrial and governmental needs. Our approach consists of two stages. The first stage calibrates and validates fine-grained physics and chemistry-based models using data from other efforts. The second stage calibrates and validates reduced-order models using the results from the fine-grained physics- and chemistry-based models.

\section{MISSION RELEVANCE}

This project is relevant to DOE missions in basic science. We expect the project to produce industrially relevant metal AM modeling capabilities that will be extremely attractive to many sponsors including the National Science Foundation (NSF); Sciaky, Inc.; Raytheon; the Fraunhofer Institute; DOE's advanced manufacturing programs, vehicle technology programs, and other offices participating in the Clean Energy Manufacturing Initiative; America Makes; the Digital Manufacturing and Design Innovation Institute; and the U.S. Air Force, Army, and Navy.

\section{RESULTS AND ACCOMPLISHMENTS}

We selected the $\mathrm{Ti}_{6} \mathrm{Al}_{4} \mathrm{~V}$ alloy and the Inconel nickel-chromium-based super-alloy as model materials and developed a design methodology that can accommodate both metals, as well as others.

We started the physics-based modeling by looking at the heating effect of a laser beam on a metal block to help us learn more about the computational interactions in this scenario. A detailed literature review, along with multi-scale simulations, resulted in models of varying degrees of fidelity. Further simulations evaluated the temperature field in the material under consideration. The model correlates the thermal fields and thermal gradients to microstructures and defects.
We also ran a model based on a Green's function approach using individual laser pulses. Figure 1 shows the Laser Metal Additive Manufacturing simulation using a Green's function approach matrix. The simulation results provided insights into the laser interaction with the substrate and guided the optimization of the additive manufacturing process.

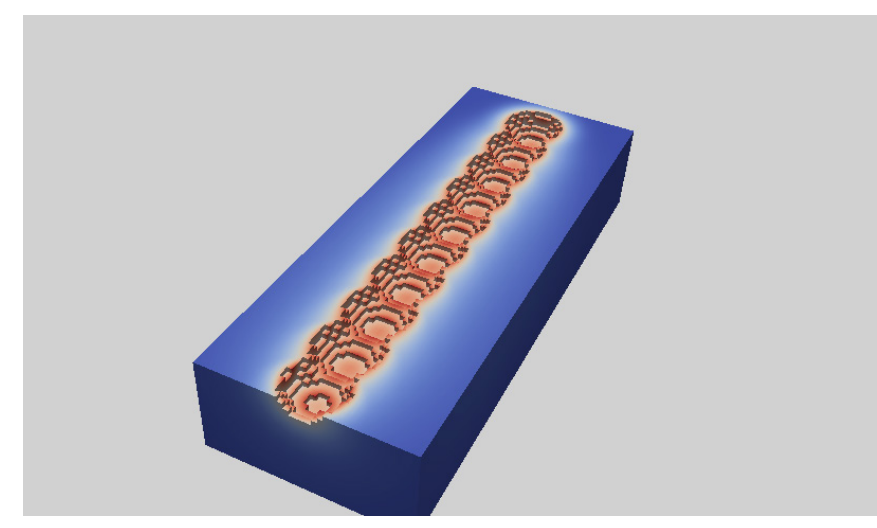

Figure 1. Example Laser Metal Additive Manufacturing simulation using a Green's function approach.

In parallel, we focused the Integrated Computational Materials Engineering (ICME) effort on the $\mathrm{Ti}_{6} \mathrm{Al}_{4} \mathrm{~V}$ alloy, which is of high relevance to additive manufacturing applications. Using the JMatPro ${ }^{\circledR}$ commercial software and a methodology developed by the project team, we could predict the phase evolution and properties of the alloy system as a function of the cooling rates. In addition, we acquired insights into the stability of relevant phases of the ternary system.

\section{PROPOSED FUTURE WORK}

In FY 2018, ICME modeling efforts will continue and will be refined to correlate with the process conditions from physics-based models and/or measured data points (spatial temperature vs. time distributions) from actual laser deposition of $\mathrm{Ti}_{6} \mathrm{Al}_{4} \mathrm{~V}$ powders. Further, we will generate data sets to feed into the reduced-order modeling efforts. Then we will analyze the entire body of data using a Bayesian algorithm implemented in a machine learning software. Furthermore, we will evaluate the uncertainty of all thermal and mechanical data generated in the project. 


\section{Improving Cost and Energy Efficiency of Nontraditional Water Desalination through Innovative Material and Process Integration}

\author{
2017-055-NO
}

Yupo J. Lin, Di-Jia Liu, and Zhi Zhou

\section{PROJECT DESCRIPTION}

Addressing the threat of an inadequate freshwater supply presents one of the most imminent challenges. Desalination of impaired water, such as brackish, reclaimed, and plant (process) water, exemplifies a resource-efficient, "fit-to-use" water service solution. The objective of this project is to develop the next-generation, highly energy-efficient water desalination technology that increases the energy efficiency to more than $50 \%$ compared to existing technologies (e.g., reverse osmosis [RO]) that have very low energy efficiency, namely, $12 \%$ for RO with impaired water. Such new technologies will have a profound impact on thermoelectric power plants, which constitute the largest users of surface water for cooling in the United States ( $40 \%$ of the entire water withdrawal). If the energy efficiency of desalination can be improved from $12 \%$ to $30 \%$ and implemented in the entire power plant industry, the energy savings would be equivalent to the production of thirty 500-MW power plants (or $\$ 6.5$ billion worth of electricity).

This project will focus on the innovative material R\&D used in electrochemical technologies to treat nontraditional water targeted initially for cooling use in thermoelectric power plants. The two electrochemical technologies evaluated in this project are ion-exchange resin wafer electrodeionization (RW-EDI) and capacitive deionization (CDI). Innovative manufacturing techniques as well as novel electrode materials are being developed to demonstrate the path forward for highly energy-efficient water desalination technologies. The deliverables in this project are to (1) advance material development of a high-surface nanofibrous electrode in a membrane-electrode-assembly specifically designed for CDI; and (2) demonstrate the electrochemical desalination performance of cooling water using RW-EDI and CDI for a power plant and the impacts of cooling water properties and treatment technology on energy production in the power plant.

\section{MISSION RELEVANCE}

This project is relevant to DOE missions in energy and the environment. The proposed material innovation tied to current water technology can provide a pathway to decrease the cost and save time to significantly improve the energy efficiency and increase the resilience of both energy production and water treatment. This project aims to address water issues in the power industry, which consumes more than $40 \%$ of total water withdrawal in the United States. The results of this research in the short term will be directly beneficial to the power plant industry without major applied technology changes while reducing energy consumption and operation costs. The increase in water treatment efficiency also benefits water conservation. Therefore, it will also be beneficial to other industries for water reuse. This project is aligned with the water research priority listed in the Multi-Year Program Plan of the DOE Advanced Manufacturing Office.

\section{RESULTS AND ACCOMPLISHMENTS}

In FY 2017, we successfully applied an innovative nanomaterial made using an advanced manufacturing technique (see example on Figure 1) on the CDI process to purify saline water. We also evaluated the desalination performance of different types of nontraditional water using RW-EDI for cooling water applications. A level of more than $30 \%$ energy efficiency of impaired water was demonstrated using RW-EDI. The process performance was used to set up property targets for innovative material development that will be tested in FY 2018.

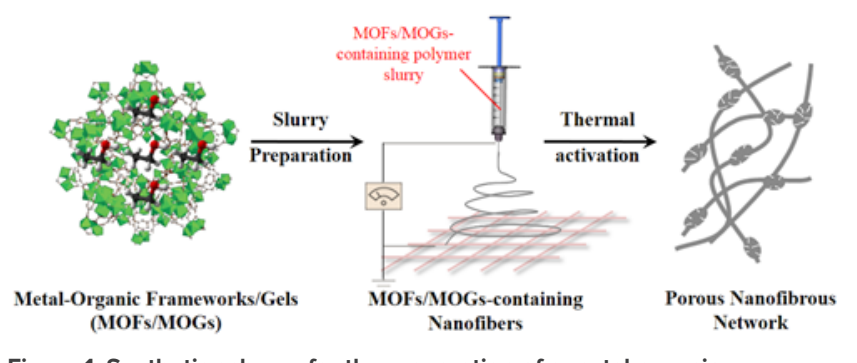

Figure 1. Synthetic scheme for the preparation of a metal-organic frameworks/gels-derived porous nanofibrous network.

\section{PROPOSED FUTURE WORK}

In FY 2018, implementation of the innovative nanomaterial in a CDI process will be conducted and evaluated for its water desalination energy efficiency. New salt adsorbent synthesis will be completed and tested in a RW-EDI device to assess its ability to achieve more than $45 \%$ energy efficiency of impaired water desalination. The research team will also evaluate the pathways for advanced manufacturing techniques to fabricate this material for future industrial applications. 
Advancing Additive Manufacturing of Metal Alloys: From Fundamental Principles to Durable Components

\author{
2017-084-NO \\ Aaron Greco, Maria De La Cinta Lorenzo Martin, \\ and Dileep Singh
}

\section{PROJECT DESCRIPTION}

The processing parameters and precursor materials of metal additive manufacturing (AM) have a significant impact on the resulting material microstructure and porosity, which in turn greatly affects the mechanical durability, corrosive susceptibility, and thermal properties of the final part. The processing parameters of metal AM generally consist of power and speed, as well as the properties of the feedstock material. These processing parameters are tunable and can be optimized for the desired performance characteristics of the final part.

The objective of this project is to establish a correlation between component performance and the processing parameters and precursor materials, through the characterization of the material as it relates to experimental evaluation of the mechanical, corrosive, and thermal properties. The ultimate goal is to establish a link between our fundamental understanding of the constitutive material processing principles and the applied component-level engineering parameters, thus enabling the industry to further unlock the potential of AM technology.

\section{MISSION RELEVANCE}

The project is relevant to DOE's missions in basic science and energy efficiency. Additive manufacturing enables a vast number of design possibilities for componentry for a range of energy, transportation, and other applications that can lead to significant improvements in energy efficiency and material waste reduction. Currently, AM is limited by its issue with controlling and limiting defect formation during the process, in addition to the limits to the materials that can be used in AM processes. These limitations restrict the use of this technology in the above-mentioned applications.

\section{RESULTS AND ACCOMPLISHMENTS}

During this phase of the project, we have focused on characterizing the feedstock metal powders that will be used to print the experimental samples for model validation. We selected the alloy we will focus on first, namely, the titanium alloy Ti-6Al-4Al (Ti 6-4). We also selected the Inconel nickel-chromium-based superalloy as a metal for future consideration. The characteristics of the powders will be used to help identify the material properties for the integrated computational materials engineering (ICME) modeling and any discrepancies in the resulting builds. Two different grades of Ti 6-4 powders were acquired from a commercial source. These powders were characterized for particle size, morphology, microstructure, and elemental analysis. Figure 1 shows an X-ray tomography image of a sample of powder where shape, size, and porosity can be observed. These characteristics will be accounted for in the computational model.

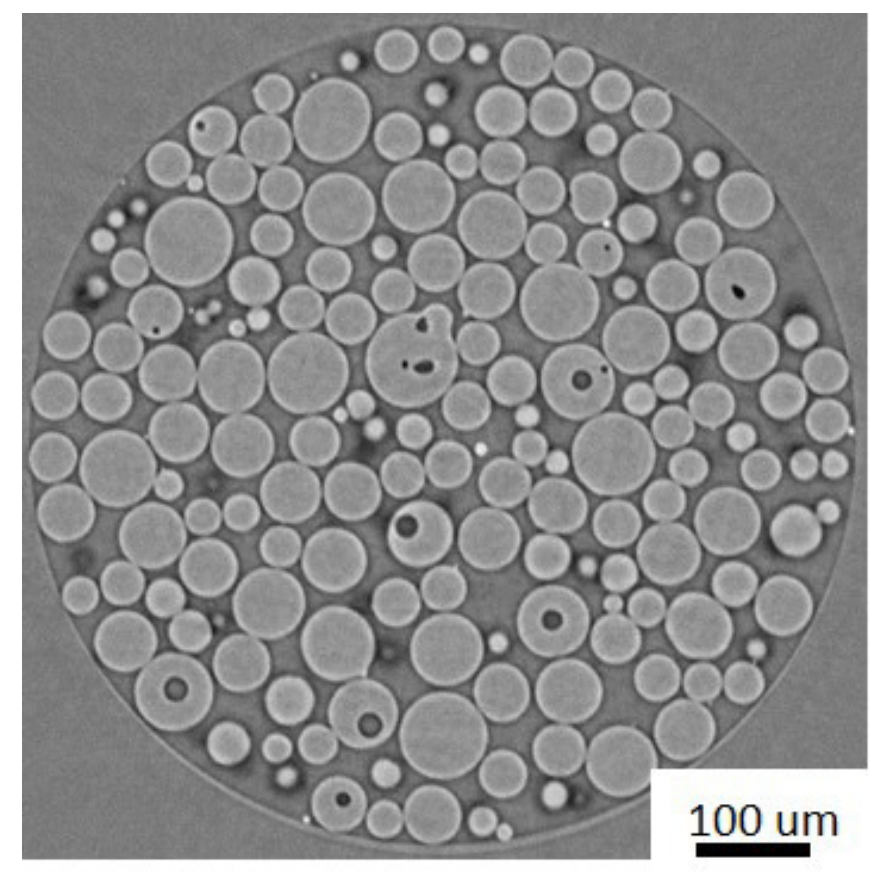

Figure 1. X-ray tomography single cross-sectional image of Ti 6-4 feedstock powder showing embedded porosity in the powder.

\section{PROPOSED FUTURE WORK}

An outcome of this effort is the development of a general methodology that can be used to extract the relevant information required to simulate the identified AM process for a specific alloy, and to evaluate the impacts that an individual process parameter would have on the end product. The characterization of the material and performance characteristics will feed into the existing ICME model, thus providing points of validation of the model. This methodology will also help in advancing this new AM technology by providing a tool that can enable the adoption of added features like graded material designs, new alloy considerations, and enhancement of the closed-loop control of the process. 


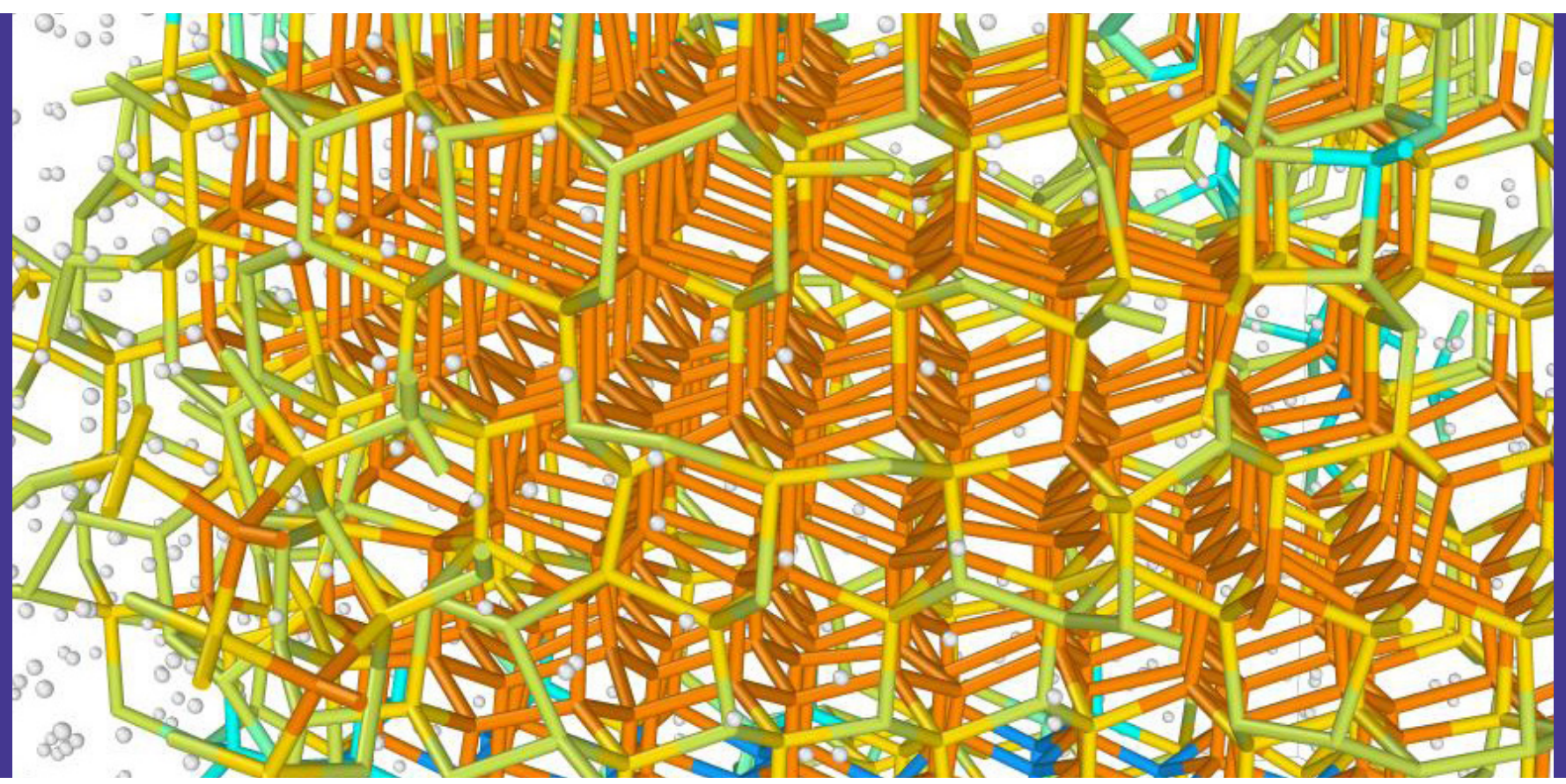

\section{LDRD PRIME -}

BIOLOGICAL AND

ENVIRONMENTAL

SCIENCE CAPABILITY

DEVELOPMENT 


\section{Genome Engineering of Environmental $P$. fluorescens to Investigate Bacterial Interactions with Plants and Other Microbes}

\author{
2015-171-R2 \\ Philippe Noirot, Gyorgy Babnigg, Christopher Henry, \\ and Marie-Francoise Gros
}

\section{PROJECT DESCRIPTION}

This project aims to advance genome engineering approaches for environmental Pseudomonas fluorescens strains, which are bacteria that associate with fungi and roots and provide benefits to plants. Although methods are currently available to modify the genome of environmental Pseudomonas, they need to be tailored for each particular strain, rendering systematic studies of the mechanisms by which these bacteria provide benefits to plants slow and labor intensive. The genome engineering tools that we are developing work across the $P$. fluorescens group, enabling the use of a single design strategy across strains and thus helping to bolster high-throughput approaches to understand the molecular mechanisms underpinning plant growth promotion (PGP).

\section{MISSION RELEVANCE}

The project has direct relevance to components of the DOE Office of Biological and Environmental Research's (BER's) Genomic Science program, specifically by addressing the first objective, "determine the molecular mechanisms, regulatory elements, and integrated networks needed to understand genome-scale functional properties of biological systems." In addition, it directly addresses two "Decadal Thrusts" from BER's Molecular Science Challenges (April 2015): "elucidate mechanisms by which plants benefit from microbiomes" and "characterize signaling or communication pathways between microbiomes and plants."

\section{RESULTS AND ACCOMPLISHMENTS}

We developed an engineering approach allowing the homologous replacement of any target sequence in the $P$. fluorescens genome by a polymerase chain reaction (PCR)-generated deoxyribonucleic acid (DNA) fragment. This approach allows the simple design of mutants and expression reporters. It was used to label four phylogenetically distinct strains of $P$. fluorescens with fluorescent protein (FP) reporters. These FP-labeled strains were used to study the colonization of the roots of aspen seedlings in a laboratory system (Figure 1). Our engineering approach was also used to validate the functions of predicted transporter systems and to study transcription regulators of sulfur utilization (two manuscripts in preparation).
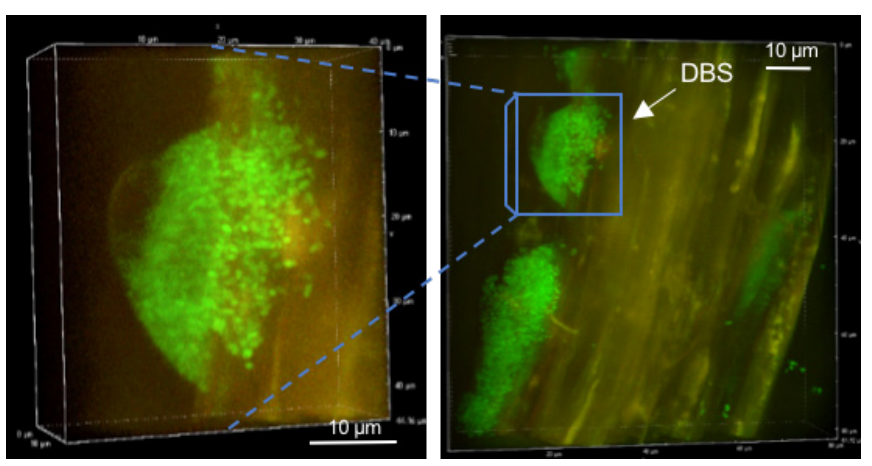

Figure 1. Colonization patterns of $P$. fluorescens strain WH6 at non-mycorrhizal aspen roots. Three-dimensional (3-D) reconstruction of bacterial biofilms at the root surface using confocal microscopy. Beehive-like structures formed by WH6 cells (green) on plant tissues were visualized using red auto-fluorescence. Scale is indicated by a white bar.

Our engineering approach leaves a selection marker in the chromosome, limiting the number of changes that can be combined in a single genome. To overcome this problem, we adapted the Clustered Regularly Interspaced Short Palindromic Repeats (CRISPRs) system to

$P$. fluorescens. We found that a CRISPR-directed cleavage of the genomic DNA leads to efficient cell killing, thus enabling the use of CRISPR as a potent counter-selection system to evict a selection marker from the genome. We are currently optimizing the CRISPR-directed cleavage and combining our engineering approach with the CRISPR counter-selection. This effort will result in tools to edit $P$. fluorescens genomes at multiple sites, enabling model-driven engineering of plant growth promotion activities in phylogenetically diverse $P$. fluorescens.

Another flavor of CRISPR, termed CRISPR interference (CRISPRi), can be used to control gene expression. CRISPRi uses a catalytically deficient Cas9 enzyme (dCas9) that will bind its target site and act as a transcriptional roadblock to downregulate genes. Preliminary assays targeting $P$. fluorescens genes with well-characterized phenotypes demonstrated that CRISPRi was a powerful approach to link the phenotype with the genotype.

\section{PROPOSED FUTURE WORK}

This project concluded in FY 2017. Application of gene editing tools to interrogate mechanisms of plant-bacteria interactions will be proposed to DOE BER. 


\section{Illuminating Linkages between Microbial Diversity and Biogeochemical Cycling in a Redox Dynamic Environment}

\author{
2015-179-R2 \\ Theodore Flynn, Jack Gilbert, Kenneth Kemner, \\ Edward O'Loughlin, and Pamela Weisenhorn
}

\section{PROJECT DESCRIPTION}

Environments such as floodplains and wetlands, which exist at the terrestrial-aquatic interface, can be both major sources and sinks of greenhouse gases. To a large extent, the flux of methane from these environments is controlled by microbial metabolism. Both methane-producing and methane-consuming organisms inhabit the water and sediments there, although the extent to which the activity of each is controlled by environmental variables (e.g., pH, temperature) is poorly understood. In particular, the dynamic biogeochemical conditions of these environments are thought to cause large shifts in both the rates of greenhouse gas emissions and, concomitantly, the structure of microbial communities.

As a result, the flux of methane from periodically flooded environments such as wetlands is a significant source of uncertainty in global climate. Our project seeks to understand the microbial processes that regulate greenhouse gas emissions in a wetland environment at spatial and temporal scales relevant to earth system modelers. We are conducting a rigorous effort involving high-frequency field sampling as well as laboratory microcosms to measure relevant biogeochemical parameters (e.g., concentrations of dissolved gases in water and sediment) and to determine the composition of the microbial community by using targeted gene sequencing and metagenomics. This effort not only improves our understanding of the feedback between microbial metabolism and greenhouse gas emissions, but also provides us with a more quantitative understanding of microbial ecology by monitoring changes in native microbial communities over space and time.

\section{MISSION RELEVANCE}

This project supports DOE's environmental quality mission. We use omics-enabled techniques to uncover basic organizing principles of biological systems in a freshwater wetland, and to learn how these principles relate to the emission of greenhouse gases such as carbon dioxide and methane in this critical environment.
We also conduct laboratory experiments to test specific hypotheses on the impact of ferric iron and organic electron shuttles on microbial methanogenesis. Combining systems biology with detailed geochemical measurements provide key variables to inform improved models of the critical biogeochemical processes that control the storage, release, and transport of carbon, nutrients, and contaminants in the critical zone, which are all critical mission challenges identified by DOE's Biological and Environmental Research program.

\section{RESULTS AND ACCOMPLISHMENTS}

We have continued high-frequency sampling of microbial communities and system biogeochemistry (e.g., methane) in the Argonne site wetland, with over 60 sampling events spanning two years. Analyses indicate that microbial communities in wetland water are significantly more variable than those in wetland sediments. Sediment microcosm experiments amended with ferric iron minerals show that the presence of organic electron-shuttling compounds affects not only the diversity and composition of the microbial community, but also the rate and extent of both iron reduction and methanogenesis. Additional microcosms were conducted where ferric iron minerals were added following the onset of methanogenesis. The results show that the production of methane is directly inhibited by the addition of ferric iron, and that the degree of inhibition is greater in less crystalline minerals. These results have been presented at several national meetings (the American Geophysical Union Annual Meeting, the spring meeting of the American Chemical Society, and the Goldschmidt geochemistry conference) and at the University of Chicago.

\section{PROPOSED FUTURE WORK}

Using microbial community samples from the wetland itself and from laboratory microcosms, we will sequence whole-genome shotgun metagenomes to better understand community dynamics at the genome-level. Led by Dr. Pamela Weisenhorn, we will construct network models of wetland time-series data to provide a further quantitative understanding of interactivity between microbial populations over time and under different biogeochemical conditions. Additional experiments investigating the impact of electron shuttles on isotopic fractionation during methanogenesis, the impact of saline groundwater discharge on microbial communities and water quality in surface wetlands, and other related topics will continue with new collaborators at Dartmouth College, the University of Illinois, and Kansas State University. 


\section{Functional Analysis of Proteins from a Key Signaling Network Involved in Plant-Growth-Promoting Bacteria}

\author{
2015-180-R2
}

Marie-Francoise Gros

\section{PROJECT DESCRIPTION}

Plant growth-promoting (PGP) rhizobacteria achieve their beneficial effects through direct and indirect interactions with plant roots. The molecular mechanisms underlying PGP effects must involve a capacity of rhizobacteria to decipher environmental stimuli and transduce signals to trigger PGP pathways. However, the protein complexes involved in these signaling pathways are poorly characterized.

In most bacterial species, the second messenger cyclic diguanylate (c-di-GMP) has emerged as a ubiquitous regulator of bacterial physiology, motility, and behavior. To understand the molecular logic underlying c-di-GMP regulatory circuitry in PGP bacteria, we are characterizing the protein-protein interaction network underpinning the bacterial response to c-di-GMP, which directs plant-root colonization in Pseudomonas fluorescens. This study combines a targeted interactome to identify protein complexes involved in c-di-GMP regulation with a systematic interrogation of phenotypes using the Clustered Regularly Interspaced Short Palindromic Repeats_interference (CRISPRi) system. Functional analysis will provide mechanistic insight into the basic principles of plant-root colonization by rhizobacteria.

\section{MISSION RELEVANCE}

This project is relevant to "Small World" and Environmental Sensing and Response - Scientific Focus Area (SFA) projects under DOE's Biological Systems Science Division, Office of Biological and Environmental Research, Office of Science, and offers a unique opportunity to interface functional and structural studies. This interdisciplinary project brings new capabilities in protein science and CRISPR-based genetic engineering to Argonne and leverages existing transcriptomics, genome engineering, and imaging capabilities developed in the Biosciences Division.

\section{RESULTS AND ACCOMPLISHMENTS}

A high-quality protein-protein interaction (PPI) map centered on c-di-GMP signaling in P. fluorescens was built from very-high-confidence interactions (Figure 1). This network is composed of 94 proteins connected by 130 interactions clustered in connected functional modules. The map reveals functional associations connecting the c-di-GMP binding proteins to metabolic pathways, and cellular machineries relevant to plant-root interactions, such as cell signaling, transcriptional regulations, cell adhesion, and transport of various nutrients.
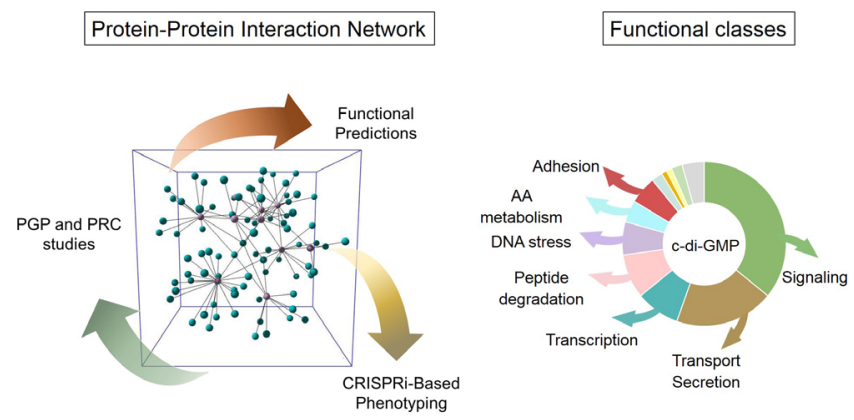

Figure 1. Protein-protein interaction network centered on c-di-GMP signaling in the PGP rhizobacteria, $P$. fluorescens, and strategy for the functional characterization of the protein complexes. (Left) Proteins are nodes (purple $=$ POI; blue $=$ interacting partner) and interactions are edges . (Right) Different functional classes are indicated by color.

The CRISPRi system is a ribonucleic acid-based method widely used in various organisms to repress the transcription of targeted genes. We purposely adapted a CRISPRi system in $P$. fluorescens to perform extensive phenotyping upon down-regulation of the c-di-GMP genes from the network. We validated this approach to study $P$. fluorescens motility and biofilm phenotypes for an extended period (up to 72 hours) and discovered novel genes from the PPI network that affect biofilm formation. This validation opens the way to a larger scale analysis of the phenotypes caused by knockdown of network proteins.

\section{PROPOSED FUTURE WORK}

We will complete the larger-scale analysis of phenotypes caused by CRISPRi knockdowns. Then, we will focus more detailed functional studies (e.g., subcellular localization of proteins) on selected protein candidates from the PPI network to understand the role of c-di-GMP signaling in biofilm formation, motility, and colonization of roots by $P$. fluorescens. We will continue to develop our genetic tools toward the goals of high-throughput functional genomics and phenotyping, with the future objective of studying Pseudomonas within multispecies communities. 


\section{Models to Observations, a Digital Atmospheric Library (MODAL)}

\author{
2016-139-R1 \\ Scott Collis, Yan Feng, Virendra Ghate, Nicki Hickmon, \\ and Robert Jacob
}

\section{PROJECT DESCRIPTION}

The aim of MODAL is to model atmospheric scenes using ultra-high-resolution atmospheric models ( 10 m), known as large eddy scale (LES) models, in order to assess the impact of environmental sampling on measurement.

The major tasks were to:

1. Develop an LES modeling framework for Argonne, leveraging large scale computing in the Argonne Leadership Computing Facility (ALCF) and the Laboratory Computing Resource Center (LCRC).

2. Apply the framework to specific examples of atmospheric phenomena to aid the understanding of the spatial and temporal scales of the phenomena.

The specific problem addressed was understanding the impact of an island in the eastern northern Atlantic on climatologies of cloud fraction (i.e., percentage of the sky covered by clouds). DOE's Atmospheric Radiation Measurement (ARM) Climate Research Facility maintains a site on Graciosa Island in the Azores archipelago to understand the climate impacts of low-altitude clouds over the ocean. Although small, the island itself can affect cloud cover. The MODAL framework is being used to address this question: does the choice of site location bias the observed cloud climatologies?

\section{MISSION RELEVANCE}

This project supports DOE environmental missions. The build of the MODAL infrastructure, including the high-resolution framework on ALCF, directly assists DOE ARM in making decisions on instrument placement and places instrument results into context.

\section{RESULTS AND ACCOMPLISHMENTS}

The LES modelling component of MODAL is fully implemented on Bebop (LCRC) and Mira (ALCF). The Cloud Model 1 (CM1) was chosen because it scaled well on both architectures. Terrain for Graciosa (shown in Figure 1) was implemented, impacting both flow and radiation (i.e., impact of the land surface albedo at visible wavelengths), and sub-100-m resolution simulations have been performed.

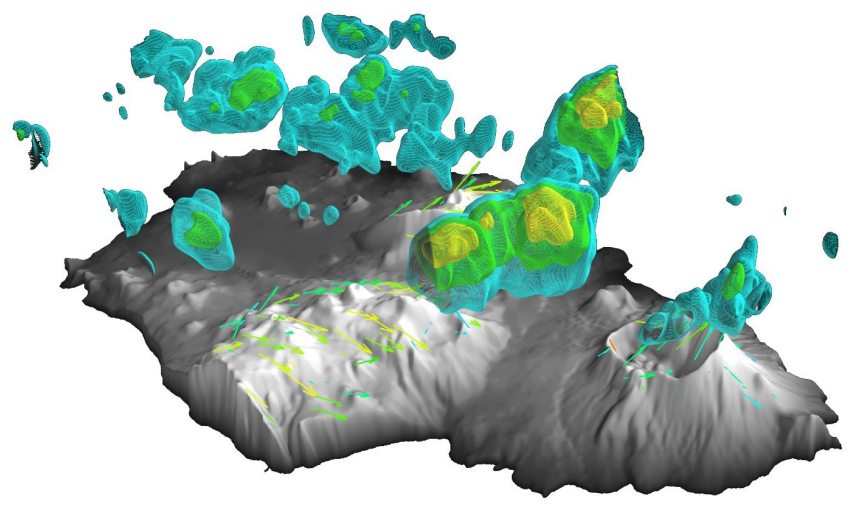

Figure 1. Terrain of Graciosa Island and isosurfaces of CM1 simulated cloudwater content.

The implementation included the design of a stretched grid that varied from coarse resolution ( $10 \mathrm{~km})$ at the edge to LES scale ( $10 \mathrm{~m}$ ) over and around the island. This treatment allowed a seamless coupling from the local clouds of interest and the larger-scale fields. It also allowed more efficient numerics and a quicker spin-up time (the time it takes for the model to achieve steady state).

K-means clustering was used to determine bulk atmospheric states (i.e., "typical" weather patterns for the island). CM1-MODAL was then run over hundreds of these atmospheric states, and the fraction of the sky over the ARM site containing clouds (as seen by a cloud radar) was calculated. The strength of MODAL is that the cloud fractions were calculated both with and without the impact of the island present in the simulation. Simulations have only recently been performed, and analysis is ongoing. Early results point to the island's increasing cloudiness even during on-shore flow periods. Results were presented at the American Geophysical Union meeting in December 2017, and a manuscript is being prepared.

\section{PROPOSED FUTURE WORK}

Work finalizing and reporting the results continues using a variety of funding sources. The investigators are actively pursuing new support to continue and apply the project, including DOE's Office of Energy Efficiency and Renewable Energy (EERE). In addition, MODAL will be used as a downscaling framework for the E3SM climate model over the Azores as part of the DOE's Climate Model Development and Validation program if funding continues in FY 2018. 


\section{Establishing a Proof-of- Concept for Protein Function Discovery Initiative}

\author{
2016-165-R1
}

Philippe Noirot, Dionysios Antonopoulos, Gyorgy Babnigg, Christopher Henry, Kenneth Kemner, Philip Laible, Peter Larsen, Tijana Rajh, and Rosemarie Wilton

\section{PROJECT DESCRIPTION}

Protein function discovery is a scientific problem of enormous scale that can only be tackled by a multidisciplinary strategy integrating experimental and computational approaches, as well as new technological developments to ensure that the strategy can be applied at large scale. Our current capabilities in protein production and characterization at Argonne's Advanced Protein Characterization Facility, our capabilities in computation and gene annotation (KBase), and our recent advances in microfluidics and nanomaterial science at the Center for Nanoscale Materials (CNM) place us in a favorable position to develop a global strategy for protein function discovery. This project aims at establishing a proof-of-concept for an integrated experimental and computational approach to the functional characterization of proteins.

\section{MISSION RELEVANCE}

The project is focused on plant-microbe interactions and is relevant to DOE's missions in science and the environment and, in particular, to a scientific theme cited in the DOE Office of Science, Office of Biological and Environmental Research (BER), Biological Systems Science Division strategic plan. Under the BER biosystems design theme, this plan addresses the goal of developing "the fundamental understanding of genome biology needed to design, modify, and optimize plants, microbes, and biomes for beneficial purposes."

\section{RESULTS AND ACCOMPLISHMENTS}

Task 1-Establish MetaboSeq, an approach coupling microfluidic cell-free assays with metabolic profiling for functional analysis

A single quadrupole liquid chromatography-mass spectrometer with a nano-electrospray ionization source was connected to an analog device and a direct sample injection system. A microfluidic chip containing a droplet splitter, a magnetic separator, a droplet sorter, a delay channel, and pneumatic flow control was designed and fabricated. The flow control hardware and software components were installed on a dedicated laptop computer. The droplet splitting and droplet partitioning behavior of the chip was characterized. The flow characteristics of the droplets in the spray needle were also characterized, such that a droplet flow compatible with the desired scan rate in the instrument was achieved.

Task 2-Apply the MetaboSeq pipeline to fill gaps in the metabolic pathways of $\boldsymbol{P}$. fluorescens

Draft models were constructed for $\sim 900$ diverse genomes, including $P$. fluorescens, providing a broader context for gaps in metabolism across all these genomes. More than 150 published metabolic models were imported to facilitate comparison with our draft models. These datasets provide a broader picture of common gaps in the metabolic networks of all microbial genomes. Our cheminformatics method was also developed and deployed in KBase, where it permits the seamless prediction and merging of novel pathways into metabolic models.

Pathways were predicted for indole-3-acetic acid (IAA) synthesis in $P$. fluorescens using model gapfilling. Protein candidates for this pathway were assayed using substrates of the tryptophan-to-IAA conversion pathway and a buffer system compatible with microfluidic droplets (Figure 1). The discovery of this novel pathway demonstrates the high potential of model-driven MetaboSeq for protein function analysis.

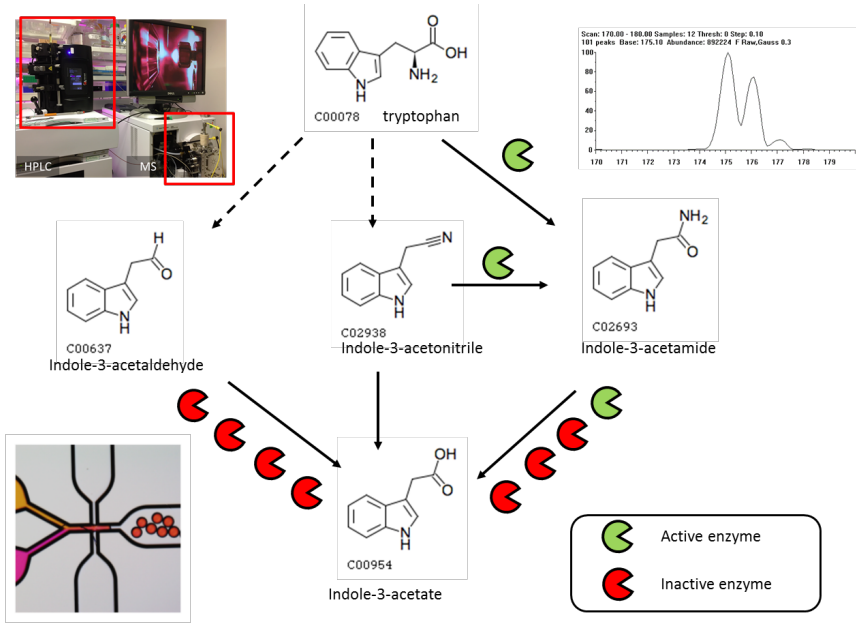

Figure 1. Activity measurements of $P$. fluorescens SBW25 gene products expressed in and purified from $E$. coli.

\section{PROPOSED FUTURE WORK}

Applications of MetaboSeq, coupled with modeling and cheminformatics and involving collaboration with CNM and the University of Chicago, will be proposed to DOE and the National Institutes of Health. 


\section{Integrating Atmospheric, Ecological, and Biogeochemical Monitoring in Wetlands}

\author{
2017-051-NO
}

Kenneth Kemner, Nicki Hickmon, and Edward O'Loughlin

\section{PROJECT DESCRIPTION}

Environments at terrestrial-aquatic interfaces are major sources of uncertainty in current Earth system models. We are developing a high-frequency environmental sampling platform that integrates biogeochemistry and omics-based approaches with atmospheric monitoring capabilities at Argonne to explore the link between the sediment-water microbial biogeochemistry of wetlands and atmospheric greenhouse gases (GHGs).

\section{MISSION RELEVANCE}

Our approach uses genomic information as a foundational basis for attaining a mechanistic and predictive understanding of plant and microbial controls on the flux of GHGs. This embodies key aspects of the mission of DOE-BER's Biological Systems Science Division (BSSD). It also addresses the goal of DOE-BER's Climate and Environmental Sciences Division (CESD) of advancing a fundamental understanding of coupled biogeochemical processes in complex environments to enable systems-level environmental prediction. Finally, it is aligned with new opportunities related to the energy-water nexus.

\section{RESULTS AND ACCOMPLISHMENTS}

Our efforts included the following:

1. Completed initial survey (data) from approximately 35 Argonne wetland sites.

2. The Southern Great Plains Atmospheric Radiation Measurement site was surveyed to identify potential sites.

3. Three focal plant species at Argonne wetland field sites were identified for study: Typha, Phragmites, and Sparganium.

4. Four focal wetlands were identified for study (two for each species; Typha + Phragmites are both in two wetlands; two for Sparganium).
5. First spring survey of pore water from focal wetlands was completed.

6. We completed Argonne wetland site history, and ecological assessments.

7. Chambers for gas analysis (without plants) were made.

8. Cu and Pt braising for construction of electrodes for redox measurement began.

9. Made iron X-ray absorption fine structure spectroscopy measurements of Argonne wetland sediments at the Advanced Photon Source. We identified reduced iron complexed mainly within the crystal structures of clays.

Additional supporting activities included, engaging at half-time wetland biogeochemist, Dr. Pamela Weisenhorn; completing the paperwork and safety analysis for work in Argonne wetlands; visiting the Smithsonian Environmental Research Center; hosting three visitors/speakers (Drs. Loretta Battaglia, Kewei Yu, and Pat Megonigal); and beginning development of a DOE Science Focus Area Science Plan.

\section{PROPOSED FUTURE WORK}

Our LDRD funded work was completed in FY 2017. The DOE Office of Biological and Environmental Research (BER) identified environments at terrestrial-aquatic interfaces (e.g., wetlands) as critical environments for the development of a new Next Generation Ecosystem Experiment (NGEE) in the next two to four years. We anticipate a DOE call for the NGEE Terrestrial-Aquatic Interface. 


\section{Linking Climate to Water: Implementing a 4-km Regional Climate Model with Hydrologic Model Coupling (WRF-Hydro) Using Argonne's HPC Resources}

\section{7-087-NO}

Rao Kotamarthi, Virendra Ghate, Preeti Malakar, Andrew Orr, Venkatram Vishwanath, Cheng Wang, and Jiali Wang

\section{PROJECT DESCRIPTION}

This project seeks to develop a comprehensive database of hydrologic and hydraulic flow parameters for historical and future projections that correspond to our existing regional-scale climate model database. It will also initiate coupled modeling simulations at convective resolving scales (with 4-km or less grid spacing) for selected cases identified from our existing regional-scale simulations at spatial scales ranging from sub-regional (e.g., Midwest, Great Plains) to the contiguous United States. This effort will result in the creation of a hydrological database that leverages the large database of regional-scale climate model products that we have built over the past few years and will establish the model's machine readiness at the Argonne Leadership Computing Facility (ALCF) and other DOE high-performance computing (HPC) clusters.

\section{MISSION RELEVANCE}

The project is relevant to DOE missions in the environment. This work will provide a fully coupled climate-hydrology model with the means to evaluate feedbacks among the atmosphere, land, and hydrology systems under current and future climate conditions. DOE is interested in furthering hydrologic model development in the context of Energy-Water Nexus science. The capabilities developed here are also relevant to the missions of the U.S. Department of the Interior and the U.S. Department of Defense.

\section{RESULTS AND ACCOMPLISHMENTS}

The proposed work will be performed as three major tasks: (1) running WRF-Hydro offline to explore the potential changes of streamflow in a future climate; (2) develop, enhance, and implement the coupled WRF-Hydro model at $200 \mathrm{~m}$ resolution and WRF model at 4-km on the ALCF HPC clusters; and (3) link an independent tool (PEST) to WRF-Hydro to conduct calibration and uncertainty analysis automatically based on a user-specified parameter range and selected parameter intervals.

In FY 2017, we achieved the following milestones by completing these tasks.

Milestone 1: We generated high-resolution routing grid files using ArcGIS tools over the contiguous United States. These files are at a spatial resolution of $300 \mathrm{~m}$, which is the spatial resolution for the hydrological component we are building for WRF-Hydro simulations.

Milestone 2: We performed a select number of offline simulations over sub-regions to establish the feasibility of using offline WRF-Hydro for climate-scale simulations (Figure 1).
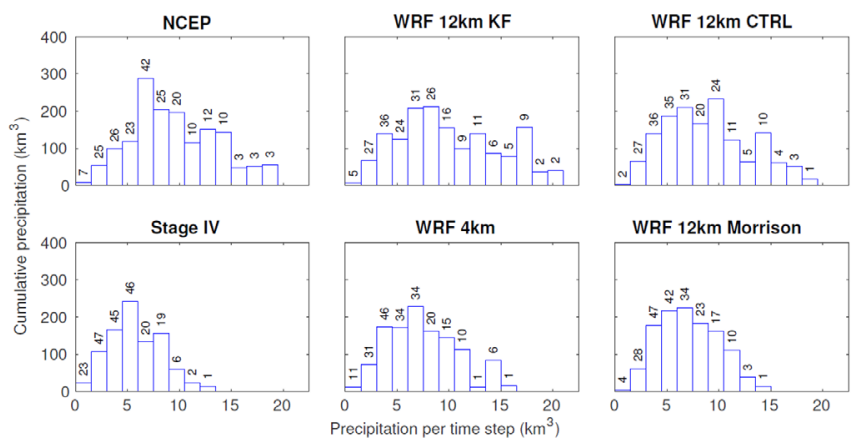

Figure 1. Contribution to total precipitation during summer 2005, classified by total rainfall per 6-hour time step to allow comparison to National Centers for Environmental Protection (NCEP) data. Numbers above each bar give the number of days in each size bin.

Milestone 3: We linked PEST to WRF-Hydro to conduct optimized parameter calibration and uncertainty analysis.

\section{PROPOSED FUTURE WORK}

In FY 2018, we will set up a WRF-Hydro simulation for the North American domain using 12-km WRF output that we generated. We will run the model driven by observations for a year, linking with PEST to obtain a set of parameters that match the observations with the smallest error and the highest previous correlations.

We will conduct a 10-year simulation for each historical and future period over the North American domain at a spatial resolution of $\sim 500 \mathrm{~m}$ for the hydrological component. In addition, we will initiate preparation of an application-oriented technical report to help users understand the methodology of WRF-Hydro and potential issues that need attention. 


\section{LDRD PRIME - HARD X-RAY SCIENCES}




\section{Development of a Compact 352-MHz/12-kW CW Solid State RF Power Amplifier System for Accelerators}

\section{5-147-R2}

Alireza Nassiri, Dave Bromberek, Aditya Goel, Douglas Horan, and Geoff Waldschmidt

\section{PROJECT DESCRIPTION}

This project focuses on exploring the development of high-power density radio frequency (RF) amplifiers operated at 352 megahertz $(\mathrm{MHz})$ utilizing highly efficient and compact solid-state power amplifier technology along with a resonant RF power combing structure. We experimentally explored improving the efficiency of the selected solid-state power devices by dynamic control of their drain voltage to maintain peak DC-to-RF conversion efficiency and minimize the transistor die temperature.

\section{MISSION RELEVANCE}

The DOE mission in science includes improving DOE user facilities. DOE has committed to meeting sustainability goals by, among other initiatives, improving energy efficiency at DOE laboratories. Among the systems with high energy consumption at DOE laboratories are the RF sources that provide RF power to accelerators. Significant energy savings can be realized if high-efficiency alternatives are installed as existing RF sources reach end-of-life or as new facilities are brought on-line. This project aims to address these issues.

\section{RESULTS AND ACCOMPLISHMENTS}

Assembly of the amplifier system has been completed (see Figures 1 and 2). This work included final assembly of the combining cavity, installation of an air blower system to provide some cooling to the cavity interior and tuner piston, and installation of the six 2-kilowatt amplifiers mounted on the common cold plate. Construction of all support electronics, including RF source, channel attenuators, pre-driver/splitter chassis, 100-watt driver amplifiers, RF level detectors, and central PLC controller, has been completed. The support electronics packages were installed in the support frame, along with both drain power supplies, and interconnect wiring was completed.

\section{PROPOSED FUTURE WORK}

This project ended in FY2017.

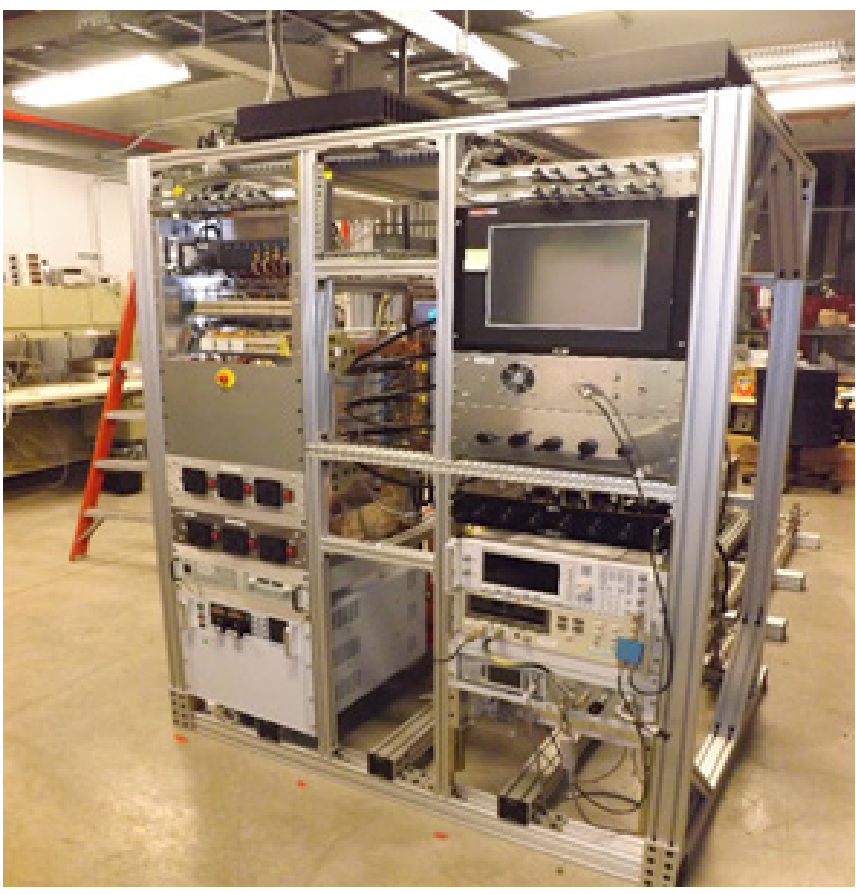

Figure 1. Photo of completed 12-kW amplifier assembly, front view.

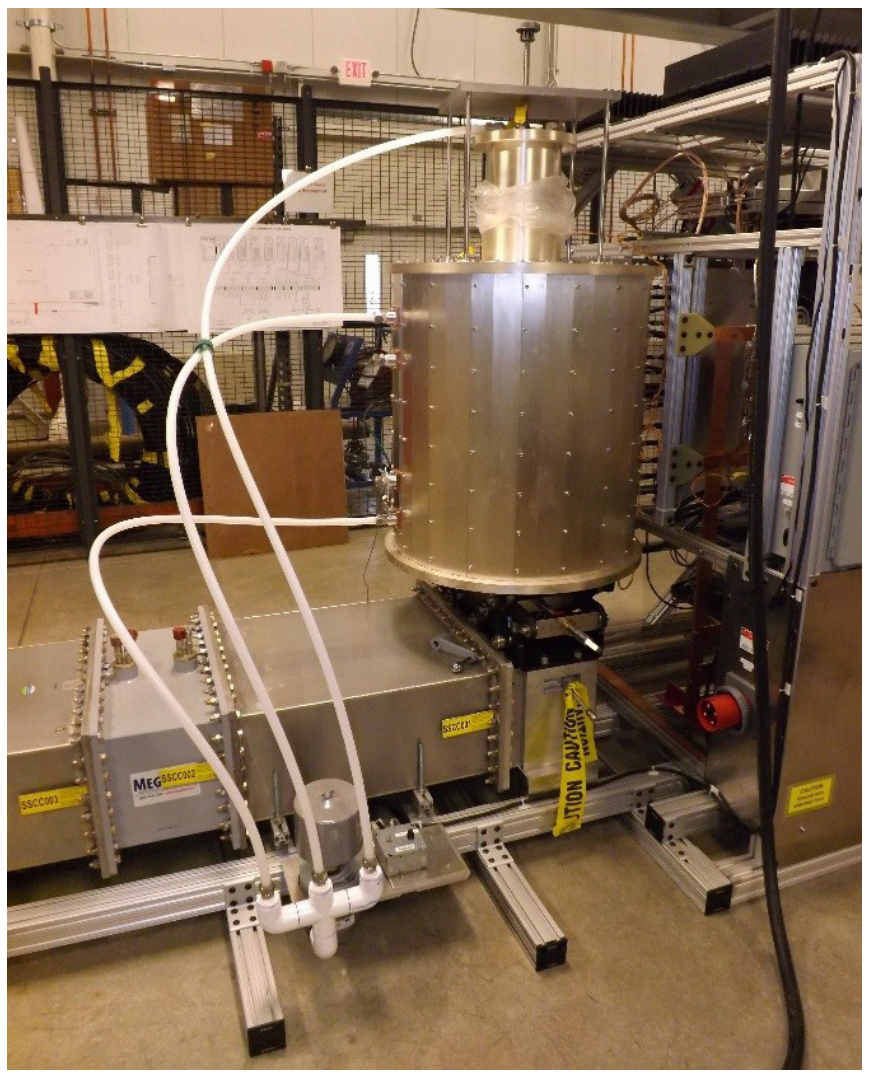

Figure 2. Photo of completed 12-kW amplifier assembly, rear view. 
Unraveling Mesoscale Spatial-Temporal Correlations in Materials Using Coherent X-ray Probes

\author{
2015-150-R2
}

Alec Sandy, Rebecca Bradford, Anthony DiChiara, Eric Dufresne, Zhang Jiang, Alan Kastengren, Meimei Li, Xiao-Min Lin, Timothy Madden, Antonino Miceli, Suresh Narayanan, Paul Nealey, Nicholas Schwarz, Joseph Strzalka, Donald Walko, Jin Wang, John Weizeorick, Haidan Wen, and Hoydoo You

\section{PROJECT DESCRIPTION}

This project is focused on (1) advancing $X$-ray photon correlation spectroscopy (XPCS) to very fast time scales, and (2) creating and deploying the infrastructure necessary to develop coherent surface-scattering imaging (CSSI) beyond its current demonstration state. The overarching goal is to develop coherent X-ray scattering capabilities-well aligned with the proposed Advanced Photon Source Upgrade (APS-U) - that will enhance understanding of the structure and dynamics of condensed matter at the mesoscale.

\section{MISSION RELEVANCE}

This project supports the large investment that DOE-BES is making to upgrade the APS into a 4th-generation light source. It is a first step in creating programs that will lead to full utilization of the increased coherent flux from the proposed APS-U. It will advance coherence-based techniques for surfaces and for measuring fast energy-dissipative dynamics. In addition, this project greatly enhances basic science capabilities that DOE-BES already supports through programmatic funding at national laboratories and universities.

\section{RESULTS AND ACCOMPLISHMENTS}

Two principal accomplishments were obtaining both (1) X-ray area-detector measurements of spontaneous nanoscale fluctuations in a gelling suspension with markedly improved time resolution, and (2) CSSI data with dynamic range far better than achieved previously.

Gelation measurements were performed in collaboration with the Detector group of the AGH University of Science and Technology in Krakow, Poland, using the ultrafast X-ray camera (UFXC) they developed. The detector achieves frame rates that are faster than any other XPCS-suitable detector in the world. As shown by the overlapping correlation functions presented in
Figure 1, obtained after three different quenches, we observed universal behavior in the nanoscale dynamic and rheological properties of nascent gels independent of their detailed formation conditions. The UFXC detector provided an unprecedented view of gel formation. Using this same detector in a newly developed burst mode, we also measured fluctuating speckle patterns at $400-\mathrm{kHz}$ frame rates, demonstrating that the dynamics of nanoparticles in aqueous suspensions can be observed with XPCS.

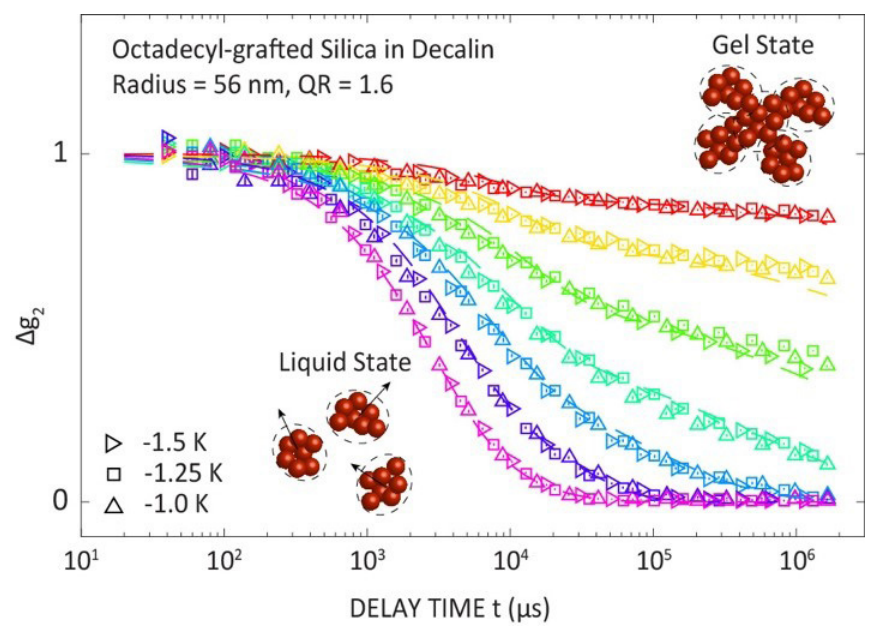

Figure 1. Overlapping correlation functions obtained after different formation times for three quenches into the gel-forming regime of a colloidal suspension. The early- and late-stage microstructures of the gel revealed by our measurements are shown schematically in the lower-left and upper-right corners, respectively, of the figure.

CSSI capabilities were advanced by measuring coherent scattering patterns from model surface structures at the P10 beamline at Petra-III. The dynamic range we obtained in these measurements is 10,000 times greater than has been measured previously. Inversion to real space of single-shot scattering patterns has been performed successfully, indicating that it will be possible with the increased coherent flux provided by APS-U to perform time-resolved CSSI measurements.

\section{PROPOSED FUTURE WORK}

The project ended at the end of FY 2017. In the future, work on this project will continue in two ways. First, with respect to XPCS extended to fast time scales, as part of the collaboration developed with the AGH University Detector group under this LDRD project, we will package the UFXC detector in a stand-alone module so that it can be used for any experiment at our beamline at the APS. With respect to CSSI, a follow-on LDRD project, "Developing Advanced Coherent Surface Scattering Reconstruction Method Incorporating Dynamical Scattering Theory," will further advance this work. 


\section{The Velociprobe: Ultra-High- Resolution Ptychographic Hard X-ray Nanoprobe}

\author{
2015-153-R2
}

Stefan Vogt, Zhonghou Cai, Junjing Deng, Barry Lai, Jörg Maser, Curt Preissner, and Chris Roehrig

\section{PROJECT DESCRIPTION}

The Velociprobe was designed to develop advanced scanning techniques and methods to take full advantage of the tremendously increased focused flux to be gained by the APS Upgrade (APS-U). The goal was to scan a $1-\mu \mathrm{m}$ by $1-\mu \mathrm{m}$ area at sub-10-nm resolution in less than 10 seconds, in atmosphere. The high spatial resolution will be achieved using ptychographic reconstruction techniques. A significant effort was made to enable the instrumentation to perform full high-resolution 3D tomographic imaging.

The Velociprobe concept is enabled by a combination of new concepts in hardware, control algorithms, and scanning approach. A novel air-bearing, guided granite, coarse-positioning stage provides an inherently stable platform as well as the alignment degrees of freedom needed while ensuring excellent mechanical and thermal stability. An in-house developed, field-programmable gate array (FPGA)-based scanning control augments the mechanical stability. This controller provides low-noise, high-bandwidth scanning capability. Key to the scanning control are the laser interferometers to measure zone plate-to-sample position. All of these technologies result in an instrument that can scan at previously unattainable rates (bandwidth), opening up new options for scan trajectories. We expect the resulting performance to meet our stated goals while demonstrating APS-U-ready solutions.

\section{MISSION RELEVANCE}

This project is relevant to DOE missions in science. The Velociprobe serves as the prototype instrument for the PtychoProbe beamline part of the APS-U project, and thus has enabled us to prove instrumentation concepts critical for the APS-U investment.

It also serves as an early instrument for large-scale ptychography of integrated circuitry, a key interest of the nation's intelligence community. See Figure 1. We anticipate that this instrument will pave the way for future work with the Intelligence Advanced Research Projects Activity (IARPA).

\section{RESULTS AND ACCOMPLISHMENTS}

The Velociprobe coarse motion system has been proven to be stable. We have measured the relative vibration between the positions of the X-ray optics and sample to be on the order of $1 \mathrm{~nm}$ in the directions transverse to the beam. This low relative motion helps achieve high resolution in the $\mathrm{X}$-ray images. We have achieved ptychographic imaging on actual samples (e.g., battery materials, photovoltaic materials) down to spatial resolution of $11 \mathrm{~nm}$, as well as demonstrated ptychographic imaging at $400 \mathrm{~Hz}$ on a test sample (data acquisition rate limited by available photon flux).

In addition, APS instrumentation engineers deployed the coarse positioning concepts developed for the Velociprobe in the new sector 32 transmission X-ray microscope, the sector 07 SNOM instrument, and the optics group's in situ Fizeau interferometer support, and are considering them for the APS-U X-ray beam position monitor supports. Other institutions have contacted the Velociprobe team about using the coarse motion concepts.

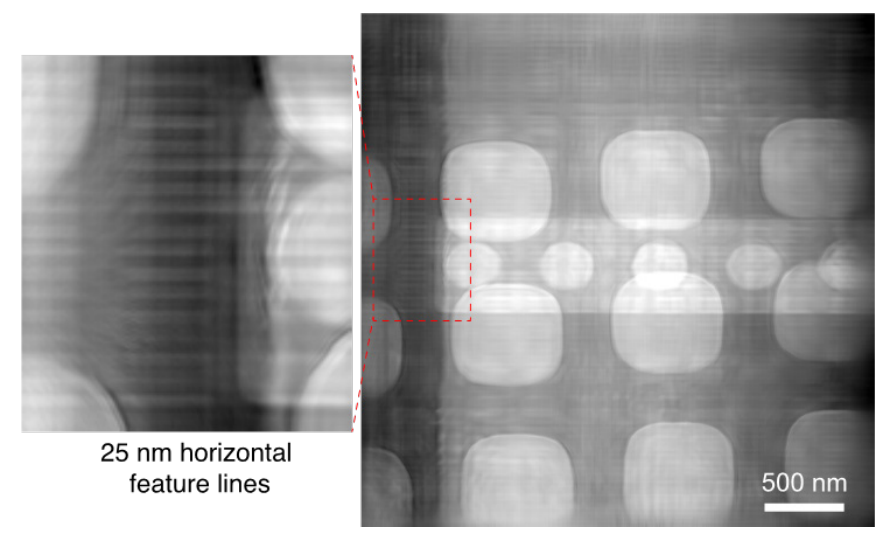

Figure 1. Ptychographic image of an integrated circuit on a $130-\mu \mathrm{m}$ silicon (Si) wafer. The scan was acquired using $10 \mathrm{keV} X$-rays delivered through a double-multilayer monochromator, and the $25-\mathrm{nm}$ circuit features are well resolved.

\section{PROPOSED FUTURE WORK}

The Velociprobe served in many ways as the prototype instrument for the PtychoProbe beamline part of APS-U project, and thus has ensured additional investment into this area. It also serves as an early instrument for large-scale ptychography of integrated circuitry, and has enabled us to become successfully involved in the IARPA/ RAVEN project.

We anticipate that this instrument will help us when applying for future funding from IARPA. 
Ion Beam Figuring with in situ Metrology: Diffraction Limited X-ray Optics and Dynamic Aperture for Three-Dimensional Control of Thin-Film Deposition and Ion-Beam Erosion

\author{
2015-161-R2
}

Ray Conley, Lahsen Assoufid, Mark Erdmann, Kurt Goetze, Elina Kasman, Tim Mooney, Jun Qian, and Bing Shi

\section{PROJECT DESCRIPTION}

This project includes two design efforts: an ion-beam figuring (IBF), in situ metrology component and a dynamic-aperture component. Both efforts leverage the large investment that the Advanced Photon Source (APS) has recently made in a new modular multilayer/ mirror deposition instrument for producing advanced multilayer-based X-ray optical elements. The machine is built around a precision in-vacuum brushless DC servo drive that provides 1-nm position resolution over a travel length of more than $4 \mathrm{~m}$. By integrating this machine with an in situ Fizeau interferometer and ion-milling equipment, the APS may be able to improve the slope error of existing mirrors or produce aspherically figured optics. The combination of ion milling, deposition, and in situ metrology will enable rapid turnaround on mirrors with figure errors appropriate for the APS-Upgrade's (APS-U) multi-bend achromat lattice. At present, such optics are fabricated by cycling between two separate instruments which is slow and introduces mirror repositioning and registration errors. By incorporating an in situ metrology station, the new machine will be uniquely positioned to handle both standard optical materials, such as silicon or glass, and materials that oxidize or form nitrides.

A second goal of this project was to develop dynamically actuated baffle arrays, comprising multiple, identical actuated baffle modules, for real-time control of thin-film deposition and/or ion-beam erosion in three dimensions. A dynamic-aperture (DA) device produces true three-dimensional, thin-film gradients by selectively shadowing, or blocking, part of the deposition flux. By controlling the exact positioning and aperture shape of the deposition flux (or ion beam), a gradient along both axes can be fabricated. Our goal was to demonstrate a reliable model device that can function long-term in an ultra-high-vacuum (UHV) deposition environment.

\section{MISSION RELEVANCE}

This work is relevant to several DOE mission areasnational security, energy, environment, and basic science-and studies at the APS, as it improves the performance of optics deployed at many beamlines at the facility and enables mirrors appropriate for the APS-U. This result will, in turn, improve the performance of all beamlines and benefit all programs relevant to DOE missions that touch the DOE light sources. This work is also relevant to the National Aeronautics and Space Administration's space-based X-ray telescope programs, because the dynamic aperture can be used to correct figure errors of slumped-glass substrates used in Wolter-geometry telescopes.

\section{RESULTS AND ACCOMPLISHMENTS}

FY 2015 and FY 2016

$\square$ Selected and purchased interferometer.

$\square$ Began design and fabrication of single-element DA, Iris, and UHV Gimbal, ion milling apparatus, and support components.

FY 2017

$\square$ Developed multiple miniaturization and packaging technologies and designs for an in-vacuum dynamic-aperture device with 5- $\mathrm{mm}$ pitch and 75- $\mathrm{mm}$ travel length.

$\square$ Developed a radial-bearing-based linear actuator for dynamic-aperture motion.

$\square$ Fabricated a multi-element dynamic-aperture device and energized it for testing.

$\square$ Demonstrated a reliable method for measuring a flat mirror within a vacuum system with a remotely situated interferometer to a root-mean-square error less than $1.5 \mathrm{~nm}$.

$\square$ Tested, and installed a UHV iris for transmission-flat protection.

$\square$ Completed the UHV gimbal with tip/tilt and rotate capabilities for transmission-flat alignment.

$\square$ Designed and procured remaining hardware for interferometry integration into the Modular Deposition System.

\section{PROPOSED FUTURE WORK}

This project ended in FY 2017. We will seek funding to complete the integration and testing of all subsystems into the MDS and begin initial IBF trials. This effort will include developing significant controls and software for firing-solution development from in situ metrology data. 


\section{A Conveyer Belt of Nanoliter to Picoliter Droplets for Hard X-ray Pump-Probe Experiments}

\author{
2016-150-R1
}

Anthony DiChiara, Chris J. Benmore, Bin Hu, Abdul Momen, and Kamlesh Suthar

\section{PROJECT DESCRIPTION}

The goal of this project is to develop a novel sample management system (SMS) by advancing acoustic trapping techniques. Acoustic trapping enables a contact-free sample environment and avoids possible contamination or unwanted nucleation events that may occur at the interface between a solution and container. Our system will provide a high degree of control for designer quantities of precious aqueous/liquid systems such as nanoparticles (NPs), bio-molecules, fuels, and photocatalytic compounds. Our developments include: automatic loading of the acoustic trap using microdispensers, using sound to translate trapped samples in one dimension, using focused and high-frequency sound waves to achieve more stable acoustic traps, and integrating acoustic levitation with small-angle X-ray scattering (SAXS).

\section{MISSION RELEVANCE}

This project supports DOE missions in basic science and discovery. The Advanced Photon Source (APS) at Argonne is unique among third-generation storage rings. Ultra-precise sample control is required as the types of systems being explored become both smaller and more exotic. The SMS here is unique in that it allows unprecedented control of solution samples in a contactfree environment. The device developed during this project will affect multiple disciplines within the APS user community and provide an alternative SMS for laboratory and industrial applications.

\section{RESULTS AND ACCOMPLISHMENTS}

During the first year of this project, our efforts were focused on understanding the noise and instability of acoustically trapped objects. We developed a combination of sound-field modeling and measurement capabilities to visualize the sound profile in ultrasonic resonators used for acoustic trapping. This realization, in turn, allowed us to draw correlations between resonator mode and particle stability. The increased stability was crucial for successfully combining SAXS with acoustic levitation of sub-millimeter droplets. We also demonstrated that acoustic traveling waves could be used to move droplets, and piezo-driven micro-dispensers can load acoustic traps (see inset of Figure 1a).

While instrumentation development continued through FY 2017, we also pursued X-ray experiments that used the developments from the previous fiscal year. In particular, we performed, to the best of our knowledge, the first laser pump X-ray probe experiment in an acoustic levitator (see Figure $1 \mathrm{~b}$ ). These experiments used a collinear pumpprobe geometry with an integrated X-ray collimator for SAXS experiments in an acoustic levitator. The results from a solution of 15-nm gold NPs suggest a cumulative damage mechanism that may be related to the laser removal of ligands.

We also began performing time-resolved small angle scattering (TRSAXS) of in situ chemical synthesis in a contact-free environment. This work leveraged our previous efforts to incorporate micro-dispensers to provide metered amounts of precursor and reagent. Figure 1a shows the results of gold NP formation using the classical citrate method. The experiment demonstrates the ability to perform real-time measurements of a synthesis process using nanoliters of material.
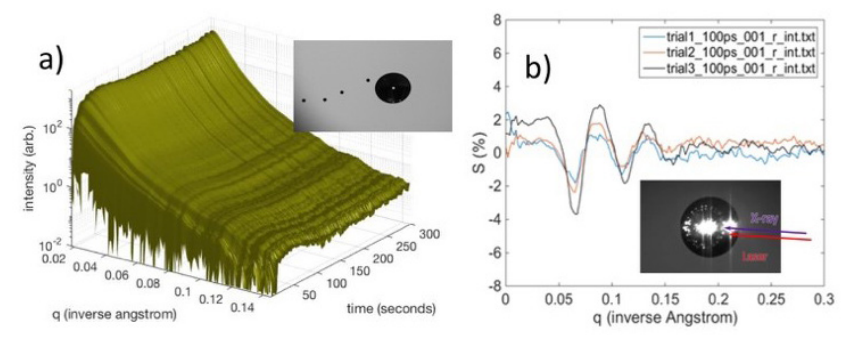

Figure 1. Panel a) shows the TRSAXS gold nanoparticle formation. The inset shows the introduction of a reducing agent into a levitated solution of gold precursor. Panel b) shows the time response of a 15-nm gold nanoparticle solution to laser excitation. The observed signal is consistent with $\sim 5 \%$ particle expansion.

\section{PROPOSED FUTURE WORK}

In FY 2018, we will continue to develop a levitator that employs acoustic lenses and operates at a variety of ultrasonic frequencies. This instrumentation will allow us to tailor the sound environment to the needs of different experiments and hold sample volumes as small as 100 microns in diameter. We will also begin experiments that use a conveyer belt mode of operation, using traveling sound waves to move droplets while continuously loading the trap with fresh samples. This operation will allow us to examine samples that undergo irreversible structural changes from exposure to either laser or X-ray beams. 


\section{Universal Superconducting Undulator}

\section{7-028-NO}

Yury Ivanyushenkov, Ethan Anliker, Joel Fuerst, Matt Kasa, and Emil Trakhtenberg

\section{PROJECT DESCRIPTION}

As part of the Advanced Photon Source (APS) Upgrade, the multi-bend achromat provides an opportunity to use a round beam vacuum chamber with a bore as small as $6 \mathrm{~mm}$ due to reduction of the horizontal dimension of the electron beam. This creates a unique opportunity to build and utilize a novel universal undulator: SuperConducting Arbitrarily Polarizing Emitter (SCAPE). As shown in Figure 1, SCAPE employs four planar-like magnetic cores around a cylindrical beam chamber. Because it uses a superconducting wire instead of a normal conductor, it can create a higher magnetic field, which helps it to generate higher fluxes of photons at higher energies. Existing Advanced Planar Polarized Light Emitted (APPLE)-type undulators cannot compete with SCAPE in terms of the magnetic field strength. The current two-year program aims to design, manufacture, and test a universal superconducting undulator magnet.

\section{MISSION RELEVANCE}

This project supports the development of brighter synchrotron radiation sources-an important part of DOE's mission in basic science. This novel undulator expands the capabilities of existing superconducting undulator technology to generate both circularly and linearly polarized photon beams at high energies and high intensities. As a result, the APS experimental program based on polarization-dependent $\mathrm{X}$-ray absorption and scattering-an essential tool in condensed matter physics to unravel the inner workings of correlated electron systems-will benefit from such a novel undulator.

\section{RESULTS AND ACCOMPLISHMENTS}

In the first year of this two-year project, we modeled the magnetic fields of SCAPE using the Radia software package. We analyzed several core shapes and chose a triangular coil for the design. We created a mechanical design concept for a 500-mm-long SCAPE undulator magnet and then created a detailed design. We fabricated parts of the undulator model shown in Figure 1. We also procured superconducting wire for magnet winding.

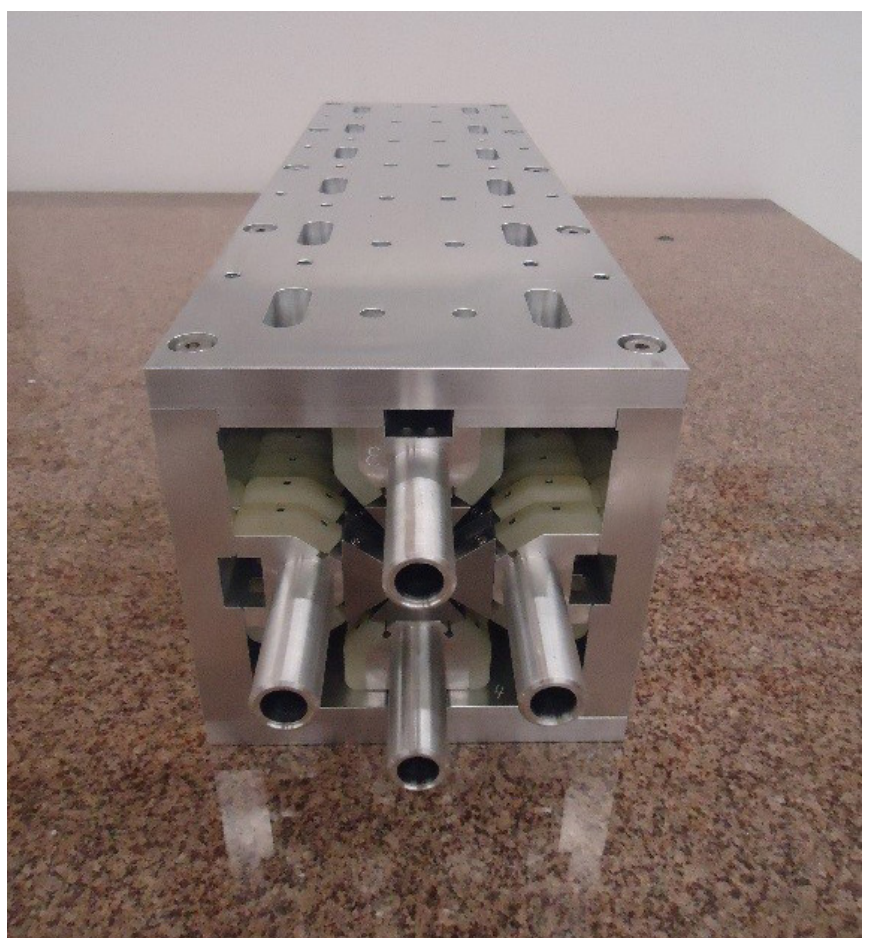

Figure 1. Structure of the SCAPE model. Four magnetic cores are mounted on a strong back structure.

\section{PROPOSED FUTURE WORK}

In the second year of the project, we will finish fabricating the SCAPE mock-up. We will test the undulator in a vertical liquid helium cryostat. 


\section{Developing Advanced Coherent Surface Scattering Reconstruction Method Incorporating Dynamical Scattering Theory}

\author{
2017-073-NO
}

Jin Wang, Wei Jiang, Zhang Jiang, and Tao Sun

\section{PROJECT DESCRIPTION}

At worldwide synchrotron X-ray sources, coherent diffractive imaging, coupled with advanced $X$-ray detectors and computational algorithms, has opened up new research frontiers in the structural sciences that were not attainable previously. Thus far, all image-recovery algorithms have used kinematic theory, assuming that the scattering from the samples is weak (Born approximation, BA). Based on this assumption, the reconstruction is only a mathematical process involving recursive Fast Fourier transform (FFT) and inverse FFT (FFT'-1). These algorithms cannot simply be applied to the coherent surface scattering imaging (CSSI) case where the weak scattering assumption is not satisfied at the grazing angles. The goal of this project is to develop a reconstruction algorithm for reconstructing the coherent scattering data obtained in this unique but powerful way.

\section{MISSION RELEVANCE}

This project is relevant to DOE's mission in basic science. Much progress has been made in the development of hard X-ray sources, resulting in significant brightness and coherence increases. Taking advantage of the high source coherence of the upgraded Advanced Photon Source, the CSSI technique will provide the ideal tools to directly observe surface/interface structures and their dynamics in responding to external stimuli.

\section{RESULTS AND ACCOMPLISHMENTS}

We developed a generalized distorted-wave BA (DWBA) theory for the calculation of coherent scattering from supported objects and embedded objects based on a similar multilayer formalism. To demonstrate the effect of the DWBA algorithm directly, we designed and performed a 1D X-ray fluorescence holography experiment (Figure 1a). The samples consisted of a monolayer of gold (Au) nanoparticles sandwiched between two ultrathin polymer layers. At an incident angle slightly above the film critical angle, one area detector recorded a conventional grazing incidence small angle X-ray scattering pattern in the forward direction, and the other detector, $90^{\circ}$ from the incident beam direction, recorded spatially dispersive gold X-ray fluorescence data. As seen in Figures $1 \mathrm{~b}$ and 1c, the fluorescence signal is highly modulated spatially. Currently, the complex phase term can be modeled to yield the position of the gold monolayer in the overall electron density profile (Figure 1d) of the sample. To demonstrate the sensitivity of the phase to the sample morphology, when we annealed the sample at above the glass transition temperature of the polymer PtBA $\left(90^{\circ} \mathrm{C}\right)$, a drastic change in the fluorescence image was observed (Figures 1e and 1f) due to the diffusion of the Au layer. Indeed, the electron density fitting revealed a wide but lower distribution of the Au layer, as shown in Figure $1 \mathrm{~g}$.
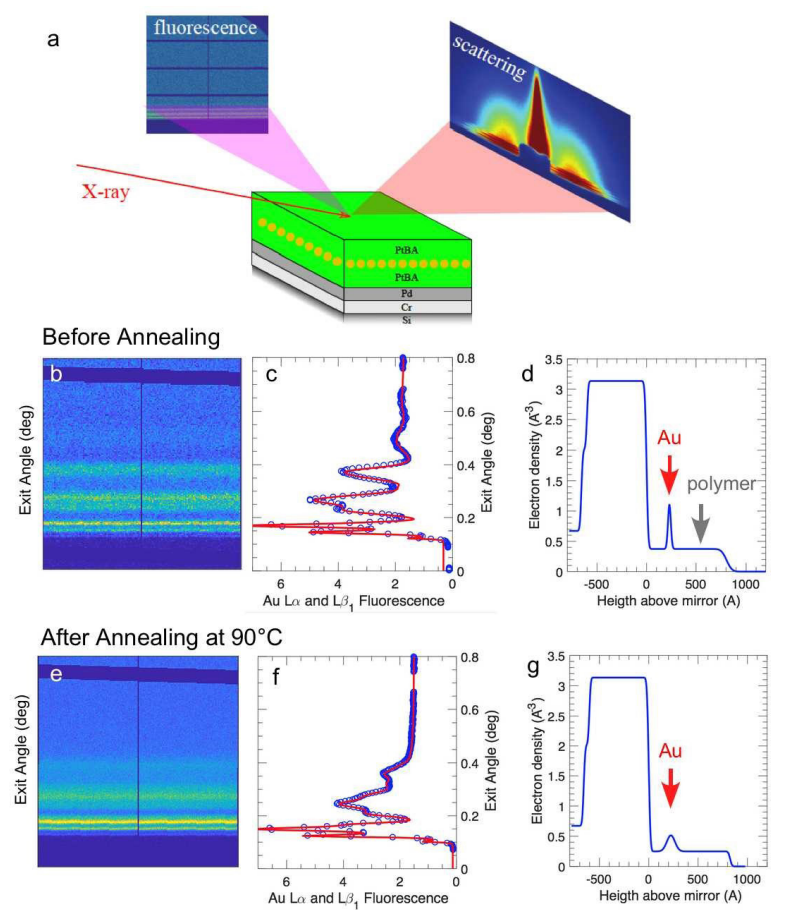

Figure 1. X-ray fluorescence and scattering experiment to demonstrate the dynamical effect at grazing incidence geometry before and after the sample was annealed at $90^{\circ} \mathrm{C}$. (a) Sample, detector layout, and scattering and measurement geometry. Before annealing: (b) and (c) spatially dispersed fluorescence signal revealing the dynamical scattering effect of the thin-film sample at grazing incidence. (d) Modeled electron density profile that yields the fit (line) to data (open circle) in panel c. After annealing: (e) and (f) spatially dispersed fluorescence signal revealing the changed dynamical scattering effect due to Au diffusion. (g) Modeled electron density profile that yields the fit (line) to data (open circle) in panel $f$, indicating the same amount of Au but in a wider distribution.

\section{PROPOSED FUTURE WORK}

Future work includes continuous development of a DWBA framework specifically for 1D reconstruction of holography in the thin-film geometry and applications in 3D CSSI cases with simple surface model patterns, and the development of a software suite for several constructive algorithms. Ultimately, we plan on applying the methods to patterns with various complexities and selecting the most effective methods for practical CSSI applications likely to be developed at the beamline. 


\section{COHED: Coherence for High-Energy Diffraction}

\section{7-080-NO}

Youssef Nashed and Stephan Hruszkewycz

\section{PROJECT DESCRIPTION}

World-leading capabilities have been developed at the Advanced Photon Source (APS) in high-energy diffraction microscopy (HEDM) and Bragg coherent diffraction imaging (BCDI). HEDM has proven ability to collect detailed strain images at micrometer resolutions (on thousands of grains), whereas BCDI collects nano-scale resolutions (on single grains). Combining these techniques presents tremendous opportunities for multi-scale imaging, but with considerable challenges from the viewpoint of both experimental design and the lack of suitable three-dimensional (3D), coherent imaging, phase-retrieval algorithms. This project addresses the problem of 3D image reconstruction from high-energy coherent X-ray Bragg diffraction patterns. This area remains largely unexplored because of the limited coherence at current high-energy $X$-ray sources and the inability of commonly used phase-retrieval algorithms to handle under-sampled datasets. We aim to overcome this challenge by designing new experimental strategies and reconstruction algorithms that bridge the gap between current BCDI methods and the constraints presented by coherent diffraction at high $\mathrm{X}$-ray energies.

\section{MISSION RELEVANCE}

This project is relevant to DOE's mission in basic science. A revolutionary capability of the Advanced Photon Source Upgrade (APS-U) will be the application of 3D strain-sensitive BCDI of nanometer and micrometer crystalline volumes using highly penetrating X-ray energies. This capability, which we term COHED, will have a far-reaching impact in critical areas of materials science and engineering. COHED will be integrated into current HEDM imaging capabilities at the APS and will rely on computational resources at the Argonne Leadership Computing Facility for iterative reconstruction of large-scale datasets. Furthermore, the approach will have an immediate impact at APS-U beamlines such as HEXM, ATOMIC, and CHEX, which emphasize in situ characterization of heterogeneous materials and require the penetrating ability of hard X-rays.

\section{RESULTS AND ACCOMPLISHMENTS}

In the first year of this project, we accomplished two of the three algorithm development objectives and reached one major experimental milestone. Using simulations, we have explored the limit of current state-of-the-art phase-retrieval algorithms under conditions relevant to high-energy BCDI. We have now developed a new data recovery scheme to treat coarsely resolved signals from $B C D I$ experiments in high-energy ranges where current $B C D I$ algorithms are ineffective. High-resolution diffraction patterns can be recovered using multiple signals acquired by in-plane sub-pixel translation of the detector and the relatively new computational tool of compressed sensing. Results of our new method are highlighted in Figure 1.

We have also successfully measured, at the APS, the first coherent diffraction dataset at high energies and are now actively working on analysis.
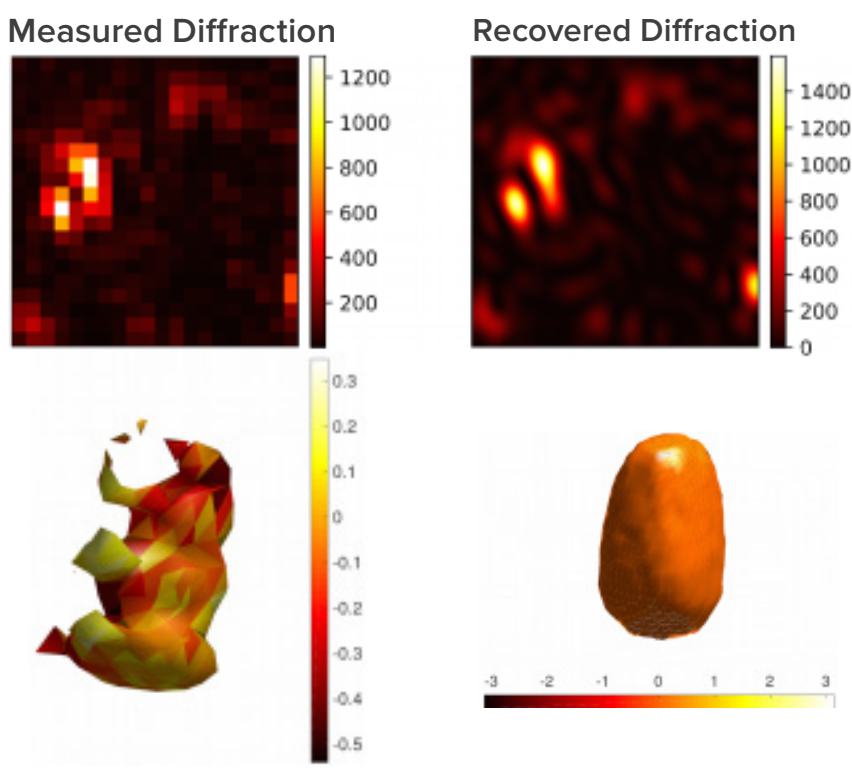

Figure 1. Phase retrieval at $54 \mathrm{KeV}$. (Left) Under-sampled diffraction pattern leading to incorrect 3D reconstruction of the gold nano-crystal sample. (Right) High-resolution diffraction patterns recovered by our method enabling a successful reconstruction.

\section{PROPOSED FUTURE WORK}

The final milestones in this project involve solving prominent challenges in the implementation of Bragg ptychography to polycrystalline materials at high X-ray energies. This goal requires further development and adaptation of phase-retrieval algorithms, for which we will build on our initial progress. This effort will inform proof-of-concept experiments on poly-grained materials. 


\section{Development of a Compact Accelerator for a High-Repetition Rate Free-Electron Laser}

\section{7-091-NO}

Alexander Zholents, Scott Doran, William Jansma, Matt Kasa, Robert Kustom, Alireza Nassiri, John Power, Kamlesh Suthar, Emil Trakhtenberg, and Geoff Waldschmidt

\section{PROJECT DESCRIPTION}

$\mathrm{X}$-ray free electron laser (XFEL) technology is profoundly impacting the $X$-ray science and synchrotron light source communities. Driving the spectacular ascent of XFEL technology is a significant improvement in the production of high-brightness electron beams in normal and superconducting radiofrequency (RF) accelerators. The next breakthrough can be made by seizing the opportunity presented by a new accelerator technology under development for a linear collider that will enable the construction of high-brightness, high-repetition-rate accelerators at potentially a significantly reduced scale and cost. For a compact accelerator, we have chosen to employ and advance a concept of a technology in which the wakefield generated by a leading high-charge drive bunch is used to accelerate a trailing low-charge witness bunch. A practical implementation is a corrugated waveguide surrounded by the focusing channel made as a quadrupole wiggler. The focus of this project is on the in-depth assessment of the feasibility of a novel and far-reaching accelerator technology based on the preliminary design of the compact collinear wakefield accelerator and on modeling and testing its components.

\section{MISSION RELEVANCE}

In line with the DOE science mission of constructing major scientific user facilities, we envision building an X-ray facility with multiple FEL-based beamlines at Argonne; each FEL will have its own designated high-repetition rate compact wakefield accelerator that will be fine-tuned to the specific performance requirements of the beamline. The electron bunches will be fed to each compact accelerator from a common high-repetition-rate, superconducting linac equipped with a high-repetition-rate electron gun.

\section{RESULTS AND ACCOMPLISHMENTS}

In FY 2017, we advanced a theory of a co-linear wakefield accelerator and highlighted intrinsic links between accelerator efficiency and an ultimate accelerating gradient. We analyzed the impact of the beam breakup instability, gained an understanding of means to control it, and defined the maximum accelerating gradient based on practical considerations such as the allowable energy chirp in the incoming drive bunch and the maximum saturation magnetic field at a tip of the quadrupole magnet.

A hybrid quadrupole wiggler was designed with a 3-mm aperture, a period of $8 \mathrm{~cm}$, and a magnetic field gradient of $\pm 0.95 \mathrm{~T} / \mathrm{mm}$. We also prepared a package of detailed drawings for fabrication of a short wiggler containing three periods and fabricated a model of one wiggler period.

We developed a pulsed wire magnetic measurement technique and demonstrated a sub-micrometer resolution in the measurement of the magnetic centers using three ultra-small-size prototype quadrupoles.

The Echo code was adapted for calculations of the wakefield and Green's function for the corrugated waveguide, and we began simulations with the goal of defining the tolerances for waveguide fabrication (see Figure 1).

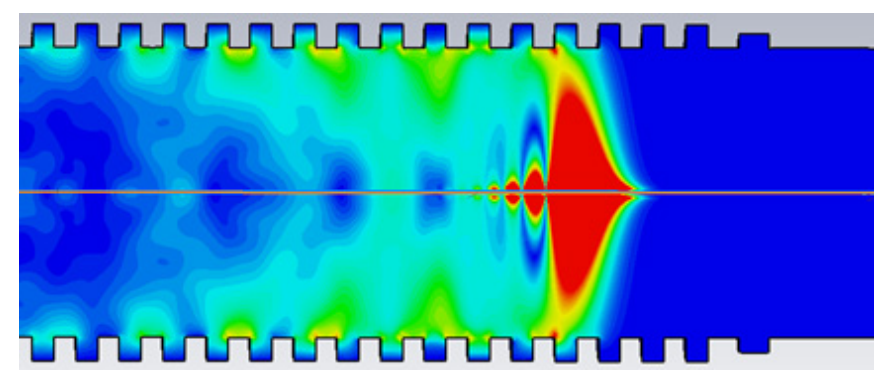

Figure 1. A calculated wakefield induced in the corrugated waveguide by the drive electron bunch.

We devised three methods for fabricating a small-size corrugated waveguide, prepared drawings, and proceeded with the fabrication, obtaining several models.

\section{PROPOSED FUTURE WORK}

In 2018 we will continue theoretical studies and consider the limitation of the accelerating gradient imposed by a beam breakup instability. We will also fabricate the quadrupole wiggler and characterize it, using the pulsed wire magnetic measurement technique, and continue experimenting with the fabrication of the corrugated waveguide. Wakefield simulations will be continued with a focus on determining guidance for tolerances. We will build a model structure operating at $22 \mathrm{GHz}$ frequency and proceed with a beam-based characterization experiment at the Argonne Wakefield Accelerator Facility. 


\section{Soft Matter Visualization and Characterization by Electron Optical Beam Lines}

\author{
2017-153-NO \\ Nestor J. Zaluzec
}

\section{PROJECT DESCRIPTION}

Imaging of materials by electron optical systems is a well established technology for structural characterization at the micro-to-nano-to-atomic scale. The scientific results from such studies have, over many decades, been instrumental in expanding our understanding of important problems in the physical sciences. Technologically important growth areas are expanding from hard (inorganic) materials to soft/hard interfaces as well as purely soft-matter systems. Importantly, today's research agendas are also expanding their focus to understanding the performance and evolution of such substances in native/operando environments rather than in vacuo studies, which have been used extensively in the past. Soft matter investigation at Argonne via electron optical systems is a challenging task that requires resources which are either nonexistent or will benefit from new technological advances yet to be realized. This project aims to determine the limits of Argonne's resources by using traditional characterization of scanning/transmission electron microscopy, as well as by exploring the feasibility of new opportunities presented by advanced technologies such as microanalytical X-ray detectors, pixelated array detectors, in situ environments, and structured probes.

\section{MISSION RELEVANCE}

This project is relevant to DOE missions in basic science. Soft matter involves a broad research community (chemistry, physics, materials) and fields of research at Argonne including:

$\square$ Molecular, colloid, and membrane frameworks.

$\square$ Complex fluids, emulsions, gels, and "amorphous" materials.

$\square$ Organic/inorganic heterostructures such as smart materials for advanced functional systems.

$\square$ Polymeric systems: self-assembly, templated growth.

$\square$ Monolayer/interfacial species.

$\square$ Hybrid/smart nanostructures for intelligent coatings for encapsulation and separation, nano-optics, sensors, liquid crystals, and photo-activated/ enhanced processes. $\square$ Single particles, macromolecular complexes, inter/ intra-cellular processes, and biosystems.

The characterization needs of soft matter research are similar in all of these fields, namely, morphology; crystallography; and elemental, chemical, and electronic structure, and involves the use of imaging, diffraction, ptychography, and spectroscopy applied from the macroscopic to the atomic regime. Electron microscopy has become particularly important for the identification and characterization of individual and/ or heterogeneous nanoscale structures, rather than ensemble averages, which are facilitated by broad-probe techniques. The ability to observe and characterize this regime using electron microscopy at the sub-nanometer level is particularly relevant in single nanoparticle characterization and has become increasing important for molecular studies.

\section{RESULTS AND ACCOMPLISHMENTS}

Prototype hardware for soft matter measurements has been designed, and initial testing of imaging and spectroscopy using differential phase contrast and X-ray microanalysis has been accomplished, an example of which is shown in Figure 1.
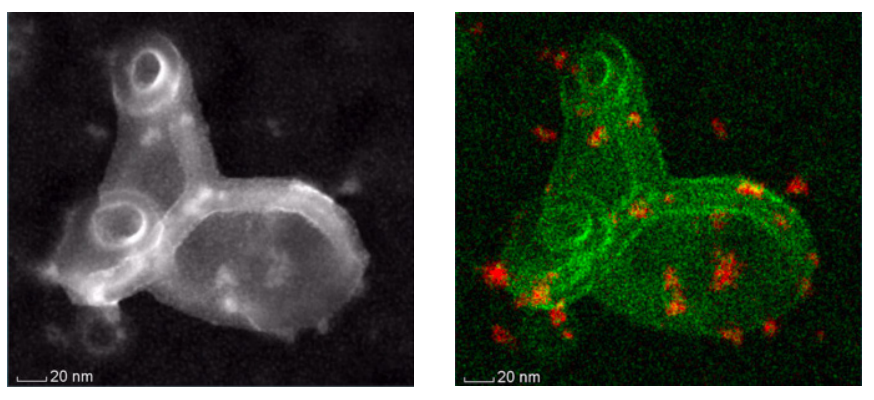

Figure 1. Electron (left) and hyperspectral (right) imaging of an organic membrane, illustrating the site-specific detection of $\mathrm{Zn}$ (green) and $\mathrm{Cu}$ (red) species accumulations.

\section{PROPOSED FUTURE WORK}

In FY 2018, we will:

$\square$ Conduct quantitative measurements of electron scattering and associated secondary signals directed to imaging and elemental characterization of soft-matter in situ gaseous and liquid environments at nanometer-scale resolutions.

$\square$ Investigate new and innovative techniques which combine the use of data mining and X-ray and energy-filtered electron scattering measurements together with position-resolved event spectroscopy and diffraction to determine optimized detectability. 


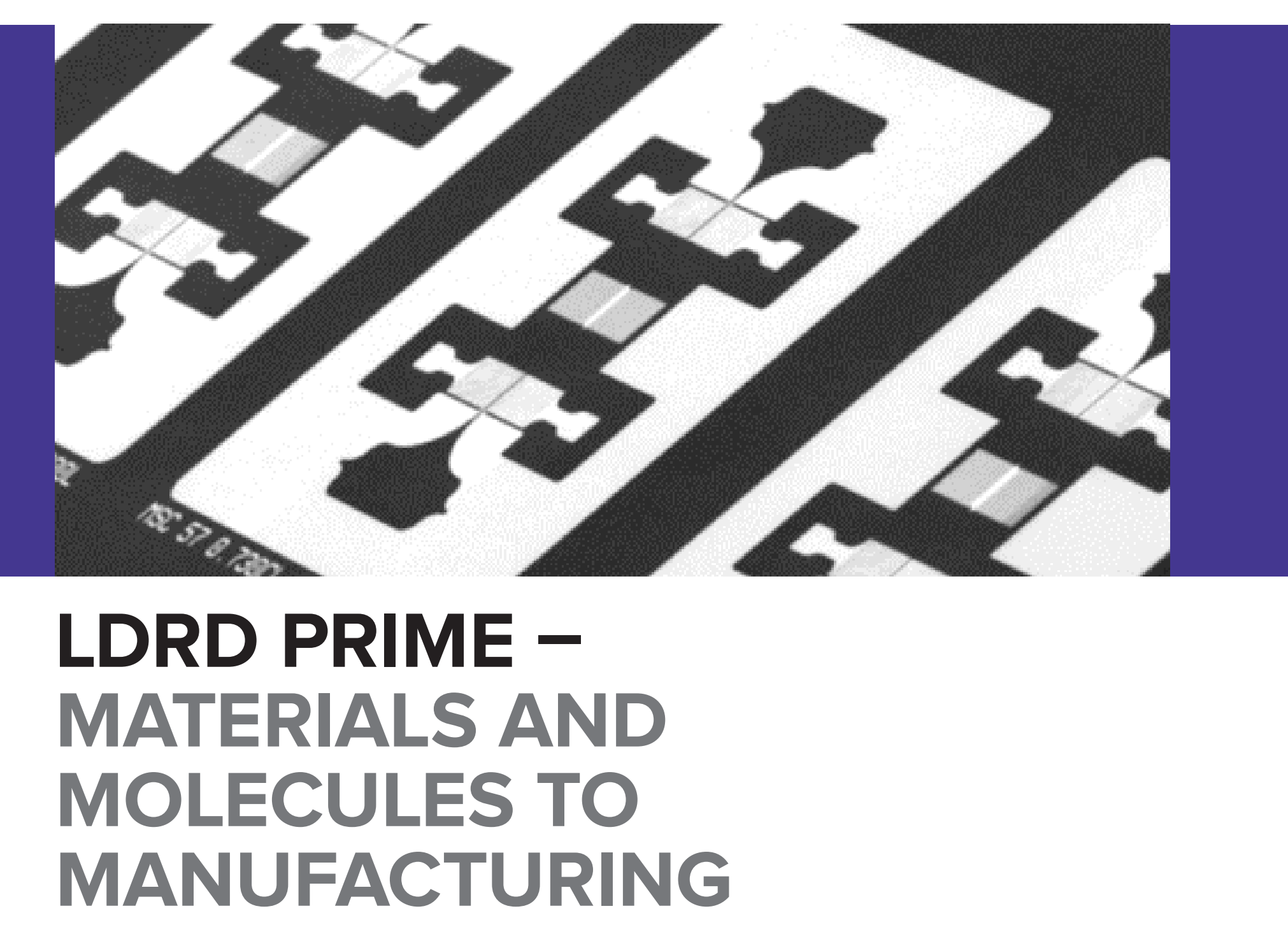




\section{Defect-Localized Spins in Semiconductors for Quantum Optoelectronics}

\author{
2014-191-R3 \\ David Awschalom
}

\section{PROJECT DESCRIPTION}

This project aims to demonstrate fully electronic control over the quantum state of spins that are bound to point defects in silicon carbide ( $\mathrm{SiC})$, gallium nitride ( $\mathrm{GaN}$ ), aluminum nitride (AIN), and diamond. This effort requires (1) the theoretical modeling and subsequent creation of new quantum states, (2) detailed spectroscopic characterization, (3) the implanting of magnetic ions in single crystal structures, (4) study to understand the resulting band structures, and (5) experimental demonstration of the existence and quantum control of these new local quantum states.

\section{MISSION RELEVANCE}

The project is relevant to DOE's missions in science. These fundamental material studies may establish a new device paradigm in which the quantum state of a material can be fluidly interconverted between electronic, magnetic, optical, and vibrational degrees of freedom via the spin of a single point defect. Such capabilities could have profound implications for energy-efficient electronics, high-speed computing (quantum information processing), and basic sensor technologies. The research also provides a pathway to creating single-ion quantum memories for high-density storage technologies.

\section{RESULTS AND ACCOMPLISHMENTS}

We successfully created and characterized new quantum spin states in $\mathrm{SiC}$ and $\mathrm{GaN}$ using chromium (Cr) ions. Specifically, we observed clear optical spin polarization and optically detected magnetic resonance (ODMR) of the $\mathrm{S}=1$ electronic ground state of $\mathrm{Cr}^{4+}$ impurities in $\mathrm{SiC}$ and gallium GaN, with spin coherence and a large (73\%) optical emission for quantum information processing. In contrast to traditional qubit states in diamond and $\mathrm{SiC}$, these new magnetic quantum states have an order of magnitude improvement in optical emission efficiency, thus offering a significant advantage in creating entanglement and single-shot readout protocols in the solid state.

Specifically, we have shown that $\mathrm{Cr}$ ion spins in $\mathrm{SiC}$ and GaN can be directly manipulated with a narrow-linewidth laser tuned to resonate with the first excited state zero phonon line optical transition. The ensemble optical linewidths we observe are similar in magnitude to the zero-field spin splitting of the electronic ground state, which enables ensemble optical spin polarization and measurement at liquid helium temperatures and low magnetic fields. This capability was used to precisely determine the $\mathrm{g}$ factor and zero-field spin splitting of all three spin species, as well as to demonstrate coherent Rabi driving and time-resolved optical spectroscopy of the two $\mathrm{Cr}$ species in $4 \mathrm{H}-\mathrm{SiC}$ (Figure 1).
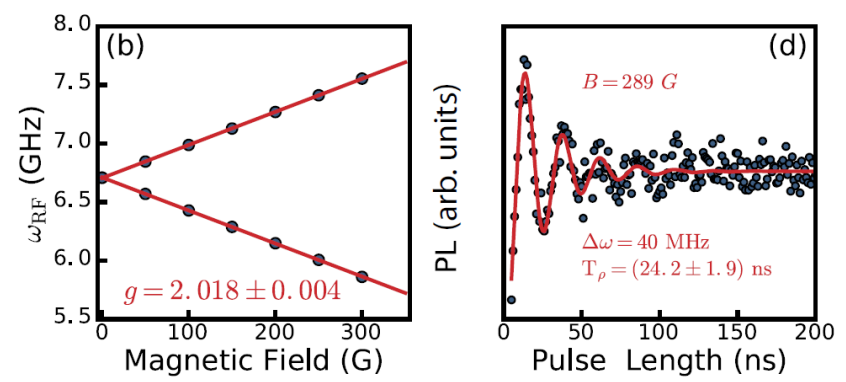

Figure 1. Left: Optically detected magnetic resonance showing $\mathrm{Cr}$-based $\mathbf{S}=1$ spin centers created in $4 \mathrm{H}-\mathrm{SiC}$ with $\mathrm{g}=\mathbf{2}$.018. Right: Rabi oscillations of the $\mathrm{Cr}$ spins at $\mathrm{T}=15 \mathrm{~K}$ and $\mathrm{B}=\mathbf{2 8 9} \mathrm{G}$ and a decoherence time of $\sim 25 \mathrm{~ns}$ (from W.F. Koehl et al., Phys. Rev. B95, 035207 [2017]).

Future improvements in materials quality should improve key optical and spin properties in this class of systems. Improvements in strain homogeneity should result in narrower optical linewidths, which would enable an even higher degree of optical spin polarization than observed here, especially if spin sublevels become fully separated within the optical spectra. In particular, a lower spin density of $\mathrm{Cr}$ ions could extend the coherence observed in this project through the increased isolation of the dopants from one another. It is expected that these times would increase to the millisecond timescale, making them competitive with state-of-the-art, solid state-qubits.

Finally, we note that the knowledge gained in our studies of $\mathrm{Cr}$ ions in $\mathrm{SiC}$ and $\mathrm{GaN}$ is transferrable to other material systems exhibiting comparable optical and spin characteristics. Similar magnetic ions are found in other common semiconductors and remain largely unexplored. These results are likely to be applicable to a range of transition metal ion systems and present exciting opportunities in the ongoing effort to exploit defect-localized spins for explorations in quantum science and engineering.

PROPOSED FUTURE WORK

This project concluded in FY 2017. 


\section{Computational Spectroscopy of Heterogeneous Interfaces}

\section{4-192-R3}

Giulia Galli, Marco Govoni, and Jonathan Skone

\section{PROJECT DESCRIPTION}

This project develops and applies large-scale quantum simulation methods to model, at the microscopic scale, the physical and chemical processes involved in photo-electrochemical (PEC) energy conversion (see Figure 1). The focus is on processes occurring at the interface between solid photoelectrodes and simple electrolytes (water with dissolved salts, acids, and bases). Although primarily applied to PEC, the study is also relevant to other problems of interest in renewable energy applications, including electrical energy storage and solar-to-thermal energy conversion.

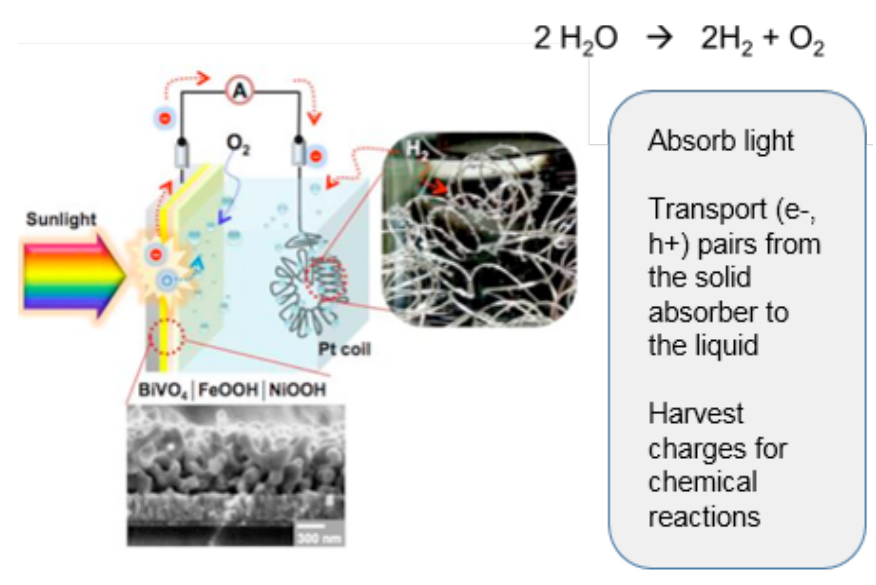

Figure 1. Interfaces and basic processes studied within this project. Interfaces between water and photoanodes are key to understanding water-splitting processes occurring upon light absorption.

This project's methods encompass ab initio molecular dynamics (MD) simulations, to obtain atomic trajectories and compute ensemble averages of thermodynamic properties, as well as the development and use of first principles methods to obtain vibrational and electronic spectra of heterogeneous interfaces. The project integrates spectroscopic techniques into existing $a b$ initio MD codes and optimizes them for use on high-performance architectures, including the IBM Blue Gene/Q (BG/Q) Mira system at Argonne.

\section{MISSION RELEVANCE}

This project is relevant to DOE missions in energy and basic science. It aims at (1) providing knowledge and computational tools to interpret a large body of ongoing experiments on fuel production from water, and (2) establishing design rules to identify Earth-abundant, non-toxic oxides and semiconductors with interfacial properties optimally suited to oxidize and reduce water. Argonne is involved in several energy projects, and this project directly supports the DOE mission to discover new materials for solar energy conversion. In addition, beneficiaries within Argonne include the Center for Nanoscale Materials, the Advanced Photon Source facility, ongoing materials design activities, and the Midwest Integrated Center for Computational Materials.

\section{RESULTS AND ACCOMPLISHMENTS}

We carried out first principles simulations ( $a b$ initio molecular dynamics coupled with many-body perturbation theory) to predict the physicochemical properties of heterogeneous interfaces for solar water-splitting applications. All of our results point to the complex interplay between the electronic and structural properties of photoabsorber/water and photoabsorber/catalyst/water interfaces and the need to account for surface chemistry and interfacial effects for realistic descriptions of PEC cells. In particular, band edge positions of photoelectrode materials were shown to depend strongly on the details of the aqueous interface structure and chemistry; therefore, predictions of these quantities that neglect the effect of liquid water may be qualitatively incorrect. Our investigation of the key role of water in determining the types of contact present at photoelectrode/catalyst interfaces, whether Ohmic contacts or Schottky barriers, highlighted the importance of the catalysts' morphology. Indeed, the catalysts' morphology may determine whether water is in direct contact with both the photoabsorber and the catalyst or just with the catalyst. In addition, we demonstrated that the inclusion of explicit layers of water at the interface with the electrode is important, especially in the case of hydrophilic surfaces with surface defects such as $\mathrm{O}$ vacancies at $\mathrm{WO}_{3}$ surfaces.

\section{PROPOSED FUTURE WORK}

This work is being transitioned into National Science Foundation and Energy Frontier Research Center proposals. 


\section{Supported Single-Site Catalysts for Selective Alkane Oxidation}

\author{
2016-131-R1 \\ Jeffrey Camacho Bunquin, Massimiliano Delferro, \\ Magali Ferrandon, Rachel Klet, Ryan Langeslay, \\ Patricia Ignacio-de Leon, Scott Nauert, SonBinh T. Nguyen, \\ Justin Notestein, and Louisa Savereide
}

\section{PROJECT DESCRIPTION}

This project aims at producing platforms of single-site, multimetallic alkane-oxidation catalysts with enhanced selectivity for early-stage oxidation products for upgrading the $\mathrm{C}_{1}$ to $\mathrm{C}_{4}$ hydrocarbon components of natural gas. Specifically, we proposed the development of well-defined multimetallic active sites on a range of modified oxide supports that enables control and tuning of local catalyst environments. By depositing discrete molecular metal cluster species on these supports, we can synthesize precisely organized multimetallic catalyst sites and use them to identify the critical factors that influence alkane oxidation reactivity and selectivity, including: (1) active-site nuclearity and dimensionality, (2) active-site composition (homonuclear versus heteronuclear active sites), and (3) local electronic structures (spin and charge states). The effects of these active-site properties on surface oxygen reactivities will be studied and applied in the development of catalysts with improved reactivity and selectivity toward the key elementary steps in catalytic alkane oxidation.

\section{MISSION RELEVANCE}

This project is relevant to DOE's basic science goal, to understand fundamental principles behind rational design of catalysts and deliberate control of chemical transformations.

\section{RESULTS AND ACCOMPLISHMENTS}

We installed isolated metal ions and/or homonuclear bimetallic clusters on modified oxide supports (silica, alumina, titania, zirconia) via atomic layer deposition (ALD) and solution-phase deposition of molecular precursors to target supported metal ions known for catalytic oxidation activity (e.g., Cu, Mn, Ni). We also investigated the effects of promoter ions-specifically, both Lewis acidic promoter ions ( $\mathrm{Ti}, \mathrm{Zr}, \mathrm{Zn}, \mathrm{Al}$ ) and redox-active promoters ( $\mathrm{V}, \mathrm{Cr}, \mathrm{Ni}$, $\mathrm{Cu}$ - - on catalyst reactivity, selectivity, and stability under hydrocarbon oxidation conditions (e.g., propylene to acrolein and propane to propylene) (Figure 1).
Propylene oxidation to acrolein. Gas-phase propylene oxidation studies showed the intrinsic reactivity of $\mathrm{MISiO}_{2}$ catalysts ( $\mathrm{M}=\mathrm{Cu}, \mathrm{Fe}, \mathrm{Co})$ as well as the effects of various metal promoters on selective oxidation (Figure 1). A comparison of the promoter effect revealed that a main group (Al) and early- to mid-transition metal promoters $(\mathrm{V}, \mathrm{Cr}, \mathrm{Zr}$ ) can stabilize the $\mathrm{Cu}$ sites from agglomeration under catalysis conditions and enhance the catalytic performance in terms of acrolein yield.

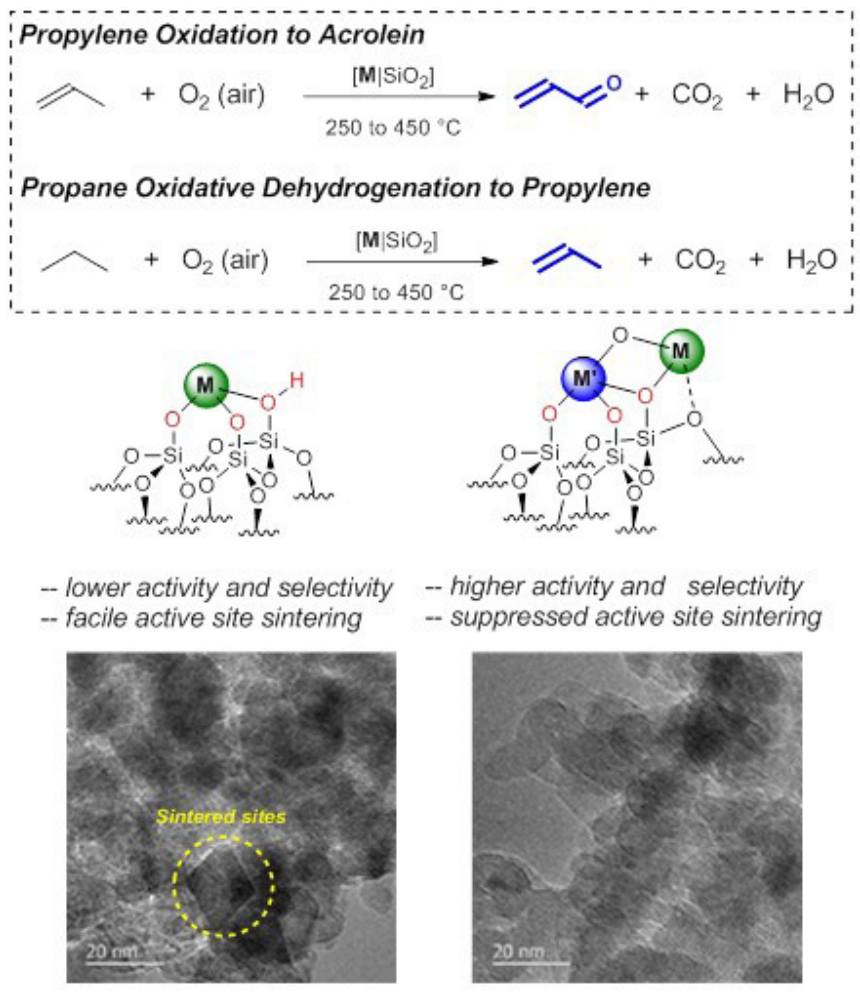

Figure 1. Enhancement of activity, selectivity, and stability of transition metal catalysts for propane oxidative dehydrogenation using metal promoters.

Propane oxidative dehydrogenation to propylene. We demonstrated the generality of the bimetallic active site design strategy described above to develop selective and stable catalysts for the oxidative dehydrogenation $(\mathrm{ODH})$ of propane to propylene (Figure 1). We investigated the effect of promoter ions on manganese-based active sites. High-throughput synthesis and catalyst evaluation showed that the composition of heterobimetallic sites and the nature of the solid catalyst supports are both critical factors for imparting high activity, high selectivity, and unprecedented sintering resistance.

\section{PROPOSED FUTURE WORK}

This LDRD project was completed in FY 2017. 


\section{Managing Emission and Thermal Absorption (META)}

\author{
2016-133-R1 \\ Pierre Darancet, Maria K. Chan, Stephen K. Gray, \\ Alex Martinson, Subramanian Sankaranarayanan, \\ Richard D. Schaller, and Gary Wiederrecht
}

\section{PROJECT DESCRIPTION}

The objective of this project is to simultaneously (1) advance our understanding of non-equilibrium heat generation and transport in nanoscale materials, and (2) develop novel spectroscopic tools and theoretical models to probe the fundamental interactions among electrons, photons, and phonons underlying thermal management in these materials. As the energy scales of the interactions lead to a wide variety of phenomena, improving nanoscale thermal science requires a trifecta of capabilities: advanced characterization, materials synthesis, and multiscale modeling. Using this synergistic approach, we aim to demonstrate how the new degrees of freedom offered by nanomaterials in controlling these interactions can lead to new paradigms in thermal management. Specifically, we will show how radiative heat can be engineered by plasmonic degrees of freedom, how non-equilibrium electron-phonon interactions lead to non-classical heat transport, and how the thermal conductivity of low-dimensional structures can be advantageously tuned by phonon-phonon interactions.

\section{MISSION RELEVANCE}

This project addresses all five of the DOE Basic Energy Sciences grand challenges, as it involves (1) control of electron dynamics, (2) design of materials with tailored properties for energy harvesting, (3) properties emerging from the complex correlations between electronic and atomic degrees of freedom, (4) new nanoscale designs of electronic circuits with improved thermal management, and (5) understanding and characterization of non-equilibrium systems.

\section{RESULTS AND ACCOMPLISHMENTS}

In FY 2017, we made significant progress on probing and understanding the role of photon-phonon, electron-phonon, and phonon-phonon interactions in thermal management. Specifically, we (1) designed and synthesized a thermal emitter with a record-breaking spectral efficiency, (2) developed the first theory of electron-phonon thermalization in semiconductors, and (3) characterized phonon scattering and ultraslow phonon thermalization in hybrid lead-halide perovskites. We also developed our capabilities in measuring thermal emission; modeling coupled non-equilibrium electron-phonon dynamics; measuring phonon propagation; computing high-temperature, phonon-phonon interactions; and computing phonon dispersions at finite temperatures.

Using a novel framework, we computed the transient dynamics of phonons in contact with high-energy "hot" electrons and holes in polar and non-polar semiconductors (Figure 1). For all materials, we found that the decay in electronic energy is concomitant with the appearance of a significant fraction of non-thermal vibrational modes. This result is in strong contrast to the current understanding of electron thermalization in metal and originates from the large heterogeneity in the time scales of electron-phonon and phononphonon interactions in these materials. We proposed a generalized two-temperature model accounting for these effects and used it to understand the nanosecond-long thermal equilibration processes we measured in hybrid perovskites.

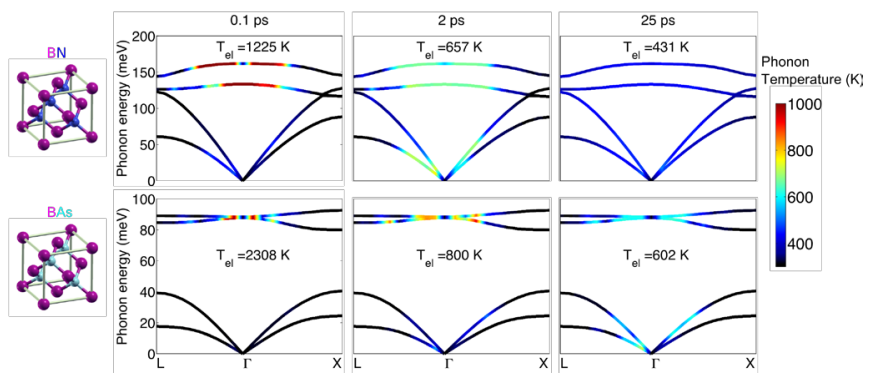

Figure 1. Snapshots of the non-equilibrium phonon distributions created by "hot" holes (temperature $=\mathbf{3 0 0 0} \mathrm{K}$ at the start of the simulation) for boron nitride (top) and boron arsenide (bottom). While the entirety of the phonon modes in boron nitride thermalizes with electrons within 25 ps, strong non-equilibrium effects are observed in boron arsenide. Corresponding electronic temperatures are indicated in each panel.

\section{PROPOSED FUTURE WORK}

On the electron-phonon thrust, we will:

$\square$ Conduct temperature-dependent, time-resolved measurements of the vibrational modes for hybrid perovskites and two-dimensional (2D) perovskites.

$\square$ Develop the theory of non-equilibrium heating in layered and anisotropic materials.

On the photon-phonon thrust, we will:

$\square$ Fabricate our second-generation emitter and evaluate its emission at $1700 \mathrm{~K}$ and its stability under different temperature ramp rates and atmospheres.

$\square$ Computationally design and experimentally fabricate a selective absorber for full thermophotovoltaic design.

On the phonon-phonon thrust, we will:

$\square$ Apply our optimized interatomic potential to the study of thermal conductivity of 2D dichalcogenide nanoribbons.

$\square$ Continue to develop a new molecular dynamics thermostat allowing for semi-classical energy distributions parametrized on our non-equilibrium phonon dynamics simulations. 


\section{Catalysts Modeled after Nature's Enzymes}

\section{7-032-NO}

\section{David Tiede, Massimiliano Delferro, Rachel Klet, Karen Mulfort, P. Raj Pokkuluri, and Lisa Utschig-Johnson}

\section{PROJECT DESCRIPTION}

This project is directed toward the development of bio-inspired and bio-hybrid approaches to catalysis that exploit multiscale confinement as a means to achieve control of chemical processes and transform matter and energy at the thermodynamic limits of efficiency. The project combines typically non-overlapping expertise in synthetic chemistry, materials, and biology to create a coordinated research capability directed at the design of bio-inspired architectures for multistep catalysis modeled after nature's enzymes. The work plan develops conceptually analogous but compositionally distinct biological and synthetic frameworks that organize a common set of catalysts for transfer hydrogenation catalysis and ultimately carbon dioxide $\left(\mathrm{CO}_{2}\right)$ and nitrogen reduction chemistries. This research effort investigates core concepts that employ spatial organization and confinement in multistep catalytic processes and develop proof-of-principle bio-hybrid and synthetic design strategies. The program targets the opportunity to link primary solar and electrochemical redox processes directly to energy-conserving catalytic chemistry.

\section{MISSION RELEVANCE}

Controlling chemical processes that transform matter and energy through the mastery of hierarchical architectures and confinement effects is a transformative opportunity identified by the DOE Basic Energy Sciences Advisory Committee report, "Challenges at the Frontiers of Matter and Energy: Transformative Opportunities for Discovery Science," which has been identified in each of the recent Basic Energy Sciences reports on research needs: "Catalysis for Energy," "Carbon Capture: Beyond 2020," and "Synthesis Science."

\section{RESULTS AND ACCOMPLISHMENTS}

1. Synthesis and Characterization of Transfer Hydrogenation Catalysts Linked to Redox Pumps. This task developed a set of transhydrogenation catalyst complexes, [LCp*M] ( $\mathrm{L}=$ bipyridyl and phenanthroline ligands; $\mathrm{Cp}^{*}=$ pentamethylcyclopentadienyl; $\mathrm{M}=\mathrm{Ir}$, $\mathrm{Rh})$, designed for integration into a broad range of host materials. The [ $\mathrm{LCp} * \mathrm{M}]$ complexes were assayed as catalysts for ketone-to-alcohol conversion activity using both photochemical and electrochemical sources of reducing equivalents. Photocatalytic reaction optimization led to complete substrate conversion under mild bio-compatible conditions of $\mathrm{pH}$ and temperature.

A significant finding from this work is the discovery of a new mechanism for transfer hydrogenation that exploits the light-excited state of the catalyst hydride (Figure 1A). Exploiting reaction landscapes using the excited states of visible light-absorbing catalysts represents a fundamentally new contribution to the field of photoredox catalysis. These results are noteworthy, given that they present an opportunity to create new catalysts following reaction pathways that are simultaneously more reactive and more selective than traditional catalytic mechanisms.

2. Synthesis and Characterization of Redox Protein Catalyst Hybrids. This task investigates the concept of coupling synthetic catalytic chemistry to biological redox chemistry. The flavin containing redox protein ferredoxin-NADP+ reductase (FNR) was developed for testing concepts toward utilizing the two-electron, proton-coupled redox chemistry of the flavin cofactor to function as a hydride donor for the catalytic chemistry. A major accomplishment of this task is the expression of FNR with engineered sites for covalently coupling the [LCp*M] catalyst at sites adjacent to the flavin cofactor (Figure 1B). This work establishes approaches to developing bio-hybrids as host environments for synthetic catalysts and spatially organized reaction sequences.
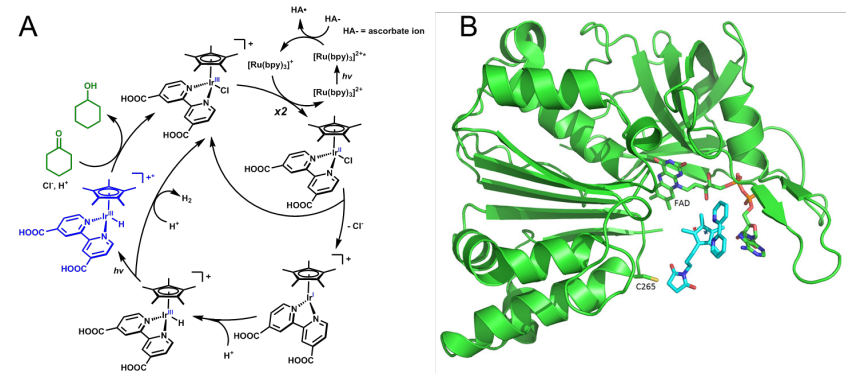

Figure 1. ( A) Proposed mechanism for the new excited-state hydride transfer reaction scheme discovered in this project for the (L)IrCp* hydrogenation catalysts. (B) FNR bio-hybrid design functionalized with a (L)IrCp* complex for covalent coupling to engineered sites adjacent to the flavin cofactor.

\section{PROPOSED FUTURE WORK}

Work in the second year will characterize electrochemical and photochemical reaction pathways for a common set of [LCP*M] hydrogenation catalysts integrated in inorganic nano- to micro-porous electrode materials and FNR redox host proteins. This work will establish building block designs for bio-inspired and bio-mimetic design for directly linking catalysis to primary redox sources. 


\section{Advanced Materials for the Energy-Water Nexus}

\section{7-033-NO \\ Seth Darling, Jeffrey Elam, Di-Jia Liu, Alex Martinson, and David Tiede}

\section{PROJECT DESCRIPTION}

This project seeks methodologies to build integrated materials that offer a flexible means of removing targeted species from water streams where it has proven challenging to address their removal efficiently with traditional techniques. We target the most energy-intensive components of purification including low-concentration metals, dilute hydrocarbons, and steroids.

Controlled nanoscale porosity, using block copolymer self-assembly, and controlled interfacial composition/ function, using sequential infiltration synthesis (SIS) and atomic layer deposition (ALD), are the core methodologies explored in this project. We will fabricate two kinds of membranes: those having extremely narrow pore-size distribution and those with innovative functionality and porous sorbents with selectivity for particular pollutants.

\section{MISSION RELEVANCE}

The energy-water nexus is one of DOE's largest growth areas, in recognition of the tremendous interconnection between these two sectors. DOE Basic Energy Sciences (BES) has existing thrusts that resonate with this project, as highlighted in the Challenges at the Frontiers of Matter and Energy report. BES held a basic research needs workshop on energy-water systems in January 2017. This workshop emphasized many aspects of materials science that align with the work undertaken here.

\section{RESULTS AND ACCOMPLISHMENTS}

Motivated by the daunting challenge seen in various industries caused by membrane fouling with oily compounds, we have explored ALD coatings on polymer membranes as a means of imparting anti-oil properties and thereby minimizing fouling. Experiments included a range of oxide coatings, and it was found that certain metal oxides exhibit far greater performance than others (Figure 1a).

Another set of membranes has been prepared in a multistep process: (1) block copolymer self-assembly of nanoscale cylindrical domains; (2) SIS to grow conductive material within the matrix surrounding the cylinders; and (3) removal of residual polymer to create pores
(Figure 1b). Next, we will characterize the electrocatalytic performance, exploring in particular the confluence of spatial confinement and chemical reactivity.
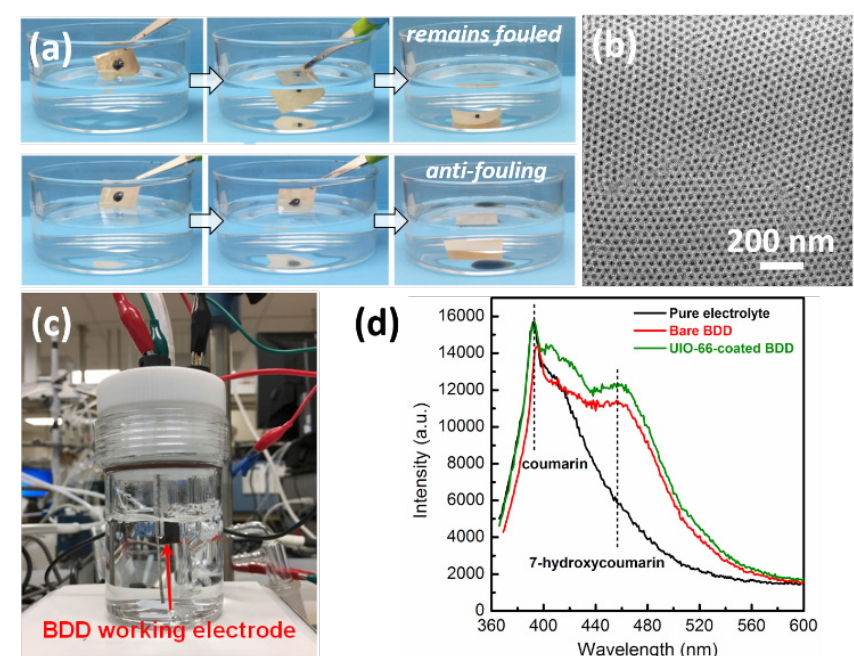

(d)

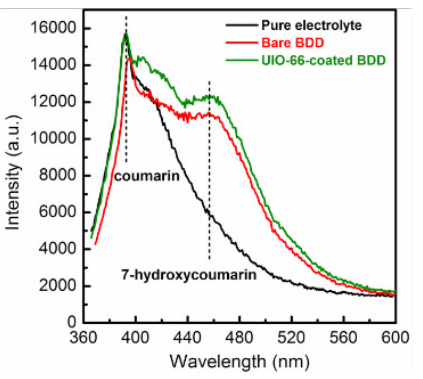

Figure 1. (a) Photographs showing anti-oil properties of ALD-coated polymer membranes; (b) a conductive isoporous membrane fabricated from block copolymer self-assembly and sequential infiltration synthesis; (c) experimental setup for anodic oxidation on boron-doped diamond working electrode in coumarin water solution; and (d) fluorescence spectra of electrolytes with and without 7-hydroxycoumarin formed.

In a parallel effort, and building on previous success in the development of the Oleo Sponge for oil-water separations, we have altered the molecular coating on SIS-treated polyurethane foams to target dissolved heavy metal ions through chelation chemistry. SIS processing conditions are being optimized to maximize the grafting density of the molecules.

To address trace hydrocarbon contaminants, we are developing materials for advanced anodic oxidation (AO). Boron-doped diamond electrodes offer high oxygen evolution overpotential and weak interactions with $\mathrm{OH}$, which we use to support a zirconium ( $\mathrm{Zr}$ )-based metal-organic framework (MOF, UIO-66) (Figure 1c). Generated $\mathrm{OH}$ was detected by fluorescence (Figure 1d), showing that the bare electrode is a potent $\mathrm{OH}$-producing electrode. Coating with UiO-66 $\left[\mathrm{Zr}_{6} \mathrm{O}_{4}(\mathrm{OH})_{4}(\mathrm{bdc})_{6}\right]$ offers an enhancement in $\mathrm{OH}$ yield, demonstrating the promise of MOFs as effective AO electrocatalysts.

\section{PROPOSED FUTURE WORK}

In the next fiscal year, we will prepare isoporous membranes with precise pore size and explore their separations performance, develop membrane materials that minimize fouling, characterize the sorption performance of the heavy-metal-capturing sponge with different dissolved metal solutions, and integrate MOFs into membrane architectures. 


\section{Self-Assembling Soft Nanostructures with Ultra- Slow Dissociation Kinetics}

\author{
2017-035-NO \\ Jeffery Hubbell

\section{PROJECT DESCRIPTION} \\ The project is focused on self-assembly of block \\ copolymers of poly(ethylene glycol) (PEG) and \\ oligo(ethylene sulfide) (OES). The OES domain is \\ hydrophobic, which drives self-assembly in water; and \\ the OES domain crystallizes, which drives very slow \\ dissociation kinetics, yielding ultra-stable self-assemblies. \\ Objectives include assembling ultra-small, ultra-stable \\ micelles for drug delivery, as well as self-assembling \\ monolayers on gold that are highly stable and highly \\ resistant to protein and cell adsorption for bioanalytical \\ purposes. A combined computational and experimental \\ approach is used to characterize these structures and \\ guide design.
}

\section{MISSION RELEVANCE}

This study of nanomaterials and nanobiomaterials supports the DOE mission in science.

\section{RESULTS AND ACCOMPLISHMENTS}

We have prepared and purified OES3-PEG44, OES4-PEG44, OES3-PEG112, OES5-PEG112, and OES7-PEG112. These polymers are now available for physical characterization of their properties as nanomaterials.

\section{Computational Modeling}

Working with the de Pablo group, we have developed computational models to (1) analyze micellization of OES-PEG, and (2) study self-assembly onto gold surfaces. Simulation of blocky co-oligomers such as OES5-PEG5 and OES3-PEG10 showed that micelles were formed with highly crystalline cores, which leads to a greatly reduced critical micelle concentration (CMC). An expected $\mathrm{CMC}$ was calculated using simulations and theory when experimental characterization proved elusive, likely due to its low value (appx. $10^{-7} \mathrm{~mol} / \mathrm{L}$ ). Computationally, these micelles are about $7 \mathrm{~nm}$ in diameter, which is consistent with experimental measurements. We have also simulated the degree of crystallinity in the core, which is approximately $40 \%$ and is also very difficult to measure experimentally. Thus, the computational model recapitulates very well what we see experimentally and sheds light on difficult-to-measure properties.

\section{Experimental and Computational Study of Adlayers on Gold}

We have thus far examined the polymers OES3-PEG44, OES5-PEG44, OES3-PEG112, and OES7-PEG112, all of which adsorb onto gold to form a very hydrophilic adlayer, with a thickness determined to be 3.5-5.0 nm by ellipsometry. Analysis by grazing-incidence wide-angle X-ray scattering (GIWAXS) demonstrated an ordered structure, with two characteristic crystalline distances, namely $3.9 \AA$ and $4.7 \AA$, consistent with computational predictions (Figure 1). Thus, the hypothesis about structure is indeed confirmed from the experimental measurements, and the computational model rationalizes the experimental measurements with high fidelity. Simulation of the adsorption and self-assembly process suggested an end-on self-assembly, (i.e., with the thiolate chemisorbing and the OES domains crystallizing). We have coated quartz crystal microbalance chips to quantify these films' resistance to protein adsorption. The OES3-PEG44 adlayer appears to limit adsorption from an order of magnitude of $100 \mathrm{ng} / \mathrm{cm}^{2}$ to the order of $1 \mathrm{ng} / \mathrm{cm}^{2}$. This coating also completely resists protein adsorption over at least two weeks, as measured by ellipsometry, and resists cell adhesion and growth for at least two weeks.

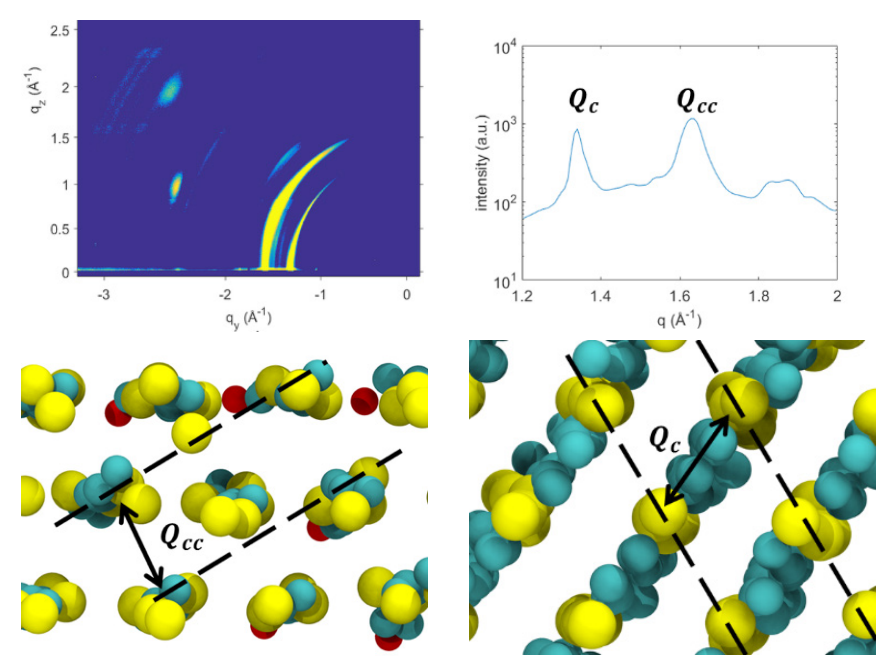

Figure 1. WAXS of PEG44-b-OES3 thin films compared to simulation results. The hexagonal ordering in two dimensions that emerged in simulations is consistent with the experimental spacing.

\section{PROPOSED FUTURE WORK}

Our objectives are to finalize synthesis of OES3PEG10 and characterize the ultra-stable and ultra-small micelles predicted by simulations, and also to study biodistribution of these micelles in mice, given that such small aggregates could uniquely navigate previously reported structures in the lymph node with size cutoffs of approximately $80 \AA$. 


\section{Oxides for Novel Computational Approaches}

\section{7-050-NO}

\section{Axel Hoffmann, David Awschalom, Anand Bhattacharya, Dillon D. Fong, Giulia Galli, and Supratik Guha}

\section{PROJECT DESCRIPTION}

This project explores two complementary strategies toward realizing more energy efficient information processing. We are exploring quantum coherence in individual defect states of oxides with the goal of harnessing these for quantum computational information processing schemes. Also, we are using the controlled manipulation of oxygen stoichiometry for the development of device concepts exploiting neuromorphic computational schemes. Both build on the recent realization of the dynamic and reversible control of defects through local electric fields, which enable a reconfigurable energy landscape for various states of condensed matter.

\section{MISSION RELEVANCE}

This work directly addresses current research challenges as they were laid out in recent DOE reports, "Neuromorphic Computing Basic Research Needs" and "Quantum Materials for Energy Relevant Technology." The need for advanced approaches for energy-efficient information technologies is becoming increasingly relevant. This is due to both the dramatic increase in power consumption in miniaturized semiconducting electronics, as well as the emergence of new types of computational workloads that value qualitative inference over scientific precision. In order to address these central challenges in today's data-driven society, we are developing new quantum materials with cognitive functionalities that can be adapted to fault-tolerant computational schemes. Thus, we aim to bridge the investigation of new materials and physical phenomena with systems integration for the development of a novel quantum or neuromorphic computational architecture.

\section{RESULTS AND ACCOMPLISHMENTS}

We demonstrated that optical excitation in strontium titanate $\left(\mathrm{SrTiO}_{3}\right)$ can be used for local gating of topological insulators. By using excitations at different wavelengths, it was possible to independently control magnetic properties and the chemical potential. Furthermore, we demonstrated that there is a more general scheme for local optical gating by combining other low-dimensional materials, such as graphene or molybdenum disulfide $\left(\mathrm{MoS}_{2}\right)$, with $\mathrm{SrTiO}_{3}$.
At the same time, we explored using first-principles calculations of the energetics of various defects in $\mathrm{SrTiO}_{3}$ that may be of interest for the above-mentioned optical gating approaches. In particular, we found that transition metal impurities form complex defect states with oxygen vacancies. Toward this end, defects based on iron (Fe) match the energetics observed in the experiments, and defects based on chromium (Cr) appear to be promising for establishing quantum bits due to the spin states associated with the defect complex.

Toward the goal of electrically manipulating resistive states, we fabricated memristive devices based on strontium cobaltate $\left(\mathrm{SrCoO}_{x}\right)$ and directly imaged the electrochemical transformations using spatially resolved diffraction and chemical mapping with advanced X-ray characterization at the Advanced Photon Source. This effort allowed us to directly trace the resistive pathways that are associated with the transformation of the $\mathrm{SrCoO}_{x}$ from a perovskite to a brownmillerite structure.

\section{PROPOSED FUTURE WORK}

We will explore the combination of optical doping and oxide electronic structures with superconducting chalcogenide layers as a means of coherently transporting localized electronic states using macroscopic quantum coherence within optically gated superconductivity. Thus, we intend to use metastable superconducting states as dissipation-free "wires" for communicating between individual quantum states associated with specific defect complexes. This experimental work will be complemented by theoretical calculations that will clarify which defect complexes are most promising for coherent quantum control. In particular, we aim to clarify the importance of the coupling of electronic motion to the crystal lattice (known as polarons) and whether it affects the stability and mobility of defects both in the bulk and at surfaces.

The other main objective for our future work will be to develop materials and systems with electrically switchable resistivities that avoid filamentary conduction, which leads to stochastic behavior that generates broad distributions in switching parameters. Toward that end, we will focus on strontium ferrite $\left(\mathrm{SrFeO}_{3-\mathrm{x}}\right)$, which is a material that, as a function of oxygen stoichiometry, undergoes a structural transition from perovskite to brownmillerite that is associated with a change of resistivity of more than 8 orders of magnitude. We aim to drive this transition by establishing an oxide $\mathrm{p}-\mathrm{n}$ junction consisting of lanthanum manganite $\left(\mathrm{LaMnO}_{3}\right) / \mathrm{SrFeO}_{3}$, where oxygen can be transferred across the interface via electric fields. In particular, we will also explore the use of epitaxial strain to optimize the functionality of these devices. 


\section{Toward an Artificial Neuron - Non-Covalent Synaptic Assemblies}

\author{
2017-061-NO \\ H. Christopher Fry, Edward Barry, Zhaowei Chen, \\ Philip Laible, Boguslaw Nocek, Tijana Rajh, Elena Rozhkova, \\ and Gleiciani de Queiros Silveira
}

\section{PROJECT DESCRIPTION}

Biological systems organize key energy components to perform their functions efficiently at low voltages (a few hundred $\mathrm{mV}$ ). Compartmentalized structures are employed in nature in the form of proteinaceous, cage-like assemblies that are used to concentrate reactants or molecules for the purposes of confined reactions and controlled transport. Our goal is to produce a compartmentalized material mimicking synaptic neuronal events. To explore this area, we are investigating hybrid, inorganic-biomolecular, self-assembled materials. We are exploring multiple avenues for developing two-dimensional (2D) and 3D nanostructures that consist of transmembrane proteins (typically light-driven ion channels/pumps) and nanoparticles (NPs) (primarily gold). The resulting materials are investigated for concentrating ions within the compartment of the material assembly.

\section{MISSION RELEVANCE}

This project's objective aligns with DOE's Basic Energy Sciences Advisory Committee report, "Challenges at the Frontiers of Matter and Energy: Transformative Opportunities for Discovery Science," through the investigation of directional ion pumping, trapping in a confined hybrid structure, and actuating a subsequent signal, all of which are beyond equilibrium phenomena. Our interests also overlap with demands outlined in the DOE report, "Neuromorphic Computing: From Materials to Systems Architecture," as our proposal addresses a fundamentally new approach to the production of a material that possesses threshold spiking, operates at low energy, and is sensitive to external stimuli.

\section{RESULTS AND ACCOMPLISHMENTS}

Our goals in FY 2017 were to develop methodologies required for obtaining $2 \mathrm{D}$ and $3 \mathrm{D}$ hybrid assemblies. We began with a well-understood light-driven protein assembly, purple membrane (PM). PM includes the light-actuated proton pump bacteriorhodopsin (bR), which is embedded in a lipid bilayer, forming a well-oriented periodic 2D nanocrystal lattice. For the development of $2 \mathrm{D}$ assemblies, we identified conditions that yielded 2D gold ( $\mathrm{Au}$ ) (15-nm) assemblies layered on top of PM
(Figure 1a). The sequential, layered addition yields regular structures that are presently being probed for their combined photocurrent response. Two methods for developing 3D hybrid assemblies have been identified. The first represents the identification of $\mathrm{pH}$-triggered PM-Au NP superstructures. As we systematically lowered the $\mathrm{pH}$, we found that the PM curls into defined tubular structures (Figure 1b). Furthermore, conditions were identified in which we co-assembled gold NPs with the tubular PM membrane, yielding the desired hybrid PM-gold NP structure. In addition, experiments to quantify the light-triggered proton pumping ability of the resulting assembly are under way. Finally, through careful method development, we have generated colloidosome assemblies through an emulsification strategy in which spherical gold NPs ( $80 \mathrm{~nm}$ ) are assembled into larger spherical structures in the presence of a positively charged polymer PDDA (Figure 1c). Subsequent functionalization of these assemblies is achieved via wrapping with PM.

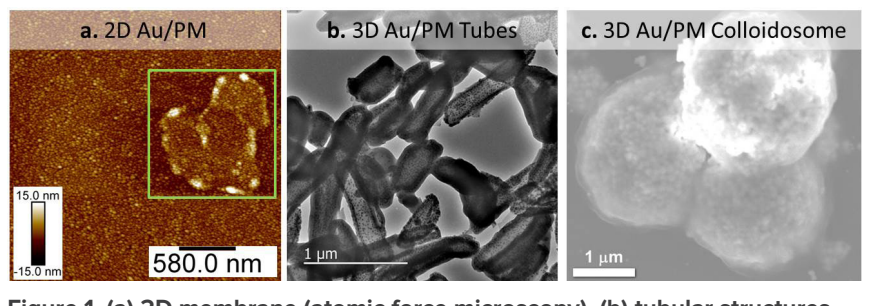

Figure 1. (a) 2D membrane (atomic force microscopy), (b) tubular structures (transmission electron microscopy), and (c) spherically shaped 3D structures (scanning electron microscopy).

\section{PROPOSED FUTURE WORK}

In light of the fact that we have three assembled hybrid structures (Figure 1), we will continue to probe their functional activity in the upcoming year by exploring the gold NP and proton pump photonic interactions. Presently, we are successfully interfacing the naturally isolated PM with Au NP assemblies. This approach ensures that we have control over the directionality of the proton pump (bR). However, one goal of this project is to develop universal strategies for transmembrane protein implantation into artificial assemblies. Most ion channels are isolated with the assistance of surfactants. Therefore, one challenge in the upcoming year is to develop methodologies that successfully take surfactant-encapsulated transmembrane ion channels with preserved functionality and assemble them into 2D and 3D structures useful for interfacing with our gold NP assemblies. This will be achieved by exploring surfactant interfacing with gold NPs. Other candidate membrane channels will be identified and assessed in nano-bio assemblies. 


\section{Developing Hierarchical Multi-Functional Hybrid Polymer-Protein Structures for Energy Applications}

\section{7-082-NO}

Tao Li, Gyorgy Babnigg, Wei Chen, Lei Cheng, Yuzi Liu, Karolina Michalska, and Randall E. Winans

\section{PROJECT DESCRIPTION}

The development of new structures that can stabilize, organize, and control the activity and stability of proteins has been well investigated but with limited success. The major challenge is that modified enzyme activity and stability are not comparable to that of the free enzyme, and enzyme recovery can be difficult. The objective of this project is to develop a method that allows protein conformation and functionality to be preserved by assembling the polymers and proteins into a hierarchical structure. The benefits derived from the success of the project are an understanding of the complex interactions and assembly for protein stabilization and development of a new method for forming reusable polymer-enzyme assemblies that will benefit the creation of novel enzyme catalysts, as shown in Figure 1.

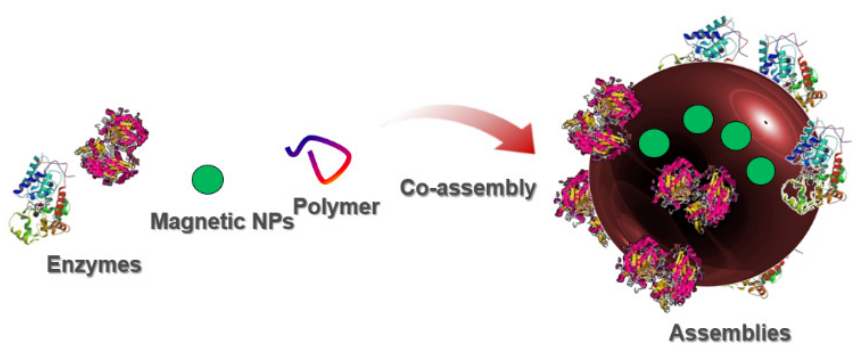

Figure 1. Schematic representation of the formation of nano assemblies from magnetic nanoparticles, an encapsulated polymer core, and an enzyme shell.

\section{MISSION RELEVANCE}

The project aligns well with DOE's mission in science, particularly its long-term mission of mastering hierarchical architectures and beyond-equilibrium matter. As stated in the DOE report, Challenges at the Frontiers of Matter and Energy: Transformative Opportunities for Discovery Science: "The emergent functionalities enabling next-generation disruptive energy technologies require mastering the design, synthesis, and control of complex hierarchical materials employing dynamic far-from-equilibrium behavior."

\section{RESULTS AND ACCOMPLISHMENTS}

We synthesized a variety of pyridine-based polymers and assembled them with enzymes under different conditions, such as $\mathrm{pH}$, temperature, and salt content. Electrostatic interactions did not play a role in the assembly. These results show that hydrogen bonding is the main driving force for the assembly. In addition, in situ X-ray scattering has been used to study the co-assembly process of poly(4-vinylpyridine) (P4VP) and protein. Once the proteins and polymers were mixed, the assembly occurred immediately, and the proteins retained their spherical shape on the surface of P4VP.

Meanwhile, several beta-glucosidases were purified on a scale of several hundred milligrams, and their activities and thermal stabilities were tested. Furthermore, one of the beta-glucosidases has been demonstrated to be assembled with P4VP to form polymer/protein core/ shell structures, which are well characterized using dynamic light scattering, small-angle X-ray scattering, and transmission electron microscopy.

We also found that solvents affect the activity of the enzyme. Five different water-soluble solvents were tested-tetrahydrofuran, dimethylformamide, dimethyl sulfoxide, methanol, and ethanol. An interesting result was that the enzyme retained its activity even at a methanol concentration of up to $30 \%$, whereas the activity for the other four solvents was lost at the same solvent concentration.

\section{PROPOSED FUTURE WORK}

Future work will include preparing the polymer-enzyme systems and testing their reusability. After that, we will screen the enzymes to evaluate the versatility of this method and perform the stability/reactivity test at different $\mathrm{pH}$, temperature, and solvent and salt content conditions. Molecular dynamics simulation will be performed to understand the effect of the solvent on enzyme activity. 


\section{Quantum Optics with Phonons}

\section{7-092-NO}

\section{Andrew N. Cleland}

\section{PROJECT DESCRIPTION}

This project is focused on demonstrating some fundamental scientific aspects associated with both the classical and the quantum behaviors of propagating, beam-split, and interfering phonons in the few-gigahertz $(\mathrm{GHz})$ range. We plan to demonstrate some of the fundamental quantum optics processes associated with microwave frequency phonons generated and detected at milliKelvin (mK) temperatures, where single-quantum control and detection should be possible. Our particular goal in this project is to design and build a system (Figure 1) capable of demonstrating the Hong-OuMandel effect with phonons, where beam-splitter-based quantum interference always forces two simultaneously generated photons (in the optics version) to emerge from the same arm of the beam splitter.

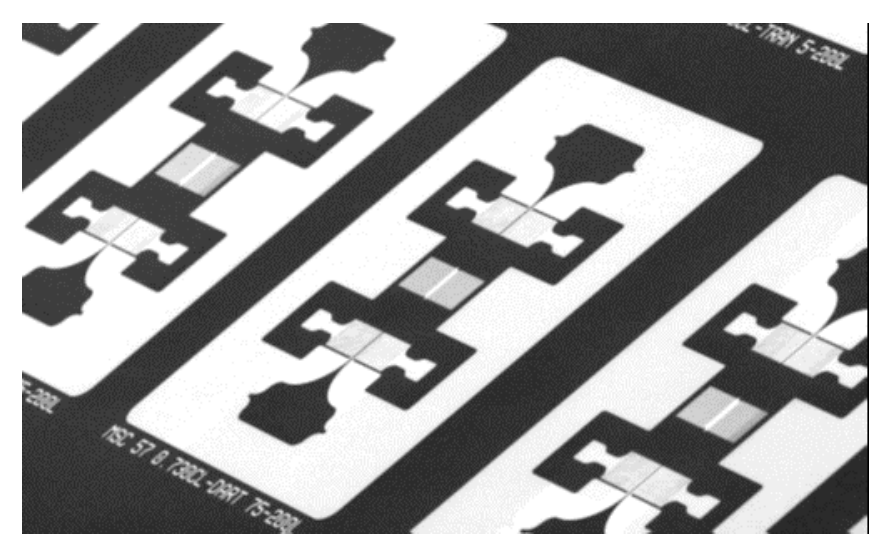

Figure 1. Image of 1.8-GHz DART transducer and beam splitter, fabricated and photographed by E. Dumur (Argonne).

\section{MISSION RELEVANCE}

This project is relevant to DOE's mission in basic science. The focus is on the specific development of one aspect within the general topic of hybrid quantum systems, where the quantum control and quantum-limited sensing afforded by superconducting and other quantum devices are applied to the development of phonons as a quantum field. Achieving quantum control over phonons similar to that of photons will open opportunities for coupling quantum systems via slow and potentially very low-loss flying qubits, which is of great utility for quantum signal processing.

\section{RESULTS AND ACCOMPLISHMENTS}

Since starting the project in January 2017, we have made excellent progress. Our experimental facilities are operating well. Our dilution refrigerator is achieving a base temperature of $7 \mathrm{mK}$ with full microwave coaxial cabling, microwave filters, and low-frequency wiring for operating up to 4 qubits, or 2 qubits and two classical phonon sources, or related configurations. In addition, we have two adiabatic demagnetization refrigerators that are set up and running, both of which can reach base temperatures of below $100 \mathrm{mK}$. These are best suited for trying to understand basic functionality using quick turn-around measurements. We are now measuring qubits fabricated in the Pritzker Nanofabrication Facility that are displaying lifetimes above a few microseconds. We have established a design for an experiment that we anticipate using to demonstrate that we can generate and receive traveling surface acoustic wave phonons, which we will beam-split with a structure-specific, beam-splitting ratio. Once characterized, first at room temperature and then at cryogenic temperatures, and with probable refinement of device details, we then intend to demonstrate the Hong-Ou-Mandel effect with phonons. It is unlikely that we will be able to test for this result in this first year of the project, because of the amount of development and characterization work needed prior to attempting the actual experiments.

\section{PROPOSED FUTURE WORK}

The next steps in this project are to fabricate test devices and measure them using the designs we have developed to date. These initial designs are actually made to operate at a lower frequency than needed for the quantum version of the experiment, as the lower-frequency designs can be fabricated much more quickly and inexpensively than the microwave frequency versions that require electron beam lithography, with much longer cycle times and lower yield. We will begin the fabrication process, which will then be debugged. 


\section{Integrated Imaging}

\author{
2017-104-NO
}

\begin{abstract}
A.K. Petford-Long, Z. Cai, M. Chan, N. Ferrier, J.R. Guest, D. Gursoy, R. Harder, S. Leyffer, Y. Liu, I. McNulty, T. Munson, T. Peterka, C. Phatak, S. Sankaranarayanan, H. Wen, and X. Xiao
\end{abstract}

\section{PROJECT DESCRIPTION}

This project aims to image structure and function of the same material under the same conditions by correlating experimental and computational imaging with modeling. For many materials, analysis over many temporal and spatial length scales is needed; thus, combining different techniques is critical. This is best achieved within a single processing and analysis framework. The project involves four closely related subtasks: (1) framework for integrated multimodal imaging of materials for energy storage (MIMES); (2) integrated modeling, analysis, and ultrafast imaging (MAUI) of energy transport in nanomaterials; (3) integrated imaging to understand and advance photocatalysis (PHOTO); and (4) smart acquisition and sampling for simulation and imaging (SASSI).

\section{MISSION RELEVANCE}

This project is relevant to DOE's mission in basic science. The methodologies and deliverables generated by this work have the potential to galvanize the development of truly integrated approaches to imaging and spectroscopy, as shown by the integration among the four subtasks and their interactions with other research groups at Argonne. This allows scientists to collaborate more freely and exploit synergistic developments, greatly benefiting Argonne's science mission and also its users.

\section{RESULTS AND ACCOMPLISHMENTS}

The MAUI and PHOTO groups worked together on photocatalyst nanoparticles to incorporate strain measurements from Bragg coherent diffractive imaging with PHOTO's other microscopy techniques (Figure 1). $\mathrm{X}$-ray images captured by PHOTO were reconstructed and analyzed using techniques developed by MAUI. Scanning tunneling microscopy (STM) measurements on the (111) surface of $\mathrm{Cu}_{2} \mathrm{O}$ crystals (Figure 2, left) have revealed the atomic-scale structure and electronic properties of surface defects that density functional theory simulations (Figure 2, right) have shown to be critical for efficient photocatalysis, which has also been quantified experimentally.

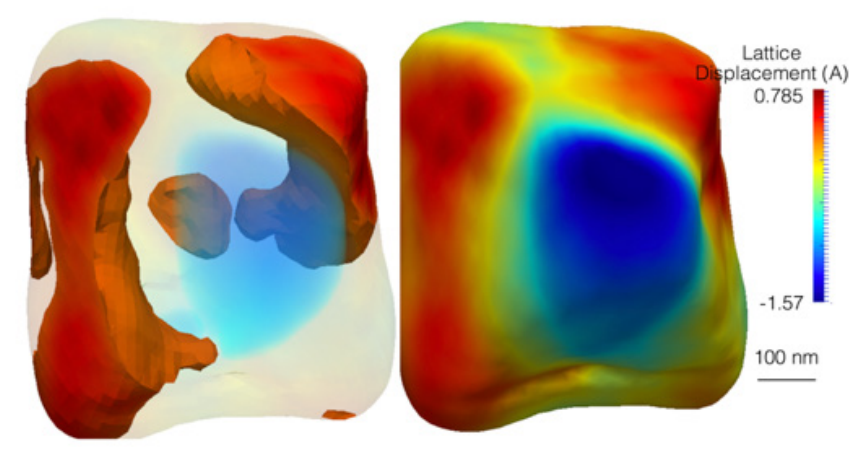

Figure 1. Reconstructed strain in the initial state of a photocatalytic nano-particle. Image courtesy of Mathew Cherukara.
Experimental STM

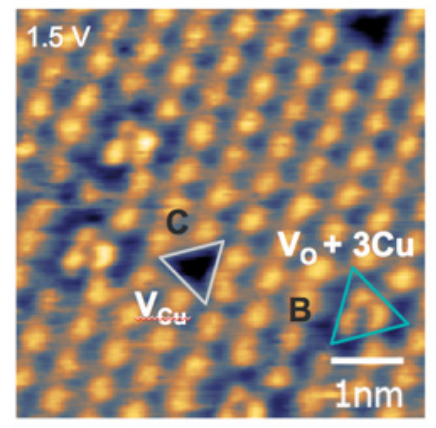

Simulated STM

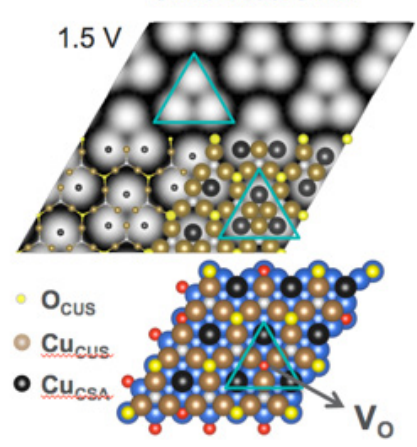

Figure 2. Left: UHV STM image of the $\mathrm{Cu}_{2} \mathrm{O}$ (111) surface. Cu vacancy (white) and $O$ vacancy (blue) are marked by trian-gles. Right: Model structure and simulated STM image for an O vacancy on the (111) surface.

Within MIMES, a new in situ battery design was tested for operando X-ray imaging and spectroscopy. To enable moving beyond the traditional raster scans and rotations used for 3D imaging, 3D phase retrieval formulations, on-the-fly data denoising, and calibration algorithms were developed. These are being applied to battery imaging to speed up data collection and reduce dose. Within SASSI, a smart hyperspectral data acquisition strategy for scanning electron microscopy was developed and implemented to acquire energy dispersive spectroscopy (EDS) composition maps. Convolutional neural networks (CNNs) were used to classify the EDS spectra into various phases.

\section{PROPOSED FUTURE WORK}

This was the final year of funding for MIMES, PHOTO, and MAUI. SASSI will develop robust computational algorithms based on CNNs and explore using continuous scanning trajectories for very fast data acquisition, which will also be implemented at the Advanced Photon Source on beam-sensitive materials such as protein-polymer complexes. 


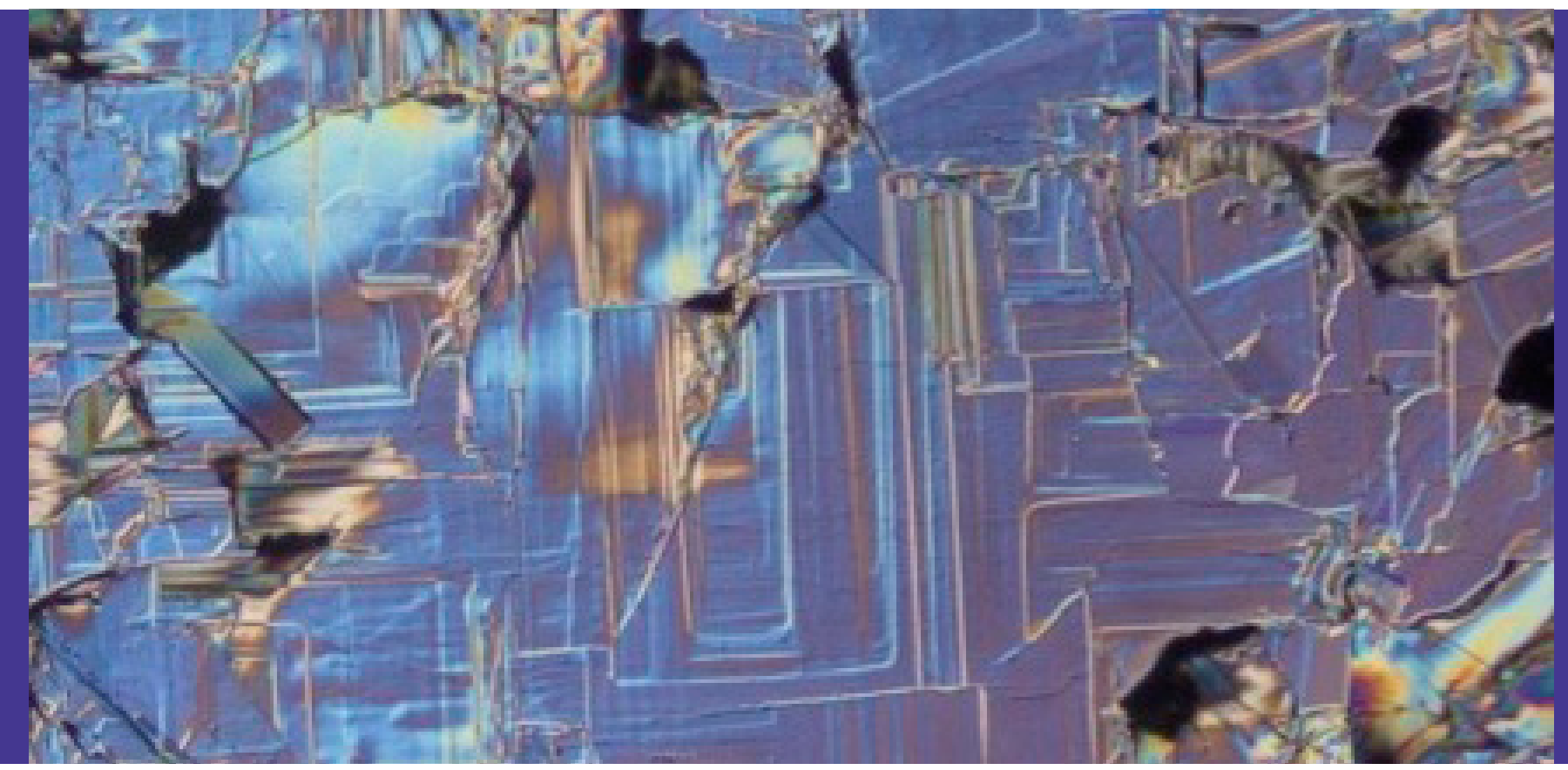

\section{LDRD PRIME - \\ NATIONAL AND \\ GLOBAL SECURITY}




\section{New ${ }^{6}$ Li-Rich Semiconductors for Neutron Detection}

\author{
2016-123-R1
}

Mercouri G. Kanatzidis and Duck Young Chung

\section{PROJECT DESCRIPTION}

This project focuses on the design, synthesis, and characterization of lithium (Li)-containing semiconductors to realize an efficient, low-cost, room-temperature, solid-state neutron detector based on ${ }^{6} \mathrm{Li}$ which produces $\alpha$ particles and ${ }^{3} \mathrm{H}$ when neutrons are absorbed. In FY 2017, project efforts focused on the screening of candidate materials and the down selection of promising materials to further optimize synthesis conditions and assess their feasibility for a semiconductor neutron detector.

\section{MISSION RELEVANCE}

Solid-state neutron detectors that are highly efficient, small, lightweight, portable, and low cost are of high importance in the DOE national security mission to detect and prevent the proliferation of illicit nuclear materials. The materials required for these devices are subject to stringent requirements as they must efficiently absorb thermal neutrons and transport the generated charge for detection. Therefore, there exists the opportunity to vastly expand the portfolio of Li-containing semiconductors and realize efficient, low-cost detectors through materials exploration and design. Lithium-based semiconductors are underexplored in the literature, and those with our target properties (i.e., with moderate band gaps and good charge transport) may also be promising for gamma-ray detection and optoelectronics.

\section{RESULTS AND ACCOMPLISHMENTS}

In 2016 we synthesized a series of Li-containing semiconducting materials (binary, ternary, and quaternary chalcogenides) by solid state reactions and characterized their physical properties to down select for further assessment. In 2017, we identified $\mathrm{Li}_{2} \mathrm{SnS}_{3}, \mathrm{Li}_{2} \mathrm{SnSe}_{3}$, $\mathrm{Li}_{2} \mathrm{ZnSnSe}{ }_{4}$, and $\mathrm{Li}_{2} \mathrm{ZnGeSe}{ }_{4}$, as candidate compounds for neutron detectors. These materials exhibit band gaps of 1.8-2.3 eV for detector applications and melt at relatively low temperatures $\left(600-800^{\circ} \mathrm{C}\right)$ which allow easy control of crystal growth for high quality single crystals and low production cost. The synthesized materials were examined by X-ray diffraction and thermal analysis, and the results showed the presence of a small fraction of an impurity phase and also difficulty in the materials process using graphite crucibles. We also explored quaternary $\mathrm{LiMP}_{2} \mathrm{Se}_{6}(\mathrm{M}=\mathrm{Bi}$ and $\mathrm{In}$ ) through solid-state synthesis using binary precursors, followed by crystal growth using a vapor transport method. These materials crystallized in two-dimensional structures and showed encouraging results: a good thermal behavior with low melting temperatures $\left(600-700^{\circ} \mathrm{C}\right)$, a band gap near $2.0 \mathrm{eV}$, and high resistivity $\left(10^{7}-10^{12} \Omega \cdot \mathrm{cm}\right)$ desirable for neutron detectors. In particular, as shown in Figure 1, Liln $\mathrm{P}_{2} \mathrm{Se}_{6}$ exhibits high resistivity and very strong photo peaks on irradiation of ${ }^{241} \mathrm{Am} \alpha$ particles, which we will further improve by enhancing the purity and quality of the crystals.
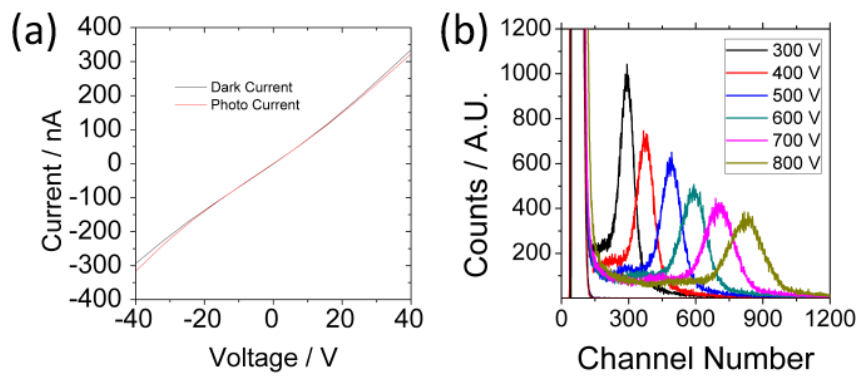

Figure 1. (a) Current-voltage data of Liln $\mathrm{P}_{2} \mathrm{Se}_{6}$ indicating resistivity of $10^{7} \Omega \cdot \mathrm{cm}$ and (b) pulse height energy spectra of $\mathrm{LilnP}_{2} \mathrm{Se}_{6}$ under ${ }^{241} \mathrm{Am} \alpha$ particles with different bias voltages applied.

\section{PROPOSED FUTURE WORK}

In FY 2018, the research plan includes production of highly pure semiconductors, continued optimization of synthesis and crystal growth conditions, and development of a purification process for precursors as well as target compounds, including lithium. We will perform a large-scale material production and crystal assay to analyze impurities present in the prepared materials. We plan to perform evaluation of the crystal quality by optical spectroscopy and study of the defects to characterize the electron and hole trap states that reduce detector performance. We will also assess the $\mathrm{Li}$ content required for the best performance by examining the materials with ${ }^{6} \mathrm{Li}$ enriched. The results from this task will feed back into the experimental process for purification and crystal growth of prepared materials suitable for complete assessment for neutron detector applications. These proposed tasks are necessary for positioning this project to obtain continued support for the development of solid-state semiconductors for neutron detector applications. 


\section{A Novel Interferometric Terahertz Phase Imager (THz-PI) for National Security Applications}

\author{
2016-136-R1
}

Shaolin "Allen" Liao and Thomas Elmer

\section{PROJECT DESCRIPTION}

We continue to make solid progress toward building an Interferometric Terahertz Phase Imager (THz-PI) setup for the strategic mission of national security. Owing to their deep penetration capability and high-resolution properties, $\mathrm{THz}$ waves (300 GHz to $30 \mathrm{THz}$ ) can play an important role in national security applications such as airport security screening, mail package scanning, and spectroscopy of hazardous chemicals such as explosives. Our goal is to develop a two-dimensional (2D)/3D scanning imaging spectroscopy system for through-barrier inspection of inaccessible/unidentified objects, such as items inside a closed container. The $\mathrm{THz}-\mathrm{PI}$ can fully obtain $\mathrm{THz}$ phase images through only one interferometry intensity measurement.

\section{MISSION RELEVANCE}

The successful development of the proposed THz-PI is important in many national security areas, such as airport screening, mail/parcel scanning, and standoff subsurface imaging. Potential users include DOE's National Nuclear Security Administration as well as the Transportation Security Administration and other offices within the U.S. Department of Homeland Security.

\section{RESULTS AND ACCOMPLISHMENTS}

In FY 2016, we obtained single-pixel spectroscopies of dielectric samples such as quartz wafer. In FY 2017, we have achieved the following:

1. We have built a $2 \mathrm{D}$ sub-THz intensity imaging setup operating at 40-120 GHz and successfully obtained the 2D THz image of an Argonne logo hidden beneath an optically opaque substrate (Figure 1a).

2. We have also built a $2 \mathrm{D} \mathrm{THz}$ intensity imaging setup operating at $0.25-0.325 \mathrm{THz}$, and it shows much higher spatial resolution compared to the 2D sub-THz imaging system.
3. We tested a novel, low-cost $\mathrm{THz}$ detector using a neon indicator lamp as a gas discharge detector (GDD).

4. We assembled an interferometric THz Doppler radar based on a neon indicator lamp GDD, and a Doppler signature has been obtained (Figure 1b).

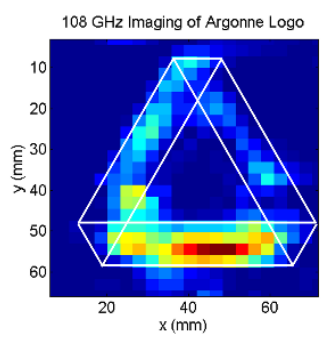

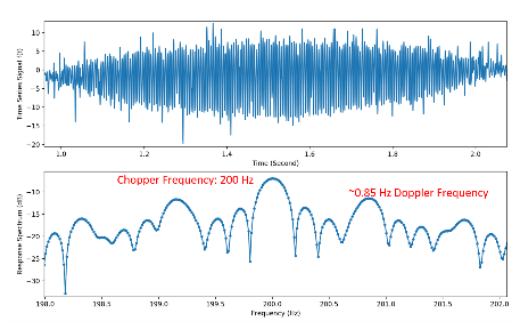

b)
Figure 1. a) 2D sub- $\mathrm{THz}$ (0.108-THz) image of an Argonne logo hidden beneath an optically opaque substrate, obtained with a standard imaging system; and b) Doppler signature detected by an interferometric radar with a novel $\mathrm{THz}$ neon indicator lamp gas discharge detector.

Finally, the work on $\mathrm{THz}$ wave generation through two-color Continuous Wave (CW) laser photo-mixing led to an invited talk at the Global Summit on Laser Optics \& Photonics, June 9-21, 2017, in Valencia, Spain, titled "Miniature DBR/DFB Fiber Lasers for CW THz Generation."

\section{PROPOSED FUTURE WORK}

We will optimize the performance of our demonstrated 2D THz-PI setup and develop a 1- to 5-THz 3D imaging spectroscopy setup. This work will include the following tasks:

1. 1- to 5-THz source: we will generate $\mathrm{THz}$ waves through two-color $\mathrm{CW}$ laser photo mixing.

2. $2 \mathrm{D} \mathrm{THz}$ imaging array: we will build a $2 \mathrm{D} \mathrm{THz}$ imaging array based on the preliminary results obtained with the $\mathrm{THz}$ neon indicator lamp GDD.

3. 3D imaging spectroscopy: we will build a 1- to $5-\mathrm{THz}$ $3 \mathrm{D}$ imaging spectrometer with the above components.

4. Spectroscopy: we will perform extensive laboratory tests of different chemicals of national security interest. 


\section{Fabrication and Testing of a Borosilicate Microchannel Plate Thermal Neutron Detector with Optimized Geometry}

\author{
2016-173-R1
}

Robert Wagner, Patrick De Lurgio, Jeffrey Elam, Anil Mane, Michael Pellin, Jingbo Wang, and Lei Xia

\section{PROJECT DESCRIPTION}

The project addresses the detection of nuclear materials via the neutrons produced by fissile materials such as uranium and plutonium. For many years, ${ }^{3} \mathrm{He}$-based detectors have been the standard for detection of thermal neutrons, but the supply of ${ }^{3} \mathrm{He}$ has been depleted and the cost has risen significantly. A less expensive alternative is ${ }^{10} \mathrm{~B}$, which also absorbs thermal neutrons efficiently. To allow the daughter products ( ${ }^{4} \mathrm{He}$ and $\left.{ }^{7} \mathrm{Li}\right)$ of ${ }^{10} \mathrm{~B}$-neutron interactions to escape to the sensing medium, ${ }^{10} \mathrm{~B}$ is typically incorporated as gaseous boron compounds or as a thin layer on the surface of the detector, both of which have low ${ }^{10} \mathrm{~B}$ density and, therefore, low probability for neutron interaction.

We seek to develop an improved ${ }^{10} \mathrm{~B}$-based neutron detector by incorporating ${ }^{10} \mathrm{~B}$ into microchannel plates (MCPs). Even when a few to 100 microns thick, they have a high probability of absorbing neutrons and allowing the daughter nuclei to escape into the MCP pores, hit the pore walls, and produce electrons that are multiplied to produce signal gain. With pores on the order of 10 microns and pore walls $\sim 1$ micron thick, the MCP has a very high open area ratio (fraction of the entire volume occupied by empty pores). The ${ }^{10} \mathrm{~B}$ in the MCP wall structure allows most thermal neutrons to be absorbed, but presents minimal material for gamma-ray interactions, which constitute a background. The fine pore structure also allows the neutron interaction location to be determined with millimeter or better precision. The path we chose for the development of ${ }^{10} \mathrm{~B}$-enriched MCPs incorporating a complex of ${ }^{10} \mathrm{~B}$ into the photoresist used to $3 \mathrm{D}$ print the capillary arrays that form the MCP support structure. We are using $\mathrm{Pu}$-Be neutron and ${ }^{60} \mathrm{Co}$ gamma ray sources to demonstrate neutron detection and measure the sensitivity to gamma rays. $A{ }^{3} \mathrm{He}$ gas tube detector is used as a comparison standard.

\section{MISSION RELEVANCE}

Thermal neutron detection is relevant to DOE missions in national security applications, such as detecting clandestine fissile nuclear materials, and basic science research applications, such as studying material structure using neutron scattering. For security applications, a portable detector with overall efficiency of at least $75 \%$ (the typical efficiency of ${ }^{3} \mathrm{He}$-based detectors) is required. The thin profile and light weight of the glass MCP detector package make it a potentially field-deployable solution for neutron detection. The potential cost for mass production of ${ }^{10} \mathrm{~B}$-loaded MCPs could be much less than using ${ }^{3} \mathrm{He}$. 3D printing of capillary arrays that are then functionalized by atomic layer deposition into MCPs by application of resistive and secondary electron emitter compounds could significantly lower fabrication costs and enable forming MCPs into novel geometries. This technique is relevant to DOE programs in high-energy and nuclear physics as well as X-ray detection. Microcapillary arrays have found use in other applications beyond MCP gain multipliers, such as filtering and gas storage. The relatively high cost of fabricating capillary arrays via gravity-drawn glass tubes has made such uses prohibitively expensive. $3 \mathrm{D}$ printing could reduce the fabrication costs to the point of opening a range of potential applications.

\section{RESULTS AND ACCOMPLISHMENTS}

During the second year of funding, we concentrated on printing 3D capillary arrays of centimeter size. While the ability to print an array of this size exists, the challenge with the current method is to reduce the print time to 1-2 days. By optimizing the pattern drawing and laser parameters, we were able to print a 1-cm-diameter, 900-micron-thick capillary array in 39 hours. We coated four samples with an $\mathrm{Al}_{2} \mathrm{O}_{3}-\mathrm{W}$ resistive coating and either $\mathrm{Al}_{2} \mathrm{O}_{3}$ or $\mathrm{MgO}$ emissive coatings to create MCP gain multipliers. We have subsequently demonstrated gain production in these devices using an ultraviolet lamp to eject electrons from the gold electrode on the device. This represented the first demonstration of gain production in a 3D-printed MCP. We also studied incorporation of complexes of ${ }^{10} \mathrm{~B}$ into the photoresist to make our thermal neutron absorber. An initial test structure with ${ }^{10} \mathrm{~B}$ loading was printed. A patent application was submitted at the end of September 2017 covering the invention of novel printing of MCPs and incorporation of ${ }^{10} \mathrm{~B}$ into the structure to produce neutron detectors.

\section{PROPOSED FUTURE WORK}

This project ended in FY 2017. We are currently in discussions with various national security agencies regarding future support for the project. Future plans for the project would include scaling the 3D printer and use of a multi-head laser beam to allow printing of larger structures. Existing test equipment allows us to continue to study gain of the MCPs we have already fabricated. 


\section{Miniaturized High-Efficiency RF Energy Harvesting}

\section{7-076-NO \\ Kaizhong Gao and Yunsong Xie}

\section{PROJECT DESCRIPTION}

Radiofrequency (RF) energy is traditionally harvested using rigid dielectric and conducting materials. The conducting materials include common low-cost metals such as copper (Cu), aluminum (Al), silver (Ag), and gold (Au). The dielectric materials include ceramics, plastics, and other polymers. This project is to develop a new generation of RF energy-harvesting devices with high efficiency. Two approaches are being taken: increasing the size of the antenna through shape transformation of a flexible substrate, and increasing the frequency response of the antenna. Just as solar cells depend on surface area for harvesting energy from sunlight, RF energy-harvesting devices depend on antenna size for capability and efficiency. The larger the antenna, the larger the device's receiving capability. The frequency response of the antenna can be enhanced by incorporating low-loss RF magnetic material into the substrate. This addition would broaden the receiving frequency band and reduce energy dissipation during harvesting.

\section{MISSION RELEVANCE}

This project is relevant to DOE's national and global security missions. It is intended to develop a high-efficiency RF energy-harvesting device. It could be widely used in self-powered devices, in conjunction with a camera or other detection device, to enable a complex command, control, communications, computers, intelligence, surveillance, and reconnaissance (C4ISR) system that supports soldiers' reliance on real-time situational awareness. Devices that power themselves by harvesting RF energy can be smaller and lighter than similar devices that do not power themselves, which can play a crucial role to protection of forces in a war zone.

\section{RESULTS AND ACCOMPLISHMENTS}

Work in FY 2017 included both infrastructure fabrication and creation of a functional RF energy-harvesting device with an integrated camera. The infrastructure mainly consists of a $6 \mathrm{ft} \times 6 \mathrm{ft} \times 7$-ft RF absorber enclosed in an anechoic chamber. The RF source and receiver are enclosed during testing. This chamber completely shields the RF radiation, ensuring that the ambient RF will not influence the test. Even more importantly, this allows the experimental work to meet RF safety requirements.
The device development led to a successful test camera integrated in an RF energy-harvesting system. This system, powered by a transformable RF antenna, is shaped differently before and after transformation. Before transformation, as shown in Figure 1a, the device is small. Only a miniature $5-\mathrm{cm} \times 4-\mathrm{cm}$ antenna is working to detect any harvestable RF energy. When the detected RF energy exceeds the predetermined threshold value, the device transforms by expanding the antenna to its full size of $20 \mathrm{~cm} \times 20 \mathrm{~cm}$ (Figure 1b). This allows it to have much larger gain and RF-harvesting capability and efficiency. The enhanced RF-harvesting capability enables the camera to take a picture and send it to the host laptop through a Bluetooth module. A picture taken by the onboard camera appears in Figure 1c.
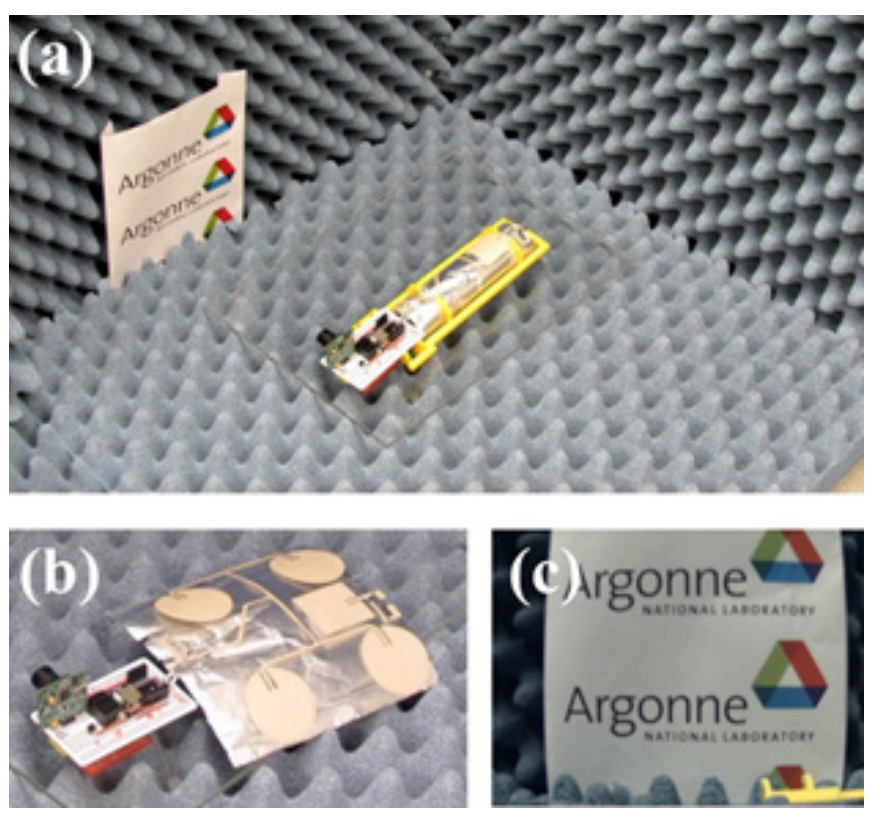

Figure 1. (a) Experimental setup for testing a transformable RF energyharvesting device, (b) RF energy-harvesting device after transformation, (c) image captured using an onboard camera powered by RF energy harvesting.

\section{PROPOSED FUTURE WORK}

Future work includes replacing the flexible substrate with a stretchable substrate. This change would enable the antenna expansion ratio to increase by a factor of more than 2. Other efforts may include implementing self-healing materials, using RF magnetic materials to make the RF device more robust to ambient mechanical stress, and improving the device's frequency response. 


\section{Understanding Resilient Infrastructure Dependencies and Interdependencies through Advanced Optimization and Simulation}

\author{
2017-088-NO
}

Charles Macal

\section{PROJECT DESCRIPTION}

The goal of this project is to develop new mathematical and computational methods for modeling lifeline infrastructure consisting of electric power, energy, water, transportation, and cyber/communications. The new methods will capture the dynamics of infrastructure interdependencies in ways that previously have not been possible. In addition to advancing the science of infrastructure resiliency, this project demonstrates new organizational models for engaging researchers to work in teams to perform cross-cutting research that spans multiple Argonne directorates and divisions.

\section{MISSION RELEVANCE}

This project is relevant to DOE's missions in national and global security. The case for strengthening U.S. infrastructure resiliency has been made in government planning documents, including Presidential Policy Directive 21. In 2015, DOE issued a Quadrennial Energy Review focused on the vulnerabilities in energy transmission, storage, and distribution infrastructure and determined policy recommendations and investments to modernize the infrastructure. A key recommendation of DOE, issued in collaboration with the U.S. Department of Homeland Security and interested infrastructure stakeholders, is to "develop comprehensive data, metrics, and an analytical framework for energy infrastructure resilience, reliability, safety, and asset security."

\section{RESULTS AND ACCOMPLISHMENTS}

In FY 2017, we developed dynamic transient natural gas flow models (to be connected to a power grid model in FY 2018). We also developed a telecommunications infrastructure model (to be connected to a power grid model in FY 2018). We demonstrated an $N-k$ security-constrained analysis of the natural gas infrastructure system and documented an infrastructure model validation study.

\section{PROPOSED FUTURE WORK}

Plans for FY 2018 include the development of logical data schema for interdependency connections among models of the electric power grid, natural gas, telecommunications, and water infrastructure elements (Figure 1).

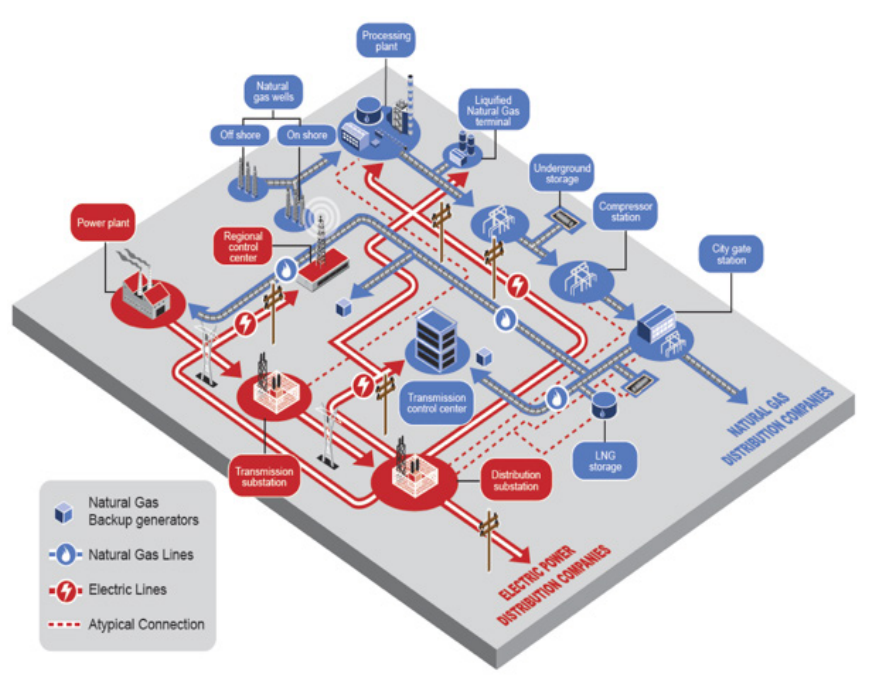

Figure 1. Interdependencies between electric power and natural gas infrastructure systems.

We will implement connected models of interdependent infrastructure systems and demonstrate an $N-k$ security-constrained analysis of an interdependent natural gas infrastructure and the electric power grid. Real-time data assimilation capabilities will be incorporated for dynamically recalibrating interdependent infrastructure models. 


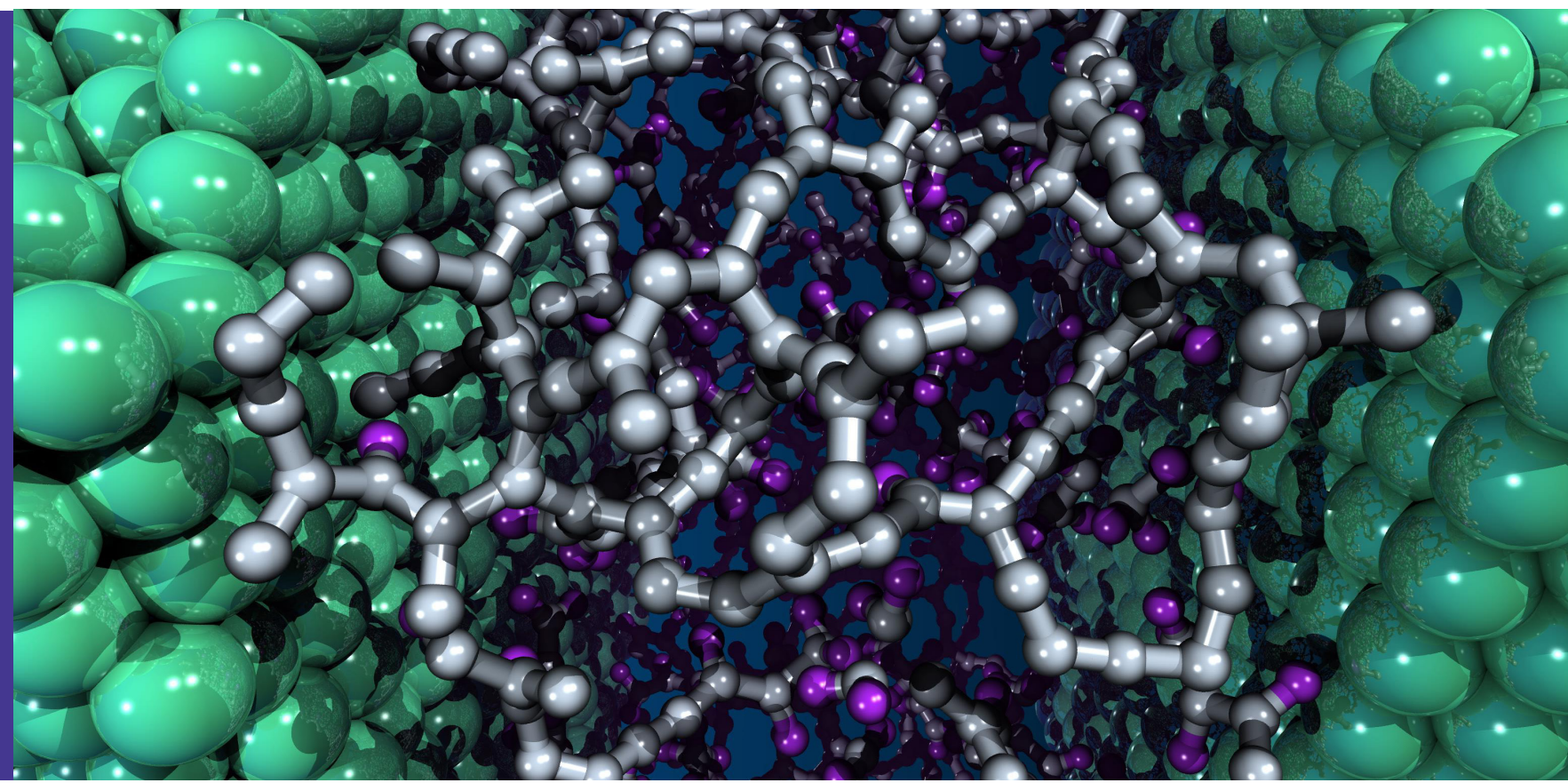

\section{LDRD PRIME - \\ NEXT GENERATION COMPUTING}




\section{Event-Based Monte Carlo Transport Methods for Next-Generation Node Architectures}

\author{
2016-135-R1
}

Paul K. Romano and Andrew R. Siegel

\section{PROJECT DESCRIPTION}

Monte Carlo (MC) particle transport methods are unique in their ability to accurately simulate complex phenomena without resorting to approximations. However, their use in many applications is often severely limited by long integration times, and so MC simulations are often confined to one-off hero calculations. Current parallel algorithms implemented in most MC codes are ill-suited for modern architectures that obtain much of their floating point performance via wide single-instruction, multiple-data (SIMD) units. The goal of this project is to improve the performance of MC particle transport simulations by exploring alternate parallel algorithms that better exploit SIMD units and a deep memory hierarchy.

In most MC codes, parallelism is achieved by assigning entire particle histories to different threads. One proposed modification of this algorithm that may better lend itself to modern architectures is to use an event-based algorithm in which the particle history is broken down into a series of "events." While the interaction physics is the same, the order in which individual events are processed and assigned to threads is completely different. The algorithm involves having many particles "in flight" simultaneously and grouping similar events together to be processed, which takes advantage of the vector units. This necessitates drastic changes in the main loop structure and significantly different data structures.

Some preliminary work in the community has demonstrated implementations of event-based algorithms in idealized, one-off research codes. Our approach is to study the algorithm using a combination of performance models and data measured from a production-level code in order to assess the merits and drawbacks of such an approach in general. A better theoretical understanding of the event-based algorithm will then help guide an implementation within a production MC.

\section{MISSION RELEVANCE}

MC particle transport methods are used in a broad range of scientific and engineering domains important to DOE missions, including nuclear engineering, radiation shielding, detection, medical dosimetry, radiation damage at the molecular level, high-energy physics, and weapons certification. In many of these domains, researchers are struggling with the question of how to best utilize next-generation node architectures. A deep understanding of performance characteristics on SIMD architectures will therefore be of great benefit.

The results of this research may be particularly relevant to the DOE Exascale Computing Project (ECP). One of the ECP application development projects, preparing production-level MC particle transport codes for application readiness at exascale, would benefit from algorithmic improvements that reduce the overall time to solution for large-scale simulations.

\section{RESULTS AND ACCOMPLISHMENTS}

A performance model with simplified assumptions about application behavior was developed and used to analyze limits on the efficiency of the event-based algorithm. Based on measured data from simulations using the OpenMC code, the performance model was used to demonstrate that in practice, an implementation of the event-based algorithm will require thousands of particles to be tracked simultaneously in order to attain acceptable efficiency, which might imply a high cost to data movement. Alongside the work of the event-based algorithm, we also investigated the potential for vectorizing cross section lookups, typically one of the most expensive operations in the particle transport algorithm. These investigations revealed opportunities for modest improvements in the performance of cross section lookups, especially when the cross sections are being evaluated using a multipole representation.

The results obtained in the first year, along with profiling data from a production MC code, showed a clear indication that dealing with memory latency was a necessary prerequisite to obtaining better use of the SIMD units on modern architectures for MC simulations. To that end, a new algorithm was developed that inherits some features of the event-based algorithm but also leverages cooperative multitasking to optimize the order in which particle events are processing and to maximize cache reuse. A prototype of the algorithm was implemented in a high-level language and shows potential for greatly improving the performance of large-scale MC simulations.

\section{PROPOSED FUTURE WORK}

Further work remains to implement this algorithm in a production code to evaluate true performance gains. This project was completed in FY 2017. 


\section{Re-form: Leveraging FPGA Reconfigurability and Floating-Point Capabilities for Next-Generation Computing Systems}

\author{
2016-148-R1 \\ Kazutomo Yoshii, Franck Cappello, Hal Finkel, \\ and Fangfang $\mathrm{Xia}$
}

\section{PROJECT DESCRIPTION}

The performance progress of microprocessors has been driven by Moore's law, with the number of transistors doubling every 18 to 24 months. This trend is expected to continue until the mid-2020s, when the complementary metal-oxide-semiconductor (CMOS) features will reach 5-7 nm in size. In the following period, performance progress for CMOS-based integrated-circuit devices will no longer come from higher levels of integration. To overcome this scaling issue, researchers are intensively investigating new technologies (e.g., carbon nanotubes). However, there is no guarantee that these new technologies will be ready for deployment in the foreseeable post-Moore era.

Our basic idea is to leverage field-programmable gate array (FPGA) reconfigurability to improve performance and energy efficiency by customizing hardware for each application workload. However, the adoption of FPGAs in scientific computing still faces two-technical challenges: (1) the lack of high-level parallel programming abstraction, and (2) the weakness in performance optimizations for scientific computing workloads. In this project, we propose to solve these technical challenges and provide a proof of concept.

\section{MISSION RELEVANCE}

This project is relevant to DOE's missions in science and energy. Since the emergence of digital computers, exponential performance growth, driven primarily by Moore's law, has been accelerating scientific discoveries and engineering breakthroughs. The end of the Moore era, which is expected in the mid-2020s, is a significant concern for DOE. Reconfigurability is one of the potential key technologies for improving the performance and/or energy efficiency of high-performance computing systems without requiring new, uncertain technologies.

\section{RESULTS AND ACCOMPLISHMENTS}

We built an FPGA development environment that includes cutting-edge FPGA platforms, such as Intel Arria10 FPGA chips; we configured related software stack components; and we developed software tools for our studies and experiments. We evaluated both the performance and usability of FPGA high-level synthesis (HLS) tools, particularly Intel FPGA SDK for OpenCL, which allows us to develop FPGA designs using a C-like programming language. To study the performance characteristics of HLS-generated FPGA designs, we ported several high-performance computing (HPC) kernels, which generally exploit parallelism using OpenMP, into OpenCL. We found that HLS tools dramatically reduce the effort required for developing FPGA designs, particularly for numerically dense codes. Because the performance is suboptimal, however, we developed microbenchmarks to identify performance bottlenecks in OpenCL FPGA designs, and we studied optimization strategies that improve both performance and energy efficiency. We demonstrated the benefits of our optimization strategies for realistic HPC workloads. We also explored a hybrid programming of OpenCL and Verilog, a hardware description language (HDL), because bit-level computing and other low-level hardware controls can be expressed only in HDL. We designed and implemented a deep-learning-influenced FPGA design based on multilayer perceptrons using the OpenCL-Verilog hybrid, and we demonstrated significant gains in both performance and energy efficiency.

As for programmability, we initiated an investigation of the translation path from OpenMP 4 to an intermediate representation using open-source LLVM compiler technology. We created a prototype compiler based on Clang, a compiler front end for LLVM. This included state-of-the-art code generation via polyhedral-loop optimizations by making use of the polyhedral parallel code generator (PPCG) project, which compiles OpenMP code using OpenMP 4 target offload capabilities to compile programs for OpenCL-enabled FPGA platforms. We experimented with this compiler using simple benchmarks.

\section{PROPOSED FUTURE WORK}

This project is now completed. Reconfigurable computing, which we investigated during this project, has a huge potential in the post-Moore era and could be a core technology for enhancing Argonne's computing capability in the near future. Thus, sustaining our efforts is crucial for Argonne and DOE. Specifically, we will prepare a proposal, including the outcomes from this project, for upcoming DOE funding opportunities involving post-Moore technologies. We will also explore other application areas in addition to HPC, such as near-sensor computing and edge/fog computing, focusing particularly on machine learning workloads. 


\section{Data-Driven Multiscale Coupled Urban Systems Modeling}

\author{
2016-157-R1 \\ Charlie Catlett, Pete Beckman, and Rajesh Sankaran
}

\section{PROJECT DESCRIPTION}

This year's effort focused on four areas: (1) develop an architecture for collecting images from Waggle nodes and keeping them secure and accessible for machine learning, (2) explore use of the Caffe image classification and deep learning framework on the Argonne Leadership Computing Facility's Athena simulation and analysis framework, (3) test classifiers on Waggle nodes in the field, and (4) explore more advanced, stream-based programming models for classifiers in Waggle nodes. We also plan to (5) explore cooling strategies for Waggle nodes in high-heat climates and (6) integrate Waggle-based data from the Array of Things deployment in terms of its utility for urban climate models.

\section{MISSION RELEVANCE}

Intelligent remote sensing is critical to many areas of DOE's mission, providing a means for field measurement in areas such as energy distribution (component performance, environmental factors), transportation (traffic and pedestrian flows), and environmental science (understanding air quality or flood patterns). As importantly, there are emerging national security needs such as detecting drone activity or airborne chemical or biological agents. The Waggle wireless sensor platform provides not only resilient remote operation in harsh conditions but also "edge computing," to enable real-time analysis of data-including images and sound-in the field. These capabilities are of major interest to several federal agencies, including the U.S. Department of Homeland Security (DHS), the U.S. Secret Service, the U.S. Department of Defense (DoD), and the U.S. Naval Research Laboratory (NRL).

\section{RESULTS AND ACCOMPLISHMENTS}

For each of the six project areas above, we provide a brief overview of progress:

1. Developed a workflow strategy to collect images from Waggle nodes and integrated it in Waggle's data management and database architecture. Defined management workflow mechanisms for turning nodes from the standard operating mode to the sampling mode.

2. Tested the Berkeley Caffe framework ported to Waggle's Odroid Linux devices and other machine learning libraries. Developed classifiers such as pedestrian count and detection of standing water.

3. Worked with the Global Security Sciences division to demonstrate Waggle's ability to execute classifiers with sufficient complexity to differentiate between a bird, a plane, and a drone. Results suggest the classifiers will also be powerful enough for initial studies of urban street flows and standing water.

4. Implemented a passive cooling strategy for Waggle nodes.

5. Worked with Urban Exascale application project personnel to place Waggle nodes for testing in Chicago. Key measurements include barometric pressure, temperature, and cloud cover.

6. Tested packaging schemes for Waggle nodes: one for Array of Things in an urban installation and the other for rooftop and non-urban deployments. Made corrections as needed (e.g., for condensation).

\section{PROPOSED FUTURE WORK}

We are pursuing funding through the National Science Foundation's (NSF's) Smart and Connected Communities program. We also proposed a configuration of Waggle with a software-defined radio as part of a multi-institutional proposal to the NSF-funded Platforms for Advanced Wireless Research program. We are also exploring potential funding sources including DHS, U.S. Secret Service, DoD, and NRL, as well as major projects such as Bloomberg's C40 Cities Climate program and potential funding sources in FY 2019 associated with the HR 3895 smart cities bill in the U.S. House of Representatives.

The team also developed a roadmap for commercial manufacture of Waggle's core platform, via a memorandum of agreement with Intel and discussion involving Argonne's Technology Development and Commercialization division about optimal licensing structures and strategies. 


\section{The Perfect Thermodynamics of Imperfect Materials}

\author{
2017-034-NO \\ Marius Stan, Chris J. Benmore, John Low, \\ and Alvaro Vazquez Mayagoitia
}

\section{PROJECT DESCRIPTION}

The goal of the project is to significantly improve the understanding and prediction of thermodynamic stability/ metastability of "imperfect" (e.g., highly defective, non-stoichiometric, or doped) oxide material phases via innovative theory (i.e., embedded uncertainty), advanced experiments at the Advanced Photon Source (APS), and "intelligent" software (i.e., software that can learn and quickly solve new problems).

\section{MISSION RELEVANCE}

This project is relevant to the DOE mission in science. We envision building the knowledge and capabilities that will allow the prediction of the thermodynamic properties of imperfect materials, with impacts on materials design, synthesis, and smart manufacturing. Furthermore, we expect this methodology to help accelerate the development of the materials genome and nextgeneration computers.

\section{RESULTS AND ACCOMPLISHMENTS}

Hafnium dioxide $\left(\mathrm{HfO}_{2}\right)$ has a high dielectric constant and is used in advanced metal-oxide-semiconductor devices. Accurate free energies have been predicted from $a b$ initio lattice dynamics (quasi-harmonic model) up to $1300 \mathrm{~K}$ for monoclinic hafnium oxide $\left(\mathrm{m}-\mathrm{HfO}_{2}\right)$. Phonon density of states from density functional perturbation theory, as implemented in ABINIT, were calculated to obtain an accurate treatment of soft phonons in oxides. Using that approach, we predicted the heat capacity of $\mathrm{HfO}_{2}$ as a function of temperature and compared it to the experimental heat capacity. We collected thermodynamic data and phase equilibria information regarding phases (element, compounds, liquid, and solid solutions) obtained from theory, experiments, the calculations obtained in this project, those published in peer-reviewed scientific literature, or available from material property databases and evaluated them for quality using Bayesian analysis. High-temperature, high-energy X-ray diffraction experiments were performed on $\mathrm{HfO}_{2}$ using an aerodynamic levitator with laser heating. Neutron diffraction experiments on the amorphous forms were performed. We found that while X-ray data are dominated by $\mathrm{Hf}$ correlations, neutrons are sensitive to oxygen interactions, such that the combination of techniques provides a rigorous test of any structural model. Figure 1 shows a comparison of the $\mathrm{X}$-ray pair distribution function (PDF) of amorphous $\mathrm{HfO}_{2}$ compared to recent classical and $a b$ initio molecular dynamics calculations, highlighting the current level of discrepancy and indicating the formation of a much more ordered structure consistent with a lower-density amorphous matrix.

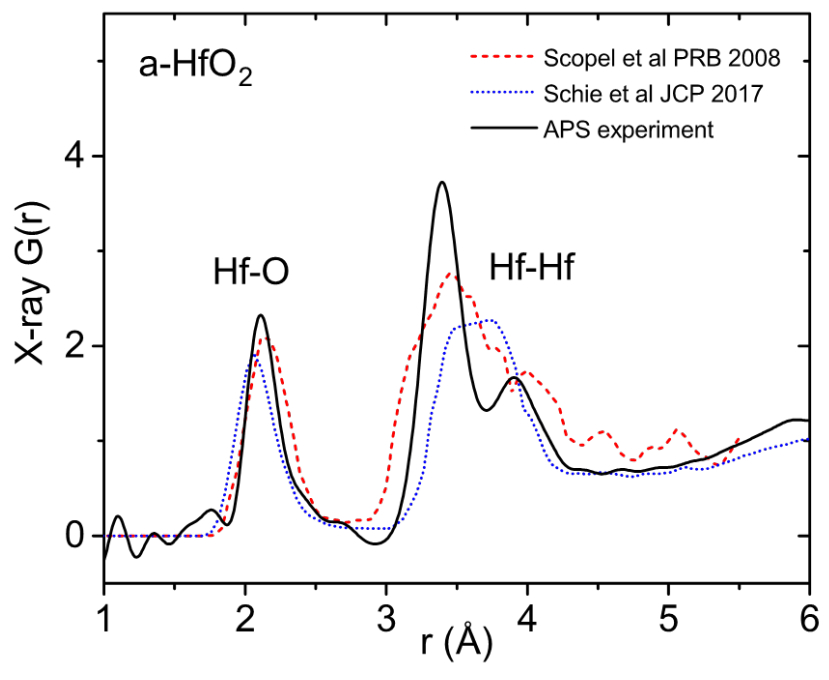

Figure 1. Comparison of the experimental X-ray Pair Distribution Function (PDF) for amorphous $\mathrm{HfO}_{2}$ measured at the APS (black line) with ab initio molecular dynamics (MD) (dashed red), and classical MD (dotted blue) simulations. $G(r)$ is the distribution of distances between pairs of atoms in a given volume.

\section{PROPOSED FUTURE WORK}

We will calculate free energy differences using $a b$ initio molecular dynamics (AIMD) on polymorphs of hafnia at high temperatures. A machine-learning algorithm using the Bennett acceptance ratio and AIMD will be used to calculate the free energy of formation of a variety of defects in the hafnium oxide polymorphs. We will also continue the analysis of phase stability in $\mathrm{Hf}-\mathrm{O}$, especially at high temperatures close to melting, and compare findings with the $\mathrm{X}$-ray results in this project. As new data come in, the Bayesian algorithm will learn and update the thermodynamic models and improve the quality of the phase stability boundaries. Furthermore, we will increase the effort to evaluate the uncertainty of all of the thermodynamic data generated in the project. 


\section{SLIK-D: Scalable Machine Learning Infrastructure for Knowledge Discovery}

\author{
2017-049-NO
}

Venkatram Vishwanath, Prasanna Balaprakash, Thomas Brettin, John Childers, Kevin Harms, Kalyanasundaram Kumaran, Vitali Morozov, Thomas Uram, Stefan Wild, and Fangfang Xia

\section{PROJECT DESCRIPTION}

Our goal is to evaluate and develop machine learning $(\mathrm{ML})$ and deep learning (DL) infrastructure that effectively scales on current and future leadership supercomputers such as Theta and Aurora. We will focus on ML and DL methods for complex and heterogeneous data, in close collaboration with application science teams. Our objective is to work closely with applications such as high-energy physics (HEP) to evaluate the efficacy of the approaches. We will also develop a knowledgebase of ML toolkits, datasets, and models and evaluate their efficacy on these systems. We will continue to maintain close collaboration with industry and foster collaboration with other application domains.

\section{MISSION RELEVANCE}

The project is relevant to the DOE mission in science. $\mathrm{ML}$ and $\mathrm{DL}$ are making revolutionary impacts on our increasingly data-driven lives, as found in such applications as self-driving cars to natural language processing. As we move toward data-driven, high-performance computing, DL and ML will be critical in the modeling of complex phenomena in synergy with current computational and experimental approaches. It is expected that by the exascale timeframe, about $20 \%-33 \%$ of science runs on leadership systems will need scalable $\mathrm{ML}$ and $\mathrm{DL}$. A robust $\mathrm{ML}$ and $\mathrm{DL}$ infrastructure will enable application teams to fully realize their science goals on the Advanced Leadership Computing Facility (ALCF) supercomputers as well as at the Advanced Photon Source.

\section{RESULTS AND ACCOMPLISHMENTS}

We have developed a hyper-parameter search for DL leveraging an asynchronous search framework that consists of sampling a small number of input parameter configurations and progressively fitting a surrogate model over the input-output space until exhausting the user-defined maximum number of evaluations. Our initial implementation is based on a message passing interface (MPI)-based scheme, and we have evaluated it on Theta on 300 nodes. In the beginning, the algorithm samples configurations at random and learns the relationship between input and output. In further iterations, it focuses on the promising regions of the space and samples more configurations from that space. Toward the end, the search converges to high-quality parameter configurations.

We have completed preliminary work on evaluating the efficacy of DL for HEP. Our current effort involves classification of features such as jets using HEP datasets.

We have started evaluating DL frameworks on the ALCF supercomputer systems. Our current focus is on data-parallel training.

\section{PROPOSED FUTURE WORK}

In the following fiscal year, we will work toward:

$\square$ Refining our hyper-parameter approach and scaling this on ALCF supercomputing systems.

$\square$ Continuing the evaluation of DL frameworks on the ALCF supercomputing systems.

$\square$ Refining models for the HEP datasets.

We expect that results from the project will attract funding opportunities spanning multiple agencies, including the DOE Office of Science, the National Nuclear Security Administration, the U.S. Department of Defense, the Defense Advanced Research Projects Agency, the National Security Agency, the National Institutes of Health, the National Strategic Computing Initiative, the U.S. Department of Homeland Security, the Veterans Administration, and the National Cancer Institute, as well as industry including drug manufacturers and engineering firms. 


\section{An Exascale Application for Simulating Urban Boundary Layers}

\author{
2017-057-NO \\ Robert Jacob, Iulian Grindeanu, Rajeev Jain, \\ Vijay Mahadevan, and Aleks Obabko
}

\section{PROJECT DESCRIPTION}

The layer of the Earth's atmosphere from the Earth's surface to about $1 \mathrm{~km}$ up is referred to as the "boundary layer." In mathematical-physical models of the atmosphere, it is important to accurately simulate the transfer of momentum, heat, water, and other substances through this layer before the models can accurately simulate weather or climate. The processes in the atmospheric boundary layer are so small that even global climate models with a very high resolution cannot simulate them explicitly. Instead, their effect is parameterized through equations that describe the average effects of boundary layer processes. This treatment is especially necessary over large urban areas where the presence of buildings, streets, and other man-made surfaces must be included. Current parameterization schemes for the urban atmospheric boundary layer are known to be deficient in representing momentum. To improve them, we are creating a model that will explicitly simulate the winds in the boundary layer. The heart of this model is an existing fluid dynamics model developed at Argonne called Nek5000. We are creating a numerical mesh for Nek5000 in the urban domain of Chicago and verifying its ability to model fluid motions there. These solutions can be used to build more accurate parameterizations. We must create the mesh, verify solutions from Nek5000, and ensure that the model will run efficiently on future high-performance computing platforms.

\section{MISSION RELEVANCE}

The techniques and knowledge developed in this project are directly relevant to the DOE mission of maintaining a vibrant effort in science and engineering. This project is also relevant to the Earth System Modeling program and Exascale Computing Project in the Office of Science and to building energy research in the Office of Energy Efficiency and Renewable Energy. Portions of the research planned for the next year have been taken up by the Exascale Computing Project.

\section{RESULTS AND ACCOMPLISHMENTS}

We have developed a mesh around the Lake Point Tower building in Chicago (Figure 1). To test the ability of Nek5000 to solve for the air motion around this tower, we are using the method of manufactured solutions. We prescribed a solution to the flow that is two-dimensional, divergence-free, and satisfies the continuity equations. At the surface of the building, the boundary condition is forced to be consistent with the rest of the solution, making the building "invisible" to the flow. With an initial velocity of zero everywhere, the forcing and Nek5000 were able to drive the flow to the target solution within a few iterations of the model and without any significant errors from the mesh.

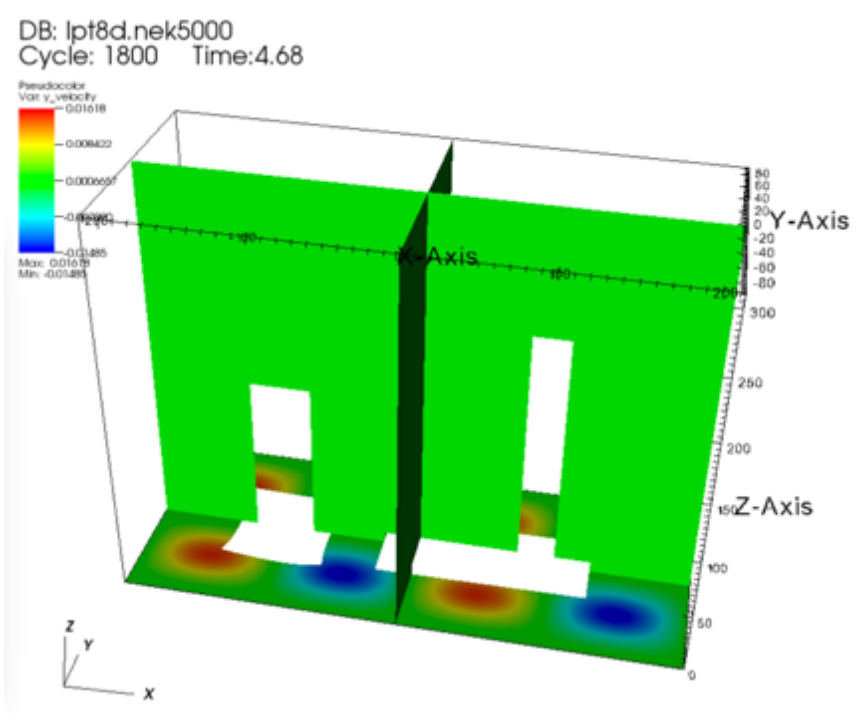

Figure 1. The horizontal slice shows the magnitude of lateral velocity close to equilibrium. The white parts in the vertical and horizontal slices are Lake Point Tower (right) and a nearby building (left).

\section{PROPOSED FUTURE WORK}

The next step is a more challenging problem: we are developing a general procedure that automatically insures physical, no-slip boundary conditions at the building wall. This procedure is based on blending any arbitrary prescribed manufactured solution with a suitable exponential function that depends on the local distance from the wall in any given element. This approach will make the flow "feel" the presence of the building. Once the no-slip condition at the wall is in place, we can add buoyance and other features needed to simulate flow in an urban boundary layer. 


\section{Enabling Multidimensional X-ray Nano-Tomography}

\section{7-063-NO}

Zichao (Wendy) Di, Si Chen, Francesco De Carlo, Doga Gursoy, Sven Leyffer, Stefan Vogt, and Stefan Wild

\section{PROJECT DESCRIPTION}

The upgraded Advanced Photon Source (APS) will deliver dramatic improvements to the brightness and coherence of the X-ray beams being generated. These will enable improved spatial resolution, which will lead to unquestionably improved clarity of sample structure. However, random and systematic errors, such as probe drift, can negatively impact spatial resolution and even the interpretation of underlying sample structures. In this project, with beamline scientists from the APS and computational scientists from the Mathematics and Computer Science division, we are formulating new physical models that capture more complex experimental setups; devising new mathematical optimization formulations and techniques for robust inversion of complex data; and providing scalable implementations of our models, formulations, and techniques that can run both on local machines and on clusters at the APS, as well as at the Argonne Leadership Computing Facility (ALCF).

The dramatic improvements made possible by an upgraded APS are requiring that we rethink how experimental setups are modeled. We must develop robust and highly efficient computational data analysis tools for reconstruction of evolving samples at nanometer resolution and provide models for multiscale and multimodal integration with the objective of delivering to our future user community a truly multidimensional suite of instruments that can take advantage of every improvement the APS upgrade will bring.

The outcome of this project will have direct benefit in reducing the data quantification time and accelerating the innovative design of hard $\mathrm{X}$-ray techniques.

\section{MISSION RELEVANCE}

This project will directly advance the goals of the next generation of hard X-ray science at Argonne, and as such is aligned with DOE's science mission and in particular with advancing photon-based science.

\section{RESULTS AND ACCOMPLISHMENTS}

1. In the context of general tomography, we have identified major drift sources and developed a mathematical abstraction to model these error sources.

2. Starting with full-field tomography, we have developed a physical model to describe the shifts of the center of rotation (COR) during an experiment and also provided an analytical formula that corrects these shifts.

3. We have developed a model-based reconstruction scheme for simultaneous tomographic reconstruction and correction of a single COR shift.

4. We have illustrated the performance of our framework on synthetic data as well as real experimental data.

5. We have proposed an objective-based policy to systematically choose the optimal reconstruction result from many local solutions.

6. We have extended the physical model to the case of multiple instances of COR shifting, which commonly shows up in scanning-probe imaging.

\section{PROPOSED FUTURE WORK}

A deliverable for the initial period will be a multilevel-based reconstruction method addressing systematic and reconstruction errors for full-field imaging (allowing for errors on a per-projection level), which will then be extended to scanning-probe imaging (allowing for errors on a per-pixel level).

Activities in FY 2018 will include:

1. Extend the uncertainty parameters to the scanning-probe images describing vertical/ lateral drifts.

2. Benchmark the scanning-probe model.

3. Design a dimensionality reduction scheme to transfer the error parameters in the high-dimensional space to a reduced space in order to lower computational complexity.

4. Document and fully implement the algorithm which simultaneously calibrates the center of rotation drift while reconstructing the sample. 


\section{End-to-End Genome Annotation and Phenotype Prediction with Deep Learning}

\author{
2017-066-NO \\ Fangfang Xia, Prasanna Balaprakash, Thomas Brettin, \\ and Venkatram Vishwanath
}

\section{PROJECT DESCRIPTION}

Genome research is generating massive amounts of data. This includes next-generation sequencing data on the one side and high-throughput phenotype screening data on the other. However, to understand how biology really works, we need to bridge the gap between these two sides. Deep learning thrives on capturing complex relationships from large training data and is the logical candidate to integrate all of this information into knowledge.

Our primary goal is to build an end-to-end, deep learning system that can outperform current shallow, manually curated techniques for genome annotation. This objective includes assigning function labels to protein encoding DNA (deoxyribonucleic acid) sequences and identifying genetic elements on multiple levels (nucleotides, motifs, genes, ribonucleic acids, operons, and pathways). While traditional bioinformatics relies on specialized modules for each feature type, the end-to-end approach is more suitable for learning such hierarchical features in a joint manner.

\section{MISSION RELEVANCE}

This project is relevant to DOE's mission in basic science. There are important differences between science domains and the fields deep learning has already revolutionized. For example, unlike an image classification task where humans have abundant intuition, biological pattern discovery requires expert knowledge and working with multiple noisy data sources. To further derive mechanistic understanding, we need to incorporate the structured biological knowledge we already have and learn even when training examples are limited. The successful execution of the project will demonstrate that we can replace scientific expert systems curated over a decade with deep learning techniques that are more generalizable and easier to maintain. Further, an interpretable sequence model will benefit anyone trying to make phenotype-genotype connections. Applications to energy production and precision medicine include predictive models for antimicrobial resistance, anticancer drug response, and bacterial growth.

\section{RESULTS AND ACCOMPLISHMENTS}

We developed a million-protein-scale genome annotation benchmark dataset leveraging decades of Argonne expertise in this area. In addition, we implemented deep, residual, convolutional neural networks that have been trained to classify 1,000 different protein functions with $96.7 \%$ validation accuracy in the top 1 class prediction (accurate on first try) and $98.4 \%$ validation accuracy in the top 5 class prediction (accurate within 5 tries). To our knowledge, deep learning on biological sequences at this scale has not been attempted before. As we have seen in image classification and other tasks, large-scale and domain-specific benchmark data and reference implementations are critical to whether deep learning can make progress in a new domain.

To demonstrate the utility of machine learning in biological pattern discovery, we have trained a detector for genomic islands, regions of a genome that have been integrated recently, using classical machine learning models as well as recurrent networks. The preliminary performance of these models is on par with existing rule-based systems. To predict bacterial genome-level phenotypes, we have implemented many sequence featurization methods. We are building attention-based deep learning models to aggregate these features so as to improve the performance of ensemble machine learning models: $90 \%$ accuracy for antimicrobial resistance prediction and $80 \%$ accuracy for bacterial growth prediction.

Success in deep learning in biology will likely depend on how we make use of the massive amount of unlabeled data available. To this end, we have begun exploring unsupervised methods to learn an effective vector representation of sequences. We have built DNA and protein sequence autoencoders that can reconstruct 100 -character sequences with $99 \%$ accuracy. These representations are being connected to supervised tasks, as well as being analyzed for biomarkers and feature interactions.

\section{PROPOSED FUTURE WORK}

Our plans for FY 2018 include improving the performance of existing neural networks and delving deeper into interpretable models. We are examining classification errors in difficult protein annotation and antimicrobial resistance cases. We will integrate gene order and protein family information and investigate their interaction with existing short sequence features. We have also identified multiple promising methods that look into the "black box" of neural networks to reveal mechanistic explanations. 


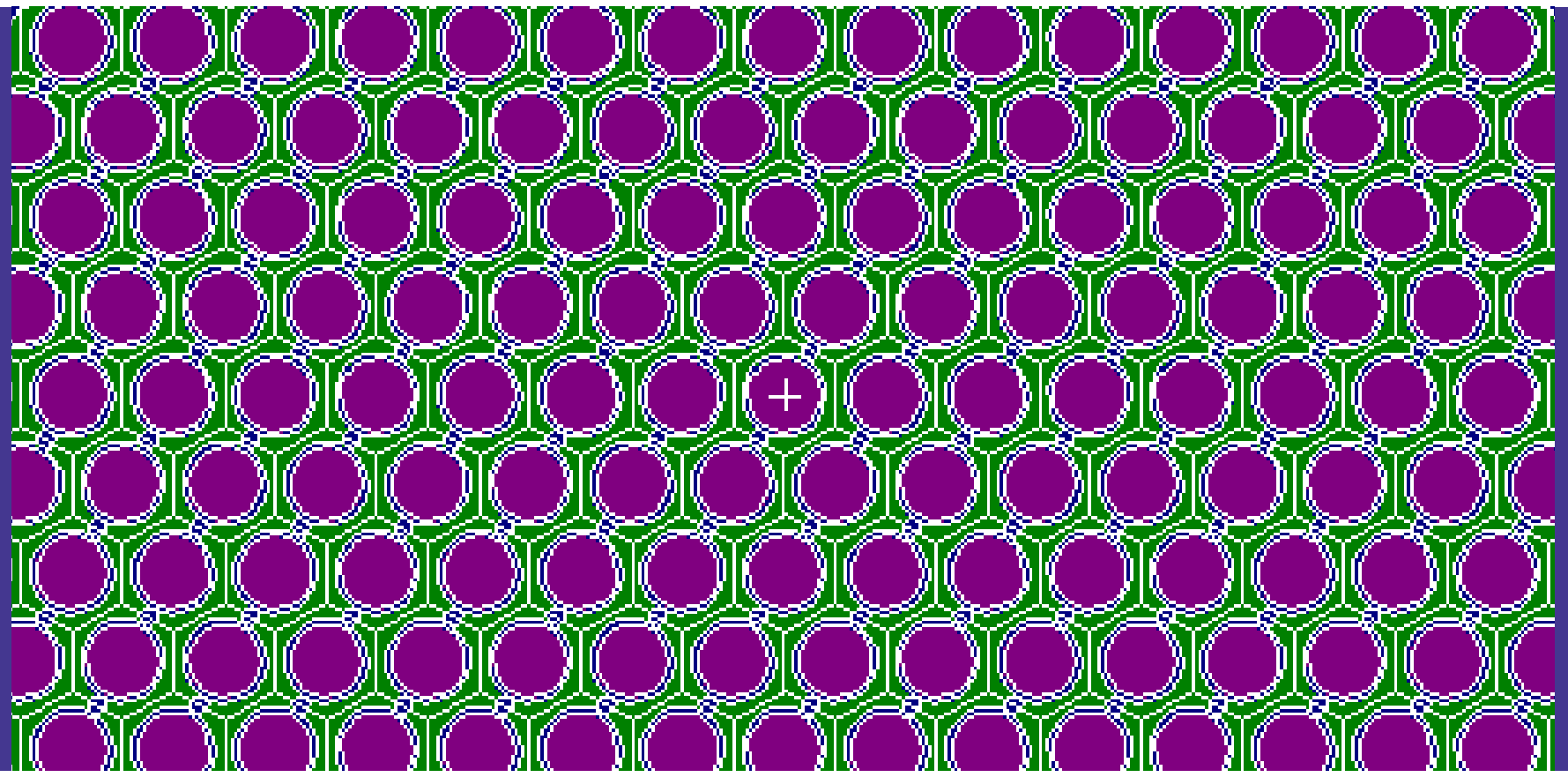

\section{LDRD PRIME -}

NUCLEAR ENERGY

SCIENCE AND

TECHNOLOGY 


\section{Development of Analysis Methods for Non-Destructive Evaluation of Concrete Degradation in Light Water Reactors}

\author{
2016-140-R1
}

Alexander Heifetz, Sasan Bakhtiari, Mitchell Farmer, Valerii Vinokur, and Peter Zapol

\section{PROJECT DESCRIPTION}

Alkali-silica reaction (ASR) has been identified by the U.S. Nuclear Regulatory Commission (NRC) as one of the causes of damage in light water reactor (LWR) structural concrete. This type of damage poses a challenge to the re-licensing of aging reactors. ASR is known as a common cause of damage in concrete structures more than 25 years old. Damage consists of concrete expansion, which leads to cracks and structure geometrical distortion. There is no remedy for preventing the occurrence of ASR, and mitigation of ASR is required for high-value structures such as LWRs. Because there is no practical quantitative nondestructive evaluation (NDE) method for identifying ASR at present, evaluation of ASR is performed with optical and electron microscopy, which requires coring of concrete structures. Developing a method for NDE of ASR would minimize the amount of coring needed for frequent monitoring of LWR concrete structures. The only reliable indicator of ASR that can be obtained from macroscopic NDE measurements is concrete length expansion. In this project, we consider applications of electro-physical NDE methods, specifically microwave backscattering and impedance spectroscopy, to discover signatures in the data that (1) provide quantitative information about ASR, and (2) correlate with length expansion reference measurements. Validation tests are performed with accelerated small-prism ASR specimens developed under controlled laboratory conditions.

\section{MISSION RELEVANCE}

Outcomes of this project are of interest to the DOE Office of Nuclear Energy's LWR Sustainability Program (LWRS), as well as to the NRC and the Electric Power Research Institute (EPRI).

\section{RESULTS AND ACCOMPLISHMENTS}

In partnership with an industrial laboratory specializing in concrete material development and testing (CTLGroup), we developed a set of concrete specimens at different stages of ASR damage. Specimens were prepared from a concrete mix known to be highly prone to ASR and placed into an environmental humidity chamber. The reaction was monitored over a period of one year, with length expansion measurements taken on specimens. Specimens were taken from the chamber at regular time intervals. Removal of a specimen from the chamber froze ASR progression. On completion of the one-year study, the specimens were brought to Argonne. Microwave backscattering in the X-band (8.2 GHz to $12.4 \mathrm{GHz}$ ) was recorded in the time domain to isolate surface scattering from bulk scattering. Readings were taken on dry and wet specimens to calculate dielectric permittivity under different conditions. Microwave backscattering data were analyzed using the Maxwell-Garnett dielectric permittivity effective medium model. A procedure was developed to calculate the fraction of ASR gel at the surface of concrete specimens. The temporal profile of this variable is the same as that of the length expansion. Figure 1 shows the normalized volume fraction of the gel and the normalized length expansion plotted as functions of time. The actual amount of surface gel fraction approximately matches the amount of gel observed by optical microscopy. A similar result was obtained for resistivity of concrete specimens, extracted from impedance spectroscopy data. In addition, thermal tomography of surfaces also revealed the presence of the ASR gel.

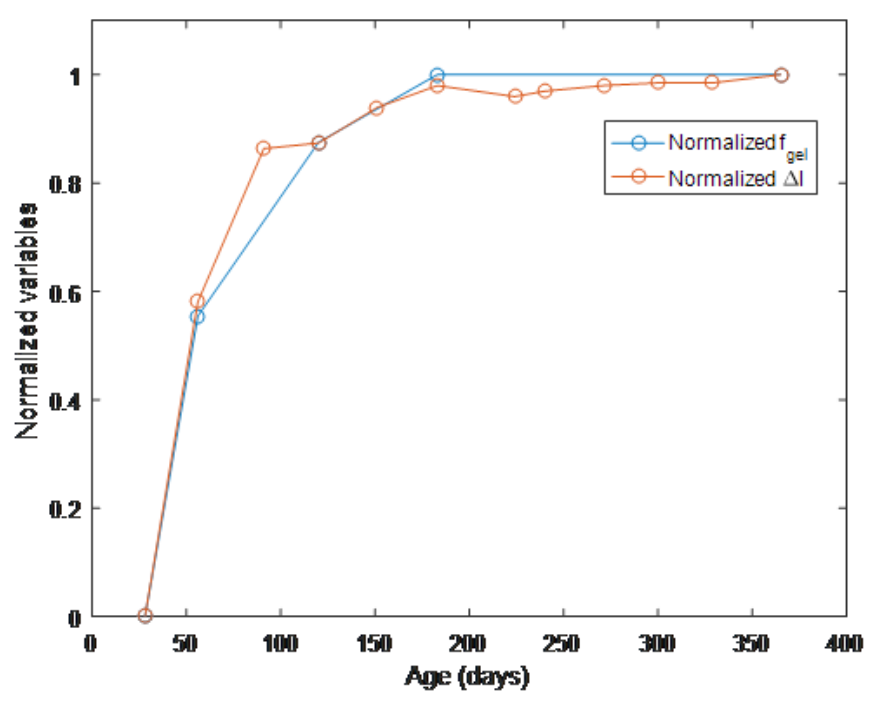

Figure 1. Comparison of normalized length expansion (the standard NDE marker of ASR) and volume fraction of ASR gel estimated from the Maxwell-Garnett model using microwave backscattering data on reactive specimens.

\section{PROPOSED FUTURE WORK}

Discussions are currently under way with the DOE LWRS, NRC, and EPRI regarding follow-on funding for this project. 


\section{The Production of Medical Isotopes Using the Argonne Electron LINAC}

\section{6-158-R1} M. Alex Brown, Jerry A. Nolen, David Rotsch,
and George F. Vandegrift

\section{PROJECT DESCRIPTION}

The 50-MeV/25-kW electron linear accelerator (LINAC) in Argonne's low-energy accelerator facility (LEAF, shown in Figure 1) is capable of producing large quantities of unique isotopes with bremsstrahlung photons. We evaluated it for the production and purification of novel, tumor-destroying therapeutic radionuclides-scandium-47 (Sc-47) and actinium-225 (Ac-225)-used in targeted radiotherapy.

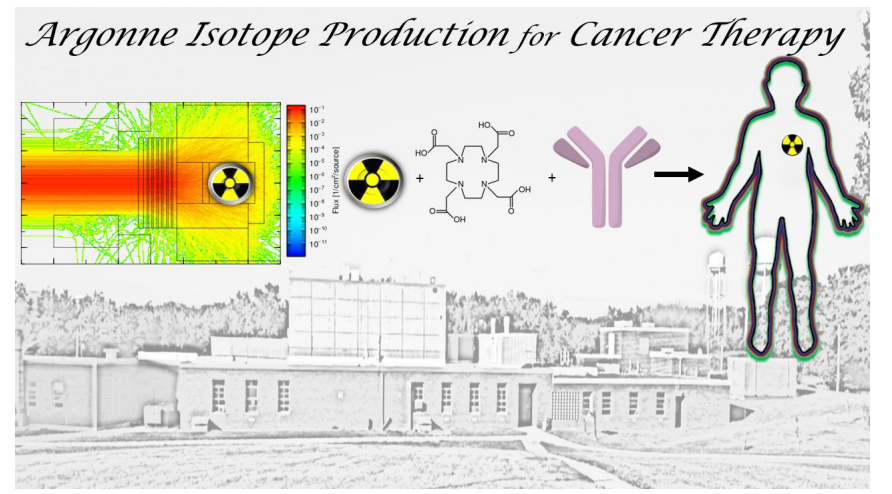

Figure 1. The Argonne LEAF (pictured) uses a high-powered, low-energy electron linear accelerator (LINAC) to produce high-priority medical isotopes for the treatment of cancers and infectious diseases. On the left is a Monte-Carlo simulation of high-powered electrons accelerating into a tantalum target. The isotope is then chemically purified and can be complexed with a bi-functional chelating agent/anti-body before delivery.

This project was directed toward the production and purification of two high-priority medical isotopes: scandium-47 and actinium-225. With titanium and radium targets, respectively, we explored the unique and challenging aspects of isotope production targetry, which demands a comprehensive understanding of materials chemistry, nuclear physics, nuclear engineering and radiochemistry. In parallel, we employed intense computational work to simulate the production yields of these isotopes.

\section{MISSION RELEVANCE}

Using safe, efficient, and cost-effective electron LINACs to produce high-demand medical isotopes that can safely destroy cancers and infectious diseases aligns with DOE's goals to expand the peaceful use of nuclear energy into commercialization, while simultaneously exploring ground-breaking science.

\section{RESULTS AND ACCOMPLISHMENTS}

To date, we have completed five irradiations at the LEAF, each producing milli-Curie amounts of high-specific-activity Sc-47. The production cycle entailed target preparation, accelerator tuning and preparation, radiometric analysis, chemical processing, and product evaluation. We explored a wide array targets-including oxides and carbide targets-each of which demands a unique chemical treatment to recover the valuable isotopes as well as costly enriched target materials. Using a novel chemical separation process developed in-house, our separation efficiencies range between $90 \%$ and $95 \%$ recovery in a chemical form that is ready for the next step of chelation studies or animal trials.

The results surrounding the production of Sc-47 are encouraging, not only on the laboratory scale but from the standpoint of large-scale production, indicating that Argonne could become a major contributor to the domestic isotope stockpile. Furthermore, a joint proposal with the University of Chicago Medical Center was recently funded to explore the production and chelation of Sc-47.

We have also explored the production of Ac-225, designated as the highest-priority isotope by the DOE Office of Nuclear Physics - National Isotope Development Center (NIDC), at the LEAF from a theoretical standpoint. Our photonuclear simulations predict that the Argonne electron LINAC could potentially meet the world's annual demand of Ac-225 in a matter of weeks.

This challenging but potentially life-saving isotope originates from an irradiated radium target. Our group has dedicated substantial time to the safe development of a unique target, target holders and stations, new efficient chemical separations and recovery processes, and photonuclear simulations that allow us to maximize the yields and recovery of Ac-225.

\section{PROPOSED FUTURE WORK}

With support for FY 2018, we will focus exclusively on the production of Ac-225. Completing two pilot irradiations is critical for the development of this unique isotope and for securing additional funds from potential sponsors. Having already completed the engineering designs for special target holders to accommodate these irradiations, we plan to fabricate these unique target holders in early FY 2018 and conduct the irradiations and analysis in the summer. 


\section{Additive Manufacturing for Nuclear Energy Applications}

\author{
2016-159-R1
}

Abdellatif M. Yacout, Peter Kozak, Kun Mo, and Michael Pellin

\section{PROJECT DESCRIPTION}

Recently, significant attention has been focused on additive manufacturing (AM) as a tool to produce precise and complex forms and/or structures, with the potential for application to large-scale manufacturing. This project considers two independent methods for low-cost, scalable 3D printing of metal for nuclear fuel applications: (1) use of metal-impregnated filament, and (2) metallic paste and/or slurry. Both of these techniques will require post-processing to remove the plastic matrix material and sinter the part, and both methods will involve the challenges of dealing with dimensional changes during sintering and porosity mitigation.

\section{MISSION RELEVANCE}

The application of AM to produce uranium molybdenum (U-Mo) foils, which are difficult to fabricate using conventional methods, will benefit the DOE National Nuclear Security Administration's material minimization efforts as well as activities related to converting high-power research reactors from the use of highly enriched uranium to low enriched uranium, as this can lead to major cost savings and reduction in waste associated with the fabrication process.

\section{RESULTS AND ACCOMPLISHMENTS}

More than 50 grams of $\mathrm{UO}_{2}$ powder have been prepared by grinding depleted $\mathrm{UO}_{2}$ pellets in a shatter box. (The depleted $\mathrm{UO}_{2}$ pellets were fabricated by the standard procedure of manufacturing light-water reactor fuel pellets.) The $\mathrm{UO}_{2}$ powder was transferred to the "glove box" and mixed with mineral oil and polylactic acid pellets. The mixture was then melted and processed in the filament extruder, which extruded the $\mathrm{UO}_{2}$ filaments. From the filaments, $\mathrm{UO}_{2}$ fuel pellets were printed out by the $3 \mathrm{D}$ printer. The success of printing $\cup_{2}$ pellets demonstrates our capability in the additive manufacturing of radioactive fuel materials.

Two other AM methods have been investigated: (1) the stereolithography assembly (SLA) method, and (2) the high-resolution AM method (based on two-photon polymerization [2PP]). Both methods provide a much higher resolution during 3D printing than the Fused Deposition Modeling (FDM) method (Figure 1). To apply the SLA method to a nuclear fuel application, the fuel powder (metal or oxide powders) is mixed with a liquid resin to produce impregnated acrylic objects. Focused ultraviolet (UV) beams are then generated by a stereolithography 3D printer to initiate polymerization of the resin. The fuel powder is encapsulated in a photopolymer resin cured by UV light. The final step is applying high-temperature annealing to remove the resin matrix but preserve the ceramic/metal fuel structures. This basic procedure was developed in FY 2017.

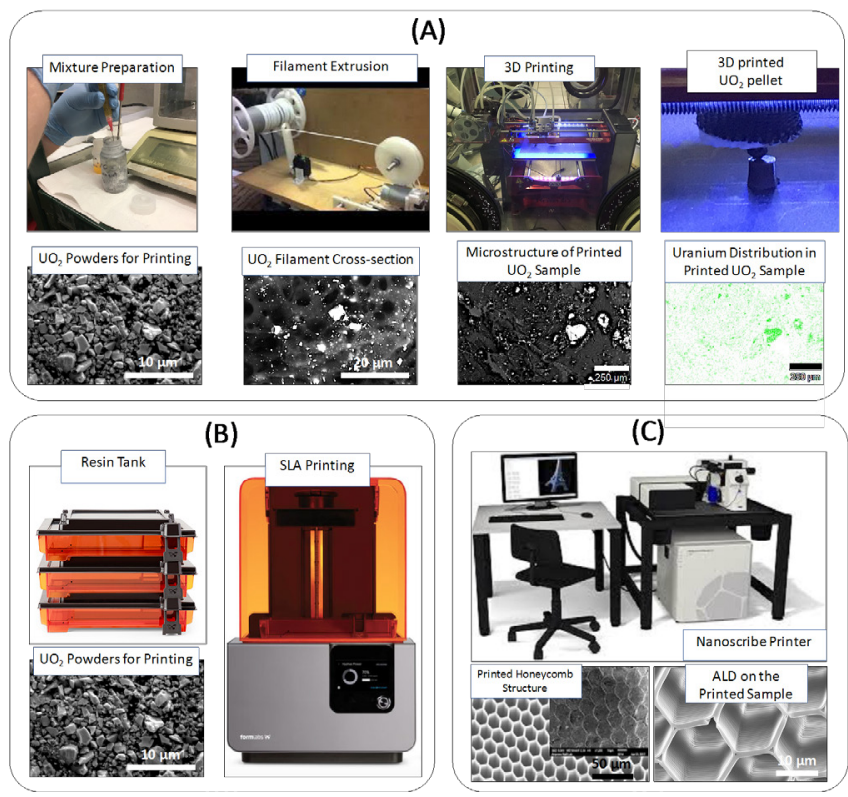

Figure 1. (A) 3D printing of $\mathrm{UO}_{2}$ based on the FDM method; (B) investigation of applying the stereolithography (SLA) method to print $\mathrm{UO}_{2}$ materials; and (C) study of a very-high-resolution AM method based on two-photon polymerization (2PP).

The other promising AM method is the 2PP technique, which can print micro- and nanostructures. To apply it to nuclear fuel AM, the 2PP technique is combined with a hybrid infiltration technique recently developed in our research group, which allows for nano-powder infiltration and agglomeration into those structures. This hybrid infiltration technique allows fuel powder to effectively fill in the printed subject.

\section{PROPOSED FUTURE WORK}

In FY 2018, we plan to continue to optimize the process parameters in 3D printing (raw powder size and sintering temperature, etc.) and demonstrate 3D-printed nuclear fuel materials by one or more AM methods. Both the stereolithography assembly method and the 2PP method will be studied further, and we will work toward printing a uranium metal alloy. 


\section{Conceptual Design of a Flexible Spectrum Test Reactor (FSTR)}

\author{
2017-030-NO \\ Bo Feng, Paul K. Romano, Dillon Shaver, and Nicolas Stauff
}

\section{PROJECT DESCRIPTION}

The goals of this project are twofold: to develop both (1) an innovative fuel and core design for a versatile test reactor, and (2) the reactor physics tools needed to accurately model such a design. Specifically, we propose using internally and externally cooled metallic annular fuel in a sodium-cooled fast reactor. This geometry would enable higher flux levels because of the higher power densities it produces, while ensuring sufficient heat removal. In addition, we envision that the core will be flexible; the user can change the neutron energy spectrum by replacing some of the fuel volume with moderating material. To capture this spectrum change, we are modifying Argonne's advanced reactor code suite so that it can model important characteristics for both fast and thermal spectrum reactors.

\section{MISSION RELEVANCE}

This project supports the DOE energy mission. DOE's Office of Nuclear Energy recently approved a program to investigate the design of a versatile test reactor (VTR). This VTR will be constructed in the United States to provide valuable fast flux for testing fuels and materials for new reactor concepts. Later versions of the VTR core can adopt the innovative fuel and core designs proposed in this work, which would greatly increase the testing volume under high flux conditions while maintaining a smaller, more economical core size.

\section{RESULTS AND ACCOMPLISHMENTS}

We made improvements to Argonne's advanced reactor deterministic code system to enable modeling of fuel depletion within a thermal spectrum. Specifically, we now have the ability to re-generate the multi-group cross sections via $\mathrm{MC}^{2}-3$ at each depletion step while running the REBUS (REactor BUrnup System) fuel cycle code. This project also supported the development of depletion capabilities for the stochastic code OpenMC, which uses continuous energy cross sections, as another means to verify the REBUS code's calculations.

These code improvements were utilized in the preliminary core designs for the thermal, fast, and mixed spectrum versions of the test reactor. Figure 1 shows a proposed fast test reactor assembly with traditional cylindrical fuel pins. We completed the geometric design of the annular fuel assembly, as shown in Figure 2, along with scoping studies for thermal-hydraulic assessment. The estimated width of the hexagonal assembly was maintained at $11.87 \mathrm{~cm}$ for both designs. To maintain at least $90 \%$ of the same fuel volume in the new design, the number of pin rows had to be reduced from 10 to 6 . As long as there is sufficient heat removal, using this annular fuel would allow the core volume to decrease by approximately $1 / 3$ (which increases the power density by approximately $50 \%$ ). This smaller core actually increases by a factor of 2.7 the total volume in which the fast flux $(E>0.1 \mathrm{MeV})$ target of $5 \times 10^{15} \mathrm{n} \mathrm{cm}^{-2} \mathrm{~s}^{-1}$ is met. With a more modest core volume reduction of approximately $1 / 6$ (and subsequent power density increase of approximately $20 \%$ ), the total fast flux testing volume can still be increased by a factor of 2.3. Thus, this innovative fuel design shows promise for reducing core size, and potentially capital costs, while increasing fast flux testing capabilities.

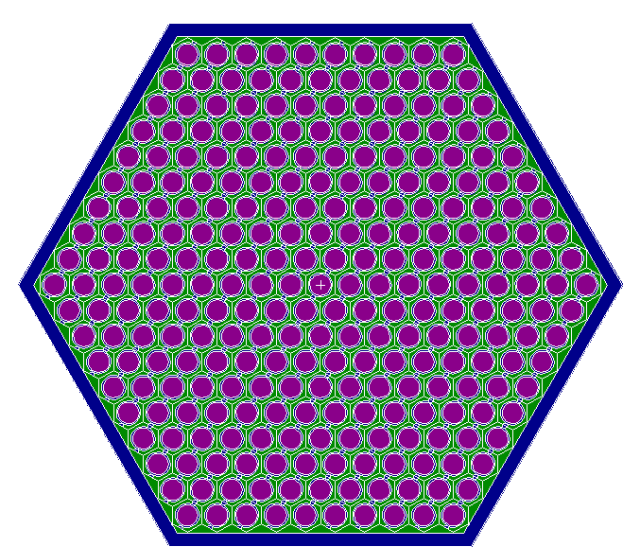

Figure 1. Test reactor assembly with traditional cylindrical metallic fuel pins (purple) with flowing sodium (green).

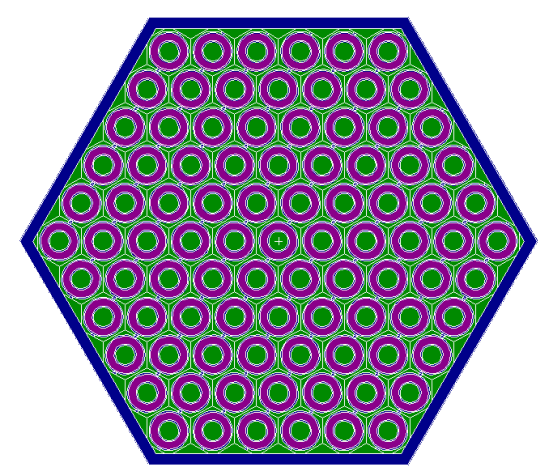

Figure 2. Test reactor assembly with innovative annular metallic fuel pins (purple) with flowing sodium (green).

\section{PROPOSED FUTURE WORK}

We will share our results with the VTR program. 


\section{Development of Molten Salt Reactor Analysis Computation Tools to Support Emerging Markets}

\section{7-040-NO}

Florent Heidet, Rui Hu, and Mark A. Williamson

\section{PROJECT DESCRIPTION}

Over the past few years, a renewed interest in molten salt reactors (MSRs) has been spreading rapidly around the world and particularly in the United States. MSRs come in two flavors. The first type uses liquid fuel circulating outside of the core, and is the focus of this project, while the second type relies on solid fuel and uses salt as a coolant. The influx of private funding enabled the creation of many startups aimed at developing MSR concepts and advancing the associated technology.

Given that MSRs have liquid fuel containing heavy metals flowing in and out of the reactor core, traditional analysis methods and tools fail to capture specific phenomena. The purpose of this project is to develop key basic capabilities that will allow the study and analysis of most kinds of MSRs. The intent is to make use of existing tools, when relevant, by expanding their capabilities, and to develop new capabilities when no appropriate tool is available.

Capabilities of interest target reactor physics, fuel composition evolution, thermal hydraulics, and molten salt properties.

The tools and methods developed as part of this project are the first stepping stone in the direction of providing a comprehensive toolset for analysis of MSRs.

\section{MISSION RELEVANCE}

With the resurging interest in MSRs, this effort is directly relevant to the Advanced Reactor Technology campaign of DOE-Nuclear Energy (NE). The tools/ methods developed here will be useful to DOE-NE programs by providing expertise on MSRs and also enabling collaborations between the DOE-NE and the MSR startups.

Other DOE-NE programs that would benefit from this work are the Nuclear Energy Advanced Modeling and Simulation (NEAMS) program, as well as the Gateway for Accelerated Innovation in Nuclear (GAIN) program.

\section{RESULTS AND ACCOMPLISHMENTS}

Tools have been developed (code written) to interface neutron flux solver Monte Carlo N-Particle Transport Code (MCNP) with depletion capabilities (ORIGEN2.2) and the basic thermal hydraulics capabilities of the System Analysis Module (SAM). Changes in salt properties as a function of temperature have also been captured directly through the SAM software.

SAM capabilities have been extended to enable radiative heat transfer modeling between two wall surfaces, to incorporate equations of state for a few salts and to implement general scalar transport capabilities.

In order to facilitate accurate modeling of the fuel cycle of MSRs, new capabilities were developed from scratch, allowing us to model the complex transit of molten salt through the reactor system and to properly account for elemental removal, fuel make-up, and different cooling/ residence times.

A salt property database containing a few of the most commonly used salts was also constructed, which will enable easy retrieval of the information needed to perform MSR simulations.

\section{PROPOSED FUTURE WORK}

Capabilities under development that will be completedpoint kinetic capabilities accounting for precursor driftwill be implemented in SAM.

Overall analysis capabilities through this project will be demonstrated by using these tools for analysis of existing or conceptual MSR designs. The objective is to publish those results and discuss their benefits over previously existing capabilities (at Argonne and elsewhere).

A future objective is also to engage with industry in order to develop partnerships and collaborations on the development of MSRs. 


\section{Technological Improvement to Increase Scalability of Ex-Vessel Melt Coolability and Concrete Interaction Experiments}

\author{
2017-041-NO
}

Jeremy Licht, Mitchell Farmer, and Craig Gerardi

\section{PROJECT DESCRIPTION}

In a core melt accident of a nuclear power plant, failure of the reactor vessel will result in core debris relocation to the reactor cavity region where its interaction with the concrete basemat can result in containment failure and release of fission products into the environment. While previous tests have significantly contributed to our knowledge base of the mechanisms for core melt/ debris cooling, there are still opportunities to increase confidence in scaling experimental results to plant conditions. A significant contributor to this scaling issue is that, in current experiments, the crust that forms over the melt-water interface can rigidly bond (or anchor) to the test section sidewalls. As a result, a void between the crust and melt can form due to melt eruptions and concrete densification of the melt into the concrete.

The goal of this project was to perform several one-dimensional (1-D) small-scale, non-radiological experiments to demonstrate an alternative test configuration for improved melt-crust contact. A floating concrete sleeve configuration was the basis for testing, as this design appeared to be a simple and effective method.

The aim of the work was to design, build, and commission the small-scale apparatus primarily utilizing existing Argonne facilities, equipment, and expertise.

\section{MISSION RELEVANCE}

This project ultimately supports DOE's light water reactor sustainability program and the Office of Advanced Reactor Technologies while benefiting from the Organisation for Economic Co-operation and Development/Nuclear Energy Agency member countries' common interests in nuclear reactor safety-thirteen countries having participated in previous experiments performed at Argonne.

\section{RESULTS AND ACCOMPLISHMENTS}

The primary goals were to prepare the lab space (containment cell) where the experiments will be performed, complete detailed design of the experimental apparatus, and perform commissioning tests.

Existing equipment in the proposed lab space (some with suspected internal contamination) was removed and a new ladder and safety railing on the containment was installed to meet current safety standards.

We also designed and installed a ventilation system for the containment to handle minute amounts of hydrogen, hot gases, and incidental airborne thermite powder.

We performed thermite ignition tests to verify the performance of the thermite material and to commission the thermite ignition system.

A 50-kW induction power supply, intended to maintain the melt at a target temperature of $\sim 2,000^{\circ} \mathrm{C}$, was designed, procured, and installed.

A data acquisition system was installed in order to monitor and record temperatures of the melt and experimental apparatus and to ensure maintenance of the melt temperature and tracking of the melt depth into the concrete.

Finally, we designed and constructed a test apparatus to perform a commissioning test for baseline experiments that demonstrates an anchored sidewall.

The technological improvements intended to Increase scalability of ex-vessel melt coolability and concrete interaction experiments were thus achieved.

\section{PROPOSED FUTURE WORK}

This project was completed in FY 2017. 


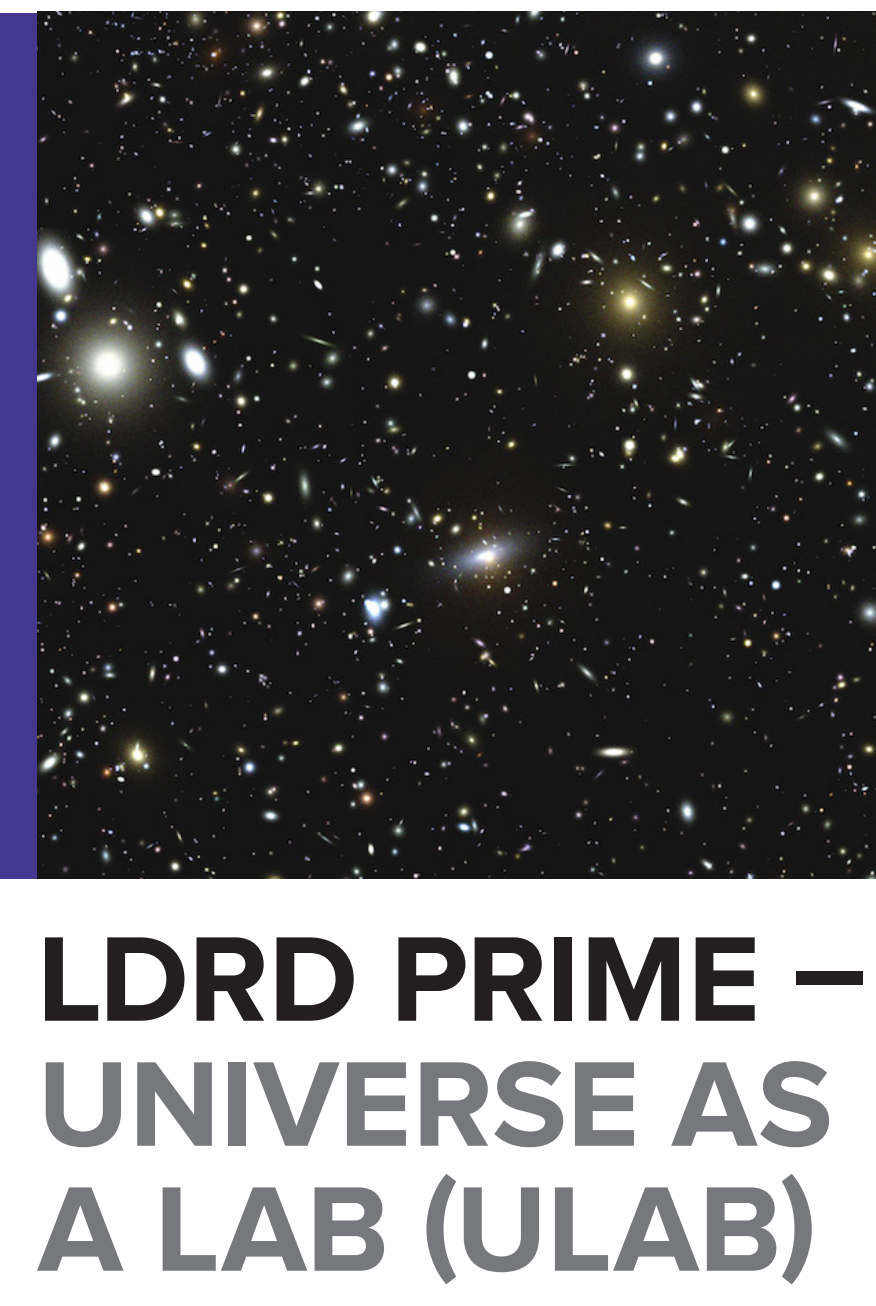




\section{A Strategic Scientific Program to Establish Argonne Leadership in the Development of the Future Electron-Ion Collider}

\author{
2017-058-NO
}

José Repond, Whitney Armstrong, Geoffrey Bodwin, Radja Boughezal, Sergei Chekanov, Ian Cloët, Zachary Conway, Kawtar Hafidi, Mohammad Hattawy, Thomas LeCompte, Stephen Magill, Jessica Metcalfe, Cédric J. Mezrag, Brahim Mustapha, Peter Ostroumov, Alexander Plastun, David Potterveld, Junqi Xie, Zhihong Ye, and Jinlong Zhang

\section{PROJECT DESCRIPTION}

The goal of this project is to pursue several specific research goals that will establish Argonne as a major contributor to the planning, development, design, and construction of the Electron-Ion Collider (EIC), a future billion-dollar facility that will study the nature of strongly interacting matter. We are tackling four tightly intertwined subtasks: theory developments (calculation of parton densities in nuclei), simulations (of various detector concepts), detector R\&D (development of MCP-PMT and ultra-fast silicon detectors), and accelerator R\&D (development of an alternative ion accelerator complex).

\section{MISSION RELEVANCE}

The EIC was identified in the 2015 Long Range Plan for Nuclear Science as the highest priority for new facility construction, with mapping of the gluon content of nucleons and nuclei as the central goal.

\section{RESULTS AND ACCOMPLISHMENTS}

Logistics. Three postdoctoral research assistants were hired and a graduate student joined the EIC accelerator design effort. The computing hardware required for carrying out the simulation effort has been acquired and commissioned.

Task 1: Theory. The primary objective of the EIC is to measure the three-dimensional (3D) structure of atomic nuclei in terms of quarks and gluons, as expressed in general parton distributions (GPDs) and transverse momentum distributions (TMDs). We have initiated a theoretical investigation of GPDs and TMDs for light nuclei by constructing a Poincaré-invariant Lagrangian characterized by a contact interaction between the nucleons. As a first step, results for the deuteron $\mathrm{F}_{2 \mathrm{D}}(\mathrm{x})$ structure function were obtained, showing excellent agreement with available experimental data.
Task 2: Simulation. We assembled a complete toolchain for the simulation of EIC colliding beam events in the various EIC detector concepts under development. We developed a novel concept of a multipurpose $4 \pi$ detector with $5 \mathrm{D}$ readout, which is the TOPSIDE concept (see Figure 1). Utilizing the toolchain, we reconstructed the $F_{2}$ structure function used to generate the events by analyzing fully simulated, digitized, and reconstructed events in TOPSIDE.

Task 3: Detector R\&D. We tested $6 \times 6 \mathrm{~cm}^{2}$ microchannel plate photomultipliers (MCP-PMTs) in magnetic fields up to 1.8 Tesla. These devices are deemed essential for the EIC. We also initiated the development of a time distribution system for ultra-fast silicon sensors, with the goal of limiting the time jitter to less than 10 ps.

Task 4: Accelerator. We studied the feasibility of using the JLab EIC electron storage ring as a large booster for the ions in an attempt to reduce both the overall risk of the project and its cost. We initiated the characterization of pulsed quarter wave resonators.

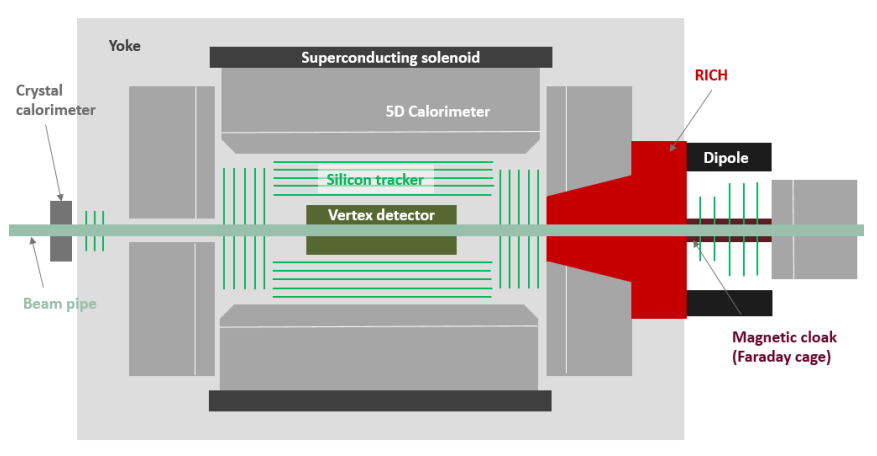

Figure 1. Argonne's concept of a multipurpose $4 \pi$ detector for the EIC.

\section{PROPOSED FUTURE WORK}

We will calculate the GPDs and TMDs for light nuclei within a covariant contact model. We will replace and update all obsolete and non-maintainable tools in our physics and detector simulation tool chest. We will optimize our detector concept using processes particularly important at the EIC. We will also optimize the design of the MCP-PMTs to be operational in magnetic fields. We will join existing collaborations to develop ultra-fast silicon sensors and further the development of a time distribution system with extremely low time jitter. We will develop a design concept for the new ion injection scheme. Our efforts will naturally result in strong collaborations with outside institutions that share our scientific goals. 


\section{Superconducting Detectors for Future CMB Experiments}

\section{7-105-NO}

Stephen Padin, Clarence Chang, and Valentine Novosad

\section{PROJECT DESCRIPTION}

This project advances the science and technology of superconducting detectors, with the goal of demonstrating detector performance appropriate for the upcoming Cosmic Microwave Background Stage 4 experiment (CMB-S4). CMB-S4 will make exquisitely sensitive observations of the CMB in order to measure the energy scale of inflation, or rule out the more compelling models of inflation. CMB-S4 will also search for evidence of weakly interacting light particles beyond the Standard Model.

Superconducting detectors are the key technology for CMB observations, and transition edge sensor (TES) bolometer arrays are currently the most successful superconducting detectors; thus, the focus of this project is on materials and devices for TES arrays.

CMB-S4 will need many observing bands to support removal of foreground signals from our galaxy, and the bands will be narrower than in existing CMB experiments. Narrower bands will require more sensitive detectors, which in turn will require TESs with lower transition temperature and bolometer thermal isolation structures with lower thermal conductivity. CMB-S4 will also need a large number of detectors, which will require fabrication techniques that yield stable detectors with consistent performance. A particular concern is oxidation of the $\mathrm{Nb}$ wiring and microstrip lines that are used for readout and signal connections.

The major tasks for this project are to demonstrate good control of both the TES transition temperature and the thermal conductivity of the long, thin legs that support the bolometers, and to demonstrate a protective nitride coating for $\mathrm{Nb}$ connections.

\section{MISSION RELEVANCE}

This project is relevant to DOE's mission in basic science. It aims to demonstrate detector components with performance appropriate for the upcoming CMB-S4 experiment. CMB-S4 will be the next major project for the DOE High Energy Physics Cosmic Frontier Program.

\section{RESULTS AND ACCOMPLISHMENTS}

We developed a gold/titanium/gold (Au/Ti/Au) TES structure that gives steep, smooth transitions (a prerequisite for high sensitivity) and allows good control of transition temperature, $T_{c}$, in the 400 - to $500-\mathrm{mK}$ range. The Au layers protect the Ti TES from the substrate and atmosphere and provide control of $T_{c}$ through the proximity effect. We mapped $T_{c}$ versus the thickness of $\mathrm{Au}$ and $\mathrm{Ti}$, and the South Pole Telescope Group used this information to design new TES arrays for CMB observations from the South Pole. We fabricated and tested aluminum/ manganese (Al/Mn) TES films and demonstrated control of $\mathrm{T}_{\mathrm{c}}$ in the 350 - to 500 - $\mathrm{mK}$ range by adjusting the $\mathrm{Mn}$ content and by heating the films. We also fabricated and tested molybdenum/cobalt (Mo/Co) films with $T_{c}$ in the 100 - to $150-\mathrm{mK}$ range.

We developed fabrication processes that allow better control of the release of the bolometer structures from the Si substrate. The legs that support the bolometers are especially important because they set the thermal conductivity, G, which in turn sets the bolometer operating power and sensitivity. Repeatable $\mathrm{G}$ requires careful control of the amount of etching of the legs during release. Thus, we developed endpoint detection procedures based on optical microscope measurements and demonstrated the use of a thin $\mathrm{SiO}_{2}$ layer to protect the bolometer legs during release. We also developed bolometer designs with thin features that release quickly, so there is less time to etch the legs. We measured the thermal conductivity contributions from the various films on the bolometer legs and tested the thermal transport model, which supports predictions of $\mathrm{G}$ for new bolometer designs.

We demonstrated low loss in niobium nitride ( $\mathrm{NbN}$ ) coplanar waveguide resonators and fabricated and tested a prototype TES array with a thin $\mathrm{NbN}$ coating on the $\mathrm{Nb}$ microstrip ground layer. Adding the $\mathrm{NbN}$ coating did not degrade the optical efficiency, which makes this a promising approach for improving the resilience of our TES arrays.

\section{PROPOSED FUTURE WORK}

The project will continue through FY 2018. We will incorporate what has been learned about $T_{c}$ and $G$ into complete detector pixel designs. Our goal is to demonstrate detector pixels that meet the most challenging bandwidth and sensitivity requirements expected for CMB-S4. Separate detector work in parallel with this project will be supported by CMB-S4 pre-project funds in FY 2018. 


\section{Exploring the Universe: Large-Scale Structure to the First Stars}

\section{7-106-NO}

Katrin Heitmann, John Anderson, Melina Avila Coronado, Ramesh Balakrishnan, Lindsey Bleem, Michael Carpenter, Salman Habib, Eve Kovacs, and Ravi Madduri

\section{PROJECT DESCRIPTION}

Cosmology has come a long way in the last three decades. Starting from a rough picture of the evolution of the universe, we have now established a precise cosmological model that describes the content and evolution of the universe at a level of accuracy that we believe is within a few percentage points. We have arrived at this point by understanding the physics of the cosmic microwave background (CMB) and by combining CMB observations with measurements from large-scale structure surveys that map out the distribution of galaxies in the universe. This project will deliver a combined approach to address the different challenges we are facing in cosmology-from the handling of very large datasets, to sophisticated simulations including detailed physics descriptions, to cross-correlations of different probes, and experiments to help us understand the formation of the first stars.

\section{MISSION RELEVANCE}

The project addresses key areas in domains relevant to DOE's science mission: specifically, to High Energy Physics (Cosmic Frontier), DOE Nuclear Physics (nuclear astrophysics), and DOE Advanced Scientific Computing Research (data-intensive computing). There is significant interest in enabling the three offices to work together on problems of mutual interest in the identified domains.

\section{RESULTS AND ACCOMPLISHMENTS}

As part of this project, we developed a data-intensive computing system for Cosmic Frontier Experiments that has already been used for a range of tasks (e.g., analysis of CMB data, analysis of simulated data, and the generation of synthetic sky catalogs). We have developed the Pipeline for Images of Cosmological Strong lensing (PICS), worked on a machine learning approach to identify strong lensing systems, started the investigation of strong lensing clusters that have been found by the South Pole Telescope (SPT), implemented a full end-to-end modeling capability to generate synthetic galaxy catalogs (Figure 1 shows images of the galaxies that are stored in such a catalog), and enhanced the muon science innovative channel (MuSIC) detector.
Among several accomplishments, our work has led to improved constraints on cosmological parameters derived from the SPT sample of clusters of galaxies. Clusters of galaxies are the largest, gravitationally bound objects in the universe. Their abundance provides a powerful probe of cosmology. The major challenge is to estimate the mass of each cluster. We have implemented an improved analysis of available X-ray data, which allows for important progress in sharpening scaling relations that in turn lead to improved mass estimates and therefore tighter cosmological constraints. We have also completed the incorporation of weak-lensing data into the cosmological analysis pipeline, another way to determine cluster masses. The combination of data from different wavelengths is a central aim of this project and provides powerful means to improve our understanding of the make-up of the universe.

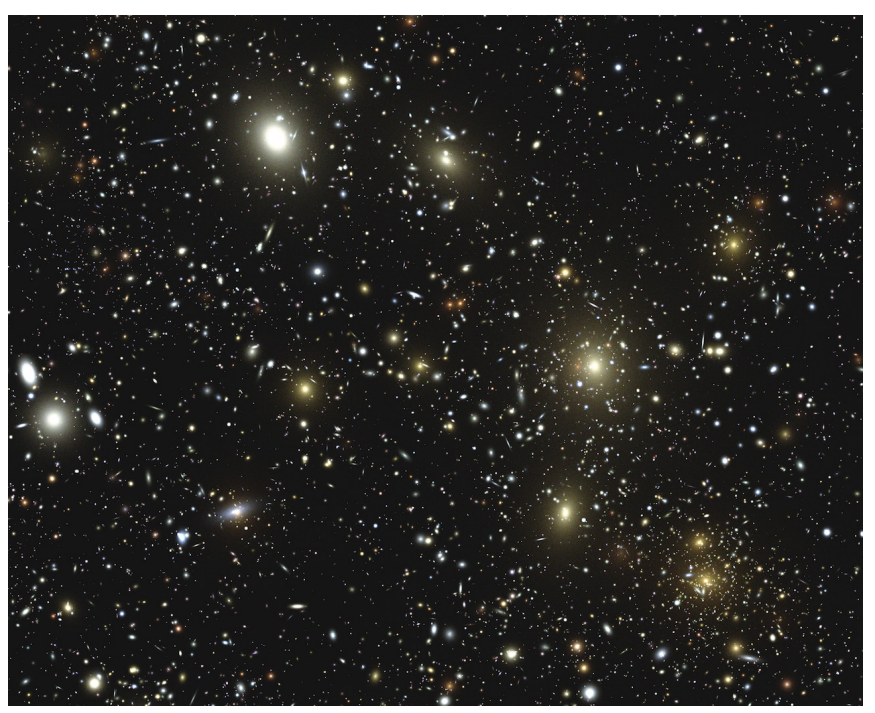

Figure 1. Simulated images of galaxies. The brightness of each image is connected to the luminosity of the galaxy shown and the distance of the galaxy to the observer (faint objects are further away). The galaxy properties are stored in a catalog that closely resembles actual observations carried out with a large telescope. Researchers can use the simulated images to test pipelines, develop new cosmological probes, and study fundamental new physics ideas by changing the assumptions that are made when generating the catalog.

\section{PROPOSED FUTURE WORK}

We will continue our work on cross-correlating optical and CMB measurements to solidify Argonne's position as the lead laboratory for SPT-3G cluster science and to CMB Stage 4 (CMB-S4) in key science areas. We will continue to build up our data-intensive computing capabilities to become a leading Cosmic Frontier Data Hub. We will continue to enhance our capabilities to build the most detailed simulated sky maps available worldwide. We will also continue to enhance the MuSIC detector and broaden the scope of possible physics measurements. 


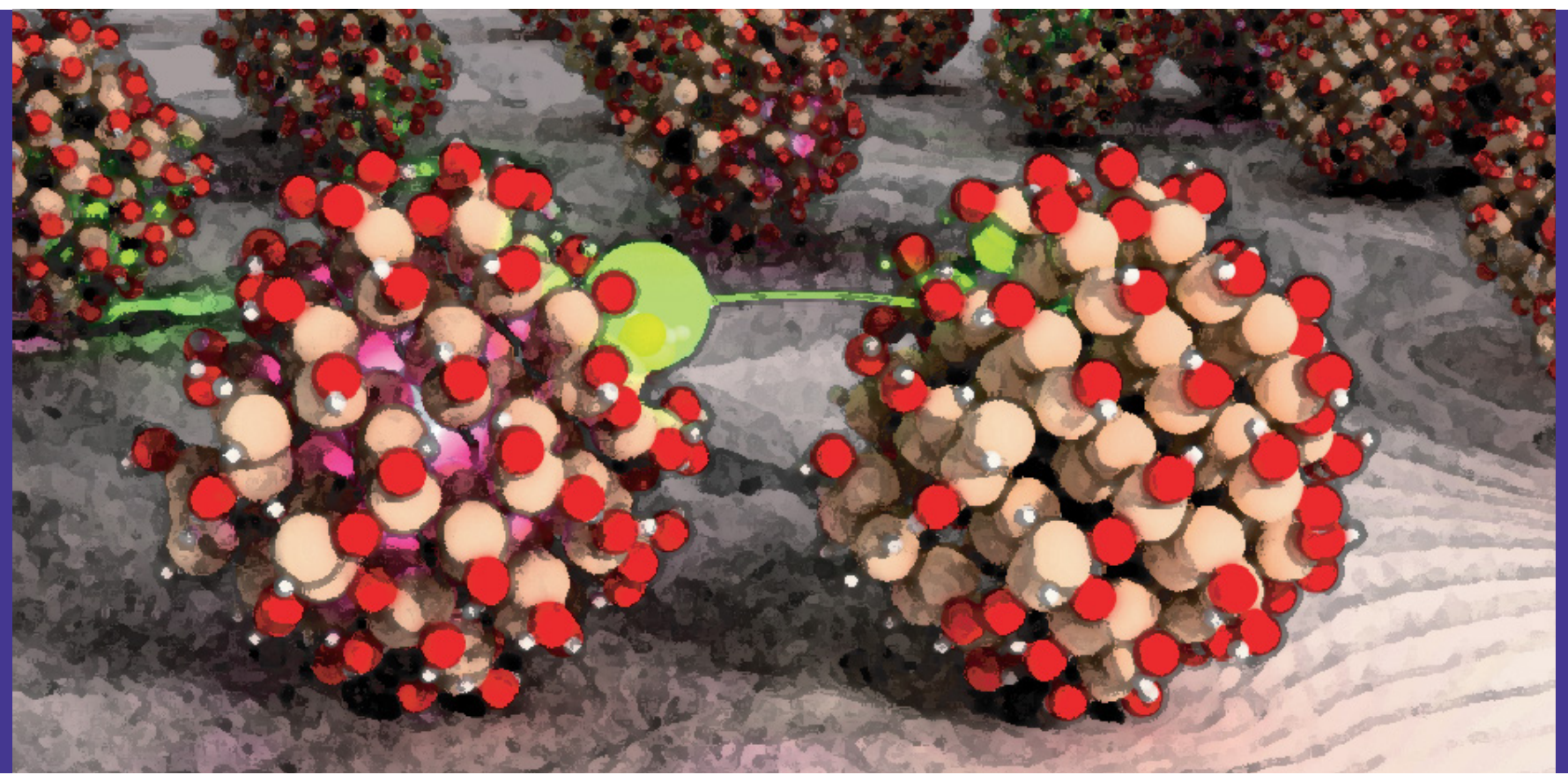

\section{OTHER NOVEL R\&D}




\section{The Computational Design of New Functional Materials from Complex Transition Metal Oxides}

\author{
2015-168-R2
}

Hyowon Park

\section{PROJECT DESCRIPTION}

The goal of this project is to study theoretically novel electronic and structural phases of complex transition metal oxide materials in which the strong correlation of electrons in transition metal ions plays an important role. Strongly correlated oxides exhibit novel electronic behaviors intimately coupled to their spin, charge, orbital, and lattice degrees of freedom. For this project, we adopted the state-of-the-art $a b$ initio method: density functional theory plus dynamical mean field theory (DFT+DMFT). The main objectives were to (1) calculate various spectroscopic quantities of complex oxides in both bulk and hetero-structured forms, and (2) develop a code for computing interatomic forces within DFT+DMFT to investigate the structural properties and energetics of oxides. The output of DFT+DMFT calculations will be directly compared to experimental measurements, including the photoemission, X-ray scattering, X-ray absorption, and neutron scattering spectra. Ultimately, our project can lead to the computational design of functional oxide materials or devices with a desired functionality.

\section{MISSION RELEVANCE}

This project is relevant to the DOE science mission. It addresses the fundamental understanding of the relationship among charge, spin, orbital, and lattice degrees of freedom in strongly correlated materials. Ultimately, the knowledge of electronic correlations among different degrees of freedom and their energetics will provide the ability to control and design new functional materials, including electronic and spintronic devices or energy materials such as batteries.

\section{RESULTS AND ACCOMPLISHMENTS}

During FY 2017, we have implemented the atomic force calculation within the DFT+DMFT method and applied it to the force calculation of strongly correlated lanthanum nickel oxide $\left(\mathrm{LaNiO}_{3}\right)$ in a rhombohedral structure as functions of oxygen position distortions. We found that phonon frequencies computed using DFT+DMFT are consistent with the experimental Raman spectroscopy measurement exhibiting the decease of the phonon frequency in the A1g rotational mode as a function of temperature. The phonon frequency in the A1g mode computed using DFT only is underestimated when compared to using DFT+DMFT, as DFT alone cannot capture the proper electronic correlation effect.
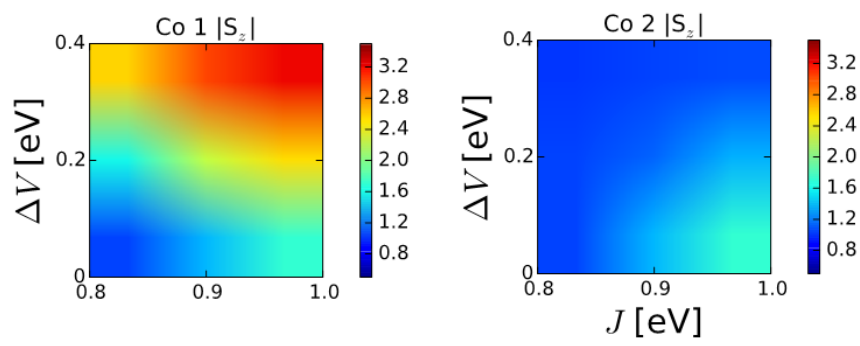

Figure 1. The averaged spin moment magnitude ( $|\mathrm{Sz}|[\mu \mathrm{B}])$ of two Co ion sites in $\mathrm{LaCoO}_{3}$ as functions of the Hund's coupling $\mathrm{J}$ and the Co $\mathrm{d}-\mathrm{O} \mathrm{p}$ charge transfer energy $\Delta \mathrm{V}$. We used DFT+DMFT with Coulomb interaction $\mathrm{U}=6 \mathrm{eV}$ and temperature $\mathrm{T}=\mathbf{1 1 6 K}$.

We have also studied the nature of mixed spin states in $\mathrm{LaCoO}_{3}$ using DFT+DMFT. The recent X-ray scattering measurements in tensile-strained $\mathrm{LaCoO}_{3}$ show evidence of rock-salt type charge ordering associated with the spin-state ordering. While there is much experimental evidence of the mixed spin states in strained samples of this material, the nature of the excited spin states in bulk $\mathrm{LaCoO}_{3}$ is still a matter of controversy. Here, we adopted DFT+DMFT to reveal the nature of spin states in bulk $\mathrm{LaCoO}_{3}$. We found that the mixed spin state (the combination of the low-spin (|Sz| 0.5 $\mu \mathrm{B}$ ) and the high-spin $(|\mathrm{Sz}| \sim 3.5 \mu \mathrm{B}))$ is indeed stable owing to the strong correlations of Co d electrons in a slightly distorted structure with the $\mathrm{Co}-\mathrm{O}$ bond length difference ( $\Delta \mathrm{a}=0.05 \AA$ ) (Figure 1 top panel: larger bond length; bottom panel: smaller bond length). This mixed spin state becomes energetically stable as the Hund's coupling $(\mathrm{J})$ increases and also the hybridization between Co $\mathrm{d}$ and O p orbitals (controlled by the charge transfer energy, $\Delta \mathrm{V}$ ) is reduced (see Figure 1). This mixed spin state exhibits a Mott insulating state and also becomes stable at the expanded volume, compared to ground-state low spin volume consistent with the volume expansion measured in experiment during the spin-state transition.

\section{PROPOSED FUTURE WORK}

This LDRD project was completed in FY 2017. The future direction will be the application of DFT+DMFT for the study of correlated electronic structure and energetics of various artificially structured oxides. 


\section{Agent-Based Behavioral Modeling of Ebola Spread in Chicago and Other Large Urban Areas}

\author{
2015-169-R2
}

Charles Macal

\section{PROJECT DESCRIPTION}

This project was initiated to understand the threat that the Ebola Virus Disease (EVD) outbreak in West Africa poses to U.S. national security and emergency preparedness. After the Ebola threat was addressed, several new diseases emerged with their own sets of questions concerning the threat to the U.S. population. These questions are of such a granular nature that they can only be addressed with large-scale computational models of disease transmission for entire populations. The resulting model, the Chicago Social Interaction Model (chiSIM), has been successfully scaled to the city (Chicago), county (Cook), and Chicago metropolitan area.

\section{MISSION RELEVANCE}

In October 2014, then-Secretary of Energy Moniz pledged his support to former President Obama and to then-Secretary of Health and Human Services Sebelius in responding to the Ebola outbreak in West Africa and its consequences, under the President's One Government Initiative. The Secretary asked the national laboratories to develop technical proposals based on each of their unique capabilities. Argonne proposed adapting an existing infectious-disease model-the Chicago methicillin-resistant staphylococcus aureus agent-based modelling (MRSA ABM) developed for community associated MRSA (CA-MRSA), a virulent bacterial pathogen-to EVD.

\section{RESULTS AND ACCOMPLISHMENTS}

In FY 2015, we developed the generalized chiSIM framework that allows one to build specific disease models as well as models of more general social interaction processes. We applied it to modeling influenza in Chicago as a test. In FY 2016, taking a hybrid modeling approach, we developed a more aggregate compartmental model of an Ebola outbreak in Chicago and identified promising intervention strategies in case an Ebola outbreak occurred in the city.

In FY 2017, our research focused on implementing computer models to run faster, more efficiently, and at larger scales. We developed new algorithms for efficiently partitioning and distributing models across distributed computing resources so that a large number of different computer runs could be done in parallel. Software and code were completed for ultra-large-scale simulation and analysis to log model events and results at the agent level, and to perform statistical analysis of model-generated contact networks and characterization of the networks by their topological properties.

Figure 1 shows the network of contacts occurring in a given hour of the simulation. The network links individuals with the individuals who had contact with them in the course of a typical 24-hour day. Individuals are represented as nodes, and contacts between individuals are represented as lines. Each contact has the potential for an infected individual to infect any other individuals in its contact network.

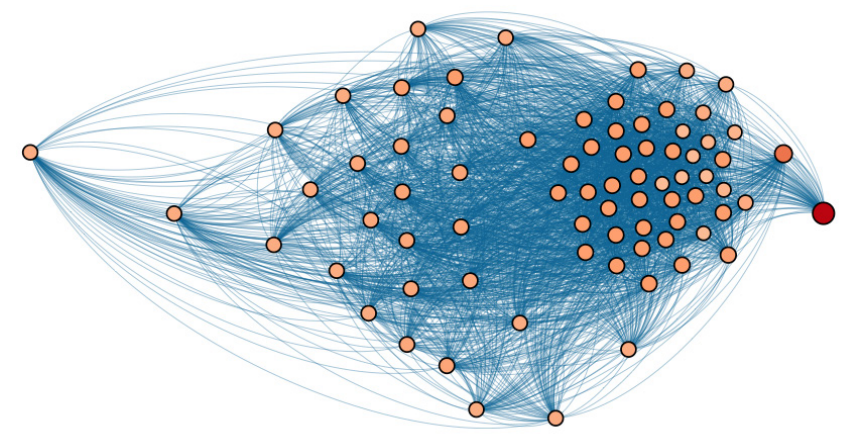

Figure 1. Contact networks generated by the chiSIM model.

A behavioral ontology was completed for EVD and other pandemic diseases. The ontology is the knowledge base for models of people's dynamic response to disease outbreaks and interventions. The dynamic response models will be included in the next implementation of the chiSIM model.

An Ebola compartmental model was developed. This work is novel in its exhaustive exploration of the disease transmission parameter space using workflows and computational approaches. The compartmental model was used to identify candidate solutions to explore in the large-scale ABM.

\section{PROPOSED FUTURE WORK}

FY 2017 was the final year of this project. Proposed future work consists of modeling Hepatitis $\mathrm{C}$ and the use of opioids in Chicago, as well as developing an infectious disease model that combines human populations and vector-borne pathogens. Continued work will be performed under grants from the $\mathrm{NIH}$ and funding from private foundations will be pursued. 


\section{Biomimetic Approaches for Water-Smart Landscapes}

\author{
2015-170-R2 \\ M. Cristina Negri, Jules Cacho, Patty Campbell, \\ and Colleen Zumpf
}

\section{PROJECT DESCRIPTION}

This project, a collaboration with Israel's Ben Gurion University (BGU), seeks to understand how vegetation self-arranges in arid environments, and mimics this strategy to develop vegetative systems that can survive drought. We develop experimental data to study the spatial self-organization patterns of vegetation in green infrastructure (GI). The experimental data we are generating help to validate the models developed at BGU, and provides guidance on how to develop more efficient GI systems for U.S. cities. GI is vulnerable to drought even in temperate regions as its shallow soil limits water storage and exposes vegetation to potentially long dry spells. It is important to develop efficient $\mathrm{Gl}$ as it is an energy-efficient, low-cost, resilient approach to managing storm water and reducing ambient temperature in cities. This work will also be useful in improving agroforestry systems in arid regions and xeriscapes in arid cities such as those in the western United States.

\section{MISSION RELEVANCE}

This work is relevant to several DOE missions: ensuring energy efficiency and scientific discovery and developing solutions for the energy-water nexus. By increasing understanding of $\mathrm{Gl}$ drought resilience, this project provides tools to better design for drought and flood management, both in urban and rural environments; and ultimately to develop ways to efficiently manage storm water, obtain better biomass production, and increase primary productivity.

\section{RESULTS AND ACCOMPLISHMENTS}

The last year of funding was dedicated to development of a controlled study that would better determine the relationship between plant survival and soil moisture conditions. The experimental set up at the Chicago Botanic Garden provided very important information about trends in moisture following precipitation and plant survival. It provided the large majority of the model inputs for our collaborators in Israel, and it was limited by the rainfed conditions which limited our control on water dosing. Therefore, we completed the study with an experiment in which we could accurately dose each plant treatment with specific amounts of water and follow their growth and survival-and ultimately drought resiliencewith more precision. See Figure 1.
Two functionally different plants were tested that differed in physiological characteristics, including root structure and type, as well as in their responses to water limitations. The two functional plants, Big Bluestem grass and sedum, were grown separately and together to compare their productivity and to measure their impacts on water loss. When mixed with sedum, Big Bluestem was found to have a slightly higher above- and belowground biomass per individual plant than when grown alone, whereas sedum had the opposite response (higher productivity when grown alone). The higher productivity of Big Bluestem, after the second year of growth, while mixed with sedum may suggest a benefit derived from the association of the two different plants under water-limited conditions. Sedum was suggested to serve as a nurse plant under these conditions for neighboring plants, where sedum's greater soil coverage reduces soil temperatures, thereby reducing heat stress. Plant percent coverage assessment revealed that by the second year sedum provided much greater soil coverage, as a result of its low-lying creeping growth habit, than Big Bluestem. Therefore, sedum was found to have greater aboveground biomass productivity overall as compared to Big Bluestem. Our results also show the benefit for mixed functional groups in waterlimited environments for both plant productivity and water usage, including storm water impact mitigation.

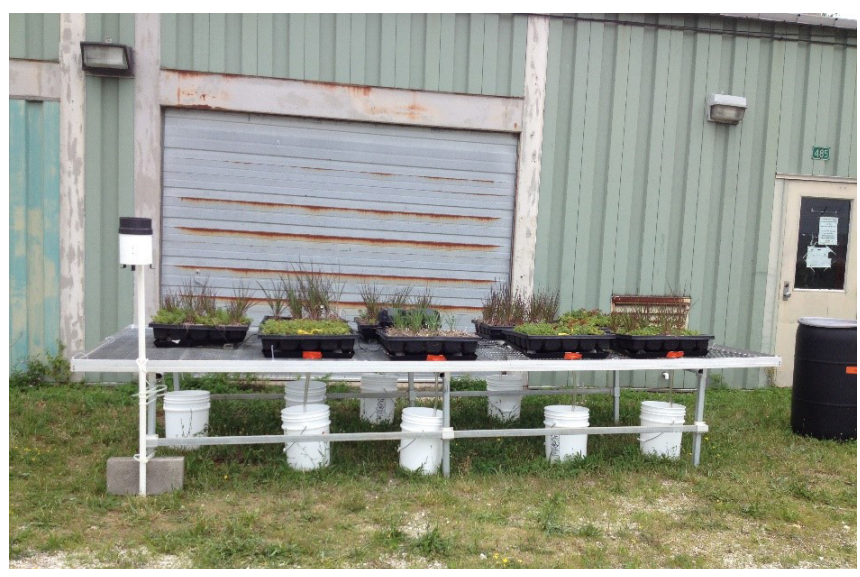

Figure 1. A view of the summer 2017 experimental setup at Argonne Area 485, showing (1) the green roof trays growing only grass, only sedum, and a combination of the two; and (2) the collection system to capture runoff flow and a rainfall gauge. To obtain a planned dosing of moisture, the setup was covered with an impermeable cover when rainfall was expected.

\section{PROPOSED FUTURE WORK}

Our LDRD funded work was completed in FY 2017. We are pursuing opportunities so we can continue developing this science. A joint manuscript with BGU will be submitted summarizing the data collected and how these data contribute to the development of a validated model representing a two-plant system's adaptation to drought. 


\section{Isotope Geochemistry via Sn Isotope Fractionation Using Inelastic X-ray Scattering of Synchrotron Radiation}

\author{
2015-173-R2
}

Esen Alp and Michael Hu

\section{PROJECT DESCRIPTION}

Siderophile (iron-loving) elements provide clues to the conditions under which terrestrial planets differentiated into metallic cores and silicate mantles. Concentrations of such elements (e.g., nickel [Ni], cobalt [Co], tungsten [W], and tin [Sn]) in the Earth's mantle were set by metal-silicate equilibrium in an early magma ocean. The thermodynamic conditions that obtained prior to this differentiation (pressure, temperature, and $\mathrm{fO}_{2}$ [fugacity or effective partial pressure of oxygen]), however, are a matter of intense debate.

Tin is generally classified as a chalcophilic (a sulfur-loving) element. Of course, we note that siderophilic or chalcophilic determinations are a matter of degree and depend on the nature of the host matrix. For example, there is a large variation in the thermochemical behavior of $\mathrm{Ni}$-Sn and $\mathrm{Ni}$-iron (Fe) alloys. Very large changes occur in the trace element activity coefficient of $\mathrm{Sn}$ in Fe-Ni alloys as a function of composition versus in pure Fe. Furthermore, the behavior of two stable oxidation states of $\mathrm{Sn}$ as $\mathrm{Sn}^{+2}$ and $\mathrm{Sn}^{+4}$ create further complications in interpreting oxygen fugacity data. For example, it is reported that $\mathrm{Ni}$-Fe meteorites contain up to $100 \mathrm{ppm}$ Sn, whereas iron meteorites contain 0.2 to 7 ppm Sn. The variation in the $\mathrm{Sn}$ content in rocks and meteorites indicates the siderophilic nature of Sn.

\section{MISSION RELEVANCE}

This project is relevant to the DOE mission of advancing the basic science of minerals, which are natural resources. The more we learn about the nature of phase separation during the formation of the Earth, the easier it is to develop the best geological practices for minerals search and exploitation.

\section{RESULTS AND ACCOMPLISHMENTS}

We measured Sn and Fe phonon density of states in several Fe-Ni-Sn compounds under pressure to determine the force constant and isotope fractionation. Different isotopes of the same element segregate or fractionate as if they are different chemical elements due to the differences in mass. To establish a firm relationship between the valence and force constant, we measured the synchrotron Mössbauer spectra of anorthite, basalt, and rhyolite glasses oxidized or reduced in air or using an iron wüstite buffer.

We have synthesized three compounds of Fe-Ni-Sn, isotopically enriched with ${ }^{119} \mathrm{Sn}$ and ${ }^{57} \mathrm{Fe}$. These are $\mathrm{Fe}_{0.93} \mathrm{Ni}_{0.04} \mathrm{Sn}_{0.03}, \mathrm{Fe}_{0.96} \mathrm{Ni}_{0.03} \mathrm{Sn}_{0.01}$, and $\mathrm{Fe}_{0.97} \mathrm{Sn}_{0.03}$. We measured the phonon density of states, and determined the force constants of these three compounds in November 2015. We are now in a position to determine the temperature-dependent isotope fractionation for $\mathrm{Sn}$ in these compounds.

We developed a synchrotron-based method to circumvent the difficulties that are related to the determination of isotope fractionation. It is quite valuable to develop a new proxy for isotope geochemistry studies that will indicate redox conditions at the time of formation. We successfully exploited nuclear resonant inelastic $X$-ray scattering to study iron isotope fractionation in minerals silicate melts relevant to rock formation. From the phonon excitation spectrum, force constant and phonon density of states (pDOS) could be calculated. These quantities were then used to derive thermoelastic properties such as specific heat, vibrational entropy, sound velocity, and isotope fractionation factors.

PROPOSED FUTURE WORK

This project concluded in FY 2017. 


\section{Conversion of $\mathrm{C}_{2}$ and $\mathrm{C}_{3}$ Paraffins into Liquid-Phase Products}

\author{
2015-174-R2 \\ Christopher L. Marshall
}

\section{PROJECT DESCRIPTION}

This project attempted to create tandem catalysts for converting gas-phase molecules directly into higher value liquids. The work coupled two of our previous successes in atomic layer deposition (ALD) synthesis at Argonne: (1) ALD overcoating of metallic catalysts, and (2) high-precision synthesis of controlled acidic functions for creating a tandem catalyst that will convert light gases directly into $C_{4}$ to $C_{6}$ liquids. ALD overcoating stabilizes the catalytic metal nanoparticles boosting the yield of heavier olefins. By designing a bimetallic catalyst consisting of a primary alumina coating and a secondary silica coating, an acidic site will be created inside the catalytic pore capable of dimerizing olefins (Figure 1). The catalysts' uniform, restricted pore dimensions should limit olefin oligomerization to $\mathrm{C}_{4}$ to $\mathrm{C}_{6}$ olefins with little or no coking. The modular design of this catalyst/reactor system will allow transportability so as to monetize stranded and remote resources that are not easily accessible to existing large commercial plants, which must be built adjacent to vast gas fields.

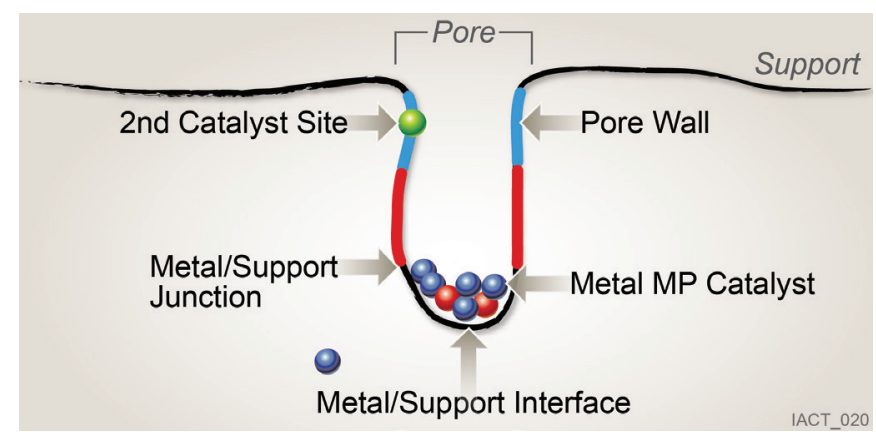

Figure 1. Concept of a dual catalyst function with a dehydrogenation catalyst at the bottom of a pore (in red) and an acidic dimerization catalyst (in green) close to the pore opening.

\section{MISSION RELEVANCE}

This project aligns well with the DOE goal of creating new materials that can lower energy costs for the production of fuels and chemicals. If successful, the program would monetize light hydrocarbon gases that are currently flared. The work should be of interest to both the chemical and refining industries.

\section{RESULTS AND ACCOMPLISHMENTS}

In FY 2017, we concentrated specifically on examining overcoating of platinum $(\mathrm{Pt})$ catalysts made from standard incipient wetness impregnation (IWI). Using IWI, we controlled the total amount of Pt added to the support. A stock material was made of $\mathrm{Pt}$ on silicon dioxide $\left(\mathrm{SiO}_{2}\right)$ for use in all reactions. We also moved from ODH, which is dehydrogenation in the presence of oxygen, to $\mathrm{DH}$, which is dehydrogenation with no oxygen. We believe that the $\mathrm{ODH}$ was less selective than desired, leading to deep oxidation, the wasting of hydrocarbon, and the decreasing of the availability of olefins for the second reaction, olefin dimerization. Finally, we moved in steps for the aluminum oxide $\left(\mathrm{Al}_{2} \mathrm{O}_{3}\right)$ overcoating to minimize both the formation of pores that are too small and completely burying the Pt catalyst.

Catalyst tests show that a reaction begins at $550^{\circ} \mathrm{C}$. No catalysis occurs at either $350^{\circ} \mathrm{C}$ or $450^{\circ} \mathrm{C}$. The reaction observed is not strictly dehydrogenation (breaking $\mathrm{C}-\mathrm{H}$ bonds) but involves the breaking of one of the $\mathrm{C}-\mathrm{C}$ bonds. When the feed is propane, this reaction leads to methane $\left(\mathrm{CH}_{4}\right)$ and ethylene $\left(\mathrm{C}_{2} \mathrm{H}_{4}\right)$. The mass balance between these two should be 1:1 if no other reactions are occurring. However, the ethylene yield was lower than expected. Examination of the GC trace showed that the deficit in ethylene is attributable to the formation of a $\mathrm{C}_{4}$ product (probably an isomer of butylene, $\left.\mathrm{C}_{4} \mathrm{H}_{8}\right)$ ). The $\mathrm{C}_{4}$ product demonstrates that the second reaction, olefin coupling, is possible. It is important to lower the amount of $\mathrm{C}-\mathrm{C}$ bond breakage while increasing the amount of $\mathrm{C}-\mathrm{H}$ bond breakage to preserve carbon in the process.

\section{PROPOSED FUTURE WORK}

The results of this LDRD project were incorporated into the work plan for a new proposal for FY 2018 funding with the DOE Advanced Manufacturing Office (AMO). The proposal was submitted in May 2017. Both Honeywell UOP and Forge Nano, our corporate sponsors, included letters of intent for working on the program, agreeing to a $25 \%$ cost share. The work will examine the effect of the commercial scale-up of ALD overcoating on catalyst longevity and catalyst selectivity. 


\section{Towards lonotronics: First- Principles Strategies for Coupling Electronic and Ionic Properties in Complex Oxides}

\author{
2015-178-R2
}

James Rondinelli

\section{PROJECT DESCRIPTION}

This project aims to deliver strategies for controlling defects (e.g., vacant cation or anion sites) in a designed fashion to establish a materials platform for an ionics-based electronic device. Anion-deficient $\mathrm{ABO}_{3-\delta}$ oxides, so-called brownmillerites, based on the perovskite structure, could be the ideal channel materials for an ionics-based field-effect transistor. Although they have been studied experimentally, the main focus has been on exploring polymorphic crystallography based on redox chemistry rather than on phase stability or electronic properties. The polymorphic flexibility of these materials is the focus of this project.

\section{MISSION RELEVANCE}

This project relates to the scientific discovery and innovation aspects of DOE's science mission. The ability to manipulate the ion concentration in an ultrathin oxide film via electric fields represents an exciting new operational platform for solid-state devices because of the sensitive coupling between stoichiometry and functional properties. The main thrust of the project is to develop an understanding of suitable pathways to manipulate the electronic structure of complex oxides through engineered static and dynamic control of the anion structure. The incorporation of such novel materials into advanced nanoelectronics has implications for development of superior computing technology and reduced power consumption, while forging pathways to new state variables beyond temperature or voltage that would represent a game-changing advance in computing.

\section{RESULTS AND ACCOMPLISHMENTS}

In FY 2017, we focused on understanding the energy landscape arising from changes in the metal-oxygen coordination polyhedra. Building on FY 2016 results, we studied $\mathrm{Ca}_{2} \mathrm{Al}_{2} \mathrm{O}_{5}$ and $\mathrm{Sr}_{2} \mathrm{Fe}_{2} \mathrm{O}_{5}$ and successfully clustered density functional energies describing the stabilities of various polymorphs of these compounds based solely on the polyhedral coordination environments. The cluster analyses enabled us to formulate a point-charge model sufficient to capture these phase stability trends in the aluminate, but importantly, not for the ferrate. This finding indicated the importance of interactions beyond Coulombic electrostatics in governing the stability of brownmillerites comprising late transition metal elements. A new model including covalency interactions and orbital hybridization was constructed. Such models will allow us to assess the likelihood of realizing polymorphic (and property) changes from polyhedral coordination environment changes at fixed stoichiometry without numerous comprehensive density functional theory calculations.

We examined pressure-dependent polymorphic changes in $\mathrm{Sr}_{2} \mathrm{Fe}_{2} \mathrm{O}_{5}$ and formulated a model for the change in magnetic order when $\mathrm{Sr}_{2} \mathrm{Fe}_{2} \mathrm{O}_{5}$ transforms from brownmillerite $\left(\mathrm{FeO}_{4}\right.$ tetrahedra and $\mathrm{FeO}_{6}$ octahedra) to a high-pressure phase with only $\mathrm{FeO}_{5}$ square pyramidal coordination. At high pressure and finite temperature, $\mathrm{Sr}_{2} \mathrm{Fe}_{2} \mathrm{O}_{5}$ is paramagnetic. Our electronic structure calculations show that a ferromagnetic metallic phase is more stable than a G-type (checkerboard spin arrangement) antiferromagnetic (G-AFM) semiconducting phase at high pressures, demonstrating the utility of using pressure to induce a magnetic transition. Last, we examined isoelectronic $\mathrm{Sr}_{2} \mathrm{Mn}_{2} \mathrm{O}_{5}$ under similar pressure ranges. We find that the change in coordination environment strongly stabilizes an E-type AFM spin ordering, in contrast to conventional $\mathrm{ABO}_{3}$ perovskite behavior.

The implications of this work extend well beyond the ferrate and manganite structures explored, as pressureand strain-induced phase transitions can be accessed broadly in brownmillerite $\mathrm{ABO}_{2.5}$ compounds and related crystal families that exhibit layered vacancy structures. Our calculations demonstrate how to access various functional properties of the $\mathrm{ABO}_{2.5}$ materials through changes in local coordination chemistry; these changes permit an additional degree of control in multifunctional property design, enabling robust magnetic ordering for spintronics, semiconducting band gaps for photovoltaic applications, and ionic conductivity for battery and fuel cell technologies.

\section{PROPOSED FUTURE WORK}

A proposal is pending at the National Science Foundation that leverages results obtained here and focuses on the effects of incorporating fluorine into the lattice of $\mathrm{SrMnO}_{3-\delta}$. Our preliminary calculations center on understanding changes in optical properties, complemented by experimental synthesis and characterization of related films of fluorine-doped $\mathrm{SrMnO}_{3-\delta}$. 


\section{Fine-Resolution Reconstruction of Large Volumes of a Brain}

\author{
2015-181-R2 \\ Narayanan Kasthuri
}

\section{PROJECT DESCRIPTION}

This project proposes to explore research using both electron and high-energy X-ray microscopy for imaging the fine structure of the brain. There is a current push to develop approaches to mapping all the neurons and connections at the nanometer $(\mathrm{nm})$ scale in a large volume of a brain and to accomplish the same for entire brains ("connectomics"). The current approach is to use serial-section electron microscopy. This research project will continue those investigations, along with determining how synchrotron-based, high-energy X-ray microscopy of the same brains can complement and extend the capabilities for connectomics. Technical objectives include producing: (1) a map of an entire mouse brain at sub-micron resolution ( $100-n m$ voxels), where the goal is to map all the cells in the brain, their long-distance projections, and the blood vessels; (2) a map of a "functional module" of a single brain at the nanometer scale ( $10 \mathrm{~nm} /$ voxel over a cubic millimeter), where the goal is to find every neuronal connection in that volume; and (3) both maps in the same brain.

\section{MISSION RELEVANCE}

The project is relevant to the DOE mission in basic science. The National Strategic Computing Initiative Executive Order promotes a whole-of-government approach to bringing the unique national computing capabilities at DOE facilities to transform how a partner agency executes its mission. The study of the brain, perhaps unlike any other biological system, requires possibly the largest datasets and simulation capacities for a fuller understanding. In this project, by partnering with multiple academic collaborators nationwide, we will be leveraging the high-performance computing, big-data analytics, and machine learning tools of the national labs with the domain knowledge and instrumentation experience of our academic partners to create a unique national resource for mapping neuronal circuits in healthy and diseased brains. The problems are difficult but will have a considerable impact on multiple DOE programs, including neuromorphic and low-energy computing. An exciting possibility is that exascale-assisted brain mapping will reveal new generations of software and hardware architectures for future national lab computing resources well beyond the exascale.

\section{RESULTS AND ACCOMPLISHMENTS}

There were several achievements during the past year:

1. We developed protocols for imaging entire mouse brains using the Advanced Photon Source, leading to a scientific paper. In addition, we have now mapped an entire mouse brain and are preparing a second manuscript for submission.

2. We are developing and deploying automated algorithms based on machine learning in collaboration with the Argonne Leadership Computing Facility and Argonne's high-performance computing. These algorithms will be used to automatically trace neurons and their connections, and these algorithms are now the fastest and most powerful computational resources (potentially) in the country for brain mapping.

\section{PROPOSED FUTURE WORK}

FY 2018 objectives include efforts to:

$\square$ Map entire mouse brains with synchrotron radiation at sub-micron resolution: this effort will allow us to reconstruct every neuron, blood vessel, and long-range projection in single brains. It will be the first-of-its-kind atlas and a reference for the entire neuroscience community.

$\square$ Map sub-volumes of entire mouse brains at nanometer resolution ( $\sim 1 \mathrm{~mm}^{3}$ at $10-\mathrm{nm}$ resolution, or 1 billion gigabytes of data). This effort will map every connection between every neuron in a biologically relevant volume. As such, it will result in one of the largest datasets ever collected about any subject matter. It will also serve as a reference atlas (see item above) that will ultimately enable comparison to diseased brains and young and aged brains, among others. 


\section{New Thin Film Oxide, Chalcogenide and Oxy-Chalcogenide Materials Discovery}

\author{
2016-120-R1
}

\section{Supratik Guha}

\section{PROJECT DESCRIPTION}

This project focuses on the development of novel resistive switching materials and devices for application in solid-state memories and neuromorphic circuits, as well as for artificial synapses and neurons for neuromorphic information processing. The focus is on new oxide materials with ultra-low power operation. The activities involve materials synthesis using ultra-high vacuum deposition and atomic layer deposition, nanoscale device fabrication, materials characterization, and electrical measurements. Materials studied include new polymer-metal oxide nanocomposite films, $\mathrm{VO}_{2}, \mathrm{CeO}_{2}$, $\mathrm{Y}_{2} \mathrm{O}_{3}, \mathrm{HfO}_{2}$, and their heterostructures.

Materials with the property of an electrically driven insulator-to-metal transition (E-IMT) are a promising candidate to be used for neuromorphic circuit components, such as an integrate-and-fire artificial neuron. However, to date, E-IMT material is limited in performance. This work investigates the physics and technology to improve E-IMT performance and its suitability for constructing artificial neuron and neuromorphic circuits.

\section{MISSION RELEVANCE}

This research into next-generation, low-power, efficient information processing is relevant to DOE's mission in discovery science. As computing workloads have changed and we arrive towards the end of Moore's Law scaling, there is interest in new computing architectures such as neuromorphic computing, where the computing hardware elements are akin to the functionality of neurons and synapses. This work addresses the underlying materials science and physical phenomena of materials that could be used for such energy efficient elements for future computing.

\section{RESULTS AND ACCOMPLISHMENTS}

A novel resistive memory device has been developed with an ultra-porous metal-oxide/polymer nanocomposite material, polymethyl methacrylate (PMMA)- $\mathrm{Al}_{2} \mathrm{O}_{3}$. The nonvolatile switches we developed operate at very low voltage $(<500 \mathrm{mV}$ ) and with high reliability (Figure 1).
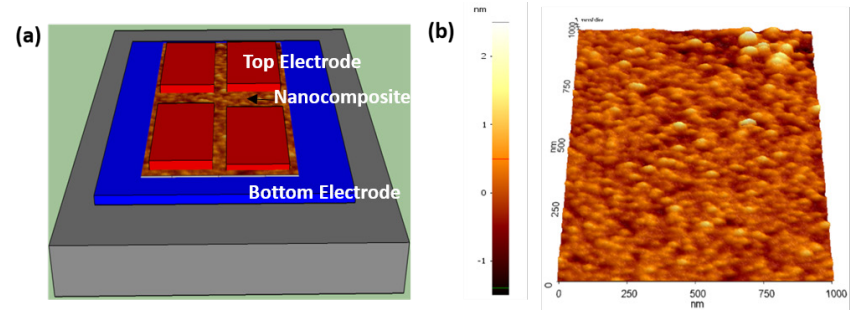

(c)
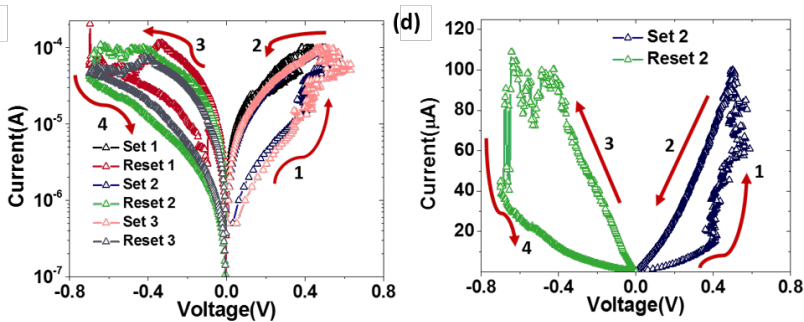

Figure 1. (a) Three-dimensional schematic of a sample with $\mathrm{PMMA-Al}{ }_{2} \mathrm{O}_{3}$ nanocomposite switching layer. (b) Atomic force microscope image of the PMMA-Al $\mathrm{O}_{3}$ nanocomposite showing a uniform film. (c,d) Current-voltage curves for bi-directional, nonvolatile resistive switching at ultra-low voltage in $\mathrm{Ag} / \mathrm{PMMA}-\mathrm{Al}_{2} \mathrm{O}_{3}(10 \mathrm{~nm}) / \mathrm{Pt}$ devices.

The current on/off ratio and voltage scalability were also investigated in nanoscale E-IMT structures fabricated in the Center for Nanoscale Materials at Argonne. For the first time, a nanoscale E-IMT structure with length of $40 \mathrm{~nm}$ and width of $200 \mathrm{~nm}$ was demonstrated and studied.

In addition, a recovery strategy and model of the recovery mechanism were developed for conductive-bridge resistive memories.

\section{PROPOSED FUTURE WORK}

Proposed future work includes further development and refining of nanoporous polymer-dielectric composites for memory, continued refinement of E-IMT-based ultra-low voltage structures, development of new oxide-oxide heterostructures, and examination of their reactive chemistry to explore memory states. 


\section{Development of a} Pre-Conceptual Design of a New Tracking System for CLAS12 Detector

\author{
2016-179-R1 \\ Kawtar Hafidi and Whitney Armstrong
}

PROJECT DESCRIPTION

The goals of this project are to identify the luminosity limitations of the Continuous Electron Beam Accelerator Facility (CEBAF) Large Acceptance Spectrometer "CLAS12" at DOE's Thomas Jefferson National Accelerator Facility and propose a new tracking detector that will allow the machine to run at luminosities an order of magnitude higher than those achievable now. This effort requires a detailed simulation to understand the limitations of the current design and how it can be improved.

\section{MISSION RELEVANCE}

This project is relevant to DOE's mission in basic science. The current CLAS12 configuration has large-area, wire drift-chamber detectors that are used to reconstruct particle tracks. When the rates of particles through the drift chambers are very high, the track reconstruction efficiency drops, owing to uncorrelated hits and multiple tracks going through the same region of the detector. Increasing the luminosity, and thus the particle rates, will allow a rapid completion of the backlog of planned measurements which, at current luminosity limitations, consists of roughly nine years' worth of approved CLAS12 experiments. Therefore, improving the detectors to handle higher rates will dramatically increase the scientific throughput of the CLAS12 physics program over the next decade.

\section{RESULTS AND ACCOMPLISHMENTS}

We developed a full Geant4 (GEometry ANd Tracking code) simulation of CLAS12 with an optimization for accurately extracting the drift chamber wire hit occupancies (see Figure 1). We developed a realistic event generator and cross-validated it with Geant4 results. This event generator allows for a fast and efficient simulation by only producing events that will land in the CLAS12 detectors. The main contribution of background events comes from Moller electron scattering. Other sources of background include electron-proton elastic scattering and inclusive pion production. All of these processes, and more, are included in the event generator to produce a realistic sample of events. We have identified small scattering angles as the location for high drift chamber occupancies. This will be the initial region we select to improve with an initial design that can mitigate the high rates and maintain a high tracking efficiency. Recently, the event generator code was re-factored to accommodate incoherent physics processes on nuclei, which will be part of the new physics program accessible with the upgraded detector system.

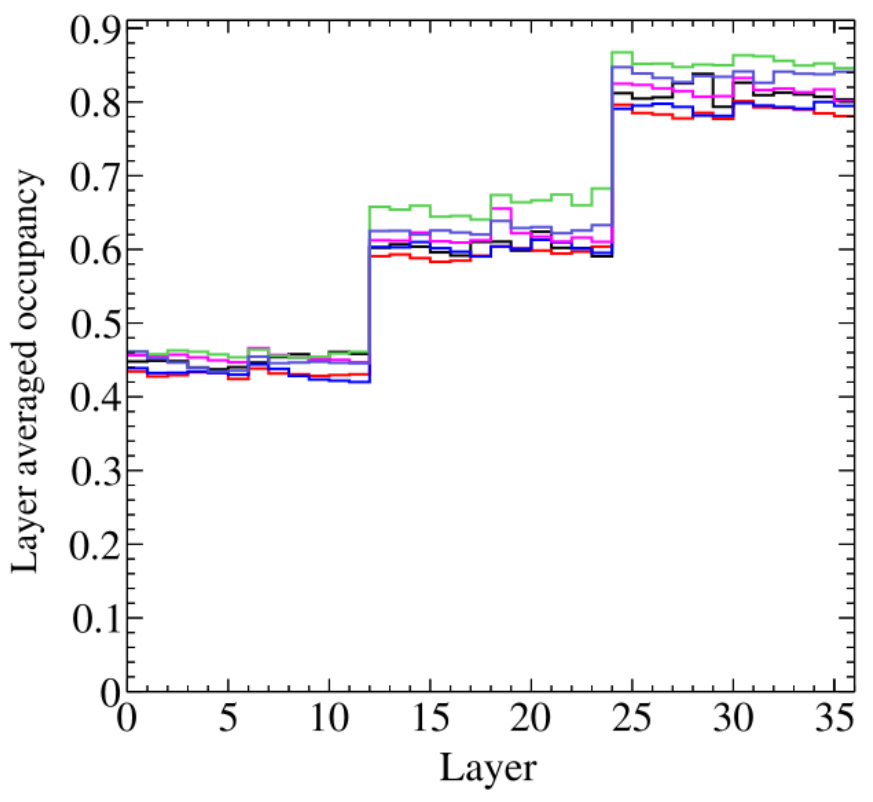

Figure 1. Drift chamber occupancies running at a luminosity of $1.3 \times 10^{35} \mathrm{~cm}^{-1} \mathrm{~s}^{-1}$ from a beam-on-target simulation that uses the Geant 4 physics. Each line shows one of CLAS12's six sectors. This result was used to cross-check the much more efficient event generator we developed.

\section{PROPOSED FUTURE WORK}

We will validate the predicted drift chamber occupancies with the measured occupancies in the upcoming engineering run. This engineering run will provide the first data collected with the full CLAS12 detector system and will allow us to normalize our predicted drift chamber occupancies. With these verified results, we can confidently move forward with an initial design proposal, which we will submit to DOE in coordination with Jefferson Lab management. In addition, the validated simulation will allow us to lay out the scope of new physics accessible with the proposed upgrade. 


\section{Core-Shell Nanowire Magnetic/Ferroelectric Multiferroic Heterostructure for Voltage-Tunable RF Devices}

\author{
2016-182-R1
}

Yunsong Xie, Kaizhong Gao, and John Hryn

\section{PROJECT DESCRIPTION}

Obtaining voltage control of magnetism through multiferroics (materials exhibiting ferroelectric and ferromagnetic traits simultaneously) is of great fundamental interest and technical importance for fast, compact, and energy-efficient next-generation tunable magnetoelectric (ME) devices, such as radiofrequency (RF) circuit components, memory devices, and sensors. This project will synthesize scalable core-shell nanowire (iron cobalt-hafnium oxide/zirconium oxide $\left[\mathrm{CoFe} / \mathrm{HfO}_{2}-\right.$ $\mathrm{ZrO}_{2}$ ] or PZT [lead zirconate titanate/CoFe]) structures as multiferroic substrates for ME devices using the electrospinning technology we have developed. $\mathrm{HfO}_{2}-\mathrm{ZrO}_{2}$ is an insulator (dielectric), and PZT has a large piezoelectric effect, which enables the material to change shape in an electric field.

The core-shell nanostructured material is synthesized by producing an electrospun nanofiber (core) and coating it using atomic layer deposition (shell). The resulting core-shell structure is a ferroelectric nanowire with a ferromagnetic shell. This material has the potential to be used as multiferroic substrates in advanced voltagetunable RF devices, because its ME coupling strength can be ten times stronger than in conventional ME devices.

\section{MISSION RELEVANCE}

This project is relevant to DOE's mission in science and also has applications relevant to energy and national security. Based on the new materials and processes developed in this project, new applications such as voltage-tunable RF and memory devices can be realized. The new materials and technology developed will enable a smaller and more energy-efficient ME device. We can also envision this technology enabling applications in space, satellite, and defense-relevant technologies.

\section{RESULTS AND ACCOMPLISHMENTS}

In 2016, we have demonstrated the multiferroics performance of the developed CoFe/PVDF-HFP core shell material by achieveing $\sim 10$ Oe ferromagnetic resonance field shift with applied an E-field of $20 \mathrm{kV} / \mathrm{cm}$. This year we demonstrated integration of the core-shell nanowires developed last year into a functional device (antenna) in order to demonstrate the performance enhancement that the uniqueness of the material (the $\mathrm{HfO}_{2}-\mathrm{ZrO}_{2}$-coated CoFe nanofiber) brings to the device. We explored the possibility of using a 3D printer to fabricate the antenna. The ideal solution for fabricating this magnetic-composite material-based antenna is to use the $3 \mathrm{D}$ printer to print the magnetic-composite substrate, followed by printing the metal antenna pattern on top of it, as shown in Figure 1. The pattern accuracy of the antenna is essential to achieving the designed radiation performance of this antenna. An anechoic chamber was constructed to characterize antenna properties.
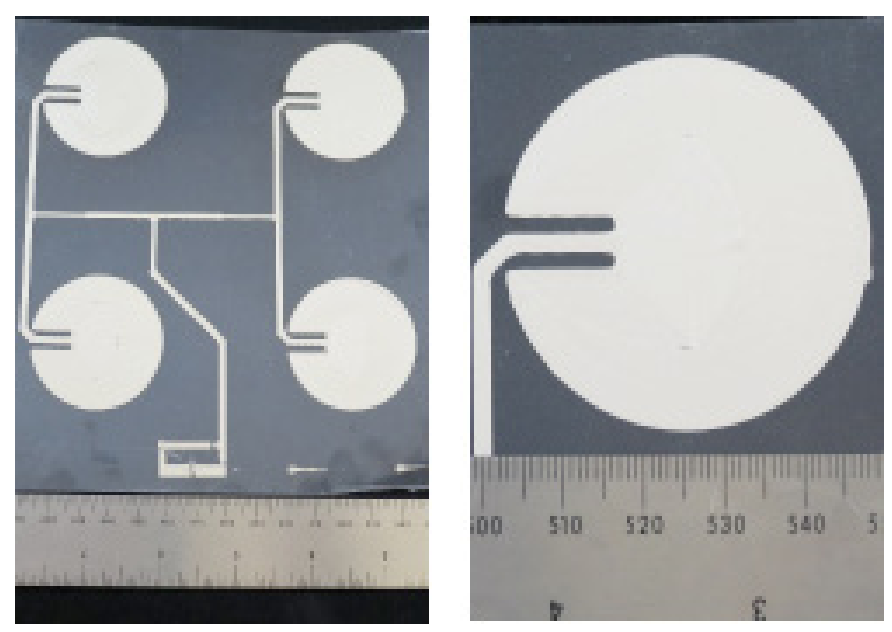

Figure 1. 3D printed patch antenna array using a customized 3D printer and 0.7-mm needle outlet.

\section{PROPOSED FUTURE WORK}

In future studies, we will characterize the antenna properties using the anechoic chamber and will investigate additional materials and process conditions to optimize this technology to realize improved multiferroic materials. In addition, we will explore uses of these materials in RF device applications. We anticipate engagement with the Defense Advanced Research Projects Agency (DARPA) for a new opportunity based on this technology for RF/microwave applications. 


\section{Hybrid Silicon Nanolasers}

\author{
2016-190-R1
}

\section{Chad Husko}

\section{PROJECT DESCRIPTION}

In this project, our team demonstrated a hybrid silicon nanolaser. Nanolasers are critical elements in the rapidly growing integrated photonics field. Nanolasers like ours are expected to completely cover optoelectronic chips, much like transistors do in electronic integrated circuits today. Ideally, we would like this laser to be made of silicon to leverage the tremendous investment in complementary metal-oxide-semiconductor electronics. While silicon has excellent electrical properties, its optical properties have proven much more challenging to master. Specifically, silicon is a very poor light emitter due to its indirect bandgap. The way to overcome silicon's intrinsic limit is to create a hybrid structure, in which light emission is provided by the complementary direct gap emitter material. Our approach is to use a two-dimensional film made of phosphorene.

This project is part of the University of Chicago's France and Chicago Collaborating in The Sciences (FACCTS) program (http://fcc.uchicago.edu/FACCTS), which links researchers in Chicago to researchers in France. Our collaborator is Prof. Xavier Checoury (Université Paris- Süd). Prof. Mark Hersam's group at Northwestern University is contributing on synthesis of the emitter. The project began in FY 2016. The two-year goals for the program were:

$\square$ Nano-cavity resonators: fabrication and characterization of silicon resonators using the existing nanofabrication recipe at Paris-Süd.

$\square$ Light emitter: development of a scalable transfer technique for the light-emitting material.

$\square$ Optical lasing: demonstration of the optically pumped hybrid silicon nanolaser.

\section{MISSION RELEVANCE}

Our hybrid silicon laser covers several areas. First, it covers the DOE basic science mission, as it is proof-of-concept for a new material for lasing. This feeds into a key energy objective because of its role in energy-efficient advanced computing. In particular, this research has the potential to greatly enhance U.S. energy and manufacturing competitiveness. In fact, the target here is directly identified under point 3.2, Emerging and Cross-cutting Areas of the Advanced Manufacturing Office (AMO) multi-year program plan. In Section 3.2.2,
Energy-efficient Advanced Computing, the broad goals are stated as: "The manufacturing focus area will leverage DOE expertise in nano-manufacturing methods, including extreme ultraviolet lithography, heterogeneous integration of advanced photonics." More specifically, Target 16.4 states: "Develop and demonstrate optoelectronic interconnects with a chip footprint one-tenth of 2015 technology." This target relies on miniaturized lasers.

\section{RESULTS AND ACCOMPLISHMENTS}

The project has been a success. We have been able to measure signatures of stimulated emission and lasing from this material combination for the first time. Moreover, we showed this at room temperature and in continuous wave operation, both of which are technologically significant milestones. We achieved a bonus result as well, by showing emission bandwidths over $70 \mathrm{~nm}$. This covers at least parts of two major telecommunications bands and was limited only by our experimental geometry. Figure 1 shows a schematic of our hybrid silicon-phosphorene nanolaser. The right panel shows the experimentally measured narrow laser linewidth emission compared to the broad spectrum without the optical nanocavity.

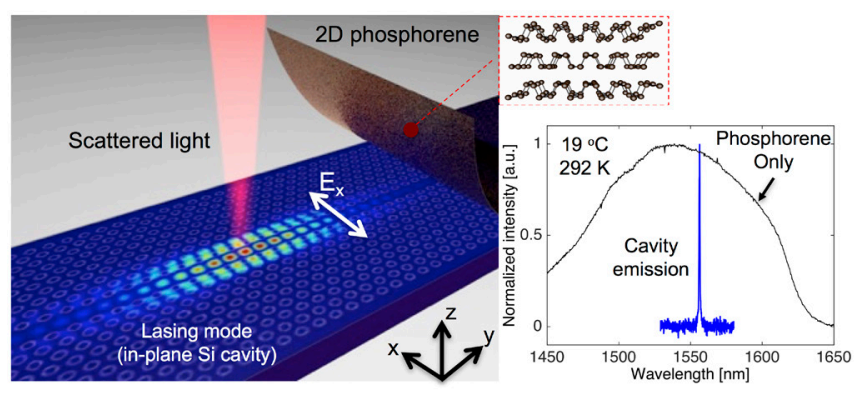

Figure 1. Schematic of the hybrid silicon nanolaser composed of a silicon optical cavity and two-dimensional phosphorene emitter. The laser emits in-plane with an $E_{x}$ polarization. Inset: atomic structure of the few-layer 2D phosphorene. Right: measured laser resonance compared to native emission of uncoupled phosphorene film normalized to their respective peak intensities.

To communicate these results, a manuscript has been submitted and is currently under review. We submitted an invention disclosure (IN-16-173), which led to the submission of a patent application in September 2017.

\section{PROPOSED FUTURE WORK}

We will seek funding to prepare new samples to directly measure the coherence of the laser, to scale the power emitted from the laser, and to improve the material quality to achieve better emission efficiency.

An application has been submitted to the Chain Reaction Innovations program at Argonne to push this technology toward commercial applications. 


\section{Exploring Next-Generation Coherent X-ray Science}

\section{6-191-R1}

Linda Young

\section{PROJECT DESCRIPTION}

DOE has invested in accelerator-based, next-generation X-ray light sources that aim for complete coherence in both longitudinal and transverse dimensions. After the stunning success of the Linac Coherent Light Source (LCLS), the world's first hard X-ray free electron laser (XFEL), in 2009, many countries have followed suit and constructed XFELs (Japan, Italy, Korea, Switzerland, Europe). China is moving rapidly toward this goal. The United States will be upgrading the LCLS to a high-repetition-rate version (from $120 \mathrm{~Hz}$ to $1 \mathrm{MHz}$ ) by 2020. Pulses from XFELs provide enough fluence per pulse in a timescale sufficiently short to enable single-shot imaging and freezing the fastest molecular vibrationsand thus monitoring atomic and molecular motions on their natural timescales. This project aims to define the science problems that make use of these revolutionary properties so that the United States can reap scientific benefit from the facility investment.

\section{MISSION RELEVANCE}

This project supports DOE's basic science mission. Expanding the available scientific application portfolio for next-generation coherent $X$-ray light sources will provide input for future accelerator-based facilities and capitalize on the current and planned investments (e.g., LCLS-II high energy upgrade). These scientific applications are enabled not only by brighter, faster coherent sources, but also by detector, data, and algorithm developments.

\section{RESULTS AND ACCOMPLISHMENTS}

We assembled an international team of investigators to address the long-standing problem of understanding the birth and fate of an excess electron in liquid water created by ionization. Ultrafast $X$-ray pulses shall be used to track valence hole dynamics and electronic coherence in strong-field ionized water. Surprisingly, the initially created cation $\mathrm{H}_{2} \mathrm{O}^{+}$remains undetected owing to its femtosecond lifetime and unclear spectroscopic signature in the ultraviolet/visible region (Figure 1). Our calculations revealed a clear signature for this and other relevant species in the soft $X$-ray regime, thus providing a handle on the electronic and chemical dynamics that extend to the earliest timescales. Beamtime at LCLS was granted for a proposal entitled "Transient Spectroscopy in the Water Window: Dynamics and Coherence in Strong-field Ionized Water."

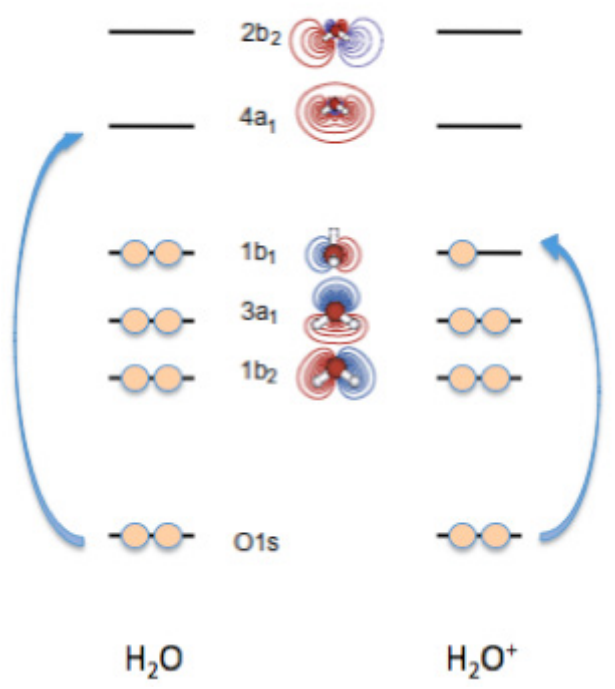

Figure 1. Schematic for X-ray transient absorption of the water cation following strong-field ionization. Strong absorption resonance in the cation appears in the water window corresponding to the HOMO-LUMO gap.

We also assembled a cross-laboratory and international team to address a fundamental question in pressure-induced phase transitions-what moves first, the atoms or the electrons? Simultaneous X-ray diffraction and emission spectroscopy can monitor the structural and electronic dynamics. Our calculations revealed that the valence-to-core emission spectrum when undergoing a wurtzite-to-rock-salt transition is a viable signature for the electronic dynamics. A proposal submitted to the European XFEL was given the highest rating but not beamtime in this first round, because the photon beam energy requested was not available.

We assembled a group of 17 world-leading scientists to contribute their perspectives to the timely roadmap in 2017, when three new XFELs host their first experiments. This roadmap has been published in the Journal of Physics $B$.

\section{PROPOSED FUTURE WORK}

The ultrafast $X$-ray experiment on transient spectroscopy in the water window will be performed at the LCLS. An exciting first sighting of the cationic partner of the solvated electron and information on the mysteriously long electronic coherence times observed in ionized liquid water are anticipated. Our proposal for understanding pressure-induced phase transitions will be resubmitted. A proposal to DOE following the expected call in ultrafast XFEL science will be submitted. This proposal will likely focus on the paradigm-changing combination of high-repetition-rate snapshots and big-data analyses to obtain a microscopic understanding of chemical dynamics in the solution phase, allowing a leap beyond standard transition state theories and kinetic descriptions. 


\section{Coherent X-Ray Studies of Phase Transitions in the Complex Oxides}

\author{
2017-155-NO \\ Dillon D. Fong
}

\section{PROJECT DESCRIPTION}

Materials are continually in a state of flux, particularly when the temperature is near that of a phase transition. A simple example is that of water as it is cooled below the freezing point: as an ensemble, the liquid gradually turns into ice, and the kinetics describing how this occurs are reasonably well understood. However, there is much happening within the ensemble during the crystallization process: some ice nanocrystals rapidly nucleate out of the liquid and grow, while others nucleate and re-melt; others may even nucleate with the "wrong" structure before evolving into the right one. Such ensemble fluctuations are not only scientifically fascinating but also technologically important: understanding the dynamics of phase transitions can lead to the discovery of materials with dramatically different and potentially exotic properties.

While there has been a fair amount of theoretical study into the subject of fluctuations near phase transitions, experimental investigations have lagged behind, owing to the practical difficulties of probing such phenomena at the relevant spatial and temporal scales. An experimental technique called X-ray photon correlation spectroscopy (XPCS) has recently been developed that enables direct investigations into the dynamics of both equilibrium and non-equilibrium fluctuations. The aim of this project is to exploit third-generation synchrotron sources like the Advanced Photon Source (APS) at Argonne and the European Synchrotron Radiation Facility (ESRF) in Grenoble, France, to conduct fundamental studies on the dynamics of phase transitions in complex oxide materials - materials known to exhibit a wide variety of phases ranging from superconducting to ferroelectric and antiferromagnetic to multiferroic. These behaviors originate from the close coupling between crystal structure and electronic structure: slight changes to either one by variations in temperature, stress, chemistry, or an electromagnetic field can result in an enormous change in properties. This behavior has made complex oxides candidate materials for brain-inspired computing-a possible "beyond Moore" technology with the potential to deliver unprecedented computational power with minimal power consumption. Success will depend, however, on our ability to spatially and dynamically control the phase behavior of these multifunctional materials.

In this two-year project, we will focus on understanding the dynamics of electrochemically driven phase transitions in the cobaltites and nickelates, both correlated electron systems with properties that vary from insulating to metallic or antiferromagnetic to ferromagnetic, depending on the Co or Ni oxidation state.

\section{MISSION RELEVANCE}

This project will benefit two major communities in DOE: one interested in the utilization of coherent X-ray techniques for understanding material processes, and the other dealing with the phase behavior of complex oxide heterostructures. There also will be benefits to those aiming to move to "beyond Moore" technologies, as our program will provide new insight into the temporal dynamics occurring during phase transitions.

\section{RESULTS AND ACCOMPLISHMENTS}

Figure 1 shows the behavior of the two-time correlation function during the $\mathrm{SrCoO}_{2.5} \rightarrow \mathrm{SrCoO}_{3}$ phase transition (left) and the $\mathrm{SrCoO}_{3} \rightarrow \mathrm{SrCoO}_{2.5}$ transition (right). This correlation function is used to analyze non-equilibrium fluctuations and here depicts the dynamics of the $\mathrm{SrCoO}_{2.5}$ phase as it dissolves into the matrix (left) or nucleates and grows (right). These are the first such measurements on complex oxide materials, demonstrating the unique ability of coherent $X$-rays to probe phase dynamics at the atomic scale.

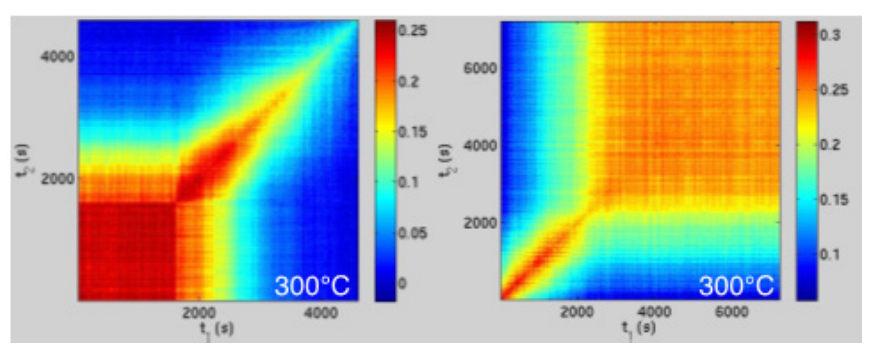

Figure 1. Two-time correlation maps for oxidation $\left(\mathrm{SrCoO}_{2.5} \rightarrow \mathrm{SrCoO}_{3}\right)$ on the left and reduction $\left(\mathrm{SrCoO}_{3} \rightarrow \mathrm{SrCoO}_{2.5}\right)$ on the right at $300^{\circ} \mathrm{C}$. Data were taken at the superlattice reflection for $\mathrm{SrCoO}_{2.5}$, which is sensitive to the nucleation or dissolution of the phase.

\section{PROPOSED FUTURE WORK}

In FY 2018, we aim to build on the work described above and investigate charge dynamics using resonant XPCS in an electrochemical cell. We will also work with theorists on modeling of transition dynamics. 


\section{Scalable Data Movement for Data-Centric Supercomputing}

\author{
2017-156-NO \\ Venkatram Vishwanath and Francois Tessier
}

\section{PROJECT DESCRIPTION}

Data-centric applications executing on supercomputing systems, such as future exascale systems, require the ability to rapidly and reliably compute, move, and manage large amounts of data through a deep and complex network interconnect and memory hierarchy and with diverse sources and destinations, including scientific instruments, storage systems, supercomputers, and analysis systems. Existing data movement solutions on supercomputers for scientific applications are based on decades-old designs for sequential systems and are not suitable to handle the deep memory, interconnect, and storage hierarchies with diverse performance characteristics expected in these systems. Our objective is to model and abstract the resource characteristics (e.g., topology and nonvolatile memory) and the application's data flow behavior and patterns (including input/output [I/O] and communications), and develop cross-layer optimal transformations for mapping flows effectively to underlying resources. We are collaborating on this project with Dr. Emmanuel Jeannot and Dr. Guillaume Aupy at INRIA in Bordeaux, France.

\section{MISSION RELEVANCE}

Data-centric supercomputing is important to DOE's mission in science. Supercomputing is becoming an increasingly integral part of scientific domains such as climate, material science, medicine, cosmology, engineering, combustion, and astrophysics. This project will help accelerate the transformation of raw data into insights and discoveries, and improve our understanding of the data-centric infrastructures needed at exascale and future facilities. This transformation will help facilitate coupling multiphysics simulation codes to better model complex phenomena, large-scale machine learning to predict various outcomes, and tightly coupling experimental and computational science to understand and design new materials and drugs, among other applications.

\section{RESULTS AND ACCOMPLISHMENTS}

We have developed TAPIOCA, a message passing interface (MPI)-based library implementing an efficient topology-aware, two-phase I/O algorithm. TAPIOCA's design features include double-buffering and one-sided communication to reduce (as much as possible) the idle time for an application during data aggregation. We have developed a performance cost model leading to a topology-aware aggregator placement for optimizing data movement. We evaluated our approach on two leadership-class supercomputers at the Argonne Leadership Computing Facility (ALCF): Mira (IBM BG/Q) and Theta (Cray XC40). We have obtained performance results with TAPIOCA on a micro-benchmark and the I/O kernel of a large-scale simulation. This work has resulted in peer-reviewed papers. On both architectures, we showed a substantial improvement of I/O performance compared with the default MPI I/O implementation. On BG/Q+GPFS, for instance, our algorithm lead to a performance improvement by a factor of twelve, while on the Cray XC40 system associated with a Lustre filesystem, we achieved an improvement factor of four.

\section{PROPOSED FUTURE WORK}

In FY 2018, we will work toward:

$\square$ Refining our resource abstractions and models to more accurately model current and expected future systems. Our first target is to include a deeper memory hierarchy such as high-bandwidth memory, dynamic random-access memory, and non-volatile memory, among others.

$\square$ Extending our library to account for additional application patterns.

$\square$ Evaluating the library on the ALCF supercomputing systems. 
PROGRAM ASSESSMENT 


\section{PERFORMANCE METRICS}

The LDRD Program Office collects statistical data on current and completed LDRD projects. The data cover various items such as inventions (see appendix to this report), publications (see appendices to this report), follow on sponsorship, and the enhancement of staff by postdoctoral appointees and new hires. In the aggregate, these metrics provide a picture of overall program productivity. Summaries of the most recently collected data are provided here in Tables 1 and 2. The first table displays project outcomes realized only during FY 2017 but derived from projects active in any year or years from FY 2014 forward. The data in Table 1 are subdivided by LDRD Program component. For the same prior and currently active projects, the second table displays those metrics for which cumulative results are monitored, with no restriction as to when the accomplishments occurred.

Table 1. Aggregate FY 2017 Outcomes for Recently Completed and Current Projects

\begin{tabular}{|c|c|c|c|c|c|c|}
\hline LDRD Component & $\begin{array}{l}\text { Number of } \\
\text { Refereed } \\
\text { Publications }\end{array}$ & $\begin{array}{l}\text { Number of } \\
\text { Students/ } \\
\text { Postdocs } \\
\text { Supported }\end{array}$ & $\begin{array}{c}\text { Number of } \\
\text { New Staff } \\
\text { Hires }\end{array}$ & $\begin{array}{l}\text { Number of } \\
\text { Non-Publication } \\
\text { Copyrights }\end{array}$ & $\begin{array}{l}\text { Number of } \\
\text { Invention } \\
\text { Disclosures }\end{array}$ & $\begin{array}{l}\text { Number } \\
\text { of Patents } \\
\text { Issued/ } \\
\text { Pending }\end{array}$ \\
\hline \multicolumn{7}{|l|}{2014} \\
\hline Competitive Grants & 0 & 0 & 0 & 0 & 1 & 1 \\
\hline Strategic Initiative & 0 & 0 & 0 & 0 & 0 & 1 \\
\hline \multicolumn{7}{|l|}{2015} \\
\hline Competitive Grants & 9 & 0 & 0 & 0 & 0 & 1 \\
\hline Strategic Initiative & 23 & 0 & 0 & 2 & 3 & 5 \\
\hline \multicolumn{7}{|l|}{2016} \\
\hline Competitive Grants & 16 & 0 & 0 & 0 & 1 & 1 \\
\hline Strategic Initiative & 0 & 0 & 0 & 0 & 0 & 0 \\
\hline Swift & 0 & 0 & 0 & 0 & 0 & 0 \\
\hline Prime & 42 & 0 & 0 & 1 & 1 & 5 \\
\hline \multicolumn{7}{|l|}{2017} \\
\hline Innovate & 27 & 53 & 2 & 0 & 3 & 4 \\
\hline Named Fellows & 37 & 2 & 3 & 0 & 1 & 1 \\
\hline Swift & 3 & 23 & 0 & 1 & 6 & 0 \\
\hline Prime & 73 & 133 & 23 & 0 & 31 & 16 \\
\hline Totals: 283 Projects & 230 & 211 & 28 & 4 & 47 & 35 \\
\hline
\end{tabular}


Table 2. Aggregate Outcomes for All Years of Recently Completed and Current Projects (2014-2017)

\begin{tabular}{|c|c|c|c|c|}
\hline $\begin{array}{l}\text { Most Recent } \\
\text { Project Year }\end{array}$ & $\begin{array}{c}\text { Number of } \\
\text { Projects } \\
\text { Receiving } \\
\text { Follow-on Funds }\end{array}$ & $\begin{array}{l}\text { Number of } \\
\text { Proposals } \\
\text { Submitted to } \\
\text { Sponsors }\end{array}$ & $\begin{array}{l}\text { Number of } \\
\text { Intellectual } \\
\text { Property } \\
\text { Events }\end{array}$ & $\begin{array}{c}\text { Number of } \\
\text { External } \\
\text { Reviews* }\end{array}$ \\
\hline & & & & $8 \mathrm{DOE}$ \\
\hline \multirow[t]{4}{*}{2014} & 13 & 96 & 39 & 19 UofC \\
\hline & & & & 2 Other \\
\hline & & & & 29 Total \\
\hline & & & & $10 \mathrm{DOE}$ \\
\hline \multirow[t]{4}{*}{2015} & 20 & 129 & 46 & 35 UofC \\
\hline & & & & 3 Other \\
\hline & & & & 48 Total \\
\hline & & & & $7 \mathrm{DOE}$ \\
\hline \multirow[t]{4}{*}{2016} & 27 & 152 & 85 & 34 UofC \\
\hline & & & & 6 Other \\
\hline & & & & 47 Total \\
\hline & & & & $7 \mathrm{DOE}$ \\
\hline \multirow[t]{4}{*}{2017} & 28 & 200 & 70 & 20 UofC \\
\hline & & & & 12 Other \\
\hline & & & & 39 Total \\
\hline & & & & 32 DOE \\
\hline \multirow[t]{3}{*}{ Totals: 283 Projects } & 88 & 577 & 240 & 108 UofC \\
\hline & & & & 23 Other \\
\hline & & & & 163 Total \\
\hline
\end{tabular}

*UofC refers to UChicago Argonne, LLC. 


\section{LDRD PROJECT CHARACTERISTICS}

\section{MISSION RELEVANCE}

Evaluating the relevance of LDRD projects with respect to DOE mission areas is of utmost importance during the review and selection process. All LDRD projects have demonstrable ties to one or more of the science, energy, environment, and national security missions of the U.S. Department of Energy (DOE), and many are also relevant to the missions of other federal agencies that sponsor work at Argonne. Figure 1 represents the number of FY 2017 LDRD projects supporting one or more of the four DOE mission areas.

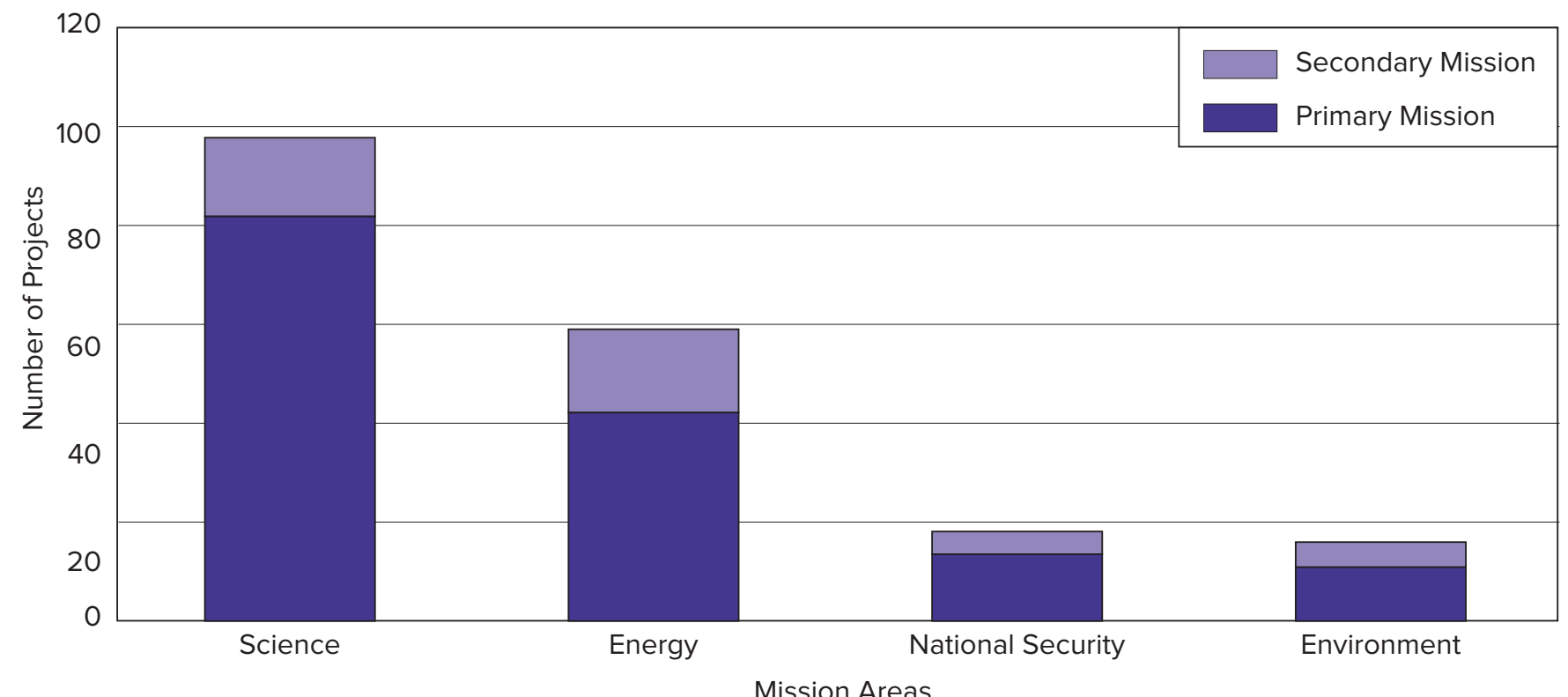

Figure 1. Number of LDRD Projects Supporting the DOE Mission Areas

\section{LEVEL OF FUNDING}

Figure 2 depicts the funding distribution for the 146 projects funded in FY 2017. Around 34\% of the projects were in the $\$ 100 \mathrm{~K}$ to $\$ 199 \mathrm{~K}$ range, $24 \%$ of the projects were in the $\$ 200 \mathrm{~K}$ to $\$ 299 \mathrm{~K}$ range with a little more than $21 \%$ receiving $\$ 100 \mathrm{~K}$ or less. About $24 \%$ of the projects received between $\$ 300 \mathrm{~K}$ and $\$ 599 \mathrm{~K}$, while only $3 \%$ of projects were in the range of $\$ 600 \mathrm{~K}$ to $\$ 999 \mathrm{~K}$. About $1 \%$ of projects received more than $\$ 1 \mathrm{M}$. The average funding level of projects in FY 2017 was approximately $\$ 225 \mathrm{~K}$.

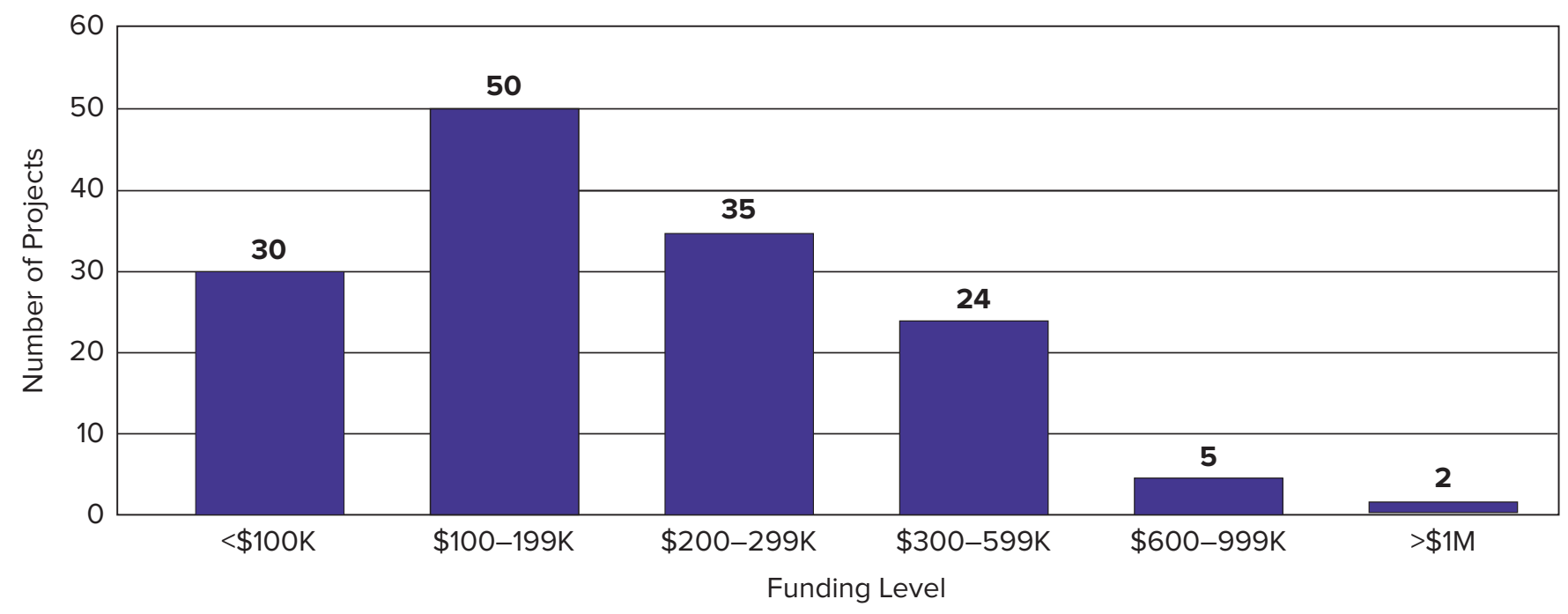

Figure 2. Number of Projects and Levels of Funding 
APPENDIX 


\section{INTELLECTUAL PROPERTY EVENTS OCCURING IN FY 2017}

\section{(As a Result of LDRD Projects and Subsequent Related Sponsored Research) INVENTION DISCLOSURES}

\section{8-195}

PATENT GRANTED

Gosztola, D.J., D. Lopez, D. Mancini, and G. Wiederrecht. "Microelectromechanical (MEMS) Manipulators for Control of Nanoparticle Coupling Interactions." Patent No. 9,548,677 granted January 2017. [ANL-IN-09-050B]

\section{0-185}

\section{PATENTS GRANTED}

Amine, K. and Z. Zhang. "Lithium Air Battery Having a Cross-Linked Polysioloxane Separator." Patent No. 9,478,782 granted October 2016. [ANL-IN-10-004]

Amine, K., J-J. Woo, and Z. Zhang. "Advanced Separators Based on Aromatic Polymer for High Energy Density Lithium Batteries.” Patent No. 9,598,545 granted March 2017. [ANL-IN-11-063]

Amine, K., L. Curtiss, K.C. Lau, J. Lu, Y-K. Sun, and Z. Zhang. "Lithium Air Batteries Having Ether-Based Electrolytes." Patent No. 9,478,837 granted October 2016. [ANL-IN-11-129B]

Amine, Khalil, L. Curtiss, P. Du, J. Lu, and J. Wen. "Porous Graphene Nanocages for Battery Applications."

Patent No. 9,590,248 granted March 2017. [ANL-IN-12-060]

\section{1-210}

\section{PATENT GRANTED}

Amine, K., L. Curtiss, J. Lu, E. Tyo, and S. Vajda. "Lithium-Air Batteries, Method for Making Lithium-Air Batteries."

Patent No. 9,496,590 granted November 2016. [ANL-IN-13-032]

\section{2-072}

\section{INVENTION DISCLOSURE}

Sumant, A., A. Erdemir, and D. Berman. "Low Friction Wear Resistant Graphene Films." Invention report disclosed January 2017. [ANL-IN-14-027B]

\section{PATENT APPLICATION}

Sumant, A., A. Erdemir, and D. Berman, "Low Friction Wear Resistant Graphene Films." Patent Application No. 15/408,137 filed January 2017. [ANL-IN-14-027B]

\section{PATENT GRANTED}

Sumant, A., A. Erdemir, and D. Berman. "Low Friction Wear Resistant Graphene Films." Patent No. 9,561,526 granted February 2017. [ANL-IN-14-027]

\section{3-116}

\section{PATENT GRANTED}

Yacout, A., M. Pellin, D. Yun, and M. Billone. "ALD Coating of Nuclear Fuel Actinides Materials." Patent No. 9,754,687 granted September 2017. [ANL-IN-12-042] [Also see 2013-152.] 


\section{3-147}

\section{PATENT APPLICATION}

Novosad, V., E. Rozhkova, and M. Nikitin. "Ferromagnetic Particles as Ultra-Sensitive Non-Linear Response Labels for Magnetic Particles Imaging (MPI) and Sensing Applications.” Patent Application filed August 2017. [ANL-IN-16-045]

\section{3-148}

\section{NON-PUBLICATION COPYRIGHTS}

Som, S., P. Kundu, M.M. Ameen, and U. Unnikrishnan. “TESF (Tabulated Equivalent SDR Flamelet).” Software copyright issued June 2017. [ANL-SF-16-159]

Som, S. and M. Ameen. "Parallel Pertubation Model for Cycle to Cycle Variability (PPM4CCV)." Software copyright issued June 2017. [ANL-SF-17-030]

\section{3-152}

\section{INVENTION DISCLOSURE}

Bhattacharya, S., M. Pellin, and A. Yacout. "Composite Matrix Using a Hybrid Deposition Technique." Invention report disclosed November 2016. [ANL-IN-16-143] [Also see 2016-159.]

\section{PATENT APPLICATION}

Bhattacharya, S., M. Pellin, and A. Yacout. "Composite Matrix Using a Hybrid Deposition Technique." Patent Application filed June 2017. [ANL-IN-16-143] [Also see 2016-159.]

\section{PATENT GRANTED}

Yacout, A., M. Pellin, D. Yun, and M. Billone. “ALD Coating of Nuclear Fuel Actinides Materials.” Patent No. 9,754,687 granted September 2017. [ANL-IN-12-042] [Also see 2013-116.]

\section{3-154}

\section{INVENTION DISCLOSURE}

Elam, J., J. Libera, and A. Yanguas-Gil. “Method and System for Continuous Atomic Layer Deposition.” Invention report disclosed February 2017. [ANL-IN-13-042B]

\section{PATENT APPLICATION}

Elam, J., J. Libera, and A. Yanguas-Gil. "Method and System for Continuous Atomic Layer Deposition."

Patent Application No. 15/426,789 filed February 2017. [ANL-IN-13-042B]

\section{PATENTS GRANTED}

Elam, J., J. Libera, and A. Yanguas-Gil. "Method and Systems for Continuous Atomic Layer Deposition." Patent No. 9,598,769 granted March 2017. [ANL-IN-13-042]

Elam, J. and A. Yanguas-Gil. "Fast Method for Reactor and Feature Scale Coupling in ALD and CVD." Patent No. 9,727,672 granted August 2017. [ANL-IN-14-026]

\section{4-051}

INVENTION DISCLOSURE

Liu, D-J. and L. Chong. "Nanofiber Electrocatalysts.” Invention report disclosed January 2017. [ANL-IN-17-002]

\section{PATENT APPLICATION}

Liu, D-J. and L. Chong. "Nanofiber Electrocatalysts.” Patent Application filed August 2017. [ANL-IN-17-002] 


\section{4-133}

\section{INVENTION DISCLOSURE}

Weimer, M., T. Proslier, J. Klug, and A. Hock. "Amorphous Elemental Germanium Thin Films Grown by Self-Limiting Vapor Deposition at Low Temperature." Invention report disclosed March 2017. [ANL-IN-17-021]

\section{4-145}

\section{INVENTION DISCLOSURE}

Beckman, P., C. Catlett, and J. Gilbert. "Automated Microbial Detection and Quantification." Invention report disclosed November 2016. [ANL-IN-16-155]

\section{PATENT APPLICATION}

Beckman, P., C. Catlett, and J. Gilbert. "Automated Microbial Detection and Quantification." Patent Application filed April 2017. [ANL-IN-16-155]

\section{4-161}

\section{PATENT APPLICATION}

Chan, H., M. Cherukara, B. Naraayanan, and S. Sankaranarayanan. "Marchine Learning Technique to Identify Grains in Polycrystalline Materials Samples." Patent Application filed August 2017. [ANL-IN-16-126] [Also see 2015-149.]

\section{4-169}

\section{PATENTS GRANTED}

Chen, X. and Z. Zhou. "Magnetic Nanofiber Composite Materials and Devices Using Same." Patent No. 9,620,839 granted April 2017. [ANL-IN-14-097]

Chen, X. and R. Stoddard. "Image Processing Tool for Automatic Feature Recognition and Quantification." Patent No. 9,639,926 granted May 2017. [ANL-IN-14-107]

\section{5-091}

\section{INVENTION DISCLOSURES}

Liu, D-J. and D. Rebollar. "Carbon Dioxide Reduction Electro Catalysts Prepared for Metal Organic Frameworks." Invention report disclosed March 2017. [ANL-IN-17-028]

Liu, D-J. and D. Rebollar. "Innovative lonomers with Intrinsic Porosity in Promoting Charge/Mass Transfer in Membrane Electrodes." Invention report disclosed September 2017. [ANL-IN-17-108]

\section{PATENT APPLICATION}

Liu, D-J. and D. Rebollar. "Carbon Dioxide Reduction Electro Catalysts Prepared for Metal Organic Frameworks." Patent Application filed August 2017. [ANL-IN-17-028]

\section{5-121}

\section{PATENT APPLICATION}

Li, J., I. Castano, S. Dull, L.B. Guzowski, R. Muehleisen, Y. Sun, and A. Yan. "Continuous Flow Synthesis of VO2 Nanoparticles for Nanorods by Using a Microreactor.” Patent Application No. 15/487, 276 filed April 2017. [ANL-IN-15-033]

\section{5-149}

\section{PATENT APPLICATION}

Chan, H., M. Cherukara, B. Naraayanan, and S. Sankaranarayanan. "Marchine Learning Technique to Identify Grains in Polycrystalline Materials Samples." Patent Application filed August 2017. [ANL-IN-16-126] [Also see 2014-161.] 


\section{5-151}

\section{INVENTION DISCLOSURES}

Elam, J. and A. Mane. "Method of Creating Boron Comprising Layer." Invention report disclosed October 2016. [ANL-IN-16-142]

Elam, J. and A. Mane. "Synthesis of Non-Toxic 10-B Containing Biocompatible Nanoparticle Based Nanonmedicines for Boron Neutron Capture Therapy." Invention report disclosed December 2016. [ANL-IN-16-170]

Mane, A. and J. Elam. "Methods to Deposit and Etch Controlled Thin Layers of Transition Metal Dichalcogenides." Invention report disclosed May 2017. [ANL-IN-17-061]

Graugnard, E., J. Elam, S. Letourneau, and A. Mane. "Methods to Control the Interface of Thin Layers of Transition Metal Dichalcogenides and Dielectrics." Invention report disclosed May 2017. [ANL-IN-17-062]

Mane, A. and J. Elam. "Methods to Control the Interface of Thin Layers of Transition Metal Dichalcogenides and Conducing Contact Materials." Invention report disclosed May 2017. [ANL-IN-17-063]

Mane, A. and J. Elam. "Apparatus and Method for Efficient Coating of Powder Materials." Invention report disclosed August 2017. [ANL-IN-17-095]

\section{PATENT APPLICATIONS}

Elam, J. and A. Mane. "Enhanced Electron Amplifier Structure and Method of Fabricating the Enhanced Electron Amplifier Structure." Patent Application filed August 2017. [ANL-IN-15-123]

Elam, J. and A. Mane. "Enhanced Neutron Detector and Electron Amplifier Structure Q and A Method of Fabricating the Enhanced Neutron Detector and Electron Amplifier Structure." Patent Application filed August 2017. [ANL-IN-16-041]

Elam, J. and A. Mane. "Method of Creating Boron Comprising Layer." Patent Application filed August 2017.

[ANL-IN-16-142]

\section{6-020}

\section{INVENTION DISCLOSURE}

Aronson, I., R. Divan, P. Laible, M. Michalska, P. Noirot, and A. Sokolov. "Antimicrobial Black Silicon." Invention report disclosed June 2017. [ANL-IN-17-059]

\section{PATENT APPLICATION}

Aronson, I., R. Divan, P. Laible, M. Michalska, P. Noirot, and A. Sokolov. "Antimicrobial Black Silicon." Patent Application No. 62/562,244 filed September 2017. [ANL-IN-17-059]

\section{6-054}

\section{PATENT APPLICATION}

Chan, M., A. Martinson, and M. Sampson. "Substituted Lead-Halide Perovskite Intermediate Band Absorbers." Patent Application filed January 2017. [ANL-IN-16-092]

\section{6-131}

\section{INVENTION DISCLOSURE}

Bunquin, J.C. and M. Ferrandon. "Supported Multimetallic Catalysts for Oxidative Dehydrogenation of Alkanes." Ferrandon, Magali. "Supported Multimetallic Catalysts for Oxidative Dehydrogenation of Alkanes." Invention report disclosed May 2017. [ANL-IN-17-006]

\section{PATENT APPLICATION}

Bunquin, J.C. and M. Ferrandon. "Supported Multimetallic Catalysts for Oxidative Dehydrogenation of Alkanes." Patent Application No. 15/688,699 filed August 2017. [ANL-IN-17-006] 


\section{6-133}

\section{INVENTION DISCLOSURE}

Jeon, N., J. Foley, S. Gray, and A. Martinson. "High Temperature Selective Emitters via Critical Coupling of Weak Absorbers." Invention report disclosed August 2017. [ANL-IN-17-086]

\section{6-140}

\section{INVENTION DISCLOSURE}

Lu, J., S. Bakhtiari, and A. Heifetz. "Microwave and Impedance Spectroscopy Methods for in situ Nondestructive Evaluation of Concrete Degradation." Invention report disclosed November 2016. [ANL-IN-16-149]

\section{PATENT APPLICATION}

Bakhtiari, S., A. Heifetz, and R. Vilim. "Transmission of Information by Acoustic Communication Along Metal Pathways in Nuclear Facilities.” Patent Application filed February 2017. [ANL-IN-16-050]

\section{6-148}

\section{INVENTION DISCLOSURE}

Jin, Z. “A Customizable Open CL Library for FPGA Platforms." Invention report disclosed August 2017. [ANL-IN-17-075]

\section{6-158}

INVENTION DISCLOSURES

Brown, M., S. Chemerisov, W. Henning, J. Nolen, and D. Rotsch. "Compact Assembly for Efficient Production of Medical Isotopes via Photonuclear Reactions.” Invention report disclosed May 2017. [ANL-IN-16-136]

Nolen, J., J. Bailey, D. Rotsch, N. Smith, and S. Chemerisov. “Triple Containment Targets for Particle Irradiation.” Invention report disclosed June 2017. [ANL-IN-17-043]

Grudzinski, J., D. Rotsch, J. Bailey, R. Kmak, D. Ehst, S. Chemerisov, J. Nolen, N. Smith, and M. Brown "Radioisitope Target Station (RITS)." Invention report disclosed August 2017. [ANL-IN-17-091]

\section{PATENT APPLICATIONS}

Brown, M., S. Chemerisov, W. Henning, J. Nolen, and D. Rotsch. "Compact Assembly for Efficient Production of Medical Isotopes via Photonuclear Reactions.” Patent Application filed September 2017. [ANL-IN-16-136]

Nolen, J., J. Bailey, D. Rotsch, N. Smith, and S. Chemerisov. "Triple Containment Targets for Particle Irradiation." Patent Application filed September 2017. [ANL-IN-17-043]

\section{6-159}

\section{INVENTION DISCLOSURE}

Bhattacharya, S., M. Pellin, and A. Yacout. "Composite Matrix Using a Hybrid Deposition Technique." Invention report disclosed November 2016. [ANL-IN-16-143] [Also see 2013-152.]

\section{PATENT APPLICATION}

Bhattacharya, S., M. Pellin, and A. Yacout. "Composite Matrix Using a Hybrid Deposition Technique." Patent Application filed June 2017. [ANL-IN-16-143]

\section{6-173}

\section{PATENT APPLICATION}

Elam, J., A. Mane, H. Nicholson, M. Pellin, R. Wagner, J. Wang, L. Xia, and J. Xie. "3D Printed Micro Channel Plate, Method of Making and Using 3D Printed Micro Channel Plate.” Patent Application filed September 2017. [ANL-IN-16-104] 


\section{6-180}

\section{PATENT APPLICATION}

Acik, M. and S. Darling. "One Step in situ Solution Growth Method for Lead Perovskite." Patent Application filed June 2017. [ANL-IN-16-118]

\section{6-182}

\section{INVENTION DISCLOSURES}

Gao, K. and Y. Xie. "Wearable Wireless Motion Tracking Sensor and System." Invention report disclosed May 2017. [ANL-IN-17-046] [Also see 2017-076 and 2017-166.]

Hryn, J. and Y. Xie. “Improved FMR Power Supply.” Invention report disclosed September 2017. [ANL-IN-17-111] [Also see 2017-076 and 2017-166.]

\section{6-186}

\section{INVENTION DISCLOSURE}

Schaller, R. and B. Diroll. "Method for Fabrication of Superlattices and Aperiodic Layered Structures Using Solution Deposition." Invention report disclosed December 2016. [ANL-IN-16-171]

\section{PATENT APPLICATION}

Schaller, R. and B. Diroll. "Method for Fabrication of Superlattices and Aperiodic Layered Structures Using Solution Deposition.” Patent Application filed July 2017. [ANL-IN-16-171]

\section{6-187}

\section{PATENT APPLICATION}

Fuoss, P., S. Hryszkewycz, A. Tripathi, A. Ulvestad, and S. Wild. "Method for Phase Retrieval to Reduce a Sampling Requirement When Imaging a Dynamic Process.” Patent Application filed October 2016. [ANL-IN-15-146]

\section{6-190}

\section{INVENTION DISCLOSURE}

Husko, C. "Hybrid Silicon Lasers and Amplifiers with 2D Phosphorene Film." Invention report disclosed January 2017. [ANL-IN-16-173]

\section{PATENT APPLICATION}

Husko, C. "Hybrid Silicon Lasers and Amplifiers with 2D Phosphorene Film." Patent Application filed September 2017. [ANL-IN-16-173]

\section{7-055}

\section{INVENTION DISCLOSURE}

Lin, Y. "Electrochemical Method of Simultaneous CO2 Delivery and H2 Production for Waste to Energy." Invention report disclosed September 2017. [ANL-IN-17-109]

\section{7-076}

\section{INVENTION DISCLOSURES}

Gao, K. "Technology to Enable a Self-Biasing Magnetic Template." Invention report disclosed February 2017.

[ANL-IN-17-005]

Gao, K. "Plasmonic on Chip for Near Field Optics and Other Technologies." Invention report disclosed February 2017. [ANL-IN-17-009] 
Gao, K. "Fiber on Chip Technology to Enable Fully Intewgrated Communication Devices." Invention report disclosed February 2017. [ANL-IN-17-010]

Gao, K. and S. Zhang. "Technology Solutions Enable Higher Sensitivity and Resolution on Magnetic Force Microscopy (MFM).” Invention report disclosed February 2017. [ANL-IN-17-011]

Gao, Kaizhong, Y. Xie. “A Mechanical Structure Reversibly Deformable Conductive Ink." Invention report disclosed February 2017. [ANL-IN-17-012] [Also see 2017-116.]

Zhang, Y. and K. Gao. "Novel Permanent Magnet Design to Enable Higher Magnetic Flux Density." Invention report disclosed February 2017. [ANL-IN-17-017]

Gao, K. and Y. Xie. "A Template Based Method to Self-Biased Magnetic Device." Invention report disclosed February 2017. [ANL-IN-17-023]

Gao, K. "Energy Storage Technology with Extreme High Energy Density Capability." Invention report disclosed April 2017. [ANL-IN-17-033] [Also see 2017-161.]

Gao, K. and Y. Xie. "Wearable Wireless Motion Tracking Senor and System." Invention report disclosed May 2017. [ANL-IN-17-046] [Also see 2016-182 and 2017-116.]

Gao, K. "Magnetic Nanowire to Enable Bio-Labeling." Invention report disclosed July 2017. [ANL-IN-17-074]

Hryn, J. and Y. Xie. "Improved FMR Power Supply." Invention report disclosed September 2017. [ANL-IN-17-111] [Also see 2016-182 and 2017-116.]

\section{PATENT APPLICATIONS}

Zhang, Y. and K. Gao. "Novel Permanent Magnet Design to Enable Higher Magnetic Flux Density." Patent Application filed August 2017. [ANL-IN-17-017]

Gao, K. “Energy Storage Technology with Extreme High Energy Density Capability." Patent Application filed June 2017. [ANL-IN-17-033]

\section{7-104}

\section{INVENTION DISCLOSURE}

Liu, Y., I. McNulty, T. Rajh, and Y. Wu. "Controlled Photocatalytic Facet Dependent CO2 Reduction to Methanol or Carbon Dioxide Using Cu2O Particles." Invention report disclosed August 2017. [ANL-IN-17-073]

\section{7-107}

\section{INVENTION DISCLOSURE}

Spangenberger, J., K. Richa, L. Gaines, A. Hubaud Heidet, M. Wang, Q. Dai, S. Ahmed, J. Kelly, and J. Dunn. "A Battery Recycling Model." Invention report disclosed April 2017. [ANL-IN-17-035]

\section{7-116}

\section{INVENTION DISCLOSURES}

Gao, K. and Y. Xie. "A Mechanical Structure Reversibly Deformable Conductive Ink." Invention report disclosed February 2017. [ANL-IN-17-012] [Also see 2017-076.]

Gao, K. and Y. Xie. "Wearable Wireless Motion Tracking Sensor and System." Invention report disclosed May 2017. [ANL-IN-17-046] [Also see 2016-182 and 2017-076.]

Hryn, J. and Y. Xie. "Improved FMR Power Supply." Invention report disclosed September 2017. [ANL-IN-17-111] 
2017-160

INVENTION DISCLOSURE

Yanguas-Gil, A. “Neuromorphic Systems.” Invention report disclosed September 2017. [ANL-IN-17-022]

\section{NON-PUBLICATION COPYRIGHT}

Yanguas-Gil, A. "SNEURON: A State-Based Implementation of Spiking Neural Networks." Software copyright issued June 2017. [ANL-SF-17-022]

\section{7-161}

\section{INVENTION DISCLOSURE}

Gao, K. "Energy Storage Technology with Extreme High Energy Density Capability." Invention report disclosed April 2017. [ANL-IN-17-033] [Also see 2017-076.]

\section{PATENT APPLICATION}

Gao, K. “Energy Storage Technology with Extreme High Energy Density Capability." Patent Application filed June 2017. [ANL-IN-17-033] 


\section{PUBLICATIONS AND PRESENTATIONS I}

\section{(Derived from LDRD Projects Active in Fiscal Year 2017)}

[Dates following author names indicate the fiscal year of appearance.]

\section{LDRD INNOVATE}

\section{5-078-R2 \\ REFEREED PUBLICATIONS}

Benseman, T.M., A.E. Koshelev, V. Vlasko-Vlasov, Y. Hao, W.K. Kwok, U. Welp, C. Keiser, B. Gross, M. Lange, D. Kölle, R. Kleiner, H. Minami, C. Watanabe, and K. Kadowaki (2015). "Current Filamentation in Large $\mathrm{Bi}_{2} \mathrm{Sr}_{2} \mathrm{CaCu}_{2} \mathrm{O}_{8+} \delta$ Mesa Devices Observed via Luminescent and Scanning Laser Thermal Microscopy.” Physical Review Applied 3(4).

Benseman, T.M., H. Yang, V.K. Vlasko-Vlasov, U. Welp, A.E. Koshelev, W.-K. Kwok, R. Divan, C. Keiser, C. Watanabe, and K. Kadowaki (2017). "High-Resolution Thermal Micro-Imaging Using Europium Chelate Luminescent Coatings." Jove-Journal of Visualized Experiments (122): e53948.

Simsek, Y., V. Vlasko-Vlasov, A.E. Koshelev, T. Benseman, Y. Hao, I. Kesgin, H. Claus, J. Pearson, W.-K. Kwok, and U. Welp. "Thick $\mathrm{Bi}_{2} \mathrm{Sr}_{2} \mathrm{CaCu}_{2} \mathrm{O}_{8+} \delta$ Films Grown by Liquid-Phase Epitaxy for Josephson $\mathrm{THz}$ Applications." Superconductor Science and Technology. (To be published.)

\section{NON-REFEREED PUBLICATIONS}

Benseman, T., A. Koshelev, V. Vlasko-Vlasov, U. Welp, W.-K. Kwok, Y. Hao, B. Gross, M. Lange, D. Koelle, R. Kleiner, and K. Kadowaki (2016). "The Cavity Resonance Mode of $\mathrm{Bi}_{2} \mathrm{Sr}_{2} \mathrm{CaCu}_{2} \mathrm{O}_{8}$ Mesa Terahertz Source as Probed by Scanning Laser Thermal Microscopy.” 2016 March Meeting of the American Physical Society, Baltimore, MD, March 14-18, 2016.

Hao, Y., U. Welp, A. Koshelev, V. Vlasko-Vlasov, W.-K. Kwok, K. Kadowaki, and T. Benseman (2016). "Artificially Induced Hotspots in $\mathrm{Bi}_{2} \mathrm{Sr}_{2} \mathrm{CaCu}_{2} \mathrm{O}_{8}$ Mesa Tetrahertz Sources." 2016 March Meeting of the American Society, Baltimore, MD, March 14-18, 2016.

Simsek, Y., V. Vlasko-Vlasov, A. Koshelev, T. Benseman, I. Kesgin, W.-K. Kwok, and U. Welp (2017). “Growth and Characterization of Epitaxial $\mathrm{Bi}_{2} \mathrm{Sr}_{2} \mathrm{CaCu}_{2} \mathrm{O}_{8+} \delta$ Thin Films for Large-Scale Fabrication of Josephson Plasma Wave-Based THz Devices." American Physical Society March Meeting, New Orleans, LA, March 13-17, 2017.

\section{PRESENTATIONS}

Benseman, T.M., A.E. Koshelev, V. Vlasko-Vlasov, Y. Hao, W.-K. Kwok, U. Welp, C. Keiser, B. Gross, M. Lange, D. Koelle, R. Kleiner, and K. Kadowaki (2015). "Comparison of Luminescent and Scanning Laser Thermal Micro-Imaging of Self-Heating in $\mathrm{Bi}_{2} \mathrm{Sr}_{2} \mathrm{CaCu}_{2} \mathrm{O}_{8+} \delta$ Mesa THz Sources." American Physical Society March Meeting, San Antonio, TX, March 2-6, 2015.

Benseman, T.M., A.E. Koshelev, V. Vlasko-Vlasov, Y. Hao, U. Welp, W.-K. Kwok, B. Gross, M. Lange, D. Koelle, R. Kleiner, and K. Kadowaki (2015). "Terahertz Radiation from $\mathrm{Bi}_{2} \mathrm{Sr}_{2} \mathrm{CaCu}_{2} \mathrm{O}_{8+} \delta$ Interlayer Josephson Junctions: Progress and Future Strategies.” AMN-7: Advanced Materials and Nanotechnology Conference, Nelson, New Zealand, February 8-12, 2015.

Benseman, T.M., A.E. Koshelev, V. Vlasko-Vlasov, Y. Hao, U. Welp, W.-K. Kwok, B. Gross, M. Lange, D. Koelle, R. Kleiner, and K. Kadowaki (2015). "Terahertz Radiation from $\mathrm{Bi}_{2} \mathrm{Sr}_{2} \mathrm{CaCu}_{2} \mathrm{O}_{8+} \delta$ Interlayer Josephson Junctions: Progress and Future Strategies." City of University of NY-Queens College Colloquium, New York, NY, February 25, 2015.

Hao, Y., T.M. Benseman, A.E. Koshelev, V. Vlasko-Vlasov, W.-K. Kwok, U. Welp, C. Keiser, B. Gross, M. Lange, D. Koelle, R. Kleiner, and K. Kadowaki (2015). "The Stability of Current Filaments in $\mathrm{Bi}_{2} \mathrm{Sr}_{2} \mathrm{CaCu}_{2} \mathrm{O}_{8+} \delta$ Observed Via Luminescent Thermal Microscopy.” American Physical Society March Meeting, San Antonio, TX, March 2-6, 2015. 
Simsek, Y., V. Vlasko-Vlasov, A. Koshelev, T. Benseman, I. Kesgin, J. E. Pearson, W.-K. Kwok, and U. Welp (2017). "Growth and Characterization of Epitaxial $\mathrm{Bi}_{2} \mathrm{Sr}_{2} \mathrm{CaCu}_{2} \mathrm{O}_{8+} \delta$ Thin Films for Large-scale Fabrication of Josephson Plasma Wave-based THz Devices." American Physical Society March Meeting, New Orleans, LA, March 13-17, 2017.

Welp, U., T. Benseman, Y. Hao, A.E. Koshelev, V. Vlasko-Vlasov, W.-K. Kwok, H. Minami, C. Watanabe, K. Kadowaki, B. Gross, M. Lange, D. Koelle, and R. Kleiner (2015). "Current Filamentation in Large $\mathrm{Bi}_{2} \mathrm{Sr}_{2} \mathrm{CaCu}_{2} \mathrm{O}_{8+} \delta$ Mesas Observed by Luminescent and Scanning Laser Thermal Microscopy.” Plasma 2014, Kyoto, Japan, November 30-December 3, 2014.

2015-091- R2

REFEREED PUBLICATIONS

Chong, L., X. Zeng, W. Ding, D.-J. Liu, and J. Zou (2015). “NaBH ${ }_{4}$ in 'Graphene Wrapper:' Significantly Enhanced Hydrogen Storage Capacity and Regenerability through Nanoencapsulation." Advanced Materials 27(34): 5070-5074.

Zou, J., L. Chong, D.-J. Liu, X. Zeng, L. Peng, and W. Ding (2016). “Wrapped by Graphene: An Efficient Way to Achieve High Capacity, Reversible Hydrogen Storage through Nanoencapsulated Hydride." Science 351(6278): 1223.

\section{PRESENTATIONS}

Liu, D.-J. (2015). "Recent Developments in New Materials and Characterization Techniques for Energy Storage and Conversion." University of Illinois at Chicago, Chicago, IL, February 26, 2015.

Liu, D.-J. (2015). "Recent Developments in New Materials and Characterization Techniques for Energy Storage and Conversion." University of Nebraska, Lincoln, NE, October 20, 2014.

Yuan, Y., and D.-J. Liu (2017). "Cobalt-Containing Porous Organic Polymer (POP) as Precursor of New Electrode Catalyst for Oxygen Reduction Reaction." 253rd American Chemical Society National Meeting and Exposition, San Francisco, CA, April 2-6, 2017.

\section{5-096-R2}

\section{REFEREED PUBLICATIONS}

Alderman, O.L.G., C.J. Benmore, J.K.R. Weber, L.B. Skinner, A.J. Tamalonis, S. Sendelbach, A. Hebden, and M.A. Williamson (2017). "The Structure of Liquid $\mathrm{UO}_{2-x}$ in Reducing Gas Atmospheres." Applied Physics Letters 110(8).

Benmore, C.J., and J.K.R. Weber (2017). "Aerodynamic Levitation, Supercooled Liquids and Glass Formation." Advances in Physics: X 2(3): 717-736.

Benmore, C.J. (2017). "Nuclear Materials at Extreme Conditions on 6-ID-D." Journal of Synchrotron Radiation 24(1).

Benmore, C.J., L.B. Skinner, B. Lee, J.R. Weber, J.B. Parise, and M.A. Williamson (2016). "Topological Ordering in Liquid $\mathrm{UO}_{2}$." Journal of Physics: Condensed Matter 28(1).

Guthrie, M., C.J. Benmore, L.B. Skinner, O.L.G. Alderman, J.K.R. Weber, J.B. Parise, and M. Williamson (2017). “Thermal Expansion in $\mathrm{UO}_{2}$ Determined By High-Energy X-ray Diffraction." Journal of Nuclear Materials 479: 19-22.

Skinner, L.B., C.J. Benmore, J.R. Weber, M.A. Williamson, A.J. Tamalonis, A.S. Hebden, T. Wiencek, O.L.G. Alderman, M. Guthrie, L. Leibowitz, and J.B. Parise (2015). "Molten Uranium Dioxide Structure and Dynamics." Science 346(6212): 984-987.

Weber, J.K.R., A.J. Tamalonis, C.J. Benmore, O.L.G. Alderman, S. Sendelbach, A. Hebden, and M.A. Williamson (2016). "Aerodynamic Levitator for in situ X-ray Structure Measurements on High Temperature and Molten Nuclear Fuel Materials." Review of Scientific Instruments 87(7): 073902-073902.

\section{NON-REFEREED PUBLICATION}

Benmore, C.J. (2017). "Aerodynamic Levitator for High-Energy X-ray Scattering from High-Temperature Nuclear Materials.” APS Science 2017. Argonne National Laboratory, ANL-17/09, May 2017. 


\section{5-121-R2}

\section{REFEREED PUBLICATIONS}

Liu, Q., G. Tan, P. Wang, S.C. Abeyweera, D. Zhang, Y. Rong, Y. Wu, J. Lu, C.-J. Sun, Y. Ren, Y. Liu, R.T. Muehleisen, L.B. Guzowski, J. Li, X. Xiao, and Y. Sun (2017). "Revealing Mechanism Responsible for Structural Reversibility of Single-Crystal $\mathrm{VO}_{2}$ Nanorods upon Lithiation/Delithiation.” Nano Energy 36: 197-205.

Yan, X., I. Castano, Y. Sun, R. Muehleisen, L. Guzowski, and J. Li. "Continuous-Flow Synthesis of Thermochromic M Phase $\mathrm{VO}_{2}$ Particles via Rapid One-step Hydrothermal Reaction." Powder Technology. (To be published.)

\section{PRESENTATION}

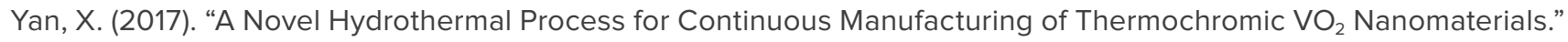
8th AIChE Midwest Regional Conference, Chicago, IL, April 3-4, 2017.

\section{6-001-R1}

\section{REFEREED PUBLICATION}

Sen, A., S.T. Pratt, and K.L. Reid (2017). "Circular Dichroism in Photoelectron Images from Aligned Nitric Oxide Molecules." Journal of Chemical Physics 147(1): 013927.

\section{PRESENTATION}

Sen, A., K.L. Reid, and S.T. Pratt (2016). "Circular Dichroism of Angular Distributions (CDAD) by Using Velocity Map Photoelectron Imaging." Advanced Particle Imaging Techniques: 1986-2016 and Beyond, Telluride, CO, August 8-12, 2016.

\section{6-010-R1}

\section{REFEREED PUBLICATIONS}

Galda, A. and V.M. Vinokur (2017). "Linear Dynamics of Classical Spin as Möbius Transformation." Scientific Reports 7(1): 1168.

Galda, A., and V.M. Vinokur (2016). "Parity-Time Symmetry-Breaking in Magnetic Systems." Physical Review B: Condensed Matter 94(2).

Rademaker, L., V.M. Vinokur, and A. Galda (2017). "Universality and Critical Behavior of the Dynamical Mott Transition in a System with Long-Range Interactions." Scientific Reports 7: 44044.

Tripathi, V., A. Galda, H. Barman, and V.M. Vinokur (2016). "Parity-Time Symmetry-Breaking Mechanism of Dynamic Mott Transitions in Dissipative Systems." Physical Review B: Condensed Matter 94(4).

\section{PRESENTATIONS}

Galda, A., and V.M. Vinokur (2017). "PT Symmetry Breaking in Magnetic Systems." American Physical Society March Meeting 2017, New Orleans, LA, March 17, 2017.

Galda, A., and V.M. Vinokur (2017). "Spin-Transfer Torque-Induced Parity-Time Symmetry Breaking in Magnetic Systems.” Superstripes 2017, Ischia, Italy, June 9, 2017.

Vinokur, V.M. (2016). "Parity-Time Symmetry and Dynamic Phase Transitions." 5th International Conference on Superconductivity and Magnetism, Fenthieye, Turkey, April 24-30, 2016.

Vinokur, V.M. (2016). "Parity-Time Symmetry and Dynamic Phase Transitions." Transport in Interacting Disordered Systems-2016, Granada, Spain, August 21-25, 2016.

Vinokur, V.M. (2016). "Parity-Time Symmetry and Dynamic Phase Transitions." 12th International Conference on Superconductivity and Magnetism, Coma Ruga, Spain, July 4-7, 2016.

Vinokur, V.M. (2016). "Parity-Time Symmetry and Dynamic Phase Transitions." Superstripes-2016, Ischia, Italy, June 23-29, 2016. 
Vinokur, V.M. (2016). "Parity-Time Symmetry Mechanism of Out-of-Equilibrium Phase Transitions." Superconducting and Magnetic Systems, Natal, Brazil, September 3-6, 2016.

Vinokur, V.M. (2017). “PT Symmetry and Dynamic Vortex Mott Transition.” Vortex 2017, Natal, Brazil, May 30, 2017.

Vinokur, V.M. (2016). “Vortex Mott Transition: Experiment and Theory.” International Conference on Quantum Condensed Matter, Engelberg, Switzerland, February 10-12, 2016.

\section{6-020-R1}

REFEREED PUBLICATION

Michalska, M., F. Gambacorta, R. Divan, I.S. Aranson, A. Sokolov, P. Noirot, and P.D. Laible. "Tuning Antimicrobial Properties of Biomimetic Nanopatterned Surfaces." ACS Applied Materials \& Interfaces. (To be published.)

\section{6-023-R1}

REFEREED PUBLICATION

Zhao, C., K. Fezzaa, R.W. Cunningham, H. Wen, F. De Carlo, L. Chen, A.D. Rollett, and T. Sun (2017). "Real-Time Monitoring of Laser Powder Bed Fusion Process Using High-Speed X-ray Imaging and Diffraction." Scientific Reports 7(1): 3602.

\section{PRESENTATIONS}

Sun, T. (2017). "Studying Processing and Deformation of Additive Manufactured Materials Using High-Speed X-ray Imaging and Diffraction Techniques." The 23rd International Conference on Plasticity, Damage, and Fracture, Puerto Vallarta, Mexico, January 3-9, 2017.

Sun, T. (2017). "X-ray Vision of Laser Powder Bed Fusion Process." Eastern New York Chapter of ASM International 2017 Spring Symposium, Niskayuna, NY, June 6-7, 2017.

\section{6-027-R1}

\section{PRESENTATION}

Wang, J., A.U. Mane, S. Liao, R.G. Wagner, J.W. Elam, and D. Haskel (2016). “X-ray Polarization Analysis with Gas-Filled Microchannel Plates: GF-MCP.” 65th Annual Conference on Applications of X-ray Analysis, Rosemont, IL, August 1-5, 2016.

\section{6-054-R1}

\section{REFEREED PUBLICATION}

Sampson, M.D., J.S. Park, R.D. Schaller, M.K.Y. Chan, and A.B.F. Martinson (2017). "Transition Metal-Substituted Lead Halide Perovskite Absorbers." Journal of Materials Chemistry A: Materials for Energy and Sustainability 5(7): 3578-3588.

\section{NON-REFEREED PUBLICATION}

Schaller, R.D., M.K.Y. Chan, and A.B.F. Martinson (2017). "Toward Lead Halide Perovskite-Based Intermediate Band Absorbers.” 2017 IEEE Photovoltaic Specialists Conference (PVSC 44), Washington, D.C., June 25-30, 2017.

\section{PRESENTATIONS}

Martinson, A.B.F. (2017). "Lead Halide Perovskite-Based Intermediate Band Absorbers." 2017 Materials Research Society Spring Meeting, Phoenix, AZ, April 17-21, 2017.

Martinson, A.B.F. (2017). “Toward Lead Halide Perovskite-Based Intermediate Band Absorbers.” 2017 IEEE Photovoltaic Specialists Conference (PVSC-44), Washington, D.C., June 25-30, 2017. 


\section{6-069-R1}

\section{REFEREED PUBLICATIONS}

Duan, Y., B. Zhang, J. Zheng, J. Hu, J. Wen, D.J. Miller, P. Yan, T. Liu, H. Guo, W. Li, X. Song, Z. Zhuo, C. Liu, H. Tang, R. Tan, Z. Chen, Y. Ren, Y. Lin, W. Yang, C.-M. Wang, L.-W. Wang, J. Lu, K. Amine, and F. Pan (2017). “Excess Li-lon Storage on Reconstructed Surfaces of Nanocrystals To Boost Battery Performance." Nano Letters 17(10): 6018-6026.

Kinaci, A., B. Narayanan, F.G. Sen, M.J. Davis, S.K. Gray, S.K.R.S. Sankaranarayanan, and M.K.Y. Chan (2017). "Unraveling the Planar-Globular Transition in Gold Nanoclusters through Evolutionary Search." Scientific Reports 6: 34974.

Sun, C., T. Paulauskas, F.G. Sen, G. Lian, J. Wang, C. Buurma, M.K.Y. Chan, R.F. Klie, and M.J. Kim (2016). "Atomic and Electronic Structure of Lomer Dislocations at CdTe Bicrystal Interface." Scientific Reports 6: 27009.

Tan, G.Q., R. Xu, Z.Y. Xing, Y.F. Yuan, J. Lu, J.G. Wen, C. Liu, L. Ma, C. Zhan, Q. Liu, T.P. Wu, Z. L. Jian, R. Shahbazian-Yassar, Y. Ren, D.J. Miller, L.A. Curtiss, X.L. Ji, and K. Amine (2017). "Burning Lithium in $\mathrm{CS}_{2}$ for High-performing Compact Li ${ }_{2} \mathrm{~S}-$ Graphene Nanocapsules for Li-S Batteries.” Nature Energy 2(7).

Tao, J., K. Sun, W.-G. Yin, L. Wu, H. Xin, J.G. Wen, W. Luo, S.J. Pennycook, J.M. Tranquada, and Y. Zhu (2017). “Direct Observation of Electronic-Liquid-Crystal Phase Transitions and Their Microscopic Origin in $\mathrm{La}_{1 / 3} \mathrm{Ca}_{2 / 3} \mathrm{MnO}_{3}$." Scientific Reports 6: 37624.

Wang, H., J. Wen, D.J. Miller, Q. Zhou, M. Chen, H.N. Lee, K.M. Rabe, and X. Wu (2016). "Stabilization of Highly Polar $\mathrm{BiFeO}_{3}$-like Structure: A New Interface Design Route for Enhanced Ferroelectricity in Artificial Perovskite Superlattices." Physical Review X 6(1).

Wen, J.G., Y. Lin, H. Sheng, L. Wang, D.J. Miller, Z. Wu, K.R. Poeppelmeier, and L.D. Marks (2016). "Atomic Surface Structures of Oxide Nanoparticles with Well-defined Shapes." Proceedings of Microscopy and Microanalysis 22(S3): 360-361.

Xia, Z., G. Liu, J. Wen, Z. Mei, M. Balasubramanian, M.S. Molokeev, L. Peng, L. Gu, D.J. Miller, Q. Liu, and K.R. Poeppelmeier (2016). "Tuning of Photoluminescence by Cation Nanosegregation in the $(\mathrm{CaMg})_{\times}(\mathrm{NaSc})_{1-x} \mathrm{Si}_{2} \mathrm{O}_{6}$ Solid Solution." Journal of the American Chemical Society 138(4): 1158-1161.

Xie, Y., H. Wang, G. Xu, J. Wang, H. Sheng, Z. Chen, Y. Ren, C.-J. Sun, J. Wen, J. Wang, D.J. Miller, J. Lu, K. Amine, and Z.-F. Ma (2016). "In Operando XRD and TXM Study on the Metastable Structure Change of $\mathrm{NaNi}_{1} /{ }^{3} \mathrm{Fe}_{1} /{ }^{3} \mathrm{Mn}_{1} /{ }^{3} \mathrm{O}_{2}$ under Electrochemical Sodium-Ion Intercalation." Advanced Energy Materials 6(24): 1601306.

\section{PRESENTATIONS}

Chan, M.K.Y. (2017). "First Principles Modeling and Optimization of Nanocatalysts." Gordon Research Conference on Clusters and Nanostructures, South Hadley, MA, July 9-14, 2017.

Chan, M.K.Y. (2017). "Nanocatalysts: Understanding Structure and Optimizing Activity Using First Principles Modeling, Experimental Characterization and Machine Learning." 10th International Conference on Computational Physics, Macao, China, January 16-20, 2017.

Chan, M. (2016). "Theory Meets Reality: Combined First Principles Modeling and Characterization Studies of Renewable Energy Materials." Massachusetts Institute of Technology, Cambridge, MA, April 7, 2016.

Chan, M. (2016). "Theory Meets Reality: Combined First Principles Modeling and Characterization Studies of Renewable Energy Materials." Nano and Advanced Materials Institute Limited, Hong Kong, December 2, 2015.

Chan, M.K.Y. (2017). "Towards High Performance Energy Materials - Experimentally-informed First Principles Materials Design.” 10th International Conference on Computational Physics, Macao, China, January 16-20, 2017.

Chan, M. (2016). "Where Are the Atoms and What Do They Do? Using Computational Modeling to Understand and Improve Renewable Energy Materials." White House Office of Science and Technology Policy, March 2, 2016.

Hills, S.T., A. Kinaci, F.G. Sen, and M.K.Y. Chan (2017). "Combined Experimental-Computational Approach to Determining Nanoscale Structures." APS/CNM User Meeting, Argonne, IL, May 8-11, 2017. 
Hills, S., E. Schwenker, F. Sen, A. Kinaci, K. Letchworth-Weaver, and M. Chan (2017). "Machine Learning Approaches for Experiment-Guided Atomistic Structure Determination." Materials Research Society Spring Meeting, Phoenix, AZ, April 17-21, 2017.

Schwenker, E., F. Sen, C. Wolverton, and M. Chan (2017). "Automatic Segmentation and Fingerprint Matching for Atomic Resolution TEM Images." Materials Research Society Spring Meeting, Phoenix, AZ, April 17-21, 2017.

Schwenker, E., F. Sen, S. Hills, T. Paulauskas, C. Sun, L. Li, A. Kinaci, K. Letchworth-Weaver, M. Kim, R.F. Klie, J. Wen, and M.K.Y. Chan (2017). "Leveraging First Principles Modeling for X-Ray Data Inversion." Advanced Photon Source Scientific Computation Seminar, Argonne, IL, February 20, 2017.

Schwenker, E., F. Sen, T. Paulauskas, C. Sun, J. Wen, M. Kim, R.F. Klie, and M. Chan (2016). "Prediction of Atomic Structure of Interfaces Using Electron Microscopy and Atomistic Simulations." 3rd International Congress on 3D Materials Science (3DMS), St. Charles, IL, July 10-13, 2016.

Schwenker, E., F.G. Sen, J. Wen, and M.K.Y. Chan (2016). "Towards Robust Prediction of 3D Atomic Arrangements from Electron Microscopy Images." Data Science and Optimal Learning for Material Discovery and Design, Santa Fe, NM, May 16-18, 2016.

Sen, F., E. Schwenker, T. Paulauskas, C. Sun, C. Buurma, M. Kim, R.F. Klie, and M. Chan (2017). "Atomic-Scale Study of Grain Boundaries in CdTe." Materials Research Society Spring Meeting, Phoenix, AZ, April 17-21, 2017.

Sen, F., S. Hills, A. Kinaci, B. Narayanan, M. Davis, S. Gray, S. Sankaranarayanan, and M. Chan (2017). "Combining First Principles Modeling, Experimental Inputs, and Machine Learning for Nanocatalysts Design." American Physical Society March Meeting, New Orleans, LA, March 13-17, 2017.

Sen, F.G., A. Kinaci, B. Narayanan, M.J. Davis, S.K. Gray, S.K.R.S. Sankaranarayanan, and M.K.Y. Chan (2017). “Designing $\mathrm{IrO}_{2}$ Nanoparticles for Oxygen Evolution.” Electrochemical Society Fall Meeting, Honolulu, HI, October 2-7, 2016.

Sen, F.G., C. Buurma, T. Paulauskas, C. Sun, M. Kim, S. Sivananthan, R.F. Klie, and M.K.Y. Chan (2016). "First Principles Modeling of Grain Boundaries in CdTe." The 43rd IEEE Photovoltaic Specialists Conference, Portland, OR, June 5-10, 2016.

Sen, F.G., K. Letchworth-Weaver, A. Wey, A. Kinaci, B. Narayanan, M.J. Davis, S.K. Gray, S.K.R.S. Sankaranarayanan, and M.K.Y. Chan (2017). "Optimizing $\mathrm{IrO}_{2}$ Nanoclusters for Oxygen Evolution." Materials Research Society Fall Meeting, Boston, MA, November 27-December 2, 2016.

Wen, J. (2016). "Amplitude Contrast High Resolution Electron Microscopy of a-Site-Associated Oxygen Octahedral Rotations in Artificial Perovskite Superlattices: Stabilization of Highly Polar BiFeO3-like Structure." XXV International Materials Research Congress, Cancun, Mexico, August 14-19, 2016.

\section{6-082-R1}

\section{REFEREED PUBLICATIONS}

Chan, H., M.J. Cherukara, B. Narayanan, K. Sasikumar, S. Gray, and S.K.R.S. Sankarananayanan. "Machine Learnt Models for Transition Metal Cichalcogenide 2-D Materials." Nano Letters. (To be published.)

Cherukara, M.J., B. Narayanan, A. Kinaci, K. Sasikumar, S.K. Gray, M.K.Y. Chan, and S.K.R.S. Sankaranarayanan (2016). "Ab initio-Based Bond Order Potential to Investigate Low Thermal Conductivity of Stanene Nanostructures." Journal of Physical Chemistry Letters 7(19): 3752-3759.

Cherukara, M.J., B. Narayanan, H. Chan, and S.K.R.S. Sankaranarayanan (2017). "Silicene Growth through Island Migration and Coalescence." Nanoscale 9(29): 10186-10192.

Cherukara, M.J., D.S. Schulmann, K. Sasikumar, A.J. Arnold, H. Chan, S. Sadasivam, W. Cha, J. Maser, S. Das, S.K.R.S. Sankaranarayanan, and R.J. Harder (2018). "Three-Dimensional Integrated X-ray Diffraction Imaging of a Native Strain in Multi-Layered WSe . $_{2}$ Nano Letters: DOI: 10.1021/acs.nanolett.1027b05441. 
Das, S., M.K. Bera, S. Tong, B. Narayanan, G. Kamath, A. Mane, A.P. Paulikas, M.R. Antonio, S.K.R.S. Sankaranarayanan, and A.K. Roelofs (2016). "A Self-Limiting Electro-Ablation Technique for the Top-Down Synthesis of Large-Area Monolayer Flakes of 2D Materials." Scientific Reports 6: 28195.

Sumant, A.V., D. Berman, S. Deshmukh, B. Narayanan, S.K.R.S. Sankaranarayanan, Z. Yan, A. Balandin, A. Zinovev, and D. Rosenmann (2016). "Metal-Induced Rapid Transformation of Diamond into Single and Multilayer Graphene on Wafer Scale." Nature Communications 7: 12099.

\section{PRESENTATIONS}

Cherukara, M.J., B. Narayanan, A. Kinaci, K. Sasikumar, S. Gray, M. Chan, and S.K.R.S. Sankarananayanan (2017). "Development of an Inter-Atomic Potential for Molecular Dynamics Simulations of Stanene Using a Genetic Algorithm Based Framework." American Institute of Chemical Engineers (AICHE) Annual Meeting, San Francisco, CA, November 13-18, 2016.

Cherukara, M.J., B. Narayanan, and S.K.R.S. Sankaranarayanan (2018). "Molecular Dynamics Simulations of Silicene Monolayer Growth." 232nd Eletrochemical Society Meeting, National Harbor, MD, October 1-5, 2017.

Narayanan, B., M.J. Cherukara, A. Kinaci, K. Sasikumar, S.K. Gray, M.K.Y. Chan, and S.K.R.S. Sankaranarayanan (2017). "Bond Order Potential for 2D Stanene to Probe Thermal Transport Using Molecular Dynamics Simulations." 2016 Pacific Rim Meeting on Electrochemical and Solid-State Science-Joint Meeting 230 Electrochemical Society, 2016 Electrochemical Society of Japan, 2016 Fall Korean Electrochemical Society, Honolulu, HI, October 2-7, 2016.

Narayanan, B., F.G. Sen, A. Kinaci, M.J. Davis, S.K. Gray, Z.-G. Mei, M.K.Y. Chan and S.K.R.S. Sankaranarayanan (2016). "Evolutionary Strategy for Developing Interatomic Potentials to Bridge the Electronic and Atomistic Length Scales." 2015 Materials Research Society Fall Meeting, Boston, MA, November 29-December 4, 2015.

Sankarananayanan, S.K.R.S. (2018). "Macroscale Superlubricity Enabled by Ensembles of Graphene on Diamond Nanoscrolls." 232nd Electrochemical Society Meeting, National Harbor, MD, October 1-5, 2017.

\section{6-092-R1}

\section{REFEREED PUBLICATIONS}

Ding, J., P. Lapa, S. Jain, T. Khaire, S. Lendinez, W. Zhang, M.B. Jungfleisch, C.M. Posada, V. G. Yefremenko, J.E. Pearson, A. Hoffmann, and V. Novosad (2016). "Spin Vortex Resonance in Non-planar Ferromagnetic Dots." Scientific Reports 6: 25196.

Stebliy, M.E., S. Jain, A.G. Kolesnikov, A.V. Ognev, A.S. Samardak, A.V. Davydenko, E.V. Sukovatitcina, L.A. Chebotkevich, J. Ding, J. Pearson, V. Khovaylo, and V. Novosad (2017). "Vortex Dynamics and Frequency Splitting in Vertically Coupled Nanomagnets." Scientific Reports 7(1): 1127.

\section{PRESENTATIONS}

Ding, J., P.N. Lapa, S. Jain, T. Khaire, S. Lendinez, W. Zhang, M.B. Jungfleisch, C.M. Posada, V.G. Yefremenko, J.E. Pearson, A. Hoffmann, and V. Novosad (2017). "Controlling of Gyrotropic Frequency in Ferromagnetic Dots using Nanoscale Vortex Barrier." 61st Annual Conference on Magnetism and Magnetic Materials, New Orleans, LA, October 31-November 4, 2016.

Ding, J. (2016). “Vortex Gyrotropic Motion in Patterned Ferromagnetic Dots.” American Physical Society March Meeting 2016, Baltimore, MD, March 14-18, 2016.

Lendinez, S., J. Ding, P. Lapa, K. Goran, A. Smirnov, and V. Novosad (2017)."Magnetic Vortex Resonance in Hybrid Ferromagnetic/Superconducting Structures." IEEE International Magnetics Conference, Dublin, Ireland,

April 24-28, 2017.

Lendinez, S., J. Ding, S. Jain, P. Lapa, T. Khaire, M.B. Jungfleisch, J.E. Pearson, A. Hoffmann, and V. Novosad (2017). "Reprogrammable Dynamics of Interacting Spin Vortices." 2017 Frontier Forum Conference on Nanoscience, Dubrownik, Croatia, May 3-7, 2017. 
Lendinez, S., J. Ding, T. Polakovic, J. Pearson, A. Hoffmann, and V. Novosad (2018). "Study of the Thermal Behavior of a Single Magnetic Vortex using Magnetotransport." 62nd Annual Conference on Magnetism and Magnetic Materials, Pittsburgh, PA, November 6-10, 2017.

Lendinez, S., J. Ding, P. Lapa, J. Pearson, A. Hoffmann, and V. Novosad (2017). “Temperature Evolution of the Anisotropic Magnetoresistance in a Magnetic Vortex." 2017 International Workshop on Magnetism and Superconductivity at the Nanoscale, Coma-Ruga, Spain, July 2-7, 2017.

Novosad, V. (2016). “Dynamic Control of Interacting Mesoscale Ferromagnets.” 2016 International Conference on Nanomaterials: Application and Properties, Lviv, Ukraine, September 14-19, 2016.

Novosad, V. (2016). "Dynamic Control of Interacting Spin Vortices.” 2016 Materials Research Society Spring Meeting and Exhibit, Phoenix, AZ, March 28-April 1, 2016.

Novosad, V. (2016). "Geometrical Confinement and Spin Vortex Resonance." 2016 Materials Research Society Spring Meeting and Exhibit, Phoenix, AZ, March 28-April 1, 2016.

Novosad, V., and E.A. Rozhkova (2016). "Magnetic Particles: Applications and Properties." 2016 International Conference on Nanomaterials: Application \& Properties, Lviv, Ukraine, September 14-19, 2016.

\section{6-094-R1}

\section{PRESENTATIONS}

Wang, J., R. Divan, Y. Xie, L.E. Ocola, Y. Zhang, A.M. Aragon, M. Ahmed, P.A.A. Ignacio-de Leon, and K. Gao

(2017). "High-Aspect-Ratio Magnetic Nanowires by Electroplating and Electrospinning with Controlled Sizes and Compositions." 5th Nano Today Conference, Waikoloa Beach, HI, June 16, 2017.

Zhang, Y., A.M. Aragon, M. Ahmed, Y. Xie, J. Wang, M. Puga, and K.-Z. Gao (2018). "Synthesis and Properties of FeCo-Based Anisotropic Magnetic Nanocomposites." 62nd Annual Conference on Magnetism and Magnetic Materials, Pittsburgh, PA, November 6-10, 2017.

\section{6-098-R1}

\section{REFEREED PUBLICATIONS}

Carrillo-Serrano, M.E., W. Bentz, I.C. Cloët, and A.W. Thomas (2016). "Baryon Octet Electromagnetic Form Factors in a Confining NJL Model." Physics Letters B 759: 178-183.

Hutauruk, P.T.P., I.C. Cloët, and A.W. Thomas (2016). "Flavour Dependence of the Pion and Kaon Form Factors and Parton Distribution Functions." Physical Review C: Nuclear Physics 94(3): 035201.

Ninomiya, Y., W. Bentz, and I.C. Cloët (2017). "TMDs of Spin-One Targets: Formalism and Covariant Calculations." Physical Review C: Nuclear Physics 96: 045206. (Also see 2017-058.)

\section{PRESENTATIONS}

Cloët, I.C. (2017). "DSE Studies of Nucleon Structure and Its Resonances." Spectrum and Structure of Excited Nucleons from Exclusive Eletroproduction, Institute for Nuclear Theory Workshop (INT-16-62W), Seattle, WA, November 14-18, 2017.

Cloët, I.C. (2016). "Dyson-Schwinger Equation Approaches to TMDs." Parton Transverse Momentum Distributions at Large X: A Window into Parton Dynamics in Nucleon Structure within QCD, Trento, Italy, April 11-15, 2016.

Cloët, I.C. (2016). “The EMC Effect: Exposing the Partonic Structure of Nuclei.” Next Generation Nuclear Physics with JLab12 and EIC, Florida International University, Miami, FL, February 10-13, 2016.

Cloët, I.C. (2017). "EMC Effect: Isospin Dependence and PVDIS." Quantitative Challenges in EMC and SCR Research and Data-Mining, Massachusetts Institute of Technology, Cambridge, MA, December 2-5, 2016. 
Cloët, I.C. (2017). "EMC Theory: The Polarized EMC Effect." Quantitative Challenges in EMC and SRC Research and Data-Mining, Massachusetts Institute of Technology, Cambridge, MA, December 2-5, 2016.

Cloët, I.C. (2017). "Exploring the Multi-Dimensional Structure of Hadrons." Colloquium at the College of William and Mary, Williamsburgh, VA, March 31, 2017. (Also see 2017-058.)

Cloët, I.C. (2016). "Hadron and Nuclear Structure from the DSEs." A New Era for Hadro-Particle Physics, Newport News, VA, June 23-24, 2016.

Cloët, I.C. (2017). "Hadron Imaging." Colloquium at the Technological Institute of Aeronautoics, Sao Paulo, Brazil, May 15, 2017. (Also see 2017-058.)

Cloët, I.C. (2017). "Impacts of Dynamical Chiral Symmetry Breaking on Hadron Masses." The Proton Mass: At the Heart of Most Visible Matter at the European Center for Theoretical Studies in Nuclear Physics and Related Areas (ECT*), Trento, Italy, April 3-7, 2017. (Also see 2017-058.)

Cloët, I.C. (2018). "Nucleon Elastic \& Transition Form Factors from the DSEs." DNP 2017 Fall Meeting of the Division of Nuclear Physics of the American Physical Society - Theoretical Approaches to Hadron Structure, Pittsburgh, PA, October 25-28, 2017.

Cloët, I.C. (2016). "Nucleon Transverse Structure from the DSEs." Probing Transverse Nucleon Structure at High Momentum Transfer, Trento, Italy, April 18-22, 2016.

Cloët, I.C. (2016). "PVDIS and QCD in Nuclei." Physics Beyond the Standard Model and Precision Nucleon Structure Measurements with Parity-Violating Electron Scattering, Trento, Italy, August 1-5, 2016.

Cloët, I.C. (2016). "Role of DCSB in the Pion \& Nucleon." 26th International Nuclear Physics Conference, Adelaide, Australia, September 9-11, 2016.

Cloët, I.C. (2017). "SBS Science Update and Overview." Super BigBite Spectrometer Review, DOE, Germantown, MD, November 7-8, 2016.

Cloët, I.C. (2017). “TMDs of a Spin-1 Target.” QCD Evolution 2017, Newport News, VA, May 22-26, 2017.

(Also see 2017-058.)

Cloët, I.C. (2017). “Tomography of Hadrons.” University of Sao Paulo, Sao Paulo, Brazil, May 15, 2017.

(Also see 2017-058.)

Cloët, I.C. (2017). “Tomography of Spin-one Hadrons." Electron Ion Collider User Group Meeting 2017, Trieste, Italy, July 18-22, 2017. (Also see 2017-058.)

Cloët, I.C. (2016). “Toward Continuum QCD Quark Fragmentation Functions.” From 1D Fragmentation Towards 3D Correlated Fragmentation, Trento, Italy, October 26-30, 2015.

\section{7-004-NO REFEREED PUBLICATION}

Kersell, H., N. Shirato, M. Cummings, H. Chang, D. Miller, D. Rosenmann, S.-W. Hla, and V. Rose (2017). "Detecting Element Specific Electrons from a Single Cobalt Nanocluster with Synchrotron X-ray Scanning Tunneling Microscopy." Applied Physics Letters 111(10).

\section{PRESENTATIONS}

Rose, V. (2017). "Elemental and Magnetic Fingerprinting of Materials at the Nanoscale by Synchrotron X-ray Scanning Tunneling Microscopy." LASSP and A\&EP Fall 2017 Seminar, Ithaca, NY, September 12, 2017.

Rose, V. (2018). "Synchrotron X-ray Scanning Tunneling Microscopy." PETRA IV Workshop Imaging of Surfaces, Interfaces and Nano-Objects, Hamburg, Germany, October 9-12, 2017. 
Rose, V. (2018). "Synchrotron X-ray Scanning Tunneling Microscopy: A Novel Approach for the Nanoscale Characterization of Materials with Chemical and Magnetic Contrast." Dutch Scanning Probe Microscopy Day 2017, Leiden, The Netherlands, November 8, 2017.

Shirato, N., M. Fisher, R. Reininger, S.-W. Hla, and V. Rose (2018). "XTIP - A Dedicated Beamline for Synchrotron X-ray Scanning Tunneling Microscopy." AVS 64th International Symposium and Exhibition, Tampa, FL, October 29-November 2, 2017.

\section{7-007-NO}

\section{REFEREED PUBLICATIONS}

Ha, G., J.G. Power, M. Conde, D.S. Doran, and W. Gai (2017). "Limiting Effects in Double EEX Beamline." Journal of Physics: Conference Series 874(1).

Ha, G., M. Conde, D. Doran, W. Gai, and J.G. Power (2017). "Preliminary Simulations on Chirpless Bunch Compression using Double-EEX Beamline." Proceedings of the 8th International Particle Accelerator Conference (IPAC17), Copenhagen, Denmark, May 14-19, 2017, Paper THPAB062: 3862-3864.

Ha, G., J.G. Power, M. Conde, D.S. Doran, and W. Gai (2017). "Simultaneous Generation of Drive and Witness Beam for Collinear Wakefield Acceleration." Journal of Physics: Conference Series 874(1).

\section{7-012-NO} REFEREED PUBLICATION

Wang, Y., H. Chan, B. Narayanan, S.P. McBride, S.K.R.S. Sankaranarayanan, X.-M. Lin, and H.M. Jaeger (2017). “Thermomechanical Response of Self-Assembled Nanoparticle Membranes." ACS Nano 11(8): 8026-8033.

\section{PRESENTATIONS}

Chan, H., B. Narayanan, Y. Wang, X.M. Lin, H.M. Jaeger, and S.K.R.S. Sankarananayanan (2017). "Coarse-Grained Modeling of Ultra-Thin Nanoparticle Membranes: Effect of Temperature, Humidity, and Ligand Dynamics.” Materials Research Society Fall Meeting, Boston, MA, November 27-December 2, 2016.

Chan, H., B. Narayanan, Y. Wang, X.M. Lin, H.M. Jaeger, and S.K.R.S. Sankarananayanan (2017). “Thermo-Mechanical Behavior of Nanoparticle Membranes.” American Physical Society March Meeting, New Orleans, LA, March 13-17, 2017.

\section{7-013-NO}

\section{PRESENTATIONS}

Mei, Z.-G., S. Bhattacharya, and A.M. Yacout (2018). "Accelerated Searching of Potential Precursors for Silicon Carbide-atomic Layer Deposition from ab initio Machine Learning Methods.” 2017 American Vacuum Society International Symposium and Exhibition, Tampa, FL, October 29-November 3, 2017.

Yanguas-Gil, A. (2017). "The Role of Surface Reactivity on the Coating of 3D Substrates and Nanostructured Materials in CVD and ALD.” Joint EuroCBD 21-Baltic ALD 15, Linkoping, Sweden, June 11-14, 2017.

\section{7-016-NO}

\section{REFEREED PUBLICATION}

Seo, S., A. Amer, P. Balaji, C. Bordage, G. Bosilca, A. Brooks, P. Carns, A. Castello, D. Genet, T. Herault, and P. Beckman (2018). "Argobots: A Lightweight Low-Level Threading and Tasking Framework." IEEE Transactions on Parallel and Distributed Systems 29(3): 512-526.

\section{PRESENTATIONS}

Amer, A. (2018). “BOLT: An Improved OpenMP Runtime.” SuperComputing 2017, Denver, CO, November $12-17,2017$. 
Balaji, P. (2017). "Some Thoughts on the Interoperability of MPI and OpenMP." 13th International Workshop on OpenMP (IWOMP) 2017, Stony Brook, NY, September 21-22, 2017.

Iwasaki, S., A. Amer, P. Balaji, and K. Taura (2017). “Optimistic Threading Techniques for MPI+ULT.” EuroMPI/USA 2017, Chicago, IL, September 25, 2017.

Seo, S., and K.A. Horkey (2017). "BOLT: OpenMP over Lightweight Threads." SuperComputing 2016, Salt Lake City, UT, November 13-18, 2016.

Seo, S., and K.A. Horkey (2017). “User-Level Threads and OpenMP.” 4th XcalableMP Workshop, Tokyo, Japan, November 7, 2016.

\section{7-022-NO}

\section{PRESENTATIONS}

Mandia, D.J., A. Yanguas-Gil, S. Letourneau, J.A. Libera, and J.W. Elam (2018). "Atomic Layer Deposition of $\mathrm{HfO}_{2} / \mathrm{Al}_{2} \mathrm{O}_{3}$ Nanolaminates on Single-Crystal $\mathrm{GaN}$ and $\mathrm{Ga}_{2} \mathrm{O}_{3}$ : Investigation of Device Degradation in Power Semiconductor Devices." 64th AVS International Symposium and Exhibition, Tampa, FL, October 29-November 3, 2017.

Yanguas-Gil, A. (2018). "The Structure of Sub-nm Oxides Synthesized by Atomic Layer Deposition: From Isolated Cations to the Emergence of Crystallinity." 64th AVS International Symposium and Exhibition, Tampa, FL,

October 29-November 3, 2017.

Yanguas-Gil, A. (2017). "When There Is No Bulk: Growth and Structure of Dielectric and Semiconductor Oxide Nanolaminates." 17th International Conference on Atomic Layer Deposition (ALD 2017), Denver, CO, July 15-18, 2017.

\section{LDRD SWIFT}

\section{7-098-NO \\ REFEREED PUBLICATION}

Mason-Smith, N., D.J. Duke, A.L. Kastengren, D. Traini, P.M. Young, Y. Chen, D. Lewis, “D.M. Edgington-Mitchell, and D.R. Honnery (2017). "Revealing pMDI Spray Initial Conditions: Flashing, Atomisation, and the Effect of Ethanol. Pharmaceutical Research 34(4): 718-729.

\section{7-102-NO}

\section{REFEREED PUBLICATIONS}

Hall, G.N., M. Stuckelberger, T. Nietzold, J. Hartman, J.-S. Park, J. Werner, B. Niesen, M.L. Cummings, V. Rose, C. Ballif, M.K.Y. Chan, D.P. Fenning, and M.I. Bertoni (2017). "The Role of Water in the Reversible Optoelectronic Degradation in Hybrid Perovskites at Low Pressure.” Journal of Physical Chemistry C 121(46): 25659-25665.

Park, J.-S., and M.K.Y. Chan. "Mechanism of Na Accumulation at Extended Defects in Si from First Principles." Journal of Applied Physics. (To be published.)

\section{PRESENTATION}

Hall, G., M. Stuckelberger, B. West, J. Hartman, J.-S. Park, J. Werner, B. Niesen, C. Ballif, M. Chan, D. Fenning, and M.I. Bertoni (2017). "Extent of Methylammonium Lead lodide Hydration by Atmosphere in Full Devices." Materials Research Society Spring Meeting, Phoenix, AZ, April 17-21, 2017. 


\section{7-116-NO}

\section{REFEREED PUBLICATION}

Xie, R., Y. Xie, C.R. López-Barrón, K. Gao, and N.J. Wagner. "Ultra-Stretchable Conductive lono-Elastomer and Motion Strain Sensor System Developed Therefrom." Technology and Innovation. (To be published.)

\section{PRESENTATION}

Xie, Y., K. Gao, and J. Hryn (2017). "RF Powered Transformable Sensor on a Flexible Substrate." 2017FLEX, Monterey, CA, June 19-23, 2017.

\section{7-118-NO}

\section{REFEREED PUBLICATION}

Miao, Y., T.K. Yao, J. Lian, J.-S. Park, J. Almer, S. Bhattacharya, A.M. Yacout, and K. Mo (2017). "In situ Synchrotron Investigation of Grain Growth Behavior of Nano-Grained UO ." Scripta Materialia 131: 29-32.

\section{7-160-NO}

\section{PRESENTATIONS}

Yanguas-Gil, A. (2018). "Fast, Smart Neuromorphic Sensors Based on Heterogeneous Networks and Mixed Encodings." Government Microcircuit Applications and Critical Technology Conference (GomacTech), Miami, FL, March 12-15, 2018.

Yanguas-Gil, A., and J.W. Elam (2018). “Neuromorphic Architectures for Smart Sensing Applications: Lessons from the Insect Brain.” 2018 Materials Research Society Spring Meeting, Phoenix, AZ, April 2-6, 2018.

\section{LDRD NAMED FELLOWS}

\section{6-180-R1}

REFEREED PUBLICATIONS

Acik, M., and S.B. Darling (2016). "Graphene in Perovskite Solar Cells: Device Design, Characterization, and Implementation.” Journal of Materials Chemistry A: Materials for Energy and Sustainability 4(17): 6185-6235.

Acik, M., I.K. Park, R.E. Koritala, G. Lee, and R.A. Rosenberg. “Oxygen-Induced Defects at the Lead Halide Perovskite/ Graphene Oxide Interfaces.” Journal of Materials Chemistry A. (To be published.)

Acik, M., T.M. Alam, F. Guo, Y. Ren, B. Lee, R.A. Rosenberg, J.F. Mitchell, I.K. Park, G. Lee, and S.B. Darling (2017). "Substitutional Growth of Methylammonium Lead lodide Perovskites in Alcohols." Advanced Energy Materials: 1701726.

\section{PRESENTATIONS}

Acik, M., R.A. Rosenberg, S.W. Hla, and S.B. Darling (2017). "Graphene Oxide/Perovskite Interfaces for Hybrid Lead Halide Perovskite/Graphene Solar Cells: An in situ Spectroscopic Evaluation.” 2017 Materials Research Society Spring Meeting, Phoenix, AZ, April 17-21, 2017.

Acik, M., Y. Ren, F. Guo, T.M. Alam, B. Lee, R.A. Rosenberg, A. Kinaci, B.T. Diroll, G. Xu, M. Chan, R.D. Schaller, S.W. Hla, and S.B. Darling (2017). "Green-Solvent-Processable in situ Methyl Ammonium Lead Halide Perovskite Growth for Energy Harvesting." The International Conference Asia-Pacific Hybrid and Organic Photovoltaics, Yokohama-shi, Japan, February 2-4, 2017.

Acik, M., and S.B. Darling (2017). "Impact of Stability Factors in Graphene-based Perovskite Hybrid Solar Cells Studied by in situ Spectroscopy.” 2016 Materials Research Society Fall Meeting, Boston, MA, November 27-December 2, 2016.

Acik, M., and S.B. Darling (2016). "In situ Characterization of Perovskite Formation in Graphene-Based Perovskite Solar Cells.” XXV International Materials Research Congress, Cancun, Mexico, August 14-19, 2016.

Acik, M., and S.B. Darling (2017). "In situ Growth and Degradation Mechanisms in Hybrid Lead Halide Perovskite/ Graphene Solar Cells." 2016 Pacific Rim Meeting on Electrochemical and Solid-state Science - Joint Meeting 230th ECS Prime Meeting, 2016 Fall Electrochemical Society of Japan, 2016 Fall Korean Electrochemical Society, Honolulu, HI, October 2-7, 2016. 
Acik, M., and S.B. Darling (2017). "In situ Growth and Degradation Mechanisms in Methylammonium Lead Halide Perovskites for Graphene-Based Solar Cells." Pacific Rim Symposium on Surfaces, Coatings, and Interfaces (PacSurf), Kohala Coast, HI, December 11-15, 2016.

Acik, M., and S.B. Darling (2017). "In situ Lead Halide Perovskite Growth and Degradation Mechanisms in Perovskite/ Graphene Hybrid Solar Cells." Materials Reserach Society Fall Meeting, Boston, MA, November 27-December 2, 2016.

Acik, M., Y. Ren, F. Guo, T.M. Alam, B. Lee, R.A. Rosenberg, A. Kinaci, B.T. Diroll, G. Xu, M. Chan, R.D. Schaller, S.W. Hla, and S.B. Darling (2017). "In situ Methylammonium Lead Halide Perovskite Growth: A Novel Approach for Solar Energy Technology.” 2017 Materials Research Society Spring Meeting, Phoenix, AZ, April 17-21, 2017.

Acik, M., and S.B. Darling (2017). "In situ Spectroscopic Analysis of Perovskite/Graphene Hybrids for Graphene-based Perovskite Solar Cells." AVS 63rd International Sypmposium and Exhibition, National, TN, November 6-11, 2016.

Acik, M., S.B. Darling, and M.M. Segovia Monrroy (2016). "Interfaces in Graphene-Organolead Halide Nanohybrid Perovskite Solar Cells Examined by in situ Spectroscopy." Materials Research Society Spring Meeting, Tucson, AZ, March 28-April 1, 2016.

Acik, M. (2017). "Interfacial Interactions at Reduced Graphene Oxide/Perovskite Interfaces for Photovoltaics." 59th Electronic Materials Conference, South Bend, IN, June 28-30, 2017.

Acik, M., and S.B. Darling (2017). "Perovskite Film Growth and Degradation Mechanisms in Graphene-Based Perovskite Solar Cells by in situ Spectroscopy." AVS 63rd International Symposium \& Exhibition, Nashville, TN, November 6-11, 2016.

Acik, M., G. Lee, and R.A. Rosenberg (2017). "Role of Halide Anions in Perovskite/Graphene Oxide Photovoltaics." The 17th IEEE International Conference on Nanotechnology (IEEE Nano 2017), Pittsburgh, PA, July 25-28, 2017.

Acik, M., and R.A. Rosenberg (2017). "Spectroscopic Evolution of Graphene Oxide/Perovskite Interfaces for Solar Energy.” 254th American Chemical Society National Meeting \& Expo, Washington, D.C., August 20-24, 2017.

Acik, M., G. Lee, and R.A. Rosenberg (2018). "Spectroscopic Evolution of Halide Perovskite Growth on Graphene Oxide Surfaces for Photovoltaics." AVS 64th International Symposium and Exhibition, Tampa, FL,

October 29-November 3, 2017.

Acik, M., and S.B. Darling (2016). "Spectroscopic Evolution of in situ Perovskite Film Growth on Reduced Graphene Oxide for Graphene-Perovskite Solar Cells.” 2016 APS/CNM Users Meeting, Argonne, IL, May 9-12, 2016.

Acik, M., and S.B. Darling (2017). "Stability Factors in Graphene-Based Perovskite Solar Cells Studied by in situ Spectroscopy." 2016 Pacific Rim Meeting on Electrochemical and Solid-State Science - Joint Meeting 230th ECS Prime Meeting, 2016 Fall Electrochemical Society of Japan, 2016 Fall Korean Electrochemical Society, Honolulu, HI, October 2-7, 2016.

Acik, M., and S.B. Darling (2017). "Surfaces and Interfaces of Methylammonium Lead Halide Perovskite/Graphene Oxide Films Studied by in situ Spectroscopy." Pacific Rim Symposium on Surfaces, Coatings, and Interfaces (PacSurf), Kohala Coast, HI, December 11-15, 2016.

Acik, M. (2017). "Surfaces and Interfaces of Nanostructured Materials for Energy Efficient Processing." Ohio State University, Columbus, OH, February 22, 2017.

Acik, M., and S.B. Darling (2016). "Unraveling Degradation Mechanisms in Graphene-Based Perovskite Solar Cells by in situ Spectroscopy.” XXV International Materials Research Congress, Cancun, Mexico, August 14-19, 2016. 


\section{6-181-R1}

\section{REFEREED PUBLICATIONS}

Ghimire, N.J., D. Phelan, H. Zheng, and J.F. Mitchell (2016). "Magnetotransport of Single Crystalline YSb." Journal of Physics: Condensed Matter 28(23): 235601.

He, J., C. Zhang, N.J. Ghimire, T. Liang, C. Jia, J. Jiang, S. Tang, S. Chen, Y. He, S.-K. Mo, and J.F. Mitchell (2017). "Distinct Electronic Structure for the Extreme Magnetoresistance in YSb." Physical Review Letters 117(26): 267201.

Xu, J., N.J. Ghimire, J.S. Jiang, Z.L. Xiao, A.S. Botana, Y.L. Wang, Y. Hao, J.E. Pearson, and W.K. Kwok (2017). “Origin of the Extremely Large Magnetoresistance in the Semimetal YSb." Physical Review B 96(7).

\section{PRESENTATIONS}

Ghimire, N.J., and J.F. Mitchell (2017). “Large Anomalous Hall Effect in a Chiral Antiferromagnet." 2017 Gordon Research Conference on Topological and Correlated Matter, Hong Kong, China, June 18-23, 2017.

Ghimire, N., J. He, H. Zheng, S. Rosenkranz, Z.-X. Shen, and J. Mitchell (2017). "Negative Longitudinal Magnetoresistance in ErSb." American Physical Society March Meeting 2017, New Orleans, LA, March 13-17, 2017.

\section{6-183-R1}

\section{REFEREED PUBLICATION}

Jungfleisch, M.B., Q. Zhang, W. Zhang, J.E. Pearson, R.D. Schaller, H. Wen, and A. Hoffmann. "Control of Terahertz Emission by Ultrafast Spin-charge Current Conversion at Rashba Interfaces." Physical Review Letters. (To be published.)

\section{PRESENTATIONS}

Zhang, Q., M.B. Jungfleisch, W. Zhang, J.E. Pearson, R. Schaller, H. Wen, and A. Hoffmann (2017). "Terahertz Emission from Ultrafast Spin and Charge Currents at a Rashba Interface." 42nd International Conference on Infrared, Millimeter, and Terahertz Waves, Cancun, Mexico, August 26-September 1, 2017.

Zhang, Q., M.B. Jungfleisch, W. Zhang, J.E. Pearson, R. Schaller, H. Wen, and A. Hoffmann (2017). "Terahertz Emission from Ultrafast Spin and Charge Currents at a Rashba Interface." American Physical Society March Meeting, New Orleans, LA, March 5-9, 2017.

\section{6-184-R1}

\section{REFEREED PUBLICATIONS}

Letchworth-Weaver, K., R. Sundararaman, and T.A. Arias. "First Principles Free-Energy Theory of Solvation with Atomic Scale Liquid Structure." Chemical Physics. (To be published.)

Nicolau, B.G., A. Petronico, K. Letchworth-Weaver, Y. Ghadar, R.T. Haasch, J.A.N.T. Soares, R.T. Rooney, M.K.Y. Chan, A.A. Gewirth, and R.G. Nuzzo. "Controlling Interfacial Properties of Lithium-Ion Battery Cathodes with Alkylphosphonate Self-Assembled Monolayers.” Advanced Materials Interfaces. (To be published.)

Plaza, M., X. Huang, J.Y.P. Ko, M. Shen, B.H. Simpson, J. Rodriguez-Lopez, N.L. Ritzert, K. Letchworth-Weaver, D. Gunceler, D.G. Schlom, T.A. Arias, J.D. Brock, and H.D. Abruna (2016). "Structure of the Photo-Catalytically Active Surface of $\mathrm{SrTiO}_{3}$." Journal of American Chemical Society 138(25): 7816-7819.

Sundararaman, R., K. Letchworth-Weaver, K.A. Schwarz, D. Gunceler, Y. Ozhabes, and T.A. Arias (2018).

"JDFTx: Software for Joint Density-Functional Theory." SoftwareX 6: 278-284.

\section{PRESENTATIONS}

Letchworth-Weaver, K., R. Sundararaman, C.K. Umbright and T.A. Arias (2016). "Benchmarking Joint DFT Predictions of the Structure and Energetics of the Electrode/Electrolyte Interface." Materials Research Society Spring Meeting and Exhibit, Phoenix, AZ, March 28-April 1, 2016.

Letchworth-Weaver, K., D. Gunceler, X. Huang, M. Plaza, J. Rodriguez-Lopez, J.D. Brock, H.D. Abruna, and T.A. Arias (2016). "Determining Operando Surface Structure: Combining First Principles Theory with Experimental Data."

2nd Functional Oxide Thin Films for Advanced Energy and Information Technology Conference, Cancun, Mexico, March 5-8, 2016. 
Letchworth-Weaver, K., A. Gaiduk, I.-C. Tung, D. Fong, G. Galli, P. Fenter, and M.K.Y. Chan (2017). “Determining Operando Surface Structure: Combining First Principles Theory with Experimental Data." Center for Nanoscale Materials Users Meeting, Argonne, IL, May 9, 2017.

Letchworth-Weaver, K., R. Warburton, Y. Ghadar, C. Knight, J. Greeley, P. Fenter, and M.K.Y. Chan (2017). "Electrolyte-Induced Changes in Stability and Structure of Metal-Oxide Battery Cathodes." Materials Research Society Fall Meeting, Boston, MA, November 27-December 2, 2016.

Letchworth-Weaver, K. (2017). "Integrating First Principles Theory and Experimental Characterization at the Solid/Liquid Interface." 254th American Chemical Society National Meeting and Exposition, Washington, D.C., August 20-24, 2017.

Letchworth-Weaver, K. (2017). "Integrating First Principles Theory and Experimental Characterization at the Solid/Liquid Interface." Rensselaer Polytechnic Institute, Troy, NY, September 19, 2017.

Letchworth-Weaver, K. (2017). "Integrating First Principles Theory and Experimental Characterization at the Solid/Liquid Interface." National Institute of Standards and Technology, Gaithersburg, MD, September 29, 2017.

Letchworth-Weaver, K., R. Sundararaman, M.K.Y. Chan, P. Fenter, J.D. Brock, H.D. Abruna, and T.A. Arias (2016). "Joint Density-Functional Theory for Multiscale Modeling of the Electrode-Electrolyte Interface." Gordon Research Conference on Electrochemistry, Ventura, CA, January 10-15, 2016.

Letchworth-Weaver, K., C.K. Umbright, M. Chan, P. Fenter, and T.A. Arias (2016). "Joint Density-Functional Theory for the Electrode/Electrolyte Interface: Benchmarking Liquid Structure with Experiment and ab initio Molecular Dynamics." American Physical Society March Meeting, Baltimore, MD, March 14-18, 2016.

Letchworth-Weaver, K., R. Warburton, J. Greeley, C.K. Umbright, T.A. Arias, and M.K.Y. Chan (2016). “Joint Density-Functional Theory Investigations of Atomic-Scale Structure and Energetics at the Solid-Liquid Interface." 28th Annual Workshop on Recent Developments in Electronic Structure Theory, Albuquerque, NM, June 26-29, 2016.

Letchworth-Weaver, K. (2016). "Joint Density-Functional Theory: Atomically Detailed Structure of the Electrode-Electrolyte Interface.” University of California-Berkeley, Berkeley, CA, August 10, 2016.

Letchworth-Weaver, K. (2016). "Joint Density-Functional Theory: Atomically Detailed Structure of the Electrode-Electrolyte Interface.” Lawrence Livermore National Laboratory, Livermore, CA, August 11, 2016.

Letchworth-Weaver, K. (2016). "Joint Density-Functional Theory: Atomically Detailed Structure of the Electrode-Electrolyte Interface.” Joint Center for Artificial Photosynthesis, California Institute of Technology, Pasadena, CA, January 19, 2016.

Letchworth-Weaver, K. (2016). "The Role of Liquids in Clean Energy Technology." Argonne Outloud Public Lecture, Argonne, IL, September 15, 2016.

Letchworth-Weaver, K., C.K. Umbright, M.K.Y. Chan, and T.A. Arias (2017). "Theoretical Investigation of Atomic-Scale Structure and Energetics of the Metal-Electrolyte Interface." Pacific Rim Meeting of the Electrochemical Society, Honolulu, HI, October 2-7, 2016.

Letchworth-Weaver, K., R. Warburton, J. Greeley, C. Umbright, T.A. Arias, and M.K.Y. Chan (2016). "Theoretical Investigations of Atomic-Scale Structure and Energetics at the Solid/Liquid Interface." Nanoscale Materials Users Meeting, Argonne National Laboratory, May 9-12, 2016.

Letchworth-Weaver, K., A. Gaiduk, M.K.Y. Chan, P. Fenter, and G. Galli (2017). "Validating First Principles Molecular Dynamics Calculations of Solid/Liquid Interfaces with X-ray Reflectivity." American Physical Society March Meeting, New Orleans, LA, March 13-17, 2017.

Letchworth-Weaver, K., A.I.P. Gaiduk, G. Galli, M.K. Chan, and P. Fenter (2017). "Validating Structure of the Solid/Liquid Interface with First Principles Molecular Dynamics and X-Ray Reflectivity." Materials Research Society Spring Meeting and Exhibit, Phoenix, AZ, April 17-21, 2017. 
Warburton, R., B.G. Nicolau, K. Letchworth-Weaver, K.-S. Chen, X. Chen, A. Petronico, Y. Ghadar, M. Chan, R.G. Nuzzo, A.A. Gewirth, J.P. Greeley, M.C. Hersam, and P. Fenter (2016). "Understanding and Controlling the Reactivity of $\mathrm{LiMn}_{2} \mathrm{O}_{4}$-Electrolyte Interfaces." Department of Energy Principal Investigators Meeting, Washington, D.C., July 25, 2016.

\section{6-185-R1 \\ REFEREED PUBLICATIONS}

Brawand, N., M.B. Goldey, M. Vörös, and G. Galli (2017). "Defect States and Charge Transport in Quantum Dot Solids." Chemistry of Materials 29(3): 1255-1262.

Brawand, N., M. Vörös, M. Govoni, and G. Galli (2017). "Generalization of Dielectric-Dependent Hybrid Functionals to Finite Systems.” Physical Review X 6(4): 041002. (Also see 2014-192.)

Brawand, N.P., M. Govoni, M. Vörös, and G. Galli (2017). "Performance and Self-Consistency of the Generalized Dielectric Dependent Hybrid Functional." Journal of Chemical Theory and Computation 13(7): 3318-3325.

Galli, A., T. Demján, M. Vörös, G. Thiering, E. Cannuccia, and A. Marini (2016). "Electron-Vibration Coupling-Induced Renormalization in the Photo-Emission Spectrum of Diamondoids.” Nature Communications 7: 11327.

Giberti, F., M. Vörös, and G. Galli (2017). "Design of Heterogeneous Chalcogenide Nanostructures with Pressure-Tunable Gaps and without Electronic Trap States.” Nano Letters 17(4): 2547-2553.

Goldey, M.B., N.P. Brawand, M. Vörös, and G. Galli (2017). "Charge Transport in Nanostructured Materials: Implementation and Verification of Constrained Density Functional Theory." Journal of Chemical Theory and Computation 13(6): 2581-2590.

Kroupa, D.M., M. Vörös, N.P. Brawand, B.W. McNichols, E.M. Miller, J. Gu, A.J. Nozik, A. Sellinger, G. Galli, and M.C. Beard (2017). "Tuning Colloidal Quantum Dot Band Edge Positions through Solution-Phase Surface Chemistry Modification." Nature Communications 8: 15257.

Qu, L., M. Vörös, and G.T. Zimanyi (2017). "Metal-Insulator Transition in Nanoparticle Solids: Insights from Kinetic Monte Carlo Simulations." Scientific Reports 7(1): 7071.

Wang, L., N.P. Brawand, M. Vörös, P.D. Dahlberg, J.P. Otto, N.E. Williams, D.M. Tiede, G. Galli, and G.S. Engel. "Excitations Partition into Two Distinct Populations in Bulk Perovskites Due to Polaron Formation." Energy \& Environmental Science. (To be published.)

Wang, L., N.P. Brawand, M. Voros, P.D. Dahlberg, J.P. Otto, N.E. Williams, D.M. Tiede, G. Galli, and G.S. Engel. "Excitations Partition into Two Distinct Populations in Bulk Single-Domain-Single-Crystal $\mathrm{CH}_{3} \mathrm{NH}_{3} \mathrm{PbBr}_{3}$ Perovskites Due to Polaron Formation." Advanced Energy Materials. (To be published.)

Wippermann, S., Y. He, M. Vörös, and G. Galli (2017). "Novel Silicon Phases and Nanostructures for Solar Energy Conversion." Applied Physics Reviews 3(4).

\section{PRESENTATIONS}

Vörös, M., N. Brawand, and G. Galli (2016). "Ligand Engineering of the Optoelectronic Properties of PbS Nanoparticles." National Renewable Energy Laboratory, Golden, CO, December 21, 2015.

Vörös, M. (2016). "Molecular Design of Nanocrystals for Third Generation Photovoltaics Using First Principles Simulations." University of Wisconsin, Madison, WI, December 9, 2015.

Vörös, M. (2016). "Molecular Engineering of Nanoparticle Solar Cells." American Physical Society March Meeting, Baltimore, MD, March 14-18, 2016. 


\section{6-186-R1}

\section{REFEREED PUBLICATIONS}

Chang, A.Y., Y.-J. Cho, K.-C. Chen, C.-W. Chen, A. Kinaci, M.K.Y. Chan, B.T. Diroll, M.J. Wagner, H.-W. Lin, and R.D. Schaller (2016). "Slow Organic-to-Inorganic Sub-Lattice Thermalization in Methylammonium Lead Halide Perovskites Observed by Ultrafast Photoluminescence." Advanced Energy Materials 6(15): 1600402.

Diroll, B.T., R.D. Schaller, D. Talapin, V. Srivastava, and E. Janke (2016). "Facile, Economic, and Size-Tunable Synthesis of Metal-Arsenide Nanocrystals." Chemistry of Materials 28(18): 6797-6802.

Diroll, B.T., G. Nedelcu, M. Kovalenko, and R.D. Schaller (2017). "High-Temperature Photoluminescence of CsPbX 3 (X=Cl, Br, I) Nanocrystals." Advanced Functional Materials 27(21): 1606750.

Diroll, B.T., P. Guo, R.P.H. Chang, and R.D. Schaller (2017). "Large Transient Optical Modulation of Epsilon-Near-Zero Colloidal Nanocrystals." ACS Nano 10(11): 10099-10105.

Diroll, B.T., R.D. Schaller, and P.T. Darancet (2016). "Surface-Area-Dependent Electron Transfer between Isoenergetic 2D Quantum Wells and a Molecular Acceptor." Journal of the American Chemical Society 138(35): 11109-11112.

Diroll, B.T., K. Schramke, P. Guo, U.R. Kortshagen, and R.D. Schaller (2017). “Ultrafast Silicon Photonics with Visible to Mid-Infrared Pumping of Silicon Nanocrystals." Nano Letters 17(10): 6409-6414.

Diroll, B.T., D.V. Talapin, and R.D. Schaller (2017). “Violet-to-Blue Gain and Lasing from Colloidal CdS Nanoplatelets: Low-Threshold Stimulated Emission Despite Low Photoluminescence Quantum Yield.” ACS Photonics 4(3): 576-583.

Guo, P., M.S. Weimer, J.D. Emery, B.T. Diroll, X. Chen, A.S. Hock, R.P.H. Chang, A.B.F. Martinson, and R.D. Schaller (2017). "Conformal Coating of a Phase Change Material on Ordered Plasmonic Nanorod Arrays for Broadband All-Optical Switching." ACS Nano 11(1): 693-701.

Guo, P., R.D. Schaller, L.E. Ocola, B.T. Diroll, J.B. Ketterson, and R.P.H. Chang (2016). "Large Optical Nonlinearity of Indium Tin Oxide Nanorods for Sub-Picosecond All-Optical Modulation of the Full-Visible Spectrum." Nature Communications 7: 12892.

Kirschner, M.S., D.C. Hannah, B.T. Diroll, X. Zhang, M.J. Wagner, D. Hayes, A.C. Chang, C.E. Rowland, C.M. Lethiec, G.C. Schatz, L.X. Chen, and R.D. Schaller (2017). "Transient Melting and Recrystallization of Semiconductor Nanocrystals under Multiple Electron-Hole Pair Excitation.” Nano Letters 17(9): 5314-5320.

Ma, X., B.T. Diroll, I. Fedin, W. Cho, R.D. Schaller, D.V. Talapin, S.K. Gray, G.P. Wiederrecht, and D.J. Gosztola (2017). "Size-Dependent Biexciton Quantum Yields and Carrier Dynamics of Quasi-Two-Dimensional Core/Shell Nanoplatelets." ACS Nano 11(9): 9119-9127.

Wu, L., J.J. Willis, I.S. McKay, B.T. Diroll, J. Qin, M. Cargnello, and C.J. Tassone (2017). “High-Temperature Crystallization of Nanocrystals into Three-Dimensional Superlattices." Nature 548(7666): 197-201.

\section{PRESENTATION}

Diroll, B.T., P. Guo, R.D. Schaller, and R.P.H. Chang (2017). "All-Optical Switching of Doped Semiconductor Nanocrystals." 2017 Materials Research Society Spring Meeting and Exhibits, Pheonix, AZ, April 17-21, 2017.

\section{6-187-R1}

\section{REFEREED PUBLICATIONS}

Cha, W., Y.H. Liu, H. You, G.B. Stephenson, and A. Ulvestad (2017). "Dealloying in Individual Nanoparticles and Thin Film Grains: A Bragg Coherent Diffractive Imaging Study." Advanced Functional Materials 27(25).

Liu, Y., P.P. Lopes, W. Cha, R. Harder, J. Maser, E. Maxey, M.J. Highland, N. Markovic, S. Hruszkewycz, G.B. Stephenson, H. You, and A. Ulvestad (2017). "Stability Limits and Defect Dynamics in Ag Nanoparticles Probed by Bragg Coherent Diffractive Imaging." Nano Letters 17(3): 1595-1601. 
Ulvestad, A., M.J. Welland, S. Collins, R. Harder, E. Maxey, J. Wingert, A. Singer, S. Hy, P. Mulvaney, P. Zapol, and O.G. Shpyrko (2016). "Avalanching Strain Dynamics during the Hydriding Phase Transformation in Individual Palladium Nanoparticles.” Nature Communications 6: 10092.

Ulvestad, A., A. Tripathi, S.O. Hruszkewycz, W. Cha, S.M. Wild, G.B. Stephenson, and P.H. Fuoss (2016). "Coherent Diffractive Imaging of Time-Evolving Samples with Improved Temporal Resolution." Physical Review B: Condensed Matter 93(18).

Ulvestad, A., K. Sasikumar, J.W. Kim, R. Harder, E. Maxey, J.N. Clark, B. Narayanan, S. Deshmukh, N. Ferrier, P. Mulvaney, S.K.R.S. Sankaranarayanan, and O.G. Shpyrko (2016). "In situ 3D Imaging of Catalysis-Induced Strain in Gold Nanoparticles." Journal of Physical Chemistry Letters 7(15): 3008-3013.

Ulvestad, A., M.J. Welland, W. Cha, Y. Liu, J.W. Kim, R. Harder, E. Maxey, J.N. Clark, M.J. Highland, H. You, P. Zapol, S.O. Hruszkewycz, and G.B. Stephenson (2017). "Three-Dimensional Imaging of Dislocation Dynamics during the Hydriding Phase Transformation.” Nature Materials 16(5): 565-571.

\section{6-188-R1 \\ REFEREED PUBLICATIONS}

Berthou, B., D. Binosi, N. Chouika, M. Guidal, C. Mezrag, H. Moutarde, F. Sabatie, P. Sznajder, and J. Wagner (2016).

"PARTONS: PARtonic Tomography of Nucleon Software." European Physical Journal C: Particles and Fields: arXiv:1512.06174.

Binosi, D., C. Mezrag, J. Papavassiliou, C.D. Roberts, and J. Rodriguez-Quintero (2017). "Process-Independent Strong Running Coupling." Physical Review D 96(5).

Mezrag, C., H. Moutarde, and J. Rodriguez-Quintero (2016). "From Bethe-Salpeter Wave Functions to Generalized Parton Distributions." Few-Body Systems 57(9): 729-772.

Qin, S.-X., C. Chen, C. Mezrag, and C.D. Roberts (2018). "Off-Shell Persistence of Composite Pions and Kaons." Physical Review C: Nuclear Physics 97(1).

Roberts, C.D., and C. Mezrag (2017). "Emergent Phenomena and Partonic Structure in Hadrons." EPJ Web of Conferences 137: 01017.

\section{NON-REFEREED PUBLICATIONS}

Roberts, C.D. (2018). "N* Structure and Strong QCD." Contribution to the proceedings of the 11th International Workshop on the Physics of Excited Nucleons, August 20-23, 2017, University of South Carolina, Columbia, SC, January 30, 2018.

Segovia, J., C. Mezrag, L. Chang and C.D. Roberts (2018). “Nucleon Viewed as a Borromean Bound-State.” Contribution to the Proceedings of the Workshop: Critical Stability of Quantum Few-Body Systems (Crit17). October 16-20, 2017.

Dresden, Germany.

\section{PRESENTATIONS}

Chouika, N., C. Mezrag, H. Moutarde, and J. Rodriguez-Quintero (2016). “An Algebraic Model for the Pion's Valence-Quark GPD.” Lightcone 2016, Lisbon, Portugal, September 5-8, 2016.

Mezrag, C. (2017). "Continuum Calculations of the PDAs of the Nucleon and Its Resonances." INT Workshop INT-16-62W: Spectrum and Structure of Excited Nucleons from Exclusive Electroproduction, Seattle, WA, November 14-18, 2016.

Mezrag, C. (2017). "Distribution Amplitudes of the Nucleon and the Roper Resonance." Nucleon and Resonance Structure with Hard Exclusive Probes, Paris, France, May 29-31, 2017.

Mezrag, C. (2017). "Nucleon Distribution Amplitude from a Diquark-Quark Approach." 3rd Workshop on Non-Perturbative QCD (NPQCD16), Seville, Spain, October 17-21, 2016. 
Roberts, C.D. (2017). "Continuum Computation of Parton Distributions." Forum on the Calculation of Parton Distributions, Nanjing, China, July 28-29, 2017.

Roberts, C.D. (2017). "Continuum QCD at JLab12 and an EIC." VI International Workshop on Nonperturbative Aspects of Field Theories: QCD and Hadron Physics, Terán Tuxtla Gutiérrez, Chiapas, Mexico, April 24-28, 2017.

Roberts, C.D., and C. Mezrag (2016). "Emergent Phenomena and Partonic Structure in Hadrons.” XIlth Quark Confinement and the Hadron Sepctrum, Thessaloniki, Greece, August 28-September 3, 2016.

Roberts, C.D. (2017). “Emergent Phenomena and Partonic Structure in Hadrons." Effective Field Theories and Nuclear Phenomena Workshop, Saclay, France, January 16-20, 2017.

Roberts, C.D. (2016). "Exposing the Mass at the Heart of Visible Matter." International Nuclear Physics Conference (INPC16), Adelaide, Australia, September 11-16, 2016.

Roberts, C.D. (2017). "N* Physics: A Window onto Strong QCD." N* Workshop with Experimentalists and JLab Theory Center, Newport News, VA, June 6, 2017.

Roberts, C.D. (2017). "N* Structure: A Window on Strong QCD." NStar 2017 11th International Workshop on the Physics of Excited Nucleons, Columbia, SC, August 20-23, 2017.

Roberts, C.D. (2017). "Perspective on the Origin of Hadron Masses." The Proton Mass: At the Heart of Most Visible Matter Workshop, Trento, Italy, April 3-7, 2017.

Roberts, C.D. (2018). "QCD - Our Weight on Its Shoulders." HEP 2018 - 7th International Conference on High Energy Physics in the LHC Era, Valparaiso, Chile, January 8-12, 2018.

Roberts, C.D. (2018). "QCD: Our Weight on Its Shoulders." Third Sino-Americas Workshop and School on the Bound-State Problem in Continuum QCD, Tianjin, China, October 16-20, 2017.

Roberts, C.D. (2017). "Strong QCD: Atlas for the Standard Model and, Potentially, Beyond." 9th Workshop on Hadron Physics in China and Opportunities Worldwide, Nanjing, China, July 24-29, 2017.

Roberts, C.D. (2017). "Why Hadron Physics in the LHC Era?" Novel Theory for New Facilities in Hadron Physics, Nankai University, Tianjin, China, December 10-11, 2016.

Segovia, J. (2018). "Is the Nucleon a Borromean Bound-State?" International School and Workshop on Critical Stability of Quantum Few-Body Systems, Location, October 9-20, 2017.

\section{7-029-NO}

\section{NON-REFEREED PUBLICATION}

Lin, J., L.E. Ocola, S. Datta, S. Ramanathan, and S. Guha (2018). "Physics and Technology of Electronic Insulator-to-Metal Transition (E-IMT) for Record High On/Off Ratio and Low Voltage in Device Applications." IEEE International Electron Devices Meeting (IEDM), San Francisco, CA, December 2-6, 2017. (Also see 2016-120.)

\section{7-093-NO}

\section{REFEREED PUBLICATION}

Si, M., A.J. Pena, J. Hammond, P. Balaji, M. Takagi, and Y. Ishikawa. "Dynamic Adaptable Asynchronous Progress Model for MPI RMA Multiphase Applications." IEEE Transactions on Parallel and Distributed Systems. (To be published.)

\section{NON-REFEREED PUBLICATION}

Hori, A., M. Si, B. Gerofi, M. Takagi, J. Dayal, P. Balaji, and Y. Ishikawa (submited). "Process-in-Process: Techniques for Practical Address-Space Sharing." 23rd ACM SIGPLAN Annual Symposium on Principles and Practice of Parallel Programming, Vienna, Austria, February 24-28, 2018. 


\section{PRESENTATIONS}

$\mathrm{Si}, \mathrm{M}$. (2017). "Data Locality Challenges in Irregular Applications for Exascale Programing." 10th International Workshop on Parallel Programming Models and Systems Software for High-End Computing in Conjunction with the 46th International Conference on Parallel Processing (ICPP 2017), Bristol, United Kingdom, August 14, 2017.

Si, M., J. Hammond, M. Takagi, and Y. Ishikawa (2017). "Towards Portable and Adaptable Asynchronous Communication for One-Sided Applications." 10th International Workshop on Parallel Programming Models and Systems Software for High-End Computing in Conjunction with the 46th International Conference on Parallel Processing (ICPP 2017), Bristol, United Kingdom, August 14, 2017.

\section{7-094-NO}

\section{REFEREED PUBLICATIONS}

Antony, L.W., N.E. Jackson, I. Lyubimov, V. Vishwanath, M.D. Ediger, and J.J. de Pablo (2017). "Influence of Vapor Deposition on Structural and Charge Transport Properties of Ethylbenzene Films." ACS Central Science 3(5): 415-424.

Jackson, N.E., B.K. Brettmann, V. Vishwanath, M. Tirrell, and J.J. De Pablo (2017). "Comparing Solvophobic and Multivalent-Induced Collapse in Polyelectrolyte Brushes.” ACS Macro Letters 6(2): 155-160.

Yu, J., N.E. Jackson, X. Xu, M.Y. Kaufman, M. Ruths, J.J. de Pablo, and M. Tirrell. "Multivalent Counterions Impede Lubrication of Polyelectrolyte Brushes." Science. (To be published.)

Yu, J., N.E. Jackson, X. Xu, B.K. Brettmann, M. Ruths, J.J. de Pablo, and M. Tirrell (2018). "Multivalent lons Induce Lateral Structural Inhomogeneities in Polyelectrolyte Brushes." Science Advances 3(12).

\section{PRESENTATIONS}

Jackson, N. (2017). "Modulating Charge Transport in Physical Vapor-Deposited Molecular Glasses." 2017 Regulating the Interfacial Physiochemical Processes of Organic Semiconductors by Design, Telluride, CO, July 17-21, 2017.

Jackson, N.E. (2018). "Towards a Strongly Coarse-Grained, Charge-Fluctuating Model for Polyelectrolytes." 2017 AIChE Annual Meeting, Minneapolis, MN, October 29-November 3, 2017.

\section{7-095-NO}

\section{REFEREED PUBLICATIONS}

Brodsky, C.N., R.G. Hadt, D. Hayes, B.J. Reinhart, N. Li, L.X. Chen, and D.G. Nocera (2017). "In situ Characterization of Cofacial $\mathrm{Co}(\mathrm{IV})$ Centers in a $\mathrm{CO}_{4} \mathrm{O}_{4}$ Cubane: Modeling the High-Valent Active Site in Oxygen Evolving Catalysts." Proceedings of the National Academy of Sciences of the United States of America 114(15): 3855-3860.

Hayes, D., R.G. Hadt, J.D. Emery, A.A. Cordones, A.B.F. Martinson, M.L. Shelby, K.A. Fransted, P.D. Dahlberg, J. Hong, X. Zhang, Q. Kong, R.W. Schoenlein, and L.X. Chen (2017). "Electronic and Nuclear Contributions to Time-Resolved Optical and X-ray Absorption Spectra of Hematite and Insights into Photoelectrochemical Performance." Energy \& Environmental Science 9(12): 3754-3769.

Hayes, D., L. Kohler, R.G. Hadt, X. Zhang, C. Liu, K.L. Mulfort, and L.X. Chen (2018). “Excited State Electron and Energy Relays in Supramolecular Dinuclear Complexes Revealed by Ultrafast Optical and X-ray Transient Absorption Spectroscopy." Chemical Science 9(4): 860-875.

Kelley, M.S., M.L. Shelby, M.W. Mara, M.K. Haldrup, D.K. Hayes, R.G. Hadt, X. Zhang, A.B. Stickrath, R. Ruppert, J.-P. Sauvage, D. Zhu, H.T. Lemke, M.C. Chollet, G.C. Schatz, and L.X. Chen (2017). "Ultrafast Dynamics of Two Copper Bis-phenathroline Complexes Measured by X-ray Transient Absorption Spectroscopy." Journal of Physics B: Atomic, Molecular, and Optical Physics 50(15): 154003.

Kohler, L., R.G. Hadt, D. Hayes, L.X. Chen, and K.L. Mulfort (2017). "Synthesis, Structure, and Excited State Kinetics of Heteroleptic Cu(I) Complexes with a New Sterically Demanding Phenanthroline Ligand." Dalton Transactions 46(38): 13088-13100. 
Li, N., D.K. Bediako, R.G. Hadt, D. Hayes, T.J. Kempa, F. von Cube, D.C. Bell, L.X. Chen, and D.G. Nocera (2017). "Influence of Iron Doping on Tetravalent Nickel Content in Catalytic Oxygen Evolving Films." Proceedings of the National Academy of Sciences of the United States of America 114(7): 1486-1491.

\section{PRESENTATIONS}

Chen, L.X., M. Shelby, D. Hayes, R.G. Hadt, P. Kim, S. Brown-Xu, J. Hong, and M. Kelly (2017). "Electronic and Geometric Dynamics of Transition Metal Complexes for Solar Energy Conversion." 253rd American Chemical Society National Meeting and Exposition, San Francisco, CA, April 2-6, 2017.

Hadt, R.G. (2017). "High-Valent States in Cobalt and Nickel Oxygen-Evolving Catalysts and Their Role in O-O Bond Formation." American Crystallographic Association Meeting, New Orleans, LA, May 26-30, 2017.

Hadt, R.G., C.N. Brodsky, D. Hayes, L.X. Chen, and D.G. Nocera (2017). "High-Valent States in Cobalt Cubanes and Clusters: Relevance to O-O Bond Formation." 253rd American Chemical Society National Meeting and Exposition, San Francisco, CA, April 2-6, 2017.

Hadt, R.G., C.N. Brodsky, T. Kroll, D. Hayes, N. Li, D.K. Bediako, L.X. Chen, and D.G. Nocera (2017). "High-Valent States in Molecular and Heterogeneous Oxygen-Evolving Catalysts and Their Role in O-O Bond Formation." 254th American Chemical Society National Meeting and Exposition, Washington, D.C., August 20-24, 2017.

Mulfort, K.L., L. Kohler, D. Hayes, R.G. Hadt, and L. Chen (2017). "Tuning Triplet Excited State Lifetimes of CuHETPHEN Complexes." 254th American Chemical Society National Meeting and Exposition, Washington, D.C., April 20-24, 2017.

\section{7-096-NO}

\section{PRESENTATION}

Merwin, A., C. Benmore, and M.A. Williamson (2018). "Advanced Characterization of Molten Salts." 232nd Meeting of the Electrochemical Society, National Harbor, MD, October 1-5, 2017.

\section{7-097-NO}

\section{NON-REFEREED PUBLICATIONS}

Chard, R.L., K. Chard, J. Alt, D.Y. Parkinson, S. Tuecke, and I. Foster (2017). "Ripple: Home Automation for Research Data Management." 37th IEEE International Conference on Distributed Computing Systems, Atlanta, GA, June 5-8, 2017.

Chard, R., K. Chard, S. Tuecke, and I. Foster (2018). "Software-Defined Cyberinfrastructure for Data Management." 13th IEEE International Conference on eScience 2017, Aukland, New Zealand, October 24-27, 2017.

Foster, I., B. Blaiszik, K. Chard, and R. Chard (2017). "Software Defined Cyberinfrastructure." 37th IEEE International Conference on Distributed Computing Systems, Altanta, GA, June 5-8, 2017.

Paul, A.K., R. Chard, K. Chard, S. Tuecke, A.R. Butt, and I. Foster (2018). "Toward Scalable Monitoring on Large-Scale Storage for Software Defined Cyberinfrastructure." 2nd Joint International Workshop on Parallel Data Storage and Data-Intensive Scalable Computing Systems, Denver, CO, November 12-16, 2017.

\section{7-157-NO}

\section{REFEREED PUBLICATIONS}

Diroll, B.T., K. Schramke, P. Guo, U.R. Kortshagen, and R.D. Schaller (2017). “Ultrafast Silicon Photonics with Visible to Mid-Infrared Pumping of Silicon Nanocrystals." Nano Letters 17(10): 6409-6414.

Diroll, B.T., P. Guo, and R.D. Schaller (2018). “Unique Optical Properties of Methylammonium Lead lodide Nanocrystals Below the Bulk Tetragonal-Orthorhombic Phase Transition." Nano Letters.

Gong, J., P. Guo, S.E. Benjamin, P.G. Van Patten, R.D. Schaller and T. Xu. "Cation Engineering on Lead lodide Perovskites for Stable and High-Performance Photovoltaic Applications.” Journal of Energy Chemistry. (To be published.) 
Guo, P., B.T. Diroll, W. Huang, B. Wang, M.J. Bedzyk, A. Facchetti, T.J. Marks, R.P.H. Chang, and R.D. Schaller. "Low-Loss Near-Infrared Hyperbolic Metamaterials with Epitaxial ITO/ $\mathrm{In}_{2} \mathrm{O}_{3}$ Multilayers." ACS Photonics. (To be published.)

Guo, P., J. Gong, S. Sadasivam, Y. Xia, T.-B. Song, B.T. Diroll, C.C. Stoumpos, J.B. Ketterson, M.G. Kanatzidis, M.K.Y. Chan, P. Darancet, T. Xu, and R.D. Schaller. "Slow Thermal Equilibration in Hybrid Organic-Inorganic Perovskites Revealed by Transient Mid-Infrared Spectroscopy.” Science Advances. (To be published.) (Also see 2016-133.)

Liu, G., L. Kong, P. Guo, C.C. Stoumpos, Q. Hu, Z. Liu, Z. Cai, D.J. Gosztola, H.-K. Mao, M.G. Kanatzidis, and R.D. Schaller (2017). "Two Regimes of Bandgap Red Shift and Partial Ambient Retention in Pressure-Treated Two-Dimensional Perovskites." ACS Energy Letters 2(11): 2518-2524.

\section{7-158-NO}

\section{PRESENTATION}

Rimer, S., and C. Macal (2018). “Using Agent-Based Modeling to Enhance System-Level Real-Time Control of Urban Stormwater Systems.” Argonne Annual Postdoctoral Reserach and Career Symposium, Argonne, IL, November 2, 2017. 


\section{LDRD PRIME}

\section{APPLIED ENERGY AND SUSTAINABLE TRANSPORTATION}

\section{5-151-R2 \\ REFEREED PUBLICATIONS}

Babar, S., A.U. Mane, A. Yanguas-Gil, R. Haasch, and J.W. Elam (2016). "Bandgap Tailoring of W: $\mathrm{Al}_{2} \mathrm{O}_{3} \mathrm{Nanocomposite}$ Thin Films Prepared by Atomic Layer Deposition.” Chemistry of Materials 120(27): 14681-14689.

Berman, D., S. Guha, B. Lee, J.W. Elam, S.B. Darling, and E.V. Shevchenko (2017). "Sequential Infiltration Synthesis for the Design of Low Refractive Index Surface Coatings with Controllable Thickness.” ACS Nano 11(3): 2521-2530.

Biswas, M., J.A. Libera, S.B. Darling, and J.W. Elam (2015). "Kinetics for the Sequential Infiltration Synthesis of Alumina in Poly(methyl methacrylate): An Infrared Spectroscopic Study." Journal of Physical Chemistry C 119(26): 14585-14592.

Lee, A., J.A. Libera, R.Z. Waldman, A. Ahmed, J.R. Avila, J.W. Elam, and S.B. Darling (2017). "Conformal Nitrogen-Doped $\mathrm{TiO}_{2}$ Photocatalytic Coatings for Sunlight-Activated Membranes." Advanced Sustainable Systems 1(1-2): 1600041.

\section{PRESENTATIONS}

Elam, J.W. (2017). "Refractory Solar Selective Coatings." 2017 Materials Research Society Spring Meeting, Phoenix, AZ, April 17-21, 2017.

Elam, J.W. (2017). "Refractory Solar Selective Coatings Synthesized by Atomic Layer Deposition." AVS 63rd International Symposium, Nashville, TN, November 6-10, 2016.

Elam, J.W. (2017). "Refractory Solar Selective Nanocomposite Coatings for Concentrated Solar Power Receivers." AVS 17th International Conference on Atomic Layer Deposition (ALD 2017), Denver, CO, July 16-18, 2017.

Elam, J.W. (2017). "Sequential Infiltration Synthesis for Nanoscale Patterning." 253rd American Chemical Society Meeting, San Francisco, CA, April 2-6, 2017.

Elam, J.W. (2017). "Synthesis, Characterization, and Application of Tunable Nanocomposite Coatings Prepared by Atomic Layer Deposition." 3rd International Conference on ALD Applications and 2016 China ALD Conference, Suzhou, China, October 16-19, 2016.

\section{5-159-R2}

\section{REFEREED PUBLICATIONS}

Campos, E., and J. Wang (2016). "Numerical Simulation and Analysis of the April 2013 Chicago Floods." Journal of Hydrology 531(Part 2): 454-474.

Chen, C., J. Wang, F. Qiu, and D. Zhao (2016). "Resilient Distribution System by Microgrids Formation after Natural Disasters.” IEEE Transactions on Smart Grid 7(2): 958-966.

Lei, S., J. Wang, C. Chen, and Y. Hou (2016). "Mobile Emergency Generator Pre-positioning and Real-Time Allocation for Resilient Response to Natural Disasters." IEEE Transactions on Smart Grid PP(99): 1-12.

Qiu, F., J. Wang, C. Chen, and J. Tong (2015). "Optimal Black Start Resource Allocation." IEEE Transactions on Power Systems 31(3): 2493-2494.

Qiu, F., and P.J. Li (2017). "An Integrated Approach for Power System Restoration Planning." Proceedings of the IEEE 105(7): 1234-1252.

Wang, J., and F. Qiu (2017). "Robust Optimization-Based Resilient Distribution Network Planning against Natural Disasters." IEEE Transactions on Smart Grid 7(6): 2817-2826. 


\section{PRESENTATIONS}

Qiu, F. (2016). "An Electric Grid Resilience Improvement Program (EGRIP)." IEEE Power and Energy Society General Meeting (IEEEPES), Boston, MA, July 17-21, 2016.

Qiu, F., P. Li, J. Xu, and J. Wang (2016). "Mitigating Cascading Outages under Severe Weather Using Simulation-Based Optimization.” INFORMS Annual Meeting, Philadelphia, PA, November 1-4, 2015.

Qiu, F. (2016). "Modeling and Optimization for Power Grid Resilience - Towards More Robust Infrastructure and More Reliable Electricity Service." IEEE Innovative Smart Grid Technologies, Minneapolis, MN, September 6-9, 2016.

Qiu, F. (2016). “Power Grid Resilience.” Resilience Week 2016, Chicago, IL, August 16-18, 2016.

Qiu, F., P. Du, and J. Wang (2017). "Power System Restoration with Renewable Generation Participation." INFORMS Annual Meeting, Nashville, TN, November 13-16, 2016.

Qui, F., and J. Wang (2015). "Power System Restoration with Integrated Sectionalization and Generator Start-Up Sequencing, International Symposium on Mathematical Programming." 22nd International Symposium on Mathematical Programming (ISMP), Pittsburgh, PA, July 12-17, 2015.

Wang, J. (2016). “Decision Support Tools for Power Grid Resilience Improvement.” IEEE Power and Energy Society General Meeting (IEEEPES), Boston, MA, July 17-21, 2016.

Wang, J. (2016). "Grid Restoration through Advanced Optimization and Microgrids Formation." IEEE Power and Energy Society General Meeting (IEEEPES), Boston, MA, July 17-21, 2016.

\section{5-176-R2}

\section{NON-REFEREED PUBLICATION}

Karbowski, D., V. Sokolov, and A. Rousseau (2016). "Vehicle Energy Management Optimization through Digital Maps and Connectivity." 22nd ITS World Congress, Bordeaux, France, October 5-9, 2015.

\section{PRESENTATIONS}

Auld, J., D. Karbowski, V. Sokolov, and N. Kim (2016). "A Disaggregate Model System for Assessing the Energy Impact of Transportation at the Regional Level." 95th Annual Meeting of the Transportation Research Board, Washington, D.C., January 10-14, 2016.

Luo, Q., J. Auld, and V. Sokolov (2016). "Addressing Some Issues of Map-Matching for Large-Scale, High-Frequency GPS Data Sets." 95th Annual Meeting of the Transportation Research Board, Washington, D.C., January 10-14, 2016.

Rousseau, A., P. Michel, D. Karbowski, J. Auld, and V. Sokolov (2017). "Energy Impact of Connected and Automated Vehicles." ITS World Congress, Melbourne, Australia, October 10-14, 2016.

Rousseau, A., P. Michel, D. Karbowski, J. Auld, and V. Sokolov (2016). "Impact of Connection and Automation on Electrified Vehicle Energy Consumption." SAE 2016 Hybrid \& Electric Vehicle Technologies Symposium, Anaheim, CA, February 9-11, 2016.

Sokolov, V., J. Auld, N. Kim, D. Karbowski, and A. Rousseau (2016). "Modeling Framework for Transportation Simulation and Energy Assessment." 22nd ITS World Congress, Bordeaux, France, October 5-9, 2015.

\section{6-126-R1}

\section{REFEREED PUBLICATIONS}

Larson, J., T. Munson, and V. Sokolov (2017). "Coordinated Platoon Routing in a Metropolitan Network." Proceedings of the 2016 SIAM Workshop on Combinatorial Scientific Computing, Albuquerque, NM, October 10-12, 2016: 10.

Luo, F., J. Larson, and T. Munson. "Coordinated Platooning with Multiple Speeds." Transportation Research, Part C: Emerging Technologies. (To be published.) 
Sokolov, V., J. Larson, T. Munson, J. Auld, and D. Karbowski (2017). "Platoon Formation Maximization through Centralized Routing and Departure Time Coordination.” Transportation Research Record: 15.

\section{PRESENTATIONS}

Larson, J., T. Munson, and V. Sokolov (2017). "Coordinated Platoon Routing in a Metropolitan Network." 2016 SIAM Workshop on Combinatorial Scientific Computing, Albuquerque, NM, October 10-12, 2016.

Munson, T., V. Sokolov, F. Luo, and J. Larson (2017). "Minimizing Collective Fuel Use by the Centralized Coordination of Vehicle Platoons.” IMA Smart Urban Transportation Forum, Minneapolis, MN, May 15, 2017.

Stan, M. (2017). "Certainty and Uncertainty at High Temperatures." Quantification of Uncertainty in Materials Science Workshop, Gaithersburg, MD, January 14, 2017.

\section{6-152-R1}

\section{NON-REFEREED PUBLICATIONS}

Kuiper, J.A., Y. Demissie, R.W. Gentry, E. Yan, J. Wang, and A.M. Jared (2016). "Quantifying and Informing Extreme Climate and Hydrologic Events." 2016 Society for Conservation GIS Annual Conference, Monterey, CA,

June 22-25, 2016.

Yan, E., Y. Demissie, and G. Betrie (2018). "Integrated Assessment of Water-Power Grid Systems under Changing Climate." American Geophysical Union Fall Meeting, New Orleans, LA, December 11-15, 2017.

Zhou, Z., Y. Wang, G. Betrie, and E. Yan (2018). "Energy-Water Nexus Modeling and Analysis: From Cooling Water Efficiency and Electric Grid Operation Perspectives.” INFORMS Annual Meeting, Houston, TX, October 22-25, 2017.

\section{PRESENTATIONS}

Betrie, G., E. Yan, and C. Clark (2017). "Simulating the Water Use of Thermoelectric Power Plants in the United States: Model Development and Verification." 2016 American Geophysical Union Fall Meeting, San Francisco, CA, December 12-16, 2016.

Kuiper, J., Y. Demissie, R.W. Gentry, A.M. Jared, J. Wang, and E. Yan (2016). "Quantifying and Informing Extreme Climate and Hydrologic Events.” 2016 Society for Conservation GIS Annual Conference, Monterey, CA, June 22-25, 2016.

Mahat, V., and E. Yan (2017). "Stream Water Temperature Model for Upper Mississippi River Basin." 2016 American Geophysical Union Fall Meeting, San Francisco, CA, December 12-16, 2016.

Wang, J., Z. Zhou, T. Veselka, F. Qui, A. Schreiber, and E. Yan (2017). "Economic Assessment of Correlated Energy-Water Impacts Using Computable General Equilibrium Modeling." American Geophysical Union Fall Meeting, San Franscisco, CA, December 12-16, 2016.

Yan, E., T. Veselka, Z. Zhou, V. Koritarov, M. Mahalik, F. Qui, V. Mahat, G. Betrie, and C. Clark (2017). “Integrated Modeling for Assessment of Energy-Water System Resilience under Changing Climate." 2016 American Geophysical Union Fall Meeting, San Francisco, CA, December 12-16, 2016.

Zhou, Z., Y. Xia, T. Veselka, G. Betrie, F. Qui, and E. Yan (2017). "Modeling and Economic Analysis of Power Grid Operations in a Water-Constrained System." American Geophysical Union Fall Meeting, San Francisco, CA, December 12-16, 2016.

\section{6-175-R1 REFEREED PUBLICATIONS}

Fischer, P., and A.G. Tomboulides (2018). "Direct Numerical Simulation of the Flow in the Intake Pipe of an Internal Combustion Engine." International Journal of Heat and Fluid Flow 68: 257-268.

Fischer, P., M. Schmitt, and A. Tomboulides (2017). "Recent Developments in Spectral Element Simulations of Moving-Domain Problems." Recent Progress and Modern Challenges in Applied Mathematics, Modeling and Computational Science. R. Melnik, R. Makarov, and J. Belair (eds.). Springer, New York, NY, Field Institute Communications. 79: 213-244. 
Patel, S., P. Fischer, M. Min, and A. Tomboulides. "A Characteristic-Based, Spectral Element Method for Moving-Domain Problems." Journal of Scientific Computing. (To be published.)

\section{NON-REFEREED PUBLICATIONS}

Giannakopoulos, G.K., C.E. Frouzakis, P.F. Fischer, A.G. Tomboulides, and K. Boulouchos (2016). "Direct Numerical Simulation of the Flow in the Intake Pipe of an Internal Combustion Engine." The 11th International ERCOFTAC Symposium on Engineering Turbulence Modelling and Measurements (ETMM11), Palermo, Italy, September 21-23, 2016.

Kodavasal, J., Y. Pei, K. Harms, S.A. Ciatti, A. Wagner, P.K. Senecal, M. Garcia, and S. Som (2016). "Global Sensitivity Analysis of a Gasoline Compression Ignition Engine Simulation with Multiple Targets on an IBM Blue Gene/Q Supercomputer." 2016 SAE World Congress, Detroit, MI, April 12-14, 2016.

Kodavasal, J., N. Van Dam, Y. Pei, K. Harms, K. Maheshwari, A. Wagner, M. Garcia, S. Ciatti, P.K. Senecal, and S. Som (2016). "Sensitivity Analysis on Key CFD Model Inputs for GCl on an IBM BG/Q Supercomputer." 2016 THIESEL Conference on Thermo-and Fluid Dynamic Processes in Direct Injection Engines, Valencia, Spain, September 13-16, 2016.

Patel, S.S., P.F. Fischer, M. Min and A.G. Tomboulides (2018). "An Operator-Integration-Factor Splitting (OIFS) Method for Incompressible Flows in Moving Domains.” Argonne National Laboratory, ANL/ALCF-17/8, October 21, 2017.

\section{7-042-NO}

\section{PRESENTATION}

Stan, M. (2017). "From Atoms to Materials: Humans and Machines." 2017 E-CAM Workshops: From the Atom to the Molecule, Cambridge, Great Brittian, September 18-20, 2017.

\section{7-055-NO}

\section{REFEREED PUBLICATIONS}

Pan, S.-Y., S.W. Snyder, H.-W. Ma, Y.J. Lin, and P.-C. Chiang (2017). "Development of a Resin Wafer Electrodeionization Process for Impaired Water Desalination with High Energy Efficiency and Productivity." ACS Sustainable Chemistry and Engineering 5(4): 2942-2948.

Pan, S.-Y., S.W. Snyder, H.-W. Ma, Y.J. Lin, and P.-C. Chiang (2018). “Energy-Efficient Resin Wafer Electrodeionization for Impaired Water Reclamation.” Journal of Cleaner Production 174: 1464-1474.

\section{PRESENTATION}

Lin, Y.J. (2017). “Urban Resource Recovery and Reuse: Fit-for-Use Water Technology.” International Collaboration R\&D Workshop on Sustainable Water Environment, Taipei, Taiwan, August 5-6, 2017.

\section{BIOLOGICAL AND ENVIRONMENTAL SCIENCE CAPABILITY DEVELOPMENT}

\section{5-171-R2}

\section{REFEREED PUBLICATION}

Noirot-Gros, M.-F., S. Shinde, P.E. Larsen, S. Zerbs, P. Korajczyk, K.M. Kemner, and P.H. Noirot. "Temporal study of Aspen Roots Colonization by Pseudomonas Reveals Strain-Specific and Mycorrhizal-Specific Patterns of Biofilm Formation." Frontiers in Microbiology. (To be published.) (Also see 2015-180.)

\section{5-179-R2}

\section{PRESENTATIONS}

Flynn, T.M., M.F. Sladek, Z.D. Jensvold, D.A. Antonopoulos, J.C. Koval, C.W. Marshall, K.M. Kemner, and E.J. O’Loughlin (2017). "Effect of Soluble Electron Shuttles on Iron(III) Reduction, Methane Production, and Microbial Community Dynamics in Wetland Sediment Microcosms." 253rd American Chemical Society National Meeting, San Francisco, CA, April 2-6, 2017. 
Flynn, T.M., M.F. Sladek, Z.D. Jensvold, C.W. Marshall, D.A. Antonopoulos, J.C. Koval, K.M. Kemner, and E.J. O’Loughlin (2017). "Microbial Community Development under Iron-Reducing Conditions in Wetland Sediment Microcosms Amended with Electron Shuttles.” 27th Goldschmidt Geochemistry Conference, Paris, France, August 13-18, 2017.

Flynn, T.M. (2017). "Shuttling Electrons Around: Quinone-Mediated Fe(III) Reduction and Its Relation to Methanogenesis.” The University of Chicago Department of Geophysical Sciences, Chicago, IL, February $17,2017$.

Flynn, T.M., M.F. Sladek, Z. Jensvold, D.A. Antonopoulos, J.C. Koval, K. Kemner, and E.J. O’Loughlin (2017). "Soluble Electron Shuttles Control Microbial Community Development under Iron-Reducing Conditions in Wetland Sediment." American Geophysical Union Fall Meeting, San Francisco, CA, December 12-16, 2016.

Graham, R.L., K.M. Kemner, and T.M. Flynn (2016). “ANL: The Terrestrial - Aquatic Interface.” DOE Program Officers, Office of Biological and Environmental Research, U.S. Department of Energy, Germantown, MD, July 11, 2016.

Johnson, L.S., Z. Jensvold, E.J. O’Loughlin, and T.M. Flynn (2017). "Investigating the Effects of Fe(III) Inputs into Methanogenic Environments.” 2016 Midwest Geobiology Symposium, Cincinnati, OH, October 11-15, 2016.

Marquart, K.A., J.M. Paper, B.R. Haller, G. Shodunke, T.M. Flynn, Q. Jin, and M.F. Kirk (2017). "pH as a Control on Interactions of Methanogens and Iron Reducers.” 27th Goldschmidt Geochemistry Conference, Paris, France, August 13-18, 2017.

O’Loughlin, E.J., M.F. Sladek, D.A. Antonopoulos, T.M. Flynn, J.C. Koval, C.W. Marshall, and K.M. Kemner (2016). "Effects of Soluble Electron Shuttles on Microbial Fe(III) Reduction and Methanogenesis in Wetland Sediments." 251st ACS National Meeting, San Diego, CA, March 13-17, 2016.

\section{5-180-R2 \\ REFEREED PUBLICATION}

Noirot-Gros, M.-F., S. Shinde, P.E. Larsen, S. Zerbs, P. Korajczyk, K.M. Kemner, and P.H. Noirot. "Temporal Study of Aspen Roots Colonization by Pseudomonads Reveals Strain-Secific and Mycorrhizal-Specific Patterns of Biofilm Formation." Frontiers in Microbiology. (To be published.) (Also see 2015-171.)

\section{PRESENTATION}

Noirot, M.-F. S., P. Larsen, R. Wilton, S. Forrester, G. Malato, and P.H. Noirot (2017). "A Protein-Protein Interaction Network Centered on c-di-GMP Signaling in the PGP Rhizobacteria P. fluorescens." 2017 Microbial and Plant Systems Modulated by Secondary Metabolites Meeting, Walnut Creek, CA, July 24-26, 2017.

\section{6-139-R1 \\ PRESENTATIONS}

Sever, G., S. Collis, V. Ghate, Y. Feng, and J. Helmus (2017). “Interacting with Large-Eddy Simulation Data Using Python.” 97th American Meteorological Society Annual Meeting, Seattle, WA, January 22-26, 2017.

Sever, G., S. Collis, and V. Ghate (2017). "Large-Eddy Simulations of Airflow Dynamics and Physics over the Island of Graciosa." 18th Weather Research and Forecasting Workshop, Boulder, CO, June 12-16, 2017.

Sever, G., S. Collis, and V. Ghate (2017). "Large-Eddy Simulations of Airflow Dynamics and Physics over the Island of Graciosa." 2016 Atmospheric Radiation Measuremeent (ARM)/Atmostpheric System Research (ASR) Joint User Facility and Principal Investigator Meeting, Vienna, VA, March 13-17, 2017.

\section{7-087-NO}

\section{PRESENTATION}

Wang, J., C. Wang, and R. Kotamarthi (2018). "A Parallel Calibration Utility for WRF-Hydro on High Performance Computers." 2017 American Geophysical Union Fall Meeting, New Orleans, LA, December 11-15, 2017. 


\section{HARD X-RAY SCIENCES}

\section{5-147-R2}

\section{PRESENTATIONS}

Horan, D. (2016). "Solid State RF Amplifier Development at the Advanced Photon Source." 9th International Workshop on CW and High Average Power, ESRF, Grenoble, France, June 21-24, 2016.

Nassiri, A. (2016). "Growth of $\mathrm{MgB}_{2}$ Films on Large Area Cu Discs and RF Characterization." 7th International Workshop on Thin Films and New Ideas for Pushing the Limits of RF Superconductivity, Newport News, VA, July 27-29, 2016.

Nassiri, A. (2016). "Some Thoughts on the Advanced Photon Source Upgrade (APS-U) 352 MHz RF System." 9th International Workshop on CW and High Average Power, ESRF, Grenoble, France, June 21-24, 2016.

Nassiri, A. (2015). "Large Area Superconducting Magnesium Diboride on Copper Substrates for SRF Applications Using HPCVD.” 17th International Conference on RF Superconductivity, British Columbia, Canada, September 13-18, 2015.

\section{5-150-R2}

\section{REFEREED PUBLICATIONS}

Dufresne, E.M., S. Narayanan, A.R. Sandy, D.M. Kline, Q.T. Zhang, E.C. Landahl, and S. Ross (2016). "Pushing X-ray Photon Correlation Spectroscopy Beyond the Continuous Frame Rate Limit." Optics Express 24(1): 355-364.

Rumaiz, A.K., D.P. Siddons, G. Deptuch, P. Maj, A.J. Kuczewski, G.A. Carini, S. Narayanan, E.M. Dufresne, A. Sandy, R. Bradford, A. Fluerasu, and M.D. Sutton (2016). "First Experimental Feasibility Study of VIPIC: A Custom-Made Detector for X-ray Speckle Measurements." Journal of Synchrotron Radiation 23(2).

Rumaiz, A.K., D.P. Siddons, G. Deptuch, P. Maj, A.J. Kuczewski, G.A. Carini, S. Narayanan, E. Dufresne, A. Sandy, R. Bradford, A.A. Fluerasu, and M. Sutton (2016). "VIPIC: A Custom-made Detector for X-ray Speckle Measurements." Journal of Synchrotron Radiation 23(2): 404-409.

Zhang, Q., D. Bahadur, E.M. Dufresne, P. Grybos, P. Kmon, R.L. Leheny, P.G. Maj, S. Narayanan, R. Szczgiel, S. Ramakrishnan, and A.R. Sandy (2018). "Dynamic Scaling of Colloidal Gel Formation at Intermediate Concentrations." Physical Review Letters 119(17).

Zhang, Q., E.M. Dufresne, S. Narayanan, P.G. Maj, A. Koziol, R. Szczygiel, P. Grybos, and A.R. Sandy. "Microsecond-Resolved Multi-Speckle X-ray Photon Correlation Spectroscopy with a Pixel Array Detector." Journal of Synchrotron Radiation. (To be published.)

Zhang, Q., E.M. Dufresne, P. Grybos, P. Kmon, P. Maj, S. Narayanan, G.W. Deptuch, R. Szcygiel, and A. Sandy (2016). "Submillisecond X-ray Photon Correlation Spectroscopy from Pixel-Array-Detector with Fast Dual Gating and No Readout Deadtime." Journal of Synchrotron Radiation 23(Part 3): 679-684.

Zhang, Q., E.M. Dufresne, P. Chen, J. Park, M. Cosgriff, M. Yusef, Y. Dong, D. Fong, H. Zhou, Z. Cai, R. Harder, S.J. Callori, M. Dawber, P.G. Evans, and A.R. Sandy (2017). "Thermal Fluctuation of Ferroelectric Nanodomains in a Ferroelectric/ Dielectric $\mathrm{PbTiO}_{3} / \mathrm{SrTiO}_{3}$ Superlattice.” Physical Review Letters 118(9): 097601.

\section{NON-REFEREED PUBLICATION}

Shu, D., S. Kearney, A. Jayson, Z. Jiang, T. Sun, J. Maser, A. Sandy, and J. Wang (2017). “Mechanical Design of a Sample Manipulator for X-ray Coherent Surface Scattering Imaging with Flexure Stages for 10-NM-Scale and 10-NRAD-Scale Positioning." 31st American Society for Precision Engineering Annual Meeting, Portland, OR, October 23-28, 2016.

\section{PRESENTATIONS}

Sandy, A. (2018). "Prospects for XPCS at 4th Generation Synchrotron Light Sources." ALS Small-Angle Coherent Scattering Workshop, Berkeley, CA, October 3-4, 2017.

Sandy, A. (2017). "Prospects for XPCS at 4th Generation Synchrotron Light Sources." PETRA IV Coherence Workshop, Hamburg, Germany, June 26-28, 2017. 


\section{5-153-R2}

\section{NON-REFEREED PUBLICATIONS}

Deng, J., C. Preissner, C. Roehrig, M. Wojcik, S. Sullivan, D. Vine, B. Lai, and S. Vogt (2017). "The Velociprobe: A Fast Hard X-ray Nanoprobe for Ptychographic Imaging." 2017 SPIE X-ray Nanoimaging: Instruments and Methods III, San Diego, CA, August 6-10, 2017.

Mashrafi, S.T., C. Preissner, and S.M. Salapaka (2017). "Fast Scanning of X-ray Optics: An Optimal Control Approach." 31st Annual Meeting American Society for Precision Engineering (ASPE), Porland, OR, October 23-28, 2016.

Mashrafi, S.T., C. Preissner, and S.M. Salapaka (2018). "The Velociprobe: Pushing the Limits with Fast and Robust Control." Proceedings of the American Society for Precision Engineering (ASPE) 32nd Annual Meeting, Charlotte, NC, October 29-November 3, 2017.

\section{PRESENTATIONS}

Preissner, C., S. Sullivan, C. Roehrig, S. Vogt, C. Jacobsen, J. Maser, J. Deng, D. Vine, S.M. Salapaka, S.T. Mashrafi, and F. Marin (2016). "APS Velociprobe: Earth, Wind, and Fire." Three-Way Meeting DESY, Hamburg, Germany, September 14-16, 2016.

Preissner, C., S. Sullivan, T. Mashrafi, C. Roehrig, J. Maser, B. Lai, C. Jacobsen, J. Deng, F. Marin, and S. Vogt (2016). "Earth, Wind, and Fire: The New Fast Scanning Velociprobe." (MEDSI) Mechanical Engineering Design of Synchrotron Radiation Equipment and Instrumentation 2016, Barcelona, Spain, September 11-16, 2016.

Preissner, C., J. Deng, C. Roehrig, S.T. Mashrafi, M. Wojcik, S.Z. Sullivan, Z. Cai, D.J. Vine, B. Lai, and S. Vogt (2017). "Exceeding the Speed Limit." 2017 APS/CNM Users Meeting, Argonne, IL, May 8-11, 2017.

Preissner, C. (2016). "The Velociprobe and Some Conventional Facilities Issues for APS-U." 5th Diffraction Limited Storage Ring (DLSR) Workshop, Hamburg, Germany, March 9-11, 2016.

Preissner, C., S. Sullivan, C. Jacobsen, J. Maser, B. Lai, and C. Roehrig (2015). “The VelociProbe Instrument Design." NSLS-II Hard X-ray Nanoprobe Team, Upton, NY, September 9, 2015.

\section{5-161-R2}

\section{NON-REFEREED PUBLICATION}

Windt, D., and R. Conley (2015). "Two-Dimensional Differential Deposition: Figure Correction of Thin-Shell Mirror Substrates for X-ray Astronomy." Proceedings of the SPIE, Optics for EUV, X-Ray, and Gamma-Ray Astronomy VII, San Diego, CA, August 9, 2015 9603: 96031H.

\section{PRESENTATIONS}

Conley, R., B. Shi, S. Bean, M. Erdmann, L. Assoufid, J. Qian, E. Kasman, S. Izzo, K. Goetze, R. Sluiter, K. Lauer, and D. Nocher (2017). "APS Modular Deposition System." 2017 SPIE Optics + Photonics Conference, San Diego, CA, August 6-10, 2017.

Conley, R., J. Qian, S.J. Izzo, E. Kasman, K. Goetze, T. Mooney, M. Erdmann, B. Shi, and L. Assoufid (2016). "In situ Surface Figure Measurement for UHV Optics Processing Applications." 2016 SPIE Optics and Photonics Conference, San Diego, CA, August 28-September 1, 2016.

\section{6-150-R1}

REFEREEED PUBLICATION

Hu, B., J.E. Lerch , A.H. Chavan, J.K.R. Weber, A. Tamalonis, K.J. Suthar, and A.D. DiChiara (2017). "Characterization of the Acoustic Field Generated by a Horn-Shaped Ultrasonic Transducer." Applied Physics Letters 111(10). 


\section{PRESENTATIONS}

Chavan, A., P. Den Hartog, K.J. Suthar, B. Hu, and A.D. DiChiara (2016). "Characterization of the Acoustic Field Generated by the Single-Axis Acoustic Levitator." 9th International Conference on Mechanical Engineering Design of Synchrotron Radiation Equipment and Instrumentation (MEDSI 2016), Barcelona, Spain, September 11-16, 2016.

Chavan, A.H., B. Hu, A.D. DiChiara, P. Den Hartog, and K.J. Suthar (2016). "Characterization of the Acoustic Field Generated by the Single-Axis Acoustic Levitator." 9th International Conference on Mechanical Engineering Design of Synchrotron Radiation Equipment and Instrumentation (MEDSI 2016), Barcelona, Spain, September 11-16, 2016.

Suthar, K., J.L. Clough, J.E. Lerch, A. Di Chiara, and B. Hu (2016). "Acoustic Levitation.” Western Michigan University, Kalamazoo, MI, March 31, 2016.

Suthar, K., A.D. DiChiara, J.E. Lerch, B. Hu, A. Chavan, and C.J. Benmore (2017). "Acoustically Levitated Liquid Droplet." Illinois Institute of Technology Physics Colloquium, Chicago, IL, October 20, 2016.

\section{7-028-NO}

REFEREED PUBLICATION

Ivanyushenkov, Y., J. Fuerst, Q. Hasse, M. Kasa, Y. Shiroyanagi, E. Trakhtenberg, and E. Gluskin (2017). "Conceptual Design of a Novel SCAPE Undulator." Proceedings of the 8th International Particle Accelerator Conference (IPAC 2017), Copenhagen, Denmark, May 14-19, 2017 874: p.TUPAB117.

\section{7-080-NO \\ PRESENTATIONS}

Hruszkewycz, S. (2017). "Opportunities for Materials Science with New 3D Bragg Ptychography Methods.” Materials Science and Technology (MS\&T16), Salt Lake City, UT, October 23-27, 2016.

Maddali, S., I. Calvo-Almazan, Y. Nashed, J. Almer, P. Kenesei, J.S. Park, R. Harder, and S. Hruszkewycz (2017). "Exploring Sparse Recovery Strategies for High-Energy Coherent Diffraction Data through Simulations." Gordon Research Conference on X-ray Science, Easton, MA, July 30-August 4, 2017.

\section{7-091-NO}

\section{REFEREED PUBLICATIONS}

Baturin, S.S., and A. Zholents. "Stability Condition for the Drive Bunch in a Collinear Wakefeild Accelerator." Physical Review Accelerators and Beams. (To be published.)

Baturin, S., and A. Zholents (2017). "Upper Limit for the Acceleration Gradient in the Collinear Wake Field Accelerator as a Function of the Transformer Ratio." Physical Review Accelerators and Beams 20(6).

\section{7-153-NO}

\section{PRESENTATION}

Zaluzec, N.J. (2017). “If You Can't Detect It Then You Can't Measure It.” Microscopy at the Frontiers of Science, Zaragoza, Spain, September 9, 2017.

Zaluzec, N.J. (2017). "Microanalysis During in situ Operando Environmental Cell Studies in the Analytical Scanning/ Transmission Electron Microscope." Australian Microscopy \& Microanalysis Society Conference (AMAS 2017), Queensland, Brisbane, Australia, February 10, 2017.

Zaluzec, N.J. (2018). "XEDS Applied to Soft-Hard/Matter Measurements at the Nanoscale in the Next Generation AEM." International Workshop on Advanced and in situ Microscopies of Functional Nanomaterials and Devices, Singapore, Singapore, November 13, 2017. 


\section{MATERIALS AND MOLECULES TO MANUFACTURING}

\section{4-191-R3}

\section{REFEREED PUBLICATIONS}

Koehl, W.F., H. Seo, G. Galli, and D.D. Awschalom (2016). “Designing Defect Spins for Wafer-Scale Quantum Technologies." MRS Bulletin 40(12): 1146-1153.

Koehl, W.F., B. Diler, S.J. Whiteley, A. Bourassa, N.T. Son, E. Janzén, and D.D. Awschalom (2017). "Resonant Optical Spectroscopy and Coherent Control of Cr4+ Spin Ensembles in SiC and GaN." Physical Review B 95(3).

\section{NON-REFEREED PUBLICATIONS}

Koehl, W.F. (2015). “Near Unity Optical Spin Polarization of ${ }^{29}$ Si Nuclei in Silicon Carbide.” American Physical Society March Meeting 2015, San Antonio, TX, March 2-6, 2015.

Koehl, W.F. (2016). "Optical and Spin Signatures of Transition Metal Impurities in Silicon Carbide." American Physical Society March Meeting 2016, Baltimore, MD, March 14-18, 2016.

\section{4-192-R3}

\section{REFEREED PUBLICATIONS}

Brawand, N., M. Vörös, M. Govoni, and G. Galli (2017). "Generalization of Dielectric-Dependent Hybrid Functionals to Finite Systems." Physical Review X 6(4): 041002. (Also see 2016-185.)

Gaiduk, A.P., and G. Galli (2017). "Local and Global Effects of Dissolved Sodium Chloride on the Structure of Water." Journal of Physical Chemistry Letters 8(7): 1496-1502.

Gaiduk, A.P., M. Govoni, R. Seidel, J.H. Skone, B. Winter, and G. Galli (2016). "Photoelectron Spectra of Aqueous Solutions from First Principles.” Journal of the American Chemical Society 138(22): 6912-6915.

Goldsmith, Z.K., A.K. Harshan, J.B. Gerken, M. Vörös, G. Galli, S.S. Stahl, and S. Hammes-Schiffer (2017).

"Characterization of NiFe Oxyhydroxide Electrocatalysts by Integrated Electronic Structure Calculations and Spectroelectrochemistry." Proceedings of the National Academy of Sciences of the United States of America 114(12): 3050-3055.

Pham, T.A., M. Govoni, R. Seidel, S.E. Bradforth, E. Schwegler, and G. Galli (2017). “Electronic Structure of Aqueous Solutions: Bridging the Gap Between Theory and Experiments.” Science Advances 3(6): e1603210.

Pham, T.A., D. Lee, E. Schwegler, and G. Galli (2015). “Interfacial Effects on the Band Edges of Functionalized Si Surfaces in Liquid Water.” Journal of the American Chemical Society 136(49): 17071-17077.

Pham, T.A., Y. Ping, and G. Galli (2017). "Modelling Heterogeneous Interfaces for Solar Water Splitting." Nature Materials 16(4): 401-408.

Ping, Y., W.A. Goddard, and G.A. Galli (2015). "Energetics and Solvation Effects at the Photoanode/Catalyst Interface: Ohmic Contact Versus Schottky Barrier.” Journal of American Chemical Society 137(16): 5264-5267.

Seo, H., M. Govoni, and G. Galli (2016). “Design of Defect Spins in Piezoelectric Aluminum Nitride for Solid-State Hybrid Quantum Technologies.” Scientific Reports 6(20803).

Skone, J.H., M. Govoni, and G. Galli (2016). "Nonempirical Range-Separated Hybrid Functionals for Solids and Molecules." Physical Review B 93(23).

Wan, Q., and G. Galli (2015). "First-Principles Framework to Compute Sum-Frequency Generation Vibrational Spectra of Semiconductors and Insulators." Physical Review Letters 115(24): 246404. 


\section{PRESENTATIONS}

Galli, G. (2016). "Ab Initio Studies of Heterogeneous Interfaces for Water Photocatalysis." Gordon Research Conference Renewable Energy: Solar Fuels, Lucca, Italy, February 28-March 4, 2016.

Galli, G.A. (2016). "Aqueous Interfaces: Insights From First Principles Calculations.” Materials Research Society Fall Meeting (2015 MRS), Boston, MA, November 29-December 4, 2015.

Galli, G. (2016). “Computational Characterization of Solar Interfaces: Coupling ab initio Molecular Dynamics and First Principles Spectroscopies.” American Chemical Society 2016, San Diego, CA, March 13-17, 2016.

Galli, G. (2016). “Earth Abundant Materials for Solar Energy Conversion.” University of Central Florida, Orlando, FL, January 20, 2016.

Galli, G. (2014). "Electronic Properties of Aqueous Interfaces: Coupled ab initio Molecular Dynamics and GW Calculations." International Center for Materials Research (ICMR) Workshop on Ab initio Description of Charged Systems and Solid/Liquid Interfaces for Semiconductors and Electrochemistry, University of California, Santa Barbara, CA, July 7-11, 2014.

Galli, G. (2016). “Energetics and Solvation Effects at the Photoanode/Catalyst Interface." American Chemical Society 2016, San Diego, CA, March 13-17, 2016.

Galli, G. (2016). “Engineering Materials for Sustainable Energy Sources.” Materials Science and Engineering (MSE) Colloquium, Stanford University, Stanford, CT, April 8, 2016.

Galli, G. (2016). “Engineering Materials for Sustainable Energy Sources.” The Frontiers of Materials Modeling, TYC 10th Anniversary Symposium, London, UK, February 17-19, 2016.

Galli, G.A. (2015). "First Principles Spectroscopy of Heterogeneous Systems: GW and Hybrid Functionals." 250th American Chemical Society National Meeting, Boston, MA, August 16-20, 2015.

Galli, G. (2014). “Heterogeneous Interfaces: Insights From First Principles Calculations.” American Conference on Theoretical Chemistry (ACTC) 2014, Telluride, CO, July 21-24, 2014.

Galli, G. (2016). “Heterogeneous Materials for Energy and Quantum Information Technologies: First Principles Predictions.” Physical Science Seminar, IBM, Yorktown Heights, NY, June 3, 2016.

Galli, G. (2014). "Light Absorbers for Photo-Electrochemical Energy Conversion: First Principles Calculations.” 248th American Chemical Society (ACS) National Meeting, San Francisco, CA, August 10-14, 2014.

Galli, G. (2016). “Materials Discovery and Scientific Design by Computation: What Does It Take?” University of Central Florida, Orlando, FL, January 20, 2016.

Galli, G. (2015). “Materials Discovery and Scientific Design by Computation: What Does It Take?” Psi-K Conference, San Sebastien, Spain, September 6-10, 2015.

Galli, G. (2016). “Materials for Solar Energy Conversion and Storage.” American Chemical Society 2016, San Diego, CA, March 13-17, 2016.

Galli, G. (2014). "Photo-Excitations in Nanostructured Semiconductors." American Physical Society (APS) March Meeting, Denver, CO, March 3-7, 2014.

Galli, G. (2015). “Predicting Complex Materials from First Principles.” Gordon Research Conference: Nanomaterials for Applications in Energy Technology, Ventura, CA, February 22-27, 2015.

Galli, G. (2016). “Predicting Materials for Solar Energy Conversion.” American Physical Society March Meeting 2016, Baltimore, MD, March 14-18, 2016. 
Galli, G. (2014). "Predicting Materials for Water Splitting: Condensed Matter Physics Meets Electrochemistry." 248th American Chemical Society (ACS) National Meeting, Presidential Symposium Photocatalytic Conversion of Water to Hydrogen and Oxygen, San Francisco, CA, August 10-14, 2014.

Galli, G. (2016). "Predicting the Properties of Interfaces Between Electrolytes and Electrodes: Condensed Matter Physics Meets Electrochemistry." 228th Electrochemical Society Meeting, Phoenix, AZ, October 11-15, 2015.

Galli, G. (2016). "Spectroscopic and Transport Properties of Heterogeneous Materials." Theory and Applications of Computational Chemistry (TACC) Conference, Seattle, WA, August 28-September 2, 2016.

Galli, G. (2016). "Spectroscopic and Transport Properties of Heterogeneous Materials." International Union of Material Research Societies: International Conference on Electronics Materials (IUMRS-ICEM), Suntec, Singapore, July 4-8, 2016.

Galli, G. (2016). "Structural and Electronic Properties of Heterogeneous Interfaces from First Principles." Materials Research Society Meeting, Phoenix, AZ, March 28-April 1, 2016.

Galli, G. (2016). "Structure-Function Relationships in Materials for Energy Applications." American Chemical Society 2016, San Diego, CA, March 13-17, 2016.

Galli, G. (2016). "Understanding and Predicting Materials for Sustainable Energy Sources: The Key Role of Density Functional Theory.” University of California, San Diego, CA, March 31, 2016.

Galli, G. (2016). “Understanding and Predicting Materials for Sustainable Energy Sources: The Key Role of First Principles Simulations.” California Institute of Technology, Pasadena, CA, May 31, 2016.

Galli, G. (2014). "Water at Surfaces: Insights from First Principles Calculations." Water and Aqueous Solutions Gordon Research Conference, Holderness, NH, July 27-August 1, 2014.

Govoni, M., and G. Galli (2016). "Large Scale GW Calculations.” 2016 CAMD Summer School on Electronic Structure Theory and Materials Design, Technical University of Denmark, Lyngy, Denmark, August 14-19, 2016.

\section{6-131-R1}

\section{PRESENTATION}

Bunquin, J.C., M.S. Ferrandon, U. Das, C. Liu, L.A. Curtiss, J.T. Miller, A.S. Hock, S.T. Nguyen, C.L. Marshall, and P.C. Stair (2016). "Supported Organometallic Sites for Olefin Hydrogenation." Gordon Research Conference (GRC) on Catalysis: From Theory to Commercialization, New London, NH, June 12-17, 2016.

\section{6-133-R1}

\section{REFEREED PUBLICATIONS}

Chang, A.Y., M.K.Y. Chan, and R.D. Schaller (2016). "Evidence of Slow Organic-to-Inorganic Sub-Lattice Thermalization in Methylammonium Lead Halide Perovskites Observed by Ultrafast Photoluminescence." Advanced Energy Materials 6(15).

Cherukara, M.J., B. Narayanan, A. Kinaci, K. Sasikumar, S.K. Gray, M.K.Y. Chan, and S.K.R.S. Sankaranarayanan (2016). "Ab initio-Based Bond Order Potential to Investigate Low Thermal Conductivity of Stanene Nanostructures." Journal of Physical Chemistry Letters 7(19): 3752-3759.

Guo, P., Y. Xia, J. Gong, C.C. Stoumpos, K. McCall, G.C.B. Alexander, Z. Ma, H. Zhou, D.J. Gosztola, J.B. Ketterson, M.G. Kanatzidis, T. Xu, M.K.Y. Chan, and R.D. Schaller (2017). "Polar Fluctuations in Metal-Halide Perovskites Uncovered by Acoustic Phonon Anomalies." ACS Energy Letters 2(10): 2463-2469.

Kirschner, M.S., D.C. Hannah, B.T. Diroll, X. Zhang, M.J. Wagner, D. Hayes, A.C. Chang, C.E. Rowland, C.M. Lethiec, G.C. Schatz, L.X. Chen, and R.D. Schaller (2017). "Transient Melting and Recrystallization of Semiconductor Nanocrystals under Multiple Electron-Hole Pair Excitation.” Nano Letters 17(9): 5314-5320. 
Sadasivam, S., M.K.Y. Chan, and P. Darancet (2017). "Theory of Thermal Relaxation of Electrons in Semiconductors." Physical Review Letters 119(13).

\section{PRESENTATIONS}

Chan, M., Y. Xia, S. Sadasivam, P. Guo, A. Kinaci, P. Darancet, R. Schaller, A.Y. Chang, and H.-W. Lin (2017). "Phonon Properties and Slow Organic-to-Inorganic Sub-Lattice Thermalization in Hybrid Perovskites." 2017 Materials Research Society Spring Meeting, Phoenix, AZ, April 17-21, 2017.

Chan, M., Y. Xia, S. Sadasivam, P. Guo, A. Kinaci, P. Darancet, R. Schaller, A.Y. Chang, and H.-W. Lin (2017). "Phonon Properties and Slow Organic-to-Inorganic Sub-Lattice Thermalization in Hybrid Perovskites." 2017 American Physical Society March Meeting, New Orleans, LA, March 13-17, 2017.

Chan, M. (2016). "Theory Meets Reality: Combined First Principles Modeling and Characterization Studies of Renewable Energy Materials.” Massachusetts Institute of Technology, Cambridge, MA, April 7, 2016. (Also see 2016-069.)

Guo, P., M.S. Kirschner, B.T. Diroll, L.X. Chen, S.K. Gray, A.B.F. Martinson, X.M. Lin, R.P.H. Chang, and R.D. Schaller (2017). "Transient Extinction in Gold Bipyramids and Doped Oxide Nanostructures." 8th International Conference on Metamaterials, Photonic Crystals and Plasmonics, Incheon-Seoul, Korea, July 25-28, 2017.

Narayanan, B., M.J. Cherukara, A. Kinaci, K. Sasikumar, S.K. Gray, M.K.Y. Chan, and S.K.R.S. Sankaranarayanan (2017). "Bond Order Potential for 2D Stanene to Probe Thermal Transport Using Molecular Dynamics Simulations." 2016 Pacific Rim Meeting on Electrochemical and Solid-State Science - Joint Meeting 230 Electrochemical Society, 2016 Electrochemical Society of Japan, 2016 Fall Korean Electrochemical Society, Honolulu, HI, October 2-7, 2016.

(Also see 2016-082.)

Sadasivam, S., Y. Xia, M.K.Y. Chan, and P. Darancet (2017). "First-Principles Simulations of Non-Equilibrium Phonon Dynamics in III-V Materials." 2017 American Physical Society March Meeting, New Orleans, LA, March 13-17, 2017.

Schaller, R.D. (2016). "Investigations of Non-Equilibrium Electron-Phonon Coupling and Phonon-Phonon Coupling for Understanding Relaxation and Dissipation." 2016 International Conference on Energy, Materials \& Photonics (EMP16), Troyes, France, July 10-13, 2016.

Wiederrecht, G. (2016). "Ultrafast Energy and Heat Flow in Nanoscale Materials." 2016 International Conference on Energy, Materials \& Photonics (EMP16), Troyes, France, July 10-13, 2016.

\section{7-033-NO}

REFEREED PUBLICATIONS

Yang, H.-C., R. Waldman, M.-B. Wu, J. Hou, L. Chen, S.B. Darling, and Z.-K. Xu. "Dopamine: Just the Right Medicine for Membranes." Advanced Functional Materials: 1705327. (To be published.)

Yang, H.-C., M.-B. Wu, J. Hou, S.B. Darling, and Z.-K. Xu (2018). "Nanofilms Directly Formed on Macro-Porous Substrates for Molecular and Ionic Sieving." Journal of Materials Chemistry A 6(7): 2908-2913.

\section{7-050-NO}

\section{REFEREED PUBLICATION}

Yeats, A.L., P.J. Mintun, Y. Pan, A. Richardella, B.B. Buckley, N. Samarth, and D.D. Awschalom (2017). “Local Optical Control of Ferromagnetism and Chemical Potential in a Topological Insulator." Proceedings of the National Academy of Sciences of the United States of America 114(39): 10379-10383.

\section{7-082-NO}

\section{PRESENTATIONS}

Li, T. (2017). "Characterization of Nanocatalyst Using Small Angle X-ray Scattering." 2017 North American Catalysis Society Meeting, Denver, CO, June 4-9, 2017. 
Li, T. (2017). "Watching Nanoparticle Growth with Tandem in situ Small Angle X-ray Scattering and X-ray Absorption Spectroscopy." 253rd American Chemical Society National Meeting, San Francisco, CA, April 2-6, 2017.

Liu, Y., Y.A. Wu, T. Rajh, B. Smith, X.-M. Lin, L. Li, R. Zhang, M.K. Chan, J.R. Guest, I. McNulty, Z. Cai, and T. Li (2017). "Observation of Materials Transformation by a Nano-Reactor in TEM." 2017 International in situ TEM Workshop, Hangzhou, Zhejiang, China, May 25-26, 2017.

\section{7-104-NO}

\section{REFEREED PUBLICATION}

Cherukara, M.J., B. Narayanan, A. Kinaci, K. Sasikumar, S.K. Gray, M.K.Y. Chan, and S.K.R.S. Sankaranarayanan (2017). "Ab initio-Based Bond Order Potential to Investigate Low Thermal Conductivity of Stanene Nanostructures." Journal of Physical Chemistry Letters 7(19): 3752-3759.

Cherukara, M.J., K. Sasikumar, A.D. DiChiara, S.J. Leake, W. Cha, E.M. Dufresne, T. Peterka, I. McNulty, D.A. Walko, H. Wen, S.K.R.S. Sankaranarayanan, and R.J. Harder (2018). "Ultrafast Three-Dimensional Integrated Imaging of Strain in Core/Shell Semiconductor/Metal Nanostructures." Nano Letters 17(12): 7696-7701.

Cherukara, M.J., K. Sasikumar, W. Cha, B. Narayanan, S.J. Leake, E.M. Dufresne, T. Peterka, I. McNulty, H. Wen, S.K.R.S. Sankaranarayanan, and R.J. Harder (2017). "Ultrafast Three-Dimensional X-ray Imaging of Deformation Modes in ZnO Nanocrystals." Nano Letters 17(2): 1102-1108.

Ching, D.J., and D. Gürsoy (2017). “XDesign: An Open-Source Software Package for Designing X-ray Imaging Phantoms and Experiments." Journal of Synchrotron Radiation 24(Pt 2): 537-544.

Gürsoy, D. (2017). “Direct Coupling of Tomography and Ptychography.” Optics Letters 42(16): 3169-3172.

Gürsoy, D., Y.P. Hong, K. He, K. Hujsak, S. Yoo, S. Chen, Y. Li, L. Miller, Y.S. Chu, V. De Andrade, K. He, O. Cossairt, A. Katsaggelos, and C. Jacobsen (2017). "Rapid Alignment of Nanotomography Data Using Joint Iterative Reconstruction and Reprojection." Scientific Reports 7(1): 11818.

Liu, Q., G.Q. Tan, P. Wang, S.C. Abeyweera, D.T. Zhang, Y.C. Rong, Y.M.A. Wu, J. Lu, C.-J. Sun, Y. Ren, Y.Z. Liu, R.T. Muehleisen, L.B. Guzowski, J. Li, X.H. Xiao, and Y.G. Sun (2017). "Revealing Mechanism Responsible for Structural Reversibility of Single-Crystal $\mathrm{VO}_{2}$ Nanorods Upon Lithiation/Delithiation." Nano Energy 36: 197-205.

Yang, X., F. De Carlo, C. Phatak, and D. Gürsoy (2017). "A Convolutional Neural Network Approach to Calibrating the Rotation Axis for X-ray Computed Tomography." Journal of Synchrotron Radiation 24(Pt 2): 469-475.

Zhang, Y., G.M.D. Godaliyadda, N. Ferrier, E.B. Gulsoy, C.A. Bouman, and C. Phatak (2018). "Reduced Electron Exposure for Energy-Dispersive Spectroscopy using Dynamic Sampling." Ultramicroscopy 184(Part B): 90-97.

Zhang, Y., Y.S.G. Nashed, N. Ferrier, E.B. Gulsoy, and C. Phatak (2017). "Under-Sampling and Image Reconstruction for Scanning Electron Microscopes." Proceedings of the 2017 Miscroscopy and Microanalysis Meeting, St. Louis, MO, August 6-10, 2017 23(Supplement 1).

Zhu, Y.G., Q. Liu, Y. Rong, H. Chen, J. Yang, C. Jia, L.-J. Yu, A. Karton, Y. Ren, X. Xu, S. Adams, and Q. Wang (2017). "Proton Enhanced Dynamic Battery Chemistry for Aprotic Lithium-Oxygen Batteries." Nature Communications 8: 14308.

\section{PRESENTATIONS}

Guest, J.R. (2017). “Atomic-Scale Imaging of Optically Active Nanoscale Systems.” North Carolina State University, Raleigh, NC, February 1, 2017.

Guest, J.R. (2017). "Atomic-Scale Imaging of Optically Active Nanoscale Systems." University of Texas, Austin, TX, September 10, 2017.

Guest, J.R. (2017). "Atomic-Scale Imaging of Optically Active Nanoscale Systems." Paul Scherrer Institute Seminar, Villigen, Switzerland, September 1, 2017. 
Guest, J.R. (2017). "Atomic-Scale Imaging of Optically Active Nanoscale Systems." University of Tennessee, Knoxville, TN, May 1, 2017.

Guest, J.R., R. Zhang, G.R. Clark, P. Darancet, and X. Xu (2017). "Characterizing and Manipulating the Charge State of Defects in WSe $e_{2}$." QMol2017, Ascona, Switzerland, September 10-14, 2017.

Li, L., R. Zhang, Y. Wu, Y. Liu, I. McNulty, J. Vinson, E.L. Shirley, J.R. Guest, and M.K.Y. Chan (2017). " $\mathrm{CO}_{2}$ Reduction on $\mathrm{Cu}_{2} \mathrm{O}(110)$ Surface: A Combined Theoretical and Experimental Study." 26th International Materials Research Congress, Cancun, Mexico, August 20-25, 2017.

Li, L., R. Zhang, Y. A. Wu, Y. Liu, I. McNulty, T. Rajh, J.R. Guest, and M.K. Chan (2017). "Reduction of $\mathrm{CO}_{2}$ on $\mathrm{Cu}_{2} \mathrm{O}(110)$ Photocatalyst: A Combined DFT and STM Study." 2016 Fall Material Research Society Meeting, Boston, MA, November 27-December 2, 2016.

Liu, Y., Y.A. Wu, T. Rajh, B. Smith, X.-M. Lin, L. Li, R. Zhang, M.K. Chan, J.R. Guest, I. McNulty, Z. Cai, and T. Li (2017). "Observation of Materials Transformation by a Nano-Reactor in TEM." 2017 International in situ TEM Workshop, Hangzhou, Zhejiang, China, May 25-26, 2017.

McNulty, I. (2017). “Unveiling New Materials Science with Nano-focused Coherent X-rays.” Brazilian Materials Research Society, Gramado, Brazil, September 10-14, 2017.

Zhang, Y., M. Crowley, J. Hinkle, C. Phatak, and L. Makowski (2017). "Machine Learning Analysis on X-ray Scattering Data of Cellulose Microfibrils." 2017 APS/CNM User Meeting, Argonne, IL, May 2017.

Zhang, R., L. Li, L. Frazer, M.K. Chan, and J. Guest (2017). "STM Study of $\mathrm{Cu}_{2} \mathrm{O}$ Surface Facets." 2017 APS March Meeting, New Orleans, LA, March 13-17, 2017.

Zhang, Y., M.D. Godaliyadda, Y.S.G. Nashed, N. Ferrier, E.B. Gulsoy, and C. Phatak (2017). “Under-Sampling and Image Reconstruction for Scanning Electron Microscopes.” 2017 Miscroscopy and Microanalysis Meeting, St. Louis, MO, August 6-10, 2017.

\section{NATIONAL AND GLOBAL SECURITY}

\section{6-136-R1}

\section{PRESENTATIONS}

Liao, S.A. (2017). "Miniature DBR/DFB Fiber Lasers for CW THz Generation.” 2017 Global Summit on Laser Optics and Photonics, Valencia, Spain, June 9-21, 2017.

Liao, S., and H.-T. Chien (2017). "THz Phase Extraction Algorithms for a Novel THz Modulating Interferometric Doppler Radar." 18th International Conference on Microwave and Terahertz Technology, New York City, NY, October 10-11, 2016.

\section{7-076-NO}

\section{REFEREED PUBLICATION}

Xie, R., Y. Xie, C.R. López-Barrón, K. Gao, and N.J. Wagner. "Ultra-Stretchable Conductive lono-Elastomer and Motion Strain Sensor System Developed Therefrom." Technology and Innovation. (To be published.)

\section{PRESENTATIONS}

Aragon, A.M., Y. Zhang, M.B. Jungfleisch, M. Ahmed, Y. Xie, J. Wang, P. Yang, M. Puga, and K.-Z. Gao (2018). "Comparative Magnetic Studies on the Ordered and Disordered Magnetic Nanowires Arrays." 62nd Annual Conference on Magnetism and Magnetic Materials, Pittsburgh, PA, November 6-10, 2017.

Wang, J., R. Divan, Y. Xie, L.E. Ocola, Y. Zhang, A.M. Aragon, M. Ahmed, P.A.A. Ignacio-de Leon, and K. Gao (2017). "High-Aspect-Ratio Magnetic Nanowires by Electroplating and Electrospinning with Controlled Sizes and Compositions." 5th Nano Today Conference, Waikoloa Beach, HI, June 16, 2017. 
Xie, Y., K. Gao, and J. Hryn (2017). “RF-Powered Transformable Sensor on a Flexible Substrate.” 2017 FLEX, Monterey, CA, June 19-22, 2017.

Zhang, Y., A.M. Aragon, M. Ahmed, Y. Xie, J. Wang, M. Puga, and K.-Z. Gao (2018). "Synthesis and Properties of FeCo-Based Anisotropic Magnetic Nanocomposites." 62nd Annual Conference on Magnetism and Magnetic Materials, Pittsburgh, PA, November 6-10, 2017.

\section{7-088-NO}

\section{REFEREED PUBLICATIONS}

Clifford, M. (2017). “The Resilient Infrastructure Initiative.” Natural Hazards Observer XL(7): 28-29.

Jalving, J., S. Abhyankar, K. Kim, M. Hereld, and V.M. Zavala (2017). "A Graph-Based Computational Framework for Simulation and Optimization of Coupled Infrastructure Network." IET Generation, Transmission \& Distribution 11(12): 3163-3176.

Muehleisen, R.T., D. Verner, K. Kim, and F. Petit. “A New Framework for Identifying and Prioritizing Failure Points in Critical Urban Infrastructure." CIP Report. (To be published.)

Petit, F., and D. Verner (2017). “Critical Infrastructure Interdependencies Assessment.” World Security Report 2016.

Pfeiffer, K.B., C.A. Burdi, and S.O. Schlueter (2017). "Local Supply Chains: The Disaster Management Perspective." International Journal of Safety and Security Engineering 7(3): 399-405.

Portante, E.C., J.A. Kavicky, B.A. Craig, L.E. Talaber, and S.M. Folga (2018). "Modeling Electric Power and Natural Gas Systems Interdependencies: Application to Natural Hazards.” Journal of Infrastructure Systems 23(4).

\section{NON-REFEREED PUBLICATIONS}

Finster, M., J. Phillips, and K. Wallace (2017). “Front-Line Resilience Perspectives: The Electric Grid.” Argonne National Laboratory, ANL-GSS-16/2, November 2016.

Folga, S., E. Portante, S. Shamsuddin, A. Tompkins, L. Talaber, M. McLamore, J. Kavicky, G. Conzelmann, and T. Levin (2017). "U.S. Natural Gas Storage Risk-Based Ranking Methodology and Results." Argonne National Laboratory, ANL-16/19, October 2016.

Phillips, J., M. Finster, J. Pillon, F. Petit and J. Trail (2017). “State Energy Resilience Framework." Argonne National Laboratory, ANL/GSS-16/4, December 1, 2016.

Portante, E., J. Kavicky, B. Craig, L. Talaber, and S. Folga (2018). "Simulation of the January 2014 Polar Vortex and Its Impacts on Interdependent Electric-Natural Gas Infrastructure.” 2017 Winter Simulation Conference, Las Vegas, NV, December 3-6, 2017.

Verner, D., K. Kim, and F. Petit (2017). "Incorporating Prioritization in Critical Infrastructure Security and Resilience Programs.” 10th Anniversary Homeland Defense/Security Education Summit, Arlington, VA, March 23-24, 2017.

Verner, D., K. Kim, and F. Petit (2017). "Prioritizing High-Consequence Failure Points in Critical Infrastructure Adaptation and Protection Program.” 2017 Critical Infrastructure Protection and Resilience Europe, The Hague, Netherlands, May 9-11, 2017.

\section{PRESENTATIONS}

Kavicky, J., E. Portante, F. Petit, and M. Clifford (2017). "Modeling Electric Power and Natural Gas Systems Interdependencies: Application to Climate Change and Natural Hazards." 2016 Society for Risk Analysis Annual Meeting, San Diego, CA, December 11-15, 2016.

Kim, H.C. (2017). "Resilient Infrastructure Initiative." 2016 Advanced Materials for Transformative Changes to the Defense, Aerospace, and Civil Environments, Oxford, MS, November 16-17, 2016. 
Muehleisen, R.T., D. Verner, K. Kim, and F. Petit (2017). "A New Framework for Identifying and Prioritizing Failure Points in Critical Urban Infrastructure.” 2017 Frontiers in Resilience Symposium, Arlington, VA, May 10-11, 2017.

Petit, F., D. Verner, and J. Kavicky (2017). "Critical Infrastructure Interdependency Assessment.” 2017 Critical Infrastructure Protection and Resilience Europe, The Hague, Netherlands, May 9-11, 2017.

Petit, F. (2017). “Critical Infrastructure Resilience and Interdependencies." 2016 CIP Workshop, Practice, Review, and Prospect, Taipei, Taiwan, December 1-2, 2016.

Petit, F. (2017). "Critical Infrastructure Resilience and Interdependencies." University of Illinois-Chicago, Chicago, IL, March 17, 2017.

Verner, D., K. Kim, and F. Petit (2017). "Incorporating Prioritization in Critical Infrastructure Security and Resilience Programs." 10th Anniversary Homeland Defense/Security Education Summit, Arlington, VA, March 23-24, 2017.

Verner, D., K. Kim, and F. Petit (2017). "Prioritizing High-Consequence Failure Points in Critical Infrastructure Adaptation and Protection Program." 2017 Critical Infrastructure Protection and Resilience Europe, The Hague, Netherlands, May 9-11, 2017.

\section{NEXT GENERATION COMPUTING}

\section{6-135-R1 \\ REFEREED PUBLICATION}

Romano, P.K., and A.R. Siegel (2017). "Limits on the Efficiency of Event-Based Algorithms for Monte Carlo Neutron Transport." Nuclear Engineering and Technology 49(6): 1165-1171.

\section{NON-REFEREED PUBLICATION}

Romano, P.K., and A.R. Siegel (2017). "Limits on the Efficiency of Event-Based Algorithms for Monte Carlo Neutron Transport." International Conference on Mathematics and Computational Methods Applied to Nuclear Science and Engineering, Jeju, South Korea, April 16-20, 2017.

\section{6-148-R1 REFEREED PUBLICATION}

Leyffer, S., S.M. Wild, M. Fagan, M. Snir, K. Palem, K. Yoshii, and H. Finkel. "Doing Moore with Less - Leapfrogging Moore's Law with Inexactness for Supercomputing." Proceedings of the National Academy of Sciences of the United States of America. (To be published.)

\section{NON-REFEREED PUBLICATIONS}

Cappello, F., K. Yoshii, H. Finkel, and J. Cong (2017). "Re-Form: FPGA-Powered True Codesign Flow for High-Performance Computing in the Post-Moore Era." 2016 Post-Moore's Era Supercomputing (PMES) Workshops, Salk Lake City, UT, November 14, 2016.

Fagan, M., J. Schlachter, K. Yoshii, S. Leyffer, K. Palem, M. Snir, S.M. Wild, and C. Enz (2016). "Overcoming the Power Wall by Exploiting Application Inexactness and Emerging COTS Architectural Features." The 29th International IEEE System-on-Chip Conference, Seattle, WA, September 6-9, 2016.

Jin, Z., H. Finkel, K. Yoshii, and F. Cappello (2017). "A Customizable OpenCL Library for FPGA Platform." Argonne National Laboratory, ANL/ALCF-17/5, July 3, 2017.

Jin, Z., H. Finkel, K. Yoshii, and F. Cappello (2017). "Evaluation of a Floating-Point Intensive Kernel on FPGA A Case Study of Geodesic Distance Kernel." The 10th Workshop on UnConventional High Performance Computing (UCHPC 2017), Santiago de Compostela, Spain, August 29, 2017.

Jin, Z., K. Yoshii, H. Finkel, and F. Cappello (2017). "Evaluation of CHO Benchmarks on the Arria 10 FPGA Using Intel FPGA SDK for OpenCL.” Argonne National Laboratory, ANL/ALCF-17/4, May 23, 2017. 
Jin, Z., H. Finkel, K. Yoshii, and F. Cappello (2017). "Evaluation of the FIR Example Using Xilinx Vivado High-Level Synthesis Compiler.” Argonne National Laboratory, ANL/ALCF-17/6, July 28, 2017.

Jin, Z., K. Yoshii, H. Finkel, and F. Cappello (2017). "Evaluation of the OpenCL AES Kernel Using the Intel FPGA SDK for OpenCL." Argonne National Laboratory, ANL/ALCF-17/3, April 20, 2017.

Jin, Z., K. Yoshii, H. Finkel, and F. Cappello (2017). "Evaluation of the Single-Precision Floatingpoint Vector Add Kernel Using the Intel FPGA SDK for OpenCL.” Argonne National Laboratory, ANL/ALCF-17/2, April 20, 2017.

Yoshii, K., H. Finkel, and F. Cappello (2017). "Benchmarking under the Hood of OpenCL FPGA Platforms." 2nd International Workshop on Heterogeneous High-Performance Reconfigurable Computing, Salt Lake City, UT, November 14, 2016.

\section{PRESENTATIONS}

Cappello, F. (2016). "Reconfigurable Computing: An Ingredient of Post-Moore Scientific Computing?" Argonne Training Program on Extreme-Scale Computing (ATPESC), St. Charles, IL, August 4, 2016.

Yoshii, K. (2016). "Leveraging Modern SoC FPGAs for Next-Generation High-Performance Computing Systems." Workshop on FPGAs for Scientific Simulation and Data Analytics, Argonne, IL, January 21-22, 2016.

Yoshii, K., F. Cappello, F. Xia, and H. Finkel (2016). "Re-form: Leveraging FPGA Reconfigurability and Floating-Point Capabilities for Next-Generation Computing Systems." Greater Chicago Area Systems Research Workshop (GCASR) Chicago, IL, April 27, 2016.

Zhang, K., S. Ogrenci-Memik, G. Memik, K. Yoshii, R. Sankaran, P. Beckman, and P. Llopis (2017). “COOLR: A New System for Dynamic Thermal-Aware Computing." 2016 Greater Chicago Area Systems Research Workshop, Chicago, IL, April 27, 2016.

\section{7-034-NO}

REFEREED PUBLICATION

Gallington, L.C., Y. Ghadar, L.B. Skinner, J.K.R. Weber, S.V. Ushakov, A. Navrotsky, A. Vazquez-Mayagoitia, J. Neuefeind, M. Stan, J.J. Low, and C.J. Benmore (2018). “The Structure of Liquid and Amorphous Hafnia.” Materials (Basel) 10(11).

\section{PRESENTATIONS}

Stan, M. (2017). "Certainty and Uncertainty at High Temperatures." Quantification of Uncertainty in Materials Science Workshop, Gaithersburg, MD, January 14, 2017.

Stan, M. (2017). "Certainty and Uncertainty at High Temperatures." 2016 International Research Conference on Structure and Thermodynamics of Oxides at High Temperature, Davis, CA, October 20-22, 2016.

Stan, M. (2017). "From Atoms to Materials: Humans and Machines." 2017 E-CAM Workshops: From the Atom to the Molecule, Cambridge, Great Brittian, September 18-20, 2017.

Stan, M. (2017). “Uncertainty of Thermodynamic Data: Humans and Machines." 9th International Conference on Advanced Materials, Bucharest, Romania, July 11-14, 2017.

\section{7-049-NO}

\section{PRESENTATIONS}

Romero, N.A., and V. Vishwanath (2017). "ALCF Data Science Program." American Physical Society March Meeting, New Orleans, LA, March 13-17, 2017.

Vishwanath, V. (2017). "Artificial Intelligence for Transportation and Mobility." Tech Hub, SAE World Congress 2017, Detroit, MI, May 2017.

Vishwanath, V. (2017). "Automatic Multi-Objective Modeling with Machine Learning." Argonne Training Program on Extreme-Scale Computing (ATPESC), St. Charles, IL, July 29-August 10, 2017. 
Vishwanath, V. (2017). "Automatic Multi-Objective Modeling with Machine Learning." Workshop on Optimization and Machine Learning (ACNTW 17), Chicago, IL, May 18, 2017.

Vishwanath, V. (2017). "Generative Adversarial Networks." The 3rd International Workshop on Data Science in High Energy Physics (DS@HEP 2017), Batavia, IL, May 8-12, 2017.

Vishwanath, V. (2017). “Generative Adversarial Networks.” US ATLAS Workshop 2017, Argonne, IL, July 25-28, 2017.

Vishwanath, V. (2017). “Need for Data Locally in Machine/Deep Learning." Fourth Workshop on Programming Abstractions for Data Locality (PADAL'17), Chicago, IL, August 2-4, 2017.

\section{7-057-NO}

PRESENTATION

Obabko, A., R. Jain, and R. Jacob (2017). "Nek5000 Urban Modeling and NEAMS Activities." CEED Annual Review Meeting, Livermore, CA, July 31-August 3, 2017.

\section{NUCLEAR ENERGY SCIENCE AND TECHNOLOGY}

\section{6-140-R1}

\section{REFEREED PUBLICATIONS}

Bakhtiari, S., H.T. Chien, A. Heifetz, and T.W. Elmer. "Nondestructive Testing Research and Development Efforts at Argonne National Laboratory-An Overview." Materials Evaluation. (To be published.)

Heifetz, A., S. Bakhtiari, J. Lu, and A. Bentivegna (2017). "Nondestructive Evaluation of Alkali Silica Reaction in High-Strength Concrete for Aging Structures Sustainability." Transactions of American Nuclear Society 116: 453-456.

Heifetz, A., S. Bakhtiari, J. Lu, I.S. Aranson, V.M. Vinokur, and A.F. Bentivegna (2016). "Development of Microwave and Impedance Spectroscopy Methods for in situ Nondestructive Evaluation of Alkali Silica Reaction in Concrete." AIP Conference Proceedings 1806(1).

\section{NON REFEREED PUBLICATION}

Heifetz, A., S. Bakhtiari, I. Aronson, V. Vinokour, J. Lu, and A. Bentivegna (2016). "Development of Non-destructive Methods for Detection of Alkali Silica Reaction in Concrete Structures." 43rd Annual Review of Progress in Quantitative Nondestructive Evaluation, Atlanta, GA, July 17-22, 2016.

\section{PRESENTATIONS}

Heifetz, A., S. Bakhtiari, P. Zapol, and A. Bentivegna (2018). "Analysis of Alkali-Silica Reaction with Concrete with Microwave Backscattering and Impedance Spectroscopy." Department of Civil and Materials Engineering University of Illinois at Chicago, Chicago, IL, December 1, 2017.

Heifetz, A., S. Bakhtiari, Y. Liu, E.R. Koehl, A. Bentivegna, and C. Arboleda (2017). "Development of Electrochemical and Electrophysical Methods for Nondestructive Evaluation of Chemo-mechanical Damage in Concrete." 44th Annual Review of Progress in Quantitative Nondestructive Evaluation, Provo, UT, July 16-21, 2017.

Heifetz, A., S. Bakhtiari, J. Lu, and A. Bentivegna (2017). "Nondestructive Evaluation of Alkali-Silica Reaction in High-Strength Concrete for Aging Structures Sustainability." American Nuclear Society Annual Meeting (ANS 2017), San Francisco, CA, June 11-15, 2017.

\section{6-158-R1}

\section{REFEREED PUBLICATIONS}

Rotsch, D.A., M.A. Brown, J.A. Nolen, T. Brossard, W.F. Henning, S.D. Chemerisov, R.G. Gromov, and J. Greene (2018). "Electron Linear Accelerator Production and Purification of Scandium-47 from Titanium Dioxide Targets." Applied Radiation and Isotopes 131: 77-82. 
Rotsch, D.A., K. Alford, J.L. Bailey, D.L. Bowers, T. Brossard, M.A. Brown, S.D. Chemerisov, D. Ehst, J. Greene, R.G. Gromov, J.J. Grudzinski, L. Hafenrichter, A.S. Hebden, W. Henning, T.A. Heltemes, J. Jerden, C.D. Jonah, M. Kalensky, J.F. Krebs, V. Makarashvili, B. Micklich, J. Nolen, K.J. Quigley, J.F. Schneider, N.A. Smith, D.C. Stepinski, Z. Sun, P. Tkac, G.F. Vandegrift, M.J. Virgo, K.A. Wesolowski, and A.J. Youker (2017). "Production of Medical Isotopes with Electron Linacs." Proceedings of the North American Particle Accelerator Conference (NAPAC) Chicago, IL, October 9-14, 2016.

Rotsch, D.A., M.A. Brown, J.A. Nolen, R. Gromov, S.D. Chemerisov, and G.F. Vandegrift (2016). "Purification of Electron LINAC Produced Scandium-47." Proceedings of the 16th International Workshop on Targetry and Target Chemistry (WTTC16), Santa Fe, NM, August 29-September 1, 2016.

\section{PRESENTATIONS}

Brown, M.A. (2016). "Radiochemistry \& Medical Isotope Production at Argonne." Oregon State University, Corvallis, OR, July 19, 2016.

Chemerisov, S., D.A. Rotsch, J. Bailey, M.A. Brown, J.A. Nolen, R. Gromov, C.D. Jonah, D. Ehst, W.F. Henning, and G.F. Vandegrift (2017). "Electron Linac Based Production of Cu-67 and Sc-47." 13th International Topical Meeting on the Nuclear Applications of Accelerators, Quebec City, Quebec, Canada, July 31-August 4, 2017.

Rotsch, D.A., M.A. Brown, J.A. Nolen, W.F. Henning, R. Gromov, S.D. Chemerisov, J. Greene, and G.F. Vandegrift (2017). "Electron LINAC Production and Subsequent Purification of Scandium-47." 62nd Conference on Radiobioassay and Radiochemical Measurements, Honolulu, HI, February 6-10, 2017.

Rotsch, D.A., M.A. Brown, J.A. Nolen, W. Henning, R. Gromov, J. Greene, S.D. Chemerisov, and G.F. Vandegrift (2017). "Electron LINAC Production and Subsequent Separation of Scandium-47." 22nd International Symposium on Radiopharmaceutical Sciences (SRS), Dresden, Germany, May 14-19, 2017.

Rotsch, D.A. (2017). "Photonuclear Production of Theranostic Isotopes: Scandium-47 and Copper-67." Society of Nuclear Medicine and Medical Imaging (SNMMI), Denver, CO, June 10-14, 2017.

Rotsch, D.A. (2017). "Production of Medical Isotopes with Electron Linacs." North American Particle Accelerator Conference (NAPAC) Chicago, IL, October 9-14, 2016.

Rotsch, D.A. (2016). "Purification of Electron LINAC Produced Scandium-47." 16th International Workshop on Targetry and Target Chemistry (WTTC16), Santa Fe, NM, August 29-September 1, 2016.

2017-040-NO PRESENTATION

Heidet, F. (2017). “Argonne's Modeling and Simulation for MSR Analysis.” 2017 Multi-Physics Modeling and Simulation of Molten Salt Reactors Workshop, Berkeley, CA, June 15, 2017.

\section{UNIVERSE AS THE LAB (ULAB)}

\section{7-058-NO}

REFEREED PUBLICATIONS

Ninomiya, Y., W. Bentz, and I.C. Cloët (2017). "TMDs of Spin-One Targets: Formalism and Covariant Calculations." Physical Review C: Nuclear Physics 96: 045206. (Also see 2016-098.)

Xie, J., M. Hattawy, M. Chiu, K. Hafidi, E. May, J. Repond, R. Wagner, and L. Xia. "Rate Capability and Magnetic Field Tolerance Measurements of Fast Timing Microchannel Plate Photodetectors." Nuclear Instruments and Methods in Physics Research Section A: Accelerators, Spectrometers, Detectors, and Associated Equipment. (To be published.) 


\section{PRESENTATIONS}

Armstrong, W. (2017). “EIC Data Model.” First EIC Software Consortium Meeting, Shirley, NY, October 17, 2016.

Armstrong, W. (2017). "Full Simulation and Reconstruction of Multiple EIC Concept Detectors." Electron Ion Collider User Group Meeting 2017, Trieste, Italy, July 18-22, 2017.

Armstrong, W. (2017). "Spectator-Tagged Exclusive Processes on Light Nuclei." Spatial and Momentum Tomography of Hadrons and Nuclei, Seattle, WA, August 28-September 29, 2017.

Armstrong, W. (2017). "TOPSiDE and Transformative Measurements at the EIC." Spatial and Momentum Tomography of Hadrons and Nuclei, Seattle, WA, August 28-September 29, 2017.

Armstrong, W. (2017). "Transformative Measurements with Nuclei." Electron Ion Collider User Group Meeting 2017, Trieste, Italy, July 18-22, 2017.

Armstrong, W. (2017). "Unified Geometry Description with DD4hep." EIC Software Consortium Meeting, Shirley, NY, February 8-10, 2017.

Armstrong, W. (2018). “Vision for ANL Software.” EIC Software Consortium Meeting, Argonne, IL, October 16-17, 2017.

Arrington, J. (2018). "A New Simulation Framework for the Electron-Ion Collider." 2017 Fall Meeting of the APS Division of Nuclear Physics, Pittsburgh, PA, October 25-28, 2017.

Blyth, D. (2018). “ProlO.” EIC Software Consortium Meeting, Argonne, IL, October 16-17, 2017.

Blyth, D. (2018). "Status of Software at ANL and Containerization." EIC Software Consortium Meeting, Argonne, IL, October 16-17, 2017.

Cloët, I.C. (2017). "Exploring the Multi-Dimensional Structure of Hadrons." Colloquium at the College of William and Mary, Williamsburgh, VA, March 31, 2017. (Also see 2016-098.)

Cloët, I.C. (2017). "Hadron Imaging." Colloquium at the Technological Institute of Aeronautoics, Sao Paulo, Brazil, May 15, 2017. (Also see 2016-098.)

Cloët, I.C. (2017). "Hadron Physics with QCD's Dyson-Schwinger Equations." Universidade Cruzeiro Do Sul and Universidade Estadual Baulista, Sao Paulo, Brazil, May 2017.

Cloët, I.C. (2017). "Impacts of Dynamical Chiral Symmetry Breaking on Hadron Masses." The Proton Mass: At the Heart of Most Visible Matter at the European Center for Theoretical Studies in Nuclear Physics and Related Areas (ECT*), Trento, Italy, April 3-7, 2017. (Also see 2016-098.)

Cloët, I.C. (2017). “TMDs of a Spin-1 Target.” QCD Evolution 2017, Newport News, VA, May 22-26, 2017.

(Also see 2016-098.)

Cloët, I.C. (2017). “Tomography of Hadrons.” University of Sao Paulo, Sao Paulo, Brazil, May 15, 2017.

(Also see 2016-098.)

Cloët, I.C. (2017). “Tomography of Spin-one Hadrons." Electron Ion Collider User Group Meeting 2017, Trieste, Italy, July 18-22, 2017. (Also see 2016-098.)

Freese, A. (2017). "Generalized Parton Distributions of Light Nuclei." Spacial and Momentum Tomography of Hadrons and Nuclei Workshop, Seattle, WA, August 28-September 29, 2017.

Freese, A. (2017). "Toward a Lorentz Covariant Calculation of Nuclear GPDs." Spatial and Momentum Tomography of Hadrons and Nuclei Workshop, Seattle, WA, August 28-September 29, 2017.

Repond, J. (2017). "Analysis of Fully Simulated EIC Events." Electron Ion Collider User Group Meeting 2017, Trieste, Italy, July 18-22, 2017. 
Repond, J. (2017). "Argonne EIC Activities." Spacial and Momentum Tomography of Hadrons and Nuclei Workshop, Seattle, WA, August 28-September 29, 2017.

Repond, J. (2018). “CALICE: Calorimetry Reinvented.” Georgia State University Colloquium, Atlanta, GA, October 10, 2017.

Repond, J. (2017). "Calorimetry for the Future." LHec and FCC-eh Workshop at CERN, Geneva, Switzerland, September 11-13, 2017.

Repond, J. (2018). “EIC Detectors: An Overview.” 12th European Research Conference on Electromagnetic Interactions with Nucleons and Nuclei, Pafos, Cyprus, October 30-November 4, 2017.

Repond, J. (2017). "Full Simulation of and Reconstruction of Concept Detectors." LHec and FCC-eh Workshop at CERN, Geneva, Switzerland, September 11-13, 2017.

Xie, J. (2018). "Development of Fast-Timing Microchannel Plate Photomultiplier." 2017 Fall Meeting of the Division of Nuclear Physics (DNP2017) of the American Physical Society, Pittsburgh, PA, October 25-28, 2017.

Xie, J. (2018). "Recent Progress on Planar Microchannel Plate Photomultiplier Development for Fast Timing and Imaging Applications.” 2017 Nuclear Science Symposium and Medical Imaging Conference, Atlanta, GA, October 21-28, 2017.

\section{7-105-NO}

\section{REFEREED PUBLICATIONS}

Carter, F.W., C.M. Posada, J. Ding, C.L. Chang, S. Padin, A.N. Bender, J.E. Carlstrom, T.W. Cecil, R.S. Divan, A.H. Harke-Hosemann, T. Khaire, S.E. Kuhlmann, V. Kutepova, C.S. Miller, V. Novosad, J.E. Pearson, L. Stan, G. Wang, and V. Yefremenko. "Tuning SPT-3G Transition-Edge-Sensor Electrical Properties with a Four-Layer Ti-Au-Ti-Au Thin-Film Stack." Journal of Low Temperature Physics. (To be published.)

Ding, J., P.A.R. Ade, Z. Ahmed, A.J. Anderson, J.E. Austermann, J.S. Avva, R. Basu Thakur, A.N. Bender, B.A. Benson, J.E. Carlstrom, T. Cecil, C.L. Chang, R. Divan, R. Gannon, A.H. Harke-Hosemann, T. Khaire, S. Kuhlmann, C.S. Miller, V. Novosad, J. Pearson, L. Stan, G. Wang, and V. Yefremenko. "Thermal Links and Microstrip Transmission Lines in SPT-3G Bolometers." Journal of Low Temperature Physics. (To be published.)

Yefremenko, V., P.A.R. Ade, Z. Ahmed, A.J. Anderson, J.E. Austermann, J.S. Avva, R. Basu Thakur, A.N. Bender, B.A. Benson, J.E. Carlstrom, F.W. Carter, T. Cecil, C.L. Chang, J. Ding, R.N. Gannon, A.H. Harke-Hosemann, J.W. Henning, T. Khaire, S. KuhImann, V. Novosad, S. Padin, J. Pearson, C.M. Posada, and G. Wang. "Impact of Electrical Contacts Design and Materials on the Stability of Ti Superconducting Transition Shape." Journal of Low Temperature Physics. (To be published.)

\section{PRESENTATIONS}

Posada, C.M., J. Ding, C.L. Chang, F. Carter, A.N. Bender, V. Yefremenko, V. Novosad, T.S. Khaire, G. Wang, S. Padin, C.S. Miller, R. Divan, D. Czaplewski, V.P. Kutepova, and L. Stan (2017). "Fabrication of the Arrays of Detectors Deployed with the SPT-3G Receiver." 17th International Workshop on Low Temperature Detectors, Kurume, Fukuoka, Japan, July 17-21, 2017.

Yefremenko, V., J. Ding, C.L. Chang, F. Carter, A. Bender, C. Posada, V. Novosad, T. Khaire, G. Wang, S. Padin, C.S. Miller, R. Divan, D. Czaplewski, V.P. Kutepova, and L. Stan (2017). "Impact of Electrical Contacts Design and Materials on the Stability of Titanium Superconducting Transition Shape." 17th International Workshop on Low Temperature Detectors, Kurume, Fukuoka, Japan, July 17-21, 2017.

\section{7-106-NO}

\section{REFEREED PUBLICATIONS}

Aylor, K., Z. Hou, L. Knox, K.T. Story, B.A. Benson, L.E. Bleem, J.E. Carlstrom, C.L. Chang, H.M. Cho, and R. Crown (2018). "A Comparison of Cosmological Parameters Determined from CMB Temperature Power Spectra from the South Pole Telescope and the Planck Satellite." Astrophysical Journal 850(1). 
Baxter, E.J., S. Raghunathan, T.M. Crawford, P. Fosalba, Z. Hou, G.P. Holder, Y. Omori, S. Patil, E. Rozo, T.M.C. Abbott, J. Annis, K. Aylor, A. Benoit-Lévy, B.A. Benson, E. Bertin, L. Bleem, E. Buckley-Geer, D.L. Burke, J. Carlstrom, A. Carnero Rosell, M. Carrasco Kind, J. Carretero, C.L. Chang, H.M. Cho, A.T. Crites, M. Crocce, C.E. Cunha, L.N. da Costa, C.B. D'Andrea, C. Davis, T. de Haan, S. Desai, J.P. Dietrich, M.A. Dobbs, S. Dodelson, P. Doel, A. Drlica-Wagner, J. Estrada, W.B. Everett, A. Fausti Neto, B. Flaugher, J. Frieman, J. García-Bellido, E.M. George, E. Gaztanaga, T. Giannantonio, D. Gruen, R.A. Gruendl, J. Gschwend, G. Gutierrez, N.W. Halverson, N.L. Harrington, W.G. Hartley, W.L. Holzapfel, K. Honscheid, J.D. Hrubes, B. Jain, D.J. James, M. Jarvis, T. Jeltema, L. Knox, E. Krause, K. Kuehn, S. Kuhlmann, N. Kuropatkin, O. Lahav, A.T. Lee, E.M. Leitch, T.S. Li, M. Lima, D. Luong-Van, A. Manzotti, M. March, D.P. Marrone, J.L. Marshall, P. Martini, J.J. McMahon, P. Melchior, F. Menanteau, S.S. Meyer, C.J. Miller, R. Miquel, L.M. Mocanu, J.J. Mohr, T. Natoli, B. Nord, R.L.C. Ogando, S. Padin, A.A. Plazas, C. Pryke, D. Rapetti, C.L. Reichardt, A.K. Romer, A. Roodman, J.E. Ruhl, E. Rykoff, M. Sako, E. Sanchez, J.T. Sayre, V. Scarpine, K.K. Schaffer, R. Schindler, M. Schubnell, I. Sevilla-Noarbe, E. Shirokoff, M. Smith, R.C. Smith, M. Soares-Santos, F. Sobreira, Z. Staniszewski, A. Stark, K. Story, E. Suchyta, G. Tarle, D. Thomas, M.A. Troxel, K. Vanderlinde, J.D. Vieira, A.R. Walker, R. Williamson, Y. Zhang, and J. Zuntz (2018). "A Measurement of CMB Cluster Lensing with SPT and DES Year 1 Data." Monthly Notices of the Royal Astronomical Society (sty305).

Bayliss, M.B., J. Ruel, C.W. Stubbs, S.W. Allen, D.E. Applegate, M.L.N. Ashby, M. Bautz, B.A. Benson, L.E. Bleem, S. Bocquet, M. Brodwin, R. Capasso, J.E. Carlstrom, C.L. Chang, I. Chiu, H.M. Cho, A. Clocchiatti, T.M. Crawford, A.T. Crites, T. de Haan, S. Desai, J.P. Dietrich, M.A. Dobbs, A.N. Doucouliagos, R.J. Foley, W.R. Forman, G.P. Garmire, E.M. George, M.D. Gladders, A.H. Gonzalez, N. Gupta, N.W. Halverson, J. Hlavacek-Larrondo, H. Hoekstra, G.P. Holder, W.L. Holzapfel, Z. Hou, J.D. Hrubes, N. Huang, C. Jones, R. Keisler, L. Knox, A.T. Lee, E.M. Leitch, A.v.d. Linden, D. Luong-Van, A. Mantz, D.P. Marrone, M. McDonald, J.J. McMahon, S.S. Meyer, L.M. Mocanu, J.J. Mohr, S.S. Murray, S. Padin, C. Pryke, D. Rapetti, C.L. Reichardt, A. Rest, J.E. Ruhl, B.R. Saliwanchik, A. Saro, J.T. Sayre, K.K. Schaffer, T. Schrabback, E. Shirokoff, J. Song, H.G. Spieler, B. Stalder, S.A. Stanford, Z. Staniszewski, A.A. Stark, K.T. Story, K. Vanderlinde, J.D. Vieira, A. Vikhlinin, R. Williamson, and A. Zenteno (2017). "Spt-Gmos: A Gemini/Gmos-South Spectroscopic Survey of Galaxy Clusters in the Spt-Sz Survey." The Astrophysical Journal Supplement Series 227(1): 3.

Bayliss, M.B., K. Zengo, J. Ruel, B.A. Benson, L.E. Bleem, S. Bocquet, E. Bulbul, M. Brodwin, R. Capasso, I.N. Chiu, M. McDonald, D. Rapetti, A. Saro, B. Stalder, A.A. Stark, V. Strazzullo, C.W. Stubbs, and A. Zenteno (2017). "Velocity Segregation and Systematic Biases in Velocity Dispersion Estimates with the SPT-GMOS Spectroscopic Survey." Astrophysical Journal 837(1).

Bocquet, S., and F.W. Carter (2017). "PYGTC: Beautiful Parameter Covariance Plots (a.k.a. Giant Triangle Confusograms)." The Journal of Open Source Software 1(6).

Gupta, N., A. Saro, J.J. Mohr, B.A. Benson, S. Bocquet, R. Capasso, J.E. Carlstrom, I. Chiu, T.M. Crawford, T. de Haan, J.P. Dietrich, C. Gangkofner, W.L. Holzapfel, M. McDonald, D. Rapetti, and C.L. Reichardt (2017). "High-Frequency Cluster Radio Galaxies: Luminosity Functions and Implications for SZE-Selected Cluster Samples." Monthly Notices of the Royal Astronomical Society 467(3): 3737-3750.

Hennig, C., J.J. Mohr, A. Zenteno, S. Desai, J.P. Dietrich, S. Bocquet, V. Strazzullo, A. Saro, T.M.C. Abbott, F.B. Abdalla, and V. Vikram (2017). "Galaxy Populations in Massive Galaxy Clusters to z =1.1: Color Distribution, Concentration, Halo Occupation Number, and Red Sequence Fraction." Monthly Notices of the Royal Astronomical Society 467(4): 4015-4035.

Henning, J.W., J.T. Sayre, C.L. Reichardt, P.A.R. Ade, A.J. Anderson, J.E. Austermann, J.A. Beall, A.N. Bender, B.A. Benson, L.E. Bleem, J.E. Carlstrom, C.L. Chang, H.C. Chiang, H.-M. Cho, R. Citron, C. Corbett Moran, T.M. Crawford, A.T. Crites, T. de Haan, M.A. Dobbs, W. Everett, J. Gallicchio, E.M. George, A. Gilbert, N.W. Halverson, N. Harrington, G.C. Hilton, G.P. Holder, W.L. Holzapfel, S. Hoover, Z. Hou, J.D. Hrubes, N. Huang, J. Hubmayr, K.D. Irwin, R. Keisler, L. Knox, A.T. Lee, E.M. Leitch, D. Li, A. Lowitz, A. Manzotti, J.J. McMahon, S.S. Meyer, L. Mocanu, J. Montgomery, A. Nadolski, T. Natoli, J.P. Nibarger, V. Novosad, S. Padin, C. Pryke, J.E. Ruhl, B.R. Saliwanchik, K.K. Schaffer, C. Sievers, G. Smecher, A.A. Stark, K.T. Story, C. Tucker, K. Vanderlinde, T. Veach, J.D. Vieira, G. Wang, N. Whitehorn, W.L.K. Wu, and V. Yefremenko (2018). "Measurements of the Temperature and E-Mode Polarization of the CMB from 500 Square Degrees of SPTpol Data." Astrophysical Journal 852(2). 
Hou, Z., K. Aylor, B.A. Benson, L.E. Bleem, J.E. Carlstrom, C.L. Chang, H.-M. Cho, R. Chown, T.M. Crawford, A.T. Crites, T. de Haan, M.A. Dobbs, W.B. Everett, B. Follin, E.M. George, N.W. Halverson, N.L. Harrington, G.P. Holder, W.L. Holzapfel, J.D. Hrubes, R. Keisler, L. Knox, A.T. Lee, E.M. Leitch, D. Luong-Van, D.P. Marrone, J.J. McMahon, S.S. Meyer, M. Millea, L.M. Mocanu, J.J. Mohr, T. Natoli, Y. Omori, S. Padin, C. Pryke, C.L. Reichardt, J.E. Ruhl, J.T. Sayre, K.K. Schaffer, E. Shirokoff, Z. Staniszewski, A.A. Stark, K.T. Story, K. Vanderlinde, J.D. Vieira, and R. Williamson (2018). "A Comparison of Maps and Power Spectra Determined from South Pole Telescope and Planck Data." Astrophysical Journal 853(1): Article 3.

Manzotti, A., K.T. Story, W.L.K. Wu, J.E. Austermann, J.A. Beall, A.N. Bender, B.A. Benson, L.E. Bleem, J.J. Bock, J.E. Carlstrom, C.L. Chang, H.C. Chiang, H.M. Cho, R. Citron, A. Conley, T.M. Crawford, A.T. Crites, T. de Haan, M.A. Dobbs, S. Dodelson, W. Everett, J. Gallicchio, E.M. George, A. Gilbert, N.W. Halverson, N. Harrington, J.W. Henning, G.C. Hilton, G.P. Holder, W.L. Holzapfel, S. Hoover, Z. Hou, J.D. Hrubes, N. Huang, J. Hubmayr, K.D. Irwin, R. Keisler, L. Knox, A.T. Lee, E.M. Leitch, D. Li, J.J. McMahon, S.S. Meyer, L.M. Mocanu, T. Natoli, J.P. Nibarger, V. Novosad, S. Padin, C. Pryke, C.L. Reichardt, J.E. Ruhl, B.R. Saliwanchik, J.T. Sayre, K.K. Schaffer, G. Smecher, A.A. Stark, K. Vanderlinde, J.D. Vieira, M.P. Viero, G. Wang, N. Whitehorn, V. Yefremenko, and M. Zemcov (2017). "CMB Polarization B-mode Delensing with SPTpol and Herschel." Astrophysical Journal 846(1).

McDonald, M., S.W. Allen, M. Bayliss, B.A. Benson, L.E. Bleem, M. Brodwin, E. Bulbul, J.E. Carlstrom, W.R. Forman, J. Hlavacek-Larrondo, G.P. Garmire, M. Gaspari, M.D. Gladders, A.B. Mantz, and S.S. Murray (2017). "The Remarkable Similarity of Massive Galaxy Clusters from Z Similar to 0 to Z Similar to 1.9." Astrophysical Journal 843(1).

Nurgaliev, D., M. McDonald, B.A. Benson, L. Bleem, S. Bocquet, W.R. Forman, G.P. Garmire, N. Gupta, J. Hlavacek-Larrondo, J.J. Mohr, D. Nagai, D. Rapetti, A.A. Stark, C.W. Stubbs, and A. Vikhlinin (2017). "Testing for X-Ray-SZ Differences and Redshift Evolution in the X-Ray Morphology of Galaxy Clusters." Astrophysical Journal 841(1).

Omori, Y., R. Chown, G. Simard, K.T. Story, K. Aylor, E.J. Baxter, B.A. Benson, L.E. Bleem, J.E. Carlstrom, and C.L. Chang. "A 2500 Square-Degree CMB Lensing Map from Combined South Pole Telescope and Planck Data." Astrophysical Journal. (To be published.)

Raghunathan, S., S. Patil, E.J. Baxter, F. Bianchini, L.E. Bleem, T.M. Crawford, G.P. Holder, A. Manzotti, and C. Reichardt (2017). "Measuring Galaxy Cluster Masses with CMB Lensing Using a Maximum Likelihood Estimator: Statistical and Systematic Error Budgets for Future Experiments." Journal of Cosmology and Astroparticle Physics 8(8).

Saro, A., S. Bocquet, J. Mohr, E. Rozo, B.A. Benson, S. Dodelson, E.S. Rykoff, L. Bleem, T.M.C. Abbott, F.B. Abdalla, S. Allen, J. Annis, A. Benoit-Levy, D. Brooks, D.L. Burke, R. Capasso, A.C. Rosell, M.C. Kind, J. Carretero, I. Chiu, T.M. Crawford, C.E. Cunha, C.B. D’Andrea, L.N. da Costa, S. Desai, J.P. Dietrich, A.E. Evrard, A.F. Neto, B. Flaugher, B. Flaugher, J. Frieman, C. Gangkofner, E. Gaztanaga, D.W. Gerdes, T. Giannantonio, S. Grandis, D. Gruen, R.A. Gruend, N. Gupta, G. Gutierrez, W.L. Holzapfel, D.J. James, K. Kuehn, N. Kuropatkin, M. Lima, J.L. Marshall, M. McDonald, P. Melchior, F. Menanteau, R. Miquel, R. Ogando, A.A. Plazas, D. Rapetti, C.L. Reichardt, K. Reil, A.K. Romer, E. Sanchez, V. Scarpine, M. Schubnell, I. Sevilla-Noarbe, R.C. Smith, M. Soares-Santos, B. Soergel, V. Strazzullo, E. Suchyta, M.E.C. Swanson, G. Tarle, D. Thomas, V. Vikram, A.R. Walker, and A. Zenteno (2017). "Optical-SZE Scaling Relations for DES Optically Selected Clusters within the SPT-SZ Survey." Monthly Notices of the Royal Astronomical Society 468(3): 3347-3360.

Schrabback, T., D. Applegate, J.P. Dietrich, H. Hoekstra, S. Bocquet, A.H. Gonzalez, A. von der Linden, M. McDonald, C.B. Morrison, S.F. Raihan, and L.E. Bleem (2018). "Cluster Mass Calibration at High Redshift: HST Weak Lensing Analysis of 13 Distant Galaxy Clusters from the South Pole Telescope Sunyaev-Zel'dovich Survey." Monthly Notices of the Royal Astronomical Society 474(2): 2635-2678. 


\section{OTHER NOVEL R\&D}

2015-168-R2

REFEREED PUBLICATIONS

Golalikhani, M., Q. Lei, R.U. Chandrasena, L. Kasaei, H. Park, J. Bai, P. Orgiani, J. Ciston, G.E. Sterbinsky, D.A. Arena, P. Shafer, E. Arenholz, B.A. Davidson, A.J. Millis, A.X. Gray, and X.X. Xi. "Nature of the Metal-Insulator Transition in Few-Unit-Cells-Thick $\mathrm{LaNiO}_{3}$ Films." Nature Communications. (To be published.)

Goremychkin, E.A., H. Park, R. Osborn, S. Rosenkranz, J.-P. Castellan, V.R. Fanelli, A.D. Christianson, M.B. Stone, E.D. Bauer, K.J. McClellan, D.D. Byler, and J.M. Lawrence (2018). "Band Excitations in CePd 3 : A Comparison of Neutron Scattering and ab initio Theory." Science 359(6372): 186-190.

Kim, C., H. Park, and C.A. Marianetti (2016). “Influence of Quantum Confinement and Strain on Orbital Polarization of Strained Four-Layer $\mathrm{LaNiO}_{3}$ Superlattices: A DFT+DMFT Study.” Physical Review B 93(23).

Kim, C., H. Park, and C.A. Marianetti (2016). "New Class of Planar Ferroelectric Mott Insulators via First Principles Design." Physical Review B 92(23).

Moon, C.-Y., H. Park, K. Haule, and J.H. Shim (2017). “Origin of Doping-Induced Suppression and Reemergence of Magnetism in LaFeAsO ${ }_{1-\mathrm{x}} \mathrm{H}_{\mathrm{x}}$." Physical Review B: Condensed Matter 94(22).

Nowadnick, E.A., J.P. Ruf, H. Park, P.D.C. King, D.G. Schlom, K.M. Shen, and A.J. Millis (2016). "Quantifying Electronic Correlation Strength in a Complex Oxide: A Combined DMFT and ARPES Study of $\mathrm{LaNiO}_{3}$." Physical Review $B$ 92(24): 245109.

Sterbinsky, G.E., R. Nanguneri, J.X. Ma, J. Shi, E. Karapetrova, J.C. Woicik, H. Park, J.-W. Kim, and P.J. Ryan. "Ferromagnetism and Charge Order from a Frozen Electron Configuration." Physical Review Letters. (To be published.)

Upton, M.H., Y. Choi, H. Park, J. Liu, D. Meyers, J. Tchakhalian, S. Middey, and J.-W. Kim (2015). “Novel Electronic Behavior Facilitating the $\mathrm{NdNiO}_{3}$ Metal-insulator Transition.” Physical Review Letters 115: 036401.

Zhu, Y., J. Hoffman, C.E. Rowland, H. Park, D.A. Walko, J.W. Freeland, P.J. Ryan, R.D. Schaller, A. Bhattacharya, and $\mathrm{H}$. Wen. "Unconventional Slowing Down of Electronic and Structural Dynamics in Photoexcited Charge-Ordered $\mathrm{La}_{1 / 3} \mathrm{Sr}_{2 / 3} \mathrm{FeO}_{3}$." Physical Review Letters. (To be published.) (135747)

\section{PRESENTATIONS}

Park, H. (2017). “The DFT+DMFT Study of Atomic-Scale Transition Metal Oxides.” 10th International Conference on Computational Physics, Macao, China, January 16-20, 2017.

Park, H. (2016). “The Dynamical Mean Field Theory Study of Rare-Earth Nickelates.” U.S.-Korea Conference, Dallas, TX, August 10-13, 2016.

Park, H. (2017). “Energetics of Spin-State Transition in $\mathrm{LaCoO}_{3}$ : DFT+DMFT Study." Study of Matter at Extreme Conditions (SMEC2017), Miami, FL, April 1-9, 2017.

Park, H. (2017). “Energetics of Spin-State Transition in $\mathrm{LaCoO}_{3}$ : DFT+DMFT Study.” American Physical Society March Meeting 2017, New Orleans, LA, March 13-17, 2017.

Park, H. (2017). "First-Principles Study of Structural, Electronic, and Magnetic Properties of Strongly Correlated Materials: DFT+DMFT Approach.” University of Illinois at Urbana-Champaign, Champaign, IL, September 8, 2017.

Park, H. (2016). "The First-Principles Study of Structural, Electronic, and Magnetic Properties of Strongly Correlated Materials: DFT+DMFT Approach.” Brookhaven National Laboratory Seminar, Upton, NY, August 25, 2016.

Park, H. (2016). "Influence of Quantum Confinement and Strain of Orbital Polarization of Four-Layer $\mathrm{LaNiO}_{3}$ Superlattices.” American Physical Society March Meeting, Baltimore, MD, March 14-18, 2016. 


\section{5-169-R2}

\section{NON-REFEREED PUBLICATIONS}

Collier, N., J. Ozik, and C.M. Macal (2015). "Large-Scale Agent-Based Modeling with Repast HPC: A Case Study in Parallelizing an Agent-Based Model." 3rd Workshop on Parallel and Distributed Agent-Based Simulations (PADABS) at the Workshop of Euro-Par 2015, Vienna, Austria, August 24-28, 2015.

Macal, C.M. (2016). "From Compartmentalized To Agent-Based Models of Epidemics." American Physical Society March Meeting, Baltimore, MD, March 14-18, 2016.

Ozik, J., N.T. Collier, and J.M. Wozniak (2016). "Many Resident Task Computing in Support of Dynamic Ensemble Computations." 8th Workshop on Many-Task Computing on Clouds, Grids, and Supercomputers (MTAGS 2015), Austin, TX, November 15, 2015.

\section{PRESENTATIONS}

Collier, N.T., and J. Ozik (2015). "High Performance Computing and Computational Modeling: Repast HPC." University College London Big Data Institute, London, England, April 30, 2015.

Macal, C.M. (2017). “Agent-Based Modeling Applications in Healthcare and Infectious Diseases." Prevention Science and Methodology Group (PSMG) Spring 2017 Virtual Grand Rounds, Northwestern University, Chicago, IL, February 14, 2017.

Macal, C.M. (2015). “Agent-Based Modeling of Ebola Spread in a Large Urban Area.” INFORMS Healthcare Conference, Nashville, TN, July 29-31, 2015.

Macal, C.M. (2018). “Zombies Apocalypse Chicago: It's Not a Matter of If, It's a Matter of When!” 2017 INFORMS, Houston, TX, October 22-25, 2017.

Murphy, J.T., J. Ozik, N. Collier, and C. Macal (2016). "Contagion Modeling with the chiSIM and ReFACE Frameworks: Agent-Based Models of Disease Transmission in Chicago, USA.” Complex Systems 2016, Amsterdam, The Netherlands, September 19-22, 2016.

Tatara, E., N. Collier, J. Ozik, and C. Macal (2017). “Endogenous Social Networks from Large-Scale Agent-Based Models." IEEE Workshop on Parallel and Distributed Processing for Computational Social Systems (ParSocial 2017), Orlando, FL, June 2, 2017.

\section{5-170-R2}

\section{PRESENTATIONS}

Negri, M.C., and H. Ssegane (2015). “Biomimetic Approaches for Water Smart Landscape.” University of Chicago Institute for Molecular Engineering (IME) Collaborative Water Research Conference, Chicago, IL, August $17,2015$.

Negri, M.C., and H. Ssegane (2015). "Efficient Green Roofs.” The Array of Things Workshop, Chicago, IL, September 3, 2015.

Zumpf, C., and M.C. Negri (2017). “Water-Smart Urban Landscape Design: Plant Organization Patterns and Water Budget of a Green Roof." Chicago Botanic Garden, Chicago, IL, June 2017.

Zumpf, C., M.C. Negri, L.M. Hernandez Gonzalez, E. Meron, O. Tse, O. Tzuk, P. Campbell, J.F. Cacho, and H. Ssegane (2017). "Water-Smart Urban Landscape Design: Plant Organization Patterns and Water Budget of a Green Roof." International Symposium on Sustainable Systems and Technology (ISIE-ISSST 2017), Chicago, IL, June $25-29,2017$.

\section{5-174-R2}

\section{PRESENTATIONS}

Marshall, C.L. (2015). "Atomic Layer Deposition Overcoating: Tuning Catalyst Selectivity for Biomass Conversion." North American Catalysis, Pittsburgh, PA, June 14-19, 2015. 
Marshall, C.L. (2016). "Enhancing the Stability/Performance of Catalysts via Atomic Layer Deposition.” ACS Meeting, Philadelphia, PA, August 23, 2016.

Marshall, C.L. (2016). "Enhancing the Stability/Performance of Catalysts via Atomic Layer Deposition." University of Kansas, Lawrence, KS, November 13, 2015.

\section{5-178-R2}

\section{REFEREED PUBLICATIONS}

Gou, G., J. Young, X. Liu, and J.M. Rondinelli (2016). "Interplay of Cation Ordering and Ferroelectricity in Perovskite Tin lodides: Designing a Polar Halide Perovskite for Photovoltaic Applications." Inorganic Chemistry 56(1): 26-32.

Gou, G., N. Charles, J. Shi, and J.M. Rondinelli (2017). "A-Site-Ordered Double Perovskite $\mathrm{CaMnTi}_{2} \mathrm{O}_{6}$ as a Multifunctional Piezoelectric and Ferroelectric-Photovoltaic Material.” Inorganic Chemistry 56(19): 11854-11861.

He, J., C. Franchini, and J.M. Rondinelli (2017). "Ferroelectric Oxides with Strong Visible-Light Absorption from Charge Ordering." Chemistry of Materials (DOI: 10.1021/acs.chemmater.6b03486).

He, J., C. Franchini, and J.M. Rondinelli (2016). "Lithium Niobate-Type Oxides as Visible Light Photovoltaic Materials." Chemistry of Materials 28(1): 25-29.

Young, J., and J.M. Rondinelli (2016). "Crystal Structure and Electronic Properties of Bulk and Thin Film Brownmillerite Oxides." Physical Review B 92(17): 174111.

Young, J., E.J. Moon, D. Mukherjee, G. Stone, V. Gopalan, N. Alem, S.J. May, and J.M. Rondinelli (2017). "Polar Oxides Without Inversion Symmetry through Vacancy and Chemical Order." Journal of the American Chemical Society 139(7): 2833-2841.

\section{5-181-R2}

\section{REFEREED PUBLICATION}

Dyer, E.L., W. Gray Roncal, J.A. Prasad, H.L. Fernandes, D. Gürsoy, V. De Andrade, K. Fezzaa, X. Xiao, J.T. Vogelstein, C. Jacobsen, K.P. Körding, and N. Kasthuri (2017). "Quantifying Mesoscale Neuroanatomy Using X-ray

Microtomography." eNeuro 4(5).

Kasthuri, N. (2017). "High-Performance Computing in Neuroscience for Data-Driven Discovery, Integration, and Dissemination.” Neuron 92(3): 628-631.

Ocola, L.E., V. Sampathkumar, N. Kasthuri, and R.P. Winarski (2017). "Contrast Enhancement of Biological Nanoporous Materials with Zinc Oxide Infiltration for Electron and X-ray Nanoscale Microscopy." Scientific Reports 7(1): 5879.

\section{PRESENTATIONS}

Kasthuri, N. (2016). "Exascale Computing and The Brain Mapping." U.S. House of Representatives, Washington, D.C., April 11, 2016.

Kasthuri, N. (2016). “Global Brain Initiatives.” Rockefeller University, New York City, NY, September 19, 2016.

Kasthuri, N. (2016). “Sci-Foo.” Google Science Fest, San Francisco, CA, July 22-24, 2016.

Kasthuri, N. (2015). “Tales From the Trenches of Connectomics.” University of lowa, lowa City, IA, September 15, 2015.

Kasthuri, N. (2016). "Towards Complete Maps of Brains with Synchrotron Source X-ray Microscopy and Automated Serial Electron Microscopy." Center for Nanoscale Materials User Meeting, Argonne National Laboratory, Argonne, IL, May 8-11, 2016.

Kasthuri, N. (2016). "Towards Complete Maps of Brains with Synchrotron Source X-ray Microscopy and Automated Serial Electron Microscopy." Fermi National Accelerator Laboratory, Batavia, IL, February 10, 2016. 
2016-120-R1

\section{REFEREED PUBLICATION}

Sonde, S., Y. Liu, K. Sasikumar, J. Lin, L. Stan, R. Divan, L.E. Ocola, D. Rosenmann, P. Choudhury, S.K.R.S. Sankaranarayanan, and S. Guha. "Predictive Framework for Electrode Selection Enables Silicon Compatible Sn-Based Resistive Switching Memory." Nanoscale. (To be pubished.)

\section{NON-REFEREED PUBLICATION}

Lin, J., L.E. Ocola, S. Datta, S. Ramanathan, and S. Guha (2018). "Physics and Technology of Electronic Insulator-to-Metal Transition (E-IMT) for Record High On/Off Ratio and Low Voltage in Device Applications." IEEE International Electron Devices Meeting (IEDM), San Francisco, CA, December 2-6, 2017. (Also see 2017-029.)

\section{PRESENTATIONS}

Lin, J., S. Guha, C. Chen, and L. Stan (2017). "Design of the Insulator-to-Metal Transition Properties in Vanadium Oxides for the Joule-Heating Devices.” Material Research Society (MRS) Spring 2017, Phoenix, AZ, April 17-21, 2017.

Sonde, S., M. Jerry, Y. Liu, N. Shukla, S. Sankaranarayanan, S. Datta, and S. Guha (2017). “Cu:HfO ${ }_{2}$ Selector Switches for 3D Crosspoint Memory." Center for Low Energy Systems Technology Annual Review, University of Notre Dame, South Bend, IN, August 10-11, 2016.

Sonde, S., K. Sasikumar, Y. Liu, J. Lin, A. Annadi, S. Sankaranarayanan, and S. Guha (2017). "Low Voltage Nano-lonics-Based Selector Devices Using Doped $\mathrm{HfO}_{2}$ For Application in 3D Crosspoint Memories." Materials Research Society (MRS) Spring Meeting, Phoenix, AZ, April 17-21, 2017.

\section{6-182-R1}

\section{REFEREED PUBLICATION}

Zhou, Z., G.L. Grocke, P. Ignacio-de Leon, and X. Chen (2016). "CoFe $/ \mathrm{Al}_{2} \mathrm{O}_{3} / \mathrm{PMNPT}$ Multiferroic Heterostructure by Atomic Layered Deposition.” Applied Physics Letters 108: 182907.

\section{6-190-R1}

\section{REFEREED PUBLICATIONS}

Husko, C., J. Kang, G. Moille, J.D. Wood, Z. Han, D. Gosztola, X. Ma, A. De Rossi, M.C. Hersam, X. Checoury, and J.R. Guest. "A Hybrid Silicon-Phosphorene Nanolaser.” Nature Communications. (To be published.)

Kang, J., S.A. Wells, J.D. Wood, J.-H. Lee, X. Liu, C.R. Ryder, J. Zhu, J.R. Guest, C.A. Husko, and M.C. Hersam (2017). "Stable Aqueous Dispersions of Optically and Electronically Active Phosphorene." Proceedings of the National Academy of Sciences of the United States of America, October 18, 2016 113(42): 11688-11693.

\section{NON-REFEREED PUBLICATION}

Husko, C., J. Kang, J.D. Wood, G. Moille, Z. Han, D. Gosztola, X. Ma, S. Combrie, A. De Rossi, M.C. Hersam, X. Checoury, and J.R. Guest (2017). "A Hybrid Silicon-Phosphorene Nanolaser FW5A.5." OSA Frontiers in Optics, Washington, D.C., September 18-21, 2017.

\section{PRESENTATIONS}

Husko, C., J. Kang, J.D. Wood, G. Moille, Z. Han, D. Gosztola, X. Ma, S. Combrie, A. De Rossi, M.C. Hersam, X. Checoury, and J.R. Guest (2017). “A Hybrid Silicon-Phosphorene Nanolaser.” University of Sydney, Sydney, Australia, October 2, 2017.

Husko, C., J. Kang, J.D. Wood, G. Moille, Z. Han, D. Gosztola, X. Ma, S. Combrie, A. De Rossi, M.C. Hersam, X. Checoury, and J.R. Guest (2017). "A Hybrid Silicon-Phosphorene Nanolaser." National Institute of Standards and Technology (NIST), Gaithersburg, MD, September 1, 2017. 
2016-191-R1

\section{REFEREED PUBLICATION}

Young, L., K. Ueda, M. Gühr, B. Phil, M. Simon, S. Mukamel, N. Rohringer, K. Prince, C. Masciovecchio, and M. Meyer. "Roadmap on Ultrafast X-ray Atomic and Molecular Physics." Journal of Physics B: Atomic, Molecular and Optical Physics. (To be published.)

\section{7-156-NO}

\section{REFEREED PUBLICATION}

Tessier, F., V. Vishwanath, and E. Jeannot (2017). “TAPIOCA: An I/O Library for Optimized Topology-Aware Data Aggregation on Large-Scale Supercomputers." Proceedings of the IEEE International Conference on Cluster Computing 2017, Honolulu, HI, September 5-8, 2017: 70-80.

\section{NON-REFEREED PUBLICATIONS}

Tessier, F., and V. Vishwanath (2018). "Reproducibility and Variability of I/O Performance on BG/Q: Lessons Learned from a Data Aggregation Algorithm.” Argonne National Laboratory, ANL/ALCF-17/9, November $28,2017$.

Tessier, F., P. Malakar, V. Vishwanath, E. Jeannot, and F.D. Isaila (2017). "Topology-Aware Data Aggregation for Intensive I/O on Large-Scale Supercomputers." 2016 Communication Optimizations in HPC Workshop in Conjunction with ACM/ IEEE SuperComputing Conference, Salt Lake City, UT, November 18, 2016.

\section{PRESENTATIONS}

Tessier, F., and V. Vishwanath (2017). "System Abstractions to Facilitate Data Movement in Supercomputers with Deep Memory and Interconnect Hierarchy." 4th Workshop on Programming Abstractions for Data Locality (PADAL'17), Chicago, IL, August 2-4, 2017.

Tessier, F., and V. Vishwanath (2017). "System Abstractions to Facilitate Data Movement in Supercomputers with Deep Memory and Interconnect Hierarchy." 7th Joint Laboratory on Extreme Scale Computing (JLESC) Workshop, Urbana-Champaign, IL, July 17, 2017.

Tessier, F., and V. Vishwanath (2017). "Toward Portable I/O Performance by Leveraging System Abstractions of Deep Memory and Interconnect Hierarchies." U.S. Department of Energy Centers of Excellence - Performance Portability Meeting, Denver, CO, August 22-24, 2017.

Tessier, F., V. Vishwanath, and E. Jeannot (2017). “Toward Taming Large and Complex Data Flows in Data-Centric Supercomputing." 6th Joint Laboratory for Extreme Scale Computing (JLESC) Workshop, Kobe, Japan, November 30-December 2, 2016. 


\section{PUBLICATIONS AND PRESENTATIONS II}

\section{(Arising from LDRD Projects Completed prior to FY 2017 but Published/ Delivered during FY 2017)}

\section{2-074 \\ PRESENTATIONS}

Matamala, R., J. Jastrow, Z. Fan, C. Liang, F. Calderon, G. Michaelson, C.-L. Ping, and U. Mishra (2017). “Predicting Potential C Mineralization of Tundra Soils Using Spectroscopy Techniques.” 2017 Environmental System Science PI Meeting, Potomac, MD, April 25-26, 2017.

Matamala, R., Z. Fan, J.D. Jastrow, C. Liang, F. Calderon, G. Michaelson, C.L. Ping, U. Mishra, and S.M. Hofmann (2017). "Using Mid-infrared Spectroscopy to Predict the Decomposability of Soil Organic Matter Stored in Arctic Tundra Soils." 2016 American Geophysical Union Fall Meeting, San Francisco, CA, December 12-16, 2016.

\section{2-206}

REFEREED PUBLICATIONS

Gonçalves, D.R.P., J.C.d.M. Sá, U. Mishra, C.E.P. Cerri, L.A. Ferreira, and F.J.F. Furlan (2017). "Soil Type and Texture Impacts on Soil Organic Carbon Accumulation in a Sub-Tropical Agro-Ecosystem." Geoderma 286: 88-97.

Mishra, U., B. Drewniak, J.D. Jastrow, R.M. Matamala, and W. Vitharana (2017). "Spatial Representation of Organic Carbon and Active-Layer Thickness of High Latitude Soils in CMIP5 Earth System Models." Geoderma 300: 55-63.

Rosemary, F., U.W.A. Vitharana, S.P. Indraratne, R. Weerasooriya, and U. Mishra (2017). "Exploring the Spatial Variability of Soil Properties in An Alfisol Catena." Catena 150: 53-61.

\section{PRESENTATIONS}

Mishra, U. (2017). "Quantifying Environmental Controls on Stocks, Uncertainties, and Scaling of Soil Organic Carbon." American Geophysical Union Fall Meeting, San Francisco, CA, December 12-16, 2016.

Mishra, U., and W. Riley (2017). "Learning from Observed and Modeled Spatial Distribution of Uncertainties in Alaskan Soil Carbon Stocks.” DOE-Regional and Global Climate Modeling PI Meeting, Rockville, MD, November 29December 1, 2016.

\section{3-016}

REFEREED PUBLICATIONS

Jungfleisch, M.B., J. Ding, W. Zhang, W. Jiang, J.E. Pearson, V. Novosad, and A. Hoffmann (2017). "Insulating Nanomagnets Driven by Spin Torque." Nano Letters 17(1): 8-14. (Also see 2014-018.)

Jungfleisch, M.B., W. Zhang, R. Winkler, and A. Hoffmann (2017). "Spin-Orbit Torques and Spin Dynamics." Spin Physics in Semiconductors. M. Dyakonov (ed.). Springer Series in Solid-State Sciences, Springer, Cham. 157: 355-385.

(Also see 2014-018.)

Saglam, H., W. Zhang, M.B. Jungfleisch, J. Sklenar, J.E. Pearson, J.B. Ketterson, and A. Hoffmann (2017). “Spin Transport through the Metallic Antiferromagnet FeMn.” Physical Review B: Rapid Communications 94(14): 140412.

Sklenar, J., W. Zhang, M.B. Jungfleisch, H. Saglam, S. Grudichak, W. Jiang, J.E. Pearson, J.B. Ketterson, and A. Hoffmann (2017). "Unidirectional Spin-Torque Driven Magnetization Dynamics." Physical Review B 95(22). 
Sklenar, J., W. Zhang, M.B. Jungfleisch, W. Jiang, H. Saglam, J.E. Pearson, J.B. Ketterson, and A. Hoffmann (2017). "Perspective: Interface Generation of Spin-Orbit Torques." Journal of Applied Physics 120(18): 180901.

(Also see 2014-018.)

\section{PRESENTATION}

Wu, S.M., W. Zhang, K.C. Amit, P. Borisov, J.E. Pearson, S. Jiang, D. Lederman, A. Hoffmann, and A. Bhattacharya (2017). "Antiferromagnetic Spin Seebeck Effect." 61st Annual Conference on Magnetism and Magnetic Materials, New Orleans, LA, October 31-November 4, 2016.

\section{3-035}

\section{REFEREED PUBLICATION}

Ahmed, A., M. Pelton, and J.R. Guest (2017). “Understanding How Acoustic Vibrations Modulate the Optical Response of Plasmonic Metal Nanoparticles.” ACS Nano 11(9): 9360-9369.

\section{3-063}

\section{REFEREED PUBLICATIONS}

Kim, H., S. Lee, S. Kim, C. Oh, J. Ryu, E. Park, S. Hong, and K. No (2017). "Phosphotungstic Acid-Nafion Composite Membranes for Direct Ethanol Fuel Cells: Membrane Crystallinity and Fuel Cross-over." Journal of Materials Science 52(903): 1-13.

Ryu, J., K. No, Y. Kim, E. Park, and S. Hong (2017). "Synthesis and Application of Ferroelectric Poly(Vinylidene Fluoride-co-Trifluoroethylene) Films Using Electrophoretic Deposition.” Scientific Reports 6: 36176.

\section{3-111}

\section{REFEREED PUBLICATION}

Withanage, W., X.X. Xi, A. Nassiri, N. Lee, M.A. Wolak, T. Tan, P.B. Welander, M. Franzi, S. Tantawi, and R.L. Kustom (2017). "Growth of Magnesium Diboride Films on 2 Inch Diameter Copper Discs by Hybrid Physical-Chemical Vapor Deposition." Superconductor Science \& Technology 30(4).

\section{3-116}

\section{REFEREED PUBLICATIONS}

Kutsaev, S.V., B. Mustapha, P.N. Ostroumov, J. Nolen, A. Barcikowski, M. Pellin, and A. Yacout (2017). "Heavy lon Linear Accelerator for Radiation Damage Studies of Materials." The Review of Scientific Instruments 88(3): 033302.

Stepnik, B., M. Grasse, C. Jarousse, D. Geslin, J. Schulthess, I. Glagolenko, A. Yacout, S. Bhattacharya, T. Wiencek, M. Pellin, S. Van den Berghe, A. Leenaers, H. Breitkreuz, T.K. Huber, T. Zweifel, W. Petry, M. Delpech, H. Palancher, Y. Calzavara, and H. Guyon (2017). "Manufacturing Progress Status of EMPIRE UMo Irradiation Experiment." 2016 RERTR International Meeting - 37th International Meeting on Reduced Enrichment for Research and Test Reactors, Antwerp, Belgium, October 23-26, 2016.

\section{3-148}

\section{REFEREED PUBLICATION}

Kodavasal, J., S.A. Ciatti, and S. Som (2017). "Analysis of the Impact of Uncertainties in Inputs on CFD Predictions of Gasoline Compression Ignition.” Proceedings of the 2016 American Society of Mechanical Engineers (ASME) Internal Combustion Engine Fall Technical Conference, Greenville, SC, October 9-12, 2016, ICEF2016-9328: V001T006A006.

Wang, Z., M.M. Ameen, S. Som, and J. Abraham (2017). “Assessment of Large-Eddy Simulations of Turbulent Round Jets Using Low-Order Numerical Schemes.” SAE International Journal of Commercial Vehicles 10(2): 572-581. 


\section{NON-REFEREED PUBLICATION}

Som, S. (2017). "Simulation of Internal Combustion Engines with High-Performance Computing Tools."

ANL/ES-C1300601: 1-8. (2013-148-R2).

\section{PRESENTATION}

Van Dam, N., S. Som, W. Zeng, and M. Sjöberg (2017). "Effects of Fuel Physical Properties on Direct-Injection Spark-Ignition Engine Performance.” IEA Combustion Agreement Fuel Spray Workshop, Detroit, MI, April 3, 2017.

\section{3-152}

\section{REFEREED PUBLICATION}

Kutsaev, S.V., B. Mustapha, P.N. Ostroumov, J. Nolen, A. Barcikowski, M. Pellin, and A. Yacout (2017). "Heavy lon Linear Accelerator for Radiation Damage Studies of Materials." Review of Scientific Instruments 88(3): 033302.

Miao, Y., J. Harp, K. Mo, S. Bhattacharya, P. Baldo, and A.M. Yacout (2017). "Short Communication on in situ TEM Ion Irradiation Investigations on $\mathrm{U}_{3} \mathrm{Si}_{2}$ at LWR Temperatures.” Journal of Nuclear Materials 484: $168-173$.

Miao, Y., J. Harp, K. Mo, S. Zhu, T. Yao, J. Lian, and A.M. Yacout (2017). “Bubble Morphology in $\mathrm{U}_{3} \mathrm{Si}_{2} \operatorname{Implanted~by~}$ High-Energy Xe Ions at $300^{\circ} \mathrm{C}$.” Journal of Nuclear Materials 495: 146-153.

Miao, Y., T. Yao, J. Lian, J.-S. Park, J. Almer, S. Bhattacharya, A.M. Yacout, and K. Mo (2017). "In situ Synchrotron Investigation of Grain Growth Behavior of Nano-Grained $\mathrm{UO}_{2}$." Scripta Materialia 131: 29-32.

Ye, B., L. Jamison, Y. Miao, S. Bhattacharya, G.L. Hofman, and A.M. Yacout (2017). "Cross Section TEM Characterization of High-Energy-Xe-Irradiated U-Mo." Journal of Nuclear Materials 488: 134-142.

\section{NON-REFEREED PUBLICATION}

Miao, Y., K. Mo, L.M. Jamison, J. Lian, T. Yao, and S. Bhattacharya (2017). "Experimental Studies of Micro- and Nano-Grained $\mathrm{UO}_{2}$ : Grain Growth Behavior, Surface Morphology, and Fracture Toughness," prepared for U.S. Department of Energy, Nuclear Energy Advanced Modeling \& Simulation Program. ANL/NE-16/12.

\section{3-184}

\section{REFEREED PUBLICATION}

Segal-Peretz, T., J. Ren, S. Xiong, G. Khaira, A. Bowen, L.E. Ocola, R.N.S. Divan, M. Doxastakis, N.J. Ferrier, J. de Pablo, and P.F. Nealey (2017). "Quantitative Three-Dimensional Characterization of Block Copolymer Directed Self-Assembly on Combined Chemical and Topographical Prepatterned Templates." ACS Nano 11(2): 1307-1319.

\section{3-194}

\section{REFEREED PUBLICATION}

Juarez-Vazquez, A.L., J.N. Edirisinghe, E.A. Verduzco-Castro, K. Michalska, C. Wu, L. Noda-Garcia, G. Babnigg, M. Endres, S. Medina-Ruiz, J. Santoyo-Flores, M. Carrillo-Tripp, H. Ton-That, A. Joachimiak, C.S. Henry, and F. Barona-Gomez (2017). "Evolution of Substrate Specificity in a Retained Enzyme Driven by Gene Loss." eLife 6.

\section{3-199}

\section{REFEREED PUBLICATION}

Harrison, R.J., G. Beylkin, F.A. Bischoff, J.A. Calvin, G.I. Fann, J. Fosso-Tande, D. Galindo, J.R. Hammond, R. Hartman-Baker, J.C. Hill, J. Jia, J.S. Kottmann, M.J. Yvonne Ou, J. Pei, L.E. Ratcliff, M.G. Reuter, A.C. Richie-Halford, N.A. Romero, H. Sekino, W.A. Shelton, B.E. Sundahl, W.S. Thornton, E.F. Valeev, Á. Vázquez-Mayagoitia, N. Vence, T. Yanai, and Y. Yokoi (2017). "MADNESS: A Multiresolution, Adaptive Numerical Environment for Scientific Simulation." SIAM Journal on Scientific and Statistical Computing 38(5): S123-S142. 


\section{3-208}

\section{PRESENTATION}

Khan, F., N. Schwarz, S. Narayanan, A. Sandy, C. Schmitz, B. Pausma, R. Aydelott, and D. Murphy-Olson (2017). "High-Performance XPCS Data Reduction Using Virtualized Computing Resources." 11th New Opportunities for Better User Group Software, Copenhagen, Denmark, October 17-19, 2016.

\section{3-213}

\section{REFEREED PUBLICATIONS}

Chunduri, S., P. Balaprakash, V. Morozov, V. Vishwanath, and K. Kumaran (2017). "Analytical Performance Modeling and Validation of Intel's Xeon Phi Architecture." Proceedings of the Computing Frontiers Conferences (CF17), ACM International Conference on Computing Frontiers 2017, Siena, Italy, May 15-17, 2017, ACM New York: 2477-2250.

Liu, Z., P. Balaprakash, R. Kettimuthu, and I. Foster (2017). "Explaining Wide Area Data Transfer Performance." Proceedings of the 26th International Symposium on High Performance Distributed Computing, Washington, D.C., June 26-27, 2017, ACM New York: 167-178.

\section{3-219}

\section{REFEREED PUBLICATIONS}

Ding, J., P.A.R. Ade, A.J. Anderson, J. Avva, Z. Ahmed, K. Arnold, J.E. Austermann, A.N. Bender, B.A. Benson, L.E. Bleem, K. Byrum, J.E. Carlstrom, F.W. Carter, C.L. Chang, D. Czaplewski, R. Divan, R. Gannon, T. Khaire, C.S. Miller, S. Padin, V. Novosad, J. Pearson, C.M. Posada, L. Stan, G. Wang, and V. Yefremenko (2017). "Optimization of Transition Edge Sensor Arrays for Cosmic Microwave Background Observations with the South Pole Telescope." IEEE Transactions on Applied Superconductivity (99).

Wang, G., J. Beeman, C.L. Chang, J. Ding, A. Drobizhev, B.K. Fujikawa, K. Han, S. Han, R. Hennings-Yeomans, G. Karapetrov, Y. Kolomensky, V. Novosad, J. Pearson, and V.G. Yefremenko (2017). "Modeling Iridium-Based Trilayer and Bilayer Transition-Edge Sensors." IEEE Transactions on Applied Superconductivity 27(4): 2100405.

\section{PRESENTATION}

Wang, G. (2017). “Iridium-Based Low-Tc Transition-Edge Sensor." 2016 Coordinating Panel for Advanced Detectors Instrumentation Frontier at Caltech: New Technologies for Discovery II, Pasadena, CA, October 8-10, 2016.

\section{4-018}

\section{REFEREED PUBLICATIONS}

Jungfleisch, M.B., J. Ding, W. Zhang, W. Jiang, J.E. Pearson, V. Novosad, and A. Hoffmann (2017). “Insulating Nanomagnets Driven by Spin Torque." Nano Letters 17(1): 8-14. (Also see 2013-016.)

Jungfleisch, M.B., J. Sklenar, J. Ding, J. Park, J.E. Pearson, V. Novosad, P. Schiffer, and A. Hoffmann (2018). "High-Frequency Dynamics Modulated by Collective Magnetization Reversal in Artificial Spin Ice." Physical Review Applied 8(6).

Norman, M.R. (2017). “Colloquium: Herbertsmithite and the Search for the Quantum Spin Liquid.” Reviews of Modern Physics 88(4).

Saglam, H., W. Zhang, M.B. Jungfleisch, J. Sklenar, J.E. Pearson, J.B. Ketterson, and A. Hoffmann (2017). "Spin Transport through the Metallic Antiferromagnet FeMn." Physical Review B 94(14): 140412.

Sklenar, J., W. Zhang, M.B. Jungfleisch, H. Saglam, S. Grudichak, W. Jiang, J.E. Pearson, J.B. Ketterson, and A. Hoffmann (2017). “Unidirectional Spin-Torque-Driven Magnetization Dynamics." Physical Review B 95(22).

Sklenar, J., W. Zhang, M.B. Jungfleisch, W. Jiang, H. Saglam, J.E. Pearson, J.B. Ketterson, and A. Hoffmann (2017). "Perspective: Interface Generation of Spin-Orbit Torques." Journal of Applied Physics 120(18): 180901.

(Also see 2013-016.) 


\section{NON-REFEREED PUBLICATION}

Jungfleisch, M.B., W. Zhang, R. Winkler, and A. Hoffmann (2017). "Spin-Orbit Torques and Spin Dynamics." Spin Physics in Semiconductors. M. Dyakonov (ed.). Springer Series in Solid-State Sciences, Springer, Cham. 157: 355-385.

(Also see 2013-016.)

\section{PRESENTATIONS}

Jungfleisch, M.B., J. Ding, J.E. Pearson, A. Hoffmann, W. Zheng, W. Jiang, J. Sklenar, and J.B. Ketterson (2017). "Spin Dynamics in $\mathrm{Ni}_{80} \mathrm{Fe}_{20} / \mathrm{Pt}$ Bilayer Antidot Lattices." 2017 American Physical Society March Meeting, New Orleans, LA, March 13-17, 2017.

Jungfleisch, M.B., W. Zhang, J. Sklenar, W. Jiang, J. Ding, H. Chang, F. Fradin, S.M. Wu, J.E. Pearson, A. Bhattacharya, V. Novosad, and A. Hoffmann (2017). "Electrically Driven Magnetization Dynamics in Yttrium Iron Garnet." 61st Annual Conference on Magnetism and Magnetic Materials, New Orleans, LA, October 31-November 4, 2016.

\section{4-051}

\section{REFEREED PUBLICATION}

Barkholtz, H.M., and D.-J. Liu (2017). "Advancements in Rationally Designed PGM-Free Fuel Cell Catalysts Derived from Metal-Organic Frameworks.” Materials Horizons 4(1): 20-37.

Barkholtz, H.M., L. Chong, Z.B. Kaiser, T. Xu, and D.-J. Liu (2017). "Enhanced Performance of non-PGM Catalysts in Air Operated PEM-Fuel Cells." International Journal of Hydrogen Energy 41(47): 22598-22604.

Chen, B., X. He, F. Yin, H. Wang, D.-J. Liu, R. Shi, J. Chen, and H. Yin (2017). “MO-Co@N-Doped Carbon (M = Zn or Co): Vital Roles of Inactive Zn and Highly Efficient Activity toward Oxygen Reduction/Evolution Reactions for Rechargeable Zn-Air Battery." Advanced Functional Materials 27(37): 1700795.

Liu, J., M. Jiao, L. Lu, H.M. Barkholtz, Y. Li, Y. Wang, L. Jiang, Z. Wu, D.-J. Liu, L. Zhuang, C. Ma, J. Zeng, B. Zhang, D. Su, P. Song, W. Xing, W. Xu, Z. Jiang, and G. Sun (2017). "High-Performance Platinum Single-Atom Electrocatalyst for Oxygen Reduction Reaction." Nature Communications 8: 15938.

Wang, H., F.-X. Yin, B.-H. Chen, X.-B. He, P.-L. Lv, C.-Y. Ye, and D.-J. Liu (2017). “ZIF-67 Incorporated with Carbon Derived from Pomelo Peels: A Highly Efficient Bifunctional Catalyst for Oxygen Reduction/Evolution Reactions." Applied Catalysis B-Environmental 205: 55-67.

\section{PRESENTATIONS}

Liu, D.-J. (2017). "New Developments in Materials and Characterization Tools for Fuel Cells and Batteries." Shanghai Jiao Tong University, Minhang Qu, China, November 14, 2016.

Liu, D.-J. (2017). "New Materials and Characterization Tools for Next-Generation Fuel Cells and Batteries." University of Alabama, Tuscaloosa, AL, October 28, 2016.

Liu, D.-J. (2017). "Rational Design and Synthesis of ORR Catalysts for Proton Exchange Membrane Fuel Cell." Wuhan University of Technology, Hubei Sheng, China, August 28, 2017.

\section{4-054}

REFEREED PUBLICATIONS

Jiang, W., G. Chen, K. Liu, J. Zang, S.G.E. te Velthuis, and A. Hoffmann (2017). "Skyrmions in Magnetic Multilayers." Physics Reports 704: 1-49.

Sklenar, J., W. Zhang, M.B. Jungfleisch, W. Jiang, H. Saglam, J.E. Pearson, J.B. Ketterson, and A. Hoffmann (2017). "Perspective on Interfacial Spin-Orbit Torques." Journal of Applied Physics 120(18).

\section{PRESENTATIONS}

Hoffmann, A. (2017). "Manipulating Room Temperature Magnetic Skyrmions." Gordon Research Conference Spin Dynamics in Nanostructures, Les Diablerets, Switzerland, July 16-21, 2017. 
Hoffmann, A. (2017). "Manipulating Room Temperature Magnetic Skyrmions." 2016 Materials Research Society Fall Meeting and Exhibit, Boston, MA, November 27-December 2, 2016.

Hoffmann, A. (2017). "Manipulating Room Temperature Magnetic Skyrmions.” 2017 Ohio State University Materials Week, Columbus, OH, May 9-12, 2017.

Hoffmann, A. (2017). "Manipulating Room Temperature Magnetic Skyrmions.” 2017 Spring Meeting of the German Physical Society, Dresden, Germany, March 19-24, 2017.

Hoffmann, A. (2017). "Manipulating Room Temperature Magnetic Skyrmions.” 2017 Symposium Bio-Convergence Spin System, Daegu, South Korea, February 9-11, 2017.

Hoffmann, A. (2017). "New Opportunities for Spintronics: Magnetic Skyrmions." Fudan University, Shanghai Shi, China, October 18, 2016.

Hoffmann, A. (2017). "New Opportunities for Spintronics: Magnetic Skyrmions." Illinois Institute of Technology, Chicago, IL, April 5, 2017.

Hoffmann, A. (2017). “New Opportunities for Spintronics: Magnetic Skyrmions.” Michigan State University, Lansing, MI, April 17, 2017.

Hoffmann, A. (2017). "New Opportunities for Spintronics: Magnetic Skyrmions.” Nanjing University, Jiangsu Sheng, China, October 17, 2016.

Hoffmann, A. (2017). "New Opportunities for Spintronics: Magnetic Skyrmions.” Texas A\&M University, College Station, TX, January 26, 2017.

Hoffmann, A. (2017). "Opportunities at the Frontiers of Spintronics.” Technische Universität München, Munich, Germany, October 10, 2016.

Hoffmann, A. (2017). "Room Temperature Generation and Manipulation of Magnetic Skyrmion." Carnegie Mellon University, Pittsburgh, PA, November 14, 2016.

Hoffmann, A. (2017). "Room Temperature Generation and Manipulation of Magnetic Skyrmions." Universidade Federal do Rio Grande do Norte, Natal, Brazil, May 3, 2017.

Hoffmann, A. (2017). "Room Temperature Generation and Manipulation of Magnetic Skyrmion." University of Florida, Gainesville, FL, November 17, 2016.

Hoffmann, A., W. Jiang, X. Zhang, Y. Zhou, G. Yu, K.L. Wang, X. Wang, X. Cheng, W. Zhang, M.B. Jungfleisch, J.E. Pearson, O. Heinonen, and S.G.E. te Velthuis (2017). "Skyrmion Hall Effect." 2017 American Physical Society March Meeting, New Orleans, LA, March 13-17, 2017.

Hoffmann, A. (2017). “Topological Quasiparticles: Magnetic Skyrmions.” 78th Japan Society of Applied Physics Autumn Meeting, Fukuoka, Japan, September 5-8, 2017.

Hoffmann, A. (2017). “Topological Quasiparticles: Magnetic Skyrmions.” Frontiers in Emergent Quantum Phenomena, New York, NY, June 28-30, 2017.

Jiang, W., X. Zhang, G. Yu, W. Zhang, X. Wang, M.B. Jungfleisch, J.E. Pearson, X.M. Cheng, O. Heinonen, K.L. Wang, Y. Zhou, A. Hoffmann, and S.G.E. te Velthuis (2017). "Experimental Observation of the Skyrmion Hall Effect." 61st Annual Conference on Magnetism and Magnetic Materials, New Orleans, LA, October 31-November 4, 2016.

Jiang, W., P. Upadhyaya, X. Zhang, W. Zhang, G. Yu, X. Wang, M.B. Jungfleisch, H. Somaily, F.Y. Fradin, J.E. Pearson, O. Heinonen, S.G.E. te Velthuis, and A. Hoffmann (2017). "Manipulating Room Temperature Magnetic Skyrmions.” 15th International Union of Materials Research Societies - International Conference on Advanced Materials, Kyoto, Japan, August 28-September 1, 2017. 
Jiang, W., P. Upadhyaya, X. Zhang, W. Zhang, G. Yu, X. Wang, M.B. Jungfleisch, H. Somaily, F.Y. Fradin, J.E. Pearson, O. Heinonen, S.G.E. te Velthuis, and A. Hoffmann (2017). "Manipulating Room Temperature Magnetic Skyrmions." International Conference on Nanomagnetism and Spintronics (Sol-SkyMag), San Sebastian, Spain, June 19-23, 2017.

Jiang, W., P. Upadhyaya, X. Zhang, W. Zhang, G. Yu, X. Wang, M.B. Jungfleisch, H. Somaily, F.Y. Fradin, J.E. Pearson, O. Heinonen, S.G.E. te Velthuis, and A. Hoffmann (2017). "Manipulating Room Temperature Magnetic Skyrmions." Trends in Nanoscience 2017, Kloster Irsee, Germany, March 27-30, 2017.

Jiang, W., X. Zhang, G. Yu, W. Zhang, M.B. Jungfleisch, J.E. Pearson, O. Heinonen, K.L. Wang, Y. Zhou, S.G.E. te Velthuis, and A. Hoffmann (2017). "Skyrmion Hall Effect." 63rd American Vacuum Society International Symposium and Exhibition, Nashville, TN, November 6-11, 2016.

Jiang, W., P. Upadhyaya, X. Zhang, X. Wang, G. Yu, W. Zhang, M.B. Jungfleisch, F.Y. Fradin, J.E. Pearson, X. Cheng, O. Heinonen, S.G.E. te Velthuis, and A. Hoffmann (2017). "Skyrmion Topological Spintronics." 2017 Skyrmionics: Materials, Phenomena, and Applications, Santa Fe, NM, August 8-10, 2017.

Jiang, W., P. Upadhyaya, X. Zhang, W. Zhang, G. Yu, X. Wang, M.B. Jungfleisch, H. Somaily, F.Y. Fradin, J.E. Pearson, Y. Tserkovnyak, K.L. Wang, Y. Zhou, X.M. Cheng, O. Heinonen, A. Hoffmann, and S.G.E. te Velthuis (2017). "Stabilizing and Controlling Room Temperature Magnetic Skyrmions." 2017 SKYMAG Challenges for Magnetic Skyrmions and Opportunities for Skyrmionic Devices Workshop, Paris, France, May 2-5, 2017.

Phatak, C., V. Brajuskovic, S. Zhang, A. Petford-Long, W. Jiang, S.G.E. te Velthuis, A. Hoffmann, O. Heinonen, and M. De Graef (2017). "Domain Behavior in Functional Materials Studied Using in situ Lorentz Transmission Electron Microscopy.” 2017 Electron Holography Workshop, Saitama, Japan, February 15-17, 2017.

te Velthuis, S.G.E., W. Jiang, P. Upadhyaya, X. Zhang, W. Zhang, G. Yu, X. Wang, M.B. Jungfleisch, H. Somaily, F.Y. Fradin, J.E. Pearson, Y. Tserkovnyak, K.L. Wang, Y. Zhou, X.M. Cheng, O. Heinonen, and A. Hoffmann (2017). "Current-Induced Creation and Manipulation of Room Temperature Magnetic Skyrmions." 2017 Spin Phenomena Interdisciplinary Center Topology Matters Workshop, Mainz, Germany, July 28, 2017.

Wang, X., W. Jiang, S. Zhang, Q. Wang, K. Buchanan, J.E. Pearson, C. Phatak, A. Petford-Long, A. Hoffmann, S.G.E. te Velthuis, and X.M. Cheng (2017). "Tuning Magnetic Skyrmions in Multilayers with Perpendicular Magnetic Anisotropy." 61st Annual Conference on Magnetism and Magnetic Materials, New Orleans, LA, October 31November 4, 2016.

\section{4-081}

\section{REFEREED PUBLICATIONS}

Hong, H., J. Kim, X.Y. Fang, S. Hong, and T.C. Chiang (2017). "Interfacial Stability of Ultrathin Films of Magnetite $\mathrm{Fe}_{3} \mathrm{O}_{4}(111)$ on $\mathrm{Al}_{2} \mathrm{O}_{3}(001)$ Grown by Ozone-Assisted Molecular-beam Epitaxy." Applied Physics Letters 110(2).

Hou, B., S. H. Kim, T.H. Kim, C. Park, C.B. Bahn, J. Kim, and S. Hong (2017). "Orientation Dependent Hydration Structures at Yttria-Stabilized Cubic Zirconia Surfaces." Journal of Physical Chemistry 120(51): 29089-29097.

Kim, J., B. Hou, C. Park, C.B. Bahn, J. Hoffman, J. Black, A. Bhattacharya, N. Balke, H. Hong, J.H. Kim, and S. Hong (2017). "Effect of Defects on Reaction of NiO Surface with Pb-Contained Solution." Scientific Reports 7.

\section{NON-REFEREED PUBLICATIONS}

Hou, B., C. Park, S. Kim, T. Kim, J.H. Kim, J. Kim, S. Hong, and C.B. Bahn (2017). "A Study of High-Temperature Stability of Zirconia Surface in Pressurized Water with High-Resolution X-ray Reflectivity." 2016 Pacific Rim Meeting on Electrochemical and Solid-State Science - Joint Meeting with 230th Electrochemical Society, 2016 Fall Electrochemical Society of Japan, 2016 Fall Korea Electrochemical Society, Honolulu, HI, October 2-7, 2016.

Hou, B., C. Park, S. Kim, T. Kim, J. H. Kim, J. Kim, S. Hong, and C.B. Bahn (2017). "Hydration Structures and Water Chemistry at Zirconia-water Interface." 2016 Pacific Rim Meeting on Electrochemical and Solid-State Science Joint Meeting with 230th Electrochemical Society, 2016 Fall Electrochemical Society of Japan, 2016 Fall Korea Electrochemical Society, Honolulu, HI, October 2-7, 2016. 
Park, C., B. Hou, S. Kim, T. Kim, J.H. Kim, C.B. Bahn, J. Kim, and S. Hong (2017). "Observation of Interfacial Hydration and Ion Adsorption at Zirconia Water Interface with High-Resolution X-ray Reflectivity." 20th Nuclear Plant Chemistry (NPC) International Conference, Brighton, United Kingdom, October 2-7, 2016.

\section{4-084}

\section{PRESENTATIONS}

Feng, Y. (2017). "Dust in ACME and Sensitivity to Model Resolution." ACME 2017 All-Hands Meeting, Potomac, MD, June 5, 2017.

Feng, Y., B. Drewniak, and A. Ito (2017). "Spatial and Seasonal Variability in Soluble Iron Deposition and Contribution to Sea-Surface Iron Distributions." 2016 American Geophysical Union Fall Meeting, San Francisco, CA, December 12-16, 2016.

Ingall, E.D., A.F. Longo, Y. Feng, and B. Lai (2017). "Influence of Atmospheric Processes on the Solubility and Composition of Iron in Saharan Dust." 2016 American Geophysical Union Fall Meeting, San Francisco, CA, December 12-16, 2016.

Ito, A., and Y. Feng (2017). "Evaluation of Labile Iron Processing in Atmospheric Models." 2017 Goldschmidt Conference, Paris, France, August 13-18, 2017.

\section{4-095}

\section{REFEREED PUBLICATION}

Fang, L., J. Im, W. DeGottardi, Y. Jia, A. Glatz, K.A. Matveev, W.K. Kwok, G.W. Crabtree, and M.G. Kanatzidis (2017). "Large Spin-Orbit Coupling and Helical Spin Textures in 2D Heterostructure $\left[\mathrm{Pb}_{2} \mathrm{BiS}_{3}\right]\left[\mathrm{AuTe}_{2}\right]$." Scientific Reports 6: 35313.

\section{4-120 \\ REFEREED PUBLICATIONS}

Krishnamurthy, D., C. Uckun, Z. Zhou, P. Thimmapuram, and A. Botterud (2017). “Energy Storage Arbitrage Under Day-Ahead and Real-Time Price Uncertainty." IEEE Transactions on Power Systems PP(99): 1-1.

Sakti, A., K.G. Gallagher, N. Sepulveda, C. Uckun, C. Vergara, F.J. de Sisternes, D.W. Dees, and A. Botterud (2017). "Enhanced Representations of Lithium-Ion Batteries in Power Systems Models and Their Effect on the Valuation of Energy Arbitrage Applications." Journal of Power Sources 342: 279-291.

Wankmueller, F., P. Thimmapuram, K.G. Gallagher, and A. Botterud (2017). "Impact of Battery Degradation on Energy Arbitrage Revenue of Grid-Level Energy Storage." Journal of Energy Storage 10: 56-66.

\section{4-127}

\section{PRESENTATION}

Kim, J. (2017). "Quartz-Based Flat-Crystal Resonant Inelastic X-ray Scattering Spectrometer (RIXS) with Sub 10-meV Energy Resolution." 10th International Conference on Inelastic X-ray Scattering, Hamburg, Germany, August 28September 1, 2017.

\section{4-128}

\section{REFEREED PUBLICATIONS}

Luo, Y., A. Benali, L. Shulenburger, J.T. Krogel, O. Heinonen, and P.R.C. Kent (2017). "Phase Stability of $\mathrm{TiO}_{2}$ Polymorphs from Diffusion Quantum Monte Carlo." New Journal of Physics 18(11): 113049.

Mookherjee, M., D. Mainprice, K. Maheshwari, O. Heinonen, and A. Hariharan (2017). "High-Pressure Elastic Softening in Albite $\left(\mathrm{NaAlSi}_{3} \mathrm{O}_{8}\right)$." Scientific Reports 6: 34815. 
Zhong, X., I. Rungger, P. Zapol and O. Heinonen (2017). "Ab initio Modeling of Transport and Thermodynamic Stability for Hafnia Memrisitve Devices.” Journal of Computational Electronics 16(4): 1066-1076.

Zhong, X., I. Rungger, P. Zapol, and O. Heinonen (2017). “Oxygen-Modulated Quantum Conductance for Ultra-thin $\mathrm{HfO}_{2}$-based Memristive Switching Devices.” Physical Review Letters 94: 165160.

\section{4-134}

\section{REFEREED PUBLICATION}

Cha, W., A. Ulvestad, M. Allain, V. Chamard, R. Harder, S.J. Leake, J. Maser, P.H. Fuoss, and S.O. Hruszkewycz (2017). "Three-Dimensional Variable-Wavelength X-Bragg Coherent Diffraction Imaging." Physical Review Letters 117(22): 225501.

\section{4-137}

\section{REFEREED PUBLICATIONS}

Kesgin, I., M. Kasa, Y. Ivanyushenkov, and U. Welp (2017). "High-Temperature Superconducting Undulator Magnets." Superconductor Science \& Technology 30(4): 04LT01.

Kesgin, I., Q. Hasse, Y. Ivanyushenkov, and U. Welp (2017). "Performance of 2G-HTS REBCO Undulator Coils Impregnated Epoxies Mixed With Different Fillers." IEEE Transactions on Applied Superconductivity 27(4).

\section{PRESENTATIONS}

Kesgin, I., M. Kasa, Y. Ivanyushenkov, and U. Welp (2017). "High-Temperature Superconducting Undulator Magnets." 2017 Low-Temperature/High Field Superconductor Workshop, Santa Fe, NM, February 27-March 1, 2017.

Kesgin, I., Q. Hasse, Y. Ivanyushenkov, and U. Welp (2017). "Performance of Vacuum Epoxy Impregnated 2G-HTS Undulator Magnets." 2017 Cryogenic Engineering Conference and International Cryogenic Materials Conference, Madison, WI, July 9-13, 2017.

\section{4-139}

\section{PRESENTATION}

Keceli, M., A. Wagner, and P. Zapol (2017). "Massively Parallel Spectrum Slicing Eigensolver for ab initio Calculations." 2017 American Physical Society March Meeting, New Orleans, LA, April 13-17, 2017.

\section{4-151}

\section{REFEREED PUBLICATIONS}

Ju, G., M.J. Highland, A. Yanguas-Gil, C. Thompson, J.A. Eastman, H. Zhou, S.M. Brennan, G.B. Stephenson, and P.H. Fuoss (2017). "An Instrument for in situ Coherent X-ray Studies of Metal-organic Vapor Phase Epitaxy of III-nitrides." Review of Scientific Instruments 88(3): 035113.

Liu, Z., R.-Z. Wang, and P. Zapol (2017). "An Atomistic Mechanism Study of GaN Step-Flow Growth on Vicinal m-plane Orientations." Physical Chemistry Chemical Physics 18(42): 29239-29248.

\section{NON-REFEREED PUBLICATION}

Ju, G., M.J. Highland, R. Sichel-Tissot, J.A. Eastman, P.M. Baldo, P. Zapol, C. Thompson, and P.H. Fuoss (2017). "In situ X-ray Studies of AIN Nucleation on Sapphire (0010) during Reactive Sputtering." 2015 Materials Research Society Fall Meeting and Exhibit, Boston, MA, November 29-December 4, 2016.

\section{4-160}

\section{NON-REFEREED PUBLICATION}

Beckman, P., R. Sankaran, C. Catlett, N. Ferrier, R. Jacob, and M. Papka (2017). "Waggle: An Open Sensor Platform for Edge Computing.” 2016 IEEE Sensors, Orlando, FL, October 30-November 2, 2016. 


\section{4-161}

\section{REFEREED PUBLICATIONS}

Kinaci, A., B. Narayanan, F.G. Sen, M.J. Davis, S.K. Gray, S.K.R.S. Sankaranarayanan, and M.K.Y. Chan (2017). "Unraveling the Planar-Globular Transition in Gold Nanoclusters through Evolutionary Search.” Scientific Reports 6: 34974.

Narayanan, B., H. Chan, A. Kinaci, F.G. Sen, S.K. Gray, M.K.Y. Chan, and S.K.R.S. Sankaranarayanan (2017). "Machine Learnt Bond Order Potential to Model Metal-Organic (Co-C) Heterostructures." Nanoscale 9(46): 18229-18239.

\section{4-166}

\section{REFEREED PUBLICATION}

Zhao, X., J. Li, X. Jiang, D. Karpeev, O. Heinonen, B. Smith, J.P. Hernandez-Ortiz, and J.J. de Pablo (2017). "Parallel O(N) Stokes' Solver Towards Scalable Brownian Dynamics of Hydrodynamically Interacting Objects in General Geometries." Journal of Chemical Physics 146(24): 244114.

\section{4-167}

\section{NON-REFEREED PUBLICATION}

Catlett, C.E., P.H. Beckman, R. Sankaran, and K.K. Galvin (2017). "Array of Things: A Scientific Research Instrument in the Public Way." 2nd International Workshop on Science of Smart City Operations and Platforms Engineering (SCOPE) in partnership with Global City Teams Challenge (GCTC), Pittsburgh, PA, April 21, 2017.

\section{4-169}

\section{PRESENTATION}

Xie, Y., K. Gao, and J. Hryn (2017). “RF Powered Transformable Sensor on a Flexible Substrate.” 2017 Flexible Electronics - Accelerating to Manufacturing (2017FLEX), Monterey, CA, June 19-23, 2017.

\section{4-175}

\section{REFEREED PUBLICATIONS}

Broecker, J., V. Klingel, W. Ou, A.R. Balo, D.J. Kissick, C.M. Ogata, A. Kuo, and O.P. Ernst (2017). "A Versatile System for High-throughput in situ X-ray Screening and Data Collection of Soluble and Membrane-Protein Crystals." Crystal Growth \& Design 16(11): 6318-6326.

Scarborough, N.M., G.M.D.P. Godaliyadda, D.H. Ye, D.J. Kissick, S. Zhang, J.A. Newman, M.J. Sheedlo, A.U. Chowdhury, R.F. Fischetti, and C. Das (2017). "Dynamic X-ray Diffraction Sampling for Protein Crystal Positioning." Journal of Synchrotron Radiation 24: 188-195.

\section{NON-REFEREED PUBLICATION}

Becker, M., D.J. Kissick, and C.M. Ogata (2017). "Locating and Visualizing Crystals for X-ray Diffraction Experiments." Protein Crystallography. Methods in Molecular Biology. A. Wlodawer, Z. Dauter, and M. Jaskolski (eds.), Humana Press, New York, NY. 1607: 143-164.

\section{4-177}

\section{REFEREED PUBLICATION}

Tentner, A., P. Vegendla, A. Tomboulides, A. Obabko, E. Merzari, and D. Shaver (2017). “Modeling of Two-phase Boiling Flow and Critical Heat Flux with the NEK-2P CFD Code." Proceedings of the 25th International Conference on Nuclear Engineering (ICONE-25), Shanghai, China, July 2-6, 2017, American Society of Mechanical Engineers 8: Computational Fluid Dynamics (CFD) and Coupled Codes; Nuclear Education, Public Acceptance and Related Issues (Paper No. ICONE25-66529): pp. V008T009A016, 9 pages. 


\section{4-181}

\section{REFEREED PUBLICATIONS}

Cappello, F., R. Gupta, S. Di, E. Constantinescu, T. Peterka, and S.M. Wild (2017). "Understanding and Improving the Trust in Results of Numerical Simulations and Scientific Data Analytics." Euro-Par 2017: Parallel Processing Workshops 10659: 545-556.

Di, S., and F. Cappello (2017). “Optimization of Error-Bounded Lossy Compression for Hard-to-Compress HPC Data.” IEEE Transactions on Parallel and Distributed Systems 29(1): 129-143.

Guhur, P.-L., E. Constantinescu, D. Ghosh, T. Peterka, and F. Cappello (2017). “Detection of Silent Data Corruption in Adaptive Numerical Integration Solvers." Proceedings of the 2017 IEEE International Conference on Cluster Computing: CLUSTER 2017, Honolulu, HI, September 5-8, 2017: 592-602.

Subasi, O., S. Di, P. Balaprakash, O. Unsal, J. Labarta, A. Cristal, S. Krishnamoorthy, and F. Cappello (2017). "MACORD: Online Adaptive Machine Learning Framework for Silent Error Detection." Proceedings of the 2017 IEEE International Conference on Cluster Computing: CLUSTER 2017, Honolulu, HI, September 5, 2017.

Tao, D., S. Di, Z. Chen and F. Cappello (2017). "Exploration of Pattern-Matching Techniques for Lossy Compression on Cosmology Simulation Data Sets.” ISC High Performance 2017: High Performance Computing 10524: 43-54.

Tao, D., S. Di, Z. Chen, and F. Cappello (2017). “Significantly Improving Lossy Compression for Scientific Data Sets Based on Multidimensional Prediction and Error-Controlled Quantization." 2017 IEEE International Parallel and Distributed Processing Symposium: DOI: 10.1109/IPDPS.2017.1115.

\section{PRESENTATIONS}

Cappello, F. (2017). “Can We Aggressively Reduce Scientific Data without Losing Science?" 12th International Conference on Parallel Processing and Applied Mathematics (PPMA 2017), Lublin, Poland, September 10-13, 2017.

Cappello, F. (2017). "Checkpoint/Restart: Why You Should Delegate It to a Specialized Library." Society for Industrial and Applied Mathematics (SIAM) Annual Meeting 2017, Pittsburgh, PA, July 10-14, 2017.

Cappello, F. (2017). “Compression of Scientific Data: From Stone Age to Renaissance.” Workshop on Clusters, Clouds, and Data for Scientific Computing (CCDSC) 2016, Lyon, France, October 3-6, 2016.

Cappello, F. (2017). "From General Purpose-Exact Computing to Tailored-Lossy Computing (Scientific Computing)." Greater Chicago Area Systems Research Workshop (GCASR 2017), Chicago, IL, April 24, 2017.

Cappello, F. (2017). "Scientific Computing and Data Analytics: How to Deal with the Flood of Data." Distinguished Lecture, Northeastern University, Boston, MA, November 8, 2016.

Cappello, F., and P. Linstrom (2017). “Compression of Scientific Data.” ISC High Performance Conference 2017, Frankfurt, Germany, June 18-22, 2017.

Di, S., D. Tao, H. Guo, Z. Chen, and F. Cappello (2017). “Towards Efficient Error-Controlled Lossy Compression for Scientific Data.” 2017 Greater Chicago Area Systems Research Workshop, Chicago, IL, April 24, 2017.

\section{4-182}

\section{NON-REFEREED PUBLICATION}

Foster, I., B. Blaiszik, K. Chard, and R. Chard (2017). “Software Defined Cyberinfrastructure." 37th IEEE International Conference on Distributed Computing Systems, Atlanta, GA, June 5-8, 2017. 


\section{4-184}

\section{REFEREED PUBLICATION}

Ivanyushenkov, Y., K. Harkay, M. Borland, R. Dejus, J. Dooling, C. Doose, L. Emery, J. Fuerst, J. Gagliano, Q. Hasse, M. Kasa, P. Kenesei, V. Sajaev, K. Schroeder, N. Sereno, S. Shastri, Y. Shiroyanagi, D. Skiadopoulos, M. Smith, X. Sun, E. Trakhtenberg, A. Xiao, A. Zholents, and E. Gluskin (2017). "Development and Operating Experience of a Full-Scale Superconducting Undulator SCU1 at the Advanced Photon Source." Physical Review Accelerators and Beams 20(10): 100701.

\section{4-185}

\section{REFEREED PUBLICATIONS}

Gutierrez, A., S. Kim, T.T. Fister, and C.S. Johnson (2017). "Microwave-Assisted Synthesis of $\mathrm{NaCoPO}_{4}$ Red-Phase and Initial Characterization as a High Voltage Cathode for Sodium-Ion Batteries." ACS Applied Materials \& Interfaces 9(5): 4391-4396.

Manikandan, P., H. Kim, S. Heo, E. Lee, and Y. Kim (2017). "Insights on Dual Electrode Characteristics of Layered $\mathrm{Na}_{0.5} \mathrm{Ni}_{0.25} \mathrm{Mn}_{0.75} \mathrm{O}_{2}$ Material for Sodium-Ion Batteries." ACS Applied Materials \& Interfaces 9(12): 10618-10625.

\section{PRESENTATION}

Johnson, C.S. (2017). "SnO Carbon Composite as Superior Anode in Sodium-Ion Batteries." 2016 PRiME Conference; 230th Electrochemical Society Meeting, Honolulu, HI, October 2-7, 2016.

\section{4-187}

\section{REFEREED PUBLICATION}

Lv, Y., C. Zhang, A. He, S.-J. Yang, G.-P. Wu, S.B. Darling, and Z.-K. Xu (2017). "Photocatalytic Nanofiltration Membranes with Self-Cleaning Property for Wastewater Treatment." Advanced Functional Materials 27(27).

\section{4-188}

\section{REFEREED PUBLICATIONS}

Gerber, C., R. Vaikmae, W. Aeschbach, A. Babre, W. Jiang, M. Leuenberger, Z.-T. Lu, R. Mokrik, P. Müller, V. Raidla, T. Saks, H.N. Waber, T. Weissbach, J.C. Zappala, and R. Purtschert (2017). "Using ${ }^{81} \mathrm{Kr}$ and Noble Gases to Characterize and Date Groundwater and Brines in the Baltic Artesian Basin on the One-Million-Year Timescale." Geochimica et Cosmochimica Acta 205: 187-210.

Yokochi, R., R. Bernier, R. Purtschert, J.C. Zappala, Y. Yechieli, E. Adar, W. Jiang, Z.-T. Lu, P. Mueller, G. Olack, and R. Ram (2017). "Field Degassing as a New Sampling Method for ${ }^{14} \mathrm{C}$ Analyses in Old Groundwater." Radiocarbon 60(1): 349-366.

Zappala, J.C., K. Bailey, P. Mueller, T.P. O’Connor, and R. Purtschert (2017). "Rapid Processing of ${ }^{85} \mathrm{Kr} / \mathrm{Kr}$ Ratios Using Atom Trap Trace Analysis.” Water Resources Research 53(3): 2553-2558.

Zappala, J.C., K. Bailey, W. Jiang, B. Micklich, P. Mueller, T.P. O'Connor, and R. Purtschert (2017). "Setting a Limit on Anthropogenic Sources of Atmospheric ${ }^{81} \mathrm{Kr}$ Through Atom Trap Trace Analysis." Chemical Geology 453: 66-71

\section{PRESENTATION}

Zappala, J.C., K. Bailey, P. Mueller, T.P. O’Connor, and R. Purtschert (2017). "Rapid Processing of ${ }^{85} \mathrm{Kr} / \mathrm{Kr}$ Ratios Using Atom Trap Trace Analysis.” 2017 Goldschmidt, Paris, France, August 13-18, 2017.

\section{4-189}

\section{REFEREED PUBLICATION}

Wardrip, N., M. Dsouza, M. Urgun-Demirtas, S.W. Snyder, J.A. Gilbert, and C. Arnusch (2017). "Printing-Assisted Surface Modifications of Patterned Ultrafiltration Membranes." ACS Applied Materials \& Interfaces 8(44): 30271-30280. 


\section{4-194}

\section{NON-REFEREED PUBLICATION}

Sydelko, P. (2017). "A Systemic Integration Approach to Designing Interagency Responses to Wicked Problems."

61st International Society for the System Sciences, Vienna, Austria, July 7-14, 2017.

\section{PRESENTATION}

Martinez-Moyano, I., P.J. Sydelko, M. North, and B. Friedman (2017). "Goldilocks and the Wicked Problem."

2016 Conference on Complex Systems, Amsterdam, Netherlands, September 19-22, 2016.

\section{5-132}

\section{PRESENTATIONS}

Kahvecioglu Feridun, O., Y. Shin, and G. Krumdick (2017). "Customized Li-Ion Cathode Materials: Particle Size Reduction and Surface Coating for Electrophoretic Deposition Process.” 2017 Argonne Battery Industry Day, Lemont, IL,

June 14, 2017.

Shin, Y., O. Kahvecioglu Feridun, and G.K. Krumdick (2017). "Scalable Advanced Chemical Reactor, Taylor Vortex Reactor, for Producing Li-ion Cathode Precursors, Collaboration with Laminar." 2017 Argonne Battery Industry Day, Lemont, IL, June 14, 2017.

\section{5-136}

\section{REFEREED PUBLICATIONS}

Alderman, O.L.G., C.J. Benmore, J.K.R. Weber, L.B. Skinner, A.J. Tamalonis, S. Sendelbach, A. Hebden, and M.A. Williamson (2017). "The Structure of Liquid $\cup_{2-x}$ in Reducing Gas Atmospheres." Applied Physics Letters 110(8).

Guthrie, M., C.J. Benmore, L.B. Skinner, O.L.G. Alderman, J.K.R. Weber, J.B. Parise, and M. Williamson (2017). “Thermal Expansion in $\mathrm{UO}_{2}$ Determined By High-Energy X-ray Diffraction." Journal of Nuclear Materials 479: 19-22.

\section{5-141}

\section{REFEREED PUBLICATION}

Shi, X., W. Xu, A. Yakovenko, G. Halder, Z. Liu, C. Kurtz, L.W. Ribaud, K.A. Beyer, K. Chapman, and P. Chaupas (2017).

"Bent Bragg-Laue Monochromator for High-Energy X-rays." Journal of Applied Crystallography 50(4): 1158-1164.

\section{5-145}

\section{REFEREED PUBLICATIONS}

Chen, Y., C. Xu, X. Zhang, W.-Y. Chen, J.-S. Park, J. Almer, M. Li, Z. Li, Y. Yang, A. Rao, B. Alexandreanu, and K. Natesan (2017). "Microstructure and Deformation Behavior of Thermally Aged Cast Austenitic Stainless Steels." Proceedings of the 18th International Conference on Environmental Degradation of Materials in Nuclear Power Systems - Water Reactors (EDM 2017). The Minerals, Metals \& Materials Series, Portland, OR, August 13-17, 2017, Springer, Cham 2: 625-641.

Chen, Y., W.-Y. Chen, B. Alexandreanu, K. Natesan, and A. Rao (2017). "Crack Growth Rate and Fracture Toughness of CF-3 Cast Stainless Steels at 3 DPA." Proceedings of the 18th International Conference on Environmental Degradation of Materials in Nuclear Power Systems - Water Reactors (EDM 2017). The Minerals, Metals \& Materials Series, Portland, OR, August 13-17, 2017, Springer, Cham 2: 673-687.

\section{NON-REFEREED PUBLICATION}

Chen, Y., W.-Y. Chen, X. Zhang, B. Alexandreanu, K. Natesan, C. Xu, Y. Yang, and A. Rao (2017). "Fracture Toughness and Deformation Behavior of Cast Austenitic Stainless Steels After Thermal Aging." 2017 ASME Pressure Vessels \& Piping Conference, Waikoloa Village, HI, July 16-20, 2017. 


\section{5-154}

\section{PRESENTATIONS}

Guest, J.R. (2017). "Atomic-Scale Imaging of Optically-Active Nanoscale Systems." North Carolina State University, Raleigh, NC, February 6, 2017.

Guest, J.R. (2017). “Atomic-Scale Imaging of Optically-Active Nanoscale Systems.” Paul Scherrer Institut, Villigen, Switzerland, September 15, 2017.

Guest, J.R. (2017). “Atomic-Scale Imaging of Optically-Active Nanoscale Systems." University of Tennessee, Knoxville, TN, May 26, 2017.

Guest, J.R. (2017). "Atomic-Scale Imaging of Optically-Active Nanoscale Systems." University of Texas, Austin, TX, September 28, 2017.

Guest, J.R., R. Zhang, G.R. Clark, P. Darancet, and X. Xu (2017). "Characterizing and Manipulating the Charge State of Defects in WSe2." 2017 Operating Quantum States in Atoms and Molecules at Surfaces (QMol), Ascona, Switzerland, September 10-14, 2017.

Li, L., R. Zhang, Y.A. Wu, Y. Liu, I. McNulty, T. Rajh, J.R. Guest, and M.K. Chan (2017). "Reduction of $\mathrm{CO}_{2}$ on $\mathrm{Cu}_{2} \mathrm{O}_{(110)}$ Photocatalyst: A Combined DFT and STM Study." 2016 Materials Research Society Fall Meeting and Exhibit, Boston, MA, November 27-December 2, 2016.

Li, L., R. Zhang, Y. Wu, Y. Liu, I. McNulty, J. Vinson, E.L. Shirley, J.R. Guest, and M.K.Y. Chan (2017). "CO 2 Reduction on $\mathrm{Cu}_{2} \mathrm{O}_{(110)}$ Surface: A Combined Theoretical and Experimental Study." 26th International Materials Research Congress, Cancun, Mexico, August 20-25, 2017.

Liu, Y., Y. A. Wu, T. Rajh, B. Smith, X.-M. Lin, L. Li, R. Zhang, M.K. Chan, J.R. Guest, I. McNulty, Z. Cai, and T. Li (2017). "Observation of Materials Transformation by a Nano-Reactor in TEM." 2017 International in situ TEM Workshop, Hangzhou, Zhejiang, China, May 25-26, 2017.

McNulty, I. (2017). “Unveiling New Materials Science with Nano-focused Coherent X-rays.” XVI Brazilian Materials Research Society Meeting, Gramado, Brazil, September 10-14, 2017.

Zhang, R., L. Li, L. Frazer, M.K. Chan, and J. Guest (2017). "STM Study of $\mathrm{Cu}_{2} \mathrm{O}$ Surface Facets." 2017 American Physical Society March Meeting, New Orleans, LA, March 13-17, 2017.

\section{5-164}

\section{NON-REFEREED PUBLICATION}

Kearney, S.P., D. Shu, and T.S. Toellner (2016). "The Design of a Precision Mechanical Assembly for a Hard X-ray Polarizer." 9th Mechanical Engineering Design of Synchrotron Radiation Equipment and Instrumentation, Barcelona, Spain, September 11-16, 2016.

\section{PRESENTATION}

Toellner, T.S. (2017). "Fast Shutters for Nuclear Resonant Scattering." PETRA-IV Project Workshop: Timing Experiments at PETRA-IV, Hamburg, Germany, March 27-29, 2017.

\section{5-177}

\section{REFEREED PUBLICATION}

Clifford, M. (2017). “The Resilient Infrastructure Initiative.” Natural Hazards Observer XL(7): 28-29.

Jalving, J., S. Abhyankar, K. Kim, M. Hereld, and V.M. Zavala (2017). "A Graph-Based Computational Framework for Simulation and Optimization of Coupled Infrastructure Networks." IET Generation, Transmission \& Distribution 11(12): 3163-3176. 
Muehleisen, R.T., D. Verner, K. Kim, and F. Petit (2017). "A New Framework for Identifying and Prioritizing Failure Points in Critical Urban Infrastructure.” The CIP Report, Track D \#2.

Pfeiffer, K.B., C.A. Burdi, and S.O. Schlueter (2017). "Local Supply Chains: The Disaster Management Perspective." International Journal of Safety and Security Engineering 7(3): 399-405.

Portante, E.C., J.A. Kavicky, B.A. Craig, L.E. Talaber, and S.M. Folga (2017). "Modeling Electric Power and Natural Gas Systems Interdependencies: Application to Natural Hazards." Journal of Infrastructure Systems 23(4).

\section{NON-REFEREED PUBLICATION}

Hereld, M., and K. Kim (2017). "Disaster = Infrastructure + Hazard." 2017 Resilience Week, Wilmington, DE,

September 18-22, 2017.

Verner, D., K. Kim, and F. Petit (2017). "Prioritizing High-Consequence Failure Points in Critical Infrastructure Adaptation and Protection Program." 2017 Critical Infrastructure Protection and Resilience Europe, The Hague, Netherlands, May 9-11, 2017.

\section{PRESENTATIONS}

Muehleisen, R.T., D. Verner, K. Kim, and F. Petit (2017). "A New Framework for Identifying and Prioritizing Failure Points in Critical Urban Infrastructure." 2017 Frontiers in Resilience: Developing Innovative Resilience Solutions at the Interface of Science, Economics and Policy, Fairfax, VA, May 9-11, 2017.

Petit, F. (2017). “Critical Infrastructure Resilience and Interdependencies." University of Illinois, Chicago, IL, March 17, 2017.

Petit, F., D. Verner, and J. Kavicky (2017). "Critical Infrastructure Interdependency Assessment.” 2017 Critical Infrastructure Protection and Resilience Europe, The Hague, Netherlands, May 9-11, 2017.

Verner, D., K. Kim, and F. Petit (2017). "Incorporating Prioritization in Critical Infrastructure Security and Resilience Programs." 10th Anniversary Homeland Defense/Security Education Summit, Arlington, VA, March 23-24, 2017.

Verner, D., K. Kim, and F. Petit (2017). "A Prioritization Algorithm to Identify Tipping Points in Critical Infrastructure Systems." 85th Military Operations Research Society Symposium, West Point, NY, June 19-22, 2017.

Verner, D., K. Kim, and F. Petit (2017). "Prioritizing High-Consequence Failure Points in Critical Infrastructure Adaptation and Protection Program." 2017 Critical Infrastructure Protection and Resilience Europe, The Hague, Netherlands, May 9-11, 2017.

Verner, D., M. Clifford, and F. Petit (2017). "Cyber-Attack on the Big City Electric Grid Scenario." 2017 World Economic Forum Workshop for Phase II of Mitigating Risks in the Innovation Economy Project, Zurich, Switzerland, September 15, 2017.

\section{6-143}

\section{REFEREED PUBLICATION}

Ding, J., P.A.R. Ade, A.J. Anderson, J. Avva, Z. Ahmed, K. Arnold, J.E. Austermann, A.N. Bender, B.A. Benson, L.E. Bleem, K. Byrum, J.E. Carlstrom, F.W. Carter, C.L. Chang, D. Czaplewski, R. Divan, R. Gannon, T. Khaire, C.S. Miller, S. Padin, V. Novosad, J. Pearson, C.M. Posada, L. Stan, G. Wang, and V. Yefremenko (2017). "Optimization of Transition Edge Sensor Arrays for Cosmic Microwave Background Observations with the South Pole Telescope." IEEE Transactions on Applied Superconductivity 27(4).

\section{PRESENTATIONS}

Posada, C.M., J. Ding, C.L. Chang, F. Carter, A.N. Bender, V. Yefremenko, V. Novosad, T.S. Khaire, G. Wang, S. Padin, C.S. Miller, R. Divan, D. Czaplewski, V.P. Kutepova, and L. Stan (2017). "Fabrication of the Arrays of Detectors Deployed with the SPT-3G Receiver." 17th International Workshop on Low Temperature Detectors, Fukuoka, Japan, July 17-21, 2017. 
Yefremenko, V., J. Ding, C.L. Chang, F. Carter, A. Bender, C. Posada, V. Novosad, T. Khaire, G. Wang, S. Padin, C.S. Miller, R. Divan, D. Czaplewski, V.P. Kutepova, and L. Stan (2017). "Impact of Electrical Contacts Design and Materials on the Stability of Titanium Superconducting Transition Shape.” 17th International Workshop on Low Temperature Detectors, Fukuoka, Japan, July 17-21, 2017. 


\section{AUTHOR INDEX}

Acik, Muge

Ahluwalia, Rajesh K.

Ahluwalia, Rajesh K.

Aithal, Shashikant

Alp, Esen

Amer, Abdelhalim

Anderson, John

Anliker, Ethan

Antonopoulos, Dionysios

Antonopoulos, Dionysios

Aragon, Ana M.

Armstrong, Whitney

Armstrong, Whitney

Arrington, John

Assoufid, Lahsen

Assoufid, Lahsen

Auld, Joshua

Auld, Joshua

Avila Coronado, Melina

Awschalom, David

Awschalom, David

Babnigg, Gyorgy

Babnigg, Gyorgy

Babnigg, Gyorgy

Bair, Ray

Bakhtiari, Sasan

Balaji, Pavan

Balaji, Pavan

Balakrishnan, Ramesh

\begin{tabular}{|c|c|}
\hline 2016-180-R1...................58 & Balaprakash, Prasanna \\
\hline 2015-091-R2 ...................... & Balaprakash, Prasanna \\
\hline 2017-113-NO .................... 42 & Barry, Edward \\
\hline 2017-042-N0................ 83 & Beckman, Pete \\
\hline 2015-173-R2 ….............148 & Beckman, Pete \\
\hline 2017-016-NO ............... 24 & Benmore, Chris J. \\
\hline 2017-106-NO ................143 & Benmore, Chris J. \\
\hline 2017-028-NO .............. 100 & Benmore, Chris J. \\
\hline 2016-165-R1.................... 91 & Benmore, Chris J. \\
\hline 2017-163-NO ................ 54 & Bera, Mrinal \\
\hline 2016-094-R1.................17 & Betrie, Getnet \\
\hline 2016-179-R1 .................153 & Bhattacharya, Anand \\
\hline 2017-058-NO ................141 & Bhattacharya, Anand \\
\hline 2016-098-R1................... 18 & Bhattacharya, Sumit \\
\hline 2015-161-R2 _.................. 98 & Bishof, Michael \\
\hline 2017-019-NO .................26 & Bleem, Lindsey \\
\hline 2015-176-R2 ….............. 79 & Bodwin, Geoffrey \\
\hline 2016-126-R1 ..................80 & Boughezal, Radja \\
\hline 2017-106-NO ...............143 & Bradford, Rebecca \\
\hline 2014-191-R3 ….............. 106 & Brettin, Thomas \\
\hline 2017-050-NO ...............113 & Brettin, Thomas \\
\hline 2015-171-R2 …............. 87 & Bromberek, Dave \\
\hline 2016-165-R1 ................... 91 & Brown, M. Alex \\
\hline 2017-082-NO ...............115 & Bunquin, Jeffrey Camacho \\
\hline 2016-175-R1 .................. 82 & Cacho, Jules \\
\hline 2016-140-R1 ................134 & Cai, Z. \\
\hline 2017-016-NO ............... 24 & Cai, Zhonghou \\
\hline 2017-093-N0................68 & Campbell, Patty \\
\hline 2017-106-NO ................143 & Cappello, Franck \\
\hline
\end{tabular}

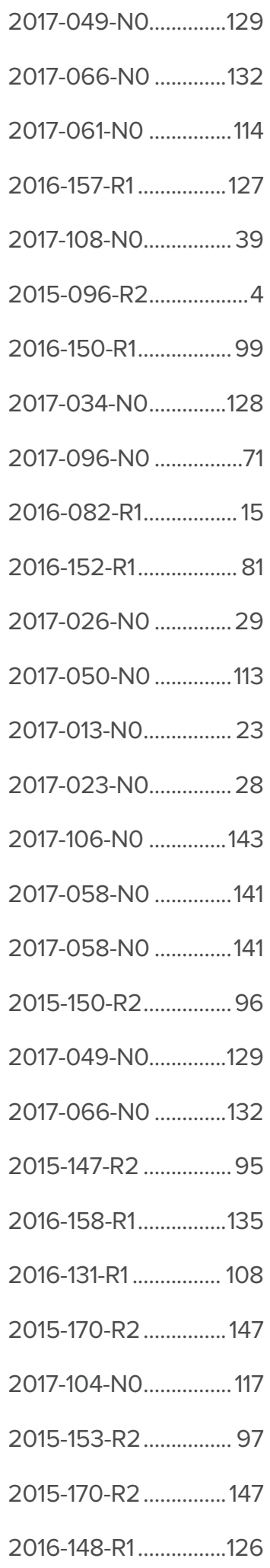




\begin{tabular}{|c|c|c|c|}
\hline Carpenter, Michael & 2017-106-NO ...............143 & Curtiss, Larry A. & 2016-185-R1_.................62 \\
\hline Catlett, Charlie & 2016-157-R1 .................127 & Curtiss, Larry A. & 2017-004-NO …............20 \\
\hline Catlett, Charlie & 2017-108-NO................... 39 & Dahl, Erik & 2017-159-NO.................50 \\
\hline Chan, M. & 2017-104-NO..................117 & Darancet, Pierre & 2016-133-R1 ................ 109 \\
\hline Chan, Maria K. & 2016-054-R1.................. 12 & Darling, Seth & 2015-151-R2 ................. 76 \\
\hline Chan, Maria K. & 2016-069-R1.................. 14 & Darling, Seth & 2017-033-NO ..................111 \\
\hline Chan, Maria K. & 2016-133-R1 .................. 109 & Davis, James & 2017-165-NO..................56 \\
\hline Chan, Maria K. & 2016-184-R1 .................... 61 & De Carlo, Francesco & 2017-063-NO................131 \\
\hline Chan, Maria K. & 2017-102-NO_.................. 36 & De Lurgio, Patrick & 2016-173-R1 .................121 \\
\hline Chang, Clarence & 2017-105-NO ................142 & De Pablo, Juan & 2017-094-NO_...............69 \\
\hline Chard, Ryan & 2017-097-NO................ 72 & de Queiros Silveira, Gleiciani & 2017-061-NO ................114 \\
\hline Chekanov, Sergei & 2017-058-NO ................141 & Delferro, Massimiliano & 2016-131-R1 ................ 108 \\
\hline Chen, Chen & 2015-159-R2 ................. 78 & Delferro, Massimiliano & 2017-032-N0................110 \\
\hline Chen, Si & 2017-063-NO................131 & Demarteau, Marcellinus & 2016-027-R1 ...................10 \\
\hline Chen, Wei & 2017-082-N0 ...............115 & Demas, Nicholaos & 2017-110-NO .................... 41 \\
\hline Chen, Wei-Ying & 2017-100-NO .................. 34 & Deng, Junjing & 2015-153-R2 _................ 97 \\
\hline Chen, Yiren & 2017-100-NO ................. 34 & Di, Zichao (Wendy) & 2017-063-NO................131 \\
\hline Chen, Zhaowei & 2017-061-N0 .................114 & DiChiara, Anthony & 2015-150-R2 _................96 \\
\hline Cheng, Lei & 2017-082-N0 ...............115 & DiChiara, Anthony & 2016-150-R1_.................99 \\
\hline Cherukara, Mathew & 2016-082-R1................... 15 & Dietrich, Matthew & 2017-023-N0.................28 \\
\hline Childers, John & 2017-049-N0..............129 & Diroll, Benjamin T. & 2016-186-R1_................63 63 \\
\hline Chung, Duck Young & 2016-123-R1 ...................119 & Doran, Scott & 2017-091-NO ...............103 \\
\hline Ciatti, Stephen & 2015-157-R2 …..............77 & Dufresne, Eric & 2015-150-R2 _...............96 \\
\hline Cleland, Andrew N. & 2017-092-N0 ...............116 & Duke, Daniel & 2017-098-N0 ................ 32 \\
\hline Cloët, lan & 2016-098-R1.................. 18 & Elam, Jeffrey & 2015-151-R2 ................. 76 \\
\hline Cloët, lan & 2017-058-N0 ................141 & Elam, Jeffrey & 2016-173-R1 ...................121 \\
\hline Collis, Scott & 2016-139-R1 ..................90 & Elam, Jeffrey & 2017-033-NO ..................111 \\
\hline Conley, Ray & 2015-161-R2 ..................98 & Elmer, Thomas & 2016-136-R1 ................ 120 \\
\hline Constantinescu, Emil & 2017-017-NO ................. 25 & Erck, Robert & 2017-110-NO .................... 41 \\
\hline Conway, Zachary & 2017-058-NO ...............141 & Erdmann, Mark & 2015-161-R2 ..................98 \\
\hline Craig, Brian & 2017-152-NO ................... 48 & Farmer, Mitchell & 2016-140-R1 ...............134 \\
\hline
\end{tabular}


Farmer, Mitchell

Feng, Bo

Feng, Yan

Fenske, George

Fenter, Paul

Ferrandon, Magali

Ferrier, N.

Fezzaa, Kamel

Finkel, Hal

Fischer, Paul

Flynn, Theodore

Fong, Dillon D.

Fong, Dillon D.

Foster, Ian

Fry, H. Christopher

Fry, H. Christopher

Fuerst, Joel

Galda, Alexey

Galli, Giulia

Galli, Giulia

Gao, Kaizhong

Gao, Kaizhong

Gao, Kaizhong

Gao, Kaizhong

Gerardi, Craig

Ghate, Virendra

Ghate, Virendra

Ghimire, Nirmal

Gilbert, Jack

Goel, Aditya

Goetze, Kurt

\begin{tabular}{|c|c|}
\hline 2017-041-NO................139 & Goldsborough, S. Scott \\
\hline 2017-030-NO ...............137 & Govoni, Marco \\
\hline 2016-139-R1 ..................90 90 & Gray, Stephen K. \\
\hline 2017-110-NO_................... 41 & Greco, Aaron \\
\hline 2016-184-R1 ..................... 61 & Grindeanu, Iulian \\
\hline 2016-131-R1 ................. 108 & Grindeanu, Iulian \\
\hline 2017-104-NO.................117 & Gros, Marie-Francoise \\
\hline 2016-023-R1....................9 & Gros, Marie-Francoise \\
\hline 2016-148-R1 ..................126 & Gros, Marie-Francoise \\
\hline 2016-175-R1 ................... 82 & Guest, J.R. \\
\hline 2015-179-R2 ................88 & Guha, Supratik \\
\hline 2017-050-NO ..............113 & Guha, Supratik \\
\hline 2017-155-NO.................157 & Guo, Peijun \\
\hline 2017-097-N0................. 72 & Gupta, Sreenath \\
\hline 2017-019-NO ................26 & Gursoy, D. \\
\hline 2017-061-N0 ................114 & Gursoy, Doga \\
\hline 2017-028-NO ............. 100 & Guzowski, Leah \\
\hline 2016-010-R1.................... 7 & Habib, Salman \\
\hline 2014-192-R3 ................107 & Hadt, Ryan G. \\
\hline 2017-050-NO ..............113 & Hafidi, Kawtar \\
\hline 2016-094-R1..................17 & Hafidi, Kawtar \\
\hline 2016-182-R1 ................154 & Hamada, Yuki \\
\hline 2017-076-NO...............122 & Harder, R. \\
\hline 2017-161-NO .................. 52 & Harms, Kevin \\
\hline 2017-041-N0...............139 & Haskel, Daniel \\
\hline 2016-139-R1 ...................90 & Hattawy, Mohammad \\
\hline 2017-087-N0 ................. 93 & Heidet, Florent \\
\hline 2016-181-R1 ...................59 & Heifetz, Alexander \\
\hline 2015-179-R2 ….............88 & Heine, Pete \\
\hline 2015-147-R2 …….........95 & Heitmann, Katrin \\
\hline 15-161-R2 …............... 98 & Henry, Christopher \\
\hline
\end{tabular}

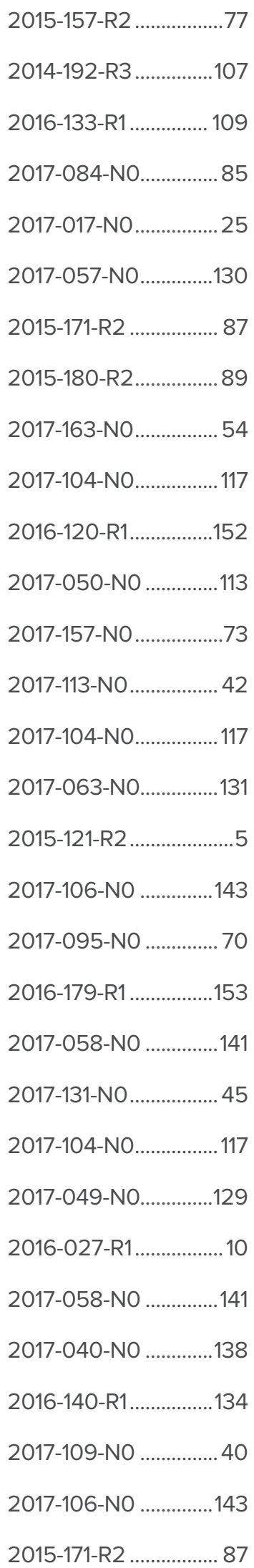




\begin{tabular}{|c|c|c|c|}
\hline Henry, Christopher & 2016-165-R1 ................... 91 & Jiang, Zhang & 2015-150-R2 _...............96 \\
\hline Hickmon, Nicki & 2016-139-R1 …................90 & Jiang, Zhang & 2017-073-N0 ..................101 \\
\hline Hickmon, Nicki & 2017-051-N0 ……….....92 & Johnson, Doug & 2017-109-NO .................. 40 \\
\hline Hla, Saw Wai & 2016-180-R1....................58 & Kanatzidis, Mercouri G. & 2016-123-R1 ..................119 \\
\hline Hla, Saw Wai & 2017-004-N0 ................20 & Karbowski, Dominik & 2015-176-R2 …............ 79 \\
\hline Hoffmann, Axel & 2017-050-N0 ...............113 & Karbowski, Dominik & 2016-126-R1_..................80 \\
\hline Horan, Douglas & 2015-147-R2 .................95 & Kasa, Matt & 2017-028-N0 ..............100 \\
\hline Hruszkewycz, Stephan & 2017-080-N0 .............. 102 & Kasa, Matt & 2017-091-N0 ................103 \\
\hline Hryn, John & 2016-182-R1 .................154 & Kasman, Elina & 2015-161-R2 ..................98 \\
\hline Hryn, John & 2016-240-R1_.................. 31 & Kastengren, Alan & 2015-150-R2 _................96 \\
\hline Hryn, John & 2017-162-NO................. 53 & Kasthuri, Narayanan & 2015-181-R2 ..................151 \\
\hline $\mathrm{Hu}, \mathrm{Bin}$ & 2016-150-R1...................99 & Kavicky, James & 2015-159-R2 …............. 78 \\
\hline Hu, Michael & 2015-173-R2 ................148 & Kemner, Kenneth & $2015-179-R 2$.................. 88 \\
\hline Hu, Rui & 2017-040-N0 ..............138 & Kemner, Kenneth & 2016-165-R1_..................91 \\
\hline Huang, XianRong & 2017-019-NO …............... 26 & Kemner, Kenneth & 2017-051-NO .................92 \\
\hline Hubaud, Aude & 2017-107-NO .................... 38 & Kemner, Kenneth & 2017-163-NO ..................54 \\
\hline Hubbell, Jeffery & 2017-035-N0................112 & Kim, Yu Jin & 2017-031-N0................. 67 \\
\hline Husko, Chad & 2016-190-R1...................155 & Klet, Rachel & 2016-131-R1 .................. 108 \\
\hline Ignacio-de Leon, Patricia & 2015-157-R2 …...............77 & Klet, Rachel & 2017-032-N0.................110 \\
\hline Ignacio-de Leon, Patricia & 2016-131-R1 .................... 108 & Klippenstein, Stephen & 2016-175-R1 …...............82 \\
\hline Ivanyushenkov, Yury & 2017-028-NO .............. 100 & Kokot, Gasper & 2016-063-R1.................13 \\
\hline Jackson, Nicholas E. & 2017-094-NO................69 & Koritarov, Vladimir & 2016-152-R1 ................... 81 \\
\hline Jacob, Robert & 2016-139-R1 ..................90 & Koshelev, Alexei & 2015-078-R2 .................... \\
\hline Jacob, Robert & 2017-017-NO ................. 25 & Kotamarthi, Rao & 2017-087-NO ................ 93 \\
\hline Jacob, Robert & 2017-057-N0 ................130 & Kotamarthi, Veerabhadra & 2017-017-NO ..................25 \\
\hline Jain, Rajeev & 2017-057-N0 ................130 & Kovacs, Eve & 2017-106-NO ................143 \\
\hline Jansma, William & 2017-091-N0 ...............103 & Kozak, Peter & 2016-159-R1_................136 \\
\hline Javanmardi, Mahmoud & 2016-126-R1 .................... 80 & Krause, Theodore & 2017-113-NO .................... 42 \\
\hline Jiang, Jidong & 2017-026-NO .................29 & Krumdick, Gregory & 2017-159-NO.................50 \\
\hline Jiang, Wei & 2017-012-N0................. 22 & Kucukgok, Bahadir & 2016-094-R1..................17 \\
\hline Jiang, Wei & 2017-073-NO .................101 & Kumaran, Kalyanasundaram & 2017-049-N0...............129 \\
\hline
\end{tabular}




\begin{tabular}{|c|c|c|c|}
\hline Kustom, Robert & 2017-091-N0 ……........103 & Liu, Y. & 2017-104-NO ..................117 \\
\hline Lai, Barry & 2015-153-R2 ................. 97 & Liu, Yuzi & 2017-012-N0................... 22 \\
\hline Laible, Philip & 2015-157-R2 …...............77 & Liu, Yuzi & 2017-082-N0 ...............115 \\
\hline Laible, Philip & 2016-020-R1_..................... & Longman, Douglas & 2016-175-R1 ...................82 \\
\hline Laible, Philip & 2016-165-R1 .................... 91 & Lorenzo Martin, Maria De La Cinta & 2017-084-N0.................. 85 \\
\hline Laible, Philip & 2017-061-N0 ….............114 & Low, John & 2017-034-N0.................128 \\
\hline Langeslay, Ryan & 2016-131-R1 ................. 108 & Macal, Charles & 2015-169-R2 ...............146 \\
\hline Larsen, Peter & 2016-165-R1 .....................91 & Macal, Charles & 2017-088-N0 ...............123 \\
\hline Larson, Jeffrey & 2016-126-R1..................80 & Macal, Charles & 2017-158-NO...................74 \\
\hline LeCompte, Thomas & 2017-058-N0 ...............141 & Macal, Charles & 2017-164-NO ...................55 \\
\hline Lee, Byeongdu & 2017-031-N0................. 67 & Madden, Timothy & 2015-150-R2 ................96 \\
\hline Lee, Jonghun & 2017-103-NO ...................... & Madduri, Ravi & 2017-106-NO ................143 \\
\hline Lendinez, Sergi & 2016-092-R1.................. 16 & Magill, Stephen & 2017-058-N0 …............141 \\
\hline Letchworth Weaver, Kendra & 2016-184-R1 .................... 61 & Mahadevan, Vijay & 2017-017-NO ..................25 \\
\hline Leyffer, S. & 2017-104-N0...................117 & Mahadevan, Vijay & 2017-057-N0................130 \\
\hline Leyffer, Sven & 2015-159-R2 .................. 78 & Mahalik, Matthew & 2016-152-R1_.................. 81 \\
\hline Leyffer, Sven & 2017-063-N0................131 & Malakar, Preeti & 2017-087-N0 .................93 \\
\hline $\mathrm{Li}$, Jie & 2015-121-R2 …................... & Mandia, David & 2017-002-N0 …................ 19 \\
\hline Li, Meimei & 2015-150-R2 _.................96 & Mane, Anil & 2016-027-R1 ................... 10 \\
\hline Li, Tao & 2017-082-N0 …............115 & Mane, Anil & 2016-173-R1 ..................121 \\
\hline Liao, Shaolin “Allen” & 2016-027-R1 ................... 10 & Marshall, Christopher L. & 2015-174-R2 ….............149 \\
\hline Liao, Shaolin “Allen” & 2016-136-R1 ................ 120 & Martinson, Alex & 2016-054-R1................. 12 \\
\hline Licht, Jeremy & 2017-041-N0................139 & Martinson, Alex & 2016-133-R1 ................ 109 \\
\hline Lin, Jianqiang & 2017-029-N0 ................66 & Martinson, Alex & 2017-026-N0 ................29 \\
\hline Lin, Xiao-Min & 2015-150-R2 _................ 96 & Martinson, Alex & 2017-033-NO.................111 \\
\hline Lin, Xiao-Min & 2017-012-N0.................... 22 & Maser, Jörg & 2015-153-R2 …............... 97 \\
\hline Lin, Xiao-Min & 2017-103-NO_....................37 & McNulty, I. & 2017-104-NO..................117 \\
\hline Lin, YuPo J. & 2017-055-NO .................. 84 & Mei, Zhi-Gang & 2017-013-NO ................... 23 \\
\hline Liu, Di-Jia & 2015-091-R2 ...................... & Merwin, Augustus & 2017-096-NO ..................71 \\
\hline Liu, Di-Jia & 2017-033-N0.................111 & Metcalfe, Jessica & 2017-058-N0 ...............141 \\
\hline Liu, Di-Jia & 2017-055-N0 …............84 & Mezrag, Cédric J. & 2016-188-R1_.................65 \\
\hline
\end{tabular}




\begin{tabular}{|c|c|c|c|}
\hline Mezrag, Cédric J. & 2017-058-NO ................141 & Nguyen, SonBinh T. & 2016-131-R1 ................. 108 \\
\hline Miao, Yinbin & 2017-118-NO .................... 44 & Nocek, Boguslaw & 2017-061-NO ..................114 \\
\hline Miceli, Antonino & 2015-150-R2 .................96 & Noirot, Philippe & 2015-171-R2 ................. 87 \\
\hline Michalska, Karolina & 2017-082-N0 ...............115 & Noirot, Philippe & 2016-165-R1_.................. 91 \\
\hline Min, Misun & 2016-175-R1 .................... 82 & Nolen, Jerry A. & 2016-158-R1_................135 \\
\hline Mitchell, John & 2016-181-R1 ...................59 & Notestein, Justin & 2016-131-R1 ................ 108 \\
\hline Mo, Kun & 2016-159-R1.................136 & Novosad, Valentine & 2016-092-R1..................16 \\
\hline Mo, Kun & 2017-118-NO ................... 44 & Novosad, Valentine & 2017-105-NO ...............142 \\
\hline Molzahn, Daniel K. & 2017-101-NO ..................... 35 & Oaks, Aaron & 2017-013-N0 .................. 23 \\
\hline Momen, Abdul & 2016-150-R1..................99 & Obabko, Aleks & 2017-057-N0 ................130 \\
\hline Mooney, Tim & 2015-161-R2 _.................98 & O’Loughlin, Edward & 2015-179-R2 .................88 \\
\hline Morozov, Vitali & 2017-049-N0...............129 & O'Loughlin, Edward & 2017-051-NO ................92 \\
\hline Muehleisen, Ralph & 2015-121-R2 ….................. & Orr, Andrew & 2017-087-N0.................93 93 \\
\hline Mueller, Peter & 2017-023-N0.................. 28 & Orzel, Ron & 2017-109-NO .................40 \\
\hline Mulfort, Karen & 2017-032-N0................110 & Ostroumov, Peter & 2017-058-N0 ................141 \\
\hline Munson, T. & 2017-104-NO_..................117 & Padin, Stephen & 2017-105-NO ................142 \\
\hline Munson, Todd & 2016-126-R1 ................... 80 & Parent, Scott & 2017-109-NO ................. 40 \\
\hline Murphy, John & 2017-152-NO.................. 48 & Park, Hyowon & 2015-168-R2 _................145 \\
\hline Mustapha, Brahim & 2017-058-N0 ................141 & Pellin, Michael & 2016-159-R1_................136 \\
\hline Narayanan, Badri & 2017-012-N0................. 22 & Pellin, Michael & 2016-173-R1 ....................121 \\
\hline Narayanan, Suresh & 2015-150-R2 _................96 & Peterka, T. & 2017-104-NO .................117 \\
\hline Narayanan, Suresh & 2017-103-N0....................37 & Petford-Long, A.K. & 2017-104-NO..................117 \\
\hline Nashed, Youssef & 2017-080-NO ............. 102 & Phatak, C. & 2017-104-NO ...................117 \\
\hline Nassiri, Alireza & 2015-147-R2 …..............95 & Plastun, Alexander & 2017-058-N0 ................141 \\
\hline Nassiri, Alireza & 2017-002-N0 ................. 19 & Pokkuluri, P. Raj & 2016-048-R1.................... 11 \\
\hline Nassiri, Alireza & 2017-091-N0 ................103 & Pokkuluri, P. Raj & 2017-032-N0.................110 \\
\hline Natesan, Krishnamurti & 2017-100-NO ................ 34 & Potterveld, David & 2017-058-N0 ................141 \\
\hline Nauert, Scott & 2016-131-R1 ................. 108 & Powell, Christopher & 2017-098-NO ................32 \\
\hline Nealey, Paul & 2015-150-R2 _................96 & Power, John & 2017-007-NO ................. 21 \\
\hline Negri, M. Cristina & 2015-170-R2 ….............147 & Power, John & 2017-091-N0 ...............103 \\
\hline Ngo, Anh & 2017-004-N0 .................20 & Power, John & 2017-091-NO ...............103 \\
\hline
\end{tabular}




\begin{tabular}{|c|c|c|c|}
\hline Pratt, Stephen T. & 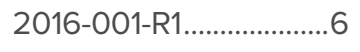 & Schaller, Richard D. & 2016-133-R1 ................. 109 \\
\hline Pratt, Stephen T. & 2016-175-R1 ….............. 82 & Schaller, Richard D. & 2016-186-R1_................63 \\
\hline Preissner, Curt & 2015-153-R2 _................. 97 & Schaller, Richard D. & 2017-157-NO ..................73 \\
\hline Pupek, Krzysztof & 2017-159-NO...................50 & Schiffer, Marianne & 2016-048-R1 .................... 11 \\
\hline Qian, Jun & 2015-161-R2 …..............98 & Schwarz, Nicholas & 2015-150-R2 _................96 \\
\hline Qiu, Feng & 2015-159-R2 ................. 78 & Sen, Ananya & 2016-001-R1...................... \\
\hline Qiu, Feng & 2016-152-R1.................... 81 & Sen, Fatih & 2016-069-R1.................. 14 \\
\hline Rajh, Tijana & 2016-165-R1 ................... 91 & Shaver, Dillon & 2017-030-NO ...............137 \\
\hline Rajh, Tijana & 2017-061-N0 ….............114 & Shevchenko, Elena & 2017-012-NO_.................. 22 \\
\hline Repond, José & 2017-058-NO ...............141 & Shi, Bing & 2015-161-R2 ..................98 \\
\hline Rimer, Sara P. & 2017-158-NO...................74 & Shirato, Nozomi & 2017-004-NO ...............20 \\
\hline Roberts, Craig D. & 2016-188-R1 ...................65 & Shukla, Maulik & 2017-165-NO..................56 \\
\hline Roehrig, Chris & 2015-153-R2 …............. 97 & Si, Min & 2017-093-NO.................68 \\
\hline Romano, Paul K. & 2016-135-R1 .................125 & Siegel, Andrew R. & 2016-135-R1 .................125 \\
\hline Romano, Paul K. & 2017-030-NO ...............137 & Singh, Dileep & 2016-094-R1..................17 \\
\hline Rondinelli, James & 2015-178-R2 ............... 150 & Singh, Dileep & 2017-042-N0................. 83 \\
\hline Rose, Volker & 2017-004-NO ...............20 & Singh, Dileep & 2017-084-N0................. 85 \\
\hline Rotsch, David & 2016-158-R1 .................135 & Sivaramakrishna, Raghu & 2015-157-R2 …..............77 \\
\hline Rousseau, Aymeric & 2015-176-R2 …............. 79 & Skone, Jonathan & 2014-192-R3 ................107 \\
\hline Rozhkova, Elena & 2017-061-NO ................114 & Snezhko, Alexey & 2016-063-R1.................. 13 \\
\hline Samsa, Michael & 2017-152-N0.................. 48 & Sokolov, Andrey & 2016-020-R1.................... \\
\hline Sandy, Alec & 2015-150-R2 .................96 & Som, Sibendu & 2016-175-R1 ................. 82 \\
\hline Sandy, Alec & 2017-103-NO.....................37 & Spangenberger, Jeffrey & 2016-240-R1.................. 31 \\
\hline Sankaran, Rajesh & 2016-157-R1 ..................127 & Spangenberger, Jeffrey & 2017-107-NO ...................38 \\
\hline Sankaran, Rajesh & 2017-108-N0...................39 & Stan, Marius & 2017-034-N0..............128 \\
\hline Sankaranarayanan, S. & 2017-104-N0..................117 & Stan, Marius & 2017-042-N0................. 83 \\
\hline Sankaranarayanan, Subramanian & 2016-082-R1..................15 & Stauff, Nicolas & 2017-030-NO ..............137 \\
\hline Sankaranarayanan, Subramanian & 2016-133-R1 ................ 109 & Stephenson, Brian & 2016-187-R1 ................... 64 \\
\hline Sankaranarayanan, Subramanian & 2017-012-N0................. 22 & Strzalka, Joseph & 2015-150-R2 _................96 \\
\hline Savereide, Louisa & 2016-131-R1 ................. 108 & Sumant, Anirudha V. & 2017-154-NO ..................49 \\
\hline Scarcelli, Riccardo & 2017-099-N0 ................ 33 & Sun, Tao & 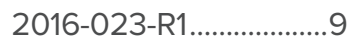 \\
\hline
\end{tabular}




\begin{tabular}{|c|c|c|c|}
\hline Sun, Tao & 2017-073-NO ..................101 & Wagner, Robert & 2016-173-R1 ...................121 \\
\hline Sun, Yine & 2017-007-NO.................. 21 & Waldschmidt, Geoff & 2015-147-R2 _................95 \\
\hline Suthar, Kamlesh & 2016-150-R1_..................99 & Walko, Donald & 2015-150-R2 _................96 \\
\hline Suthar, Kamlesh & 2017-019-NO ................ 26 & Wang, Jiali & 2017-017-NO ..................25 \\
\hline Suthar, Kamlesh & 2017-091-N0 ……........103 & Wang, Jiali & 2017-087-N0 ................. 93 \\
\hline Szymanski, Adam & 2017-137-NO .................. 46 & Wang, Jianhui & 2015-159-R2 _............... 78 \\
\hline Tessier, Francois & 2017-156-NO.................158 & Wang, Jin & 2015-150-R2 ….............96 \\
\hline Tiede, David & 2017-032-N0................110 & Wang, Jin & 2017-073-NO .................101 \\
\hline Tiede, David & 2017-033-NO.................111 & Wang, Jingbo & 2016-173-R1 ..................121 \\
\hline Tomboulides, Ananias & 2016-175-R1 .................. 82 & Weisenhorn, Pamela & 2015-179-R2 …............. 88 \\
\hline Trakhtenberg, Emil & 2017-028-NO ............. 100 & Weisenhorn, Pamela & 2017-141-NO ...................47 \\
\hline Trakhtenberg, Emil & 2017-091-NO ...............103 & Weizeorick, John & 2015-150-R2 _................96 \\
\hline Tranter, Robert & 2015-157-R2 …...............77 & Wen, $\mathrm{H}$. & 2017-104-NO ..................117 \\
\hline Ulvestad, Andrew & 2016-187-R1 .................. 64 & Wen, Haidan & 2015-150-R2 _................96 \\
\hline Uram, Thomas & 2017-049-N0................129 & Wen, Haidan & 2016-023-R1_............................ \\
\hline Utschig-Johnson, Lisa & 2017-032-N0..................110 & Wen, Haidan & 2016-183-R1 ...................60 \\
\hline Vandegrift, George F. & 2016-158-R1................135 & Wen, Jianguo & 2016-069-R1................... 14 \\
\hline Vazquez Mayagoitia, Alvaro & 2017-034-N0 ................128 & Wiederrecht, Gary & 2016-133-R1 ................. 109 \\
\hline Veselka, Thomas & 2016-152-R1 .................... 81 & Wild, Stefan & 2017-049-NO...............129 \\
\hline Vinokour, Valerii & 2016-010-R1..................... 7 & Wild, Stefan & 2017-063-NO................131 \\
\hline Vinokour, Valerii & 2016-140-R1................134 & Williamson, Mark A. & 2015-096-R2 ................. 4 \\
\hline Vishwanath, Venkatram & 2017-049-NO...............129 & Williamson, Mark A. & 2017-040-NO ..............138 \\
\hline Vishwanath, Venkatram & 2017-066-NO ..............132 & Williamson, Mark A. & 2017-096-NO .................71 \\
\hline Vishwanath, Venkatram & 2017-087-N0 ..................93 & Wilton, Rosemarie & 2016-048-R1_.................... 11 \\
\hline Vishwanath, Venkatram & 2017-094-NO...............69 & Wilton, Rosemarie & 2016-165-R1_..................91 \\
\hline Vishwanath, Venkatram & 2017-156-NO................158 & Winans, Randall E. & 2015-151-R2 ….............. 76 \\
\hline Vlasko-Vlasov, Vitalii & 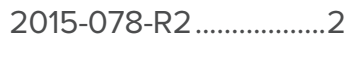 & Winans, Randall E. & 2017-082-N0 ...............115 \\
\hline Voeroes, Marton Andras & 2016-185-R1...................62 & Wu, May & 2016-152-R1 ................... 81 \\
\hline Vogt, Stefan & 2015-153-R2 ................ 97 & Xia, Fangfang & 2016-148-R1 .................126 \\
\hline Vogt, Stefan & 2017-063-NO................131 & Xia, Fangfang & 2017-049-NO...............129 \\
\hline Wagner, Al & 2016-175-R1 ……........... 82 & Xia, Fangfang & 2017-066-NO …..........132 \\
\hline
\end{tabular}


AUTHOR INDEX

\begin{tabular}{|c|c|c|c|}
\hline Xia, Lei & 2016-173-R1 ...................121 & Yanguas-Gil, Angel & 2017-160-NO .................. 51 \\
\hline Xiao, X. & 2017-104-NO_..................117 & Yanguas-Gil, Angel & 2017-162-NO................. 53 \\
\hline Xie, Junqi & 2017-058-NO ................141 & Ye, Zhihong & 2017-058-N0 ................141 \\
\hline Xie, Yunsong & 2016-182-R1................154 & Yoshii, Kazutomo & 2016-148-R1 .................126 \\
\hline Xie, Yunsong & 2017-076-N0...............122 & You, Hoydoo & 2015-150-R2 _................96 \\
\hline Xie, Yunsong & 2017-116-NO ................... 43 & Young, Linda & 2016-191-R1...................156 \\
\hline Yacout, Abdellatif M. & 2016-159-R1 .................136 & Zaluzec, Nestor J. & 2017-153-NO ................104 \\
\hline Yacout, Abdellatif M. & 2017-013-NO................. 23 & Zapol, Peter & 2016-140-R1 .................134 \\
\hline Yacout, Abdellatif M. & 2017-118-NO ................... 44 & Zhang, Jinlong & 2017-058-N0 ................141 \\
\hline Yan, Eugene & 2016-152-R1 ................... 81 & Zhang, Qi & 2016-183-R1 ..................60 \\
\hline Yan, Xiaojie & 2015-121-R2 ….................. & Zhao, Cang & 2016-023-R1 ..................... \\
\hline Yanguas-Gil, Angel & 2017-002-NO .................19 & Zholents, Alexander & 2017-091-NO ...............103 \\
\hline Yanguas-Gil, Angel & 2017-013-N0.................. 23 & Zhou, Zhi & 2016-152-R1 .................. 81 \\
\hline Yanguas-Gil, Angel & 2017-022-N0 ............... 27 & Zhou, Zhi & 2017-055-N0 ............... 84 \\
\hline Yanguas-Gil, Angel & 2017-042-N0................ 83 & Zumpf, Colleen & 2015-170-R2 …............. 147 \\
\hline
\end{tabular}




\section{ACKNOWLEDGMENTS}

Argonne's LDRD Program Office comprises the LDRD Program Manager, Vivian S. Sullivan and Marianne L. Dietz, Program Administrator. Those of us in the program's management are grateful for the critical support received throughout the year from the individuals listed below. Special appreciation is due to the many experts who perform the research and provide their project reports; to the technical and administrative management of Argonne's programmatic divisions, within which the research and development are pursued; to the many members of the several peer-review panels who evaluate LDRD proposals; and to the Laboratory's management, whose vision and understanding of the nation's technological needs help guide and refine the content of Argonne's LDRD portfolio.

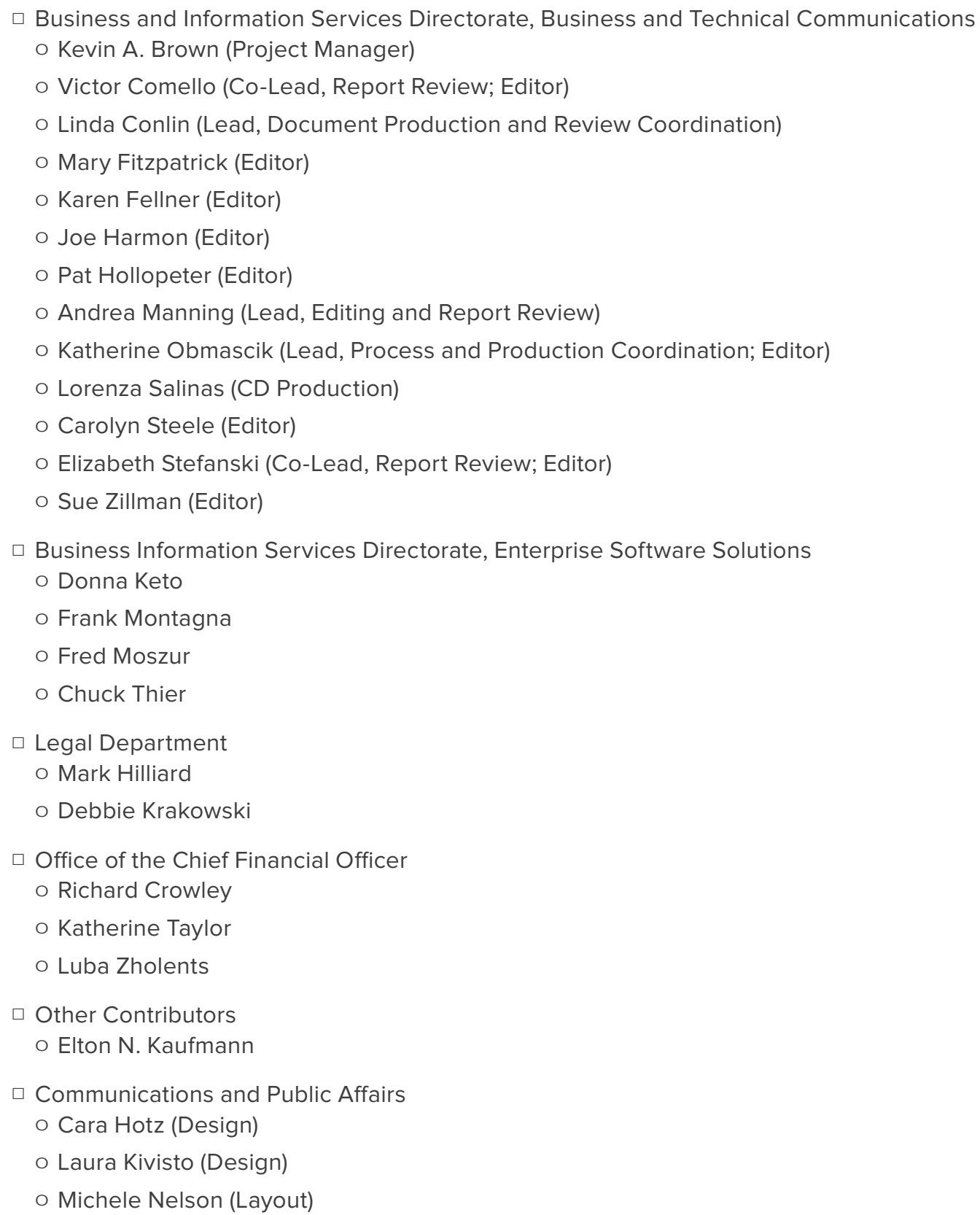




\section{ARGONNE NATIONAL LABORATORY}

$\square$ U.S. Department of Energy research facility

$\square$ Operated by the University of Chicago

$\square$ Midwest's largest federally funded R\&D facility

$\square$ Located in Argonne, IL, about 25 miles (40 km) southwest of Chicago, IL (USA)

$\square$ Conducts basic and applied research in dozens of fields

$\square$ Unique suite of leading-edge and rare scientific user facilities

\section{CONTACT}

Vivian S. Sullivan

LDRD Program Manager

Office of Strategy, Performance, and Risk; Office of the Director

Phone: (630) 252-1890

E-mail: vivian.sullivan@anl.gov

This report was prepared as an account of work sponsored by an agency of the United States Government. Neither the United States Government nor any agency thereof, nor UChicago Argonne, LLC, nor any of their employees or officers, makes any warranty, express or implied, or assumes any legal liability or responsibility for the accuracy, completeness, or usefulness of any information, apparatus, product, or process disclosed, or represents that its use would not infringe privately owned rights. Reference herein to any specific commercial product, process, or service by trade name, trademark, manufacturer, or otherwise, does not necessarily constitute or imply its endorsement, recommendation, or favoring by the United States Government or any agency thereof. The views and opinions of document authors expressed herein do not necessarily state or reflect those of the United States Government or any agency thereof, Argonne National Laboratory, or UChicago Argonne, LLC. 